\title{
Untersuchungen zum Sekundärmetabolismus mariner Pilze, Naturstoffscreening und Bioprozessoptimierung mit Hilfe eines kontinuierlichen Bioreaktors
}

\author{
Dissertation \\ zur Erlangung des Doktorgrades \\ der Mathematisch-Naturwissenschaftlichen Fakultäten \\ der Georg-August Universität zu Göttingen
}

Vorgelegt von

Peter Grzeganek

aus Hindenburg/OS

Göttingen 2003 
D7

Referent:

Prof. Dr. H. Laatsch

Korreferent:

Prof. Dr. L. Fitjer

Tag der mündlichen Prüfung: 3. 07. 2003 
Die vorliegende Arbeit wurde in der Zeit von September 1998 bis März 2003 im Institut für Organische Chemie der Georg-August Universität zu Göttingen unter der Leitung von Herrn Prof. Dr. H. Laatsch angefertigt.

Herrn Prof. Dr. H. Laatsch danke ich für die Möglichkeit zur Durchführung dieser Arbeit sowie die ständige Bereitschaft, auftretende Probleme zu Diskutieren. 
Die Nichtlinearität der Natur zeigt ihr Gesicht immer dort, wo das Ganze mehr ist als die Summe seiner Teile.

Für meine Eltern 


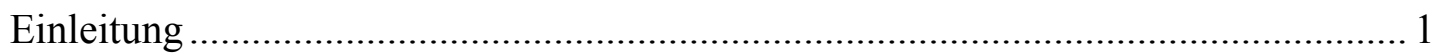

Schutzmechanismen der Bakterien................................................................ 9

Perspektiven der Antibiotikaforschung ....................................................... 12

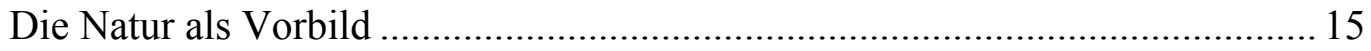

Exzentrische Mikroorganismen als Lieferanten neuer Naturstoffe..................... 18

Untersuchungen an mariner Organismen ................................................... 22

Meerespilze als eine potenzielle Quelle neuer Naturstoffe ..............................2 28

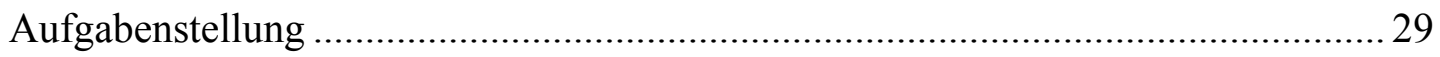

Untersuchungen von Extrakten mariner Pilze .................................................. 32

Genetische Diversität der untersuchten Pilzproben.......................................... 32

Primärscreening der Pilzextrakte ............................................................... 34

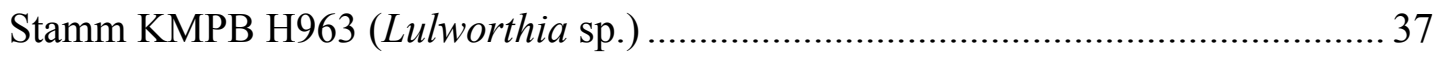

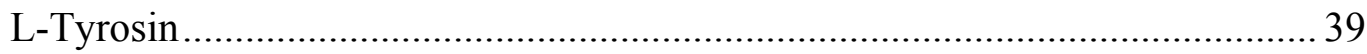

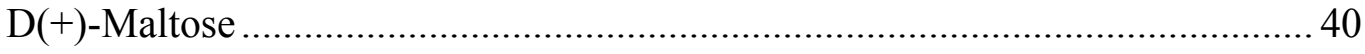

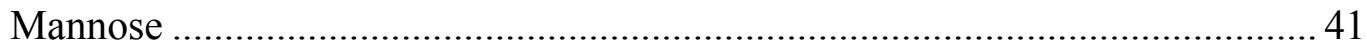

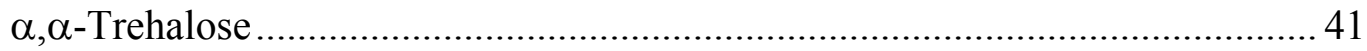

Nährmedienpotimierung anhand Genetischer Algorithmen .................................. 44

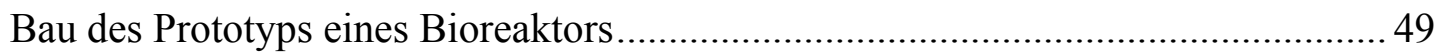

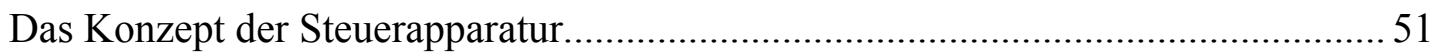

Die Arbeitsweise der Steuerungsapparatur ................................................... 52

Die Animpf- und Kultivierungsversuche .................................................... 56

Kontinuierliche Fermentation im Personal Fermenter .................................... 57

Die Kopplung eines Durchflussreaktors mit einer XAD-Säule ...............................6 60

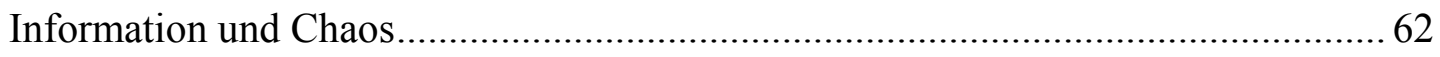

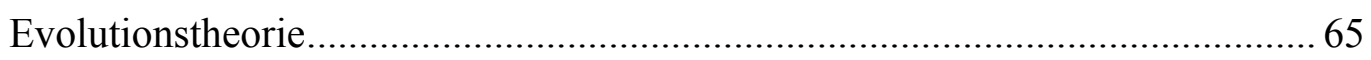

Das Konzept der unscharfen Optimierungsmethoden ...................................... 66

Genetische Algorithmen bei Optimierungsproblemen..................................... 68 


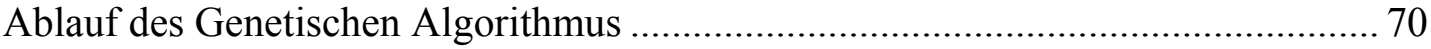

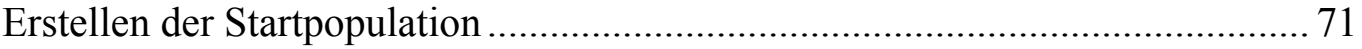

Erste Bewertung der Population ................................................................. 71

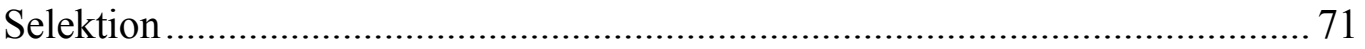

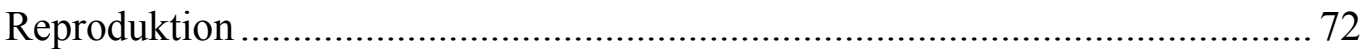

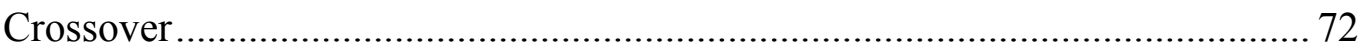

GALOP - Genetische Algorithmen zur Optimierung von Prozessen....................... 73

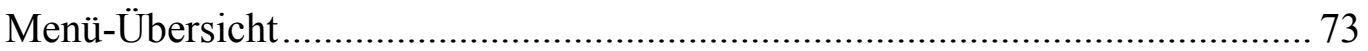

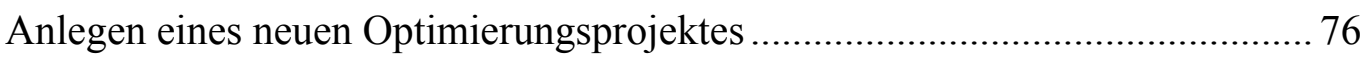

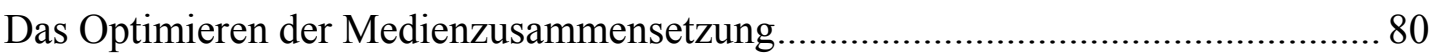

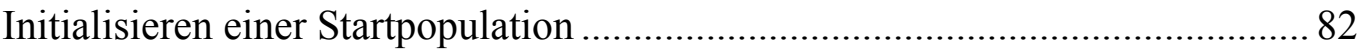

Zufallsbedingte Generierung der Anfangswerte und erste Bewertung der

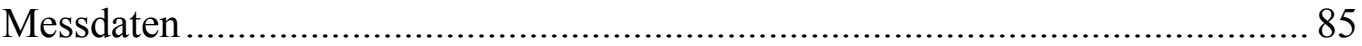

Editieren der Messwerte und Erzeugung der Tochtergeneration ....................... 86

Experimentelle Durchführung der Versuchsreihen ......................................... 87

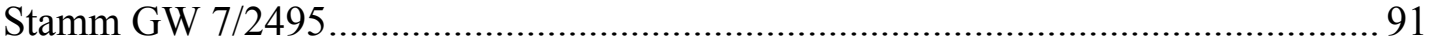

Die diskontinuierliche Anzucht von GW 7/2495 _.................................................... 91

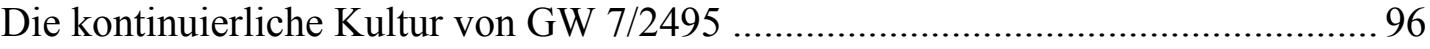

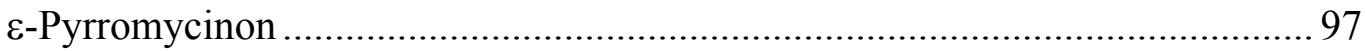



5-[2-(2-Hydroxy-3-methoxycarbonyl-propyl)-5-methyl-phenyl]-penta-2,4-dien-

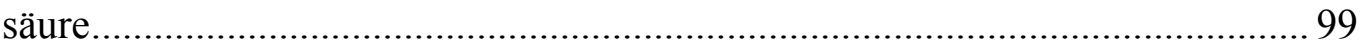

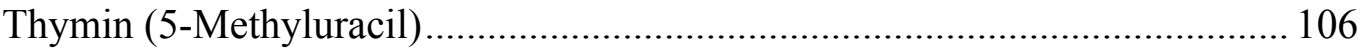

Unterschiede der beiden Fermentationsmethoden......................................... 106

Stamm B 7284

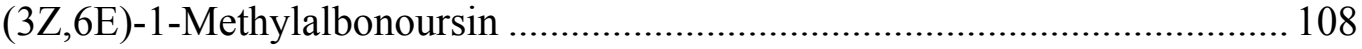

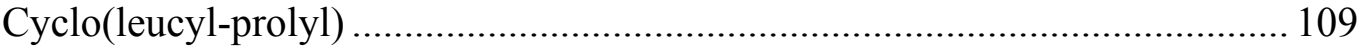


Stamm B 7939.

N-Acetyltryptamin

3-Indolylethanol

N-Acetyltyramin

4-(2-Hydroxy-ethyl)-phenol (Tyrosol)

Phthalsäure-dibutylester und Phthalsäure-diisobutylester.

Vitamin MK-7

Hydroxybuttersäure-Oligomere.

Stamm B 8927

3-Isobutylpiperazin-2,5-dion.

2-(1-Hydroxyethyl)-3H-chinazolin-4-on (Chrysogin)

Uracil

Uridin

Dodecyl-(4-methoxy-1'H-[1,2']bipyrrolyl-5-ylidenmethyl)-amin (BE-18591)126

(3Z, 6E)-1N-Methylalbonoursin (M145722) 128

Phthalsäure-di(2-ethylhexyl ester) (Diisooctyl-phthalat) 129

Stamm B 9020 130

4-(2-Hydroxy-ethyl)-phenol (Tyrosol) (39) 131

Indolyl-3-carbaldehyd

Ergosterol (Provitamin $\left.\mathrm{D}_{2}\right)$

3-Indolyl-carbonsäuremethylester

NIAX und Polyhydroxy-buttersäure

N-Acetyltryptamin und 3-Methyl-hexyl-carbonsäureamid .

2-Methyl-3H-chinazolin-4-on

2-Phenyl-acetamid 


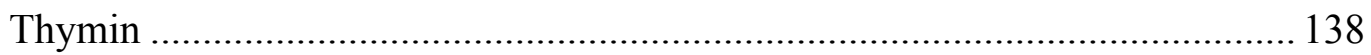

Mehrere homologe iso-Fettsäure-methylester.............................................. 138

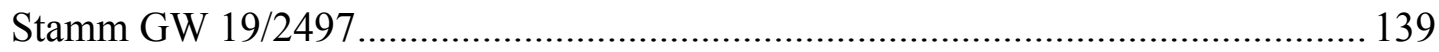

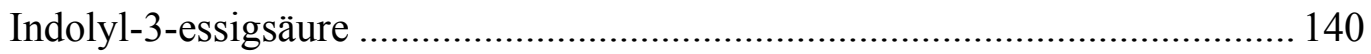

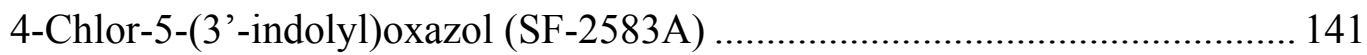

1,4-Dihydroxy-2-methyl-benzol (Toluhydrochinon) .................................... 142

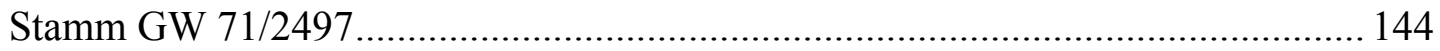

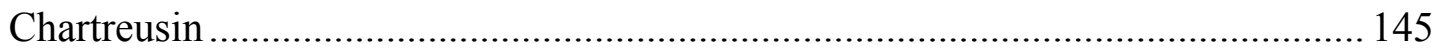

Anthranilsäure, Cyclo(leucyl-prolyl) und Uracil ........................................... 146

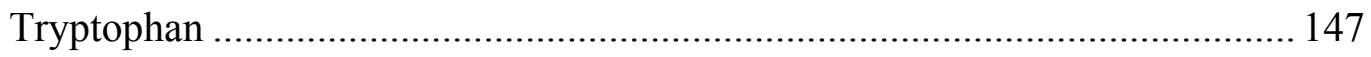

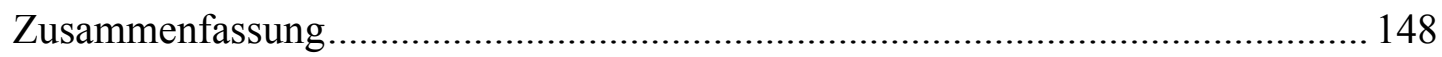

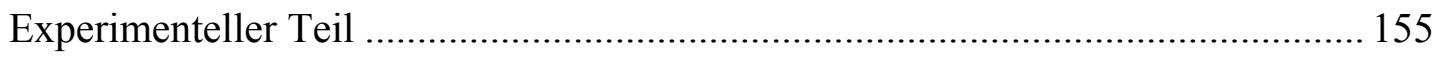

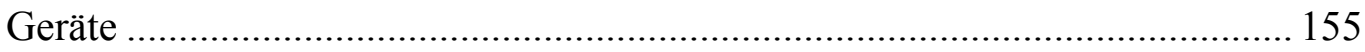

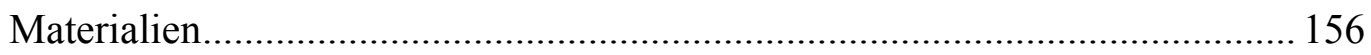

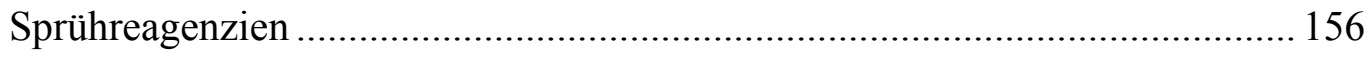

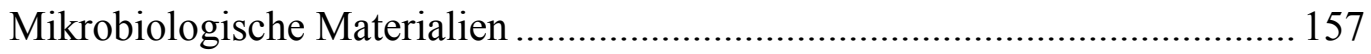

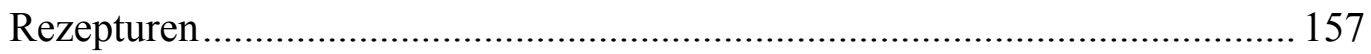

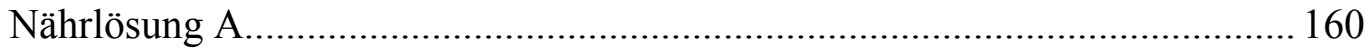

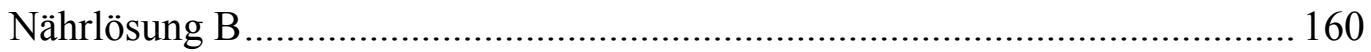

Mikrobiologische und analytische Arbeitsmethoden............................................. 162

Stammhaltung und Anzucht der Streptomyceten ......................................... 162

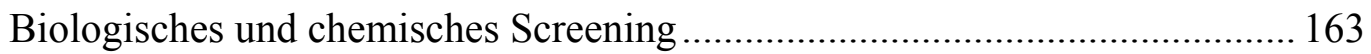

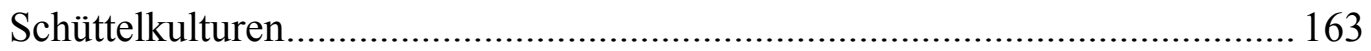

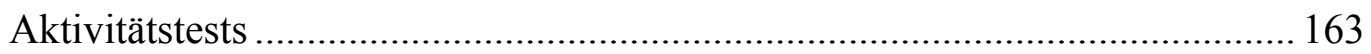

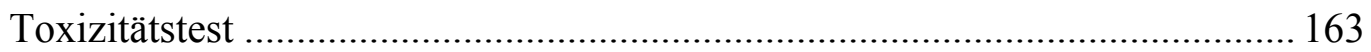

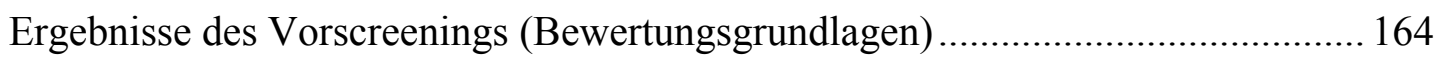

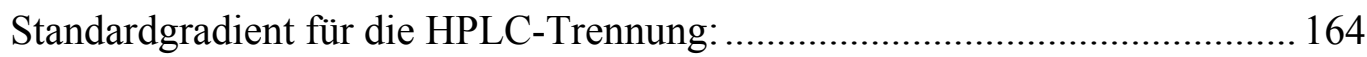


GALOP-Optimierung 165

Primärscreening der Pilzproben ...................................................................... 165

Stamm KMPB H963 (Lulworthia sp.) ................................................................. 171

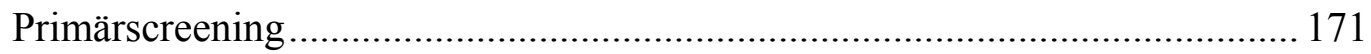

Fermentation und Aufarbeitung ..................................................................... 171

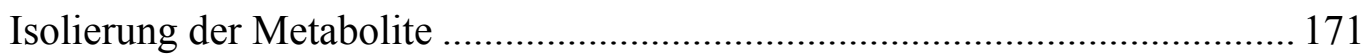

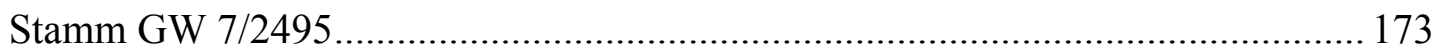

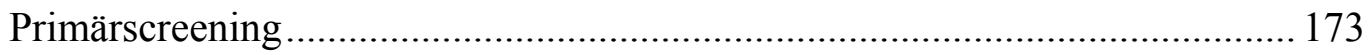

Diskontinuierliche Kultivierung von GW 7/2495 ………………………………...... 173

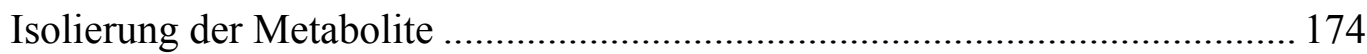

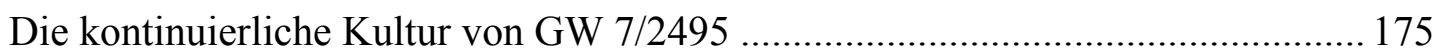

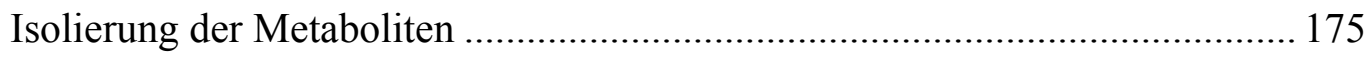

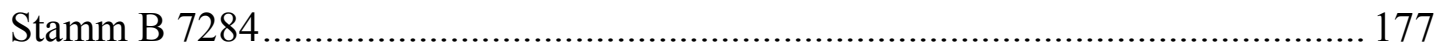

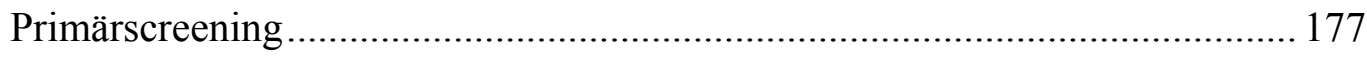

Fermentation und Aufarbeitung ..................................................................... 177

Isolierung der Metaboliten .......................................................................... 178

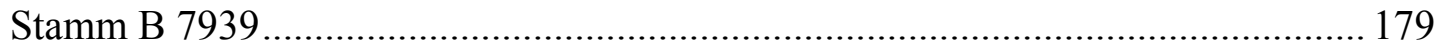

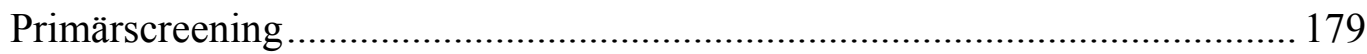

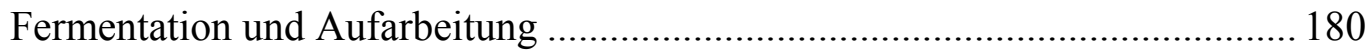

Isolierung der Metaboliten ............................................................................. 180

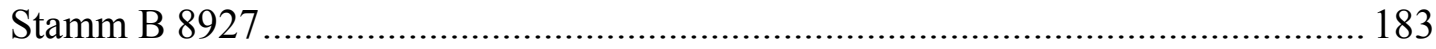

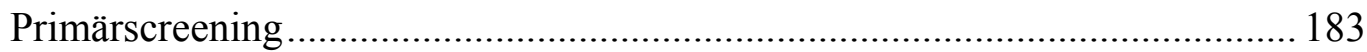

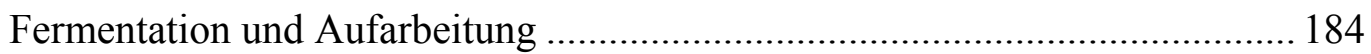

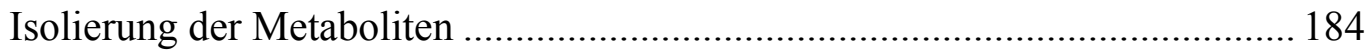

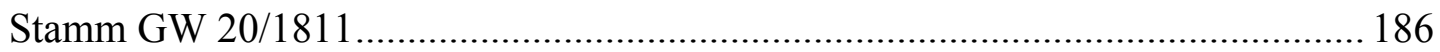

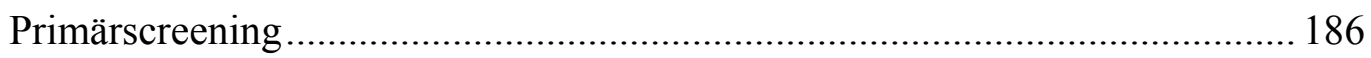

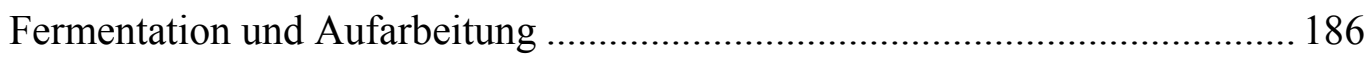




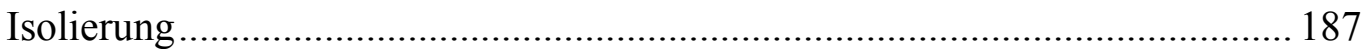

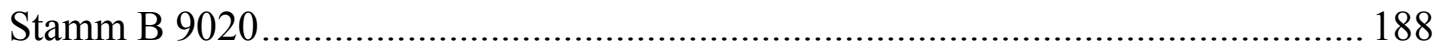

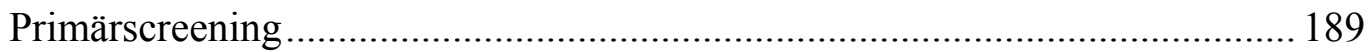

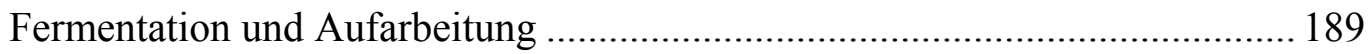

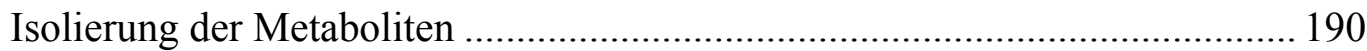

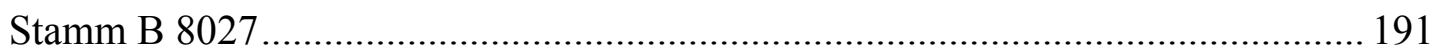

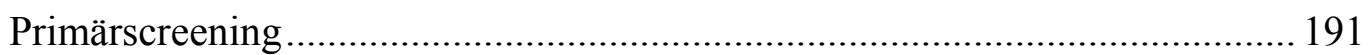

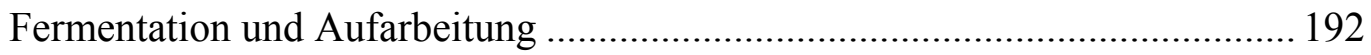

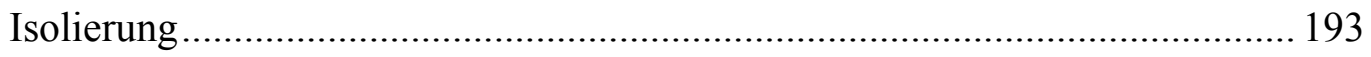

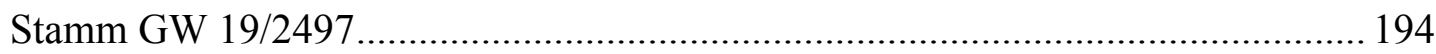

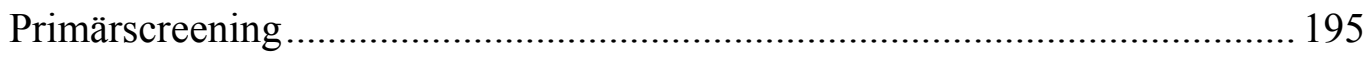

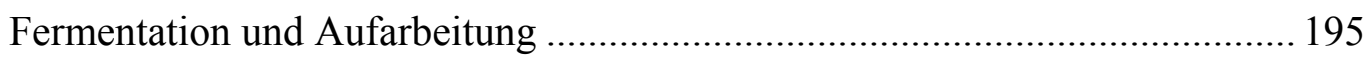

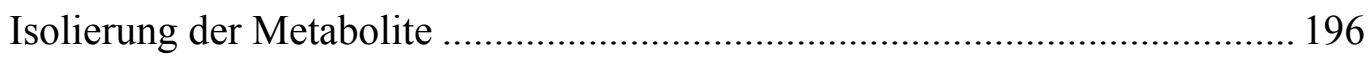

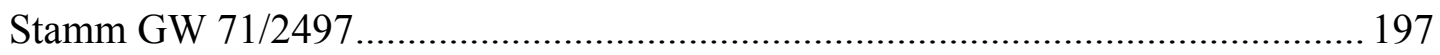

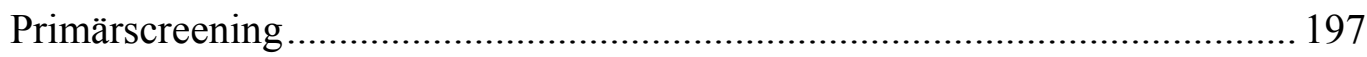

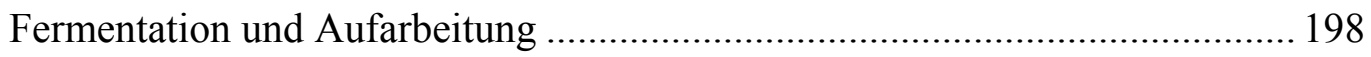

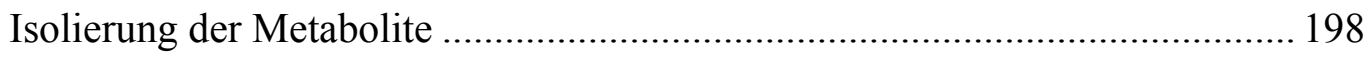

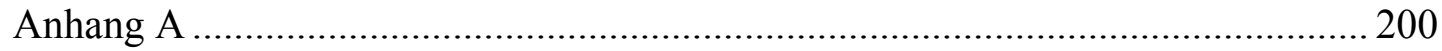




\section{Verzeichnis der Abbildungen}

$\begin{array}{lll}\text { Abbildung 1: } & \text { Einsatz der Antibiotika-Substanzklassen } & 14\end{array}$

Abbildung 2: Die Europäische Eibe (Taxus baccata) 17

Abbildung 3: Die Taxonomie und Aktivität der Pilzextrakte. 33

Abbildung 4: Chromatogramme von 26 marinen Pilzextrakten. 34

Abbildung 5: HPLC/MS-Untersuchungen von Extrakten mariner Pilze. 36

Abbildung 6: Die Aktivität der Pilzextrakte. 37

Abbildung 7: ${ }^{1} \mathrm{H}-\mathrm{NMR}-$ Spektrum von $\alpha, \alpha$-Trehalose nach $\mathrm{D}_{2} \mathrm{O}$-Austausch. 43

Abbildung 8: ${ }^{1} \mathrm{H}-\mathrm{NMR}-$ Spektrum der peracetylierten $\alpha, \alpha$-Trehalose. $\quad 44$

Abbildung 9: Die Konstruktion eines 2 1-Durchflußfermenters. 50

Abbildung 10: Die Pumpe ohne Gehäuse. 51

Abbildung 11: Darstellung der modularen Bauweise des Gerätes 53

Abbildung 12: Die Steuerungsapparatur von vorn. 55

Abbildung 13: Die Apparatur besteht aus mehreren normierten Platinen. $\quad 56$

Abbildung 14: Der Bioreaktor mit dem Träger aus Edelstahlnetz. 59

Abbildung 15: Darstellung eines Genetischen Algorithmus. 70

$\begin{array}{ll}\text { Abbildung 16: crossover-Prozesses } & 73\end{array}$

Abbildung 17: Die Crossingrate und Mutationsrate können verändert werden. $\quad 74$

Abbildung 18: Menü Project mit allen Unterfunktionen. 75

Abbildung 19: Menü Population mit allen Unterfunktionen. 76

Abbildung 20: Eingabefeld für die Funktion New. 82

Abbildung 21: Eingabefeld für die Angabe der veränderbaren Parameter. $\quad 82$

Abbildung 22: Eingabefeld für Distribution. 83

Abbildung 23: Eingabefeld für die Messgrößen. $\quad 84$

Abbildung 24: Eingabefeld für die Zieldefinition. $\quad 84$

Abbildung 25: Eingabefeld für die Gewichtsfaktoren. 85

Abbildung 26: Eingabefeld Crossing- und Mutationsrate. 85 
Abbildung 27: Eingabefeld für die Ausgangswerte. 86

Abbildung 28: Eingabefeld für die im Experiment gemessenen Werte. 87

Abbildung 29: HPLC-Chromatogramm. 90

Abbildung 30: HPLC-Chromatogramm. 90

Abbildung 33: ${ }^{1}$ H-NMR-Spektrum von 2-Methylfervenulon. 94

Abbildung 34: Mögliche Konstitutionsisomere von 29.

Abbildung 35: Die H,H-Korrelationen von $32 . \quad 100$

Abbildung 34: ${ }^{13} \mathrm{C}$-Spektrum von 32 aufgenommen in $\mathrm{CDCl}_{3}(125 \mathrm{MHz}) . \quad 101$

Abbildung 35: ${ }^{1} \mathrm{H}-\mathrm{NMR}-S p e k t r u m$

Abbildung 36: Die in der Abbildung 37 sichtbaren C-H-Korrelationen. $\quad 102$

$\begin{array}{ll}\text { Abbildung 37: HMQC-Spektrum von 32. } & 102\end{array}$

$\begin{array}{ll}\text { Abbildung 40: H,H-COSY-Spektren von 32. } & 103\end{array}$

Abbildung 39: HMBC-Spektrum von 32 aufgenommen in $\mathrm{CDCl}_{3}(500 \mathrm{MHz}) . \quad 103$

$\begin{array}{ll}\text { Abbildung 40: Die Biosynthese von } \varepsilon \text {-Pyrromycinon (30). } & 105\end{array}$

$\begin{array}{ll}\text { Abbildung 41: Ein Vorschlag zur Biosynthese von } 32 . & 105\end{array}$

Abbildung 42: ${ }^{1} \mathrm{H}-\mathrm{NMR}-S p e k t r u m$ von $\mathbf{4 3}$ in $\mathrm{CDCl}_{3}(300 \mathrm{MHz})$.

Abbildung 43: ${ }^{1} \mathrm{H}-\mathrm{NMR}-$ Spektrum von $\mathbf{5 0}$ aufgenommen in $\mathrm{CDCl}_{3}(300 \mathrm{MHz}) . \quad 128$

Abbildung 44: ${ }^{1} \mathrm{H}-\mathrm{NMR}-S p e k t r u m$ von 60 aufgenommen in $\mathrm{CDCl}_{3}(300 \mathrm{MHz}) .142$ 


\section{Verzeichnis der Tabellen}

Tabelle 1: Beispiele für extremophile Bakterien und die dazugehörigen Habitate. 21

$\begin{array}{ll}\text { Tabelle 2: Die Optimierung mit GALOP. } & 88\end{array}$

Tabelle 3: Die besten Individuen der Optimierung. $\quad 89$

Tabelle 4: Aktivitäten der entfetteten Pilzextrakte im Plattendiffusionstest. $\quad 166$

Tabelle 5: Aktivitäten der entfetteten Pilzextrakte im Plattendiffusionstest. $\quad 167$

$\begin{array}{ll}\text { Tabelle 6: Aktivitäten der entfetteten Pilzextrakte im Plattendiffusionstest. } & 168\end{array}$

$\begin{array}{ll}\text { Tabelle 7: Aktivitäten der entfetteten Pilzextrakte im Plattendiffusionstest. } & 169\end{array}$

Tabelle 9: Aktivitäten der entfetteten Pilzextrakte im Plattendiffusionstest. $\quad 170$

Tabelle 9: Aktivitäten des entfetteten Extraktes im Plattendiffusionstest. $\quad 177$

Tabelle 10: Aktivitäten des entfetteten Extraktes im Plattendiffusionstest. $\quad 180$

Tabelle 11: Aktivitäten des entfetteten Extraktes im Plattendiffusionstest. $\quad 184$

Tabelle 12: Aktivitäten des entfetteten Extraktes im Plattendiffusionstest. $\quad 186$

Tabelle 13: Aktivitäten des entfetteten Extraktes im Plattendiffusionstest. $\quad 189$

Tabelle 14: Aktivitäten des entfetteten Extraktes im Plattendiffusionstest. 192

Tabelle 15: Aktivitäten des entfetteten Extraktes im Plattendiffusionstest. 195 


\section{Einleitung}

Seit Jahrtausenden werden die Menschen mit Unheil bringenden Infektionen konfrontiert. Sie kannten zwar die Ursache der sie quälenden Krankheiten nicht, setzten jedoch trotzdem zu ihrer Bekämpfung Pflanzen, Pilze und andere Mittel in großer Vielfalt ein. Oft waren es Schamanen, die vielseitige Kenntnisse auf dem Gebiet der Naturheilkunde besaßen ${ }^{[1]}$. In vielen Regionen der Welt, in Afrika, Asien, Süd- und Mittelamerika spielt die traditionelle Heilkunde immer noch eine zentrale Rolle bei der Behandlung leidender Menschen. Das Wissen über „magische Kräfte“ verschiedener Arzneipflanzen wird mit großer Sorgfalt seit Jahrhunderten von Generation zu Generation von den Medizinmänner gepflegt und weitergegeben.

Die molekularen Wirkmechanismen solcher Methoden blieben jedoch lange Zeit im Verborgenen. Die exakten Kenntnisse über die Ätiologie der Erkrankungen sind erst seit ca. zwei Jahrhunderten wissenschaftlich belegt. Im 16. Jahrhundert stellte der Veroneser Arzt Fracastorius die Hypothese eines belebten Entzündungsstoffes auf, der von Mensch zu Mensch übertragen werden kann. Er nannte ihn „Contagium animatum“. Da es sich jedoch um mikroskopisch kleine Lebewesen handeln sollte, wurde seine Theorie erst mit dem von Leeuwenhoek im Jahre 1677 entwickelten Mikroskop bestätigt. Letzterer beschrieb erstmals verschiedene Mikroorganismen, die er unter dem Mikroskop sehen konnte ${ }^{[2]}$.

Fast hundert Jahre später entdeckte der Franzose Louis Pasteur die bakteriologischen Ursachen von Gärung und Fäulnis und legte dadurch die Grundlage für die schnelle Entwicklung der Bakteriologie. Nach ihm hat eine große Reihe von Mikrobiologen die einzelnen Bakterien und Erreger isoliert und ausführlich erforscht. Eine besondere Stellung gebührt hier sicherlich dem Bakteriologen Robert Koch, der unter anderem die Wundinfektionen, die Blutvergiftung, Milzbrand und Tuberkulose eingehend untersucht hat ${ }^{[2]}$.

Kommt es zu einer Infektion, vermehren sich die Erreger unter Schädigung des Gewebes oder sogar ganzer Organe. Nicht selten setzt der pathogene Organismus Toxine, Pyrogene oder andere Stoffwechselprodukte frei, die den Wirt schädigen können. Diese Toxine werden jedoch nicht nur während der Infektion im Menschen ausgeschieden. 1877 entdeckte Pasteur, dass andere Bakterien den Milzbranderreger ( $\mathrm{Ba}$ cillus anthracis) in der Kultur abtöten können. Diese Antibiose bedeutet, dass bestimmte Mikroorganismen in der Lage sind, Substanzen zu bilden, die das Wachstum 
anderer Mikroben negativ beeinflussen können. Ob diese Stoffe tatsächlich deswegen produziert werden, um sich im Konkurrenzkampf gegenüber anderen durchsetzen zu können, ist auch heute noch nicht eindeutig bewiesen worden.

1904 entdeckte Ehrlich das Trypanrot, einen synthetischer Farbstoff, der gegen Trypanosomen wirksam ist. Zwei Jahre danach entwickelte er zusammen mit Bertheim das Salvarsan ${ }^{\circledR}(\mathbf{1})$. Und obwohl es eine große Fülle an Nebenwirkungen aufweist, wurde es recht erfolgreich gegen Syphilis eingesetzt.

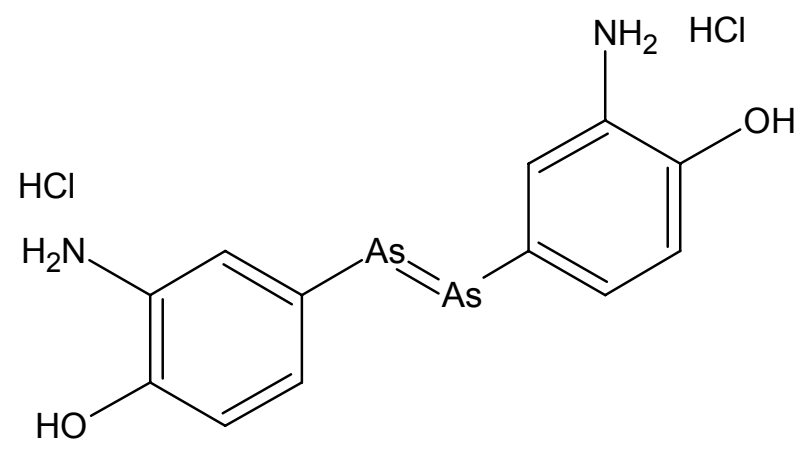

1

1928 untersuchte der Bakteriologe A. Fleming den Schimmelpilz Penicillium notatum, der in der Lage war, Staphylokokken abzutöten. Nach gründlicher Untersuchung stellte der Wissenschaftler fest, dass der Pilz einen spezifischen Naturstoff herstellt, der das Bakterienwachstum inhibiert. Fleming nannte den Wirkstoff des Pilzes Penicillin (2). Florey und Chain führten 10 Jahre später 2 in die Therapie ein. Alle drei Forscher bekamen für ihre Arbeiten 1945 den Nobelpreis für Medizin.

Nach dem II. Weltkrieg wurden ähnlich wirkende aus Mikroorganismen isolierte Substanzen in großem Stil eingesetzt. Bakterielle Durchfallerkrankungen, Typhus, Ruhr und Cholera konnten zum ersten mal erfolgreich behandelt werden. Sie haben als schnell und effektiv wirkende Medikamente den Rang von „Wundermitteln“ in der modernen Medizin erreicht. 1941 wurden sie erstmals von Waksman als Antibiotika bezeichnet.

Grundsätzlich unterscheidet man jedoch zwischen den Begriffen Chemotherapeutika und Antibiotika. Die ersten umfassen alle Arzneimittel mit einer antibakteriellen Wirkung, während Antibiotika nur diejenigen Chemoterapeutika sind, die von Mikroorganismen produziert werden. Da allerdings viele der heute eingesetzten Antibiotika aus der „chemischen Retorte“ als chemische Syntheseprodukte auf den Markt kommen, ist diese Trennung eher formal. 


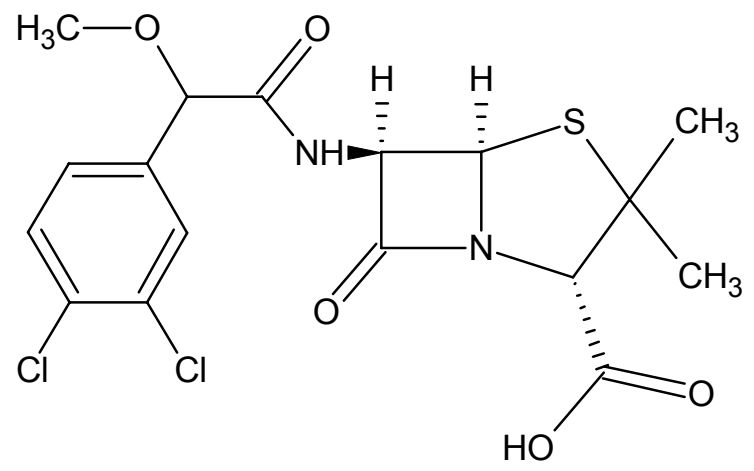

2

Man erhoffte, durch den massiven Einsatz dieser Präparate die meisten Infektionskrankheiten auf Dauer zu besiegen. Zusammen mit anderen Fortschritten in der $\mathrm{Hu}-$ manmedizin (z.B. den Schutzimpfungen) und mit besseren Lebensverhältnissen hat dies tatsächlich zum scheinbaren Verschwinden einiger Erreger geführt. Diesen positiven Erfahrungen stand jedoch eine negative Rückkopplung entgegen, nämlich die zunehmende Auslese, Anreicherung und Ausbreitung resistenter Keime, die auf derartige Behandlungsmethoden nicht mehr ansprachen. Schon bald nach der Entdeckung des Penicillins (2) fand man Bakterien, die aufgrund der Bildung eines penicillinabbauendes Enzyms, der Penicillinase, gegen 2 resistent geworden waren. Man konnte derart negative Entwicklung jedes mal nach der Einführung eines neuen Medikaments beobachten. Sporadisch kam es zum Auftreten von resistenten Varianten der Mikroorganismen, die sich schnell ausbreiten.

Zum Teil wird dies durch die Globalisierung und den weltweiten internationalen Reiseverkehr verursacht. Einem „reisenden“ Bakterium wird es ermöglicht, auch die entferntesten Orte rasch zu erreichen und zum globalen Ereignis anzuwachsen ${ }^{[3]}$. Solange zudem Tuberkulose, Diphtherie, Typhus, Scharlach und andere Infektionskrankheiten in den Ländern der Dritten Welt nicht wirksam bekämpft werden, sind sie auch für die Industrieländer eine nicht zu unterschätzende Bedrohung. ${ }^{[4]}$

Infektionskrankheiten, die erstmals an einem bestimmten Ort aufgetreten sind, nennt man Miasmen. Unter einer Endemie versteht man das Auftreten einer Infektionskrankheit in einer Region ohne zeitliche Begrenzung. Eine Epidemie ist ein örtlich und zeitlich begrenztes, gehäuftes Auftreten einer Ansteckungskrankheit. Unter dem Begriff Pandemie versteht man dagegen das zeitlich begrenzte, aber örtlich nicht 
gebundene Auftreten der Infektion, z.B. die weltweite Ausbreitung der Grippe in einzelnen Schüben.

Beispielweise ist die Therapie bei der Behandlung von Tuberkulose für die Dauer von mehreren Monaten erforderlich, da die Mycobakterien zum einen durch die speziellen Eigenschaften der Zellwand für die meisten Arzneistoffe nicht zugänglich sind, zum anderen nur sehr langsam wachsen ${ }^{[5]}$. Sehr oft behandelte man Patienten über längere Zeitabschnitte mit längst unwirksamen Mitteln. Die bereits entwickelten Resistenzen wurden oft erst spät entdeckt.

Um solche Fehler in der Zukunft zu vermeiden, wurden neue, effektivere Methoden entwickelt, um die Wirksamkeit der Antibiotika noch vor der Applikation feststellen zu können.

Für den Nachweis von Resistenzen stehen derzeit den Wissenschaftler neue molekularbiologische Techniken zur Verfügung. In vielen Fällen sind es Ergänzungen zu den meist phänotypisch orientierten und seit langem in der Diagnostik eingesetzten Screeningverfahren. Mit Hilfe der Genomanalytik lassen sich rasch und hochspezifisch Resistenzgene nachweisen. Dies ist insbesondere für schwer kultivierbare oder langsamwachsende (Tuberkulose) Bakterien von Bedeutung.

In den letzten Jahren hat die Häufigkeit der Chemotherapeutika-Resistenz bei den wichtigen Infektionserregern wie Streptokokken, Staphylokokken, Gonokokken, Enterobakterien und Mycobakterien weltweit drastisch zugenommen. Unter den grampositiven Bakterien bereiten besonders Penicillin-resistente Pneumokokken und Vancomycin (3)-resistente Enterokokken zunehmend Probleme.

Leider werden Antibiotika all zu oft nicht nur unter ärztlicher Kontrolle verwendet. In den Entwicklungsländern avancierten sie zu einer profitablen Einnamequelle und werden auch bei Straßenhändlern nicht selten in Form von einzelnen Kapseln angeboten. Die Folgen solcher dilettantischen Vorgehensweise blieben nicht aus. 1996 waren z.B. in Bangladesch 95 \% der Shigella dysenteriae, Auslöser von Ruhr, einer gefährlichen Durchfallerkrankung, gegen drei Standardantibiotika resistent. Als Mecillinam (4) 1991 auf den Markt kam, betrug die Resistenz weniger als 1 \%. Fünf Jahre später waren es 14-40 \%. Auch in den Industrieländern ist eine alarmierende Resistenzentwicklung zu beobachten. Staphylococcus aureus, ein Keim, der bei geschwächten Menschen schwere Infektionen auslösen kann, ist in Japan zu 80 \% und in Griechenland zu 40 \% gegen eine Vielzahl von Präparaten resistent ${ }^{[10]}$. 


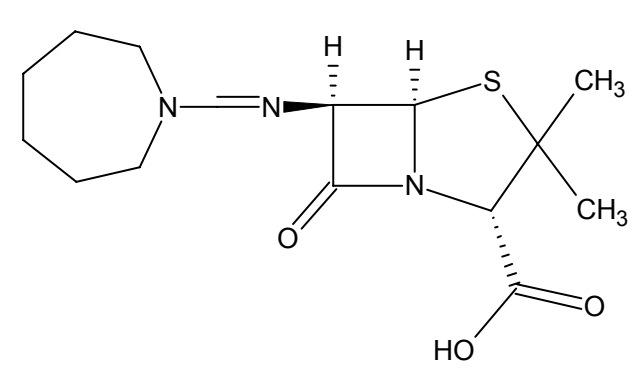

4

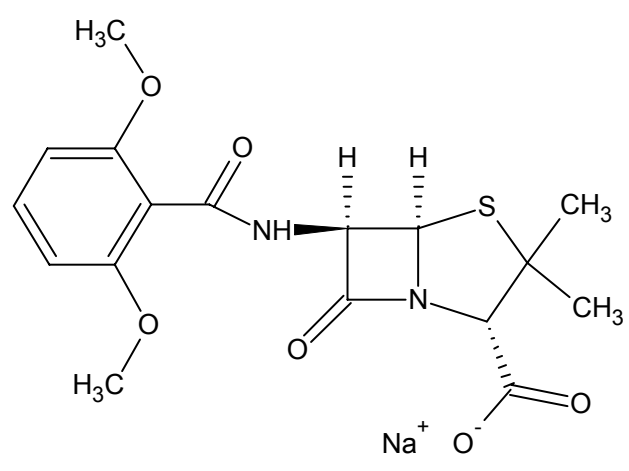

5

Zahlreiche internationale Studien zeigen eindeutig, dass bereits über $70 \%$ aller Staphylococcus aureus-Stämme gegen Penicilline eine ausgeprägte Resistenz aufweisen $^{[6]}$. Noch alarmierender ist die zunehmende Zahl der Methicillin (5)resistenten Staphylokokken-Stämme, die bereits weit verbreitet sind und in jüngster Zeit vermehrt gegen das „Reserve-Antibiotikum“ 3 widerstandsfähig wurden ${ }^{[7]}$. Ein ähnlicher Sachverhalt wird auch bei Mycobacterium tuberculosis und Plasmodium falciparum beobachtet. Letzterer, der Erreger von Malaria, hat eine weit verbreitete Unempfindlichkeit gegen Chloroquin entwickelt. Eine weitgehende Resistenz ist ebenfalls bei dem ersten Virostatikum, Azidothymidin, bei HIV-Infektionen sichtbar. Bereits bei 50 \% der Stämme führt die Behandlung zu keiner Besserung mehr. 


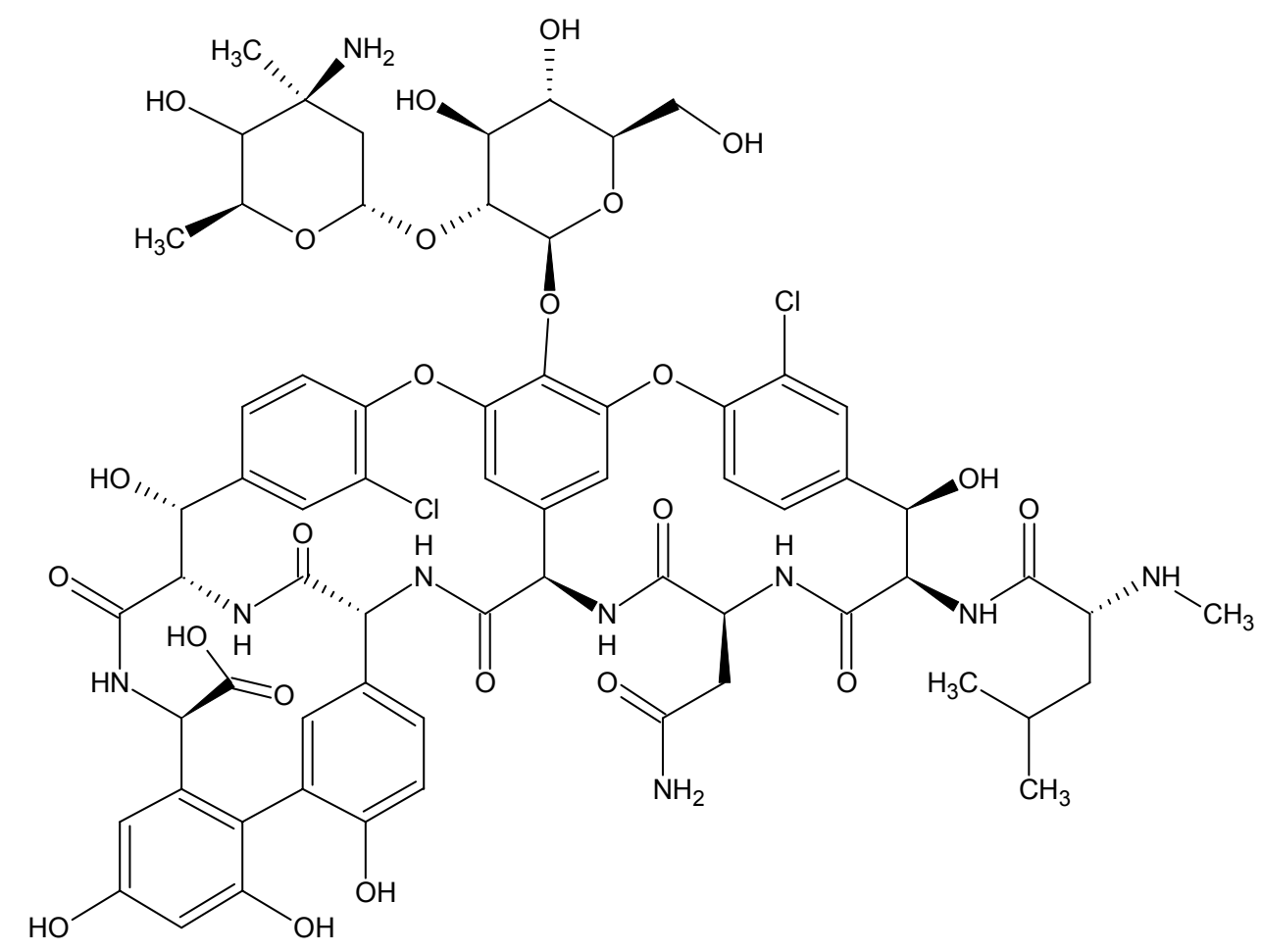

3

Eine Studie von LIBRA (Internationale Fortbildungs- und Forschungsinitiative der Bayer AG), die unter Niedergelassenen Ärzten in Westeuropa durchgeführt wurde, zeigt eindeutig, dass die erste antibiotische Behandlungsmethode bei nahezu $20 \%$ aller Patienten zu keinem Erfolgt führt, da viele Antibiotika aufgrund von Erfahrungswerten verschrieben werden. Dazu kommt, dass viele Patienten schon nach 1-2 Tagen eine deutliche Besserung verspüren, weil die Symptome weitgehend abgeklungen und bis zu $95 \%$ der Erreger bereits abgetötet sind. Sie setzen die Medikamente eigenmächtig ab. Die verkürzte Behandlung selektiert jedoch diejenigen Mikroorganismen, die deutlich überlebensfähiger sind als die meisten anderen. Diese Erscheinung stellt jedoch keine genetisch bedingte Resistenzentwicklung dar, sondern ist das Ergebnis einer unbeabsichtigten Selektion der Widerstandsfähigen.

Nicht nur die unzureichende Applikation der Medikamente, sondern auch die regelmäßige Aufnahme von Antibiotika über die Nahrungskette, besonders in Form von tierischen Lebensmittel, die Spuren von Antibiotika enthalten, führt oft zu einer Anreicherung der resistenten Bakterien ${ }^{[8]}$.

Eine ebenfalls nicht zu unterschätzende Triebkraft der Resistenzentwicklung ist die Anwendung von Wachstumsförderern in der Fleisch- und Fischproduktion. Nach Angaben der FEDESA (European Federation of Animal Health) wurden 1997 10.500.000 kg Antibiotika in der EU und in der Schweiz angewandt. Davon entfielen 
$52 \%$ auf die Humanmedizin, $33 \%$ auf Therapie und Prophylaxe in der Veterinärmedizin sowie $15 \%$ auf den Einsatz als antimikrobielle Wachstumsförderer (AMWF) in der landwirtschaftlichen Nutztierhaltung ${ }^{[4]}$. Höchst bedenklich ist, dass teilweise die gleichen Arzneimittel bei Tier und Mensch angewendet werden.

In den letzten Jahren hat sich herausgestellt, dass einige der zugelassenen AMWF Kreuzresistenzen gegen Antibiotika der Humanmedizin hervorrufen (z.B. durch chemisch verwandte Strukturen). Seit 1999 sind sie in den EU-Ländern weitgehend verboten $^{[4]}$.

Der bedenkenlose Einsatz von Antibiotika in der Landwirtschaft und Tierzucht hat dazu geführt, dass viele früher potente Substanzen mittlerweile weitgehend wirkungslos sind ${ }^{[9]}$.

Das Streptothricin-Antibiotikum Nourseothricin (NOT) wurde als Leistungsförderer seit 1982 in der ehemaligen DDR bei der industriellen Schweinemast eingesetzt. NOT zeigte keine Kreuzresistenz zu anderen Antibiotika und wurde auch nie in der Klinik angewendet. Wenige Monate später konnten resistente E. coli-Stämme aus den Schweinen isoliert werden. Bald darauf wurden die gleichen Isolate bei den Pflegern der Tiere, später bei den Familienangehörigen der Tierpfleger und dann bei Harnweginfektionen in der Bevölkerung gefunden ${ }^{[10]}$. Schließlich wurde auch bei Shigella sonnei eine Resistenzentwicklung festgestellt, einem Keim, der nur bei Menschen und nicht bei Tieren vorkommt ${ }^{[10]}$.

Aktuelle Untersuchungen belegen, dass $35 \%$ der E. coli-Populationen bei Rindern gegenüber dem Amphenicol-Derivat Florfenicol (FFA) resistent sind. Andere Beispiele sind Glycopeptid- bzw. Streptograminresistenzen der Enterokokken ${ }^{[11]}$. In den 70en Jahren wurden in der Schweinezucht und Geflügelproduktion das Glycopeptidantibiotikum Avoparcin (6) bzw. das Streptogramin Virginiamycin (7) als Wachstumsförderer in kleinen Mengen zum Futter zugegeben. Kurze Zeit darauf wurden glycopeptid- bzw. streptograminresistente Enterokokken selektiert. Aufgrund bestehender Kreuzresistenzen (Avoparcin-Vancomycin-Teicoplanin bzw. VirginiamycinQuinupristin/Dalfopristin) verloren auch die anderen Substanzen ihre Wirkung. Diese multiresistenten Bakterien konnten nun auch auf den Menschen übertragen wer$\operatorname{den}^{[12,13]}$. 


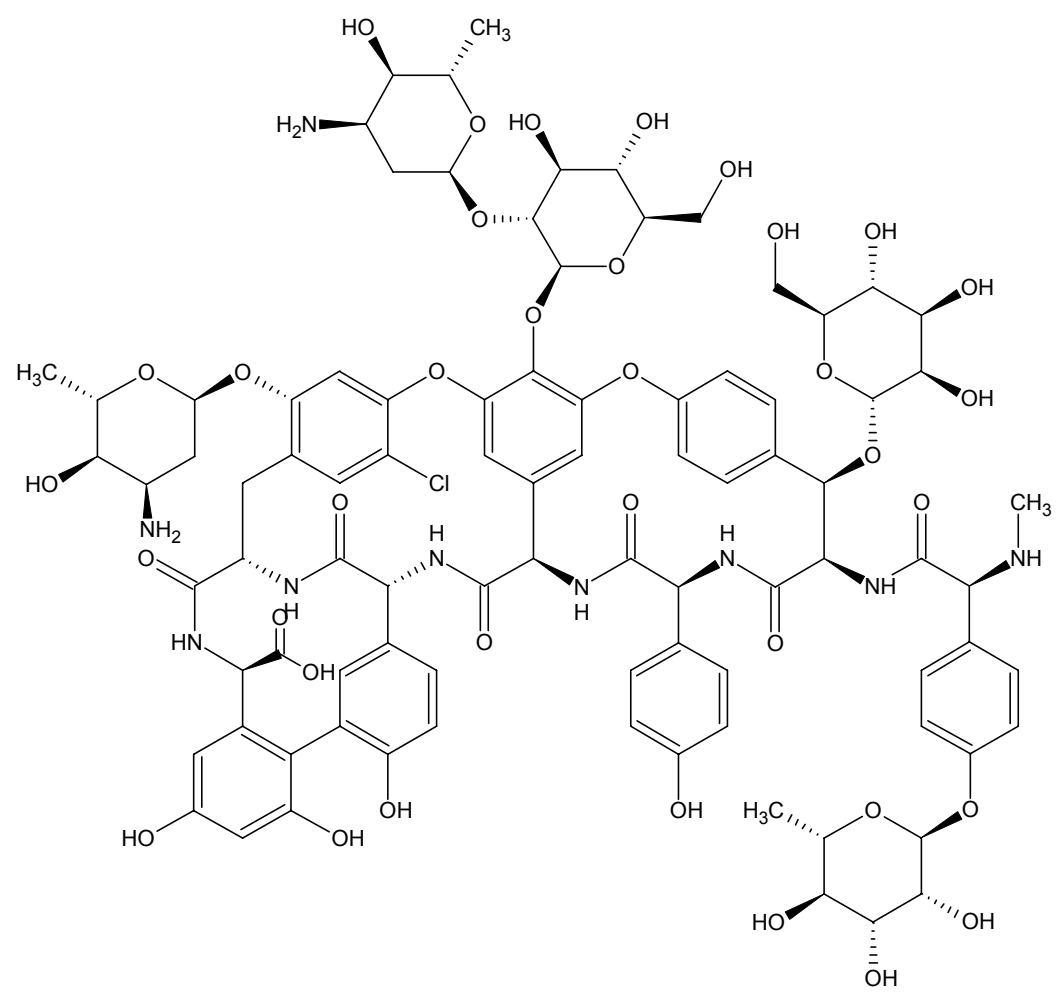

6

Die Tatsache, dass Enterokokken aus dem Tier regelmäßig in fermentierte Produkte aus der Tierhaltung gelangen, darf ebenfalls nicht bagatellisiert werden. Enterokokken zeigen eine ausgeprägte Tendenz, ihre genetische Informationen auf andere Bakterienarten zu übertragen, z.B. auf Enterobakterien, Milchsäurebakterien, Listerien, Clostridien, Staphylokokken und sogar auf Darmbakterien.

Das Vorkommen von Avoparcin/Vancomycin-resistenten Enterokokken in der Darmflora von Hühnern oder Schweinen sowie bei den Konsumenten hat in Europa zum Verbot der AMWF auf der Basis von 6 geführt ${ }^{[4]}$.<smiles></smiles> 


\section{Schutzmechanismen der Bakterien}

Durch hohe Teilungs- und Mutationsraten können sich Mikroorganismen veränderten Lebensbedienungen relativ schnell anpassen. Man spricht in diesem Zusammenhang von genetischer Variabilität.

Erworbene Resistenzen entstehen durch mutative Veränderungen des genetischen Materials eines Stammes und treten relativ selten auf. Permanente subletale Konzentrationen antimikrobieller Wirkstoffe können auf eine Population einen entsprechenden Selektionsdruck ausüben und damit entsprechenden, privilegierten Zufallsmutanten einen Wachstumsvorteil verschaffen. Wenn die seltenen Resistenzvarianten gegenüber den anderen besser an die Umgebung angepasst sind, reichern sie sich in einer Population an.

Bakterien vermehren sich durch Querteilung, da bei ihnen Paarungsprozesse nicht existieren. Das ganze genetische Material wird von der Urzelle auf die Nachkommen übertragen. Diese Art der Vermehrung hat enorme Nachteile für die Evolution und die Anpassung der Nachkommen, weil sie nur wenig genetische Variabilität zulässt. Um dieses Defizit zu kompensieren, haben Bakterien im Laufe der Evolution besondere Mechanismen entwickelt, die nicht nur die Diversität des Nachwuchses garantieren, sondern auch gleichzeitig besondere Schutzmechanismen für die Mikroorganismen darstellen. Diese Prozesse werden als Parasexualität bezeichnet. Zu diesen Mechanismen gehören die Transformation, Transduktion sowie die Konjugation.

Die meisten Bakterien haben keine Zellorganellen wie die Eukaryonten. Sie besitzen ebenfalls keinen von der Zellmembran umhüllten Zellkern. Das Erbgut dieser Prokaryonten, die chromosomale DNA, schwebt als ringförmiges Molekül frei im Cytoplasma. Diese DNA bildet zusammen mit Proteinen, die sie schützen, ein Nukleoid, ein Kernäquivalent der Eukaryontenzelle. Viele Bakterien verfügen außerdem noch über kleine extrachromosomale DNA-Ringe, die sogenannten Plasmide. Die relativ kleinen DNA-Ketten können nicht nur durch Zellteilung der Bakterien weitergegeben werden, sondern auch durch so genannte Cytoplasmabrücken oder durch Phagen (Bakterienviren) von einem Bakterium auf das andere übertragen werden (Konjugation).

Die Konjugation stellt die effizienteste Form der lateralen Ausbreitung von Resistenz-DNS dar. Das Plasmid wird während der Ausbildung einer direkten Verbindung (Cytoplasmabrücken) zwischen zwei Zellen transferiert. Derartige Plasmide bezeich- 
net man als konjugativ. Diese tragen Gene (tra-Gene), die für den Transfer verantwortlich sind. Neben den tra-Genen und Sequenzen, die eigenständige Replikationen des Plasmids steuern, können letztere noch weitere Gene beinhalten, z.B. auch Antibiotika-Resistenzgene (Resistenzplasmide). Dieser Austausch genetischer Informationen zwischen unterschiedlichen Arten (horizontaler Gentransfer), verursacht durch die Wanderung verschiedener genetischer Elemente (u.a. Plasmide, Bakteriophagen), trägt dazu bei, dass die in einem Ökosystem lebenden Mikroorganismen ihre Resistenzdeterminanten problemlos angleichen können ${ }^{[14]}$.

Bei der Transformation wird die chromosomale DNA von einer Zelle auf die andere transferiert. Bei der Transduktion übertragen Phagen eigene DNA auf die Bakterien. Teile der chromosomalen und der plasmidischen DNA können nicht nur zwischen verschiedenen Zellen einer Spezies, sonder auch zwischen verschiedenen Gattungen übertragen werden. Somit werden auch die Erbeigenschaften (z.B. Resistenzeigenschaften) weitergegeben ${ }^{[2]}$.

Einige Mikroorganismen sind von Natur aus resistent gegen manche Antibiotika, andere wiederum erwerben diese Fähigkeit erst während der Behandlung mit entsprechenden Medikamenten. Außerdem entwickeln Bakterien intelligente Abwehrmechanismen, mit denen sie sich vor der Schädigung durch unterschiedliche chemische Substanzen schützen können ${ }^{[15]}$.

Bakterien können z.B. Proteine produzieren, durch die Antibiotika enzymatisch inaktiviert werden, z.B. bei den Penicillinen und Aminoglycosiden.

Eine andere Möglichkeit der Abwehr besteht darin, die Zellwand so zu modifizieren, dass das Medikament nicht mehr ins Innere der Zelle vordringen kann. Dies ist ein häufiges Problem bei der Therapie gramnegativer Bakterien, deren äußere Membran aus einer stark lipophilen Schicht besteht, durch die ca. $80 \%$ der Antibiotika nicht eindringen können ${ }^{[5]}$.

Bestimmte Bakterien, z.B. Bazillen, sind in der Lage, Dauerformen zu bilden, so genannte Bakteriensporen. Sie dienen nicht der Fortpflanzung der Individuen, sondern stellen eine Eigenform dar, die sehr resistent gegen äußere Einflüsse, wie Hitze, Trockenheit und sogar die toxische Wirkung der Antibiotika ist.

Manche Substanzen können zu einer veränderten Zusammensetzung der Zellhülle führen, so dass die Empfindlichkeit ihnen gegenüber heruntergesetzt wird. Weiterhin könne viele Mikroorganismen in wässrigen und nährstoffarmen Lösungen extrazellu- 
lare, polymere Substanzen (EPS) ausscheiden, die ihnen eine schützende Schleimhülle verleihen und gleichzeitiges Anheften an festen Oberflächen ermöglichen. Solche sessilen Zellen bilden einen Biofilm, der die bioziden Wirkstoffe adsorbiert und inaktiviert.

Eingedrungene, zellfremde Substanzen können jedoch durch sogenannte EffluxPumpen aus der Zelle eliminiert werden. Diese Mechanismen findet man z.B. bei Resistenzen gegenüber Tetracyclinen. Außerdem können Mikroorganismen die Struktur der Antibiotika verändern. Dabei werden Gene aktiviert, die für sogenannte Bindeproteine verantwortlich sind. Diese binden das Antibiotikum und deaktivieren es gleichzeitig. So erwerben Bakterien z.B. die Resistenz gegenüber MakrolidAntibiotika. Die Resistenz gegen Sulfonamide und Trimethoprim (8) kann durch das Umgehen der kritischen Schritte im Folsäure-Stoffwechsel erzeugt werden. Die genannten Resistenzmechanismen können auch simultan auftreten ${ }^{[16,17]}$.<smiles>COc1cc(Cc2cnc(N)nc2N)cc(OC)c1OC</smiles>

8

Durch kleinere, sporadische Mutationen (antigenic drift) entstehen jährlich neue Subtypen, die einen Organismus auch dann infizieren können, wenn bereits Antikörper gegen das ursprüngliche Antigen produziert wurden. Bei einem „antigenic shift" findet eine bedeutende Mutation statt, die zu Pandemien führen kann ${ }^{[18]}$. Die Spanische Grippe (1918) verursachte z.B. 20 Millionen Todesfälle, mehr als die Anzahl der im ersten Weltkrieg gefallenen Soldaten. Andere bekannte Pandemien wurden durch die Asiatische Grippe (1957) und die Hongkong-Grippe (1968) verursacht ${ }^{[18]}$. 


\section{Perspektiven der Antibiotikaforschung}

Die Vernetzung der Biosphäre und der darin in Symbiose lebenden Makroorganismen (Menschen, Tiere, Pflanzen) und Mikroorganismen macht deutlich, dass für das Resistenzproblem keine isolierten geographischen und biologischen Lösungen existieren.

Einen einzelnen Menschen mit Antibiotika zu behandeln, bedeutet immer auch, sein Umfeld zu behandeln, was zur Selektion resistenter Bakterien und möglicherweise auch zum Austausch von genetischem Material mit anderen Bakterien führen kann. Man hat nachgewiesen, dass die Anzahl resistenter Bakterien im Kot von Tieren, die in der Nähe von Menschen leben, deutlich höher liegt, als bei wild lebenden. Der Grund dafür ist wohl in der Nahrungsaufnahme zu suchen ${ }^{[3]}$.

Die Mikroorganismen werden zunehmend gegen mehrere an unterschiedlichen Wirkorten angreifende Substanzen abwehrfähig, was auch die Kombinationstherapien erfolglos macht. Man spricht hier von Multi-Drug-Resistenzen (MDR) ${ }^{[1]}$.

Derartige Problematiken beobachtet man gehäuft in Pflegeheimen und Krankenhäusern, in denen eine verstärkte Wechselwirkung zwischen Mensch und großen Mengen an eingesetzten Medikamenten zu fürchten ist. Stationär behandelte Patienten leiden unter dem erhöhten Risiko, an nosokomialen Infektionen (Krankenhausinfektionen) zu erkranken, an denen jährlich Tausende von Menschen versterben. Um dieses Problem wieder unter Kontrolle zu bekommen, rät die Weltgesundheitsorganisation WHO dringend zu einer globalverantwortlichen Strategie im Umgang mit Antibiotika $^{[19]}$.

Da kaum physikalische Barrieren für die Bakterien in diesen Einrichtungen existieren, ist es nur eine Frage der Zeit, wann diese Resistenzen außerhalb auftreten und auf nicht behandelte Patienten übertragen werden. Aber nicht nur die Mikroorganismen können die Kliniken verlassen. Man hat festgestellt, dass auch die Konzentration an Makroliden, Trimethoprim (8), Sulfamethazol (9) und Ciprofloxacin (10) im Krankenhausabwasser besonders hoch ist ${ }^{[12,7]}$. 


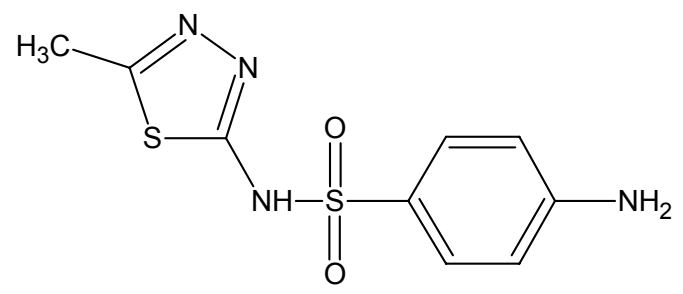

10<smiles>O=C(O)c1cn(C2CC2)c2cc(N3CCNCC3)c(F)cc2c1=O</smiles>

9

Durch die massiven Sparmassnahmen der öffentlichen Krankenkassen werden den betroffenen Patienten inzwischen in der Regel nur noch „Billigantibiotika“ verschrieben. Dies sind häufig Produkte, gegen die bereits eine partielle Resistenzentwicklung zu beobachten ist, und die meist von Firmen angeboten werden, die selbst keine Forschung an neuen Präparaten betreiben. Oft erweist sich dieser Weg als eine Sackgasse, denn die Behandlung einer resistenten Infektion ist kostspieliger als die einer nicht resistenten, sowohl hinsichtlich des Zeit- als auch Geldaufwandes.

Die Medizin verfügt jedoch nicht über eine beliebige Zahl von Arzneimitteln. Zur Zeit sind nur ca. 150 Antibiotika klinisch zugelassen, wobei bei den meisten bereits ein Wirkverlust zu beobachten ist. In den letzten 25 Jahren wurde nur eine einzige neue Wirkstoffklasse entdeckt, die Oxazolidinone, alle anderen waren eine Modifizierung der Leitstrukturen entsprechender schon existierender Verbindungen. Die Entwicklung eines neuen, noch effektiveren Medikaments dauert im Durchschnitt 10-20 Jahre und verschlingt mindestens 600 Millionen US-Dollar. Man ist daher trotz der vorhandenen Therapiemethoden und des Einsatzes moderner Technik gezwungen, nach neuen und besseren Medikamenten zu suchen ${ }^{[20]}$.

Während die bevorzugte Strategie zur Umgehung der Resistenzentwicklung in den Anfängen der Antibiotika-Ära (1928-1965) die Suche nach neuen Leitstrukturen darstellte, was zu den wichtigsten heute eingesetzten Substanzklassen geführt hat, wurden in den darauf folgenden 40 Jahren verstärkt Versuche unternommen, die Leitstrukturen chemisch zu verändern. Diese Strategie schlug jedoch teilweise fehl, denn durch die Kreuzresistenzen lagen in den meisten Fällen bereits vorselektierte Erreger vor. Die Leitstrukturveränderungen bewirkten oft nur eine Erhöhung der Stabilität oder Löslichkeit des Medikaments und wurden durch kleine Mutationen im Erbmaterial der Mikroorganismen rasch kompensiert. 


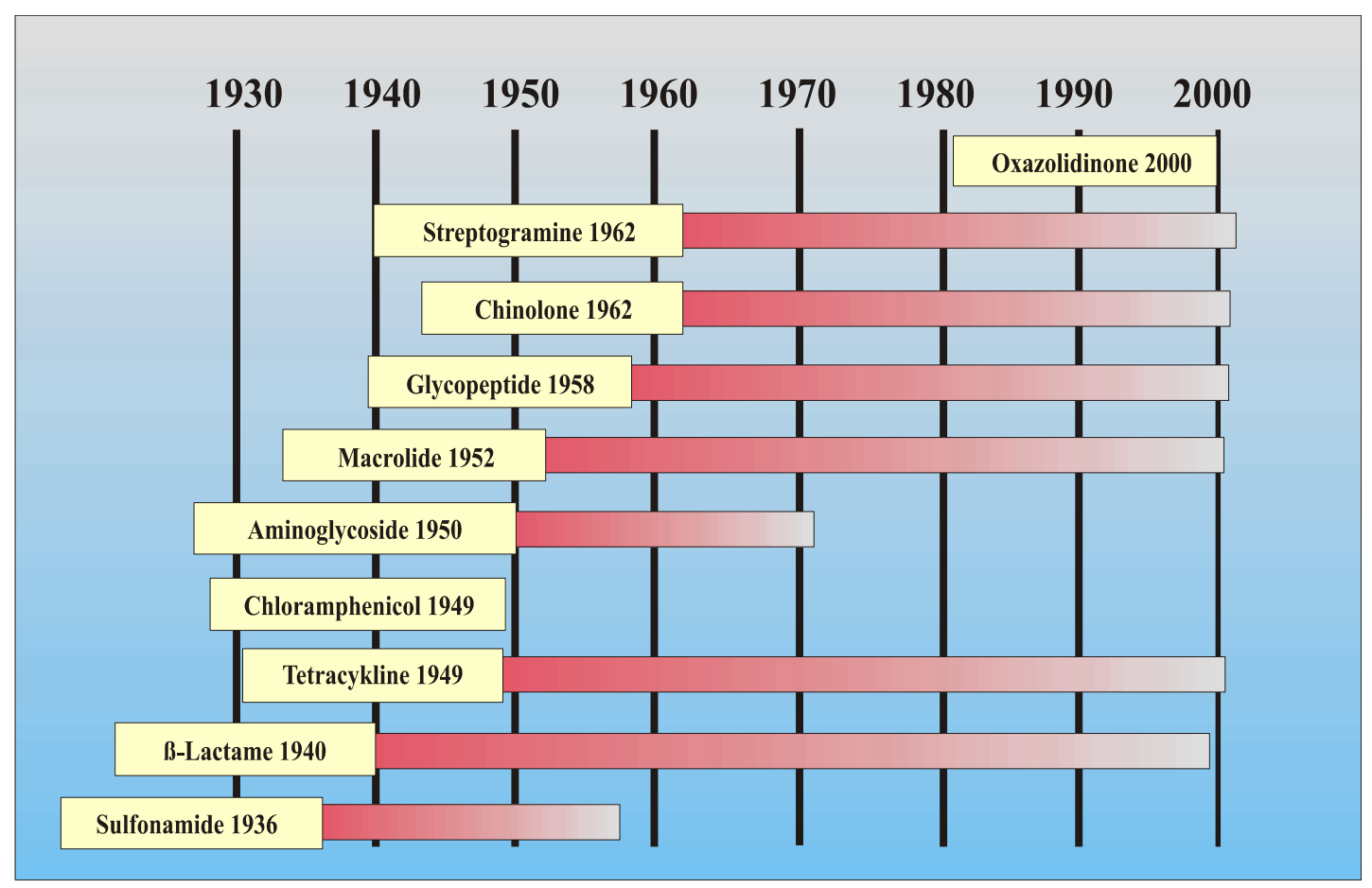

Abbildung 1: Einsatz der Antibiotika-Substanzklassen und ihre spätere Entwicklung.

Seit kurzem greift man immer öfter auf die schnell wachsenden DNA-Sequenzdatenbanken zurück, um möglich rasch neue Zielstrukturen zu identifizieren. Neue Disziplinen, wie das Protein design, Molecular modelling und Functional genomics, gehören zu den computerunterstützten Methoden, die erlauben, biochemische Vorgänge zu simulieren und so zur Entwicklung neuer Antibiotika beitragen ${ }^{[21]}$.

Mittels Hochdurchsatz-Screeningverfahren, in denen Substanzbibliotheken aus kombinatorischen Synthese- und Naturstoffscreeningprogrammen auf Aktivität und Wirksamkeit geprüft werden, sollen dann neue Leitstrukturen identifiziert werden. Erste erfolgsversprechende Ansätze bieten Inhibitoren von Efflux-Pumpen und Deformylase, Proteinsekretionsenzymen sowie der Signaltransduktionssysteme. Die Hauptproblematik der Verfahren liegt jedoch darin, dass sie zwar endlose Mengen an Ergebnissen liefern, diese jedoch nicht selbst auswerten können, denn es fehlt der entscheidende Intelligenzfaktor. Deswegen sucht man auch in der Informatik nach neuen intelligenten Verfahren, Programme zu entwickeln, die imstande wären, solche Aufgaben selbständig zu lösen, der sogenannten „künstlichen Intelligenz“. 


\section{Die Natur als Vorbild}

Trotz der Fortschritte in der synthetischen Chemie und dem großen Einsatz der Syntheseroboter ist die Natur heute immer noch eine unersetzliche Quelle für biologisch aktive Verbindungen. Die kombinatorische Chemie hat nur zum Teil die Erwartungen der Industrie erfühlt. Es hat sich gezeigt, dass die strukturelle Diversität und Komplexität der auf diesem Wege erhaltenen Produkte deutlich hinter den komplexen Formen der natürlichen Moleküle liegt. Im Vergleich sind 60 \% aller kommerziell vertriebenen oder sich in klinischen Tests befindenden Antitumormittel und Antibiotika eines natürlichen Ursprungs. Die kombinatorische Chemie zeigt jedoch ihre Stärken bei der Suche nach neuen wirksamen Derivaten bekannter Leitstrukturen. So konnte z.B. mittels Derivatisierung die Wasserlöslichkeit, Aktivität und Selektivität von Linezolid (12) deutlich erhöht werden.

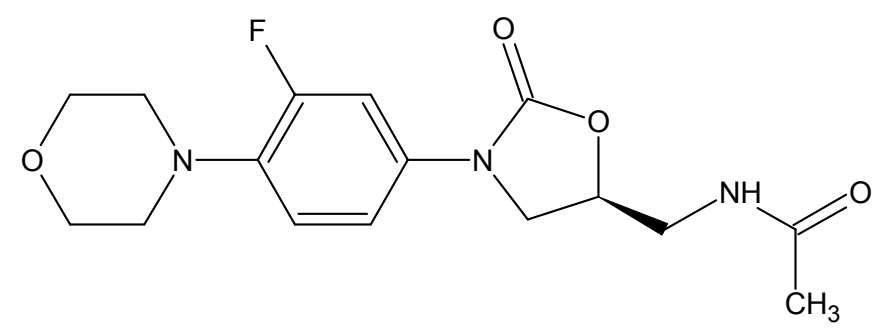

12

Die außerordentliche strukturelle Komplexität bestimmter Naturstoffe hat in vielen Fällen ihre Synthese erschwert oder sogar unmöglich gemacht. Als Beispiel seien hier das Herzglykosid Digitin (13), Taxol (14), menschliche Peptidhormone und Proteine wie Insulin sowie die zahlreichen Antibiotika angeführt ${ }^{[17]}$. Klassische biotechnologische und neue gentechnologische Methoden werden deshalb zunehmend wichtiger. 


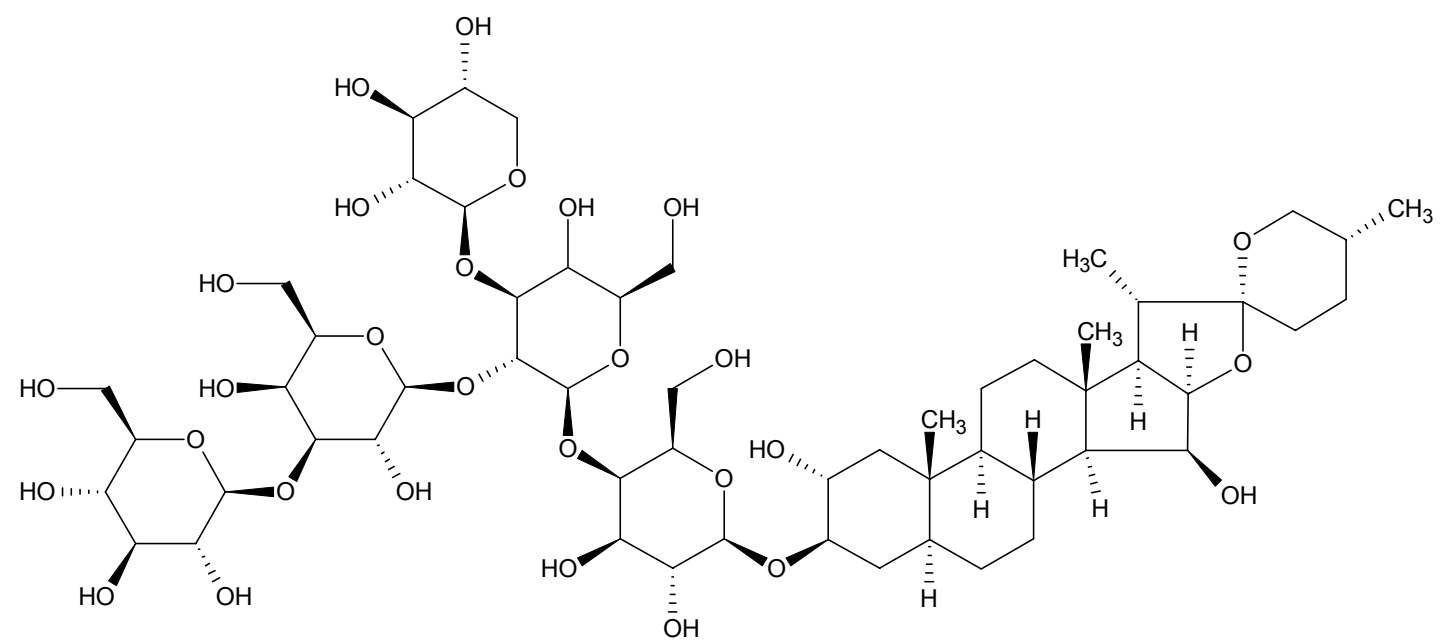

13

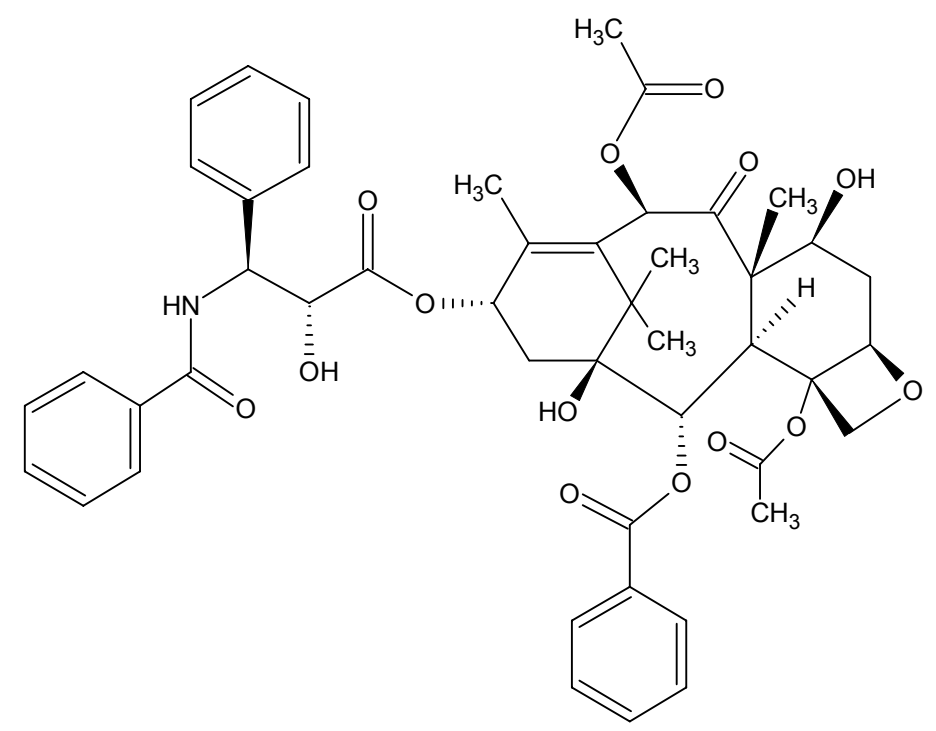

14

Die Naturstoffe finden nicht nur in der Medizin und Pharmaindustrie als Medikamente, sondern auch in der Landwirtschaft z. B. als Pflanzenschutzmittel (die Strobilurine als Fungizide) und in der Mikrobiologie eine breite Anwendung. Sie haben ermöglicht, Einblicke in den Ablauf von Lebensvorgängen zu gewinnen. Viele dieser Stoffe haben sich als Aktivatoren, Inhibitoren oder Modulatoren von Zellrezeptoren erwiesen. Zahlreiche Naturstoffe dienten daher als Leitstrukturen bei der Arzneimittel-Entwicklung: Sie wurden durch teils nur geringfügige Veränderung der ursprünglichen Strukturen auf chemischem oder mikrobiologischem Wege zu biologischen Wirkstoffen weiter entwickelt. 
Alle mikrobiellen Wirkstoffe gehören der Gruppe der sogenannten Sekundärmetabolite an. Während die Primärmetabolite für das Leben und die Vermehrung der Zelle essentiell sind, ist die Funktion der meisten Sekundärmetabolite bisher noch nicht abschließend geklärt. Sie werden meist am Ende der Wachstumsphase des Organismus gebildet und nur von einer beschränkten Anzahl der Organismen produziert. Sie spielen u.a. eine spezielle Rolle beim Überleben des Mikroorganismus in seiner Umwelt, bei der Kommunikation mit anderen Organismen und bei der Erhaltung von symbiotischen Beziehungen. Zu den Sekundärstoffen gehören unter anderem aromatische Verbindungen, Terpene, Steroide, Alkaloide und Antibiotika.

Obwohl die meisten Wirkstoffe pflanzlicher Herkunft sind, gewinnen die Mikroorganismen immer mehr an Bedeutung und Interesse. Mikroorganismen besitzen gegenüber den anderen Produzenten viele Vorteile. Ihre Kultivierung ist viel einfacher, billiger und beansprucht weniger Platz als z.B. der Pflanzenanbau. Für ihre Fortpflanzung reicht prinzipiell nur eine einzige Zelle, und die Kultivierung dauert anders als bei höheren Organismen nur wenige Stunden bis Tage. Sehr oft kann man die Produktivität durch Variation der Kultivierungsbedienungen oder Nährmedienzusammensetzung um ein Vielfaches steigern. Diese Strategie wurde von Zeeck et al. als OSMAC-Methode bezeichnet (One Strain / Many Compounds) ${ }^{[24]}$.

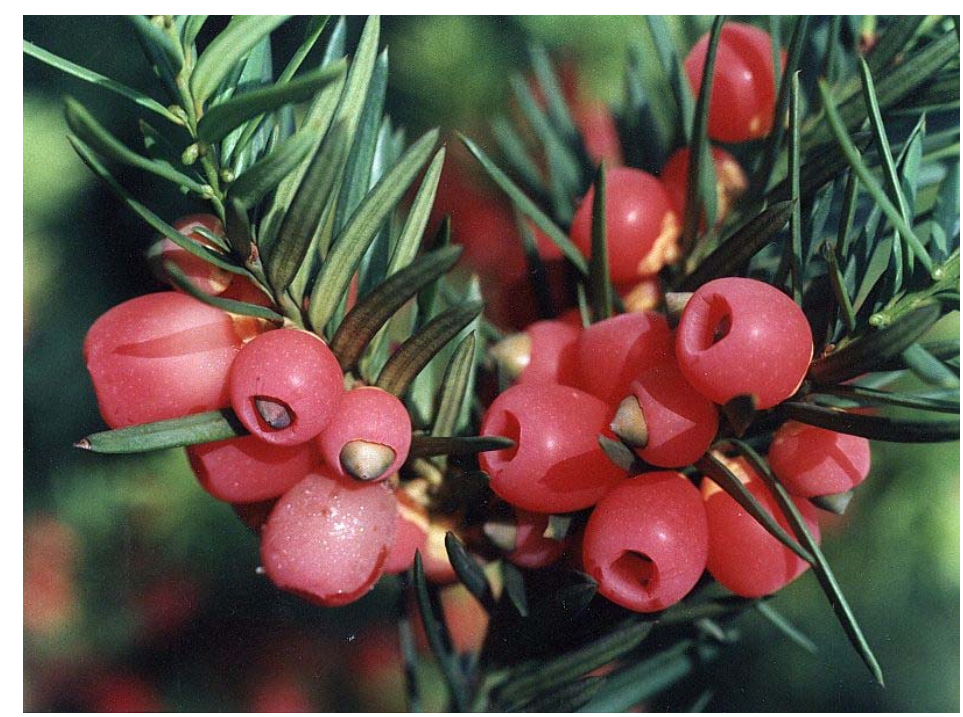

Abbildung 2: Die Europäische Eibe (Taxus baccata)

Die Optimierung wird bei den Pflanzen erst durch einen wesentlich größeren Aufwand erreicht. Man versucht, analoge Produkte durch Derivatisierung aus Substanzen zu gewinnen, die aus verwandten Pflanzenarten bereist in beträchtlichen Mengen $\mathrm{zu}$ isolieren sind. So wird z.B. das Antitumormittel Paclitaxel aus der Pazifischen 
Eibe Taxis brevifolia nur in sehr kleinen Mengen isoliert. Die Europäische Eibe (Taxus baccata) liefert dagegen 10-Deacetylcaccatin III in beträchtlichen Mengen, das sich leicht zu Paclitaxel abwandeln lässt.

Die von Mikroorganismen produzierten Naturstoffe zeichnen sich ebenfalls durch eine große strukturelle Vielfalt aus. Seit der Entdeckung von 2 im Jahre 1928 wurden etwa 25.000 mikrobielle Naturstoffe isoliert. Davon sind über 10.000 biologisch aktiv und 8.000 zeigen antibiotische und cytotoxische Wirkung. Die Zahl der in der Natur vorkommenden Metabolite ist dennoch beschränkt. Diese Tatsache spiegelt sich in der Zahl der neuentdeckten Verbindungen wieder. Zählt man die Erstbeschreibungen von Sekundärstoffen aus terrestrischen Organismen auf, bemerkt man, dass nach einem exponentiellen Zuwachs seit 1966 nun eine stationäre Phase zu folgen scheint. Dennoch waren es 1999 noch 20000 Verbindungen im Jahr. Diese Erscheinung wird aber auch zum Teil dadurch verursacht, dass zur Suche nach neuen, interessanten Strukturen andere Wege eingeschlagen werden. In den letzten Jahren beschränkte man sich hauptsächlich auf terrestrische Organismen. Die marinen Bakterien wurden nur sporadisch untersucht. Die Entwertung anderer Habitate wurde durch die Schwierigkeiten in der Beschaffung, Kultivierung und durch kleine Ausbeuten verursacht.

\section{Exzentrische Mikroorganismen als Lieferanten neuer Naturstoffe}

Lange Zeit wollte man nicht glauben, dass das Leben auch in Extrembereichen existieren kann. Heute weiß man, dass diese Extreme sogar notwendig sind, um den entsprechenden Mikroorganismen die Vegetation zu ermöglichen. Intensive Untersuchungen seit Mitte der 80er Jahre haben verdeutlicht, dass Extremophile nicht nur für die Grundlagenforschung, sondern auch für moderne biotechnologische Anwendungen von Bedeutung sind ${ }^{[22]}$.

Diese an extreme Umweltverhältnisse angepassten Lebewesen stellen eine ideale Quelle sowohl für neue Biokatalysatoren, die ihre Wirkung auch bei hohen Temperaturen entfalten können, als auch als Bezugsort für den technischen Fortschritt und neue Ideen dar.

In den heißen Quellen des Yellowstone-Nationalparks entdeckte man Bakterienagglomerate (z.B. Thermus aquaticus), die nur oberhalb von $60^{\circ} \mathrm{C}$ existieren können. Die optimalen Lebensbedienungen liegen für diese Mikroorganismen bei $80-100{ }^{\circ} \mathrm{C}$. 
Noch extremere Temperaturen von $113{ }^{\circ} \mathrm{C}$ toleriert Pyrolobus fumarii. All diese thermophilen Bakterien haben im Laufe der Evolution ihre eigenen Anpassungsapparate perfektioniert ${ }^{[23]}$. Sie produzieren Hitzeschockproteine (Chaperone) und Streßproteine, die wiederum neuentstandene Proteine abschirmen und dadurch auch schützen können. Das natürliche Umfeld, in dem sich thermophile Mikroorganismen am besten entwickeln, ist meistens geothermischen Ursprungs und steht damit in Verbindung zu tektonisch aktiven Zonen. Der große wirtschaftliche Nutzen der Enzyme aus Thermophilen wird bei der Polymerasen-Kettenreaktion sichtbar (PCR $)^{[24]}$. Auf die größte Anzahl psychrophiler (kälteliebender) Mikroorganismen trifft man in den polaren Regionen, besonders aber in den arktischen Meeren, die eine konstante Temperatur von -2 bis $+4{ }^{\circ} \mathrm{C}$ aufweisen. Unter diesen Temperaturen produzieren die Organismen unterschiedliche Osmolyte, mit denen Wasserentzug verhindert werden kann, wie z.B. Dimethylsulfoniumpropionat, die Vorstufe des flüchtigen Dimethylsulfids. Neben den Osmolyten sind besonders kälteangepasste Enzyme mit hoher katalytischer Aktivität bei niedrigen Temperaturen für die Frostresistenz essentiell. Um die Fluidität der Zellmembranen auch bei sehr niedrigen Temperaturen zu gewährleisten, stellen die Organismen mehrfach ungesättigte Fettsäuren her. Außerdem werden Polysaccharide ausgeschieden, um einer Art Schutzschicht um die Zelle zu bilden $^{[24]}$.

An der Untersuchung dieser Naturstoffquelle ist unsere Arbeitsgruppe als erste in Deutschland in Zusammenarbeit mit E. Helmke vom Alfred-Wegener-Institut für Polar- und Meeresforschung seit vielen Jahren beteiligt. In dem arktischen Proteobakterium ANT V/2 437 konnte man z.B. Daidzein (15) und in B 4710 Actinomycin D (16) nachweisen.<smiles>O=c1c(-c2ccc(O)cc2)coc2cc(O)ccc12</smiles> 


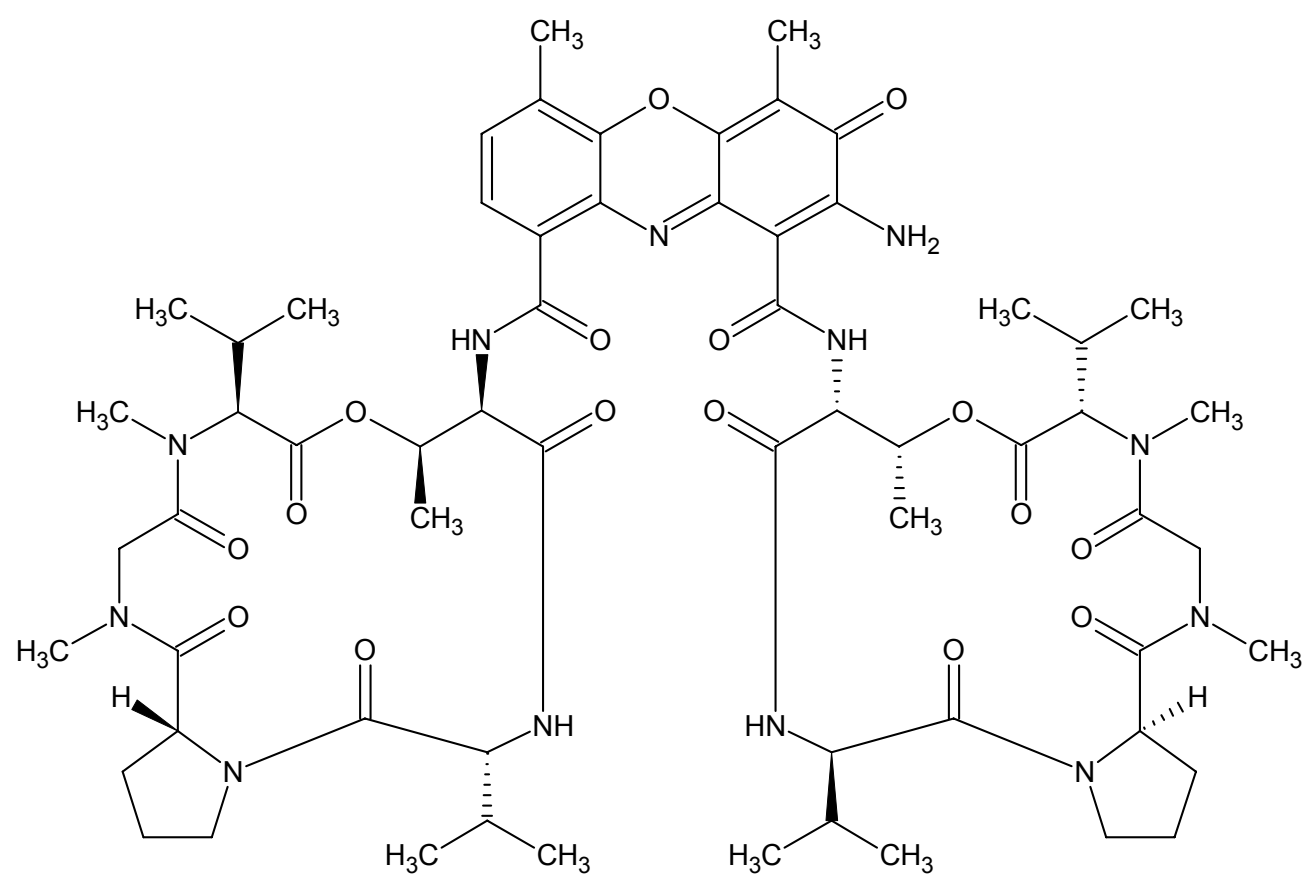

16

Die barophilen Bakterien haben sich an hohen Druck angepasst. Derartige Organismen wurden in Meerestiefen von 3000 - 10000 m gefunden.

Extrem acidophile Mikroorganismen treten in der Regel in heißen, schwefelhaltigen Quellen auf, wo sie an pH-Werte von 1.0 bis 3.5 und hohe Temperaturen von bis zu $85^{\circ} \mathrm{C}$ adaptiert sind. Somit sind sie auch zugleich als thermophil zu bezeichnen.

In den extrem saueren, durch Schwermetalle wie $\mathrm{Ni}, \mathrm{Cu}, \mathrm{As}, \mathrm{Cd}$ und Fe vergifteten und tiefrot gefärbten Wässern des spanischen Flusses Rio Tinto haben Wissenschaftler ca. 1300 verschiedene Lebensformen entdeckt: Bakterien, Pilze, Hefen, Algen und andere Einzeller, darunter auch Bakterien der Spezies Leptospirillum ferrooxidans, die Eisen oxidieren und dadurch Energie für die Biosynthese gewinnen können $^{[25,26]}$. Ebenso hat man in alkalischen Grundwässern, Salzseen und Salzwüsten Anzeichen organischen Lebens gefunden. Bestimmte Einzeller können bei pHWerten über 9.0 (alkalophile) oder bei sehr hohem Salzgehalt (halophile) existieren. Die Salzkonzentrationen des Meerwassers (3\%) bis hin zu den gesättigten Salzlösungen (35\%) des Toten Meers, von Salzlaken und Salzseen stellen einen natürlichen Lebensraum halophiler Bakterien dar.

Die terrestrischen und in den Meeren lebenden Organismen haben sich in vielen Bereichen deutlich auseinander entwickelt und durch diverse Überlebensstrategien evolutionär an ihre Umgebung angepasst. 
Tabelle 1: Beispiele für extremophile Bakterien und die dazugehörigen Habitate.

\begin{tabular}{|c|c|c|c|}
\hline Organismus & Habitat & Extrem & überlebt bis: \\
\hline Pyrolobus fumarii & $\begin{array}{l}\text { In und an Black } \\
\text { Smokers }\end{array}$ & Hitze & $+113^{\circ} \mathrm{C}$ \\
\hline $\begin{array}{c}\text { Sulfolobus acidocal- } \\
\text { darius }\end{array}$ & $\begin{array}{c}\text { Heiße, saure } \\
\text { Quellen im Yel- } \\
\text { lowstone Park }\end{array}$ & Hitze und Säure & $+85^{\circ} \mathrm{C} ; \mathrm{pH} 2$ \\
\hline $\begin{array}{l}\text { Polaromonas vacuo- } \\
\text { lata }\end{array}$ & Meereis & Kälte & $0^{\circ} \mathrm{C}$ \\
\hline Mikroalgen & $\begin{array}{l}\text { In antarktischem } \\
\text { Gestein }\end{array}$ & Kälte & $-15^{\circ} \mathrm{C}$ \\
\hline $\begin{array}{c}\text { Natronobacterium } \\
\text { pharaonis }\end{array}$ & $\begin{array}{l}\text { alkalisches Ge- } \\
\text { stein }\end{array}$ & Lauge & pH 10 \\
\hline $\begin{array}{l}\text { Agrobacterium } \\
\text { tumefaciens }\end{array}$ & Boden & Lauge & pH 12 \\
\hline $\begin{array}{c}\text { Picrophilus torridus, } \\
\text { P. oshimae }\end{array}$ & $\begin{array}{l}\text { In sauren, vulka- } \\
\text { nischen Quellen }\end{array}$ & Säure & $\mathrm{pH} 0$ \\
\hline $\begin{array}{l}\text { Bakterien und See- } \\
\text { gurken }\end{array}$ & $\begin{array}{c}\text { Boden des } \\
\text { Marianengrabens }\end{array}$ & Druck & 1.200 Atmosphären \\
\hline Bakterium & In Gestein & Tiefe & 3,2 Kilometer Tiefe \\
\hline $\begin{array}{c}\text { Deincoccus radiodu- } \\
\text { rans }\end{array}$ & $\begin{array}{l}\text { In Kuhdung, ver- } \\
\text { faultem Fleisch }\end{array}$ & Radioaktivität & 3 Millionen rad \\
\hline Arthrobacter oxydans & $\begin{array}{l}\text { In Basaltgestein } \\
\text { unterhalb von } \\
\text { Deponien }\end{array}$ & $\begin{array}{l}\text { hochgiftige } \\
\text { Schwermetalle }\end{array}$ & 6-wertiges Chrom \\
\hline $\begin{array}{c}\text { Thiobacillus fer- } \\
\text { rooxidans; Leptop- } \\
\text { sirillum ferrooxidans }\end{array}$ & $\begin{array}{l}\text { Rio Tinto in } \\
\text { Spanien }\end{array}$ & $\begin{array}{c}\text { Säure und } \\
\text { Schwermetalle }\end{array}$ & $\begin{array}{c}\mathrm{pH}<2 \text {; Arsen, Nickel, } \\
\text { Kadmium }\end{array}$ \\
\hline $\begin{array}{l}\text { Halobacterium sali- } \\
\text { narum }\end{array}$ & $\begin{array}{l}\text { Natürliche und } \\
\text { künstliche Salz- } \\
\text { seen, Totes Meer }\end{array}$ & Salz & $\begin{array}{l}5.2 \text { molare } \mathrm{NaCl}- \\
\text { Lösung }\end{array}$ \\
\hline
\end{tabular}




\section{Untersuchungen an mariner Organismen}

Nachdem sich die Wissenschaft bei der Suche nach neuen Wirkstoffen oder Leitstrukturen für Medikamente zunächst auf terrestrische Pflanzen und Tiere beschränkte, wird mittlerweile intensiv auch im Meer nach neuen Substanzen gesucht.

Man schätzt, dass etwa $60 \%$ aller auf der Erde vorkommenden Arten im Meer existiert. Die Zahl der mariner Spezies wird grob auf zwischen 3 und 500 Millionen geschätzt. Wie man sich in den vergangenen 30 Jahren Forschung zweifellos überzeugen konnte, können marine Naturstoffe ihre biologische Wirkung auch außerhalb ihrer Habitate entfalten ${ }^{[27]}$. Angesichts des Mangels an geeigneten Arzneimittel bei vielen Krankheiten und der zunehmenden Resistenzentwicklung gewinnen diese Kenntnisse besondere Bedeutung.

Unter der Annahme, dass jede der erwähnten 500 Millionen mariner Spezies nur einen einzigen arteigenen Sekundärmetaboliten herstellen kann, erscheint die Zahl der möglichen Substanzen gerade zu utopisch. Die bisher publizierten Daten deuten darauf hin, dass selbst diese Annahme viel zu rigoros gemacht wurde. In der Nordseekrabbe Crangon crangon fand man über 400 Substanzen. Darunter Kohlenwasserstoffe, Alkohole, Aldehyde, Ketone, Carbonsäuren, Carbonsäureamide, Ester, Lactone, Steroide, Diketopiperazine, Nucleoside, Aminosäuren und einfache aromatische Verbindungen. Sechs Hydroxysäureamide und vier macrocyclische Lactone waren vorher noch nie beschrieben worden. Erwähnenswert ist in diesem Zusammenhang die Entwicklung einer neuen Mikroderivatisierungsmethode für Hydroxysäureamide, bei der diese in acetylierte Nitrile übergeführt werden. Die so erhaltenen Produkte lassen sich leicht mittels GC/MS identifizieren ${ }^{[28]}$.

Zur Zeit erlebt die Erforschung der marinen Naturstoffe auch in Deutschland einen buchstäblichen Boom. Während sich Mikrobiologen, Zoologen und Botaniker mit den Aspekten der biologischen Struktur, Funktion und Dynamik von Lebensgemeinschaften befassen, suchen Mediziner, Pharmazeuten und Chemiker nach neuen, wirksamen Substanzen.

Marine Naturstoffe besitzen ein großes Potential für pharmakologische Anwendungen. Die Verbindungen aus dem Meer sind meist besser wasserlöslich und wirken äußerst effektiv in kleinsten Konzentrationen, da das wässrige Milieu, in dem sie üblicherweise eingesetzt werden, sie sehr rasch verdünnt. Diese extreme Wirksam- 
keit ist auch bei Arzneimittel erwünscht. Geringe Dosierung verringert außerdem die Gefahr der Akkumulation eines Medikamentes im Körper ${ }^{[29]}$.

Laut Angaben des National Cancer Institute (NCI) stammt mehr als die Hälfte der Naturstoffe, die aktuell auf ihre Einsetzbarkeit in der Pharmazie geprüft werden, aus dem Meer.

Ein Zellgift der Seescheide Ecteinascidia turbinata, das Ecteinascidin 743 (ET743) (17), wird zur Zeit neben dreißig anderen Substanzen „aus dem Meer“ als Antikrebsmittel in klinischen Studien erprobt ${ }^{[30]} .200$ Patente für neue Meeresmedikamente wurden in den vergangenen drei Jahren eingereicht. Insgesamt liegt dennoch die Zahl aller marinen Naturstoffe noch unter 10000.

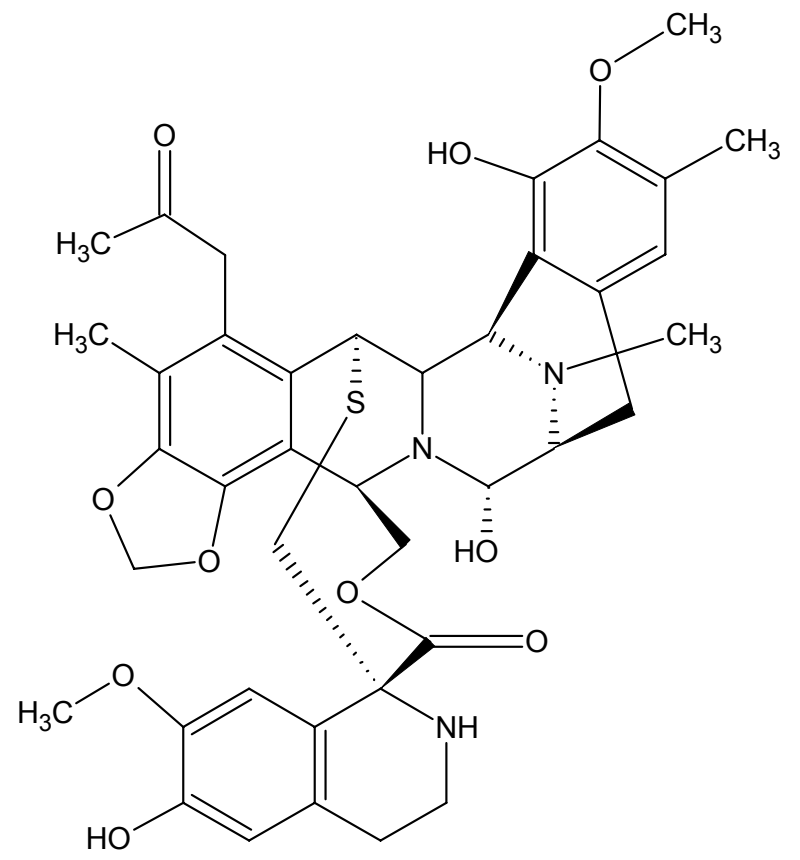

17

Saxotoxin, Tetrodotoxin und Palytoxin (18) werden bereits in der Pharmakologie eingesetzt. Der bekannteste Taxol-Konkurrent marinen Ursprungs in der AntikrebsTherapie ist Bryostatin (19), welches sich nun in der letzten Phase der klinischen Prüfung befindet.

Bereits in den fünfziger Jahren wurden sporadisch Versuche unternommen, Sekundärmetabolite aus Meeresbakterien und Schwämmen zu extrahieren. Aus dem karibischen Schwamm Cryptotethya crypta wurde Spongouridin und Spongothymidin isoliert. 
Ein hoch bromiertes Pyrrol-Derivat, das Pentabrompseudilin, wurde 1966 aus einem marinen Bakterium der Gattung Alteromonas isoliert, das auf der Oberfläche von karibischem Seegras gefunden wurde ${ }^{[31-33]}$.

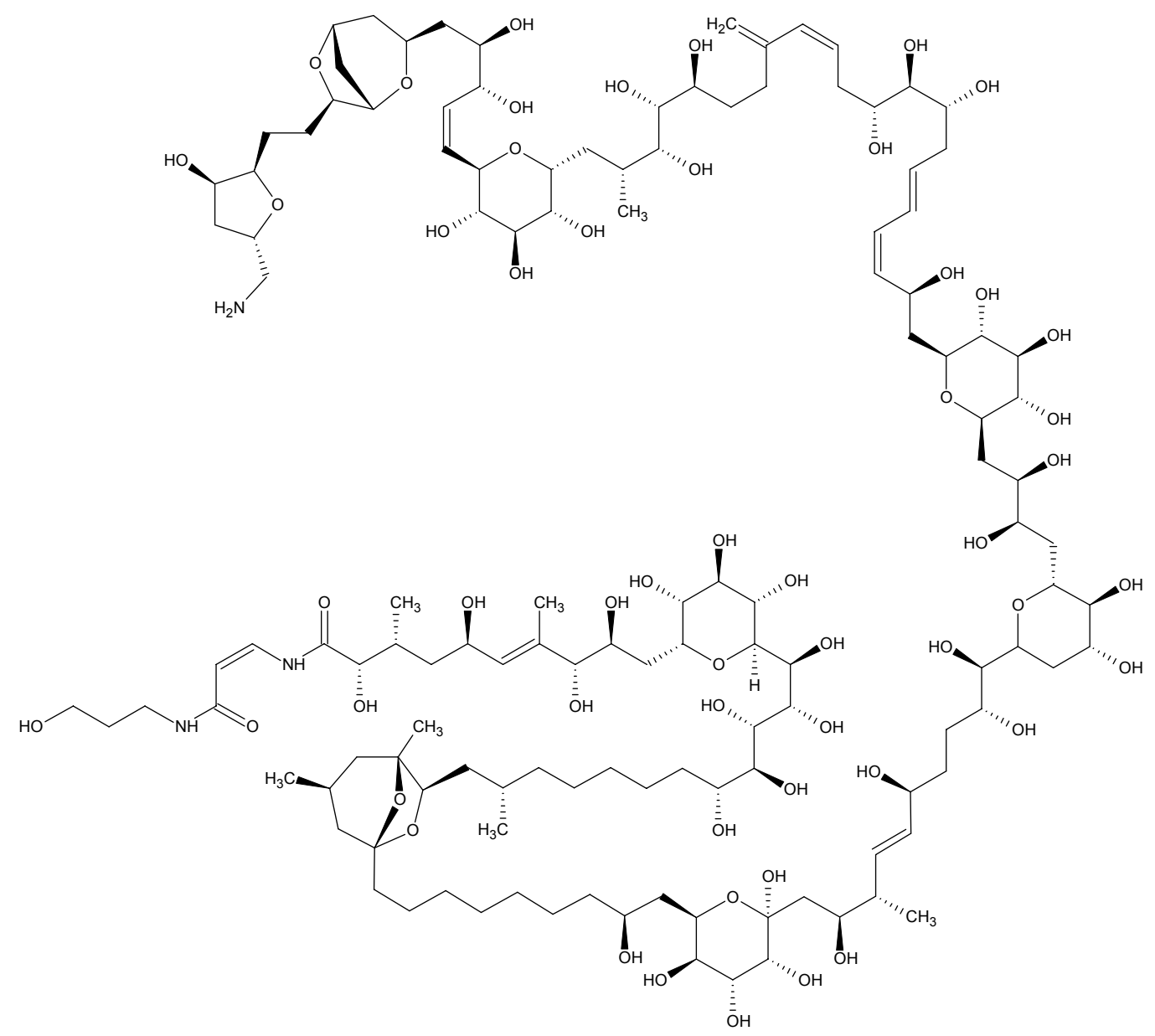

18

Aus einem Meeresschwamm (Porifera) stammt das antivirale Medikament Aciclovir $\AA$, das seit 1995 als Medikament gegen Lippenherpes und Gürtelrose mit Erfolg eingesetzt wird.

Das bislang größte bekannte nicht-peptidische Naturprodukt ist das Maitotoxin, eine Verbindung, die 1000fach giftiger als Nicotin wirkt (die tödliche Dosis für Nicotin liegt bei $50 \mathrm{mg}$ ). Es kommt in dem marinen Einzeller Gymnodiscus toxicus vor und verursacht schwere Fischvergiftungen.

Die Organismen, aus denen wirtschaftlich attraktive Naturstoffe gewonnen werden, stammen hauptsächlich aus wärmeren Gewässer. Die Polarmeere, Tiefseen und das Wattenmeer wurden auf diesem Gebiet wenig erforscht. 
Festsitzende, wirbellose Tiere, Algen und marine Mikroorganismen zählen zu den reichhaltigsten Quellen neuer, biologisch aktiver Naturstoffe. Besonders die sesshaften Tiere, die auf dem Untergrund fest verankert sind, haben besondere chemische Verteidigungsapparate entwickelt, um in den ungünstigen Umweltbedingungen überleben zu können. Das Moostierchen Bugula neritina scheidet ebenfalls 19 aus, um sich vor Feinden zu wahren ${ }^{[33]}$. Nach näheren Untersuchungen und Behandlung mit Antibiotika konnte man allerdings belegen, dass diese giftigen Substanzen nicht von den Moostierchen selbst, sonder von ihren Symbionten, dem noch nicht endgültig identifizieren Bakterium Candidatus endobugula, produziert wird ${ }^{[34]}$. Ähnliche symbiotische Systeme fand man bei Schwämmen Aplysiana aerophoba und Aplysiana cavernicola. Im Körper dieser Schwämme hat man außergewöhnlich hohe Mengen von Mikroorganismen vorgefunden, die bis zu 40 \% der Biomasse des Tieres ausmachen $^{[35]}$.

Die altchinesischen Überlieferungen bezeugen, dass Algenpräparate bereits vor 3000 Jahren in der Volksmedizin als Heil- und Vorbeugungsmittel Verwendung fanden. Die Mikroalge Spirulina wird gegenwärtig in der Begleittherapie bei Tumorerkrankungen genutzt. Aus einigen Algen isolierte man den gegen Brustkrebs vorbeugenden Wirkstoff Fucoidan. Aus den bis heute untersuchten 2000 Arten von Manteltieren gewann man eine Reihe von Cyclopeptiden, polycyclischen Alkaloiden, Indolderivaten (wie Grossularin, (20)), und Schwefelverbindungen. Neben einer antiviralen und immunsupressiven Aktivität besitzt es vor allem cytostatische Eigenschaften. Ein unter den Naturstoffen einmaliges Schwefelgerüst, ein Benzopentathiepin, zeichnet das Varacin (21) aus. Ein aus Lissoclinum vareau isoliertes, antimykotisch und tumorhemmend wirkendes Alkaloid ist das Dideminin B (22) ${ }^{[36]}$. Das Cyclopeptid 22 gehört zu den wirksamsten Verbindungen dieser Strukturklasse. 


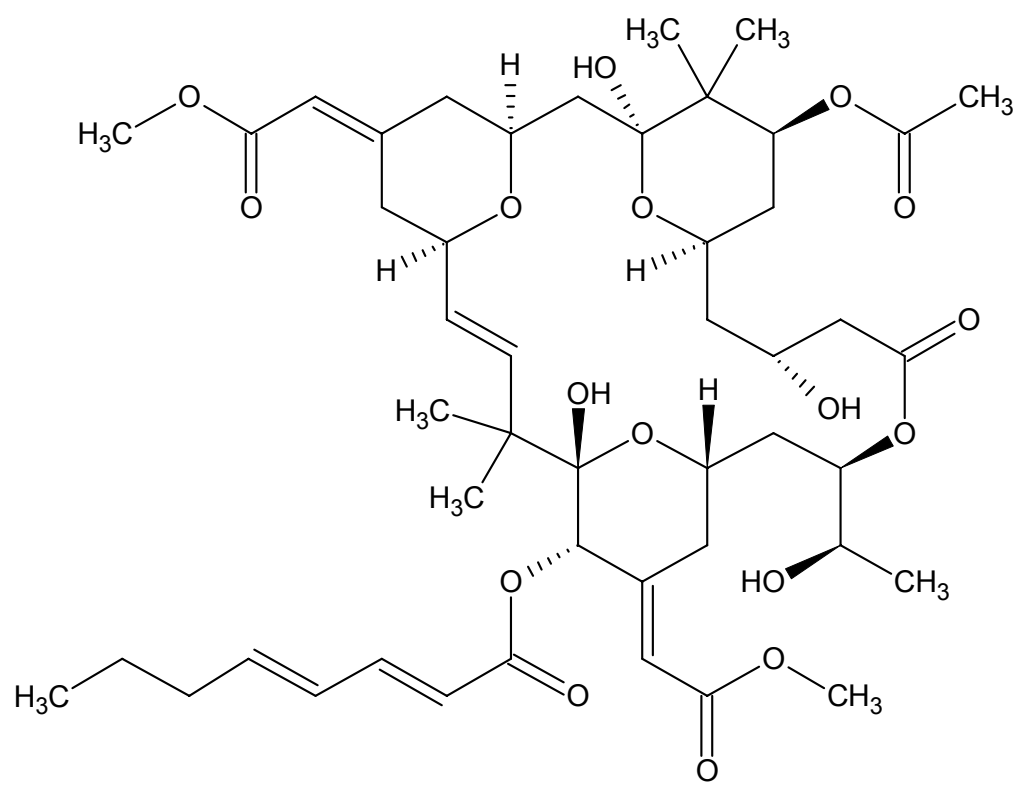

19

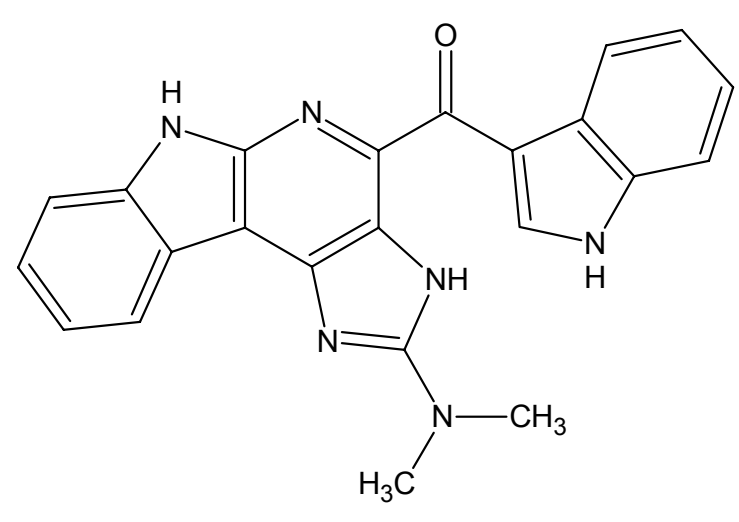

20<smiles>COc1cc(CCN)c2c(c1OC)SSSSS2</smiles>

21

Problematisch erscheint bei der industriellen Gewinnung der wirksamen Substanzen aus marinen Mikroorganismen in fast allen Fällen deren schwere Kultivierbarkeit und unzureichende Produktivität. Deren Optimierung ist daher ein zentraler Punkt bei der Erforschung dieser Klasse von Lebensformen. Oft erweist sich die elementare Aufgabe, die natürlichen Lebensbedienungen im Forschungsbetrieb zu rekonstruieren, als schwierig genug (z.B. bei Barophilen) ${ }^{[37]}$. Meist ergeben sich erst in komplexen Seewassermedien optimale Wachstumsbedingungen. Es stellte sich heraus, dass weniger als $5 \%$ aller marine Bakterien unter Standardbedingungen wachsen. Sie stellen mannigfache Ansprüche an Salzgehalt, Nährstoffe, Temperatur, Druck u.a. Es muss dennoch ein Kompromiss zwischen Reproduzierbarkeit des Mediums und der Produktivität der Stammkultur gefunden werden. 


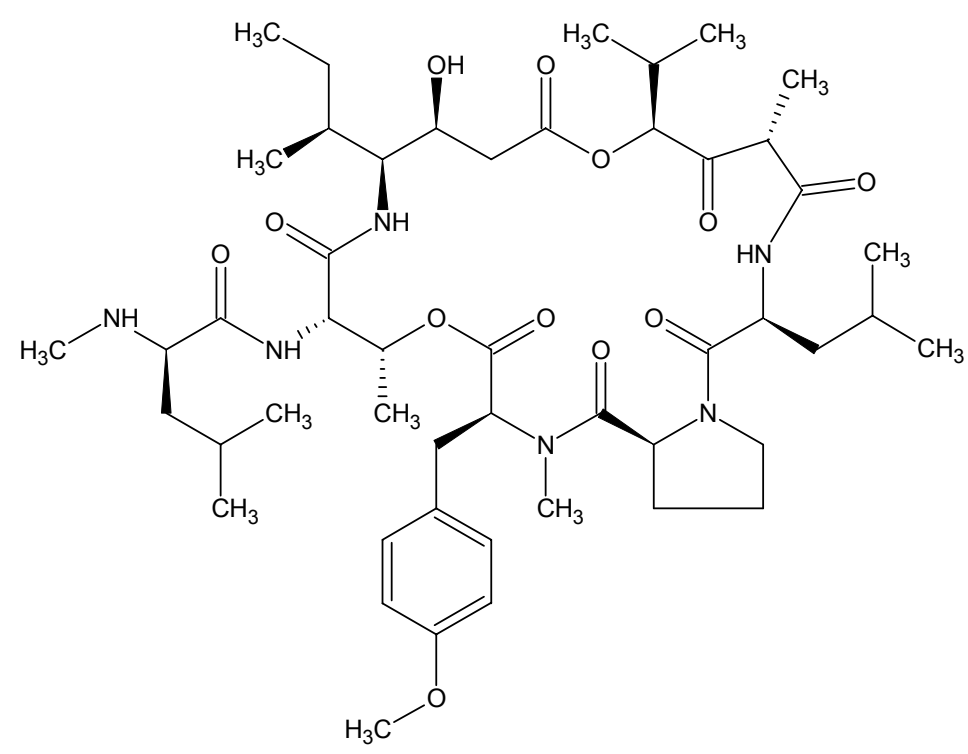

22

Oft sind die geringen Ausbeuten an Biomasse das größte Hindernis in der Identifizierung neuer Stoffe. Dadurch wächst automatisch die Nachfrage nach neuen, noch empfindlicheren analytischen Methoden. Bei der Probenaufarbeitung wird außer den etablierten Extraktionsmethoden verstärkt auch die schonende Festphasenextraktion erfolgreich eingesetzt.

Aus $600 \mathrm{~kg}$ eines Schwamms Halichondria okodai konnte man gerade $12.5 \mathrm{mg}$ der antitumorwirkender Substanz Halichondrin B1 gewinnen. Letzteres ist ein cyclisches Lacton, dessen Antitumorwirkung auf der Bindung an Proteinkinase $\mathrm{C}$ beruht.

Inzwischen kennt man mehr als 20 unterschiedliche Derivate von 19, die sich lediglich in den Säureresten unterscheiden. Angesichts der sehr niedrigen Konzentration im Zellmaterial $\left(10^{-8}\right.$ bis $10^{-3} \%$ der Biomasse) werden 500 bis $1000 \mathrm{~kg}$ Bryozoen benötigt, um wenige Milligramm dieser Verbindung zu erhalten. Inzwischen werden die Bryozoen von einer Biotechnologiefirma (CalBioMarine-Technologies) in 5000 Liter-Tanks gezüchtet.

Die große Komplexität vieler Substanzen stellt nicht nur für den Analytiker ein Problem dar. Die chemische Synthese eines Wirkstoffes ist oft die einzige Möglichkeit, wirtschaftlich und pharmazeutisch nutzbare Mengen herzustellen. Anderseits bringt eine Vereinfachung der Struktur sporadisch auch eine Steigerung der biologischen Aktivität. Eine moderne Strategie, die Produktivität zu steigern, ist die für die Produktion der Metabolite verantwortlichen Gene zu identifizieren und sie in das Genom eines gut kultivierbaren und anspruchlosen Mikroorganismus wie E. coli zu insertie- 
ren. Haygood et al. ${ }^{[30]}$ haben bei der Untersuchung von Candidatus endobugula das Gen gefunden, welches für die Bryostatinbildung kodiert. Aufgrund dieser Entdeckung hofft man auf eine effiziente biotechnologische Produktion dieser Verbindung.

\section{Meerespilze als eine potenzielle Quelle neuer Naturstoffe}

Bis heute hat man erst 1-5 \% der insgesamt im Meer vorhandenen Diversität der Pilze erforscht, sehr wenig ist auch über die von ihnen produzierten Substanzen bekannt. Neben den Sekundärmetaboliten produzieren die marinen Pilze weitere Substanzen, wie Enzyme, Peptide, und hochungesättigte Fettsäuren, die nicht nur für die Pharmaindustrie von Bedeutung sein können.

Der charakteristische Stoffwechsel der Pilze basiert wesentlich auf der Produktion bestimmter Enzyme, deren physiologische Funktionen bei marinen Pilzen nur in Einzelfällen untersucht wurden. Die Artcharakterisierung, Taxonomie, Erforschung der Naturstoffproduktion und Klärung der Rolle dieser Lebewesen in ihrem Habitat ist ein aktuelles Thema, das von vielen Forschungsgruppen bereits aufgegriffen wurde.

Fast alle bekannten Meerespilze wurden in den obersten Meeresschichten gesammelt. Die neusten Untersuchungen zeigen jedoch, dass auch eine weitreichende Adaptation des Lebenszyklus an den in den Tiefen herrschenden Druck möglich ist. Ebenso steht die symbiotisch-parasitische Anpassung der Pilze an den Stoffwechsel anderer Meereslebewesen im Mittelpunkt der Beobachtung.

Aus marinen Algen und Schwämmen wurden höhere, myzelbildende Pilze gewonnen. Bei einer Untersuchung von 600 aus Algen isolierter Pilzarten überraschte die hohe Aktivität der Extrakte in Bioassays. Im Pilz Ascochyta alicorniae, der epiphytisch auf Grünalgen in der Nordsee lebt, fand man die Ascosalipyrrolidinone A und B. Ascosalipyrrolidinon A hemmt das Enzym Tyrokinase, das an der Signaltransduktion bei der Zellteilung beteiligt ist. Im Gewebe der marinen Rotalge Liagora vicida kommt ein Pilz vor, der elf bislang unbekannte Sesquiterpenoide herstellt. Diese Verbindungen hemmen das Wachstum von Plasmodien und unterschiedlichen pathogenen Bakterien $^{[1]}$. In einer Studie von U. Höller ${ }^{[38]}$ wurden aus 16 Schwammproben von sechs verschiedenen Herkunftsstellen (u. a. aus Australien, Malta, Dominika und Helgoland) 681 Pilzstämme aus 53 Gattungen gewonnen. 92 Stämme wurden chemisch analysiert. 81 \% der Stämme zeigten eine antimikrobielle Wirkung in Agardiffusionstests, und 7 Stämme zeigten eine Hemmung der Tyrosin-Kinase (p561ck). Das 
chemische Screeningverfahren lieferte diverse Primär- und Sekundärmetabolite, darunter Microsphaeropsin, Strobilinin, Variabilin (23) und Ulocladol (24) (aus Ulocladium botyris ${ }^{[38,39]}$.

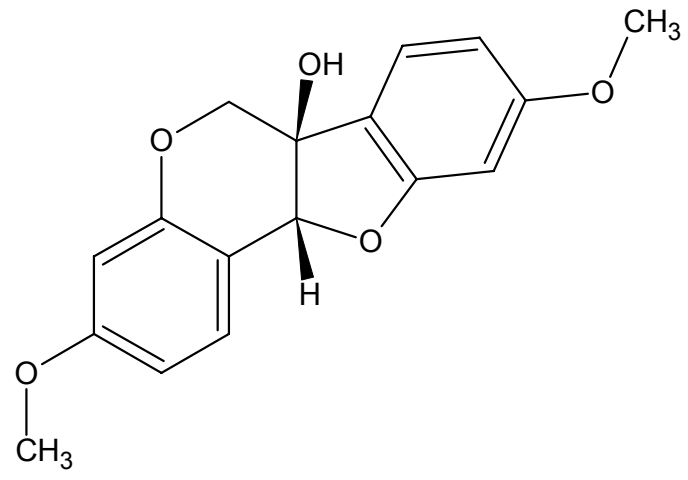

24

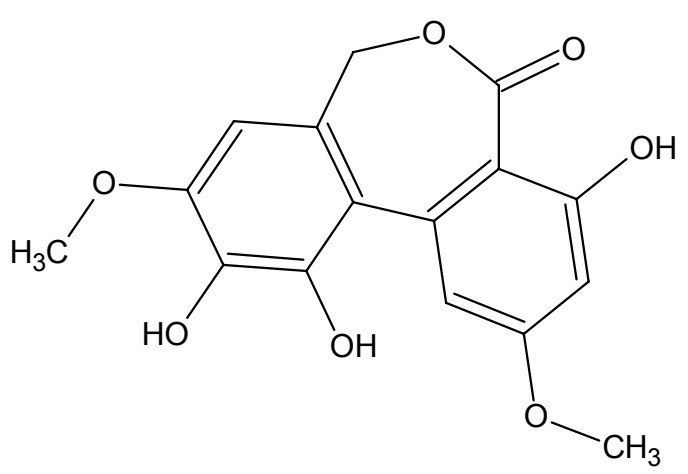

23

Die BRAIN AG isolierte aus 200 marinen Pilzen mit Hilfe moderner Trennverfahren und molekularbiologischer Methoden 120 Metabolite mit Aktivität gegen Tumorzellen. Davon wurden 18 aufgrund ihrer nachgewiesenen spezifischen Wirkung zum Patent angemeldet ${ }^{[40]}$.

\section{Aufgabenstellung}

Im Rahmen des BMBF-Verbundprojektes „Produktion biologisch aktiver Sekundärmetabolite durch marine Pilze und Meereisbakterien in Abhängigkeit von ihrer Biodiversität und Kultivierung (Förderkennzeichen 02F0233A) sollten in dieser Arbeit unter anderem auch Stoffwechselprodukte der bisher kaum untersuchten Meerespilze erforscht werden.

In einer zweijähriger interdisziplinären Pilotstudie wurden in Kooperation mit anderen Arbeitsgruppen Untersuchungen zur morphologischen, physiologischen, molekulargenetischen und chemischen Variabilität ausgewählter Gattungen, Arten und Stämme in Mehrfachisolaten mariner Pilze durchgeführt. Die gewonnenen Daten sollten zu allgemeinen Rückschlüssen im zukünftigen Naturstoffscreening mariner Pilzisolate genutzt werden.

Primär sollten die noch weitgehend unbekannten Fähigkeiten der marinen Pilze im Bezug auf die Naturstoffsynthese untersucht, ggf. die für die Aktivität verantwortli- 
chen Substanzen isoliert und identifiziert werden. Ergänzend war die Beteiligung an der Erforschung mariner Streptomyceten vorgesehen.

Bei der Kultivierung mariner Organismen erhält man oft nur sehr geringe Ausbeuten. Die Konzentrationen der produzierten Sekundärmetabolite sind im Durchschnitt viel kleiner als z.B. bei terrestrischen Streptomyceten. Dieses ungünstige Verhältnis kann durch mehrere Ansätze positiv beeinflusst werden. So könnte man etwa nur die ergiebigsten Stämme auswählen und bearbeiteten.

Einen vollständig anderen Ansatz bietet die Optimierung der Kultivierungsbedingungen und der Entwurf solcher Arbeitsmethoden, die auch die weniger effektiven Mikroorganismen zur Produktion größerer Mengen an Naturstoffen veranlassen. Dadurch sollten auch diejenigen Organismen zur Produktion neuer Leitstrukturen herangezogen werden können, die bei der üblichen Methode nicht berücksichtigt worden wären. Diese Überlegung geht auf die Tatsache zurück, dass unter normalen Wachstums- und Lebensbedingungen nur ein geringer Prozentsatz des in den Zellen vorhandenen genetischen Materials auch tatsächlich aktiv ist, exprimiert wird und so Metabolite erzeugt. Durch geeignete Anzuchtbedingungen sollten die ausgesuchten Stämme zur Aktivierung bislang inaktiver Gene angeregt werden.

Zahlreiche technische Prozesse lassen sich mathematisch nur durch nichtlineare Funktionen beschreiben; sie werden zudem oft durch zahlreiche Variable beeinflusst, was Voraussagen über den Ablauf des Vorgangs oder die Suche nach Extremwerten schwierig macht. Für die Quantifizierung wurden daher spezielle Rechenverfahren zur Prozessoptimierung entwickelt, die z. B. genetische Algorithmen oder statistische Methoden einsetzen. Derartige Verfahren eignen sich grundsätzlich auch für Fermentationsoptimierungen, haben jedoch - zumindest an Universitäten - bisher kaum Verbreitung gefunden. Es ist jedoch offensichtlich, dass die Ausbeuteoptimierung schon bei kleinen Ansätzen zu erheblichen Kosten- und Zeiteinsparungen führen würde. Genetische Algorithmen bieten zudem den einzigen Weg, den Einfluss komplexer Medienzusammensetzungen auf die Produktbildung systematisch zu erfassen. Ein weiteres Ziel der vorliegenden Arbeit war daher, diese mathematischen Modelle bei der Medienoptimierung einzusetzen.

Gleichzeitig sollte ein neues Konzept zur Anzucht der Mikroorganismen erprobt werden, das die Möglichkeit bietet, die mit Optimierungsroutinen erzeugten Daten möglichst rasch im Labor umzusetzen. Da dies im diskontinuierlichen Fermenter nur 
schwer zu realisieren ist, sollten mehrere kleine kontinuierlich zu betreibende Bioreaktoren gebaut und mit einer elektronischen Steuerung versehen werden.

Die vorliegende Arbeit gliedert sich dadurch in drei Teile: in das Screening mariner Mikroorganismen und die Isolierung und Strukturaufklärung ihrer Metabolite, die Entwicklung elektronisch gesteuerter Durchlauffermenter sowie die Erprobung genetischer Algorithmen zur Medienoptimierung. 


\section{Untersuchungen von Extrakten mariner Pilze}

\section{Genetische Diversität der untersuchten Pilzproben}

Mehrfachisolate aus insgesamt 11 Arten und 6 Gattungen aus Kultursammlungen mariner Pilze (Bremerhaven, KMPB) wurden in Pepton-Malz-HefeextraktNährlösung mit Meerwasser angezogen und in Bremerhaven taxonomisch und molekulargenetisch analysiert. Zur Bestimmung der Diversität wurden in der Arbeitsgruppe von Dr. K. Schaumann Genclusteranalysen durchgeführt. Mit Fluoreszenzmarkern verbundene Gensonden erlaubten die taxonomische Einordnung der Pilzstämme durch Fluoreszenz-in-situ-Hybridisierung (FISH).

Die Sequenzierung des D1-2-Abschnitts der LSU rDNA sowie ITS1-, 5,8S-, ITS2und 18S-Regionen der rDNA haben dabei eine größere Heterogenität offenbart, als dies anhand der bisher ausschließlich angewandten morphologischen und ökophysiologischen Identifikation der Taxa vermutet wurde ${ }^{[41]}$. Zum Teil machen die neuen Erkenntnisse sogar taxonomische Umstellungen notwendig.

Auch im biologischen und chemischen Screening fand man eine entsprechende Variabilität, die sich in den Wirkungsspektren sowie den komplexen Ergebnissen von HPLC/UV und HPLC/MS-Untersuchungen wiederspiegelt.

Langjährig in Kultur gehaltene Pilzstämme zeigten dabei deutlich weniger Strukturvielfalt und Bioaktivität als frisch isolierte und sind offenbar metabolisch verkümmert. In frischen Isolaten obligat mariner Pilze fand man dagegen zahlreiche Verbindungen mit Molmassen zwischen 150 und 1500, die in keiner Datenbank auftauchten. 


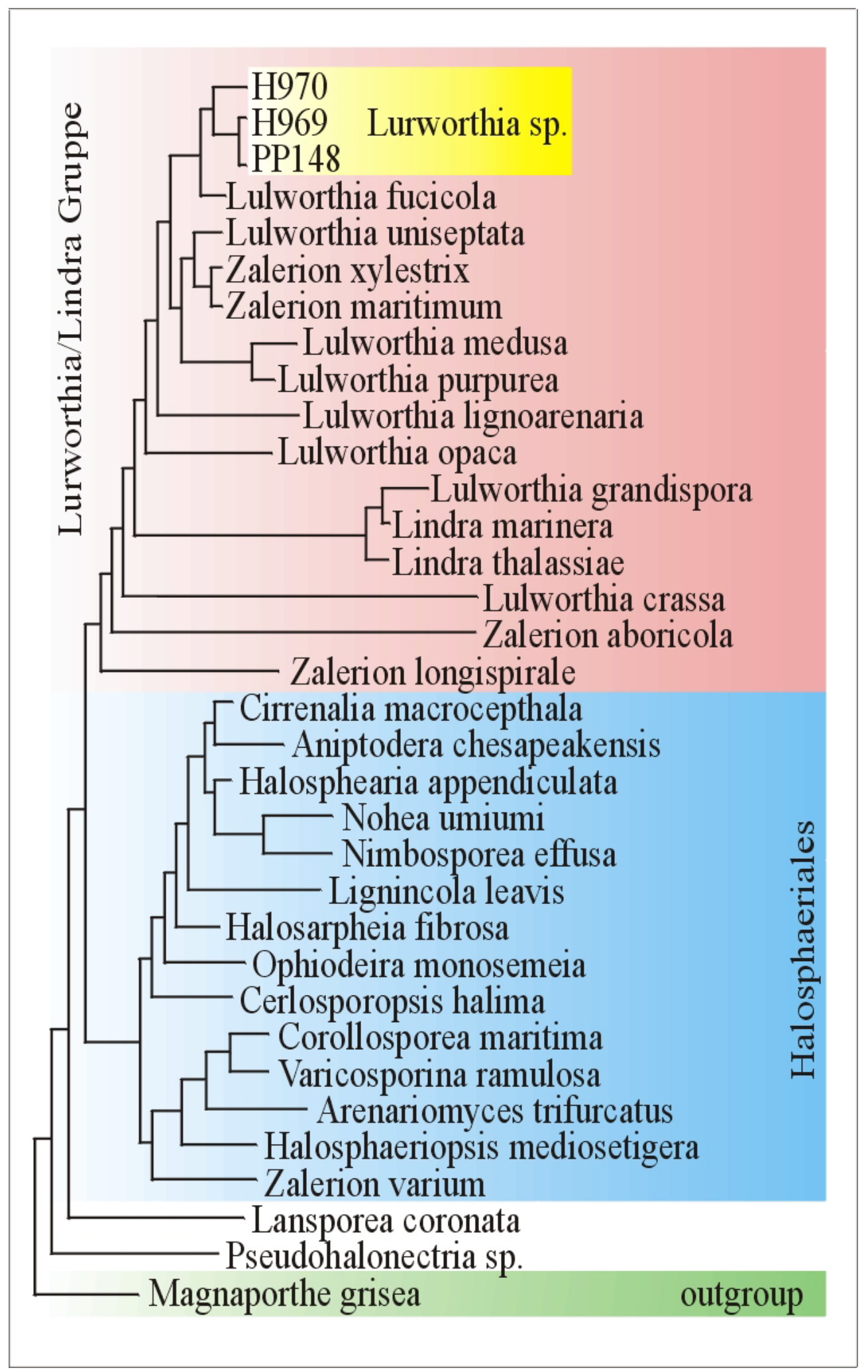

Abbildung 3: Unterschiede der Taxonomie in der Variabilität der Aktivität und chemischen Zusammensetzung der Extrakte. 


\section{Primärscreening der Pilzextrakte}

Zur chemischen Untersuchung hat K. Schaumann 92 Pilzstämme zur Verfügung gestellt und im Alfred-Wegener-Institut für Polar- und Meeresforschung in Bremerhaven in Standkulturen angezüchtet.

Das Pilzmaterial wurde mit Essigester abgetötet und in Glasflaschen aus Bremerhaven geliefert. Die organischen Reste des Pilzmaterials befanden sich in einem Gemisch aus Wasser und Essigester, was die unmittelbare Gefriertrocknung ausschloss. Abdestillieren der Flüssigkeit schien unnötig viel Zeit in Anspruch zu nehmen und wurde deswegen nicht angewendet. Der Inhalt der Behälter wurde mit dem Ultraturrax zerkleinert und anschließend dreimal mit Essigester extrahiert. Am Ende der Aufarbeitungsphase wurden die Proben mit Cyclohexan entfettet. Man erhielt 92 farblose Pilzextrakte. Die erhaltenen Mengen an Rohextrakt lagen zwischen 20 und $50 \mathrm{mg}$ je Probe.
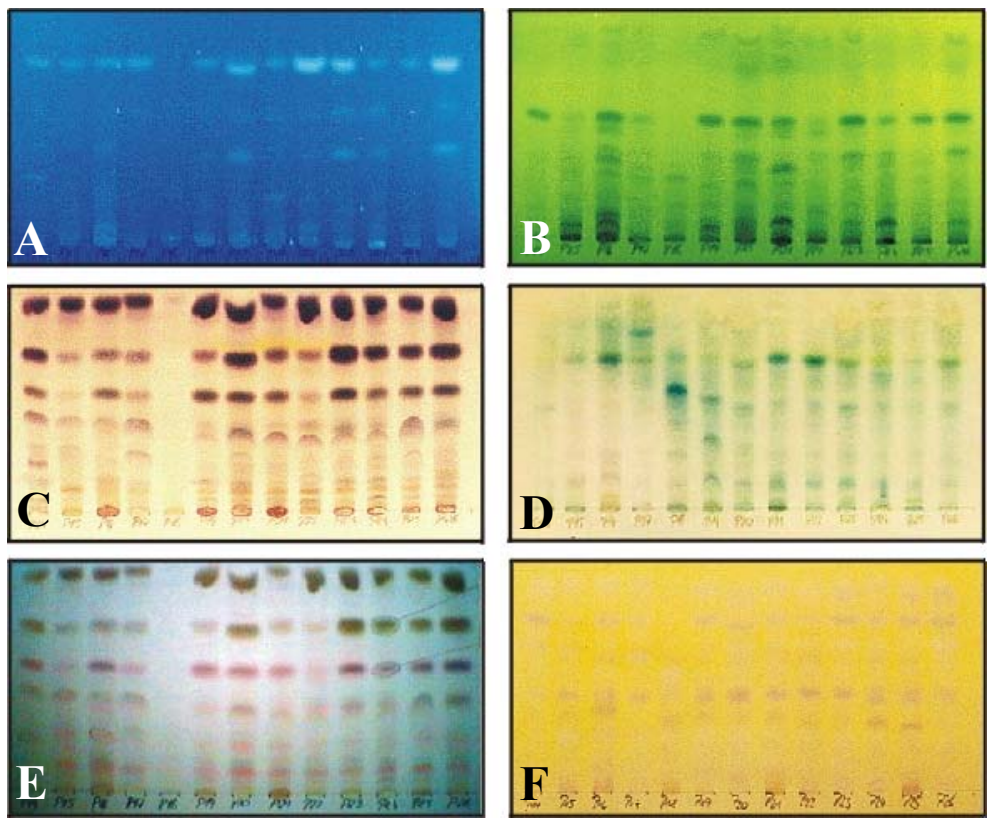

Abbildung 4: Chromatogramme von 26 marinen Pilzextrakten. A) UV 366 nm, B) UV 254 nm, C) Anisaldehyd/Schwefelsäure (mit einem Grafikprogramm veränderte Farbkontraste),

D) INPTC/KOH, E) Anisaldehyd/Schwefelsäure, F) Ehrlich/HCl .

Um möglichst viele Informationen bezüglich der chemischen Zusammensetzung der Proben zu erhalten, entwickelte man parallel zu dem HPLC-Screening auch Dünnschicht-Chromatogramme, die bei zwei unterschiedlichen Wellenlängen (254 und $366 \mathrm{~nm}$ ) photographiert und mit verschiedenen Sprühreagenzien besprüht wurden. Zusätzlich konnte man durch den Einsatz des Diodenarraydetektors im Bereich von 
$180 \mathrm{~nm}$ bis $720 \mathrm{~nm}$ im HPLC-Screening belegen, dass die untersuchten Pilze bevorzugt farblose bis leicht gelbe Substanzen produzierten, deren Absorptionsmaximum unter $350 \mathrm{~nm}$ lag.

Auf den ersten Blick sahen die erhaltenen Chromatogramme zwar ziemlich ähnlich aus. Die HPLC-Untersuchungen zeigten jedoch, dass es sich bei manchen Stämmen offensichtlich zwar um strukturell verwandte Strukturen, aber unterschiedliche Moleküle handelte.

Um diesen Verdacht zu bestätigen und gleichzeitig die Molmassen der Metabolite zu bekommen, wurden im HPLC-Screening aufgefallene Pilzstämme mittels HPLC-MS untersucht. Von den im Extrakt erhaltenen Verbindungen wurden ESI-Spektren sowohl im positiven als auch im negativen Modus aufgenommen.

Man fand nicht nur kleine Moleküle, deren Massen nicht größer als $m / z 300$ waren, sondern auch Metabolite mit Molmassen > 1000. Aus den Massenspektren mit Signalen bei $\Delta \mathrm{m}=14$ konnte man darauf schließen, dass die Pilze oft homologe Verbindungen herstellten, die sich lediglich in der Länge der Seitenketten unterschieden.

Die HPLC-Trennung des Extraktes aus Lutoworthia sp. ergab mehr als 14 Signale, von denen 7 Massenspektren gemessen wurden. Wie man am Spektrum des HPLCPeaks 2 (s. Abbildung 5) erkennt, liegt ein Gemisch von Homologen vor, deren Masse sich um jeweils 44 Dalton unterscheidet. Es könnte sich also z.B. um Kronenether aus 10-20 Ethylenglycol-Einheiten handeln. Entsprechende Naturstoffe sind bisher unbekannt, die Identität muss also noch bestätigt werden.

Auch bei den anderen Spektren wurden die erhaltenen Massen mit den in Naturstoffdatenbanken gespeicherten Daten verglichen. Die Recherchen ergaben entweder überhaupt keine oder aber nur wenige Treffer. 

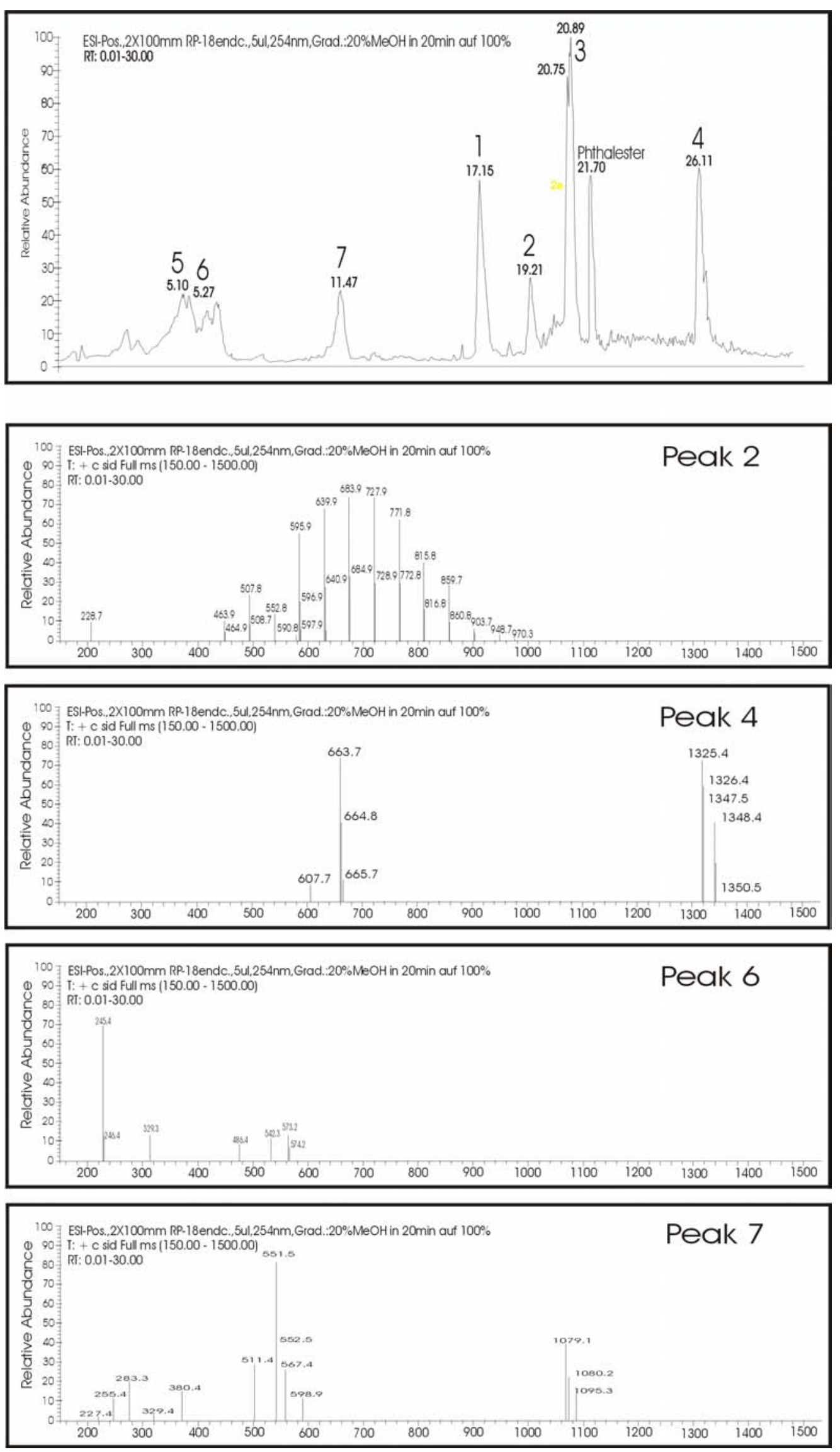

Abbildung 5: Ausgewählte Ergebnisse der HPLC/MS- Untersuchungen von Extrakten mariner Pilze. 
Die kleine Menge des zur Verfügung stehenden Pilzmaterials erlaubte nicht die präparative Trennung der Substanzen und genauere Untersuchungen der Zusammensetzung. Die gewonnenen Extraktmengen ließen dennoch zu, die Aktivität gegen verschiedene Mikroorganismen zu ermitteln.

Im biologischen Screening verwendete man Agarplattendiffusionstests mit Escherichia coli, Bacillus subtilis, Mucor miehei, Candida albicans, Streptomyces viridochromogenes (Tü57), Staphylococcus aureus und den Mikroalgen Chlorella sorokiniana, Chlorella vulgaris und Scenedesmus subspicatus.

Leider zeigten nur wenige der getesteten 92 Extrakte überhaupt eine bemerkbare Aktivität gegen die im biologischen Screening verwendeten Erreger. Die Algenaktivität wurde bei 66 Extrakten getestet und nur bei dreien fand man schwache Hemmhöfe (s. Abbildung 6).

Diese Ergebnisse diskriminieren aber noch nicht diese Substanzquelle als einen denkbaren Lieferanten neuer und interessanter Strukturen. Sicherlich ist die schwache biologische Aktivität der Pilze auch durch die sehr kleinen Konzentrationen der Substanzen im Rohextrakt bedingt. Eine andere Erklärung kann die taxonomische Ähnlichkeit der Proben liefern (s. Abbildung 3).

\begin{tabular}{|c|c|c|c|}
\hline Algen & 3 & 66 & \\
\hline Candida albicans & 3 & 66 & \\
\hline Mucor mehei & 1 & & 92 \\
\hline Escherichia coli & 10 & & 92 \\
\hline Tü57 & 7 & & 92 \\
\hline Bacillus subtilis & 5 & & 92 \\
\hline Staphylococcus aureus & 4 & & 92 \\
\hline Salinenkrebse & 2033 & & \\
\hline
\end{tabular}

Abbildung 6: Die Aktivität der Pilzextrakte. Schwarze Balken repräsentieren die Zahl der aktiven Proben, die Zahlen am Rand die Anzahl der getesteten Proben.

\section{Stamm KMPB H963 (Lulworthia sp.)}

Der Stamm KMPB H963 (Lulworthia sp.) wurde im Alfred-Wegener-Institut für Polar- und Meeresforschung in Bremerhaven auf Potato-Dextrose-Broth mit Meer- 
wasser innerhalb von 35 Tagen bei $20^{\circ} \mathrm{C}$ angezogen. Es handelt sich bei dem Isolat um einen Pilz, der zwar in den Tests keine biologische Aktivität aufwies, aber dessen Extrakte im HPLC-Chromatogramm interessante Peaks gezeigt hatte.

Die gesamte Biomasse wurde abfiltriert und gefriergetrocknet, wobei aus 20 Litern Kultur 6.3 g Mycel erhalten wurden. Die Zellmasse wurde mit dem Ultraturrax aufgeschlossen und mit Methanol behandelt. Nach dem Abfiltrieren des Lösungsmittels extrahierte man die unlöslichen Bestandteile dreimal mit Ethylacetat. Die Methanolund Essigester-Phasen wurden vereinigt und eingeengt. Das Entfetten erfolgte durch dreimaliges Ausschütteln mit Cyclohexan, wobei 340 mg methanolischer Rohextrakt zurückblieben. In dünnschichtchromatographischen Untersuchungen waren bei 254 nm zwei schwache UV-löschende Zonen zu erkennen, die sich nach Ansprühen mit Anisaldehyd/Schwefelsäure schwach braun verfärbten. Die Vortrennung des Extraktes an einer Kieselgel-Säule ergab mehrere Fraktionen, die nach dem Einengen weiße, im Dünnschichtchromatogramm nicht sichtbare Rückstände ergaben. Die präparative HPLC-Trennung ergab drei kristalline Substanzen, die sich sehr gut in Wasser und schlechter in Methanol lösten. Die Spektren bestätigten die Vermutung, dass es sich bei diesen Verbindungen um Kohlenhydrate handelte. 


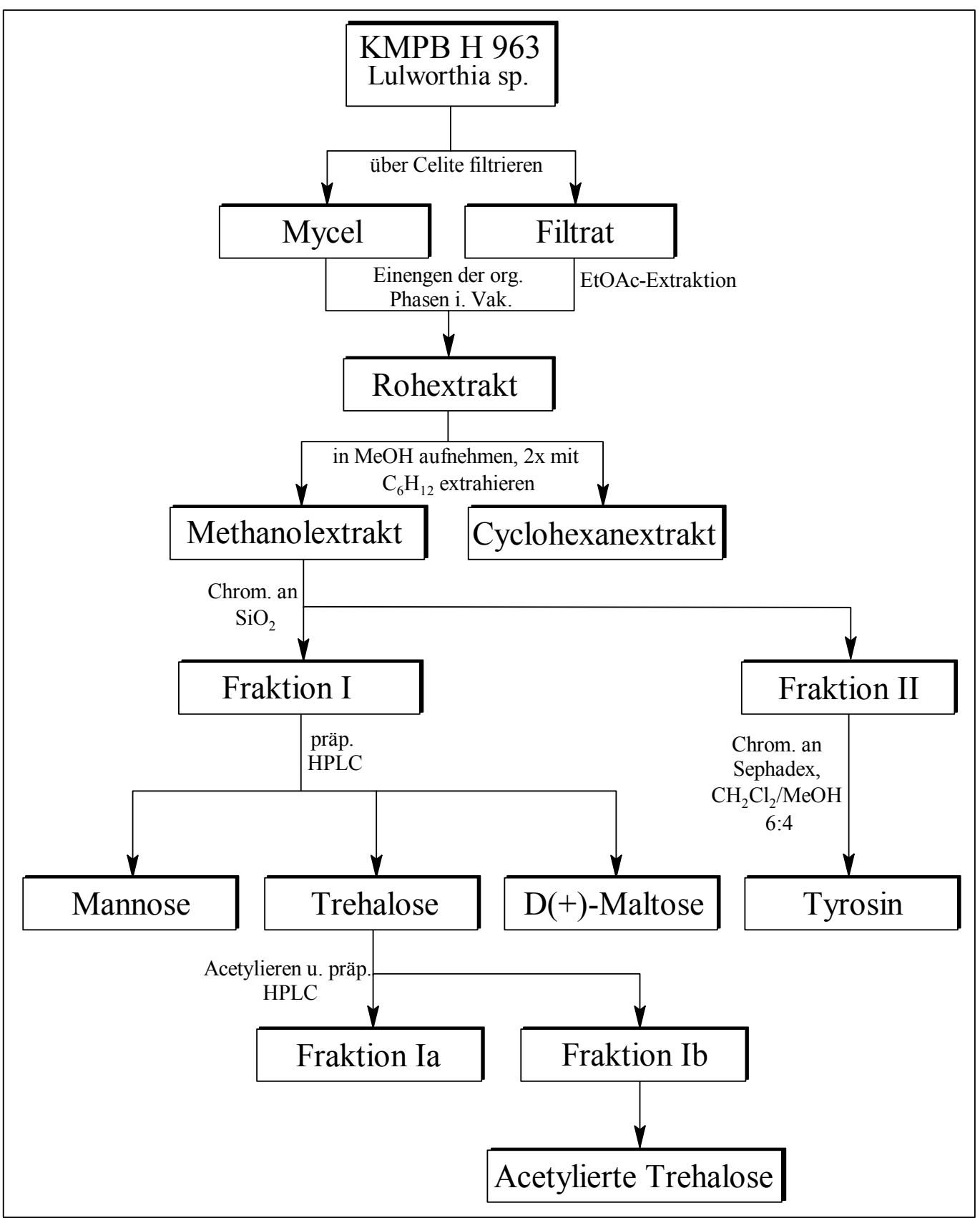

Schema 1: Aufarbeitung des Stammes KMPB H 963.

\section{L-Tyrosin}

Aus der UV-löschenden Fraktion II konnte man durch eine zusätzliche Reinigung an Sephadex LH 20 eine aromatische Verbindung mit $R_{\mathrm{f}}=0.2\left(\mathrm{CH}_{2} \mathrm{Cl}_{2} / \mathrm{MeOH}, 95: 5\right)$ gewinnen, die sich beim Besprühen mit Anisaldehyd/Schwefelsäure braun verfärbte und im negativen Modus (ESI) einen Massenpeak bei $m / z 181$ ergab. Im ${ }^{1}$ H-NMRSpektrum konnte man im aromatischen Bereich ein A,A'B,B'-Signal $(4 \mathrm{H})$ sehen, wie es charakteristisch für einen para-disubstituierten Benzolring ist. Weiterhin fand man 
bei $\delta=4.00$ ein Doppeldublett $(1 \mathrm{H})$, bei $\delta=2.80(1 \mathrm{H})$ und bei $\delta=2.60(1 \mathrm{H})$ ebenfalls je ein Dublett vom Dublett. Eine Datenbank-Suche (AntiBase) ergab Tyrosin (25), das alle geforderten Kriterien erfüllte. Ein Vergleich der ${ }^{1} \mathrm{H}-\mathrm{NMR}$ - und ${ }^{13} \mathrm{C}$ NMR-Spektren (SDBS-Spektrendatenbank sowie eigene Messungen) bestätigte diese Annahme.

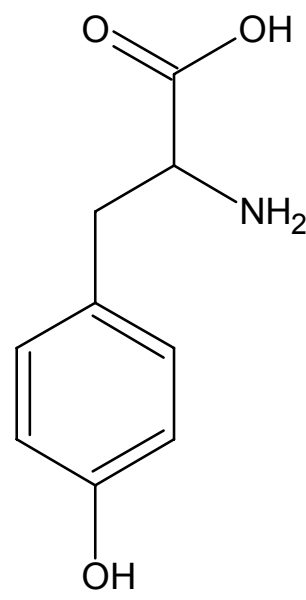

25

\section{D(+)-Maltose}

Aus der Fraktion I konnte man durch präparative HPLC 18 mg einer weißen, in Wasser löslichen Verbindung isolieren, die im Dünnschichtchromatogramm weder im UV-Licht noch durch Farbreagenzien sichtbar zu machen war.

Die ersten Vermutungen, dass es sich bei der gesuchten Verbindung um ein Kohlenhydrat handelt, haben die aufgenommenen ${ }^{1} \mathrm{H}-\mathrm{NMR}$ - und ${ }^{13} \mathrm{C}-\mathrm{NMR}-$ Spektren bestätigt. Nach der Zahl der Signale lag ein Disaccharid vor, was durch die bei ESIMassenspektrometrie gefundene Masse von 342 gestützt wurde. Wenn die glycosidische Bindung zwischen den acetalischen Kohlenstoffatomen beider Monosaccharide liegt, werden beide Ringe in der Acetalform fixiert und die Zucker verlieren ihre reduzierenden Eigenschaften; die Fehling-Probe verläuft dann negativ ${ }^{[91]}$. Wird das acetalische Kohlenstoffatom eines Monosaccharids mit einem anderen Kohlenstoffatom des zweiten verbunden, so kann es noch eine offenkettige Form annehmen und verliert dadurch seine reduzierenden Eigenschaften nicht und die Fehling-Probe fällt positiv aus. Im Falle des untersuchten Zuckers verlief die Fehling-Probe positiv.

Oligosaccharide können sich nicht nur durch die Verknüpfung der Ringe unterscheiden, sondern auch durch die Stereochemie der Acetalbindung, was manchmal drasti- 
sche Unterschiede in den spektroskopischen Daten verursacht ${ }^{[42]}$. Der ${ }^{13} \mathrm{C}-\mathrm{NMR}-$ Spektrenvergleich mit zahlreichen Disacchariden aus der Literatur (SDBSSpektrendatenbank) ergab eine Übereinstimmung mit Maltose (26), die in der Probe als Gemisch aus $\alpha$ - und $\beta$-Anomer vorlag.

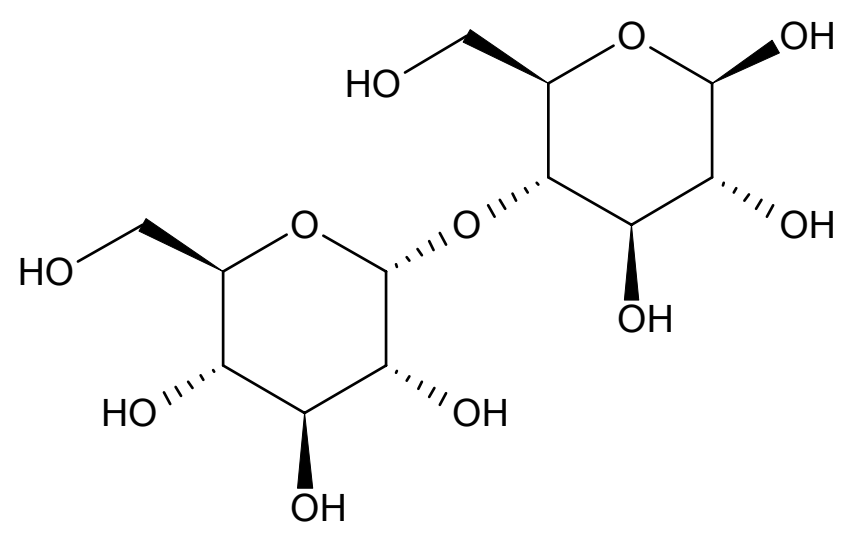

26

\section{Mannose}

Eine zweite Verbindung mit ähnlichen physikalischen Eigenschaften wie die oben beschriebene Maltose (26) wurde ebenfalls mittels präparativer HPLC in reiner Form erhalten. Die Molmasse wurde durch DCI zu 182 Dalton bestimmt. Die Anhäufung der Signale im ${ }^{1}$ H-NMR-Spektrum bei $\delta=3.5 \sim 4.0$ ließ vermuten, dass die gesuchte Verbindung ebenfalls ein Zucker, nach der Masse aber ein Monosaccharid ist. Die Suche in AntiBase lieferte sechs Strukturen, deren Vergleichsspektren die Verbindung als Mannose (27) identifizierten.

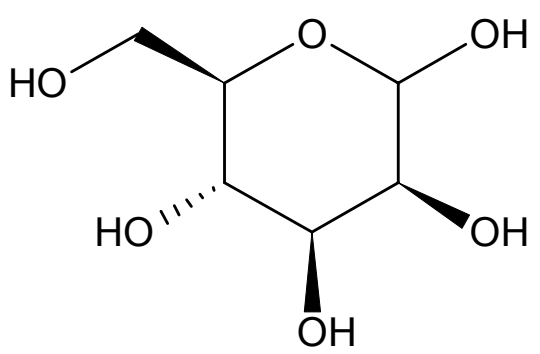

27

\section{$\alpha, \alpha$-Trehalose}

Die aus der Fraktion I isolierte Hauptkomponente (70 \% des Gesamtextraktes) fiel als weißes, kristallines Pulver an. Sie zeigte im ${ }^{1} \mathrm{H}$-NMR-Spektrum ausschließlich Multipletts im Bereich von $\delta=3 \sim 4$, von denen in [D 6 -DMSO mehrere austausch- 
bar waren. Im ${ }^{13} \mathrm{C}$-NMR-Spektrum fand man ausschließlich Signale im Bereich zwischen $\delta=60$ und 80, die man mit Sauerstoff verbundenen Kohlenstoffatomen zuordnen konnte. Fehlende Carbonylsignale schlossen Ester aus.

Die Vermutung, dass es sich auch in diesem Fall um einen Zucker handeln könnte, wurde durch die gute Wasserlöslichkeit und das Fehlen UV-absorbierender Zonen im Dünnschichtchromatogramm bestätigt. Da die Fehling-Probe negativ ausfiel, musste es sich um einen Disaccharid handeln, bei dem die beiden Ringe über die acetalischen Kohlenstoffatome verknüpft sind.

Um die Strukturbestimmung und die Interpretation der Spektren zu vereinfachen, wurden $30 \mathrm{mg}$ des Disaccharids mit Acetanhydrid in Gegenwart von Pyridin und katalytischen Mengen von 4-N,N-Dimethylaminopyridin umgesetzt. Die Reaktionsmischung wurde filtriert und vorsichtig mit Methanol/Wasser unter Eiskühlung hydrolysiert. Nach einigen Minuten fiel ein weißer, flockiger Niederschlag aus, der erneut abfiltriert und mit Wasser gewaschen wurde. Die Endreinigung erfolgte durch präparative HPLC an einer RP18-Säule. Man erhielt zu 68 \% eine in Dichlormethan gut lösliche Verbindung mit der Masse 678, die im ${ }^{1} \mathrm{H}-\mathrm{NMR}-$ Spektrum zusätzliche Signale für die eingeführten Acetatgruppen bei $\delta=2.00$ zeigte. Die gefundene Masse entspricht einem vollständig acetylierten Disaccharid der Summenformel $\mathrm{C}_{28} \mathrm{H}_{38} \mathrm{O}_{19}$. In den Protonenspektren des Acetats fand man nur vier 3H-Singuletts statt der erwarteten acht. Auch die unzureichende Anzahl der restlichen Zucker-Protonen im ${ }^{1} \mathrm{H}$ NMR-Spektrum deutete auf eine symmetrische Struktur hin. Die Vermutung wurde durch die ${ }^{13} \mathrm{C}$-NMR-Spektren bestätigt. Die vier Signale bei $\delta 170$ gehören den Carbonylkohlenstoffatomen in den entstandenen Acetatresten an, das Signal bei $\delta=92$ und vier weitere bei $\delta=66$ gehören zu den Kohlenstoffatomen des Zuckerrings. Das Signal bei $\delta=60$ kann einer Methylengruppe und das Signal bei $\delta=20$ den vier Acetat-Methylresten zugeordnet werden. Insgesamt fand man wegen der Symmetrie nur die Hälfte der erwarteten Signale.

Die Suche im Dictionary of Natural Products lieferte zwei Kandidaten, die $\alpha, \alpha-$ Trehalose (28a) und die $\alpha, \beta$-Trehalose. Erstes ist ein Disaccharid, das sehr oft aus Pilzen, Algen und verschiedenen Insekten isoliert worden ist und ebenfalls im Hefeextrakt und in Schimmelpilzen vorkommt. $\alpha, \beta$-Trehalose ist ein Bestandteil des Bienenhonigs. Wegen der aus den Spektren folgenden Symmetrie des Moleküls musste das $\alpha, \alpha$-Isomere vorliegen. 


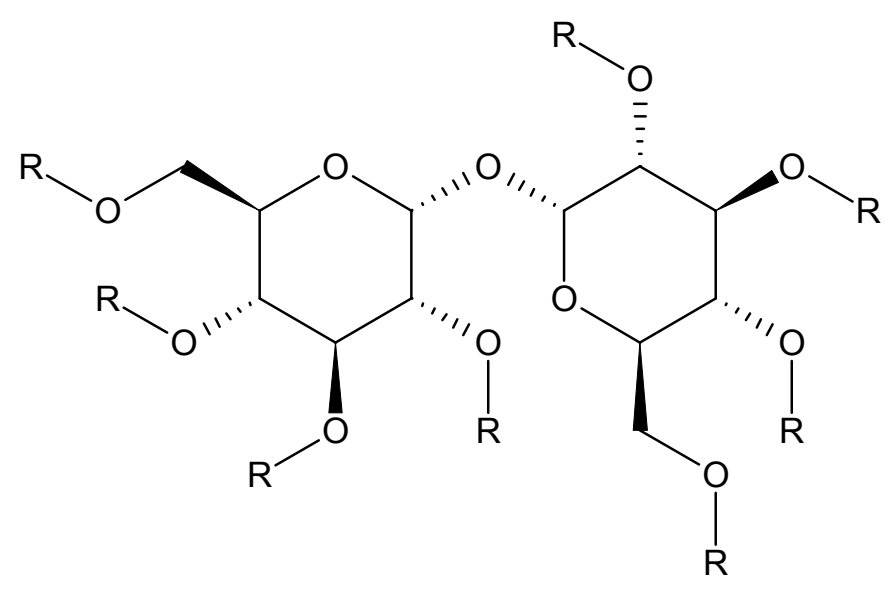

28a: $\mathrm{R}=\mathrm{H}$

28b: $\mathrm{R}=\mathrm{COCH}_{3}$

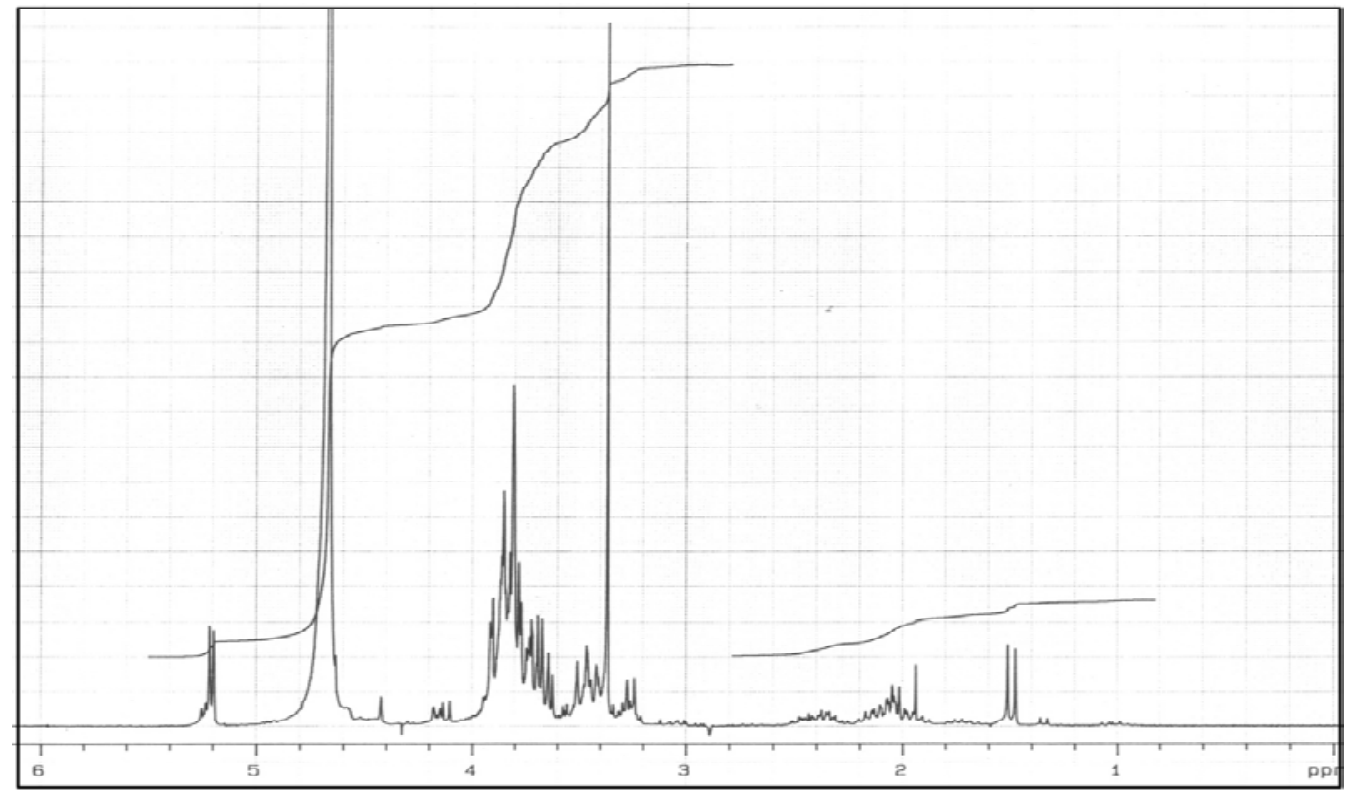

Abbildung 7: $\quad{ }^{1} \mathrm{H}-\mathrm{NMR}-$ Spektrum $\left(300 \mathrm{MHz}\right.$ in $\left.\mathrm{D}_{2} \mathrm{O}\right)$ von $\alpha, \alpha$-Trehalose nach $\mathrm{D}_{2} \mathrm{O}$-Austausch. 


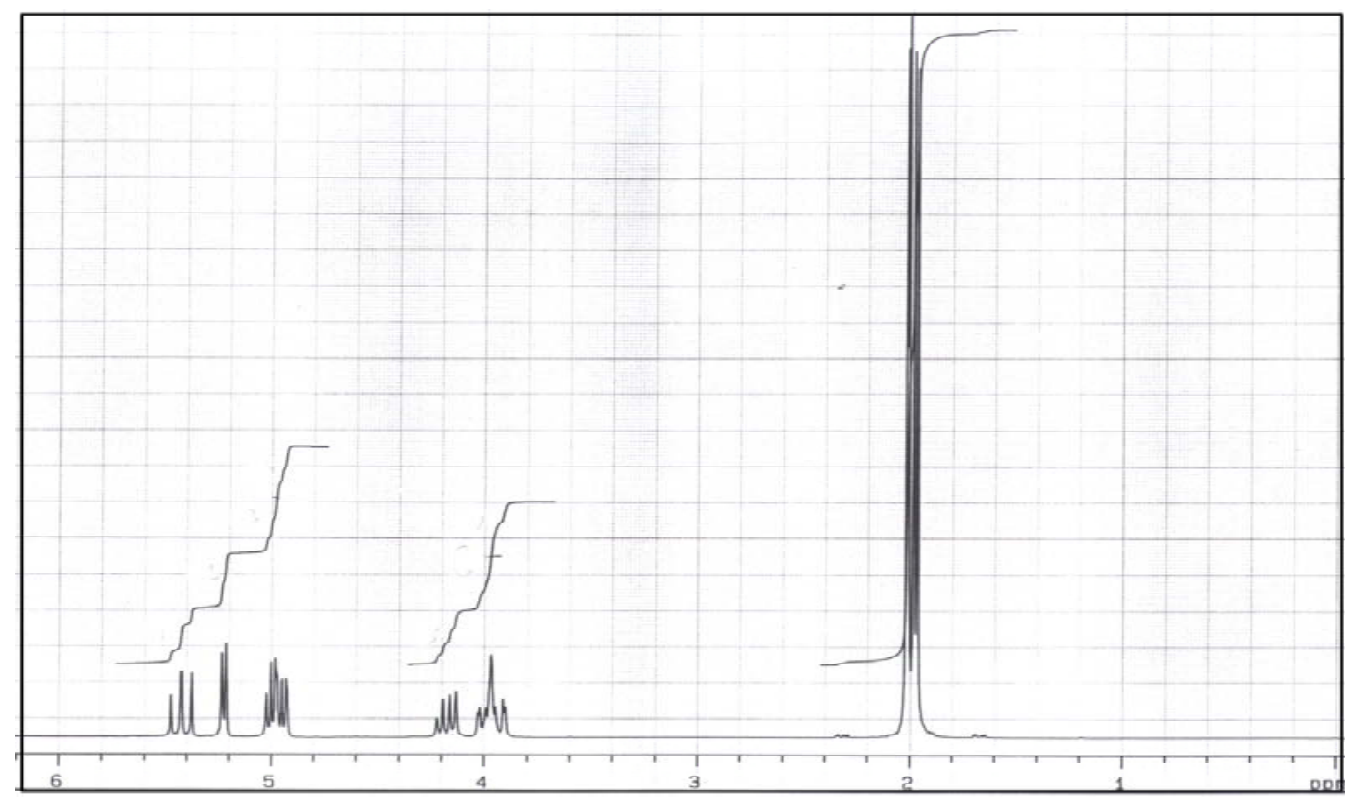

Abbildung 8: $\quad{ }^{1} \mathrm{H}-\mathrm{NMR}-$ Spektrum der peracetylierten $\alpha, \alpha$-Trehalose $\left(\mathrm{CDCl}_{3}, 200 \mathrm{MHz}\right)$.

\section{Nährmedienpotimierung anhand Genetischer Algorithmen}

Aufgrund der vielen Parameter, durch die wachsende Biokulturen beeinflusst werden können, ist die systematische Erforschung der dabei ablaufenden Vorgänge besonders problematisch ${ }^{[43]}$. Noch komplizierter erscheint die Aufgabe, die Bakterien in ihrer natürlichen Umgebung zu untersuchen. Es scheint fast unmöglich, unter Laborbedingungen die Komplexität eines Ökosystems in einem diskontinuierlichen Bioreaktor oder Schüttelkolben nachzuahmen. Man kann sich nur schwierig unter diesen Umständen Experimente vorstellen, die eine Anpassung der Mikroorganismen an neue Lebensräume mit ihren vielförmigen Lebensbedingungen untersuchen. Es wäre notwendig, außerordentlich viele Versuchsreihen durchzuführen, bei denen man die einzelnen Parameter schrittweise verändert ${ }^{[44]}$.

Die Entwicklung neurer Kultivierungsmethoden bringt nicht nur einen praktischen Gewinn in Form der Kostensenkung bei der Kultivierung, sondern verkürzt auch die zeitlich eskalierenden Prozesse.

Mit den kontinuierlichen Verfahren hat man Werkzeuge entwickelt, die die dynamische Verhaltensweise der Mikroorganismen unter den schwankenden Faktoren der Ökosysteme auch unter künstlichen Bedingungen zu studieren erlauben. Aber nicht nur die Ökosymbiose in vivo ist für die Forschung von Interesse. Manchmal geht es lediglich darum, eine höhere Ausbeute an bestimmten Sekundärstoffen zu erreichen. 
Dazu ist es nicht immer erforderlich, die Nährmedienzusammensetzung zu verändern, obwohl sie zu den entscheidenden Wachstumsfaktoren gehört ${ }^{[45,46]}$. Eine deutliche Steigerung der Produktausbeuten bei marinen Bakterien kann z.B. nicht nur durch die Variation der Medienzusammensetzung, sondern auch durch die entsprechende Auswahl der Kultivierungsbedingungen erreicht werden, wie die Untersuchungen von M. Hüners zeigen. Der Wechsel von Schüttelkolben zum Bioreaktor führte unabhängig vom Medium zu einer Steigerung der Biomassenkonzentration um einen Faktor 1.3. Auch die Kultivierungszeit bis zum Erreichen der stationären Phase verkürzte sich deutlich. Der Einsatz der Fed-Batch Technologie brachte zwar eine allgemeine Verschlechterung der Ausbeuten, erlaubte jedoch weiterführende Einblicke in den Stoffwechsel der Bakterien zu gewinnen ${ }^{[33]}$.

Ein diskontinuierlicher Bioreaktor ist ein abgeschlossenes System. Es wird während des Betriebs mit Sauerstoff versorgt, und der $\mathrm{pH}$-Wert sowie die Temperatur werden geregelt. Nährmedienzugabe und die Entfernung der Stoffwechselprodukte finden jedoch nicht statt. Diese Vorgehensweise wird als Batch-Prozess bezeichnet.

Nach einer kurzen Adaptationsphase (lag-Phase) vermehren sich die Zellen im Bioreaktor exponentiell (log-Phase). Je mehr Biomasse bereits entstanden ist, desto größer ist die weitere Wachstumsgeschwindigkeit. In der log-Phase erreicht die Verdopplungsrate den maximalen Wert. Dieser Zuwachs an Zellmasse erfolgt so lange, bis die vorhandenen Nahrungsreserven zum größten Teil verbraucht wurden oder es $\mathrm{zu}$ einer toxischen Anreicherung von Stoffwechselprodukten kommt. Erreicht die Kultur ihre maximale Zelldichte, geht die Population in die stationäre Phase über. Abschließend kommt es zum langsamen Absterben der Zellen (Absterbephase).

Mathematisch wird die log-Phase durch folgende Gleichung definiert:

$$
\frac{d X}{d t}=\mu \cdot X
$$

Am Anfang der Fermentation ist $\mathrm{X}=\mathrm{X}_{0}$ und $\mathrm{t}=\mathrm{t}_{\text {Ende der lag-Phase (Adaptation) }}$

$\mathrm{X}$ ist die Zelldichte und $\mu$ die spezifische Wachstumsgeschwindigkeit. Wenn das Nährmedium optimal mit den von den Mikroorganismen benötigten Komponenten versorgt wird, dann ist $(\mathrm{dX} / \mathrm{dt})$ der Zelldichte proportional. Nach der Umformung und Auflösung erhält man: 


$$
X=X_{0} \cdot e^{\mu t}, \text { daraus folgt }: \ln X=\ln X_{o}+\mu \cdot t \text { bzw. } \ln \frac{X}{X_{0}}=\mu \cdot t
$$

Die letzte Phase des Absterbens hat ebenso wie die log-Phase einen logarithmischen Verlauf und kann durch analoge Gleichungen definiert werden:

$$
\frac{d X}{d t}=-k \cdot X
$$

mit der Anfangsbedingung $\mathrm{X}=\mathrm{X}_{\mathrm{a}}$ und $\mathrm{t}=\mathrm{t}_{\text {Ende der stationären Phase }}$

Unter X versteht man die Zelldichte und unter k die spezifische Absterbegeschwindigkeit. Nach dem Auflösen der Gleichung erhält man:

$$
X=X_{a} \cdot e^{-k \cdot t}
$$

Da $\mathrm{X}_{\mathrm{a}}$ die Zelldichte zur Beginn der exponentiellen Absterbephase ist, kann man daraus die Halbwertszeit der Zellkonzentration ableiten:

$$
t_{\frac{1}{2}}=\frac{\ln 2}{k}
$$

Bei den semikontinuierlichen Kultivierungsmethoden (Fed-Batch-Prozesse) wird eine abgeführte Volumenmenge ersetzt oder das Volumen im Laufe der Kultivierung erhöht. Dadurch wird die Konzentration an Nährstoffen semikonstant gehalten und die Stoffwechselkonzentrationen durch Verdünnung erniedrigt. Da die Endprodukte jedoch nicht vollständig aus dem Kultivierungsmilieu entfernt werden können, wirken sie dennoch negativ auf das Zellwachstum.

Die kontinuierlichen Bedingungen erlauben einen permanenten Austausch des Kulturmediums (Fit-Prozesse). Mit einer festgelegten Geschwindigkeit, die von der Vermehrungsrate des jeweiligen Mikroorganismus abhängig ist, werden der Kultur Substrate zugeführt und die im Medium angereicherten Stoffwechselprodukten aus dem Reaktor entfernt.

Da die Konzentrationen an Metaboliten ständig klein gehalten wird, haben diese keine wachstumshemmende Effekte und stimulieren möglicherweise sogar die Bakterien in der Kultur, die fehlenden Produkte nachzubilden. Durch die konstante Konzentration an Nährstoffen bleiben die Organismen zusätzlich in der log-Phase.

Im Vergleich zu anderen biotechnologischen Verfahren eignen sich Fit-Prozesse am besten, um die natürlichen Bedingungen zu simulieren. Sie erlauben eine weitgehen- 
de Veränderung der Zusammensetzung des Mediums und gleichzeitig die Untersuchung der aus dem Bioreaktor gewonnenen Kulturbrühe.

Auf diese Weise sind auch einfache Fütterungsexperimente sehr leicht durchzuführen. Den sich vermehrenden Mikroorganismen werden bestimmte Substrate zugeführt. Nach der Extraktion einer Probe des Mediums wird durch einen simplen DCoder HPLC-Vergleich sofort ersichtlich, ob neue Metabolite entstanden sind, bzw. ob die zugesetzten Stoffe verarbeitet worden sind.

Die mathematische Beschreibung des kontinuierlichen Bioreaktors basiert auf der einfachen Annahme, dass der Fluss des Mediums durch den Reaktor mit einer konstanten Geschwindigkeit erfolgt. Er wird als Durchflussgeschwindigkeit oder Flussrate $\mathrm{V}_{\mathrm{D}}$ bezeichnet :

$$
V_{D}=\frac{\text { Mediumvolumen }}{\text { Zeit }}\left[\frac{m l}{h}\right]
$$

In der kontinuierlichen Versuchsdurchführung wird auch das Volumen V des Reaktors berücksichtigt, was zur einer spezifischen Verdünnungsgeschwindigkeit $V_{S}$ führt:

$$
V_{S}=\frac{V_{D}}{V}
$$

Da die wachsenden Zellen gleichzeitig aus dem Reaktor entfernt werden, ist die zeitliche Änderung der Zelldichte dX/dt gleich der Differenz zwischen der Wachstumsgeschwindigkeit der Zellen $\mu \cdot \mathrm{X}$ und ihrer Austragsgeschwindigkeit $\mathrm{V}_{\mathrm{S}} \cdot \mathrm{X}$ :

$$
\frac{d X}{d t}=\mu \cdot X-V_{S} \cdot X=X \cdot\left(\mu-V_{S}\right)
$$

Wenn die Durchflussgeschwindigkeit so eingestellt wird, dass die spezifische Wachstumsgeschwindigkeit $\mu$ der Zellen gleich der Verdünnungsrate $V_{S}$ ist, dann gilt:

$$
\mu=V_{S} \quad \text { und } \quad \frac{d X}{d t}=0
$$

Um möglichst große Mengen an produzierten Substanzen zu erhalten, ist es von Bedeutung, ständig maximal viele Zellen zur Produktion zu veranlassen ${ }^{[44]}$. Die gebildeten Zellen müssen jedoch im Reaktor fest verankert werden, denn sonst verlassen sie die Umgebung mit dem verbrauchten und abgeführten Medium. Der Bioreaktor 
muss also bei relativ kleinem Kulturvolumen über eine große, für die Adhäsion der Zellen nötige Fläche verfügen. Die kommerziellen Anlagen basieren auf Hohlfaser-, Festbett- oder Wirbelschichtkulturen. In Wirbelschicht-Bioreaktoren werden Zellen in offenporigen Mikroträgern aus Glas immobilisiert ${ }^{[47]}$. Das aufgewirbelte Zellkulturmedium umströmt diese kleinen Partikeln, was eine optimale Nahrungsversorgung der darin wachsenden Zellen gewährleistet ${ }^{[44]}$. Der 2 Liter Wirbelschicht-Reaktor der Firma Papaspyrou Biotechnologie besteht aus einer doppelwandigen Glasröhre, die gleichzeitig das Innere des Reaktors temperiert. Diese Anlage wird zur Produktion von monoklonalen Antikörper eingesetzt und entspricht einem 25 Liter BatchReaktor.

Eine andere Anwendung für in vivo-Experimente ist die Untersuchung von Organfunktionen. Ch. Giese beschreibt eine Studie über die Fähigkeit der Luteralzellen zur Progesteronproduktion. Die Zellen wurden in Hohlfasermodulen auf Cellulose kultiviert. Es handelt sich dabei um ca. 3000 gebündelte poröse Hohlfasern mit einer Gesamtoberfläche von $1400 \mathrm{~cm}^{2}$ und einem extrakapillaren Volumen von nur $12 \mathrm{ml}^{[45]}$.

Die kommerziellen Lösungen gehören jedoch nicht zu den kostengünstigsten. Ein weiterer Nachteil dieser Technologien liegt darin, dass die Apparaturen nicht flexibel eingesetzt werden können. Sie wurden als kontinuierliche Apparate konzipiert, und da liegt auch ihre Stärke. Sie sind z.B. als Animpfkulturen für größere Bioreaktoren nur wenig geeignet.

Die Entwicklung einer einfachen Technologie, die es erlauben würde, die hohe Leistung der kommerziellen Anlagen mit der Flexibilität der Schüttelkolben im Labormaßstab zu verbinden, sollte ermöglichen, Fütterungsexperimente bequem und kostengünstig im größeren Maßstab durchzuführen. Das Ziel dieser Arbeiten war, einen universell einsetzbaren Bioreaktor zu konstruieren und in einem Experiment auszutesten. Außerdem sollten sowohl sequentielle als auch parallele Optimierungsversuche durchgeführt werden können.

Ein besonderer Aspekt bei der Entwicklung des Bioreaktors war, die Apparatur so zu konzipieren, dass sie sowohl für die Kultivierung von Bakterien als auch für Pilze einsetzbar wäre. 


\section{Bau des Prototyps eines Bioreaktors}

Für den Bau des Gerätes verwendete man Duran-Planschliff-Glasgefäße (Schott) mit 3 mm Wandstärke und einem maximalen Füllvolumen von 21.

Die Gefäße wurden über einen Dichtring mittels einer Schnellverschlussklemme mit einem autoklavierbaren Plastikdeckel verbunden, in dem sich 16 Gewindeanschlüsse für Elektroden sowie Zu- und Ableitungen befinden. Die Kunststoff- und Metallteile der Apparatur sowie die Walzenpumpen für die Zugabe der Medienbestandteile wurden in der mechanischen Werkstatt des Instituts angefertigt.

Da der Reaktor in einem temperierten Raum eingesetzt werden sollte, konnte man auf die Wärmeisolierung verzichten. Im Fermenter befand sich ein langes, spiralförmiges Rohr, das als Wärmeaustauscher konzipiert war. Eine Zuleitung aus Edelstahl, die bis zum Boden des Reaktors reichte, diente im kontinuierlichen Betrieb der Medienzufuhr. Die Luft wurde über einen am Boden des Reaktors liegenden perforierten Kunststoffschlauch eingeleitet.

Diese Lösung erlaubte nicht nur eine ausreichende und gleichmäßige Sauerstoffversorgung der Zellen. Die aufsteigenden Luftblässchen durchwirbelten die Flüssigkeit und erzeugten dadurch auch eine gute Durchmischung des Mediums, was zusätzliche Rotoren überflüssig machte. Diese Lösung vereinfachte die Apparatur, reduzierte die Kosten und gleichzeitig die Gefahr des Verschleißes mechanischer Teile. 

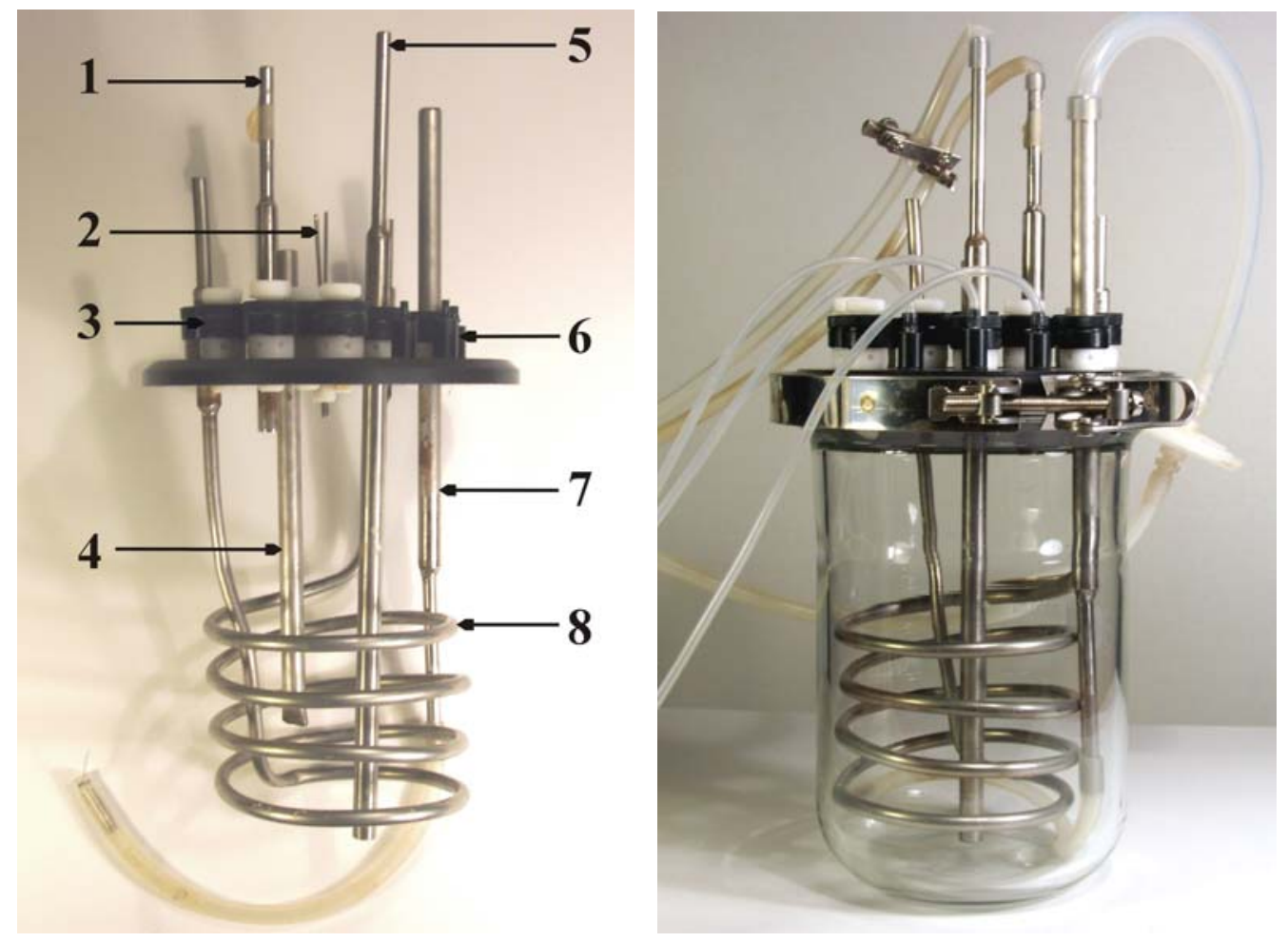

Abbildung 9: Die Konstruktion eines 2 1-Durchflußfermenters. 1 Abflussrohr für Medium, 2 Antischaumsonde, 3 Verschlüsse für die pH-, Sauerstoff- und Glucoseelektroden, 4 verschlossenes Rohr für das Thermoelement, 5 Zuleitungsrohr für Medium, 6 AS/Säure/Base Anschlüsse, 7 Sauerstoffversorgung, 8 Wärmeaustauscher.

Da der Fermenter dicht abgeschlossen war, erzeugte die Luftzufuhr einen leichten Überdruck im Inneren des Gefäßes, der über ein bis zur Flüssigkeitsoberfläche reichendes Rohr abgebaut wurde. Stieg der Flüssigkeitspegel, so wurde überschüssiges Kulturmedium aus dem Reaktor in eine Vorratsflasche gedrückt.

Um die ganze Apparatur vor Kontaminationen zu schützen, wurden alle Öffnungen sowie die Luftzufuhr mit Teflon-Membranfiltern mit $0.2 \mu \mathrm{m}$ Porengröße (Midisart, Sartorius) versehen.

Die Temperaturmessung erfolgte mit Hilfe eines kommerziellen Digital-Temperaturmessmoduls (TMB 880EXF), dessen Thermoelement in einem Blindrohr steckte. Als Antischaum-Sonde wurden zwei $2 \mathrm{~mm}$ voneinander entfernte Edelstahldrähte benutzt, deren Höhe über dem Medium frei einstellbar war.

Die Apparatur wurde mit den Vorrats- und Sammelflaschen durch Silikonschläuche (Innendurchmesser 3 bis $5 \mathrm{~mm}$, Wanddicke $2 \mathrm{~mm}$ IDL-Silikon) verbunden. Für die pH-Regelung befanden sich im Aufsatz zwei kleinere Gewindeanschlüsse für die Zugabe von Säure und Base. 
Für den kontinuierlichen Betrieb wurde ein System zur Medienzufuhr und Medienentsorgung vorgesehen. Die Vorratsflaschen konnten auch während des Betriebs steril ausgetauscht werden.

Der Bioreaktor war mit allen Komponenten im zusammengebauten Zustand autoklavierbar. Das Medium wurde separat in 10 Liter Duranflaschen (Schott) sterilisiert und vor dem Experiment an den Reaktor unter einer Sterilbank angeschlossen. Die Sammelflasche wurde so dimensioniert, dass sie das gesamte Volumen der abgeführten Flüssigkeit ohne Umtausch aufnehmen konnte.

Um die nötige Drehkraft bei den Schlauchpumpen zu erzeugen, wurden 12V Motoren mit Übersetzung verwendet. Der Kopf der Pumpe bestand aus einem Planetengetriebe mit drei sich um ihre eigene und eine geozentrische Achse drehenden Ringen aus Hartkunststoff und war mit einer durchsichtigen und abnehmbaren Schutzscheibe versehen. Die Abdeckung verhinderte ein Abrutschen der Schläuche.

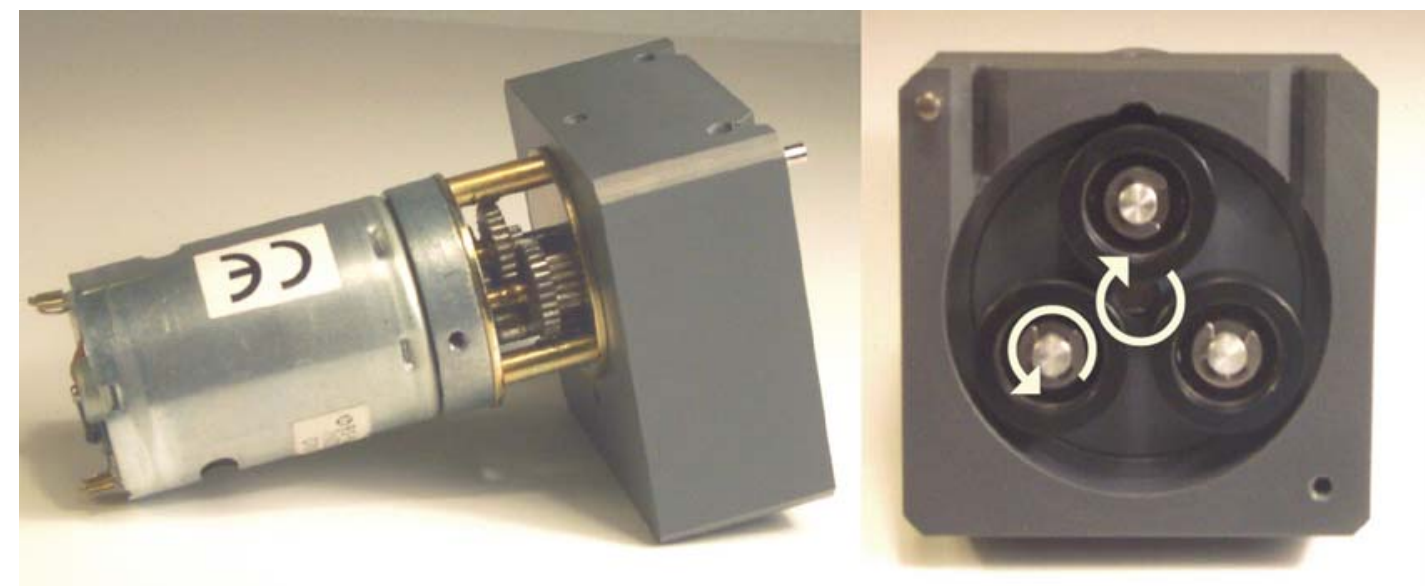

Abbildung 10: Die Pumpe ohne Gehäuse. Pumpenkopf und die Drehrichtungen der Andruckrollen.

\section{Das Konzept der Steuerapparatur}

Genauso wie der Bioreaktor sollte auch die Steuerapparatur in der Zukunft problemlos um weitere Funktionen erweiterbar und kostengünstig sein. Zusätzlich sollte sie die parallele Messungen der Signale an mehreren Fermentern gleichzeitig erlauben. Sie wurde für die Überwachung acht gleichzeitig laufender Reaktoren vorgesehen, sollte das Medium mit Nährstoffen versorgen und darüber hinaus die Antischaum-, Säure- und Basendosierung kontrollieren. 
Da für jeden laufenden Reaktor primär grundsätzlich nur drei Walzenpumpen vorgesehen waren (AS, Säure, Base), musste die Möglichkeit in Betracht gezogen werden, dass Pumpen, die unbenutzten Fermentern zugewiesen wurden, auch flexibel einzusetzen sind. Dies reduziert zwar die Zahl der parallel laufenden Bioreaktoren erheblich, erhöht aber gleichzeitig die Zahl der Medienbestandteile, die der Zellkultur variabel zugeführt werden können.

Würde man alle 24 Pumpen für die Versorgung nur eines Reaktors einsetzen, wäre es möglich, bei der Untersuchung der Wachstumsbedingungen eines Mikroorganismus gleichzeitig 24 Parameter zu verändern.

Außer dem AS-, Säure- und Basezulauf sind noch 5 zusätzliche Überwurfmuttern verfügbar, die entweder mit zusätzlichen Messsonden, Elektroden oder als Zulauf bestückt werden können.

\section{Die Arbeitsweise der Steuerungsapparatur}

Die Steuerungsapparatur ist aus einzelnen Modulen (Karten) aufgebaut. Jede Karte wird durch eine interne Adresse angesprochen und kann jeder Zeit umgetauscht oder aus dem Gerät entfernt werden. Dieser Vorgang beeinflusst auf keine Weise die Arbeit anderer Karten, die sich bereits im System befinden. Allerdings muss das Entfernen der Karte stets bei ausgeschaltetem Gerät erfolgen, um die Elektronik der Steuerungskarte nicht zu gefährden.

Die in der Steuerungsapparatur eingesetzten Platinen sowie alle Schaltpläne wurden in Rahmen dieser Arbeit selbstständig entworfen und angefertigt. Im Anhang A sind die Schaltpläne sowie die Layouts aller hergestellten Platinen abgebildet.

Die wichtigste Komponente der Apparatur ist eine zentrale Steuerungseinheit, die keine interne Adresse hat und dauerhaft im Gerät integriert ist. Sie übernimmt alle Steuerungsfunktion und spricht die anderen Komponenten an. Sie sendet und empfängt Daten und ermöglicht den Informationsaustausch zwischen den einzelnen Modulen. Die empfangenen Daten können auf die serielle Schnittstelle, mit der die Steuerungseinheit mit dem dazugehörigen Computer kommuniziert, gesendet werden. Da diese Karte die anderen Messkarten mit Spannung und Daten versorgt, dürfen die Pinbelegung und das Sendeprotokoll nicht geändert werden. 
Dank der modularen Bauform und der Adressierung erfolgt die Kommunikation zwischen dem Steuerungsprogramm und der Hardware auf einer virtuellen Ebene. Bis jetzt konnte jedoch nur die manuelle Steuerung im Einsatz getestet werden. Das Steuerungsprogramm muss noch geschrieben werden. Um den Programmieraufwand zu minimieren, könnte man für diesen Zweck grafisch orientierte Programmiersprachen, wie G, LabView von National Instruments oder HP-VEE von Hawlett-Packard verwenden. Im Gegensatz zu den klassischen Programmiersprachen wird hier auf die Eingabe von Befehlszeilen verzichtet und stattdessen durch grafische Verbindung funktionaler Unterprogrammsymbole (userfunctions, userobjects und virtuelle Instrumente) programmiert.

Die Kommunikation mit dem Computer erfolgt ausschließlich durch die serielle Schnittstelle (COM1 oder COM2). Der Transfer muss mit der höchsten Geschwindigkeit dieser Schnittstelle erfolgen.

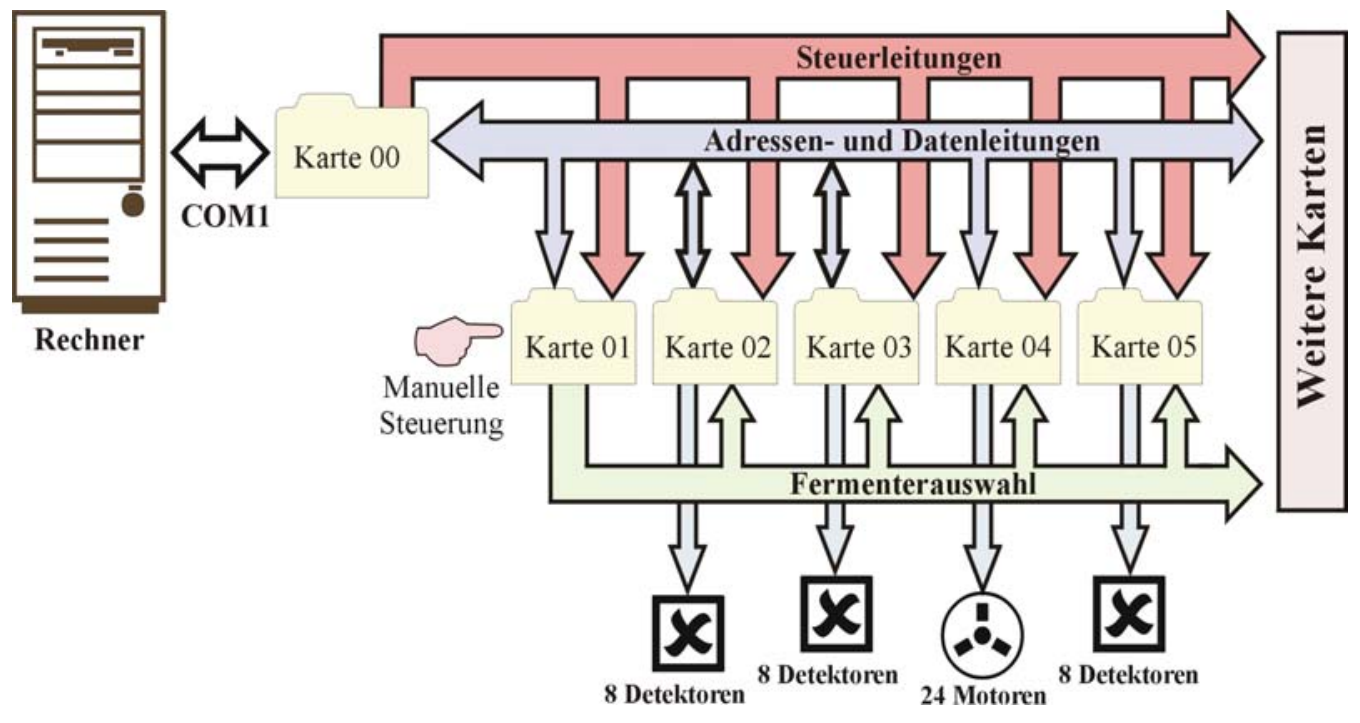

Abbildung 11: Darstellung der modularen Bauweise des Gerätes

Zur Zeit sind vier unterschiedliche Karten an das Gerät angeschlossen. Die Karte mit der Adresse 01 erlaubt das Umschalten zwischen den acht unterschiedlichen Fermenter. Da sie auch ein manuelles Umschalten über einen externen Schalter zulässt, muss dies in der zukünftigen Programmierung der restlichen Module berücksichtigt werden. Wurde ein Fermenter ausgesucht, wird dessen Zustand mit einer Leuchtdiode signalisiert, was einer Aktivierung der entsprechenden Leitung und der Deaktivierung aller sieben anderen entspricht. 
Die manuelle Umschaltung kann für die Zeit, die nötig ist, um die anderen Karten anzusprechen, per Software unterdrückt werden, in dem man einen entsprechenden Bit in den Hi-Zustand versetzt. Die gerade aktivierte Fermenterleitung ist mit einer Leuchtdiode gekennzeichnet. Diese Leitung ist direkt an alle Kartenschnittstellen gelegt und kann durch die Hardware abgefragt werden. Die Leitungen können Belastungen bis zu $0.25 \mathrm{~A}$ ausgesetzt werden.

Die Karte mit der Adresse 02 wurde zur Kontrolle des Flüssigkeitspegels im Reaktor oder als Antischaumdetektor konzipiert. Sie macht einen direkten Gebrauch aus den oben beschriebenen Fermenterwahl-Leitungen, so dass immer nur einer (der aktive) Fermenter abgefragt werden kann. Um bei einem anderen Fermenter die Schaumbildung zu überprüfen, muss die Karte mit der Adresse 01 angesprochen und ein anderer Fermenter aktiviert werden.

Auf der Karte befindet sich ein A/D-Wandler, der die von dem Feuchtigkeitsdetektor (LM 1030) ankommenden analogen Signale in das 8-Bit-Format umwandelt. Der Chip LM 1030 überprüft, ob sich die Elektroden im stromleitenden Medium befinden. Um Korrosion zu vermeiden, wird durch einen internen Generator eine Wechselspannung (ca. $2.3 \mathrm{~V}$ ) an die Elektroden angelegt. Der momentane Zustand der zu dem jeweiligen Fermenter gehörigen Elektrode wird durch zwei Leuchtdioden (Rot/Grün) signalisiert.

Die Karte mit der Adresse 03 übernimmt die pH-Überprüfung. Auch diese Karte nutzt die Fermenterwahl-Leitungen, um zwischen den einzelnen pH-Elektroden umzuschalten. Der pH-Wert wird mit Hilfe eines digitalen Displays angezeigt und zusätzlich durch einen A/D-Wandler dem Rechner zur Verfügung gestellt. Der entsprechende $\mathrm{pH}-$ Wert wird als unterschiedliche Spannung (-2,5V bis 2,5V) am Ausgang des A/D-Wandlers ausgelesen.

Die Karte mit der Adresse 04 steuert die Dosierungseinheiten: Gleichstrommotoren, die einen Pumpenkopf antreiben. Sie werde im Pulsverfahren betrieben. Dies ermöglicht es, die Geschwindigkeit mühelos zu verändern. Allerdings erfolgt dies nur manuell durch Drehen an einem Potentiometer, das die Geschwindigkeit aller Motoren gleichzeitig beeinflusst. Die 24 Motoren sind in acht Gruppen (drei Motoren in einer Gruppe) aufgeteilt, was die Zugabe von Antischaum, Säure und Base ermöglicht. 
Die auf der Karte untergebrachte interne Hardwarelogik sorgt dafür, dass immer nur ein Motor arbeiten kann. Durch diese Schutzmaßnahme wird vermieden, dass die Elektronik durch Überlastung zerstört wird.

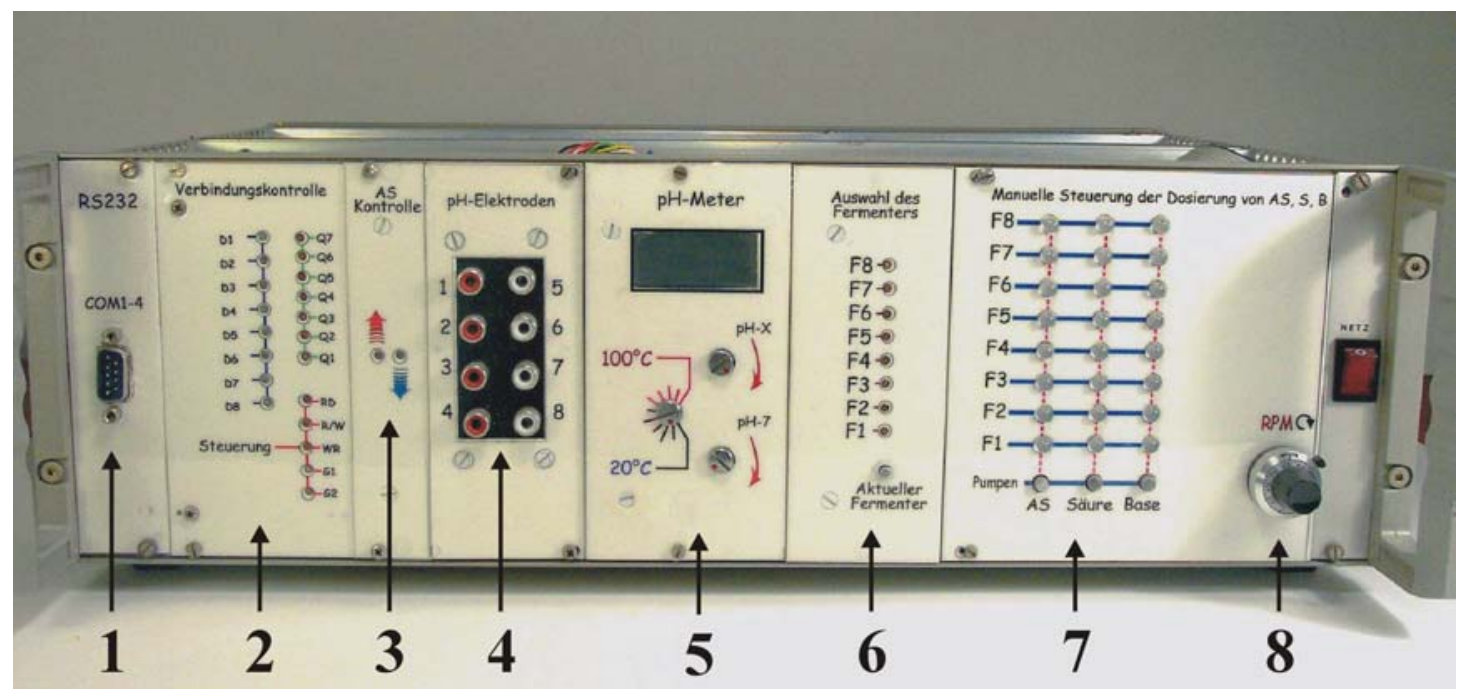

Abbildung 12: Die Steuerungsapparatur von vorn. 1 COM-Schnittstelle für die Verbindung mit dem Rechner, 2 Kontrolldioden für alle Steuerungssignale, 3 Antischaumsteuerungskarte, 4 Anschlüsse für die pH-Elektroden, 5 pH-Meterkarte, 6 Taste für die manuelle Auswahl und die Anzeige des ausgewählten Fermenters, 7 manuelle Steuerung der Pumpen und Anzeige ihres Zustandes, 8 Potentiometer für die Regelung der Geschwindigkeit der Pumpen

Auch weitere Module können durch die Fermenterwahl-Leitungen den aktiven Fermenter ähnlich wie bei der Antischaum- und pH-Meter-Messkarte abfragen. Dabei sind drei Arten der Module denkbar:

Eine Karte liest eine Messgröße ab, ein A/D Wandler digitalisiert sie und stellt sie dem Rechner zur Verfügung. Oder aber die Karte wird entsprechend initialisiert und der D/A-Wandler steuert ein Ausgabegerät an. Eine dritte Möglichkeit ist ein Hybrid aus den zwei ersten, sie kann sowohl einen Wert liefern als auch externe Geräte ansteuern. 


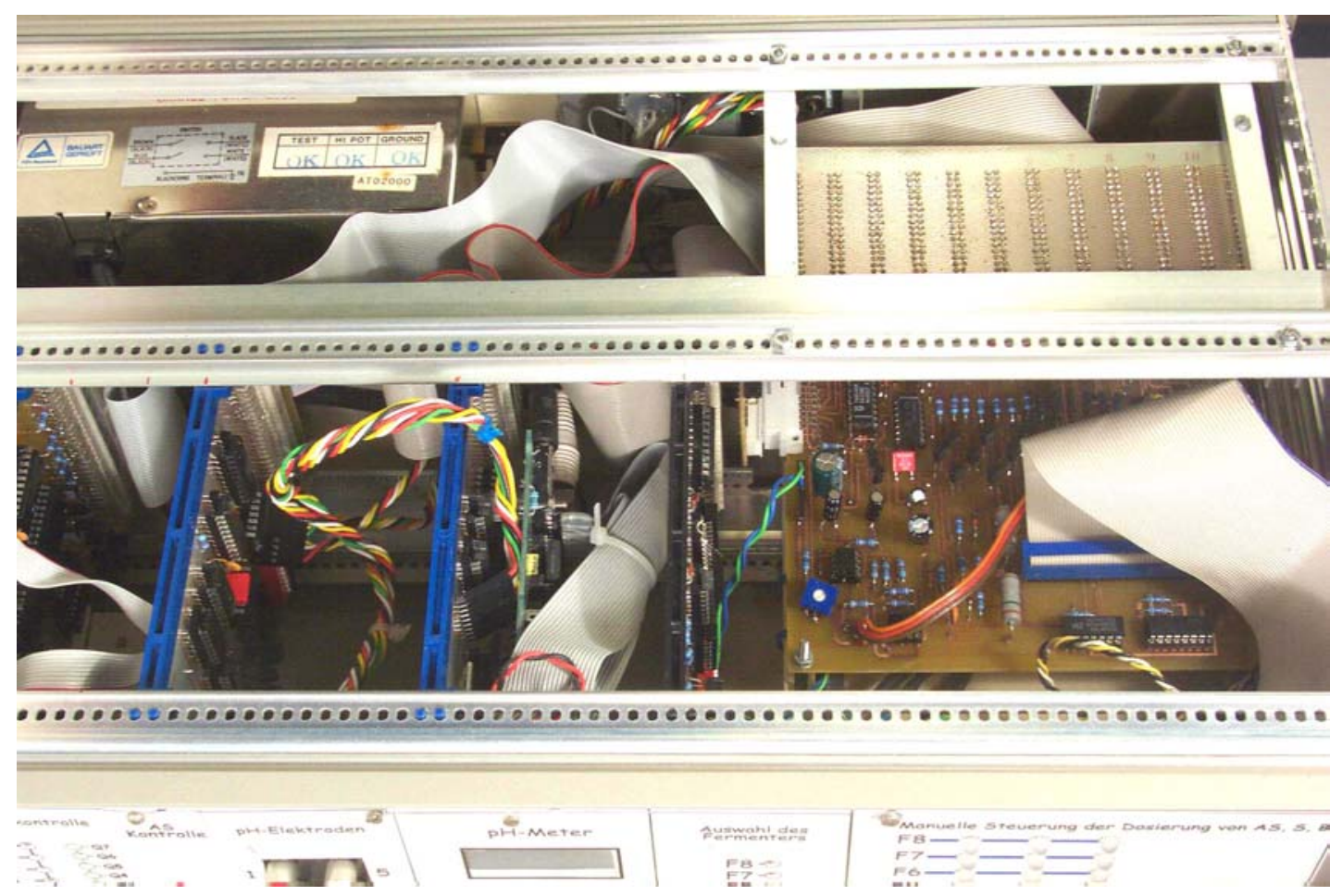

Abbildung 13: Die Apparatur besteht aus mehreren normierten Platinen.

\section{Die Animpf- und Kultivierungsversuche}

Um zu überprüfen, ob der entworfene Bioreaktor Schüttelkulturen ersetzen kann, wurde zuerst ein vereinfachter Aufbau im Einsatz getestet. Gleichzeitig wollte man auch die Tauglichkeit der Steuerungsapparatur im Dauerbetrieb prüfen.

Der Fermenter wurde über Silikonschläuche mit den Vorratsflaschen für Säure, Base und Antischaummittel verbunden. Die Luftzufuhr erfolgte über einen autoklavierbaren Membranfilter. Alle anderen Anschlüsse wurden vor dem Sterilisieren (20 min Heißdampf) verschlossen. Die Bestandteile des Nährmediums wurden in separaten Flaschen getrennt sterilisiert. Nach dem Abkühlen wurden die Flaschen mit den Medienbestandteilen unter der Sterilbank an den Bioreaktor angeschlossen.

Für den Versuch wurde der terrestrische Streptomycet GW 3/1122 ausgewählt, weil bereits aus der früheren Bearbeitung Erkenntnisse über die Wachstumsge-schwindigkeit und die Produktion von Actinomycin D (16) unter Standardbedingungen vorlagen. Es sollte geprüft werden, ob die Synthese noch anderer Naturstoffe in dem neuen Verfahren angeregt wird.

Die Animpfkultur wurde zunächst auf Agar-Platten angezüchtet, anschließend mit 1 $\mathrm{ml}$ des Mediums abgeschwemmt und unter sterilen Bedingungen in den vorbereiteten 
Reaktor übertragen. Anschließend wurden noch die Flaschen mit Säure, Base und Antischaum-Lösung unter sterilen Bedingungen aufgefüllt und die $\mathrm{pH}$-Elektrode und die Antischaumsonde mit den Messkarten verbunden.

Bereits nach wenigen Stunden bei $28^{\circ} \mathrm{C}$ wurde die Lösung im Fermenter trüb und man konnte im Medium die ersten Bakterienkolonien erkennen. Nach 24 Stunden war der Fermenter sehr stark bewachsen. Die rasante Entwicklung ist darauf zurückzuführen, dass die Bakterien bei konstantem pH-Wert ausreichend mit Sauerstoff versorgt wurden.

Der Versuch zeigte eindeutig, dass sowohl der Bioreaktor als auch die Steuerung die Erwartungen zufriedenstellend erfüllte. Die Fermenter können kleinere Schüttelkulturen ersetzen und ermöglichen die Parallelisierung der Anzucht und die schnellere Gewinnung der Bakterienkulturen. Das rasche Wachstum ermöglicht in Zukunft den Einsatz bei der Erzeugung der Animpfkulturen für größere Bioreaktoren. Da der Kleinfermenter noch zusätzliche, nicht belegte Anschlüsse besitzt, kann man sie verwenden, um die Bakterien mittels einer Walzenpumpe steril in den größeren zu überführen.

Nach dem Extrahieren der erhaltenen Kulturmasse mit Methanol und Essigester zeigte sich jedoch, dass das Assortiment der Sekundärmetabolite dem der Schüttelkolben entsprach. Es wurde nur 16 in großen Mengen gebildet.

Es stellte sich dem Experiment weiterhin heraus, dass die Sauerstoffversorgung über die Druckleitung die Temperatur des Mediums auf unerwünschte Weise drastisch herabsetzte. Dieses Problem trat nicht mehr auf, wenn man die Luft durch ein langes, in ein $50^{\circ} \mathrm{C}$ warmes Wasserbad eingetauchtes, spiralförmiges Metallrohr vorwärmte.

\section{Kontinuierliche Fermentation im Personal Fermenter}

Um den Fermenter im kontinuierlichen Betrieb einzusetzen, mussten vor allem die Zellen im Bioreaktor auf einem Trägermaterial verankert werden. Dazu wurden zunächst Versuche mit verschiedenen Trägern statisch in Batch-Kulturen durchgeführt. Als Voraussetzungen mussten die Träger vor allem kostengünstig, biologisch inaktiv, autoklavierbar sein und eine große Oberfläche besitzen, um die Bakterien festzuhalten. Vom Vorteil wäre auch die mehrmalige Verwendbarkeit. 
Zuerst wurde nach einem kostengünstigen Tongranulat gesucht, das durch seine große poröse Oberfläche einen optimalen Halt für das Mycel bieten würde. Seramis ${ }^{\circledR}$ weist im Handelsprodukt Korngrößen bis zu einem Zentimeter auf. Die Körner wurden mechanisch zerkleinert und eine Korngröße von $3 \mathrm{~mm}$ ausgesiebt. Die so erhaltenen Partikel wurden mehrmals mit Wasser ausgewaschen, ausgekocht und in den Fermenter gefüllt. Schließlich wurde der Bioreaktor mit dem Stamm GW 11/1124 angeimpft und 3 Tage bei $28{ }^{\circ} \mathrm{C}$ inkubiert. Um die Vergleichbarkeit der Ergebnisse zu gewährleisten, wurde dieser Stamm auch in den folgenden Versuchen mit anderen Materialien verwendet.

Unerwarteterweise wurde kein Zuwachs an Zellmasse beobachtet. Es lag die Schlussfolgerung nahe, dass das Tongranulat selbst eine Bioaktivität besitzt und das Wachstum behindert. In der Tat verursachte Seramis-Granulat starke Hemmhöfe auf Agarplatten, die mit dem Teststamm beimpft waren. Ob dies durch zugesetzte Antibiotika oder z. B. im Ton enthaltene Eisensalze bedingt war, wurde nicht geprüft. Diese Eigenschaften schlossen Tongranulat jedenfalls als potenziellen Träger aus.

Als nächstes wurden Glasperlen als Trägermaterial getestet. Die Kugeln erwiesen sich als zu schwer, sammelten sich am Boden und behinderten im Endergebnis die Sauerstoffversorgung erheblich. Ein weiterer Nachteil lag daran, dass sie den unter diesen Umständen nur langsam wachsenden Bakterien nicht genug Halt boten. Positive Resultate lieferten dagegen Raschig-Ringe. Der Reaktor wurde ausreichend mit Sauerstoff versorgt: Die Luftblasen verfingen sich in den Hohlräumen des Glasmaterials, die auch den Zellen ausreichend Halt boten. Die Reinigung des Materials war allerdings problematisch. Auch nach mehrmaligem Auskochen mit Wasser konnte das Mycel nicht vollständig vom Glasmaterial entfernt werden.

Eine ähnliche Struktur mit vielen kleinen Hohlräumen bieten zusammengerollte Netze. Es wurde mit den handelsüblichen Produkten experimentiert. Dabei zeigte sich jedoch, dass die meistens aus Polyethylen oder Polystyrol angefertigten Kunststoffnetze nicht autoklavierbar waren, weil sie schmolzen. Dafür schienen die Aluminiumnetze prädestiniert für die Versuche, und zusätzlich bestand wegen der Oberflächenpassivierung durch sich bildende Oxide keine Gefahr der Korrosion, was einen wiederholten Einsatz ermöglichen würde. Auch hier wuchsen die Bakterien jedoch nicht, wahrscheinlich, weil doch zu viel Aluminium freigesetzt wurde. 
Als geeignete Träger erwiesen sich schließlich Netze aus Edelstahl, die von den Bakterien nach kurzer Zeit vollständig bedeckt wurden. Eine Korrosion fand auch nach mehrmaligem Einsatz nicht statt. Die verwendeten Netzrollen konnte man schnell und problemlos mit einer Bürste unter laufendem Wasser von den Pellets befreien.

$\mathrm{Zu}$ befürchten war daher auch, dass die Bakterien im kontinuierlichen Betrieb durch die abgeführte Flüssigkeit mitgerissen und ausgetragen würden. Um den Zellen noch mehr Halt zu geben, wurde deshalb versucht, eine Agarschicht auf den Netzen zu erzeugen. Zu diesem Zweck goss man in den Bioreaktor noch vor dem Autoklavieren Nähragar hinein und schüttelte das Gefäß nach dem Sterilisieren so lange, bis der Agar erstarrte und eine einheitliche Schicht auf dem aufgespannten Netz bildete. Leider führte dies aber auch zu einer teilweisen Verstopfung der Löcher für die Sauerstoffversorgung.

Der Fermenter wurde anschließend steril mit Nährlösung befüllt und angeimpft. Zwar führte auch diese Methode zum Erfolg, in Anbetracht der Schwierigkeiten und des hohen Aufwandes war die erreichte Verbesserung der Kultivierungsbedingungen jedoch nur minimal. Deswegen verzichtete man bei den weiteren Versuchen auf den Zusatz von Nähragar.
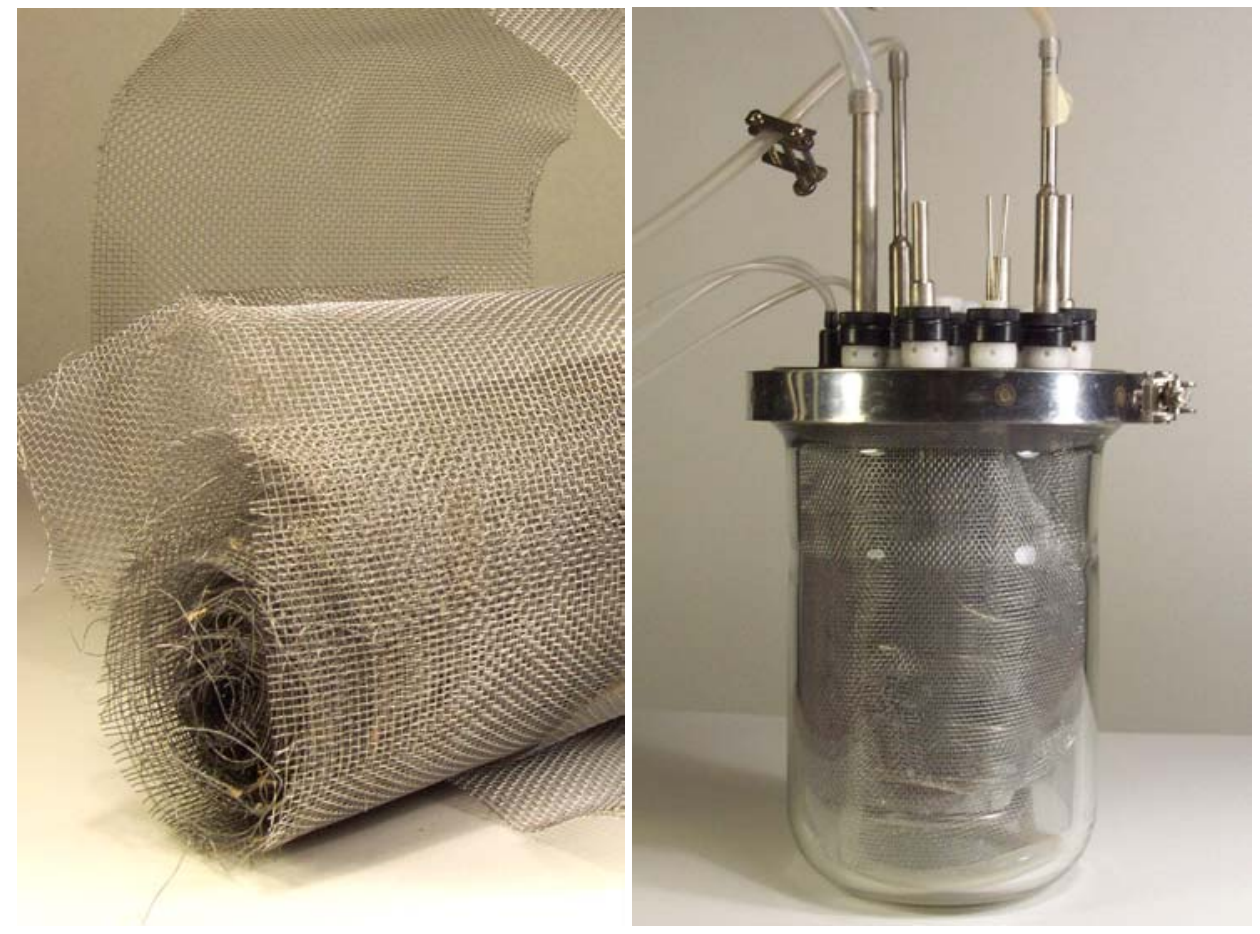

Abbildung 14: Der Bioreaktor mit dem Träger aus Edelstahlnetz. 


\section{Die Kopplung eines Durchflussreaktors mit einer XAD-Säule}

Im Durchflussbetrieb fließt kontinuierlich eine vorher festgesetzte Menge des Nähmediums durch den Reaktor, in dessen Innerem die Mikroorganismen hauptsächlich auf dem entsprechenden Trägermaterial wachsen. Dabei werden die Bestandteile der Nährmedien jedoch nur zum Teil verstoffwechselt. In den nach dieser Methode erhaltenen Rohextrakten finden sich daher stets auch Nährmedienkomponenten wieder, was aus verschiedenen Gründen nicht optimal ist. Zum einen wird die Isolierung der Metabolite durch die ebenfalls in den Extrakten befindlichen Medienbestandteile aufwendiger. Zum anderen ist gerade das Nährmedium ein wesentlicher Kostenfaktor.

Es lag daher nahe, die aus dem Reaktor fließende Kulturbrühe kontinuierlich von den Metaboliten zu befreien und das zurückerhaltene Nährmedium zumindest teilweise erneut einzusetzen.

Es ist bekannt, dass durch Adsorbtion an XAD-Harzen Sekundärmetabolite aus Kulturbrühen extrahiert werden können. Bei der darauf aufbauenden moving bedMethode wird eine Säule mit dem Adsorberharz gefüllt ${ }^{[48]}$ und die zu extrahierende Kulturbrühe langsam von unten durch die Säule gepumpt. Um die festgehaltenen Substanzen wieder vom Harz zu lösen, lässt man nach der Fermentation ein geeignetes Lösungsmittel, z. B. Methanol, in umgekehrter Richtung durch die Säule fließen. Durch die Wahl der Lösungsmittel kann dabei schon eine Vortrennung der Extrakte erreicht werden.

Ausgehend von diesen Überlegungen wurde eine weitere Pumpe an den kontinuierlichen Fermenter angeschlossen, die die Kulturbrühe durch eine mit XAD-Harz (XAD-7, XAD-16) gefüllte, an der Unterseite mit einer Glasfritte abgedichtete Chromatographiesäule pumpte. Das filtrierte und extrahierte Medium wurde dem Prozess im Kreislauf wieder zugeführt. Gleichzeitig wurde der kontinuierliche $\mathrm{Zu}$ fluss frischen Mediums und die Entnahme von Kulturbrühe reduziert oder aber diskontinuierlich gestaltet. Auf den Zufluss frischen Mediums konnte nicht ganz verzichtet werden, da man davon ausgehen muss, dass immer auch Medienbestandteile an dem Harz festgehalten werden oder sich das Medium an bestimmten Komponenten erschöpft. Gleichzeitig besteht auch die Gefahr, das XAD-Harz mit den mittelpolaren Stoffen des Mediums zu sättigen. 
Es zeigte sich jedoch, dass die Mikroorganismen nicht nur auf den Trägermaterialien in den Reaktoren, sondern auch auf dem Harz und in den Poren der Glasfritte wuchsen. Dadurch wurde der Filter- und Extraktionskreislauf sehr schnell behindert. Verschiedene Methoden, um diesen Problemen zu begegnen, wurden überprüft. Zum einen wurde eine senkrecht aufgehängte XAD-Säule von unten her langsam mit der Kulturbrühe durchflossen (moving bed-Verfahren). Zum anderen wurde die Extraktionssäule oben mit verschiedenen Filtermaterialien (Papier-, Glasfaser- und Baumwollfilter) bedeckt und mit hoher Flussrate von oben von der Kulturbrühe durchflossen. Man erhoffte, dass die Mikroorganismen dadurch nicht so rasch auf dem Harz siedeln und die Säule verstopfen würden.

Auch diese Versuche führten zu unzureichenden Ergebnissen, und es wurde von der XAD-Methode Abstand genommen. Um die Extraktionsmethode in der Zukunft einsetzen zu können, müssen weitere Lösungen, z.B. Hohlfasermodule, gründlich geprüft werden, wozu derzeit aber die Voraussetzungen fehlten. 


\section{Information und Chaos}

Die rasante Entwicklung von Computern trug dazu bei, die Information als eine Funktion der Selbstorganisation neu zu definieren. Wenn ein System keine Möglichkeit besitzt, Variationen hervorzurufen, hat es auch keine Möglichkeit, sich weiterzuentwickeln. Man könnte also entsprechend die ganze Entwicklungsgeschichte der lebenden Systeme als eine Tendenz der Optimierung im Bezug auf Speicherung und Verarbeitung relevanter Informationen auffassen. Dieser Trend führte im Endergebnis zu komplexen und differenzierten Organisationsformen. Die Informationsmenge, die ein System beinhalten kann, hängt ab von der Zahl der Bindungen, die Untereinheiten des Reservoirs untereinander ausüben können.

Die Komplexität der lebenden Systeme demonstriert sich dadurch, dass Chaos und Ordnung in einer ausgewogenen Balance existieren. Wo veränderte Bedingungen eine dynamische Anpassung eines Systems erfordern, erlaubt es dieses Gleichgewicht, auf kleinste Abweichungen mit großen Korrekturen zu antworten. Das Leben existiert in all seinen Formen am Rande des Chaos, in dem es sich auf der Suche nach emergenter Ordnung zwischen diesen zwei Zuständen bewegt ${ }^{[49,50]}$.

Um die Selbstorganisation der Natur zu begreifen, reicht es nicht, die zu simulierenden Prozesse in ihre Teilkomponenten zu zerlegen (Top-Down-Ansatz). In natürlichen Systemen, die aus einer großen Zahl gleichrangiger Einzelobjekte bestehen, ist es oft unmöglich, das Verhalten des Gesamtsystems auf dieser Weise vorauszusehen. Die so erhaltenen Resultate der Berechnungen waren oft sehr abweichend von den Beobachtungen. Vielmehr ergibt sich die komplexe Variabilität erst aus dem Zusammenspiel aller Bestandteile, auf denen ein System aufbaut. Ein Phänomen des Makrosystems, das aus vielen Interaktionen auf der Ebene der kleinsten Komponenten besteht, wird als Emergenz bezeichnet (bottom-up-Ansatz). Um solche Modelle mathematisch zu beschreiben, bedarf man einer neuen, abstrakten Sprache der Mathematik.

Die ersten Versuche, die natürlichen Interaktionen im Computer zu simulieren, waren Programme, die auf einfachen und abstrakten Anweisungen basierten (artifical life). Sie beschrieben die Wechselwirkungen beliebig vieler Teilsysteme (Zellen oder Agenten), die alle den gleichen, einfachen Regeln folgten und sich durch die unmittelbare Nachbarschaft im gemeinsamen Lebensraum gegenseitig beeinflussen konnten. Die Art der elementaren Wechselwirkungen, die durch einfache Grundsätze de- 
finiert waren, führte zu einem Reichtum mosaikartiger Ergebnisse. Diese Programme nannte man zelluläre Automaten ${ }^{[51,52]}$.

Am Ende der 60er Jahre entwickelte der Mathematiker J. Horton Conway das Programm „LIFE“. J. Conway betrachtete diese Simulation weniger unter dem Gesichtspunkt biologischer Relevanz. Vielmehr war es ein abstraktes Spiel mit all den variablen Möglichkeiten, die sich aus einfachsten Grundregeln ergeben könnten. Bis in die Mitte der achtziger Jahre galten diese „Zellulären Automaten“ als ein interessanter, aber auch unpraktischer Gedanke. Erst durch die Pionierarbeiten von J. von Neuman, J. Conway und S. Wolfram gewannen diese Computermodelle an Interesse. Immer mehr Wissenschaftler begannen, diese Modelle in ihren Untersuchungen einzusetzen. Zuerst waren es Untersuchungen zur Musterbildung. Man fand ebenfalls heraus, dass sich komplizierte Phänomene mit einer überraschenden Genauigkeit durch derart einfache Konstrukte beschreiben lassen. Die Dynamik von Gasen, die Kollision gleichartiger Teichen, und das Strömungsverhalten der Flüssigkeiten konnten nun ohne großen mathematischen Aufwand naturtreu dargestellt werden. Dem Beispiel der Physik folgten nun die ersten erfolgreichen Versuche, auch chemische Reaktionen auf diesem Wege zu koschreiber ${ }^{[53,54]}$ vorzem hielt man es für nicht möglich, dass ein chemisches System etwas anderes als ein Gleichgewicht erreichen kann. Dies würde dem zweiten Hauptsatz der Thermodynamik, also dem Zustand der maximalen Entropie, entgegenhandeln. Es zeigte sich jedoch, dass offene Systeme (dissipative Systeme) ganz neue Strukturen fern vom thermodynamischen Gleichgewicht besitzen können. Heute ist die nach den Entdeckern benannte Belusov-Zabotinsky-Reaktion (BZ-Reaktion) ein effektvolles Beispiel für oszillierende Reaktionen. Um die vielen kleinen Reaktionszentren, die an der Strukturbildung beteiligt sind, zu beschreiben, war es nötig, ein mathematisches Verfahren zu entwickeln, das eine große Zahl unabhängiger Reaktionszentren gleichzeitig berechnen kann.

Die gesamte Spannbreite der möglichen Anwendungen umfasst all diese Erscheinungen, aus denen Rückschlüsse gezogen werden müssen, welchen Einfluss mikroskopische Fluktuationen auf das makroskopische Verhalten eines Systems haben können. Auch das Leben ist ein hervorragendes Beispiel der globalen Strukturen, die sich aus kleinen, simplen Einheiten, wie Genen, Zellen, Bakterien, Mikro- und Makroorganismen ergeben. 
Die Mannigfaltigkeit des Ökosystems ist unter anderem auf die Vielfalt möglicher Musterbildungen zurückzuführen. Viele Tiere schmücken ihre Haut mit charakteristischen, mosaikartigen Strukturen, die sich ergeben, wenn benachbarte Zellen/Pigmentzellen interagieren. Diese Idee führte schließlich zu einem AktivatorInhibitor Modell, das auch unter dem Namen Volterra-Lotka-Modell bekannt ist.

Die bei der BZ-Reaktionen entstehenden chemischen Muster entsprechen noch einem ganz anderen System. Der Lebenszyklus des Schleimpilzes Dictyostelium discoideum besteht aus zwei Phasen. Solange die Nahrung in Form von Bakterien verfügbar ist, existieren die Zellen als freilebende, eigenständige, herumkriechende Amöben. Sobald sie jedoch beginnen, unter Nahrungsmangel zu leiden, organisieren sie sich zu Aggregaten. Dieses Zusammenwachsen lässt Muster entstehen, die den BZ-Mustern entsprechen. Die Kommunikation zwischen den Amöben basiert auf der Ausscheidung von cyclischem Adenosinmonophosphat (cAMP). cAMP wird auch von anderen Bakterien ausgeschieden, wenn sie unter Nahrungsmittel leiden, und wird manchmal auch Glucosemangelsignal genannt. Man konnte beweisen, dass die entstandenen Muster nicht nur durch zufällige Ähnlichkeit den BZ-Muster entsprechen. In beiden Fällen handelt es sich um mehrere Zentren einer oszillierenden Welle.

Ameisenarten der Gattung Leptothorax bewegen sich nach einem chaotischen Muster nur so lange, bis die Kolonie eine gewisse Größe erreicht. Dann verändert sich das Verhaltensmuster vom ungeordneten zum rhythmischen, mit einer erkennbaren, definierbaren Periode. Diese Gruppe verfällt in einen kollektiven Verhaltensmodus.

Nun weiß man von Bakterien, die unter Laborbedingungen wachsen, dass sie nach einer Wachstumsphase eine zweite stationäre und unproduktive Phase erreichen. Es ist seit langem bekannt, dass z.B. E. coli und viele andere Mikroorganismen am Anfang der Wachstumsphase Glucose nicht vollständig abbauen, sondern metabolische Zwischenprodukte in das Medium ausscheiden. Später werden diese Substanzen wieder aufgenommen und zum Wachstum verwendet. Ein zweiter, wichtiger Faktor ist die Tatsache, dass der Übergang von der Wachstums- zur stationären Phase nur wenige Minuten dauert.

Neben der Emergenz spielt auch die Evolution eine zentrale Rolle in den Optimierungsmethoden, die auf natürlichen Vorgängen aufbauen. 
Das Prinzip der Evolution, also auch der natürlichen Selektion, zwingt alle Individuen einer Population, von kleinsten Molekülen über Zellen bis hin zu Tieren und Menschen zum Egoismus und einer parasitären Verhaltensweise. Manchmal bringt jedoch eine symbiotische, oder sogar altruistische Kooperation einen größeren Gewinn nicht nur für die ganze Population, sondern auch für das Individuum.

In den „LIFE“-Simulationen ziehen sich kooperierende Einheiten zuerst in kleinen Gruppen zusammen, doch kommen sich zwei solche Agglomerate zu nah, werden sie wieder durch egoistische Verhaltensweisen auseinander gebahnt. Ein Beispiel ist die Mycelbildung in Form von kleinen, kugeligen Agglomeraten (Pellets), wie sie bei verschiedenen Bakterien und Pilzen beobachtet wird. Die evolutionäre Spieltheorie kann also die soziobiologischen Komponenten innerhalb evolutionärer Prozesse mathematisch analysieren.

\section{Evolutionstheorie}

Darvin hielt die Evolution für eine natürliche Auslese. Er entdeckte ebenfalls, dass die Kombination des Wettbewerbs um vorhandene Ressourcen mit einem ihm noch unbekannten Variationsmechanismus in Sinne der in einer Population geltenden Spielregeln zur Entstehung einer neuen Artenvielfalt beiträgt.

Wenn die Vermehrung von Organismen in einem Ökosystem zu vererbbaren Variationen führt, werden die besser angepassten Individuen angereichert. Die am schlechtesten angepassten Individuen werden auf dem natürlichen Weg ausselektiert. Mikroorganismen besitzen die Fähigkeit, sich exponentiell zu vermehren. Meistens ist dieser Zuwachs nur durch unzureichende Ressourcen beschränkt. Da die Nachfrage dann immer größer wird, führt sie unausweichlich zu einem Wettstreit zwischen den Konkurrenten. Durch die zufallsbedingte Variabilität der Größe, Stärke, Wachstumsgeschwindigkeit usw. kommt es zu einer zwangsläufigen Bevorzugung der tauglichsten Exemplare (survival of the fittest) ${ }^{[55]}$.

Gene vermehren sich durch Replikation. Gelegentlich in diesem Prozess auftretende kleine Fehler, die Mutationen, erlauben es, dem Konkurrenz- und Überlebensdruck entgegenzuwirken. Zudem können diese Defekte neue Organismen hervorbringen, die sich schneller an verschiedene Lebensräume und Lebensbedienungen anpassen. Die bereits besprochene Resistenzentwicklung der Mikroorganismen ist nur eine Konsequenz dieser Vorgehensweise der Natur. 
Die natürliche Evolution ist also ein ständig fortlaufendes Experiment, in dem die durch die Gene gespeicherte Information kontinuierlich optimiert wird. Sie wird durch Mutation und Rekombination validiert und ist durch die Selektion ständig einer Prüfung unterworfen. Die neodarvinistische Betrachtungsweise fügt diesem Modell zusätzlich die Überkreuzung der elterlichen DNA-Stränge (Rekombination, crossing-over) als wirksames Mittel neben der Mutation hinzu, um eine Veränderung des Organismus zu bewirken.

Die am besten angepassten Individuen einer Population stellen gleichzeitig auch das Resultat einer evolutionären Optimierung dar. Die Suche nach der maximalen Ausbeute einer Umsetzung (technologisches Optimum) oder die Minimalisierung der Herstellungskosten (wirtschaftliches Optimum) gleicht dieser natürlichen Proze$\operatorname{dur}^{[56]}$.

\section{Das Konzept der unscharfen Optimierungsmethoden}

Die Optimierung eines Raumes, der durch eine Anzahl an Parametern definiert wird, bedeutet, diese Größen so zu verändern (die bestmögliche Fitness zu finden), dass eine gestellte Aufgabe besser oder sogar optimal bewältigt werden kann. Bei zwei veränderbaren Parametern kann man sich diesen Suchraum als eine topografische Landschaft vorstellen. Die beiden Variablen werden durch x- und y-Achse repräsentiert. Für jeden Parametersatz (x, y) ist das Ergebnis der Qualitätsfunktion als Höhe des „Gipfels“ in diesem Raum zu verstehen. Der Gipfel entspricht also einem globalen - oder im Falle von mehreren - lokalen Maximum. Hat man mehr als zwei Parameter, nimmt der Raum mehrere Dimensionen an. Da diese Parameter oftmals noch von einander abhängig sind und die mathematische Definition des Suchraumes nicht vorhanden ist (Black Box-Verfahren), ist es häufig unmöglich, durch reine statistische Methoden ein solche Maximum zu finden. Nicht immer ist es jedoch notwendig, die den Raum beschreibende mathematische Funktion zu kennen. Die Menschen z.B. sind in der Lage, undeutliche Sprache zu verstehen, schlechte Schriften zu lesen, eine Situation zu analysieren und anhand der vorliegenden Information einen Entschluss zu fassen. Sie interpretieren die vorliegenden Informationen, was eng mit mathematischen Berechnungen zusammenhängt. Man arbeitet aber nicht mit Zahlen, sondern unscharfen Strukturen ohne fest definierte Grenzen. 
In den 60er Jahren fand das Konzept des survival of the fittest einen Einsatz in der Informatik. Evolutionäre Optimierungsverfahren gehören zu Algorithmen, die überall dort Anwendung finden, wo gewöhnliche, statistische Auswertungen versagen. 1965 versuchte Holland seinen Rechenmaschinen bei der Anpassung an das zu lösende Problem ein wenig von der Elastizität und Flexibilität der lebenden Organismen beibringen. Das war die Geburtsstunde der Genetischen Algorithmen.

Jedoch genauso wie die Zellulären Automaten erlebte diese Idee ihren großen Durchbruch bei der wissenschaftlichen Anwendung erst Mitte der 80er Jahre.

Evolutionäre Algorithmen (EA) orientieren sich an den biologischen Mechanismen des Evolutionsprozesses und erlauben, die Lösungen sogenannter NP-vollständiger (nonlinear-polynomical) Probleme zu finden ${ }^{[57,53]}$.

Sie teilen sich in vier Gebiete. Dazu gehören Genetische Algorithmen (GA), Genetische Programmierung (GP), Evolutionsstrategien (ES) und Evolutionäre Programmierung $(\mathrm{EP})^{[58]}$. In der Biologie wird der genetische Aufbau eines Organismus durch seinen Genotyp definiert. Der Phänotyp offenbart sich durch seine sichtbaren Eigenschaften. In EA verweist der Genotyp auf die Kodierung der für die Fitness verantwortlichen Parameter. Der Phänotyp entspricht dann also dem dekodierten Fitnesswert der Lösung.

Die eigentlichen ES verwenden keine genetischen Ereignisse. Jedes Individuum wird als Satz numerischer Werte (Allele) repräsentiert. Die Optimierung stützt sich auf eine Neubewertung der Lösungen nach dem Umformen. Diese Modifikationen finden jedoch nicht auf der Bitebene statt, sondern vielmehr werden die numerischen Werte um einen bestimmten Betrag verändert.

Bei den GA werden die Erbinformationen als binäre Matrizen in Form eines digitalen Code darstellt. Alle Veränderungen werden durch Bitmanipulationen erreicht.

EA sind allgemeine, einfach einzusetzende, stochastische Suchverfahren, die genauso wie „Neuronale Netze“ ihr Vorbild in dem Neo-Darvinismus finden. Künstliche „Neuronale Netze“ arbeiten wie ihre natürlichen Vorbilder ${ }^{[59]}$. Durch Verstärkung bzw. Dämpfung der ankommenden Signale und durch Knüpfung bzw. Lösen neuer interneuronalen Verbindungen lernen sie, ankommende Informationen zu bewerten und zu speichern. Es sind Algorithmen, die eine Darstellung unscharfer, nichtlinearer Zuordnungen durch Abstrahieren erlauben. Sie eignen sich hervorragend zur Modellierung komplexer Welten und Prozesse ${ }^{[60]}$. 
Genetische Algorithmen gehören zu den stabilsten Optimierungsverfahren, die selbst dann eine Lösung liefern, wenn alle anderen statistischen Methoden durch enorme Zahlen an Parametern scheitern müssen ${ }^{[61,62]}$.

Durch die Nachahmung der Evolution finden die genetischen Auswertemethoden eine Lösung, die besser ist, als die Startbedingung. Dabei werden mehrere Lösungen gleichzeitig angegeben und durch die Evolutionsprinzipien, wie Selektion, Kreuzung und Mutation, die besten ermittelt. Wiederholt man dieses Verfahren mehrmals, werden von Generation zu Generation immer bessere Lösungen entwickelt. Wenn es sich dabei um eine gerichtete und teilweise gesteuerte Veränderung handelt, bewegt man sich in Richtung des Optimums. Eine am Anfang ungeeignete Lösung wird Schritt für Schritt verbessert, indem die Rekombination überwiegend der Individuen mit der höchsten Fitness stattfindet. Die Tochterpopulationen werden so lange erzeugt, bis eine Abbruchbedingung erfüllt ist. Diese Bedingung kann entweder die Anzahl der Durchläufe oder aber das Erreichen des erwarteten Fitnesswertes darstellen. Ist keine Verbesserung des Fitness zu erwarten, so kann der Algorithmus ebenfalls unterbrochen werden ${ }^{[63]}$.

Dadurch erhält man typischerweise nicht die bestmögliche, sondern eine an das gestellte Problem angepasste Lösung. EA ist also ein Algorithmus, der zwar nur eine Näherung liefert, aber sehr flexibel und schnell arbeitet und dazu enorm vielseitig einzusetzen ist. Zeit- und Kostenoptimierung von parallelen Produktionsabläufen, Transportprobleme und sogar der Entwurf von innovativen technischen Maschinen ist mit Hilfe von (EA) möglich.

\section{Genetische Algorithmen bei Optimierungsproblemen}

Bevor man aber ein mathematisches Optimierungsproblem durch EA lösen kann, müssen die zu verändernden Variablen und Startwerte richtig definiert werden. Die richtige Initialisierung der Startwerte trägt dazu bei, die Anzahl der notwendigen Populationen so zu reduzieren, dass ,gute“ Werte erhalten werden. Man sollte vermeiden, von identischen Individuen am Anfang der Optimierung auszugehen, da dann die erwartete genetische Vielfalt erst erzeugt werden müsste.

Wenn man keine Erfahrungswerte über eine sinnvolle Belegung der Gene hat, werden diese standardmäßig mit Zufallswerten initialisiert. Die Gesamtheit aller Gene der Individuen einer Population nennt man den „Gen-Pool““64]. 
Hat man dagegen bereits in Experimenten die Erfahrung gesammelt, wie eine vernünftige Lösung aussehen könnte oder welche Variablen den größten Einfluss auf die Anpassung der Population haben könnten, werden auch die Anfangsparameter entsprechend angepasst. Hat man die neue Generation errechnet, sollte eine Auslese erfolgen. Meist werden die schlechtesten Exemplare aussortiert. Manchmal ist es dennoch notwendig, nicht alle minderwertigen Ergebnisse zu eliminieren, um die Vielfalt der Population nicht zu gefährden und gleichzeitig auch die Gefahr zu vermeiden, in einem lokalen Maximum zu verweilen. Bei einer von Null unterschiedenen Mutationsrate kann sich das System durch vorübergehende Erniedrigung der Gesamtfitness aus der Falle des lokalen Maximum leicht befreien, um anschließend das globale Maximum zu erreichen. Diese Methodik liefert jedoch keinen mathematischen Beweis dafür, dass die erhaltenen Maxima tatsächlich eine Antwort auf die gestellte Frage darstellen. Alles, was man im Endergebnis bekommt, sind empirische Daten. Die Auswertung dieser Daten wird dem Experimentator überlassen.

Die mikrobielle Fermentation eines Naturstoffs ist im Vergleich zur chemischen Synthese unter vielen Aspekten vorteilhafter. Mikroorganismen können schnell und kostengünstig große Moleküle aus einfachen Bausteinen unter Standardbedingungen synthetisieren. Dabei kann dazu die erwünschte Variabilität erzeugt werden. Doch dafür müssen die Kultivierungsmethoden an den jeweiligen Stamm angepasst werden. Man konnte z.B. die Produktion von 2 bei Penicillium chrysogenum lediglich durch Optimierung der Wachstumsbedingungen um den Faktor 40000 steigern. 


\section{Ablauf des Genetischen Algorithmus}

Die Zahl der Wiederholungen (N) sollte sinnvoller Weise wesentlich kleiner sein, als die Anzahl der Kombinationsmöglichkeiten der Eingangsparameter der Optimierung. Genauso wie N stellen die Wahrscheinlichkeiten für Mutationen, Rekombinationen, die Anzahl der Individuen und die Sterberate einen geschwindigkeits- und qualitätsbestimmenden Faktor dar. Sie unterliegen keinen allgemeingültigen Regeln, sondern vielmehr muss der Anwender sie experimentell an seine Fragestellung anpassen ${ }^{[65]}$.

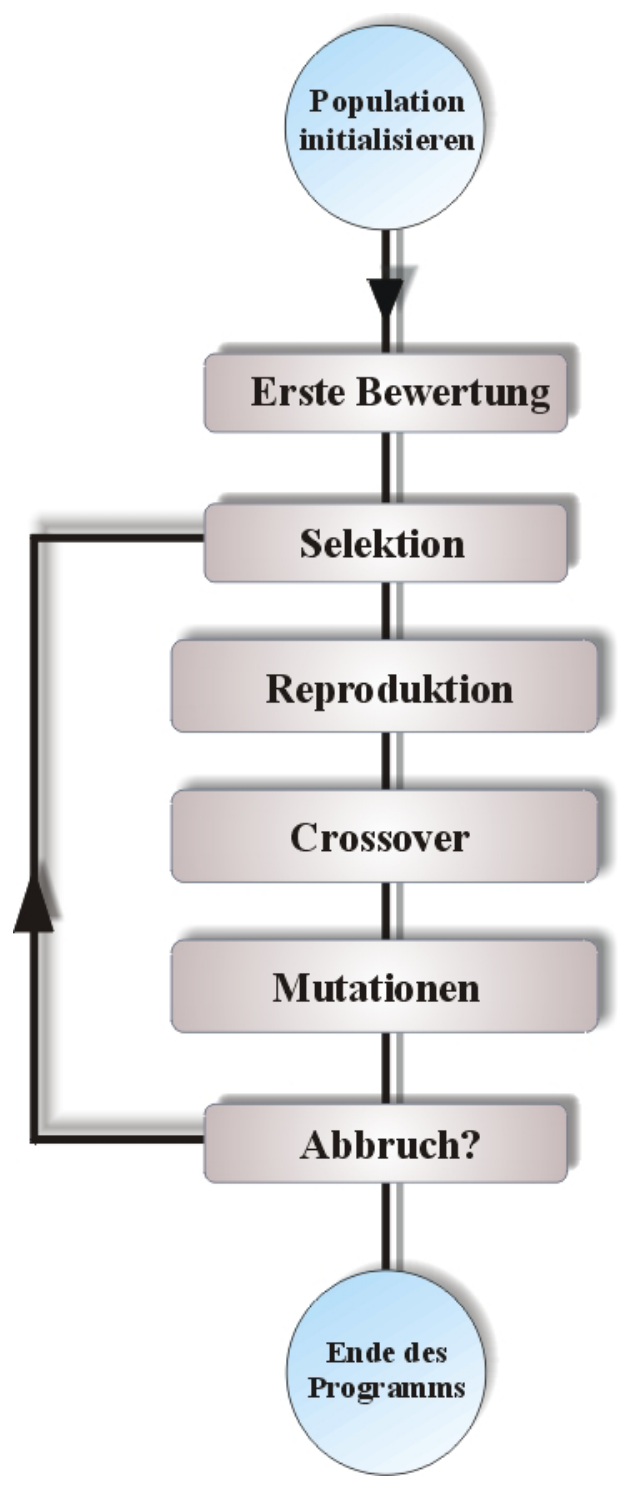

Abbildung 15: Schematische Darstellung eines auf Genetischen Algorithmen basierenden Programms.

Eine Optimierungsprozedur, die mit Hilfe von GA arbeitet, besteht aus diversen, sich zeitlich wiederholenden Unterprozeduren, wie es aus der Grafik ersichtlich wird. Das in dieser Arbeit verwendete Programm „GALOP“ (Genetische Algorithmen zur Op- 
timierung von Prozessen) vom Forschungszentrum Jülich GmbH ist ebenfalls so konzipiert und besteht aus den nachfolgend beschriebenen Unterprozeduren ${ }^{[66]}$.

\section{Erstellen der Startpopulation}

Die erste Generation wird in der Regel per Zufall erzeugt. Im Binärvektor der kodierten Parameter werden die Werte gleich Eins mit einer bestimmten Wahrscheinlichkeit gesetzt. Diese Wahrscheinlichkeit wird entweder durch den GA angegeben oder als Erfahrungswerte (bezogen auf experimentelle Daten) definiert. Erfolgt die Auswahl der ersten Population nicht zufällig, werden meist experimentelle Erkenntnisse in Betracht gezogen. Bei einer Population aus N Individuen werden im Rechner N Arrays erzeugt. Die Parameter werden in den Arrays durch Nullen und Einsen repräsentiert. Welcher von den beiden Werten gesetzt wird, hängt von der Bewertung der Parameter ab, die sich dahinter verstecken.

\section{Erste Bewertung der Population}

Die als Gencluster gespeicherten Informationen werden ausgewertet. Je nach Qualität der Anpassung erhält jedes Gen eine Bewertung, einen Fitnesswert. Automatisch wird jedem neu erzeugten Individuum anhand seines Genpools und der Anpassungsfähigkeit in Richtung Optimierungsziel eine Fitness zugeordnet. Diese Charakterisierung führt jedoch noch nicht zum Abbruch des Programms, auch wenn die Fitnesswerte über das Ziel der Optimierung hinausragen.

\section{Selektion}

Unter Selektion wird im allgemeinen die Bewertung der Fähigkeiten einer Spezies verstanden, sich an ihre Umwelt anzupassen. Durch bessere Eigenschaften kann das Individuum einen elitären Status erwerben, der ihn vor der Elimination oder Veränderung schützt. Dieser Zustand wird nur bis zu der nächsten Selektion beibehalten. Diese Überprüfung ist meist zeitaufwendiger als alle anderen Schritte der GA. Auch hier befolgt man zwei Strategien. Entweder wird die alte Population komplett durch die gerade entstandene ersetzt, die neue dient dabei nur als Ausgangspunkt für die nächste Generation, oder die besten Individuen der alten Population werden in die 
neue übertragen, um sicherzustellen, dass die guten Merkmale aufgrund der stochastischen Selektion und anderer Operatoren nicht ganz aussterben.

\section{Reproduktion}

Bei der Reproduktion entsteht eine neue Generation. Die einfachste Art der Vermehrung ist ein Kopiervorgang der alten Population, wobei absichtlich Fehler (Mutationen) gemacht werden. Bei GA werden darüber hinaus durch crossover der elterlichen Gene bessere Individuen generiert.

\section{Crossover}

Bei dem Crossover handelt es sich um einen Austausch des genetischen Materials und somit ein Informationsaustausch zwischen den Eltern, um einen neue Nachkommen mit kombinierten Eigenschaften zu produzieren.

Bei einer Ein-Punkt Replikation wird nur an einer zufällig ausgewählten Position beider Bitvektoren (je ein Bitvektor pro Elternteil) geschnitten. Die so erhaltenen vier Teile werden gekreuzt miteinander verbunden.

Crossover mit mehreren Schneidestellen wird als uniform oder als n-point crossover definiert. Dabei hat jedes Bit (Gen) des Nachkommens eine bestimmte Wahrscheinlichkeit, von einem der beiden Elternteile abzustammen. Die Erbinformationen der Eltern werden an mehreren zufälligen Stellen getrennt und neu kombiniert. Die Reihenfolge der Bits wird jedoch nicht vertauscht. Die uniformen Replikationen sind effektiver in der Vermischung des genetischen Materials. Gleichzeitig wirken sie aber bei „guten“ Genen zerstörerischer. 
Kind A

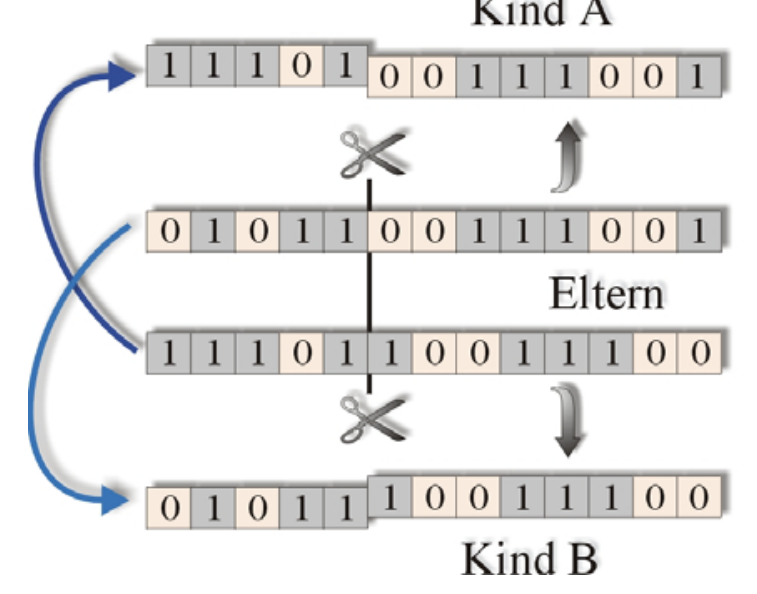

Abbildung 16: Eine Vermischung des Genmaterials während des einfachen crossover-Prozesses

\section{GALOP - Genetische Algorithmen zur Optimierung von Prozessen}

Bei Arbeiten mit GALOP ist es notwendig, die grundlegenden Ideen der Optimierung anhand der Genetischen Algorithmen zu verstehen. Es wird deswegen auf die Beschreibung der Optimierungsmethoden im Kapitel „Information und Chaos“ verwiesen.

Weiterführende Informationen bezüglich der Installation und Bedienung der fensterorientierten Oberfläche des Programms sind beim Erwerb der Anwendung mitgeliefert worden und werden in dieser Arbeit nicht weiter besprochen. Die folgenden Erläuterungen beschränken sich lediglich auf die Beschreibung der notwendigen Schritte, um eine Optimierung eines beliebigen Systems, hier eines Nährmediums, praktisch durchzuführen.

\section{Menü-Übersicht}

Das Menü Project dient der allgemeinen Kommunikation des Programms mit der Peripherie, wie Drucker, Festplatte und anderen Anwendungen. Es erlaubt die Parameter der Mutationsrate und Crossingrate zu verändern, ein neues Projekt zu generieren und die erste, zufällige Generation zu erzeugen. Es bietet ebenfalls die Möglichkeit, die bereits generierten Daten auf der Festplatte zu sichern, abzurufen und zu drucken. Es besteht aus folgenden Funktionen:

New: Alle ungespeicherten Daten werden gelöscht und ein neuer Optimierungsversuch wird gestartet. 
Open: Erlaubt das Öffnen der bereits auf der Festplatte gespeicherten Daten und Optimierungsversuchen.

Save: Bietet die Möglichkeit, die durch das Programm erzeugten Daten auf der Festplatte zu sichern.

Print: Erlaubt den Ausdruck der angelegten Daten (funktioniert nur mit Druckern, die durch eine LPT1 Schnittstelle mit dem Rechner verbunden sind).

Export to Excel: Die erzeugte Generation wird in Form einer Excel-Datei auf der Festplatte abgelegt und liegt in tabellarischer Form vor.

Init GA: Durch den Auswahl dieser Funktion wird die generierte Population gelöscht und eine neue erzeugt. Diese Funktion ist der Funktion New ähnlich. Der Unterschied besteht darin, dass ein bereits der laufenden Optimierung zugewiesener Name auf dieser Weise leicht übernommen werden kann.

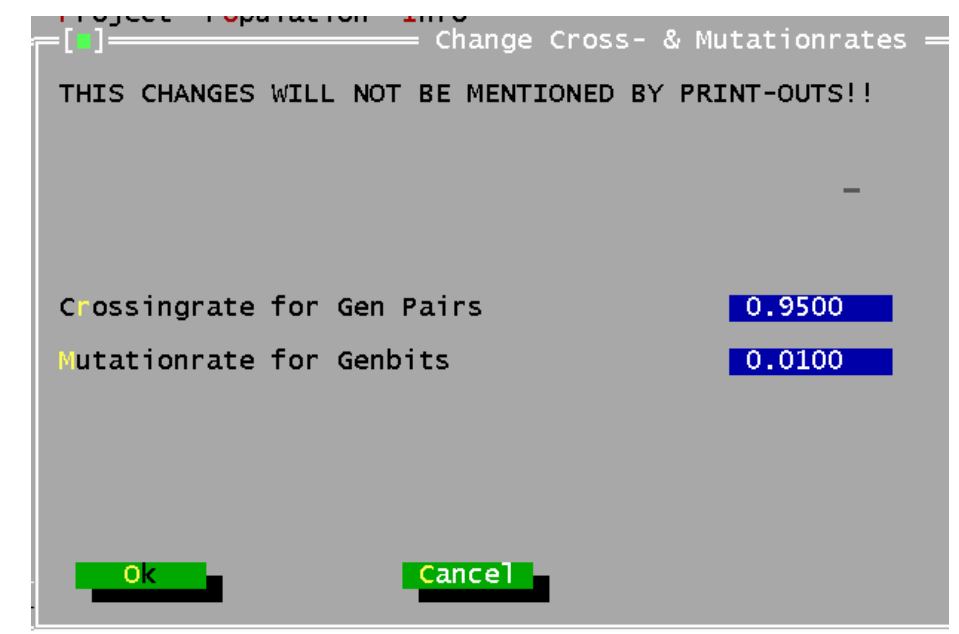

Abbildung 17: Die Crossingrate und Mutationsrate können verändert werden.

Change Rates: Das Programm erlaubt unter diesem Menüpunkt die Crossingrate für Genpaare sowie die Mutationsrate für Genbits zu verändern. Welche Auswirkung es auf die zukünftig generierten, neuen Generationen haben kann, wurde im Kapitel „Ablauf des Genetischen Algorithmus“ ausführlich besprochen. Die Crossingrate wird standardmäßig auf 0.95 und die Mutationsrate auf 0.01 gesetzt.

Randomize: Erzeugt zufällig eine Generation.

Exit: Das Programm wird durch den Aufruf diese Funktion beendet. 


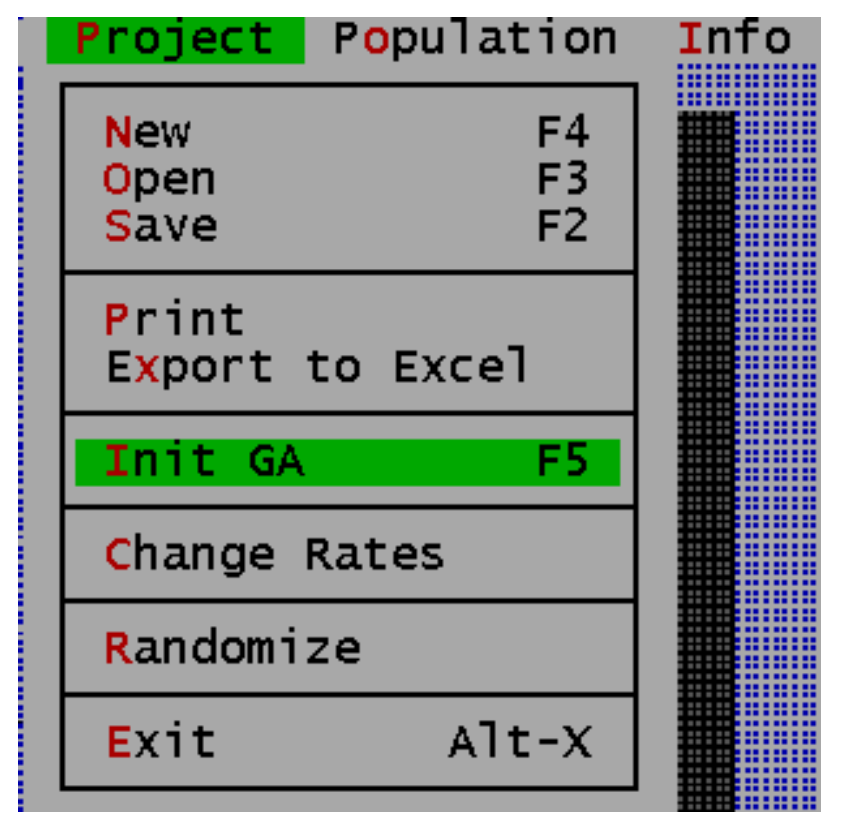

Abbildung 18: Menü Project mit allen Unterfunktionen

Das Menü Population erlaubt die Manipulation der erzeugten Population sowie die Angabe der experimentellen Daten für jedes Individuum dieser Population. Weiterhin bietet es die Möglichkeit, die Tochtergeneration zu erzeugen sowie die angegebenen Daten der aktuellen Generation zu löschen. Die Angaben können entweder gedruckt oder in Form einer Texttabelle auf dem Datenträger gesichert werden. Es besteht aus folgenden Unterfunktionen:

Edit: Erlaubt das Manipulieren der zu optimierenden Daten. Unter diesem Menüpunkt sind alle zu optimierenden Parameter anzugeben sowie die Grenzen der Optimierung (z.B. minimale und maximale Konzentrationen der Medienkomponenten) für jedes Individuum zu definieren.

Enter Results: Die im Optimierungsexperiment gewonnenen Daten können beim Aufruf dieser Unterfunktion jedem Individuum zugeordnet werden. Bei den Daten kann es sich z.B. um Mycelmenge, Peakhöhe, Lichtabsorption bei Farbstoffen oder andere physikalische Größen handeln. Diese Angaben beeinflussen die Tochtergeneration am stärksten, denn anhand dieser experimentellen Werte wird die Auswahl getroffen, welche Individuen eliminiert werden.

Next Generation: kreiert aus der Elterngeneration eine neue Tochtergeneration. Die Elterngeneration wird bei diesem Prozess unausweichlich gelöscht. Es ist also notwendig, die Daten auf dem Datenträger zu sichern, bevor man eine neue Generation erzeugt. 
Print: Erlaubt den Ausdruck der bereits erzeugten Daten. (Funktioniert nur mit Druckern, die durch eine LPT1-Schnittstelle mit dem Rechner verbunden sind).

Write as Table: Speichert die eingegebenen Daten in Form einer Tabelle auf dem Datenträger unter dem angegebenen Namen.

Remove Pop: Die aktuelle Population kann auf Wunsch gelöscht werden. Durch den Aufruf dieser Funktion kann die durch die Tochtergeneration ersetzte Elterngeneration wieder als Ausgangspopulation eingesetzt werden. Diese Funktion hebt also die Wirkung der Funktion Next Generation auf.

\begin{tabular}{|c|c|}
\hline Project & Population Info \\
\hline 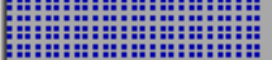 & Edit \\
\hline 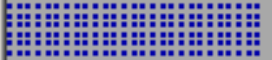 & Enter Results \\
\hline 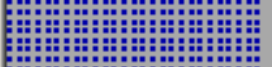 & Next Generation \\
\hline 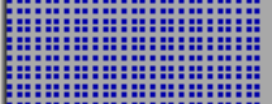 & $\begin{array}{l}\text { Print } \\
\text { Write as Table }\end{array}$ \\
\hline 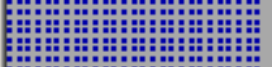 & Remove Pop \\
\hline
\end{tabular}

Abbildung 19: Menü Population mit allen Unterfunktionen

Im Menü Info können zwei aufklappbare Fenster aufgerufen werden. Die Unterfunktion „project info“ liefert eine Beschreibung des aktuellen Projektes und den $\mathrm{Na}$ men, durch den die durchgeführte Optimierung identifiziert werden kann. Dieser Name wird gleichzeitig als Dateiname auf dem Datenträger verwendet, wenn die gewonnenen Daten durch die Funktion Save gesichert wurden.

Das zweite Fenster, das durch den Auswahl der Unterfunktion ,product info“ zugänglich ist, zeigt die Informationen des Herstellers an.

\section{Anlegen eines neuen Optimierungsprojektes}

Um ein neues Optimierungsprojekt zu erzeugen, wählt man zuerst die Unterfunktion New aus dem Menü Project. Es erscheinen nacheinander einige Dialogfenster, die einer Initialisierung der ersten Population (s. Abbildung 20) gleichgesetzt werden können. Die Optimierungsmethode erhält einen eindeutigen Namen, unter dem sie auf dem Datenträger abgelegt werden kann. Es besteht jedoch die Möglichkeit, zwischen dem Namen der Optimierung und der erzeugten Datei zu unterscheiden. Bei 
der Angabe des Namens der Datei wird keine Namenserweiterung (Suffix: .xyz) angegeben, denn es wird automatisch durch das Programm erzeug. Zusätzlich besteht die Möglichkeit, das Projekt mit ergänzenden Informationen $\mathrm{zu}$ versehen (Comment). Die Bestätigung der Angaben erfolgt durch die Taste OK und führt gleichzeitig zur nächsten Eingabemaske. Es handelt sich dabei um ein Dialogfenster, in dem die zu optimierenden Parameter, die sogenannten Variationsgrößen, wie z.B. die Konzentration von Maltose, Glucose, $\mathrm{CaCl}_{2}$ und Hefe im Medium, anzugeben sind. Diese Parameter werden als Gene codiert. In diesem Eingabefenster gibt man an, aus welchen Teilstücken (bits) sich das vollständige Gen zusammensetzt ist. Es resultiert aus der Summe aller Bitfragmente. Zusätzlich bestimmt man den Bereich, in dem die gesuchten Größen verändert werden können. Dies wird durch die minimale und maximale Konzentrationsangabe definiert. Jedes Teilstück kann mit einem abstrakten Namen und einer physikalischen Einheit versehen werden. Die Berechnungen werden stets mit der angegebenen Genauigkeit durchgeführt. Die in der nächsten Generation generierten Werte der Variationsparameter sind zusätzlich durch die Option Distribution zu beeinflussen. Sie gleicht dem Erfahrungspotential des Experimentators und erlaubt die bereits bei der Laborarbeit gesammelten Kenntnisse bei der Optimierung einzusetzen.

Das folgende Fenster trägt den Namen Create Variation Elements und beinhaltet folgende Eingabemasken:

Name: In diesem Bereich werden alle Namen der Variationselemente festgelegt. Die Namen können aus Buchstaben, Zahlen, Sonderzeichen und deren jeglichen Kombinationen bestehen.

Unit: Ist ein Informationsfeld, in dem man angeben kann, in welchen physikalischen Größen die Variationselemente bei der Optimierung auftreten. Dieses Feld dient nur Informationszwecken und hat keinen Einfluss auf das Ergebnis der Optimierung.

Round res: In diesem Feld wird die kleinste Auflösung definiert, die beim Protokollieren am Bildschirm ausgegeben wird. Die Zahlen werden zwar im gewünschten Format gerundet angegeben, werden aber trotzdem mit der Rechnergenauigkeit des Computers für die weiteren Berechnungen eingesetzt.

Lower: Ist die untere Grenze des Wertebereichs, bis zu dem die zu optimierende Größe verändert wird. 
Upper: Die obere Grenze des zu optimierenden Wertes wird durch diese Option festgelegt.

Bits: Diese Spalte definiert die Zahl der Bits, die zur Bildung eines Genteilstückes verwendet werden. Die Zahl der Informationen, die durch solche Teilstücke kodiert werden können ist gleich $2^{Z}$, wobei $Z$ den in dieser Spalte angegebenen Wert repräsentiert.

D (distribution): Mit der Betätigung dieser Schaltfläche gelangt man zu einem weiteren Angabefenster, in dem der Verlauf einer Zufallsfunktion definiert werden kann. Diese Funktion ermöglicht es einen zusätzlichen, geringen Einfluss auf die Startwerte auszuüben. Man kann so bewirken, dass die generierten Anfangswerte der ersten Generation in bestimmten Bereichen öfter angenommen werden. Die horizontale Achse ist das Variationsintervall des Elementes. Die Höhe des Wertes ist mit der Maus bei niedergedrückter Maustaste zu verändern.

Die Taste Add fügt der Liste einen neuen Variationsparameter hinzu. Die Taste Remove entfernt das letzte Element aus der Liste der Parameter.

Falls man die in diesem Fenster gemachten Angaben auch bei späteren Optimierungsversuchen einsetzen möchte, besteht die Möglichkeit, sie mit der Taste Save/Load zu verwenden.

Anhand der gemessenen Versuchsgrößen wird bei der Ausgangspopulation entschieden, welche Individuen die besten Resultate liefern und eine Basis für die Erzeugung der Tochtergeneration darstellen können. Diese Ergebnisse, sei es Peakgrößen im Chromatogramm bei der Messung des Rohextraktes, optische Dichte der Zellkultur oder die erzeugte Biomasse, können intern gespeichert werden und mit einem Bedeutungswert versehen werden. Dieser Wert bestimmt, welche Bedeutung die aufgenommene Einheit für die Optimierung hat und kann optional bei der Bewertungsfunktion eingesetzt werden.

Die Messwerte sind im nächsten Fenster (Create Result Elements) einzugeben. Wird auch dieses Fenster mit der OK-Bestätigung abgeschlossen, gelangt man zum Fenster Edit Fitness Objectives.

Unter dem Gesichtspunkt „Erste Bewertung“ (s. Abbildung 27) wird in dieser Eingabemaske dem Programm mitgeteilt, nach welchen Kriterien überhaupt optimiert wird. Es ist erlaubt, mehrere Parameter parallel zu optimieren. Die „Güte“ ist ein 
Verteilungsverhältnis, das einer Generation eine Bedeutungsfunktion zuschreibt, die sich aus den zu optimierenden Parametern und allen Messgrößen zusammensetzt.

Das entsprechende Fenster wird aus mehreren Eingabefeldern aufgebaut:

Name: Der Name der Bewertungsfunktion.

Unit: Die Einheit der Bewertungsfunktion kann in diesem Eingabefeld definiert werden. Sie hat jedoch keinen Einfluss auf die berechneten Ergebnisse.

Rel. Weight: Anhand der relativen Gewichtung der Bewertungsfunktion wird eine Population in mehrere kleine Einheiten unterteilt, bei denen die Parameter und Messergebnisse sich mit der unterschiedlichen Bedeutung auf die nächste Population auswirken.

Edit: Wird die Gewichtungsfunktion benannt, muss sie noch zusätzlich definiert werden. Durch das Anklicken der Taste Edit kommt man zu einer neuen Eingabemaske Edit Function.

In der Maske Edit Function ist man in der Lage, mehrere Parameter der Gütefunktion des Programms einzustellen. Das Endergebnis ist eine Linearkombination aus Variationsgrößen und Ergebnisgrößen, die durch die unten angegebene Formel wiedergegeben wird:

$$
f\left(x_{1}, x_{2}, x_{3}, \ldots\right)=\sum_{i} a_{i} \cdot x_{i}
$$

Es ist allerdings zu beachten, dass eine größere Zahl besser ist als eine niedrigere. Sucht man nach einem Minimum, ist eine größere Zahl für $a_{i}$ ebenfalls besser als eine kleinere, muss aber zusätzlich mit einem negativen Koeffizienten versehen werden.

Weitere Eingabefelder dieses Fensters sind:

Scaling Window: Gibt an, wie viele vergangene Generationen bei der Bewertung der Güte durch den Genetischen Algorithmus berücksichtigt werden sollen. Wird die Zahl groß gehalten, arbeitet der GA sensibler, weil bei der Erzeugung der Tochtergeneration auf größere Erfahrung zugegriffen wird. Es reduziert aber auch dadurch die Wahrscheinlichkeit, ein im großen Maß mutiertes Individuum zu erzeugen.

Fitness Multiple Faktor: Wird das beste Individuum erzeugt, kann es beliebig oft ohne Veränderung in die nächste Generation übernommen werden. Der Faktor gibt an, wie oft dies passieren soll. 
Jury Decision Kind: Zur Zeit der Experimente standen vier Methoden zur Verfügung, die das Übernehmen des Individuum in die nächste Generation gesteuert haben:

Roulette: Aus dem Pool aller möglichen Individuen werden zufallsbedingt für die Tochtergeneration die gültigen bestimmt. Dabei kann es passieren, dass die am besten angepassten nicht ausgewählt werden und aussterben.

Keep best, roulette: Wie bei der Roulette-Methode werden die Individuen zufallsbedingt ausgewählt. Es wird jedoch garantiert, dass das beste Individuum auf jeden Fall ausgewählt wird.

Ball bearing: Der zufallsbedingte Auswahl erfolgt nur bei dem ersten Individuum, die restlichen befinden sich in einem bestimmten Abstand von dem ersten.

Keep best, ball bearing: Die Auswahl erfolgt wie bei Ball bearing mit der zusätzlichen Garantie, dass auch das am besten angepasste Individuum in die Tochtergeneration übernommen wird.

\section{Das Optimieren der Medienzusammensetzung}

Wie aus der Abbildung 15 folgt, besteht eine Optimierung aus mehreren Schritten und fängt mit der Erzeugung der ersten Population an. Meistens werden nur die Randbedingungen und die Zahl der Versuche (gleicht der Zahl der Individuen einer Generation) eindeutig definiert. Sodann werden die Versuche durchgeführt und die erhaltenen Werte dem Programm zur Verfügung gestellt. Nach der Auswertung werden die an die gestellte Aufgabe am besten angepassten Individuen/Ergebnisse selektiert und dienen als Ausgangsmaterial bei der Erzeugung der Tochtergeneration. Dies geschieht durch Crossover und Mutationen der informationstragenden Bits, die in diesem Fall auch als Gene bezeichnet werden. Wenn die neue Generation erzeugt wird, erfolgen weitere Experimente, bei denen die neu generierten Werte im Versuch eingesetzt werden. Diese Schritte wiederholen sich, bis eine vorausgesetzte Zahl der Generationen erreicht wird oder ein Optimum (Maximum/Minimum) der gesuchten Funktion gefunden wird. Diese Vorgehensweise fand bei den im Rahmen dieser Arbeit durchgeführten Optimierungsversuchen Anwendung. Es wurde vorgegeben, dass die Zahl der Populationen und die sich daraus ergebenen Zahl der Experimente im Einklang mit der benötigten Zeit stehen musste. Die Zahl der Optimierungsversuche wurde auf 5 Generationen mit je 15 Individuen festgesetzt. 
Für die Optimierungsarbeiten wurden die Stämme GW 7/2495, B 7284, B 7939, B 8927, GW 20/1811, B 9020, B 8027, GW 19/2497 ausgewählt.

Die Optimierung erfolgte für alle ausgewählten Stämme auf die gleiche Art und Weise und wird nun anhand des Stammes GW 7/2495 näher erläutert.

Das zu optimierende Medium sollte aus vier Komponenten bestehen, Glucose, Maltose, Hefeextrakt und $\mathrm{CaCl}_{2}$. Zum einen wurden Hefeextrakt und Glucose bereits mit großem Erfolg bei den Standardmedium $\mathrm{M}_{2}^{+}$eingesetzt. Glucose dient den Mikroorganismen als Kohlenstoffquelle und Energieträger. Hefeextrakt ist ein wichtiger, vitaminreicher Aminosäurelieferant. Maltose sollte als eine weitere Kohlenstoffquelle eingesetzt werden. Als Stressfaktor, der die Bakterien zur Produktion der Sekundärmetabolite anregen sollte, wurde $\mathrm{CaCl}_{2}$ ausgewählt. Dabei spielten folgende Überlegungen eine entscheidende Rolle: es wurde von Goodwin gefunden, dass die Calciumionenkonzentration der Nährmedien einen starken Einfluss auf die Morphogenese von Acetabularia hat ${ }^{[67-69]}$. Verschiedene Experimente zeigen, dass nicht nur die Wirkung von Calciumionen auf die Zellwand, sondern auch die Konzentration dieser Ionen im Zytoplasma von entscheidender Bedeutung ist. Calcium beeinflusst nicht nur das Elastizitätsmodul der Zellwand, sondern greift in viele andere Funktionen der Zelle ein.

$\mathrm{Ca}^{2+}$ Ionen besitzen die Fähigkeit, an Proteine zu binden und dadurch deren Aktivität zu verändern. Steigt die Calciumionenkonzentration im Zytoplasma über den Wert von $10^{-5} \mathrm{Mol}$, werden sämtliche Lebensvorgänge in der Zelle unterdrückt, und die Zelle stirbt. Um eine möglichst große Unabhängigkeit gegenüber der $\mathrm{Ca}^{2+}-$ Konzentration außerhalb der Zelle zu garantieren, wird die Konzentration von $\mathrm{Ca}^{2+}$ Ionen auf eine sehr exakte Weise kontrolliert. Zum einen sind in der Zellmembran Proteine eingelagert (Calciumpumpen), die die überschüssigen Ionen nach außen transportieren können. Zum anderen werden spezielle Calcium bindende Proteine gebildet. Sie übernehmen die Aufgabe, im Falle einer zu geringen Calciumkonzentration im Nährmedium dennoch eine ausreichende Menge dieser Ionen dem Zellplasma zur Verfügung zu stellen. Es gibt also einen Rückkopplungsmechanismus, der eine Anpassung der Bakterien an die Umgebungskonzentration garantiert.

Bei allen Medien für marine Streptomyceten wurde eine Mischung aus 50 \% künstlichem Meerwasser und $50 \%$ Leitungswasser eingesetzt. 


\section{Initialisieren einer Startpopulation}

Unter dem Menüpunkt Project wird durch den Aufruf der Funktion New ein neues Projekt angelegt und auf der Festplatte gespeichert (Save). Die so erzeugte Startpopulation muss noch mit entsprechenden Parametern initialisiert werden.

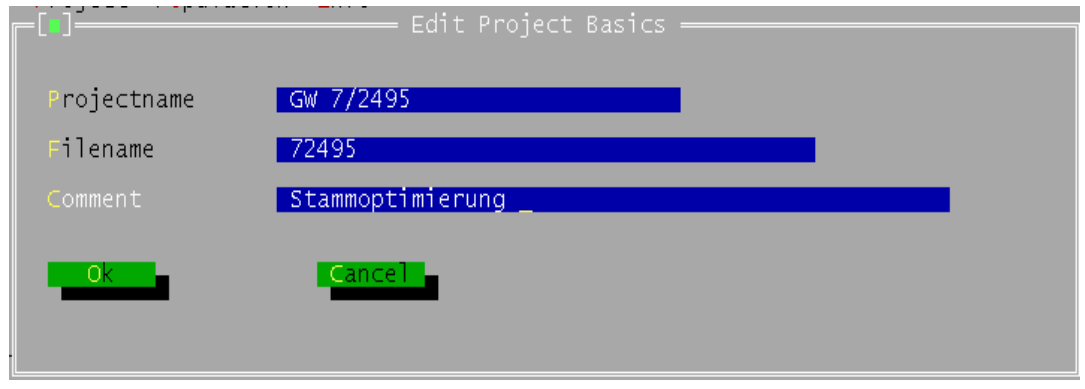

Abbildung 20: Eingabefeld für die Funktion New

Nach dem Betätigen der OK-Taste werden die zu optimierenden Parameter im Eingabefeld Name definiert und mit einer entsprechenden physikalischen Größe versehen. Weiterhin wird die Genauigkeit der Ausgabe festgesetzt und die untere und obere Grenze für die Konzentrationen der Medienbestandteile festgelegt. Die untere Grenze liegt bei 0, was dem Fehlen der entsprechenden Komponente im Nährmedium entspricht. Der obere Wert wurde für alle Komponenten auf $10 \mathrm{~g}$ pro Liter begrenzt.

Diese Parameter werden durch je 5 Bits kodiert, was dem Gehalt von $32\left(2^{5}\right)$ Informationen entspricht. Diese Bits sind dann ein Bestandteil des Bitgens, der bei der Berechnung einer neuen Generation der Mutation und dem Crossover unterliegt.

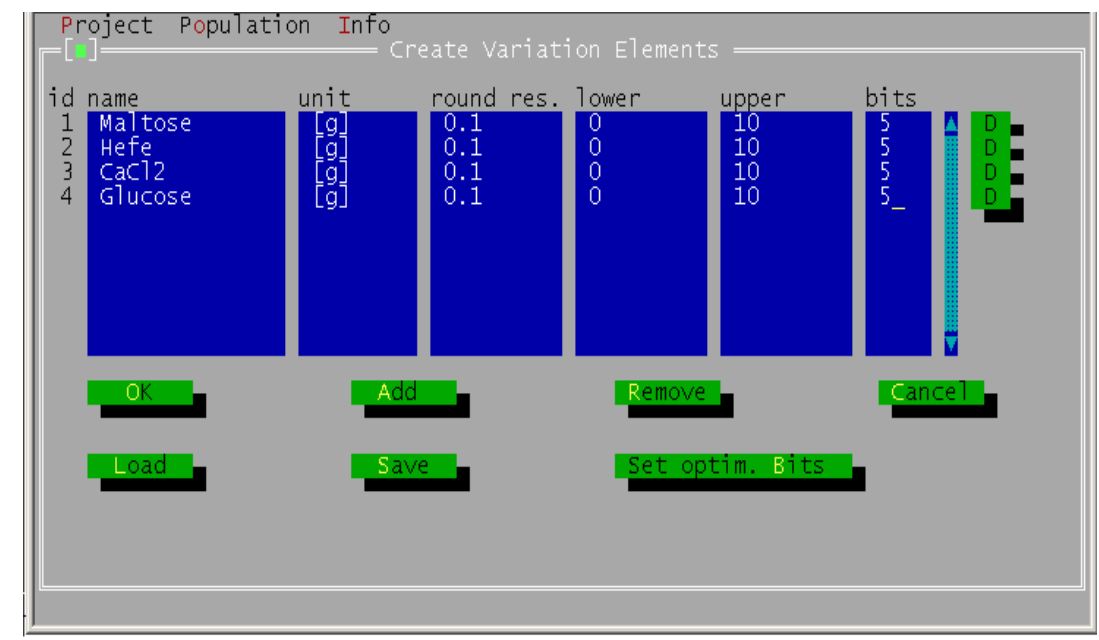

Abbildung 21: Eingabefeld für die Angabe der veränderbaren Parameter 
Ist bereits ein bestimmtes Vorwissen über den Einfluss der Konzentrationen der Medienbestandteile vorhanden, kann man durch den Aufruf der Unterfunktion Distribution die günstigen Bereiche der Variationselemente festlegen. Erfahrungsgemäß erwartet man z.B. den Anstieg der Biomassenproduktion für höhere Glucosekonzentration. Das gleiche gilt für Hefeextrakt. Es ist also vernünftig, die Verteilungsfunktion so zu definieren, dass höhere Konzentrationswerte für diese Stoffe öfter generiert werden. Der Verlauf der so definierten Funktion für Hefeextrakt ist in der Abbildung 22 dargestellt. Für Calciumkonzentration sind die Werte zu invertieren, denn bei sehr hohen Konzentrationen können die Bakterien abgetötet werden. Distribution definiert also die Wahrscheinlichkeit, mit der bestimmte Werte generiert werden.

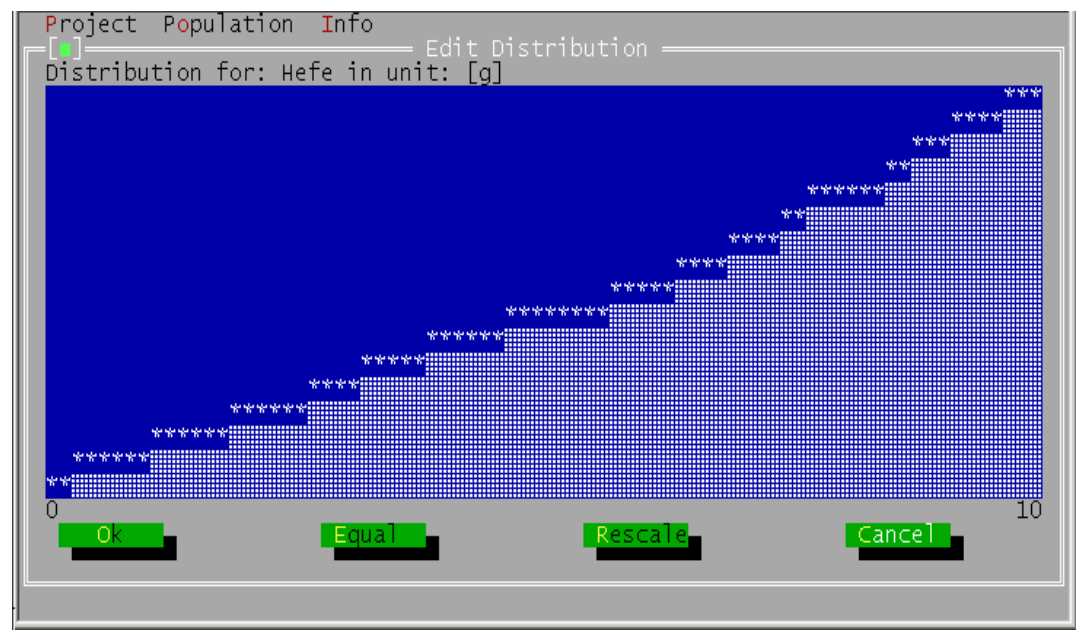

Abbildung 22: Eingabefeld für Distribution. Hohe Lage entspricht einer hohen Wahrscheinlichkeit bei der Generierung der Zufallswerte.

Im nächsten Schritt werden die Messgrößen, die im Versuch beobachtet werden, definiert. Bei der durchgeführten Optimierung basierten die Messungen auf dem HPLC-Screening der Rohextrakte. Die Peakfläche der von den Bakterien produzierten Hauptkomponenten wurde eingegeben, jedoch mit einem kleinen Gewichtsfaktor der Bewertungsfunktion versehen. Dies geschah aufgrund der Überlegung, dass bei der Optimierung nicht die Produktion der bereits von den Bakterien in größeren Mengen hergestellten bekannten Metabolite als Ziel gesetzt wurde. Vielmehr sollte bei der Gesamtproduktion der Biomasse ein Maximum erreicht werden. Gleichzeitig sollte die Produktion neuer Sekundärmetabolite angeregt werden. Die Optimierung verlief also in Richtung der maximalen Biomasseproduktion. Diesem Messwert wurde deswegen ein Gewichtsfaktor von 5 zugewiesen. 


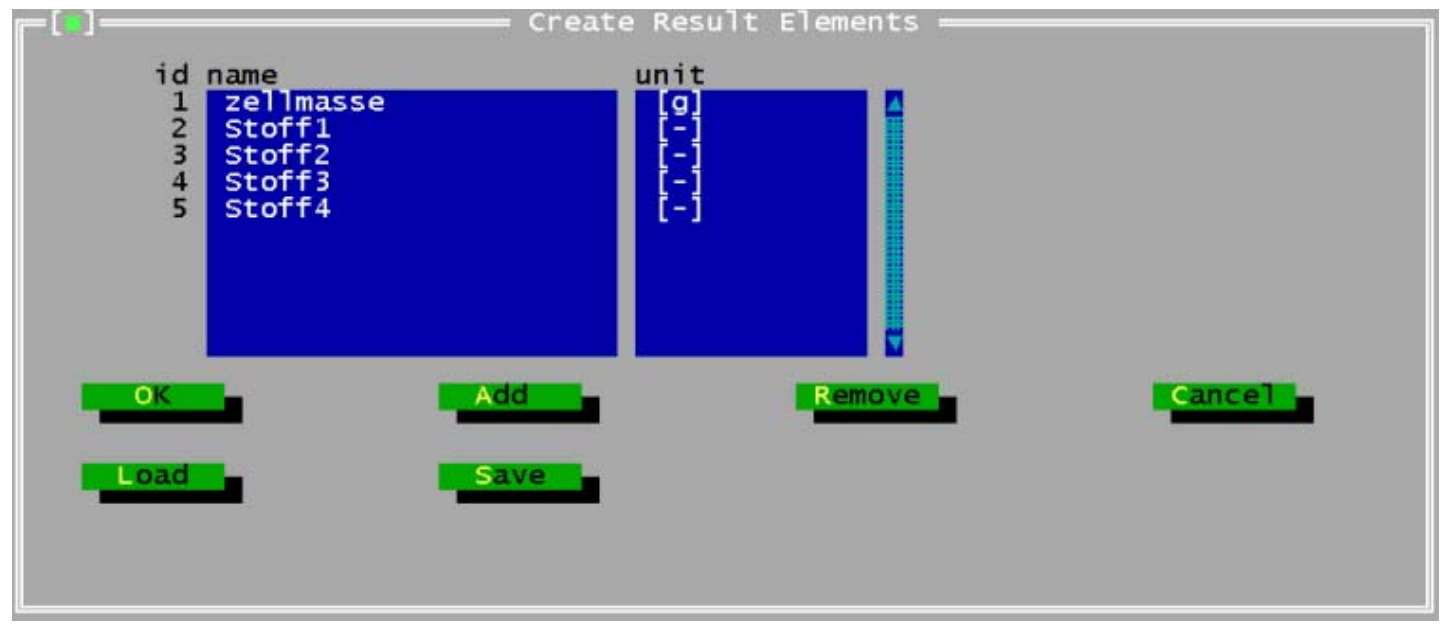

Abbildung 23: Eingabefeld für die Messgrößen

Beim Auswahl der Individuen, die als Vorlage für die Generierung der Tochtergeneration dienten, wurde durch die Schaltfläche Edit die Zufallsfunktion „keep best, roulette on rest" ausgewählt.

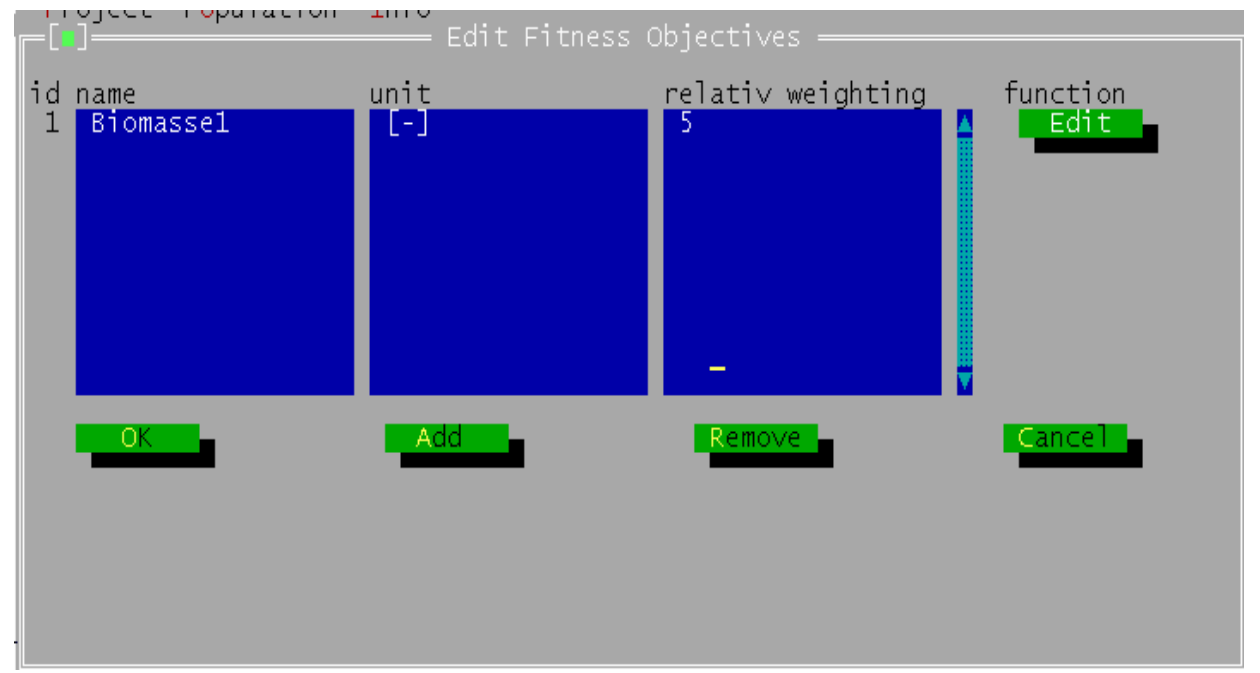

Abbildung 24: Eingabefeld für die Zieldefinition 


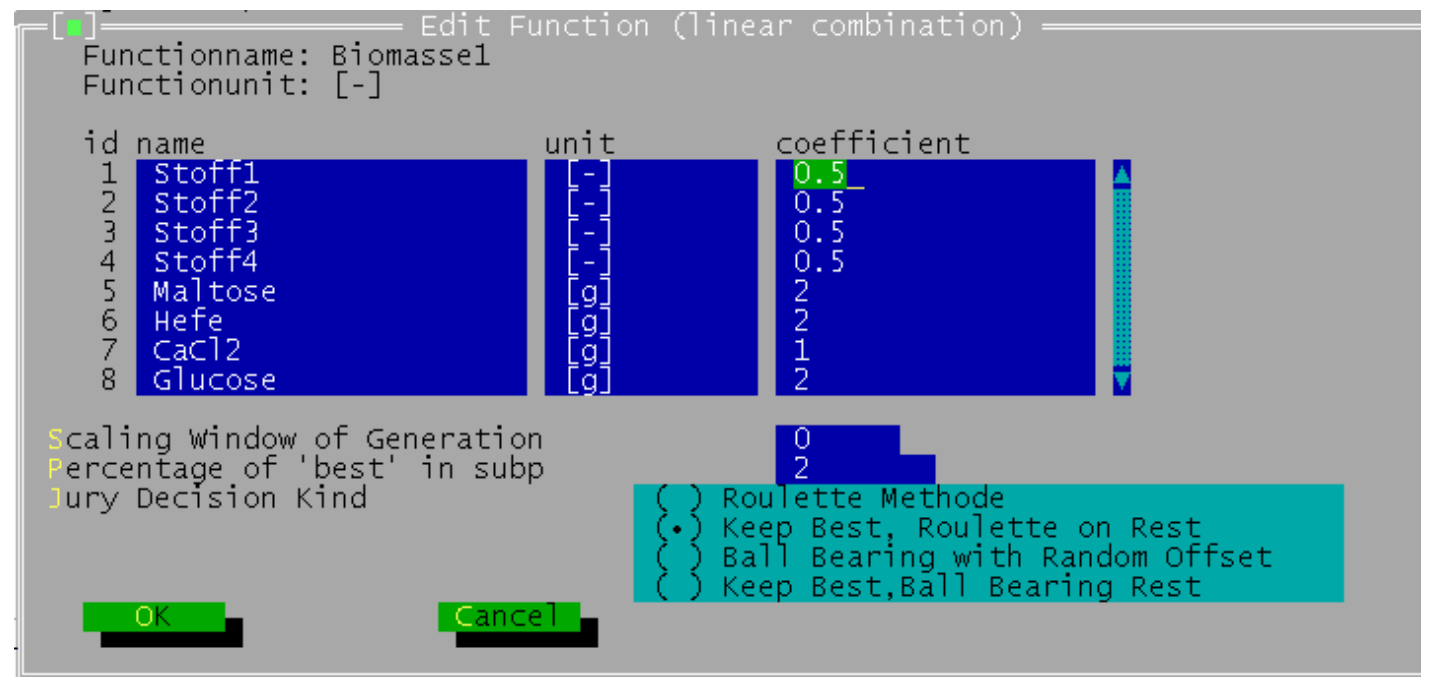

Abbildung 25: Eingabefeld für die Gewichtsfaktoren und Auswahl der Optimierungsmethode

Wurden diese Angaben gemacht und mit OK bestätigt, konnte man in der letzten Eingabemaske die Zahl der Individuen, die Crossingrate und die Mutationsrate festlegen. Damit wurde die Generierung der Startbedingungen für die erste Generation abgeschlossen.

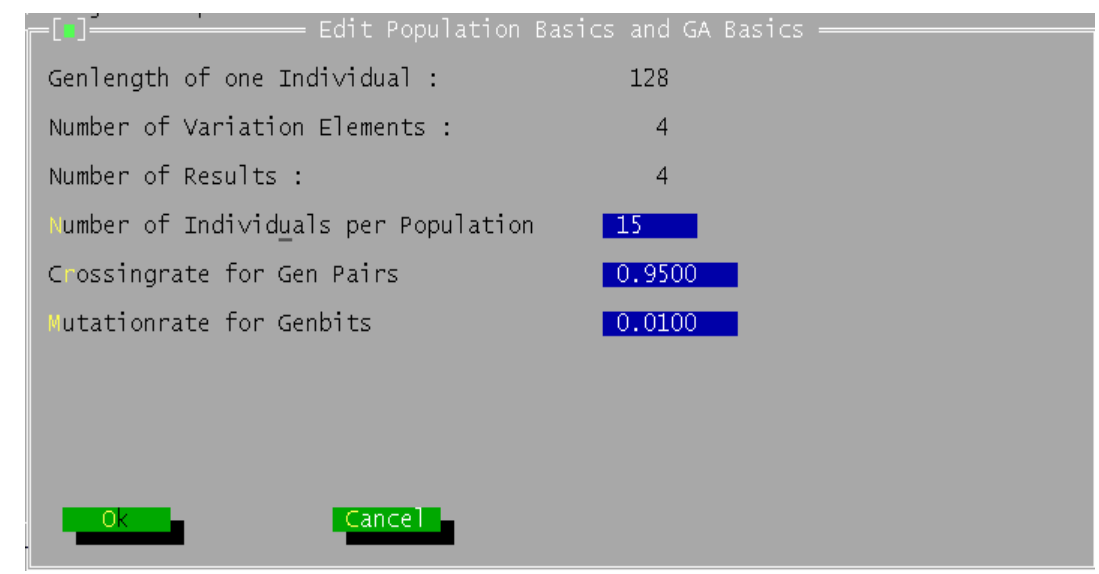

Abbildung 26: Eingabefeld für die Zahl der Populationen, Crossing- und Mutationsrate

\section{Zufallsbedingte Generierung der Anfangswerte und erste Bewertung der Mess- daten}

Unter dem Menü Population findet man zwei wichtige Unterfunktionen, die in der nächsten Phase des Optimierungsexperiments eine entscheidende Rolle spielen. Die Unterfunktion Edit öffnet eine Eingabemaske, in der die Anfangskonzentrationen aller Medienbestandteile für alle Individuen einer Generation erfolgen kann. 
Ist ein Projekt gerade neu angelegt worden, sind noch Konzentrationsangaben für die erste Population zu machen. Dies kann anhand der bereits vorhandenen Kenndaten erfolgen, oder aber auch basierend auf dem Zufallsprinzip (Normalfall bei neuen Projekten). In der ersten Generation wird durch des Betätigen der Schaltfläche Rand all für jedes Individuum ein Startwert der Konzentration für alle Parameter innerhalb der vorher gesetzten Grenzen erzeugt. Diese Generierung kann durch die Unterfunktion Change Lock Status für bestimmte Individuen oder Parameter unterdrückt werden. Im sichtbaren Fenster erscheint eine zusätzliche Information über den Schutzstatus. LOCKED pop Element besagt, dass die Werte des entsprechenden Individuums nicht verändert werden können.

Die Freigabe solcher geschützten Werte erfolgt durch erneutes Betätigen der Schaltfläche. Rand This findet zufällige Werte der Parameter für die erste Generation.

Zwei Schaltflächen Prev und Next bieten die Möglichkeit, zwischen den entsprechenden Individuen einer Generation umzuschalten. Über die Funktion Save werden die erzeugten Daten auf dem Datenträger gesichert. Die Funktion Load erlaubt die früher generierten und bereits abgespeicherten Daten vom Datenträger abzulesen.

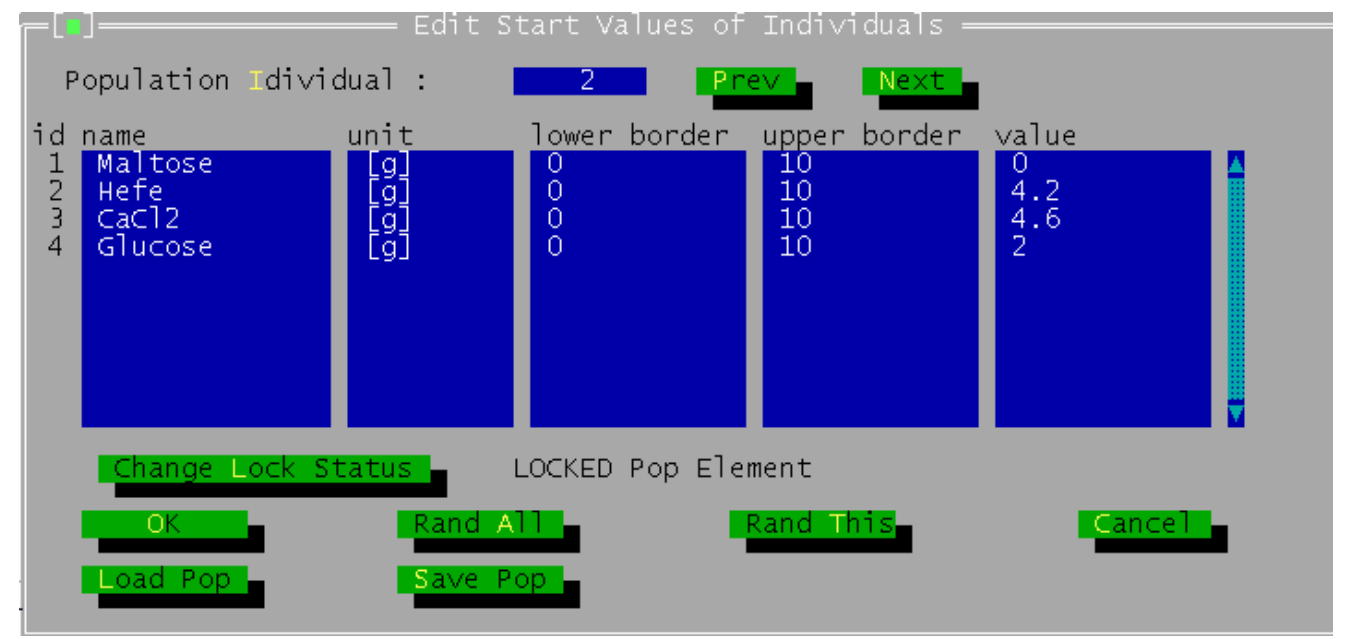

Abbildung 27: Eingabefeld für die Ausgangswerte der ersten bzw. nächsten Generation

\section{Editieren der Messwerte und Erzeugung der Tochtergeneration}

Die Unterfunktion Enter Results aus dem Menü Population erlaubt die experimentell gewonnenen Daten den Individuen zuzuordnen. Die Individuen einer Population werden mit 1 beginnend nummeriert und können durch die Schaltflächen Prev und Next ausgewählt werden. Sind alle Messdaten für jedes Individuum in den Eingabe- 
feldern eingetragen, kann eine neue Generation durch die Auswahl der Unterfunktion Next Generation im Menü Project erzeugt werden.

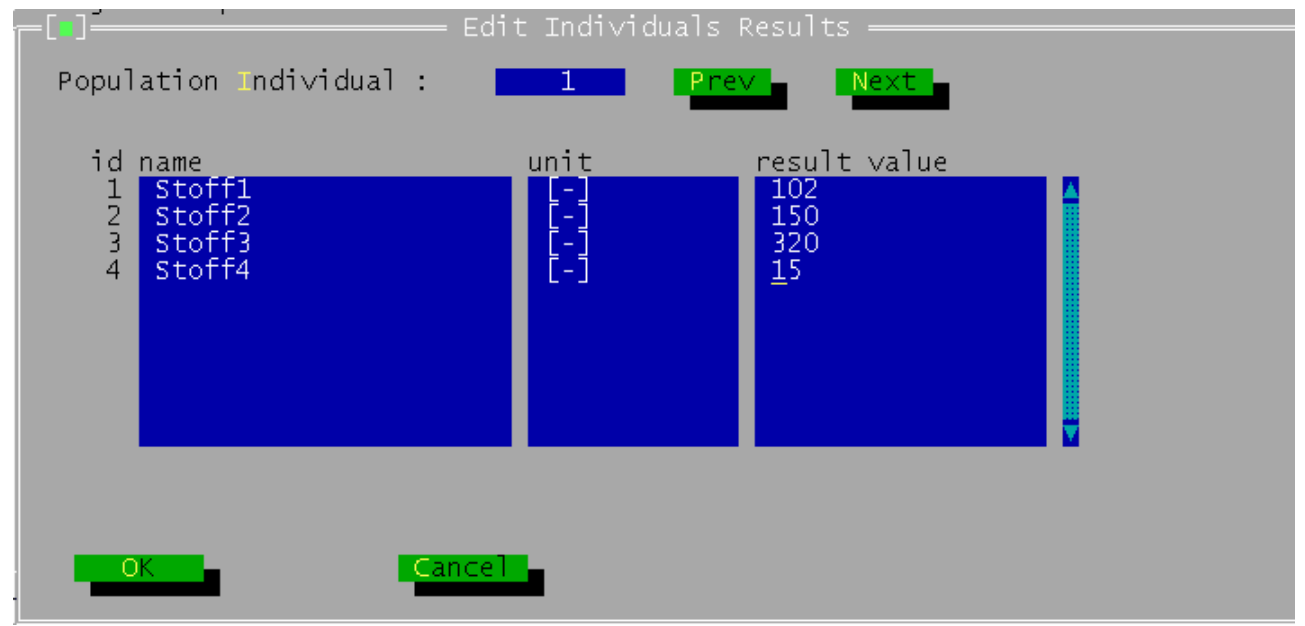

Abbildung 28: Eingabefeld für die im Experiment gemessenen Werte

Diese Vorgehensweise hat zur Folge, dass für alle Individuen neue Konzentrationswerte erzeugt werden, die im darauf folgenden Experiment eingesetzt werden sollen. Die letztgenannten Schritte werden so lange wiederholt, bis die vorausgesetzte Zahl der Tochtergenerationen erreicht wird, oder das Ergebnis der Optimierung zufriedenstellend ist.

\section{Experimentelle Durchführung der Versuchsreihen}

Für jede Generation der Optimierung mit GALOP wurden NährmedienZusammensetzungen für jeweils 15 Individuen erzeugt. Die in der Parametermaske des GALOP-Programmes angegebenen Medienbestandteile jedes Individuums wurden in $25 \mathrm{ml}$ destilliertem Wasser aufgelöst.

Sollten die Medien für marine Stämme verwendet werden, wurden zusätzlich $25 \mathrm{ml}$ künstliches Meerwasser zugesetzt; sollten die Medien für terrestrische Stämme verwendet werden, wurden zusätzlich $25 \mathrm{ml}$ Trinkwasser zugesetzt.

Die mit den Nährmedien gefüllten 100 ml Erlenmeyerkolben wurden 30 min bei 121 ${ }^{\circ} \mathrm{C}$ autoklaviert. Um die Kolben standardisiert mit den Mikroorganismen beimpfen zu können, wurde zunächst eine Kultur des entsprechenden Stammes auf einer AgarPlatte kultiviert. Sie wurde dann mit $1 \mathrm{ml}$ Wasser überschichtet. Die so entstandene Zellsuspension wurde in einen Kolben übergeführt und mit Trinkwasser auf $25 \mathrm{ml}$ 
aufgefüllt. In die vorher mit den Nährmedien gefüllten und autoklavierten Anzuchtkolben wurde jeweils genau ein Milliliter dieser Zellsuspension pipettiert. Die Kolben wurden mit einem sterilen Baumwollgewebe abgedeckt und drei Tage bei $28^{\circ} \mathrm{C}$ mit einer Frequenz von $1 \mathrm{~Hz}$ auf einer Rührplatte kultiviert. Jedes Individuum (im Sinne von GALOP, nicht im mikrobiologischen Sinne) wurde nach Aussehen, Mycelbildung und Farbe beschrieben. Die Zellkulturen wurden mit $50 \mathrm{ml}$ Methanol versetzt und bei $40{ }^{\circ} \mathrm{C}$ und $50 \mathrm{hPa}$ vollständig vom Lösungsmittel befreit. Die Trockensubstanzen je dreimal mit je $50 \mathrm{ml}$ Essigsäureethylester extrahiert. Die vereinigten Extrakte jedes Individuums wurden mittels HPLC untersucht, um die für die weitere Optimierung mit GALOP benötigten Werte vervollständigen zu können.

Tabelle 2: Die Zusammensetzung des Mediums nach dem Optimieren mit GALOP

\begin{tabular}{|c|cccc|}
\hline $\begin{array}{c}\text { Stamm- } \\
\text { bezeichnung }\end{array}$ & $\begin{array}{c}\text { Maltose- } \\
\text { menge [g] /10 I }\end{array}$ & $\begin{array}{c}\text { Hefeextrakt- } \\
\text { menge [g] /10 I }\end{array}$ & $\begin{array}{c}\text { Glucose- } \\
\text { menge [g] /10 I }\end{array}$ & $\begin{array}{c}\mathbf{C a C l}_{2} \text {-Menge } \\
\text { [g] /10 I }\end{array}$ \\
\hline GW 7/2495 & 6 & 92 & 60 & 40 \\
GW 7/2495 & 3 & 46 & 30 & 20 \\
B 7284 & 83 & 98 & 48 & 16 \\
B 7939 & 27 & 68 & 51 & 54 \\
B 8927 & 18 & 43 & 76 & 45 \\
GW 20/1811 & 66 & 87 & 68 & 22 \\
B 9020 & 38 & 55 & 61 & 19 \\
B 8027 & 54 & 77 & 21 & 37 \\
GW 19/2497 & 67 & 60 & 41 & 18 \\
GW 27/1179 & 16 & 85 & 39 & 20 \\
\hline
\end{tabular}

Wie zu erwarten, ist die Produktion der Sekundärmetaboliten nicht immer linear von der Konzentration der Medienkomponenten abhängig. Diese Tendenz ist am stärksten bei dem Chartreusinproduzenten GW 27/1179 ausgeprägt. Die Zusammensetzung der Medien, die die besten Individuen jeder Optimierungsreihe hervorbrachten, wurden in der Tabelle 3 zusammengestellt. Bei der Beurteilung der Güte wurde vor allem die Peakfläche des Chartreusin-Signals (62) bewertet.

In der ersten Generation fand man Individuen, die nur wenig 62 produzierten. Die Erhöhung der $\mathrm{CaCl}_{2}$-Konzentration und die gleichzeitige Reduktion der Maltose- und Glucose- und Hefeextrakt-Konzentration führte zu einer fast 400proz. Steigerung der Produktion von 62. Anscheinend betrafen diese Werte aber ein lokales Maximum, 
wie die Generation III zeigt. Anhand der Werte der Generation II würde man erwarten, dass die hohe Konzentration von $\mathrm{CaCl}_{2}$ für die Produktion von 62 ausschlaggebend ist. Nun ist sie aber bei der Generation III fast so niedrig, wie bei I. Generation IV und V zeigen weiterhin deutlich, dass es nicht ausreichend ist, die zu optimierenden Parameter unabhängig voneinander zu verändern. Erst das Zusammenspiel aller verwendeten Komponenten bei einer so komplexen Aufgabe, wie der Mediumoptimierung, bringt die erwünschten Resultate. Durch den Einsatz von GALOP konnte sogar die Produktion der Nebenkomponenten weitgehend reduziert werden (s. Abbildung 29 und Abbildung 30). Die Methode brachte in diesem Fall eine Steigerung der Chartreusinproduktion um mehr als $700 \%$.

Wenn man die Peakflächen über alle Generation miteinander vergleicht, findet man den größten Produktionszuwachs am Anfang der Optimierung. Der Peakflächenunterschied zwischen Generation I und II beträgt 17.09, zwischen II und III 8.66 und zwischen Generation IV und V nur noch 3.22.

Diesen Tendenz konnte man bei allen untersuchten Stämmen beobachten. Sie lässt sich damit erklären, dass das Programm zuerst grob die Richtung des Optimums bestimmt und sich dann bei der weiteren Suche bevorzugt nur im kleinen Bereich um diesen Punkt bewegt. Wird allerdings durch Stichproben ein neues Maximum entdeckt, wird die Suche in dem neuentdeckten Gebiet fortgesetzt.

Tabelle 3: Die besten Individuen der fünf folgenden Generationen. Die Peakfläche war bei der Auswahl des Individuums ausschlaggebend.

\begin{tabular}{|cccccc|}
\hline Generation & $\begin{array}{c}\text { Maltose } \\
{[\mathbf{g} / \mathbf{l}]}\end{array}$ & $\begin{array}{c}\text { Glucose } \\
{[\mathbf{g} / \mathbf{I}]}\end{array}$ & $\begin{array}{c}\text { Hefeextrakt } \\
{[\mathbf{g} / \mathbf{l}]}\end{array}$ & $\begin{array}{c}\mathbf{C a C l}_{\mathbf{2}} \\
{[\mathbf{g} / \mathbf{l}]}\end{array}$ & $\begin{array}{c}\text { Peakfläche } \\
{[-\mathbf{~ ] ~}}\end{array}$ \\
$\mathbf{1}$ & 8.1 & 7.9 & 1.9 & 0.3 & 5.7 \\
$\mathbf{2}$ & 3.1 & 5.8 & 0.6 & 1.7 & 22.79 \\
$\mathbf{3}$ & 9.0 & 8.8 & 7.5 & 0.4 & 31.45 \\
$\mathbf{4}$ & 5.8 & 8.2 & 4.1 & 1.3 & 38.87 \\
$\mathbf{5}$ & 1.6 & 8.5 & 3.9 & 2.0 & 42.09 \\
\hline
\end{tabular}




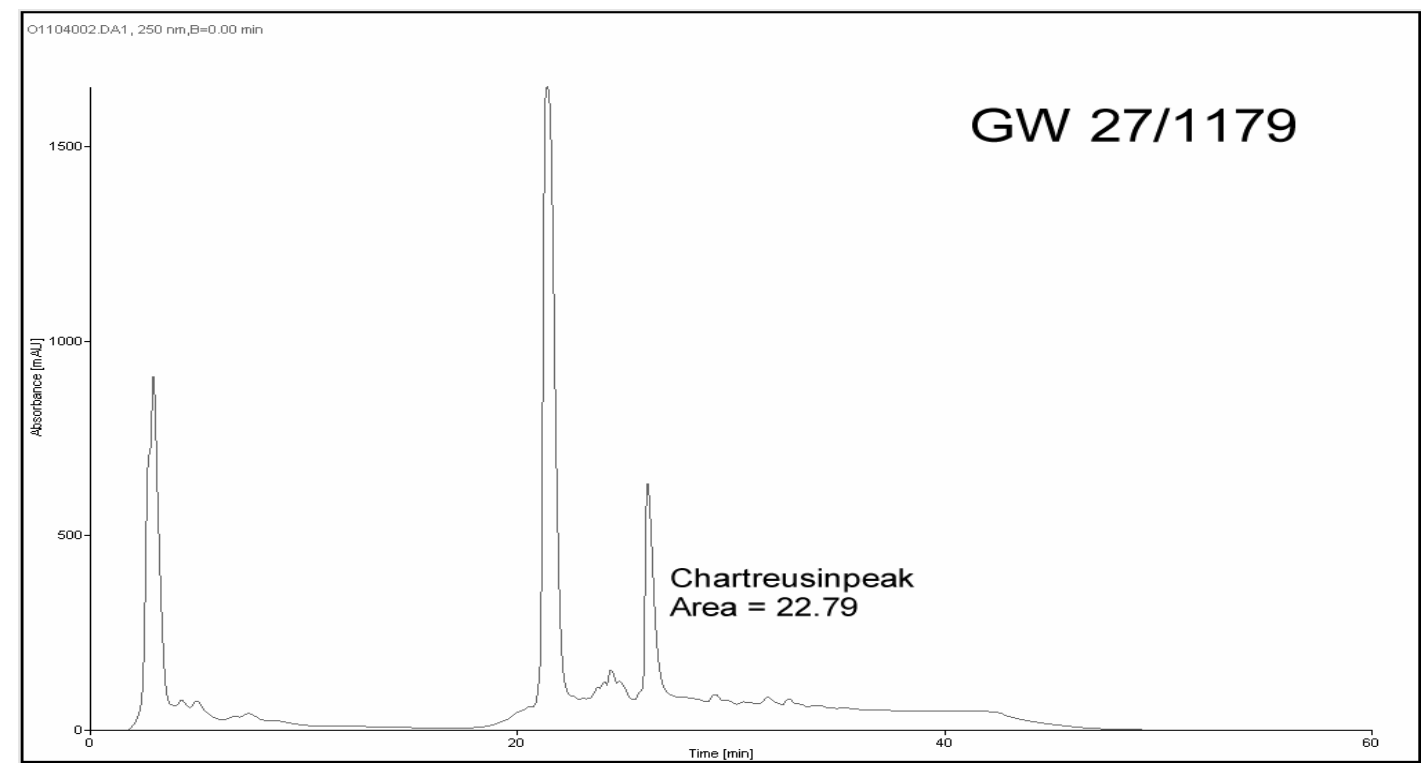

Abbildung 29: HPLC-Chromatogramm des besten Individuums der Generation II, aufgenommen bei $250 \mathrm{~nm}$.

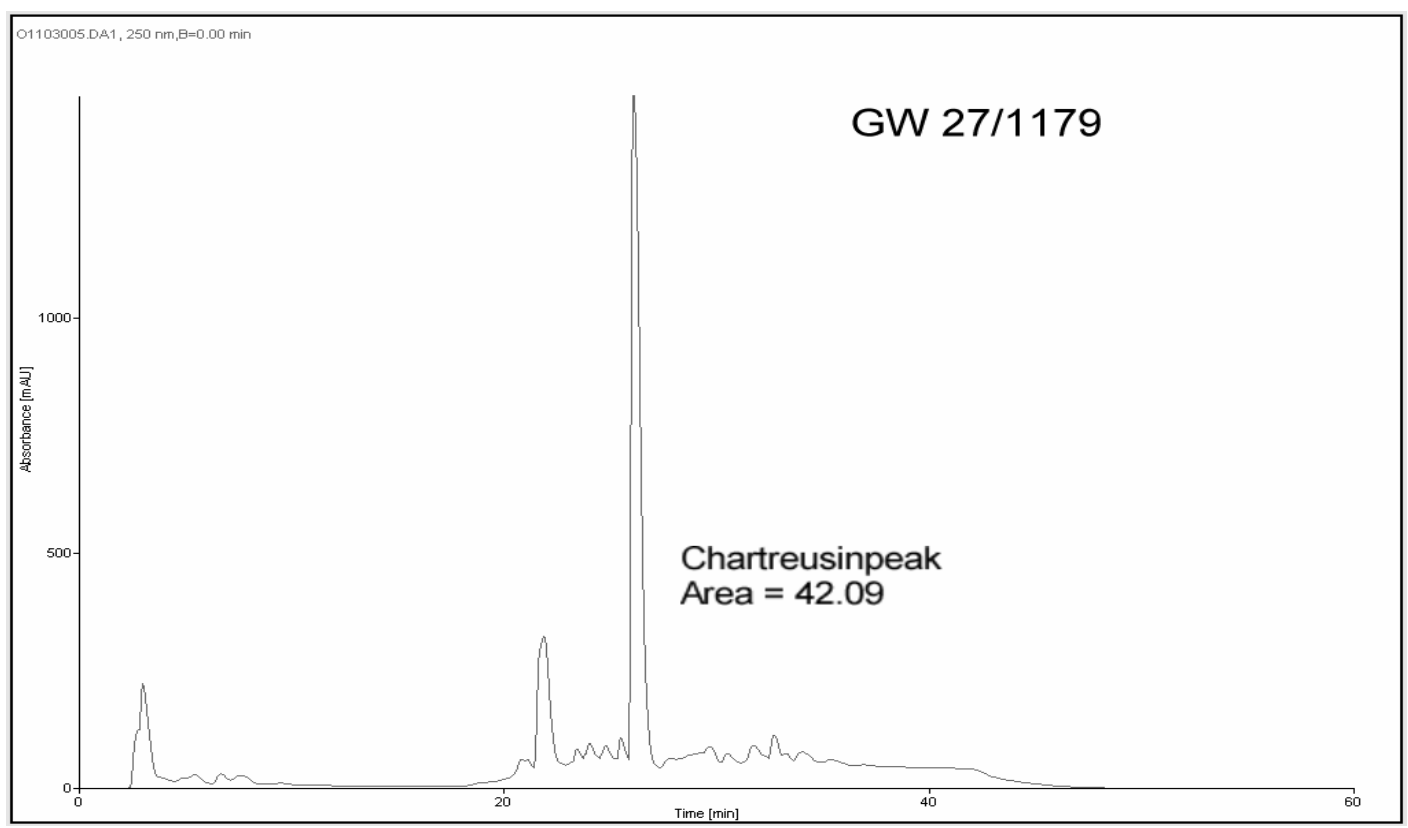

Abbildung 30: HPLC-Chromatogramm des besten Individuums der Generation V, aufgenommen bei $250 \mathrm{~nm}$. 


\section{Stamm GW 7/2495}

Der terrestrische Streptomycet GW 7/2495 zeigte lediglich eine biologische Aktivität gegen Streptomyces viridochromogenes (Tü 57). Auf Agar wuchs der Stamm in kleinen, weißen Kolonien. Die Rohextrakte zeigten im HPLC-Screening nur wenige Peaks, deren UV-Spektren auf farblose Substanzen hindeuteten. Die mit Anisaldehyd angefärbten Dünnschichtchromatogramme zeigten zwei schwache blaue und mehrere braune Flecke.

Es stellte sich die Frage, ob man diesen nach dem ersten Eindruck unspektakulären Naturstoffproduzenten durch kontinuierliche Kultivierungsverfahren und durch Medienoptimierung zur Produktion interessanter Metabolite bewegen könnte. Um die Ergebnisse der Anzucht unter diesen Bedingungen direkt mit einem Batch-Prozess vergleichen zu können, wurde gleichzeitig ein diskontinuierlicher Bioreaktor mit dem Mikroorganismus besiedelt. In beiden Fällen wurde ein mit GALOP optimiertes Medium (s. Tabelle 2), das aus 6 g Maltose, 92 g Hefeextraktextrakt, 60 g Glucose und $40 \mathrm{~g} \mathrm{CaCl}_{2}$ pro Liter bestand, eingesetzt. Zum Animpfen nahm man sowohl für den kontinuierlichen, als auch für den diskontinuierlichen Fermenter die gleiche Vorkultur, die vorher in zehn Schüttelkolben kultiviert wurde. Für die Schüttelkolben wurde das Standardmedium $\mathrm{M}_{2}$ (ohne Seewasser) verwendet.

\section{Die diskontinuierliche Anzucht von GW 7/2495}

Die Bakterien wuchsen in einem 20 Liter-Reaktor unter pH-Kontrolle (6.5 bis 7.5) bei $28^{\circ} \mathrm{C}$ über drei Tage, wobei sich das Mycel bereits nach einem Tag gelb verfärbte. Das Mycel und der Überstand wurden durch Filtration voneinander getrennt. Der Überstand wurde mit Ethylacetat extrahiert. Das Mycel wurde zunächst mit Methanol behandelt, um die Zellwände zu zerstören und den Zellinhalt für Ethylacetat zugänglich zu machen. Erst dann wurde das Mycel mit Essigester extrahiert. Schließlich wurden beide intensiv gelb gefärbten Extrakte vereinigt, mit Cyclohexan entfettet und weiter nach dem unten dargestellten Schema 2 bearbeitet. Es zeigte sich, dass eine gelbe Substanz in großen Mengen produziert wurde, die im UV-Licht stark grün fluoreszierte. 


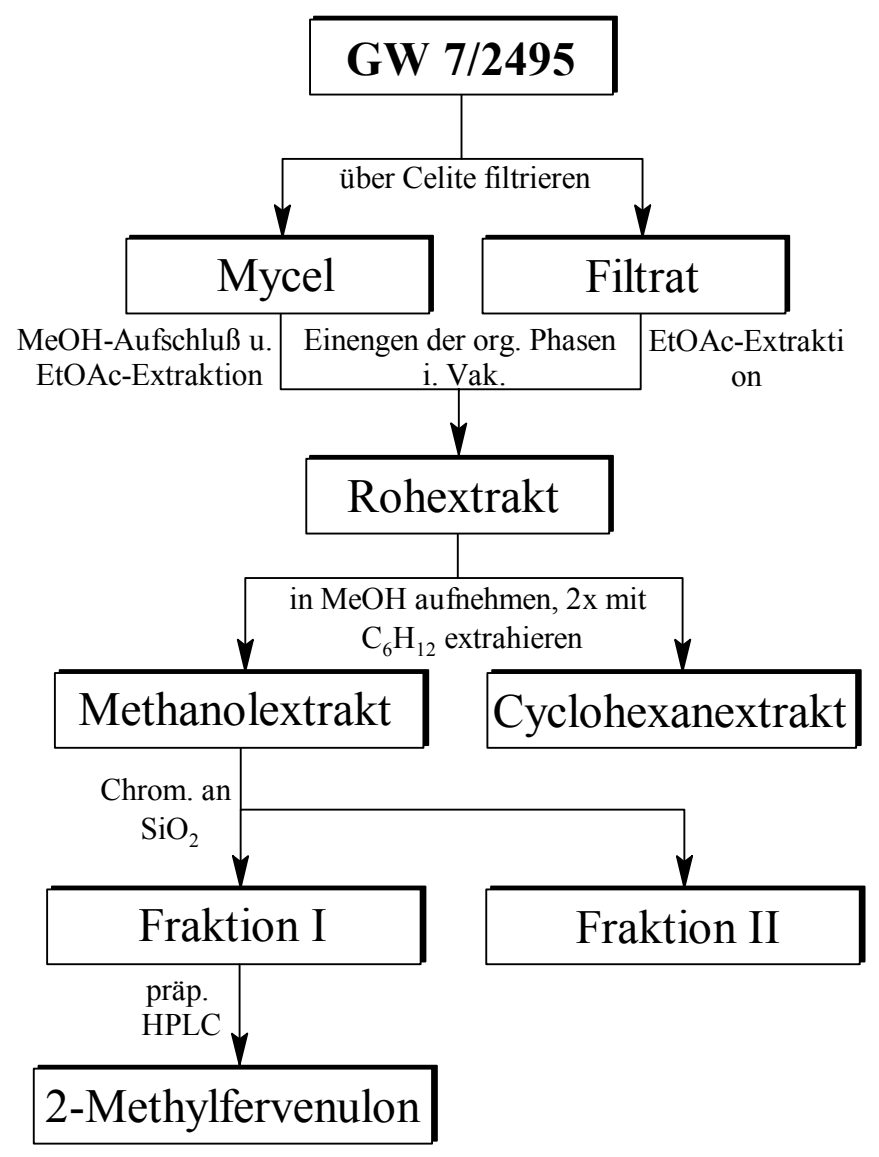

Schema 2: Aufarbeitung des diskontinuierlich angezogenen Stammes GW 7/2495

Der entfettete Rohextrakt wurde mittels Säulenchromatographie an Kieselgel vorgereinigt und in zwei Hauptfraktionen aufgeteilt. Fraktion II enthielt mehrere farblose Komponenten in geringen Mengen. Durch eine weitere HPLC-Aufreinigung konnten keine sauberen Komponenten in ausreichenden Mengen gewonnen werden, um die spektroskopischen Daten aufnehmen zu können. Die weniger polare und gelbgrünliche Fraktion I wurde durch präparative HPLC an RP18 gereinigt. Schließlich erhielt man 84 mg einer im Test gegen Streptomyces viridochromogenes (Tü 57), Escherichia coli und Staphylococcus aureus aktiven gelben kristallinen Substanz, die bei $183^{\circ} \mathrm{C}$ schmolz.

Das ${ }^{1} \mathrm{H}-\mathrm{NMR}-$ Spektrum zeigte nur drei Singuletts der relativen Intensität 1 bei $\delta=$ 3.50, 3.55 und 3.95. Bei diesen Signalen konnte es sich entweder um einzelne Protonen, um separate Methyl- oder um Methylengruppen handeln, die ein Heteroatom als Nachbarn besaßen. Das ${ }^{13}$ C-NMR-Spektrum mit Signalen bei $\delta=29.6,28.6$ und 42.1 bestätigte die Vermutung, dass diese Signale drei Methylgruppen repräsentierten. 
Das Spektrum zeigte weitere fünf Signale bei $\delta=135$ bis 160 , die auf mehrere aromatische oder heteroaromatische quartäre Kohlenstoffatome hindeuteten.

Eine Suche mit der massenspektrometrisch durch Hochauflösung ermittelten Molmasse (EI) von $\mathrm{m} / \mathrm{z} 223.0705$ in der Naturstoff-Datenbank AntiBase lieferte 26 Verbindungen, von denen nur zwei jeweils drei Methylreste und keine weiteren Protonen besaßen. Die aus den spektroskopischen Daten gewonnenen Informationen konnten eindeutig dem 2-Methylfervenulon (29) zugewiesen werden.

Die Anwesenheit der Carbonylkohlenstoffe konnte zusätzlich durch Banden bei 1666 $\mathrm{cm}^{-1}$ im IR-Spektren untermauert werden. Ein Vergleich der ${ }^{1} \mathrm{H}-\mathrm{NMR}-\mathrm{Daten}$ und der Schmelztemperatur der gefundenen Verbindung mit Literaturwerten bestätigte schließlich die vermutete Struktur ${ }^{[70-72]}$. Verbindung 29 hemmt die PyroglutamylPeptidase und ähnelt strukturell dem aus einem terrestrischen Streptomyceten bereits 1992 isolierten Pyrizinostatin ${ }^{[73]}$.<smiles>Cn1nc2c(nc1=O)c(=O)n(C)c(=O)n2C</smiles> 


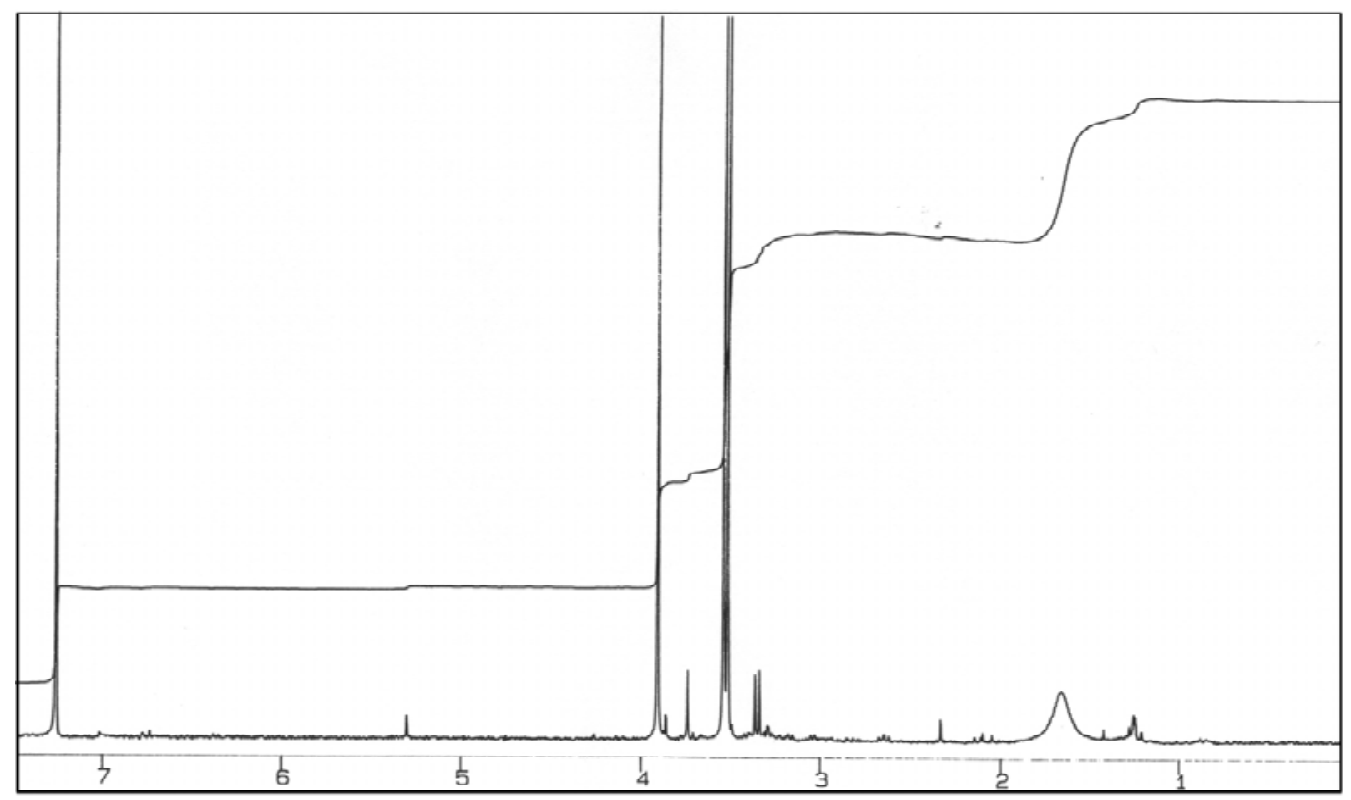

Abbildung 31: $\quad{ }^{1} \mathrm{H}-\mathrm{NMR}-S p e k t r u m\left(\mathrm{CDCl}_{3}, 200 \mathrm{MHz}\right)$ von 2-Methylfervenulon (29) mit den Satellitensignalen einer möglicherweise isomeren Verbindung.

Anhand dieses Befundes konnten auch die zusätzlichen Satellitensignale des ${ }^{1} \mathrm{H}$ NMR-Spektrums (s. Abbildung 31) erklärt werden. Dabei handelte es sich ebenfalls um drei Singuletts der Intensität 1, die leicht zu höherem Feld verschoben waren. Wegen der ähnlichen physikochemischen Eigenschaften gelang es allerdings nicht, die dazugehörige Verbindung in reiner Form zu isolieren. Es ist jedoch sehr wahrscheinlich, dass es sich hierbei um ein Konstitutionsisomeres von 29 handelte, bei dem die Methylgruppen allerdings ebenfalls an Stickstoff gebunden vorliegen, wie die chemischen Verschiebungen zeigen. Da weiterführende Erkenntnisse aus den zweidimensionalen ${ }^{n} J$-korrelierten Spektren wie HMQC und HMBC nicht gewonnen werden konnten, weil strukturbedingt keine ${ }^{2} J$ - und ${ }^{3} J$-Korrelationen zu sehen wären, muss man mehrere mögliche Strukturen in Betracht ziehen, die solche Signale ergeben können.

Alle in Abbildung 32 aufgezeigten Strukturen wurden mit dem Computerprogramm ${ }^{1}$ H-NMR-Predictor von ACD simuliert und die so erhaltenen Spektren mit den gemessenen Werten verglichen. Dabei zeigte sich, dass das Spektrum der in der Abbildung 32 fett hervorgehobenen simulierten Struktur am besten der Realität entspricht.

Die anderen aufgezeichneten Strukturen zeigten im Vergleich dazu in der Simulation größere Unterschiede bei der chemischen Verschiebung der Signale. 


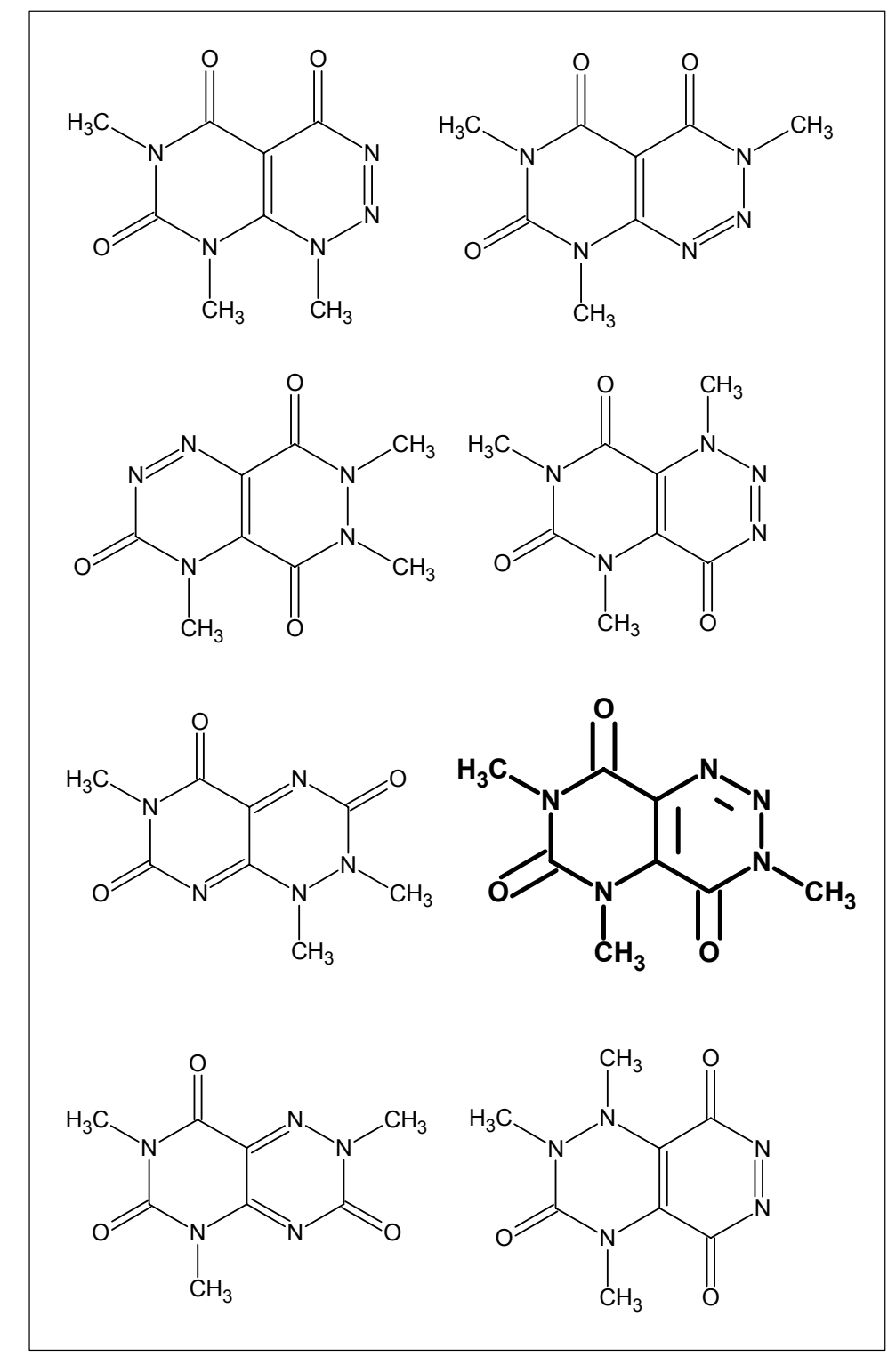

Abbildung 32: Mögliche Konstitutionsisomere von 29, die die gefundenen spektroskopischen Daten liefern könnten.

Ob die Nebenkomponente tatsächlich die postulierte Struktur hat, konnte allerdings nicht eindeutig bewiesen werden.

Der entfettete Extrakt dieses Stammes wurde noch zusätzlichen GC-MS-Analysen an der Technischen Universität Braunschweig unterzogen. Da diese Methode sehr empfindlich ist, konnte man mit der Unterstützung einer internen Datenbank vier weitere Komponenten finden. Es war allerdings nicht möglich, eine eindeutige Struktur spektroskopisch $\mathrm{zu}$ bestimmen. Außer Bromacetophenon fand man ein chloriertes Naphthalin-Derivat mit der Masse 218, eine aromatische Verbindung ohne Hetero- 
atom mit der Masse 272 und schließlich eine aromatische Komponente mit $m / z$ 533, die möglicherweise ein Ethylphenyl-Strukturelement sowie zwei Bromatome enthält.

\section{Die kontinuierliche Kultur von GW 7/2495}

Die kontinuierliche Fermentation von GW 7/2495 besaß den Vorteil, dass man die verbrauchten Medienbestandteile während der Fermentation ergänzen und gleichzeitig die entstandenen Metabolite aus dem Bioreaktor entfernen konnte. Für die Fermentation wurde eine 101 Vorratsflasche mit allen Medienkomponenten, sowie kleine Behälter für Salzsäure, Natronlauge und Antischaummittel mit dem Bioreaktor mit Silikonschläuchen verbunden. Die pH-Elektrode wurde an die Steuerungsapparatur angeschlossen. Nach dem Animpfen des Reaktors wurde 24 Stunden unter BatchBedingungen fermentiert, bis sich ein sichtbarer Mycel-Film auf dem Stahlnetz gebildet hatte. Danach wurde die Durchflussrate so eingestellt, dass am Tag 1 Liter des Mediums durch den Fermenter floss. Die Fermentation wurde nach acht Tagen beendet.

Die in einem 10 1-Behälter gesammelte und vom Mycel größtenteils freie Kulturbrühe wurde filtriert und mit Ethylacetat dreimal extrahiert. Nach dem Eindampfen der roten Lösung erhielt man 467 mg eines Extraktes, der durch Ausschütteln mit Cyclohexan entfettet wurde. Nach dem Entfetten verringerte sich das Gewicht um nur 17 mg, was auf ungewöhnlich geringe Mengen an Fetten hindeutete. Diese Beobachtung wurde durch Dünnschichtchromatogramme bestätigt. Nach dem Anfärben mit Anisaldehyd/Schwefelsäure waren schwache violette Zonen von unpolaren Substanzen mit hohem $R_{\mathrm{f}}$-Wert zu sehen. Das Mycel wurde vom Stahlnetz abgetrennt, abfiltriert, mit Methanol aufgeschlossen und erneut mit Essigester extrahiert.

Die vereinigten Extrakte trennte man an einer Kieselgel-Säule in zwei Hauptfraktionen, wobei die weniger polare Fraktion II nur wenige Verbindungen enthielt. Nach der präparativen Dünnschichtchromatographie erhielt man eine weiße Substanz mit $R_{\mathrm{f}}=0.6$.

Aus der Fraktion I isolierte man mittels präparativer HPLC-Trennung und Chromatographie an Sephadex LH-20 zwei rote und eine farblose Komponente, letztere mit einem $R_{\mathrm{f}}$-Wert von $0.5\left(\mathrm{CH}_{2} \mathrm{Cl}_{2} / \mathrm{MeOH} ; 9: 1\right)$. 


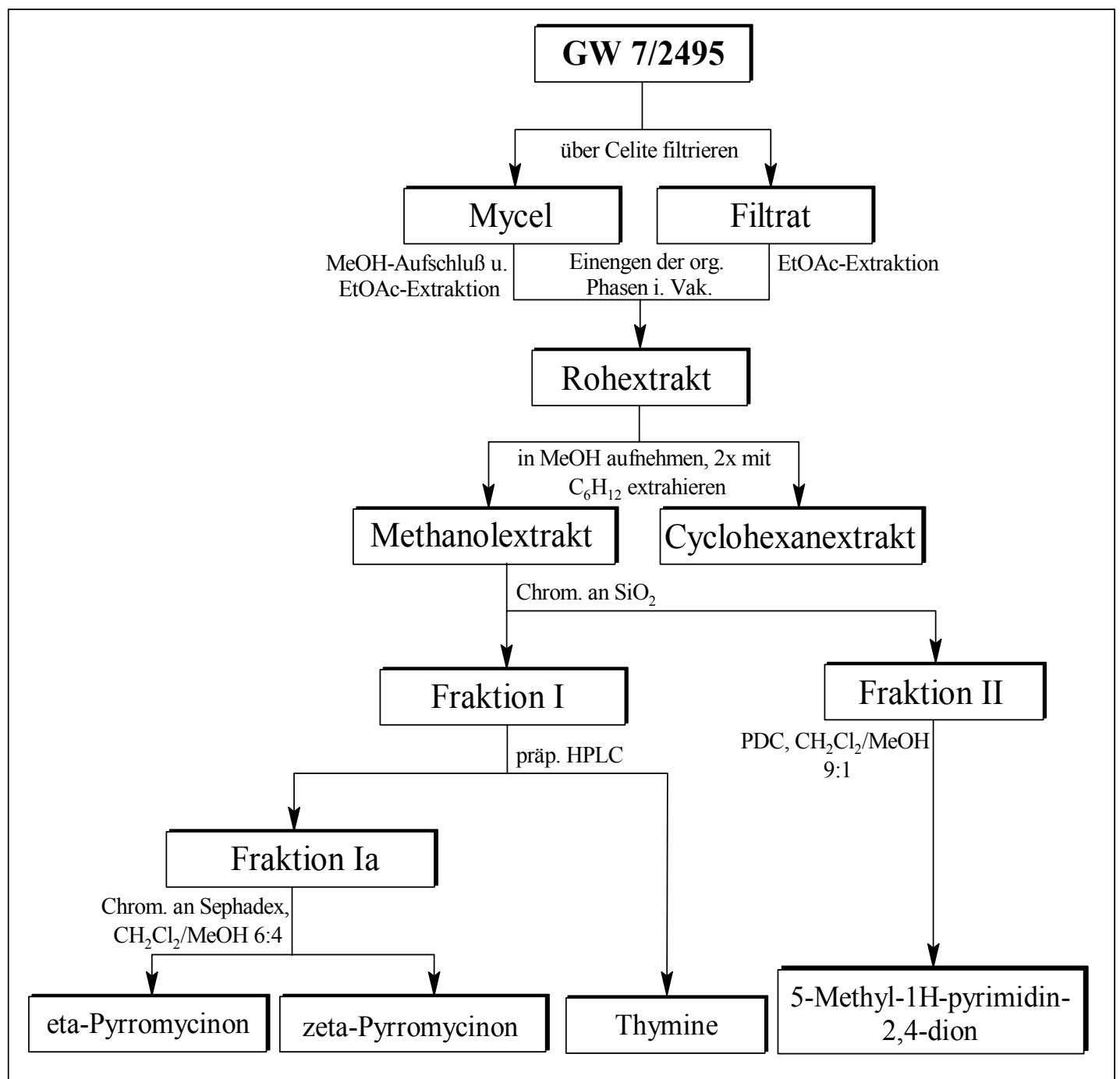

Schema 3: Aufarbeitung des Stammes GW 7/2495

\section{$\varepsilon$-Pyrromycinon}

Die erhaltene rote Verbindung mit $R_{\mathrm{f}}=0.6$ färbte sich auf der DC-Platte bei Behandlung mit Natronlauge blau, was ein Indiz für ein Chinonderivat mit chelierter Hydroxygruppe ist. Das ${ }^{1} \mathrm{H}-\mathrm{NMR}-$ Spektrum zeigt drei Signale bei $\delta=13.82,13.10$ und 12.30. Im aromatischen Bereich erkennt man ein Singulett mit der relativen Intensität 1 bei $\delta=8.33$ und ein weiteres mit der Intensität 2 bei $\delta=7.24$. Außerdem findet man bei $\delta=7.60$ und 8.45 zwei miteinander koppelnde Dubletts. Das Signal bei $\delta=$ $4.10(3 \mathrm{H})$ deutet darauf hin, dass sich eine an ein Heteroatom gebundene Methylgruppe im Molekül befindet. Die miteinander koppelnden Signale bei $\delta=2.80(2 \mathrm{H})$ und $1.36(3 \mathrm{H})$ gehören wahrscheinlich einem Ethylrest an, der an einen aromatischen Ring gebunden ist. Diese Vermutungen konnten zusätzlich durch ${ }^{13} \mathrm{C}-\mathrm{NMR}$ Spektren bestätigt werden. Eine Datenbankrecherche (AntiBase) lieferte 12 ähnliche 
Strukturen, von denen nur eine einzige alle Bedingungen der Spektren erfüllen konnte. Einen eindeutigen Beweis lieferte der Vergleich der aufgenommenen Spektren mit bereits in der Spektrenbibliothek vorhandenen. Bei der gesuchten Struktur handelte es sich um $\varepsilon$-Pyrromycinon (30).<smiles>CCc1ccc2c(O)c3c(cc2c1C(=O)OC)C(=O)c1c(O)ccc(O)c1C3=O</smiles>

30

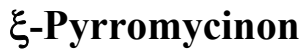

Die zweite ebenfalls rote Verbindung konnte man präparativ nicht in reiner Form isolieren. Alle Spektren enthielten daher zusätzliche Signale einer wegen der fehlenden chelierten $\mathrm{OH}-$ Gruppen offensichtlich nicht-chinoiden Nebenkomponente, die etwa im Verhältnis 1:1.2 vorlag, wie die Integrale zeigten.

Mit der Struktur des bereits isolierten 30, den drei ähnlichen chelierten Hydroxygruppen, die im ${ }^{1} \mathrm{H}-\mathrm{NMR}-$ Spektrum bei $\delta=13.0$, 12.6 und $12.3 \mathrm{zu}$ sehen waren, sowie den 2D-NMR-Korrelationen war eine ähnliche Grundstruktur zu erwarten. Die Suche in AntiBase nach entsprechenden Chinon-Grundgerüsten lieferte zuerst 434 Treffer, von denen aber der größte Teil Glycoside waren.

Da man im ${ }^{1}$ H-NMR-Spektrum keine Signale sah, die einem Zucker zugeordnet werden konnten, ließen sich alle Glycoside eliminieren. Es blieben noch 183 Moleküle übrig. Nur vier davon hatten die gefundene Masse von $m / z$ 412. Der Spektrenvergleich bestätigte, dass die gefundene Struktur dem bereits 1967 gefundenen Anthracyclin-Antibiotikum ${ }^{[74]} \xi$-Pyrromycinon (31) entspricht. Die unbekannte, nach den Signalen bei $\delta=7.83$ und 7.50 ebenfalls aromatische Komponente konnte nicht 
in reiner Form isoliert werden. Man konnte den vorliegenden Signalen keine eindeutige Struktur zuweisen.<smiles>CC[C@@]1(O)CCc2c(cc3c(c2O)C(=O)c2c(O)ccc(O)c2C3=O)C1C(=O)OC</smiles>

\section{5-[2-(2-Hydroxy-3-methoxycarbonyl-propyl)-5-methyl-phenyl]-penta-2,4-dien-} säure

Bereits in der Vortrennung des Extraktes an Kieselgel fiel eine Fraktion mit einer stark UV-löschenden Substanz an. Die präparative Dünnschichtchromatographie ergab $4.3 \mathrm{mg}$ einer farblosen und in den biologischen Tests fast inaktiven Substanz. Nur gegen Escherichia coli konnte man einen Hemmhof von $15 \mathrm{~mm}$ beobachten.

Die ${ }^{1} \mathrm{H}-\mathrm{NMR}$-Spektren zeigten mehrere aromatische Signale, aus denen allerdings kein Substitutionsmuster zu erkennen war. Weiterhin konnte man bei $\delta=6.40$ ein Triplett $(1 \mathrm{H})$, bei $\delta=6.00$ ein Dublett $(1 \mathrm{H})$ und bei $\delta=4.16$ ein Multiplett $(1 \mathrm{H})$ erkennen. Anhand der chemischen Verschiebung wurde angenommen, dass es sich bei dem Singulett $\delta=3.64(3 \mathrm{H})$ um eine Methylgruppe handelt, die mit einem Heteroatom verbunden ist. Ein weiteres Singulett $(3 \mathrm{H})$ sah man bei $\delta=2.38$. Dieses tieffeldverschobene Signal deutete auf eine separate Methylgruppe hin, die keinen Kopplungspartner besitzt und mit hoher Wahrscheinlichkeit entweder an einen Carbonyl-Kohlenstoff oder an einen aromatischen Ring gebunden ist. Zwei weitere Multiplett-Signale bei $\delta=2.40(2 \mathrm{H})$ und $2.80(2 \mathrm{H})$ waren ein Indiz für zwei weitere Methylengruppen.

Die Recherchen in AntiBase, dem Dictionary of Natural Products sowie weitführende Internetsuchen (SDBS-Spektrendatenbank) ergaben keine zu den ${ }^{1} \mathrm{H}-\mathrm{NMR}$ - und 
${ }^{13} \mathrm{C}-\mathrm{NMR}-$ Spektren passende Struktur. Es handelte sich um eine ungesättigte Verbindung (UV-löschende Flecke), die beim Besprühen des Dünnschichtchromatogramms mit Anisaldehyd /Schwefelsäure einen graubraunen Fleck mit $R_{\mathrm{f}}=0.65$ ergab.

Die hochauflösende EI-Massenspektrometrie lieferte eine Masse von 304.1311 und die entsprechende Summenformel von $\mathrm{C}_{17} \mathrm{H}_{20} \mathrm{O}_{5}$.

Mit Hilfe der zweidimensionalen HMQC-Korrelationsspektren konnten alle im ${ }^{1} \mathrm{H}$ NMR-Spektrum sichtbaren Protonen den Kohlenstoffatomen eindeutig zugewiesenen werden.

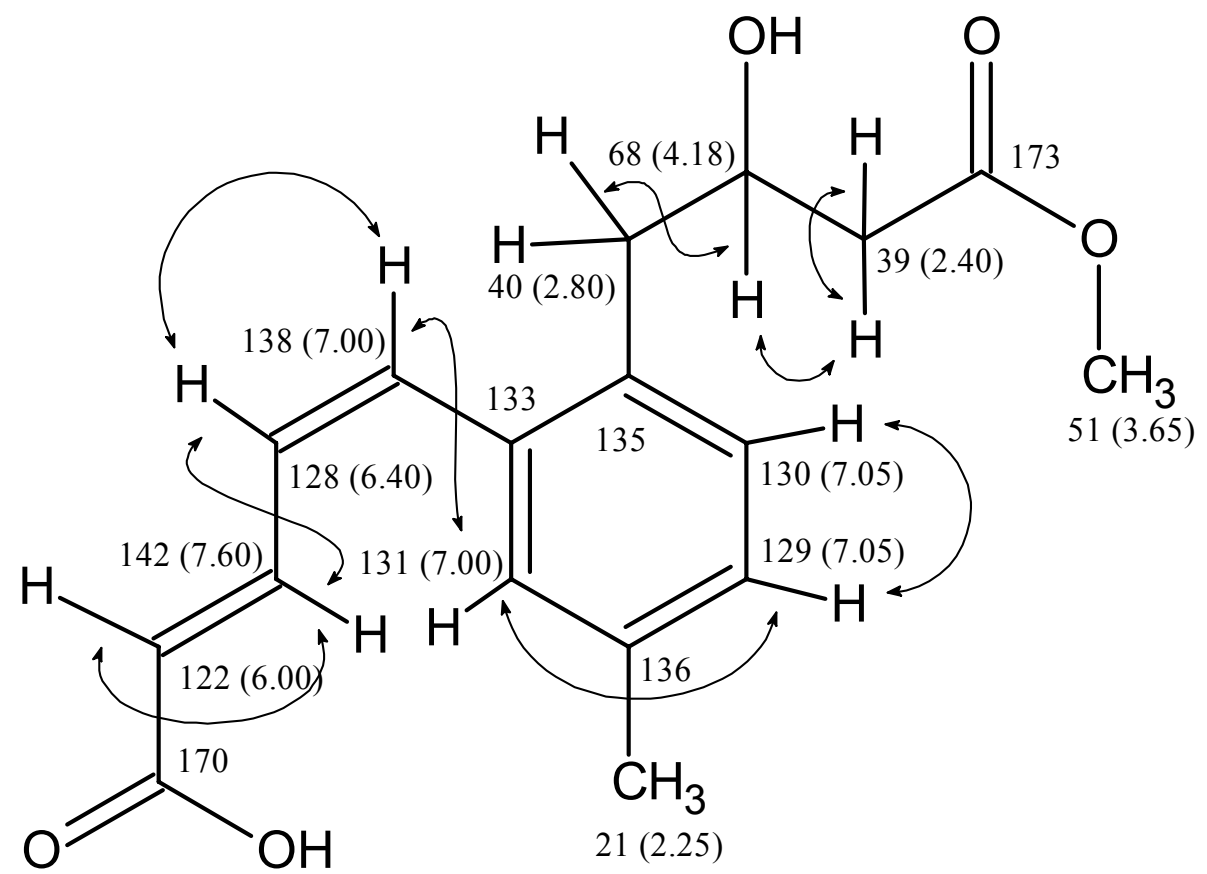

Abbildung 33: Die in der Abbildung 38 sichtbaren H,H-Korrelationen von 32

Die H,H-COSY-Korrelationen ergaben ein erstes, noch nicht eindeutiges Bild der Anordnung der H-Atome zueinander, das mit Hilfe der weiteren zweidimensionalen Spektren über ${ }^{3} J$ - und ${ }^{4} J$-Kopplungen bestätigt werden konnte. Es ergab sich die in Abbildung 33 dargestellte Struktur. Die Anordnung der Protonen der ungesättigten Seitenkette konnte anhand der Kopplungskonstanten ermittelt werden. Das H-Atom mit der Verschiebung $\delta=6.00$ koppelt mit dem H-Atom bei $\delta=7.60$ mit der Kopplungskonstante von $15.8 \mathrm{~Hz}$, was charakteristisch für eine trans-Anordnung ist. Die Kopplungskonstante von $12 \mathrm{~Hz}$ des Protons bei $\delta=6.40$ mit dem bei Proton bei $\delta=$ 7.00 könnte ein Indiz für eine cis-Anordnung sein. 


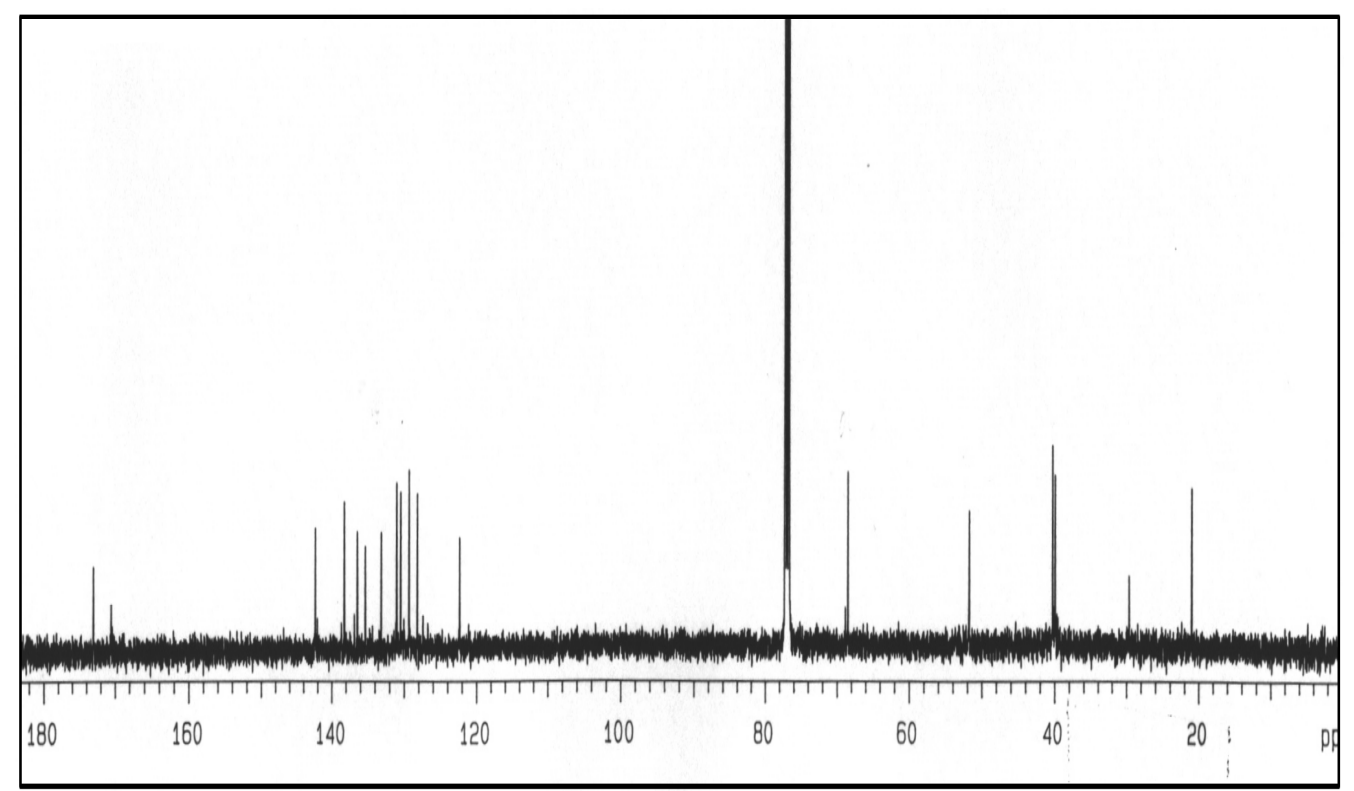

Abbildung 34: ${ }^{13} \mathrm{C}$-Spektrum von 32, aufgenommen in $\mathrm{CDCl}_{3}(125 \mathrm{MHz})$.

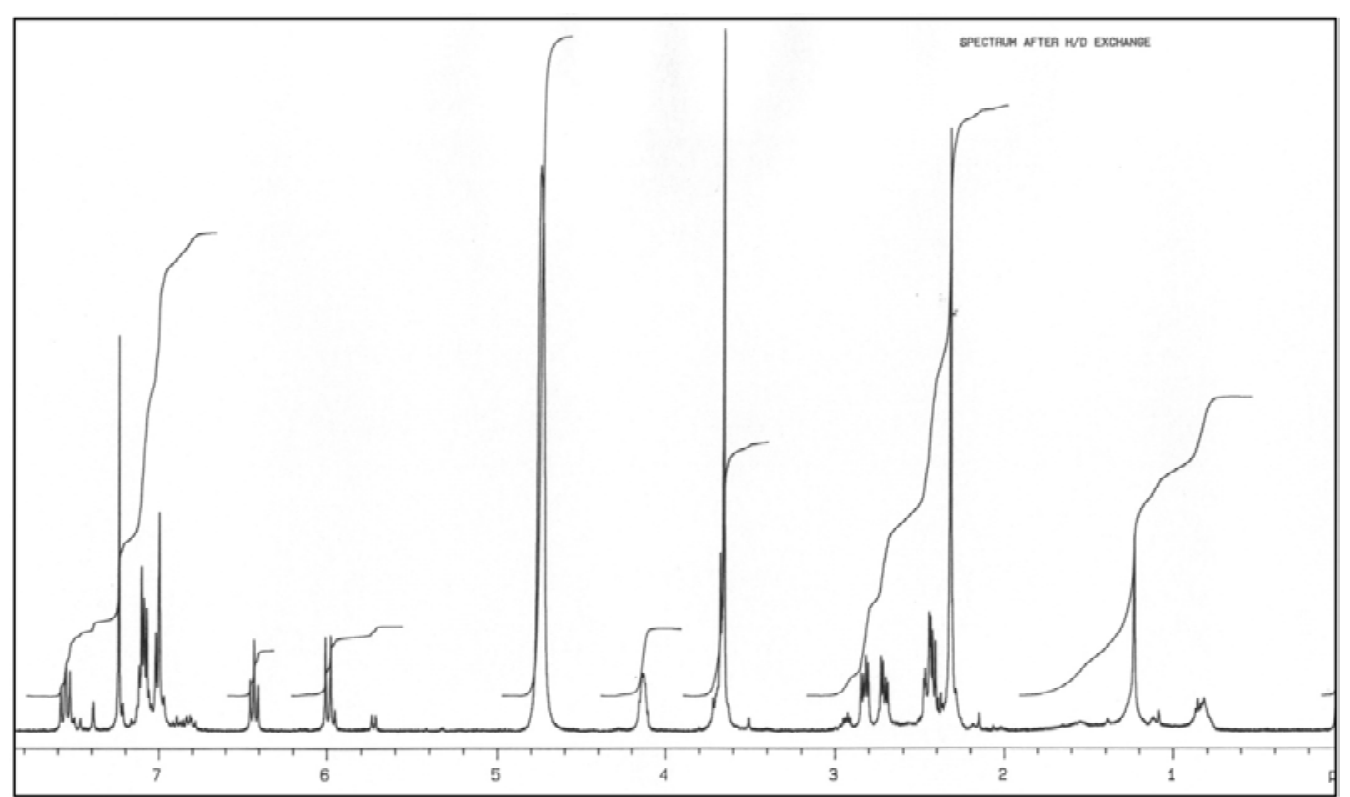

Abbildung 35: ${ }^{1} \mathrm{H}-\mathrm{NMR}-$ Spektrum von 32, aufgenommen in $\mathrm{CDCl}_{3}(300 \mathrm{MHz})$. 


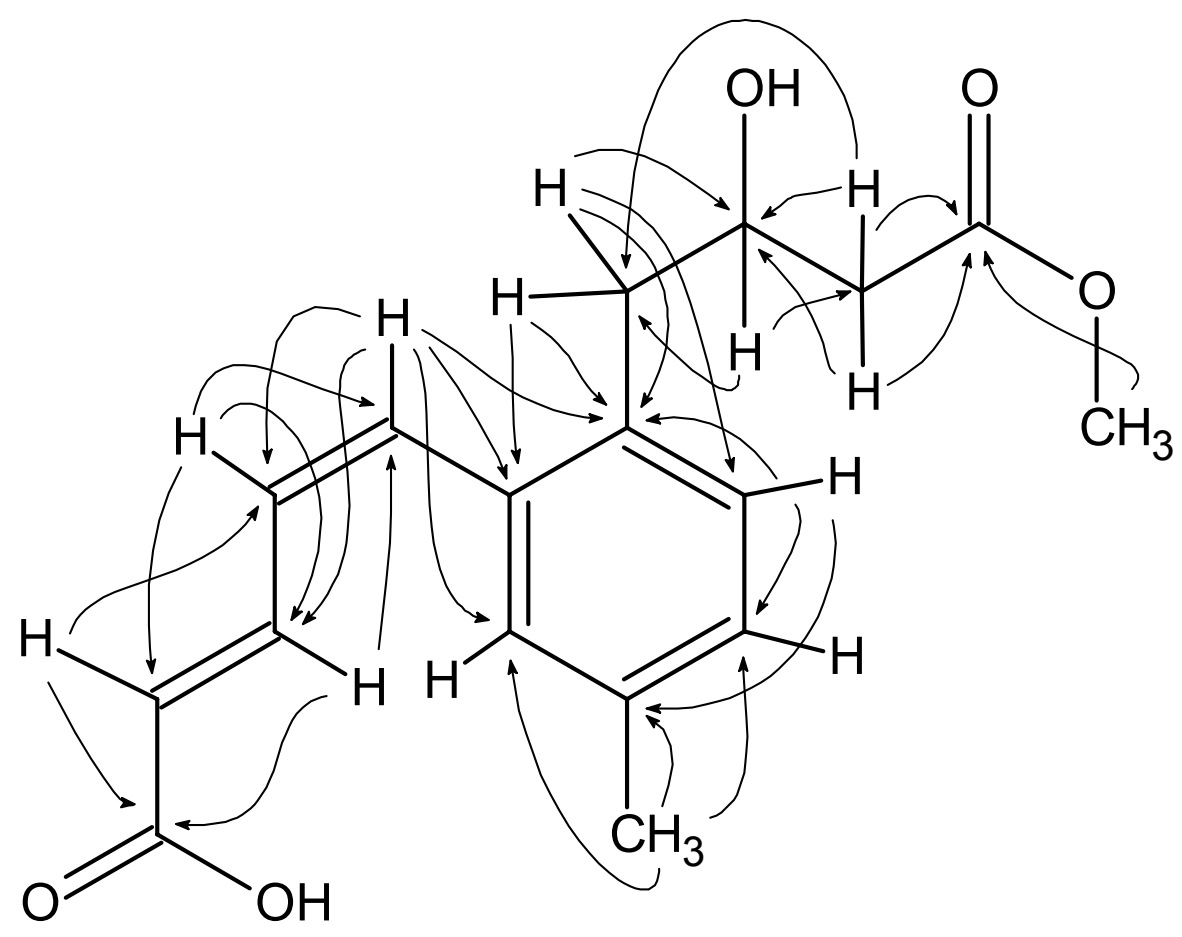

Abbildung 36: Die in der Abbildung 37 sichtbaren C-H-Korrelationen

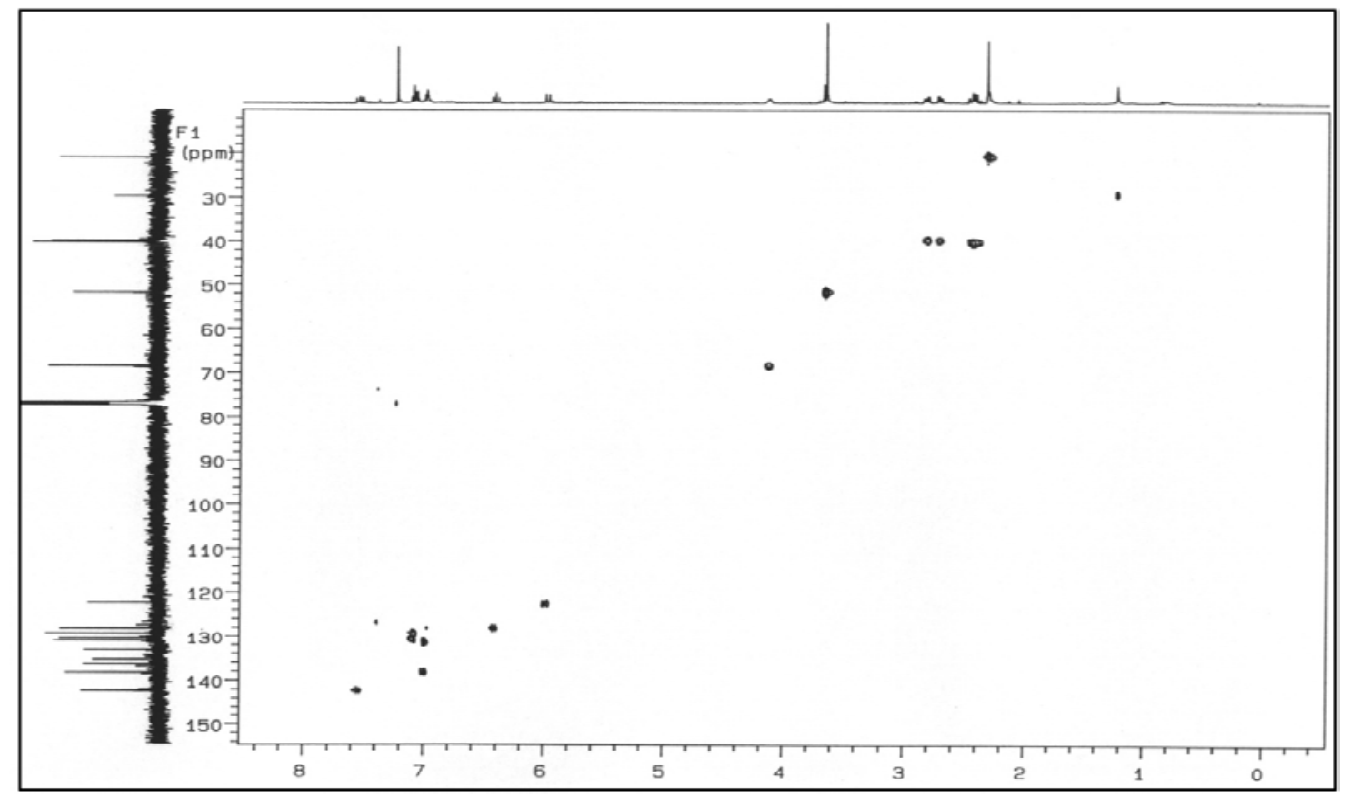

Abbildung 37: HMQC-Spektrum von 32, aufgenommen in $\mathrm{CDCl}_{3}(500 \mathrm{MHz})$. 

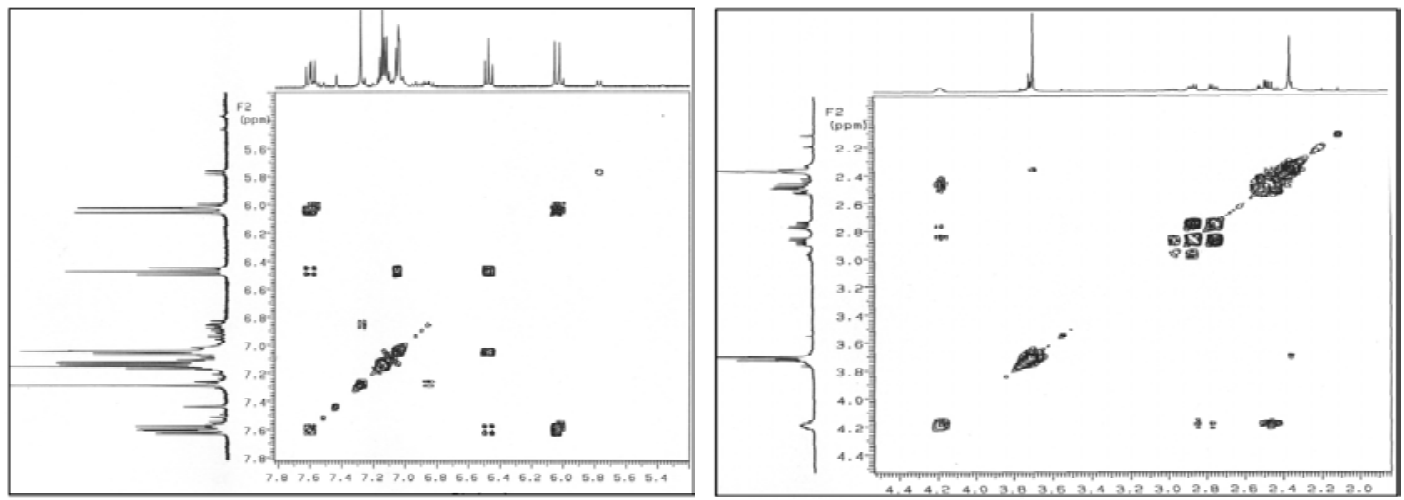

Abbildung 38: $\mathrm{H}, \mathrm{H}-\mathrm{COSY}-$ Spektren von 32 (aufgenommen in $\mathrm{CDCl}_{3}$ bei $500 \mathrm{MHz}$ ).

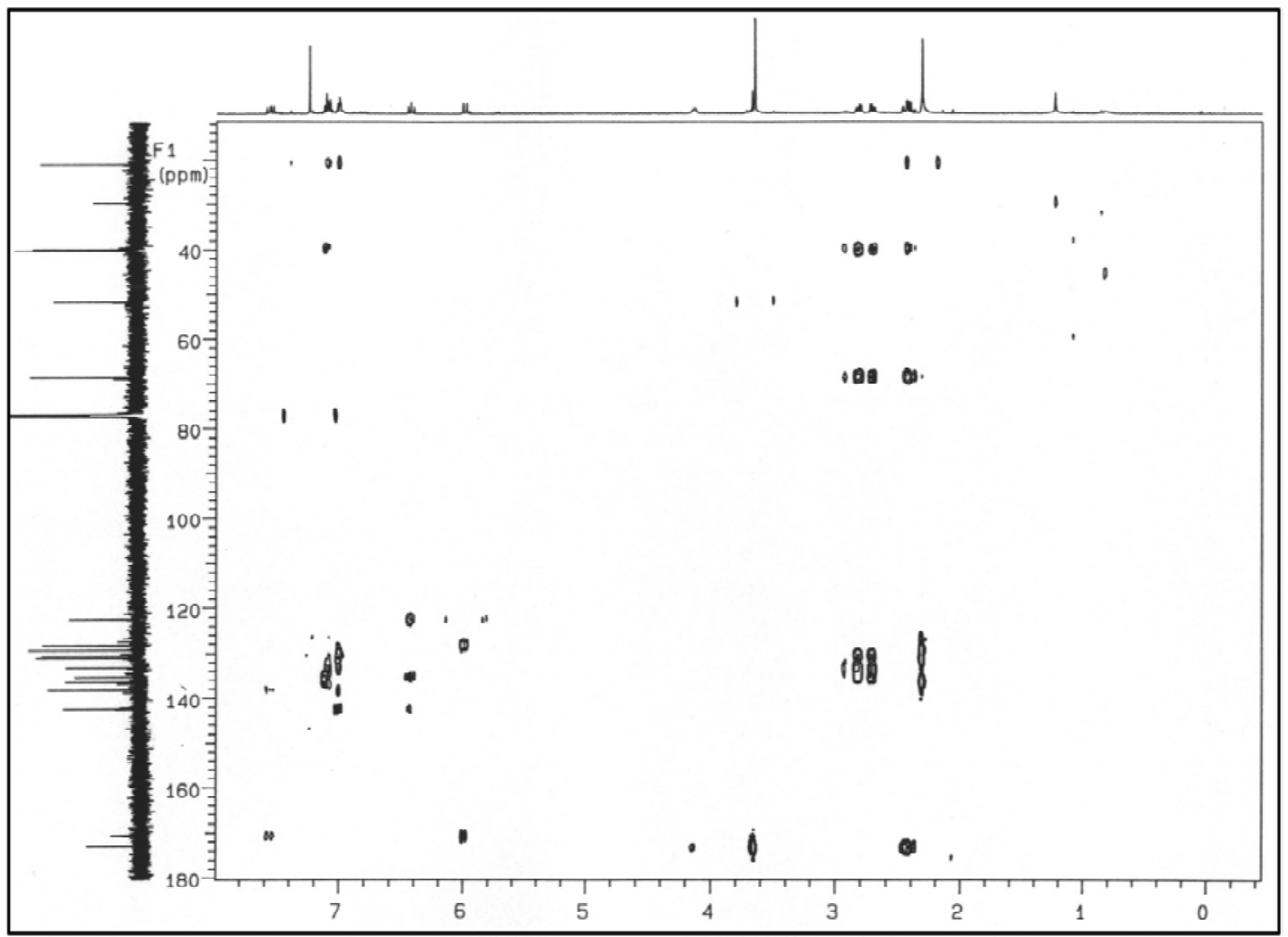

Abbildung 39: HMBC-Spektrum von 32, aufgenommen in $\mathrm{CDCl}_{3}(500 \mathrm{MHz})$.

Aufgrund der Aufspaltung der Protonen, die dem aromatischen Ring zugehörten, konnte man beweisen, dass es sich um einen 1,4,6-trisubstituierten Aromaten handeln muss. Daraus ergaben sich aber mehrere Möglichkeiten für die Zuordnung der Seitenketten. Die Protonen der an den aromatischen Ring gebundenen Methylgruppe koppelten mit zwei tertiären Kohlenstoffen, jedoch nicht mit einem der zwei anderen quartären. Daraus resultierte für die Methylgruppe die zugehörige Position 4 des Ringes. Das Proton der ungesättigten Seitenkette $[\delta=138$ (7.0)] koppelte mit dem 
Proton bei $\delta=131$ (7.0) und mit drei aromatischen Kohlenstoffatomen des Ringes ( $\delta$ $=133,135$ und 131). Wegen der fehlenden Kopplung mit dem Kohlenstoff bei $\delta=$ 130 folgte daraus die Substitution in der Position 6 des Ringes. Diese Zuordnung wurde zusätzlich durch Kopplungen zwischen den Protonen bei $\delta=2.8$ und den Kohlenstoffen 1, 2 und 6 des Ringes bestätigt. Danach handelte es sich um 5-[2-(2Hydroxy-3-methoxycarbonyl-propyl)-5-methyl-phenyl]-penta-2,4-diensäure eine bisher nicht beschriebene Verbindung. Da zu wenig Substanz isoliert werden konnte, war es nicht möglich, die Konstitution durch eine Kristallstrukturanalyse zu bestätigen.

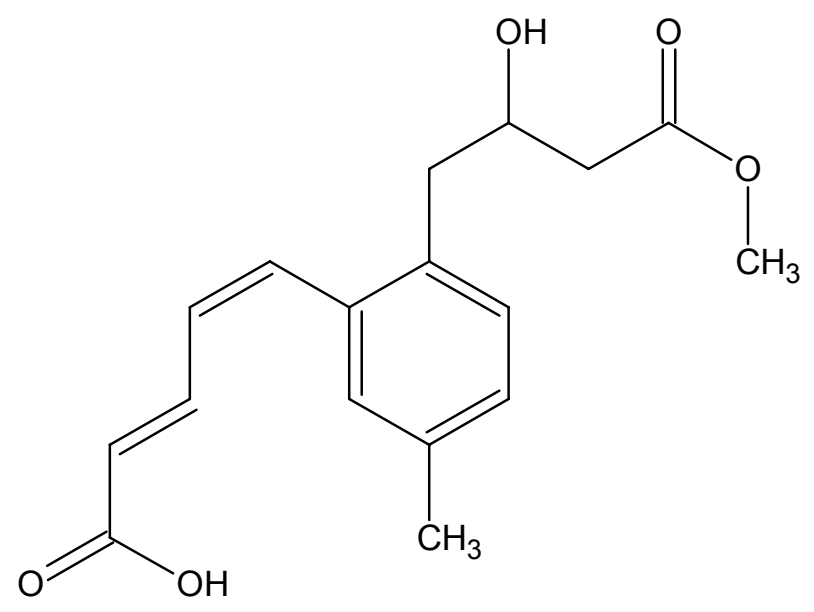

32

Die Biosynthese des Pyrromycinongerüstes in $\mathbf{3 0}$ und $\mathbf{3 1}$ geht von der Essigsäure aus und verläuft über ein Decaketid, das sich zum Tetracengerüst faltet und über Folgeschritte weiter funktionalisiert wird (s. Abbildung 40) ${ }^{[42]}$. Bei der Verbindung 32 könnte sich auf ähnliche Weise ein Octaketid falten, das schließlich zu einem $\mathrm{C}_{15^{-}}$ Gerüst decarboxyliert und im Ring C-methyliert wird (s. Abbildung 41). Bei dem Biosynthesevorschlag müsste allerdings eine endständige Methylgruppe abgespalten werden, was jedoch nicht leicht erklärbar ist. 


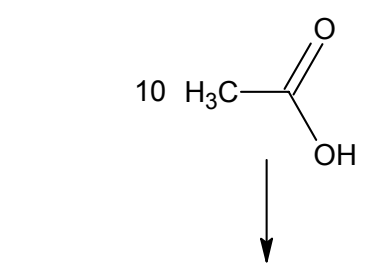

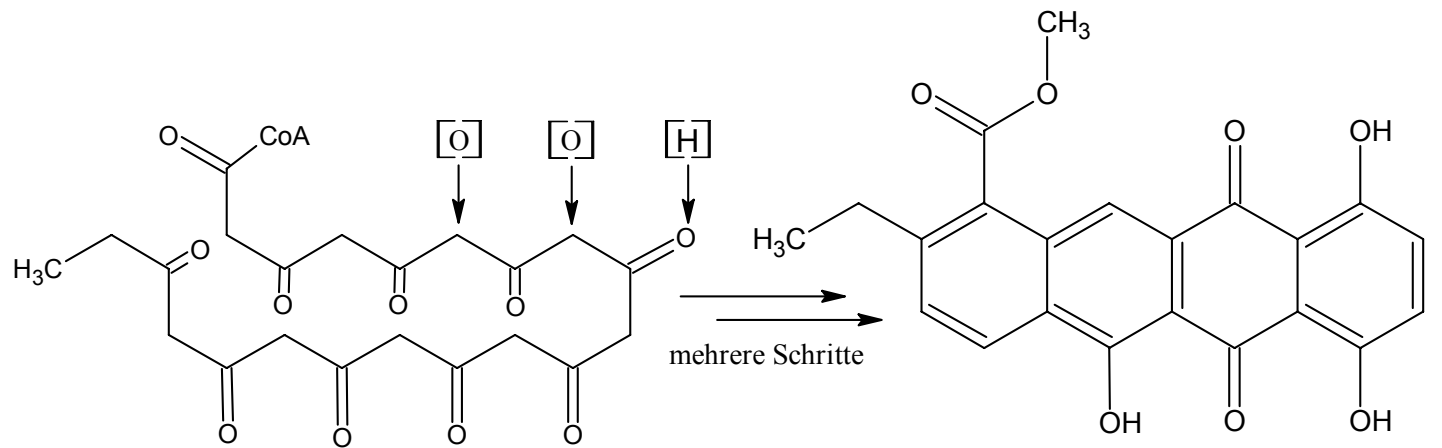

Abbildung 40: Die Biosynthese von $\varepsilon$-Pyrromycinon (30)

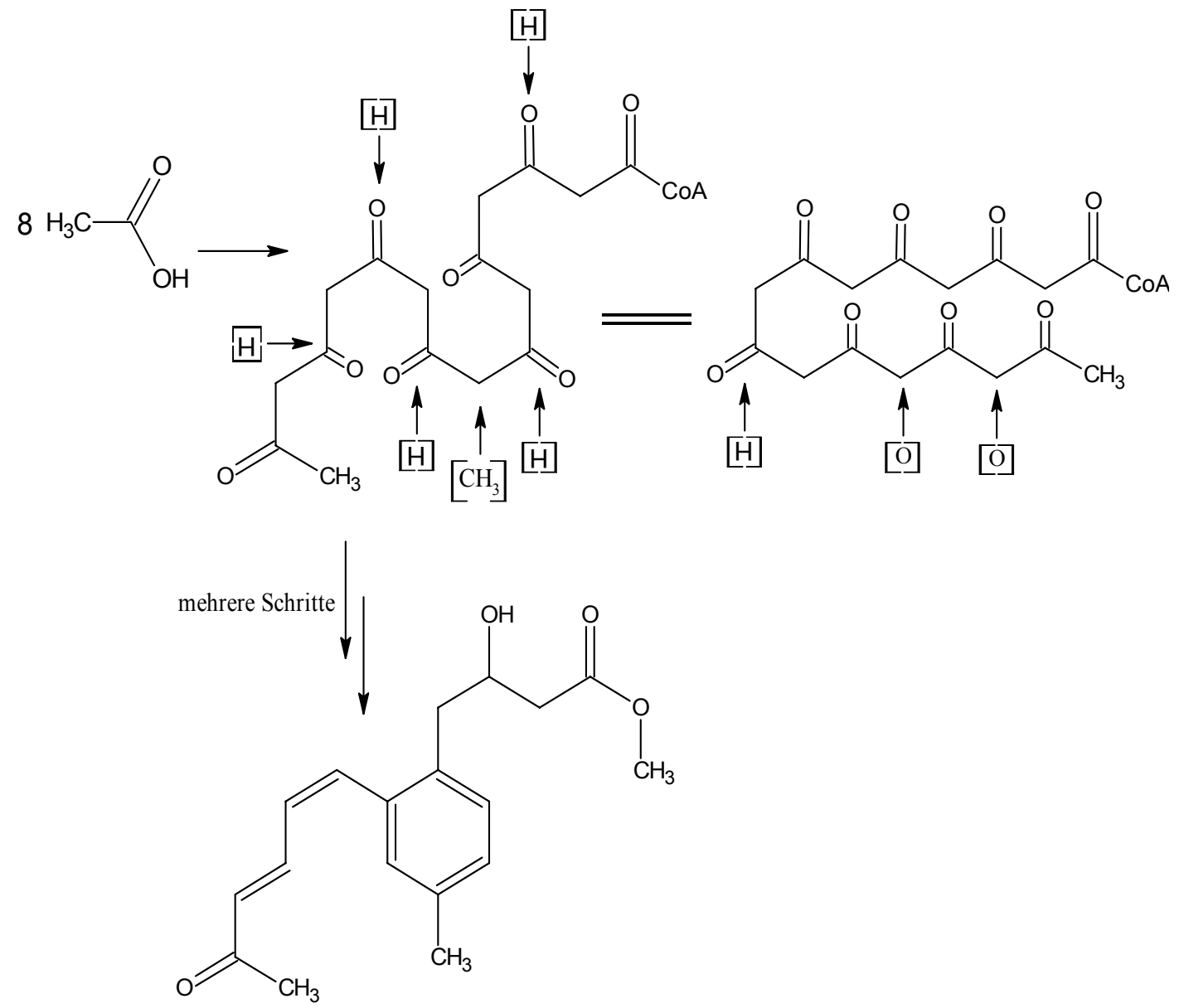

Abbildung 41: Ein Vorschlag zur Biosynthese von 32. 


\section{Thymin (5-Methyluracil)}

Aus der Fraktion I erhielt man durch präparative HPLC an RP18 eine farblose Verbindung, die im Dünnschichtchromatogramm einen $R_{\mathrm{f}}$-Wert von $0.5\left(\mathrm{CH}_{2} \mathrm{Cl}_{2} / \mathrm{MeOH}\right.$; 9:1) hatte. Unter UV-Licht (254 nm) ergab sie im Chromatogramm eine stark fluoreszierende und bei $366 \mathrm{~nm}$ löschende Zone. Dieses Verhalten war charakteristisch für Uracil (48), eine aus Mikroorganismen sehr oft isolierte Verbindung. Im Bereich der aromatischen Protonen konnte man bei $\delta=7.20$ ein Dublett der Intensität 1 sehen. Da die restlichen Signale keine Kopplungen besaßen, musste es sich um eine Kopplung mit einem austauschbaren H-Atom handeln. Solche Kopplungen sind oft bei Amino- oder Hydroxygruppen zu finden. Zwei breite Signale bei $\delta=10.5$ und 10.9 kamen als Kopplungspartner in Frage. Das letzte Singulett bei $\delta=1.7(3 \mathrm{H})$ stammte wahrscheinlich von einer zusätzlichen Methylgruppe.

Die massenspektrometrische Untersuchung (ESI) ergab eine Masse von 126. Eine Suche mit diesen Daten in AntiBase ergab nur eine Verbindung, die gleichzeitig auch das gleiche Spektrum besaß. Bei der gesuchten Verbindung handelte es sich um Thymin (33).<smiles>Cc1c[nH]c(=O)[nH]c1=O</smiles>

33

\section{Unterschiede der beiden Fermentationsmethoden}

Erstaunlicherweise brachte die kontinuierliche Fermentation einen bedeutenden Anstieg in der Zahl der produzierten Substanzen. Während bei der diskontinuierlichen Anzucht hauptsächlich 29 produziert wurde, fand man im kontinuierlichen Verfahren eine neue Verbindung 32 und zwei farbige Pyrromycinone 30 und 31. Das vorher produzierte Methylfervenulon 29 wurde nur in sehr geringen Mengen gefunden und konnte nicht in reiner Form isoliert werden. In der kontinuierlichen Fermentation wurde die Fettproduktion ebenfalls stark beschränkt. 


\section{Stamm B 7284}

Der Stamm B 7284 zeigte nur wenige Komponenten im HPLC-Vorscreening und wurde deswegen für die Optimierung mit GALOP ausgewählt. Ein weiteres Kriterium für die Auswahl war die erhöhte biologische Aktivität gegen Streptomyces viridochromogenes (Tü57), Bacillus subtilis, Escherichia coli, Mucor miehei und Staphylococcus aureus. Die Anzucht des Mikroorganismus für die Vorkultur erfolgte zuerst auf einer Agar-Platte mit $\mathrm{M}_{2}$-Medium mit 50 \% Meereswasser. Die Inkubationsdauer betrug 3 Tage. Weiterhin wurden fünf Schüttelkolben unter der Sterilbank angeimpft und weitere drei Tage bei $20^{\circ} \mathrm{C}$ geschüttelt. Die Optimierung erfolgte anhand der Genetischen Algorithmen mit dem Computerprogramm GALOP. Weitere fünf Schikanekolben wurden als Animpfmaterial für den Durchflussreaktor vorbereitet. Der Durchflussreaktor wurde mit der Schüttelkultur angeimpft und nach 24 Stunden, als sich ein Bakterienfilm am Stahlnetz gebildet hatte, das optimierte Medium (s. Tabelle 2) langsam durchgeleitet. Nach sieben Tagen wurde das verbrauchte Medium mit Essigester dreimal extrahiert. Der Rohextrakt wurde eingedampft, in Methanol aufgenommen und zwei mal mit Cyclohexan entfettet. Man erhielt $320 \mathrm{mg}$ eines braun gefärbten Extraktes.

Die Vortrennung an einer Kieselgelsäule lieferte mehrere Fraktionen, von denen nur eine für die weitere Trennung verwendet wurde. Die restlichen Fraktionen enthielten zu wenig Material und wurden verworfen.

Durch Chromatographie an Sephadex LH-20 erhielt man eine farblose Fraktion Ia und eine leicht gelbe Fraktion Ib, die von restlichen Verunreinigungen durch präparative HPLC an RP18 getrennt werden konnten. 


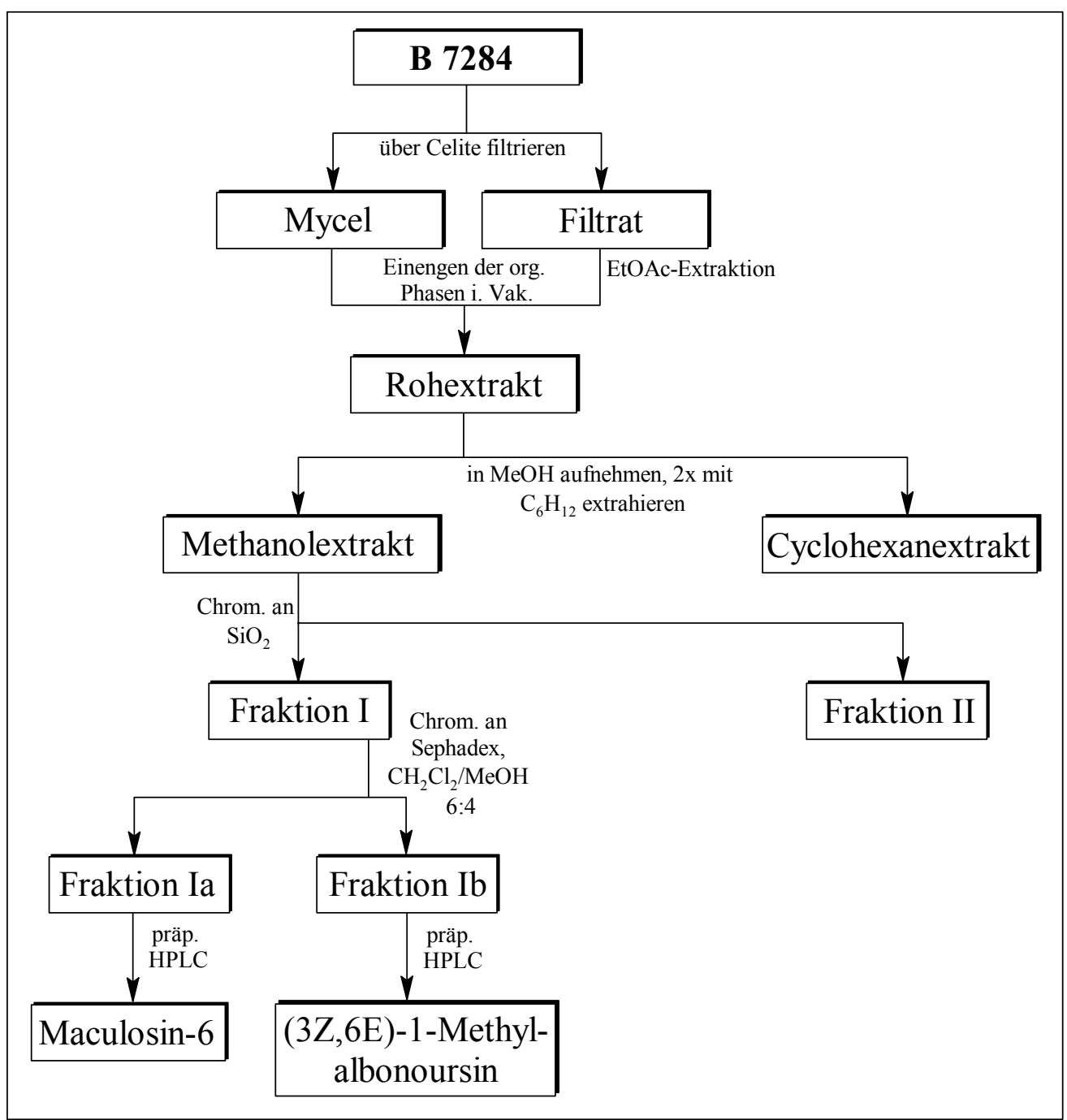

Schema 4: Aufarbeitung des Stammes B 7284

\section{(3Z,6E)-1-Methylalbonoursin}

Aus der Fraktion Ib konnte man 32 mg einer leicht gelblichen, kristallinen Substanz mit dem $R_{\mathrm{f}}$-Wert von $0.8\left(\mathrm{CH}_{2} \mathrm{Cl}_{2} / \mathrm{MeOH}\right.$ 9:1) isolieren, die im Dünnschichtchromatogramm UV-löschende Flecke ergab und sich mit Anisaldehyd/Schwefelsäure braun verfärbte. Das ${ }^{1} \mathrm{H}-\mathrm{NMR}-$ Spektrum zeigte bei $\delta=8.03$ ein breites Singulett, das einem austauschbaren Protonen zugeordnet werden konnte. Im aromatischen Bereich bei $\delta=7.20$ konnte man mehrere nahe beieinander liegende Protonensignale erkennen, was ein Indiz für einen monosubstituierten Aromaten war. Das Lösungsmittelsignal $\left(\mathrm{CDCl}_{3}\right)$ verhinderte allerdings, die Intensität und damit die Zahl der Protonen genau zu bestimmen. Ein weiteres Singulett bei $\delta=7.00(1 \mathrm{H})$ und ein Dublett bei $\delta=5.45(1 \mathrm{H})$ stammten wahrscheinlich von Protonen, die an unterschiedlichen Doppelbindungen gebunden waren. Das Multiplett bei $\delta=3.80(1 \mathrm{H})$ 
deutete darauf hin, dass dieses Proton, genauso wie eine Methylgruppe, die ein Singulett bei $\delta=3.20(3 \mathrm{H})$ verursachte, an ein elektronenziehendes Atom gebunden ist. Die charakteristische Aufspaltung der Signale bei $\delta=1.05 \mathrm{zu}$ einem Dublett der Intensität 6 wies auf ein Isopropylfragment am Ende einer Seitenkette hin. Die Massenspektrometrie (DCI) lieferte eine Masse von 270.1 Dalton. Auf dieser Grundlage konnte in AntiBase eine Struktur gefunden werden, die alle gestellten Anforderungen erfüllte. Der Vergleich der ${ }^{13} \mathrm{C}-\mathrm{NMR}$ - und ${ }^{1} \mathrm{H}-\mathrm{NMR}-$ Spektren der isolierten Verbindung mit den entsprechenden Spektren von 3-Benzyliden-6-isobutyliden-1-methylpiperazin-2,5-dion (34) ergab eine vollständige Übereinstimmung.

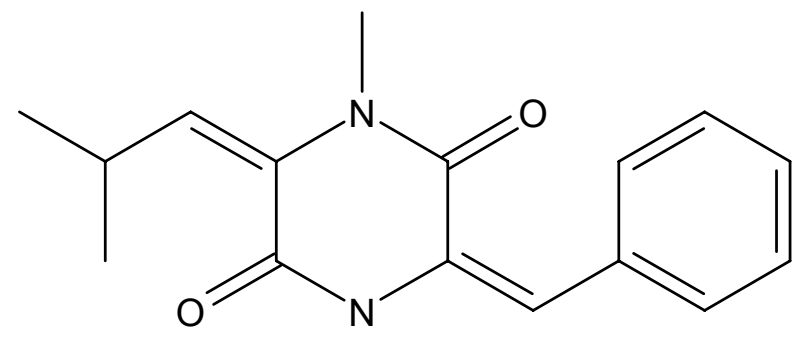

34

\section{Cyclo(leucyl-prolyl)}

Aus der Fraktion Ia konnte eine weiße, kristalline Substanz mittels präparativer HPLC-Trennung an RP18 isoliert werden. Die Verbindung war nur mäßig aktiv gegen Escherichia coli, Bacillus subtilis und Shewanella sp.

Die ${ }^{1} \mathrm{H}-\mathrm{NMR}-$ Spektren zeigten das bekannte Muster der Diketopiterazinderivate, die häufig aus Mikroorganismen isoliert worden sind. In unserer Arbeitsgruppe fand man sie in terrestrischen und limnischen Stämmen sowie in Extrakten von Mikroorganismen aus der Nordsee. Das ${ }^{1}$ H-NMR-Spektrum zeigte im aliphatischen Bereich ein Triplett bei $\delta=4.08(1 \mathrm{H})$, ein Dublett vom Dublett bei $\delta=4.04$, ein Multiplett bei $\delta$ $=3.70$ bis $\delta=3.60$. Weitere Multipletts waren im Bereich von $\delta=2.50$ bis $\delta=1.80$ und bei $\delta=1.50$ erkennbar. Die Aufspaltung der Signale bei $\delta=1.00$ deutete auf eine Isopropylgruppe hin. Die ermittelte Masse (DCI) von 210 und die bereits diskutierten spektroskopischen Befunde führten bei der Datenbanksuche (AntiBase) zu 35, einem Diketopiperazin, das auch unter dem Namen Maculosin-6 bekannt ist ${ }^{[75,79]}$.

Die meisten Diketopiperazine besitzen zwei chirale Kohlenstoffatome und können daher in vier stereoisomeren Formen auftreten. Da jedoch die Verbindungen als Na- 
turstoffe schon lange bekannt sind, wurde auf die Aufklärung der Stereochemie von $35 \mathrm{im}$ Rahmen dieser Arbeit verzichtet. Es handelt sich bei allen Stereoisomeren um Verbindungen ohne bekannte biologische Aktivität.

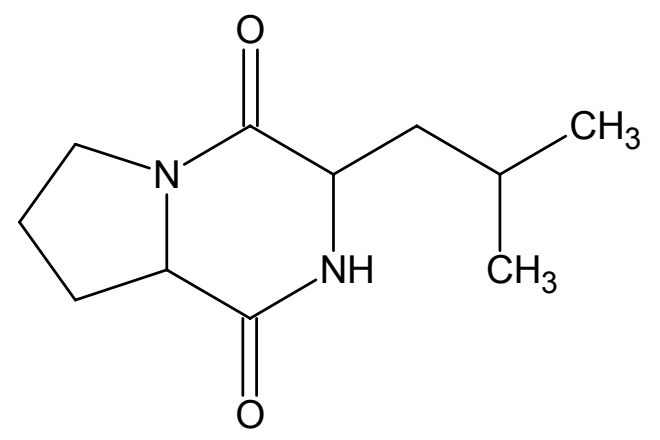

35

\section{Stamm B 7939}

Der marine Streptomycet B 7939 wuchs in kleinen weißen Kolonien auf $\mathrm{M}_{2}$-Medium mit 50 \% Meereswasser. Im HPLC-Vorscreening zeigte der Rohextrakt nur wenig auffallende Peaks. Die Rohextrakte zeigten eine geringe biologische Aktivität gegen Escherichia coli, Chlorella vulgaris, Chlorella sorokiniana und Scenedesmus subspicatus. Um den Stamm zu einer höheren Produktivität zu bringen, wurde das Medium mit dem Computerprogramm GALOP auf Glucose-, Maltose-, Hefe- und $\mathrm{CaCl}_{2}$-Gehalt optimiert (s. Tabelle 2). Die Anzucht und die Vorbereitung des Bioreaktors verliefen wie bei dem Stamm B 7284. Die kontinuierliche Fermentation dauerte sieben Tage. Während dieser Zeit wurden insgesamt 101 des Nährmediums durch den Fermenter gepumpt. Das verbrauchte Medium wurde dreimal mit je 1 Liter Essigester extrahiert und nach dem Einengen dreimal mit Cyclohexan entfettet. Man erhielt $1.2 \mathrm{~g}$ einer braungelben, öligen Flüssigkeit, die säulenchromatographisch (Kieselgel) vorgetrennt wurde. Man erhielt mehrere Fraktionen, die mehrere Flecken im Dünnschichtchromatogramm aufwiesen, nach dem Eindampfen aber keine ausreichende Mengen an Material lieferten. Sie wurden verworfen. Für die weitere Trennung wurden nur die Hauptfraktionen I und II verwendet. Aus Fraktion I wurden durch präparative Mitteldruck-Chromatographie drei Substanzen isoliert, die im Dünnschichtchromatogramm UV-löschende Eigenschaften zeigten und sich mit Anisaldehyd/Schwefelsäure blauviolett verfärbten. Die Fraktion II wurde durch präpa- 
rative Dünnschichtchromatographie und nachfolgende präparative HPLC zur Fraktion IIb aufgereinigt.

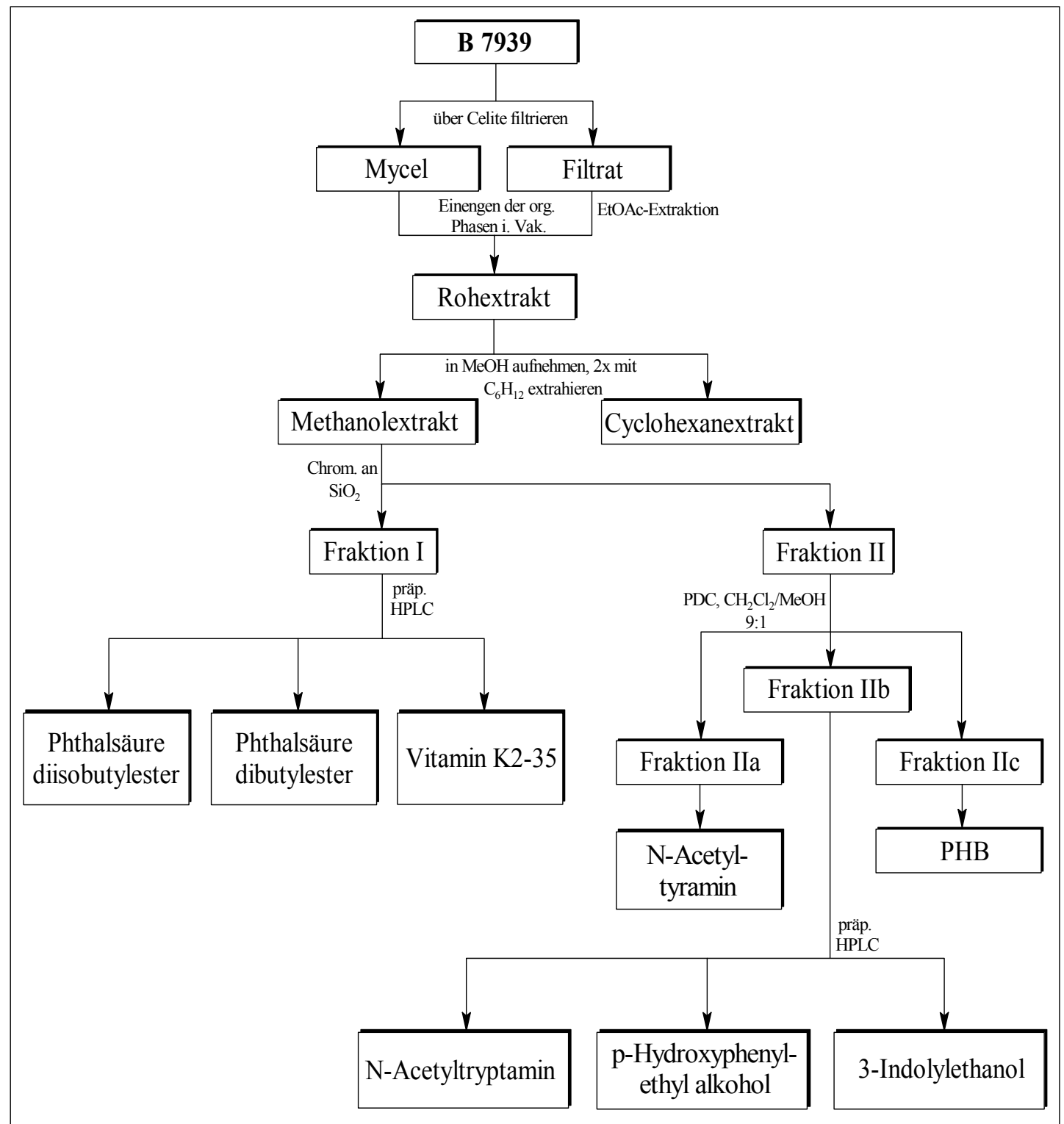

Schema 5: Aufarbeitung des Stammes B 7939

\section{N-Acetyltryptamin}

Die Substanz mit dem $R_{\mathrm{f}}$-Wert von $0.4\left(\mathrm{CH}_{2} \mathrm{Cl}_{2} / \mathrm{MeOH}\right.$; 95:5) verfärbte sich mit Anisaldehyd/Schwefelsäure zuerst orange. Nach einigen Minuten wechselte die Farbe zu Violett. Mit Ehrlichs Reagenz ergab sich eine violette Zone, was als ein Hinweis für einen Indolderivat interpretiert werden konnte.

Durch präparative HPLC erhielt man $16 \mathrm{mg}$ einer weißen Substanz, die sich gut in Chloroform löste. Im ${ }^{1} \mathrm{H}-\mathrm{NMR}-$ Spektrum konnte man im aromatischen Beriech das typische Kopplungsmuster eines 3-Indolderivates mit zwei Dubletts und zwei 
Tripletts des 1,2-disubstituierten Sechsrings erkennen. Zusätzlich sah man ein Dublett mit kleiner Kopplungskonstante, die einem Proton in meta-Position zugeordnet werden konnte. Das austauschbare Proton, das an den Stickstoff des heteroaromatischen Ringes gebunden ist, sah man in Form eines breiten Signals bei $\delta=8.06$. Ein zweites austauschbares H-Atom ergab ein ebenfalls breites Signal bei $\delta=5.50$. Weitere Signale bei $\delta=3.06$ und 2.98, die durch eine gegenseitige Kopplung zu einem Quartett und Triplett aufgespalten wurden, deuteten auf zwei benachbarte Methylengruppen hin. Wie sich bei der Betrachtung des H,H-COSY-Spektrums herausstellte, kam die Aufspaltung zu dem vorliegenden Quartett durch eine weitere Kopplung mit dem austauschbaren breiten Signal bei $\delta=5.50$ zustande. Ein scheinbar fehlender Kopplungspartner wird oft bei Imino- oder Hydroxygruppen beobachtet. Das letzte Singulett der Intensität 3 bei $\delta=1.90$ konnte durch eine Methylgruppe verursacht worden sein, deren Signal durch die Nachbarschaft eines aromatischen Ringes oder eines Carbonyl-Kohlenstoffs zu tiefem Feld verschoben war. Die Suche in AntiBase ergab die zwei ähnlichen Verbindungen N-Acetyltryptamin (36) und 5Hydroxy-N-acetyltryptamin. Die zweite Struktur wurde ausgeschlossen, da alle aromatischen Protonen von 36 im Spektrum zu finden waren. Diese Annahme wurde noch durch die Molmasse von 202 bestätigt. Letztendlich wurde die Struktur von 36 durch ${ }^{1} \mathrm{H}-\mathrm{NMR}-$ Vergleichspektren aus der internen Sammlung gesichert.

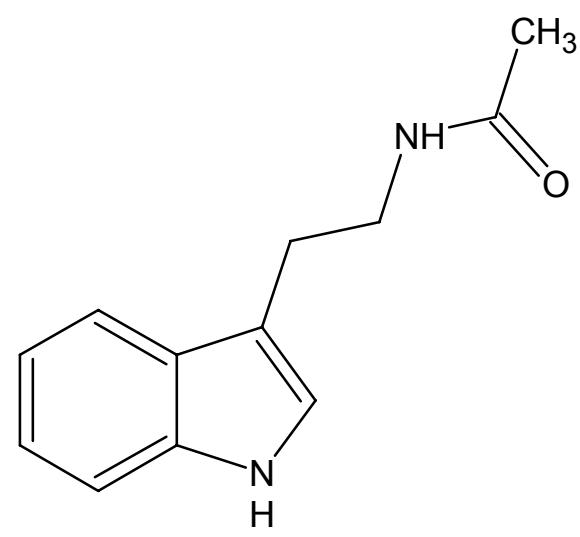

36

\section{3-Indolylethanol}

Eine weitere farblose Verbindung, die eine ähnliche Verfärbung bei der Reaktion mit Ehrlichs Reagenz sowie Anisaldehyd/Schwefelsäure ergab, wurde aus der Fraktion IIb durch präparative HPLC-Reinigung gewonnen. Das ${ }^{1} \mathrm{H}-\mathrm{NMR}-$ Spektrum zeigte 
außer den Signalen, die der Substanz eindeutig zugeordnet werden konnten, noch kleinere Verunreinigungen. Da sich aber die richtige Struktur mit Hilfe der vorhandenen Daten ableiten ließ, wurde auf die weitere Reinigung verzichtet. Im aromatischen Bereich fand man die gleichen Kopplungsmuster wie bei 36. Es handelte sich eindeutig um ein in Position 3-substituiertes Indolderivat. Auch die Aufspaltung der Signale bei $\delta=3.84$ und $3.00 \mathrm{zu}$ zwei Dubletts deutete darauf hin, dass eine Seitenkette vorliegt, die aus zwei Methyleneinheiten besteht. Die Tieffeldverschiebung der Signale deutete auf ein elektronenziehendes Heteroatom hin. Die Suche in AntiBase nach einem 3-Indolylderivat mit einer Ethylseitenkette, die zusätzlich ein Heteroatom trägt, lieferte 10 Kandidaten. Da die Hauptkomponente im Spektrum keine anderen Signale zeigte, konnten alle Strukturen ausgeschlossen werden, die eine längere Seitenkette besaßen. N,N-Diacetyltryptamin und $\mathbf{3 6}$ kamen nicht in Frage, da sich im Spektrum keine Methylsinguletts bei $\delta=1.50$ bis 2.20 finden ließen. Die resultierende Struktur des 3-Indolylethanol (37) konnte durch Spektrenvergleich bestätigt werden $^{[76]}$. Die gefundene Masse (DCI-MS) von 161 untermauert diese Struktur. 37 wurde bereits von Ehrlich aus einer Tryptophan-Fermentation isoliert und ist gegen grampositive Bakterien und Candida albicans schwach aktiv ${ }^{[77,78]}$.

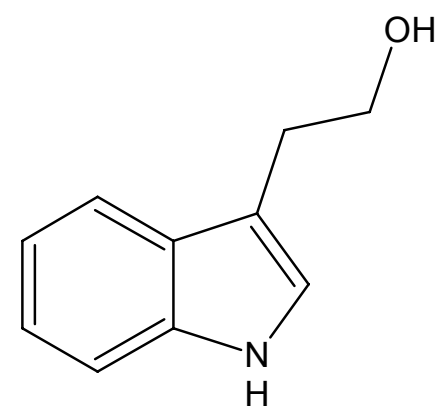

37

\section{N-Acetyltyramin}

Die in der präparativen Dünnschicht-Trennung von Fraktion II mit dem $R_{\mathrm{f}}$-Wert von 0.3 laufende Zone wurde mit Essigester extrahiert und das Filtrat vom Lösungsmittel befreit. Man erhielt eine weiße Substanz, die im Dünnschichtchromatogramm UVlöschende Zonen zeigte, die sich bei der Reaktion mit Anisaldehyd/Schwefelsäure braun verfärbten. Das ${ }^{1} \mathrm{H}-\mathrm{NMR}-\mathrm{Spektrum}$ enthielt noch einige Verunreinigungen, die aber bei der Aufklärung der Struktur außer Acht gelassen werden konnten. Auf weitere Reinigung wurde deswegen verzichtet. Im ${ }^{1} \mathrm{H}-\mathrm{NMR}$-Spektrum findet man im 
aromatischen Bereich bei $\delta=7.02$ und 6.78 zwei miteinander koppelnde Dubletts der Intensität je 2 mit einem ausgeprägten Dacheffekt, die nur einem para-disubstituierten Benzol herrühren können. Das breite Signal bei $\delta=5.42$ deutet auf ein austauschbares Proton hin. Weitere zwei Signale bei $\delta=3.44$ und 2.70 koppeln ebenfalls miteinander und werden zu einem Triplett vom Dublett aufgespalten. Das letzte Singulett bei $\delta=1.94(3 \mathrm{H})$ wird von einer Methylgruppe verursacht. Da die Verschiebungen und Kopplungsmuster der Seitenkette identisch sind mit den bereits bei 36 diskutierten, liegt es nahe, anzunehmen, dass auch in diesem Fall eine N-Ethylacetamid Seitenkette vorliegt. Leider ist es aus dem vorliegenden Spektrum nicht ersichtlich, ob der in para-Position stehende Substituent eine Hydroxygruppe ist. Diese Annahme wird jedoch durch die gefundene Molmasse (DCI-MS) von 179 wahrscheinlich gemacht. Die in AntiBase gefundene Struktur von 38 ergibt ein identisches ${ }^{1}$ H-NMR-Spektrum wie die aus dem Stamm isolierte Verbindung. Demnach handelt es sich bei der gesuchten Struktur um N-Acetyltyramin (38).

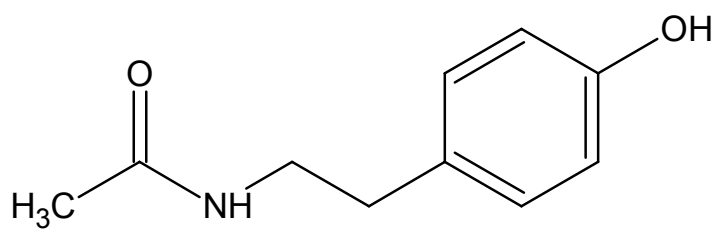

38

\section{4-(2-Hydroxy-ethyl)-phenol (Tyrosol)}

Nach der präparativen HPLC-Reinigung der Fraktion IIb erhielt man 12 mg einer weißen kristallinen Substanz mit $R_{\mathrm{f}}=0.2\left(\mathrm{CH}_{2} \mathrm{CH}_{2} / \mathrm{MeOH} 9: 1\right)$, die sich bei der Reaktion mit Anisaldehyd/Schwefelsäure braun verfärbte. Um die Löslichkeit der Substanz für die spektroskopischen Messungen im Deuterochloroform zu erhöhen, wurden der Lösung einige Tropfen $\left[\mathrm{D}_{4}\right]$-Methanol zugesetzt. Das hatte allerdings zur Folge, dass alle austauschbaren Protonen durch Deuterium ersetzt und im ${ }^{1} \mathrm{H}-\mathrm{NMR}$ Spektrum unsichtbar wurden.

Das ${ }^{1}$ H-NMR-Spektrum enthielt nur wenige Signale, davon zwei Dubletts der Intensität $2 \mathrm{im}$ aromatischen Bereich bei $\delta=7.00$ und 6.77 mit einer Kopplungskonstanten $J=9 \mathrm{~Hz}$, was auf einen para-disubstituierten aromatischen Sechsring hindeutete. Die 
zwei Tripletts bei $\delta=3.78$ und 2.76 koppeln mit einer Kopplungskonstante von $J=6$ $\mathrm{Hz}$ miteinander und stammen vermutlich von zwei Methylengruppen der Seitenkette. Die starke Tieffeld-Verschiebung wird durch ein Heteroatom oder einen Elektronenakzeptor verursacht. Die im DCI-Spektrum gefundene Molmasse betrug 138 Dalton. Die Datenbanksuche in AntiBase ergab zuerst sieben Treffer, aber nur ein Resultat erfüllte alle spektroskopischen Voraussetzungen. Dass es sich bei der gesuchten Verbindung um Tyrosol (39) handeln musste, wurde durch die Molmasse und den Vergleich mit einem Referenzspektrum aus der Sammlung verifiziert. Tyrosol (39) wurde oft auch bei der Extraktion von Pilzen und Pflanzen, wie Ceratocystis, Gibberella fujikuroi und Pyricularia oryzae gefunden ${ }^{[79-82]}$.

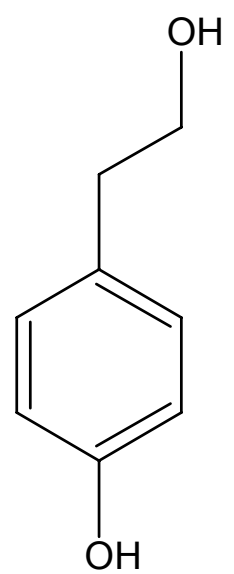

\section{Phthalsäure-dibutylester und Phthalsäure-diisobutylester}

Durch präparative HPLC-Trennung an Flash-Kieselgel konnte man aus Fraktion I einen weißen Feststoff isolieren, der im Dünnschichtchromatogramm eine UVlöschende Zone mit dem $R_{\mathrm{f}}$-Wert von $0.8\left(\mathrm{CHCl}_{3}\right)$ ergab, der sich mit Anisaldehyd/Schwefelsäure violett anfärben ließ. Im ${ }^{1} \mathrm{H}-\mathrm{NMR}$-Spektrum konnte man bei $\delta=$ 7.70 und $\delta=7.50$ zwei aromatische Signale erkennen, die eine für orthodisubstituierte Aromaten charakteristische Aufspaltung zeigten. Weiterhin konnte man bei $\delta=4.28$ ein Triplett und bei $\delta=4.03$ ein Dublett erkennen. Im aliphatischen Bereich fand man bei $\delta=2.00,1.70$ und 1.40 drei Multipletts und bei $\delta=0.98$ mehrere Signale, die endständigen Methylgruppen zugeordnet werden konnten. Anhand der Integrale war es jedoch nicht möglich, die Zahl der zugehörenden Protonen zu 
ermitteln. Vermutlich handelte es sich um eine Mischung aus zwei Isomeren, die ähnliche Grundstruktur, aber unterschiedliche Seitenketten besaßen. Bei der Auswertung des H,H-COSY-Spektrums wurde ersichtlich, dass eine Mischung vorliegt. Nach der Suche in Antibase handelte sich um Phthalsäure-dibutylester (40) und Phthalsäure-diisobutylester (41), die als Mischung im Molverhältnis 1:3 isoliert wurden. Da Phthalester oft als Weichmacher bei der Kunststoffproduktion eingesetzt werden und gelegentlich aus Plastikmaterial in die Extrakte gelangen, wurde die Mischung nicht weiter aufgetrennt.<smiles>CCCCOC(=O)c1ccccc1C(=O)OCCCC</smiles>

40<smiles>CC(C)COC(=O)c1ccccc1C(=O)OCC(C)C</smiles>

41

Um Plastik als Quelle für die der Weichmacher auszuschließen, wurden alle in Frage kommenden Teile der Apparatur mit Essigester extrahiert und dünnschichtchromatographisch mit den gefundenen Phthalaten verglichen. Hierbei wurde allerdings nur Diisooctyl-phthalat (51) gefunden. Die Ester 40 und 41 können also keine Artefakte der Isolierung sein, sondern sind Substanzen, die von den Mikroorganismen produziert wurden. Die Datenbankrecherche lieferte weitere Phthalsäurederivate, die ebenfalls aus unterschiedlichen Bakterien isoliert wurden. 3315-Af2 wurde aus Streptomyceten und der Phthalsäure-dibutylester (40) aus Penicillium bilaii isoliert ${ }^{[83]}$. Aus Streptomyceten wurde auch Phthalomycin (42) gewonnen, das ungesättigte Seitenketten besitzt ${ }^{[84]}$. 


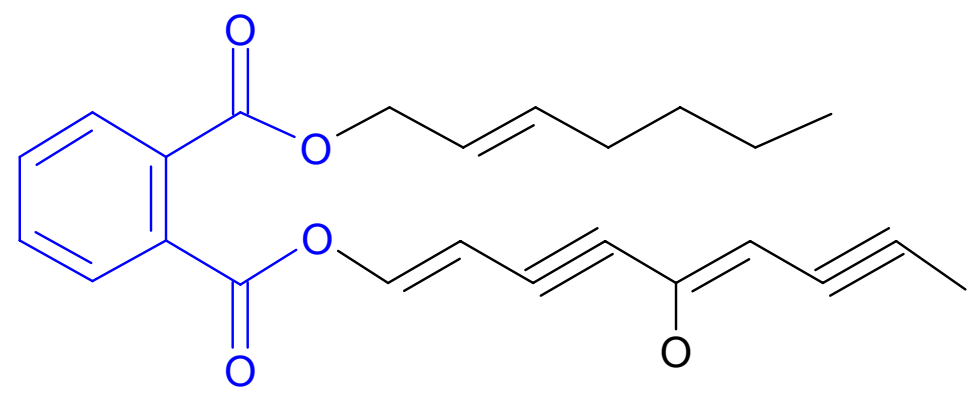

42

\section{Vitamin MK-7}

Aus der Fraktion I wurden $32 \mathrm{mg}$ einer schwachgelben Substanz mit dem $R_{\mathrm{f}}-$ Wert $0.7\left(\mathrm{CHCl}_{3}\right)$ isoliert. Das ${ }^{1} \mathrm{H}-\mathrm{NMR}-$ Spektrum zeigt im Aromatenbereich zwei charakteristische Signale der Intensität von jeweils 2 bei $\delta=8.05$ und $\delta=7.65$. Die Aufspaltung deutete darauf, dass es sich wahrscheinlich um ein Naphthochinon-Derivat handelt. Im Bereich der Doppelbindungen erkannte man ein Multiplett bei $\delta=5.00$ $(8 \mathrm{H})$. Ein Dublett findet man bei $\delta=3.28(2 \mathrm{H})$.

Auffallend war die Anwesenheit mehrerer Singuletts im aliphatischen Bereich bei $\delta$ $=2.18,1.80,1.62,1.60$ und 1.58. Die Tieffeldverschiebung dieser Signale konnte durch einen elektronenziehenden Bindungspartner verursacht werden, hier wahrscheinlich durch die im Alkenbereich sichtbaren Doppelbindungen. Ebenfalls auffallend war das Multiplettsignal bei $\delta=2$, das durch die Überlagerung mehrerer Signale zustande kam. Die Molmasse (ESI) lag bei 648.6. Zusätzlich war aus den ${ }^{13} \mathrm{C}-\mathrm{NMR}-$ und APT-Spektren ersichtlich, dass die gesuchte Verbindung ein chinoides System mit Carbonylsignalen bei $\delta=185.5$ und 184.5 besitzt. Es befanden sich $22 \mathrm{sp}^{2}$ hybridisierte Kohlenstoffatome im Aromatenbereich bei $\delta=146-116$. Die restlichen Kohlenstoffsignale waren ausschließlich im aliphatischen Teil des Spektrums bei $\delta=$ 40 bis $10 \mathrm{zu}$ finden. Eine mit den NMR-Daten durchgeführte Suche in AntiBase lieferte zuerst 28 mögliche Kandidaten, die alle Vitamin K-ähnliche Strukturen besaBen. Unter Berücksichtigung der gefundenen Masse kam nur eine Struktur in Frage. Es handelte sich bei der gesuchten Verbindung demnach um Vitamin MK-7 (43) [85,86]. 
<smiles>CC(C)=CCC/C(C)=C/CC/C(C)=C/CC/C(C)=C/CC/C(C)=C/CC/C(C)=C/CC1=C(C)C(=O)c2ccccc2C1=O</smiles>

43

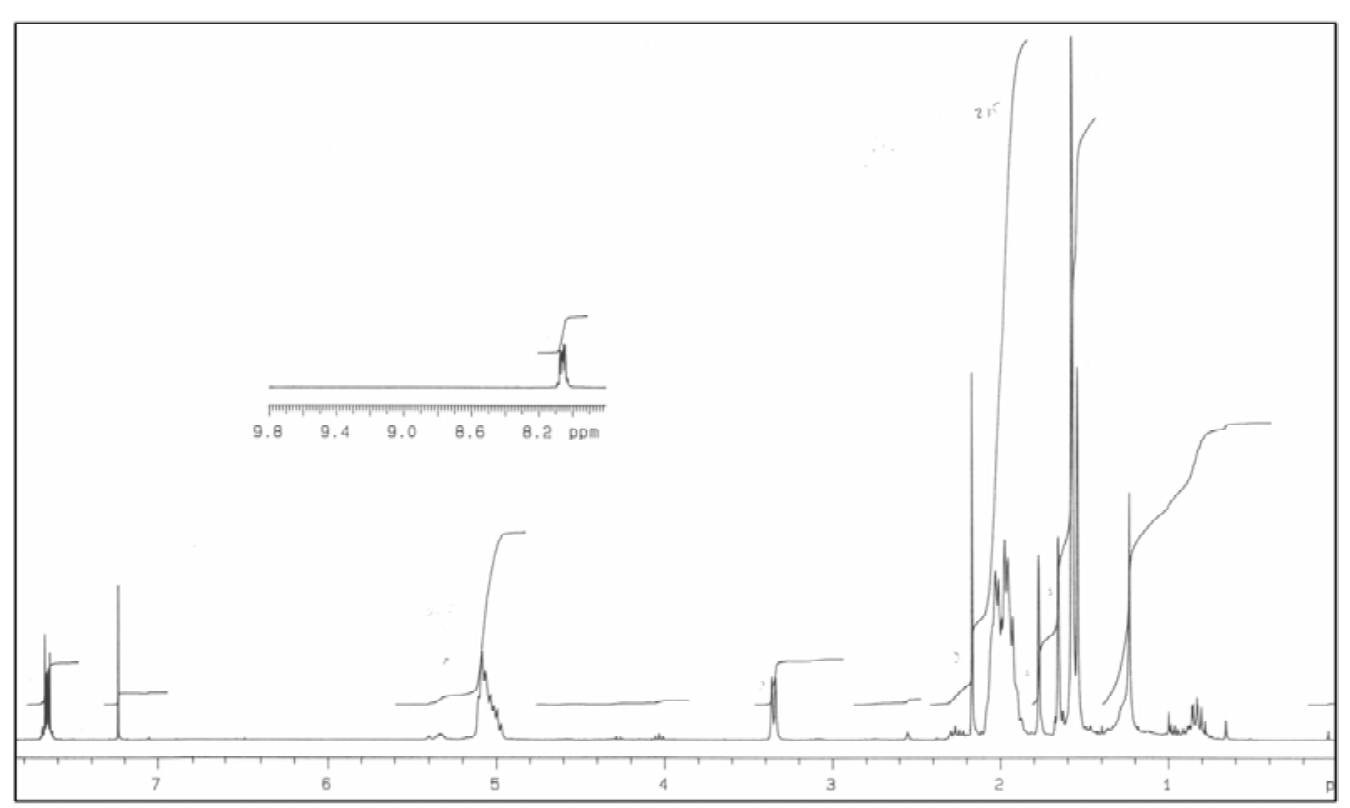

Abbildung 42: ${ }^{1} \mathrm{H}-\mathrm{NMR}-\mathrm{Spektrum}$ von $\mathbf{4 3}$ in $\mathrm{CDCl}_{3}(300 \mathrm{MHz})$.

Die Vitamine der K-Gruppe sind sehr oft aus Bakterien isoliert worden ${ }^{[87,88]}$. Es handelt sich dabei um relativ unpolare Naturstoffe, die im Dünnschichtchromatogramm keine Reaktion mit Natronlauge zeigen. In höheren Organismen übernehmen sie wichtige Funktionen, sie sind z.B. im Menschen bei der Biosynthese von Prothrombin beteiligt, wo sie notwendig für die 4-Carboxylierung von L-Glutaminsäurerestern und somit für die Bindungsfähigkeit von Calcium verantwortlich sind. Bei Bakterien spielen sie eine Rolle in der Atmungskette, und bei Algen sind sie an der Photosynthese beteiligt ${ }^{[91]}$. Verbindungen mit ähnlichen Eigenschaften sind die Ubichinone 
(44). Auch sie besitzen Redox-Eigenschaften und werden in der Atmungskette als Hilfssubstanzen für den Wasserstoffaustausch genutzt.<smiles>COC1=C(OC)C(=O)C(C/C=C(\C)CC/C=C(\C)CC/C=C(\C)CC/C=C(\C)CC/C=C(\C)CC/C=C(\C)CCC=C(C)C)=C(C)C1=O</smiles>

Ubichinon-9 (44)

\section{Hydroxybuttersäure-Oligomere}

Das ${ }^{1} \mathrm{H}-\mathrm{NMR}-\mathrm{Spektrum}$ der aus der Fraktion IIc isolierten Verbindung besaß keine aromatischen Signale, sondern lediglich ein Sextett der Intensität eins bei $\delta=5.22$, was auf ein Proton deutete, das sich in der Nähe einer elektronenziehenden Gruppe befand. Anhand der H,H-COSY-Korrelationen konnte man die zugehörigen Kopplungspartner eindeutig bestimmen. Es handelte sich dabei um einen Methylenkohlenstoff, der zwei Dubletts von Dubletts bei $\delta=2.60$ und 2.40 ergab, und um eine Methylgruppe, deren Signal bei $\delta=1.20 \mathrm{zu}$ einem Dublett aufgespalten wurde. Die gefundene Molmasse betrug 172 Dalton, was dem aus dem Ascomyceten Diplogelasinospora grovesiieinem isolierten Dimer 45 entsprach.

Wahrscheinlicher ist jedoch, dass Polyhydroxybuttersäure isoliert wurde, die in Streptomyceten in Form von nieder- und hochmolekularen Polymeren häufig ist ${ }^{[89,90]}$ und den Bakterien als Reservestoff dient.

Es gibt drei isomere Formen der Hydroxybuttersäure, 45a, 45b und 45c. 4Hydroxybuttersäure (45c), eine farblose Flüssigkeit (Sdp. $\left.178^{\circ} \mathrm{C}\right)$, wirkt blutdruckstabilisierend und betäubend, weshalb sie in der Anästhesie eingesetzt wird. Ihre 
Ammoniumsalze sind als Dauerwellmittel zugelassen. Als Naturstoff sind Hydroxybuttersäure (45b) und ihre Polymeren seit langem bekannt. ${ }^{[1]}$

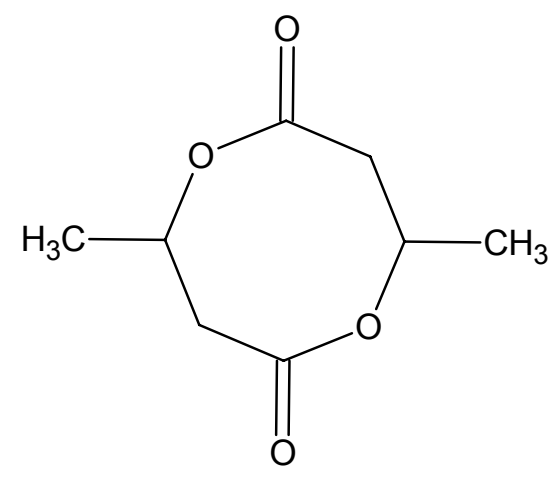

45<smiles>CC(CC(=O)CCOC(C)(C)C)OC(C)(F)F</smiles><smiles>CCC(O)C(=O)O</smiles>

$45 \mathbf{a}$<smiles>CC(O)CC(=O)O</smiles>

$45 b$<smiles>O=C(O)CCCO</smiles>

$45 \mathrm{c}$

\section{Stamm B 8927}

Der Extrakt des marine Streptomyceten B8927 fiel im biologischen Screening durch eine hohe Aktivität gegen Escherichia coli, Bacillus subtilis, Staphylococcus aureus, Streptomyces viridochromogenes (Tü57), Mucor miehei, sowie gegen zwei der im Tests eingesetzten Algen (Chlorella vulgaris und Chlorella sorokiniana) auf.

Auf der Agar-Platte wuchs der Stamm in leicht rosa verfärbten Kolonien. Nach dreitägigem Wachstum bei $20^{\circ} \mathrm{C}$ wurden Mycel und Filtrat des 20 Liter-Bioreaktors mit Essigester separat extrahiert. Die eingeengten Rohextrakte zeigten ähnliche Zusammensetzungen und wurden daher vereinigt und nach dem Entfetten durch Ausschütteln mit Cyclohexan säulenchromatographisch an Kieselgel vorgetrennt. Man erhielt drei Fraktionen, von denen die mittlere verworfen wurde. Aus der Fraktion III wurde 
Uracil (48) isoliert. Aus der Fraktion I erhielt man durch präparative Dünnschichtchromatographie eine unpolare Zone mit dem $R_{\mathrm{f}}$-Wert von $0.95\left(\mathrm{CH}_{2} \mathrm{Cl}_{2} / \mathrm{MeOH}\right.$; 9:1). Eine zweite polare Zone $\left(R_{\mathrm{f}}=0.2\right.$ bis 0.4$)$ lieferte nach HPLC zwei farblose UV-löschende Verbindungen Ic und Id.

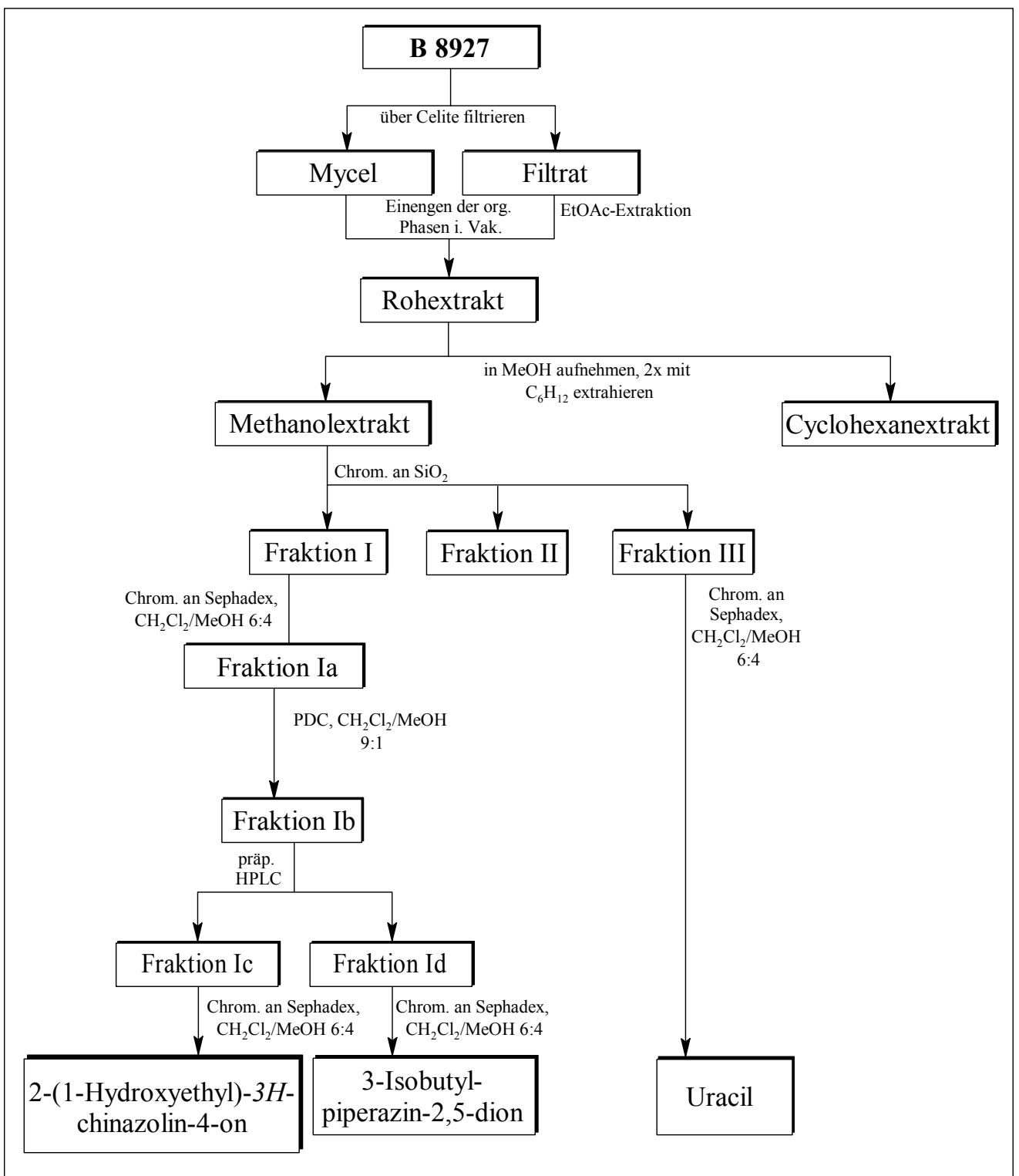

Schema 6: Aufarbeitung des Stammes B 8927

\section{3-Isobutylpiperazin-2,5-dion}

Aus der Fraktion Ic wurde eine farblose Verbindung gewonnen, die sich mit Anisaldehyd/Schwefelsäure leicht braun verfärbte. Das ${ }^{1} \mathrm{H}-\mathrm{NMR}-$ Spektrum zeigte im aromatischen Bereich nur ein Singulett bei $\delta=6.64(1 \mathrm{H})$. Bei $\delta=3.96$ beobachtete man ein Dublett vom Dublett der Intensität 1, das nach den H,H-COSY-Spektren mit 
Multipletts bei $\delta=1.80(2 \mathrm{H})$ und $1.60(1 \mathrm{H})$ koppelte. Letztere koppelten zusätzlich untereinander. Auch die Signale bei $\delta=1.30$ und 1.04 waren zu Multipletts aufgespalten. Das höchste Signal des Spektrums lag bei $\delta=0.96$ und stellte eine Dublett vom Dublett der Intensität 6 dar. Wahrscheinlich handelte es sich um einen Isopropylrest. Die Molmasse der Verbindung wurde anhand des ESI-Spektrums zu m/z 170 bestimmt. Die Suche in AntiBase lieferte mehrere Strukturen, von denen nur 3Isobutylpiperazin-2,5-dion (46) mit den spektroskopischen Daten übereinstimmte. Wegen des benachbarten Chiralitätszentrums sind die Methylgruppen nicht mehr äquivalent und ergeben daher zwei sich überlagernde Dubletts.

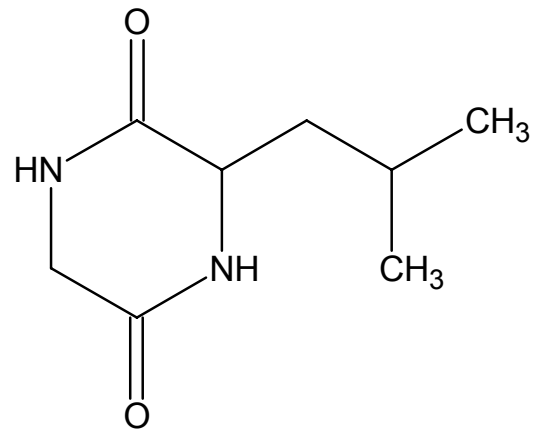

46

\section{2-(1-Hydroxyethyl)-3H-chinazolin-4-on (Chrysogin)}

Das ${ }^{1}$ H-NMR-Spektrum der aus Fraktion Ic isolierten Verbindung zeigte bei $\delta=10.6$ $(1 \mathrm{H})$ ein breites Signal, das nur einem austauschbaren Proton zugeordnet werden konnte. Im aromatischen Bereich des Spektrums fand man weitere vier Protonensignale. Bei $\delta=8.22(1 \mathrm{H})$ befand sich ein Dublett vom Dublett, das mit dem Dublett vom Triplett bei $\delta=7.68(1 \mathrm{H})$ koppelte. Anhand des H,H-COSY-Spektrums wurde ersichtlich, dass dieses Signal weiterhin mit dem aromatischen Dublett bei $\delta=7.62$ ( $1 \mathrm{H})$ und dem Triplett bei $\delta=7.4$ koppelte. Ein weiteres Signal fand man bei $\delta=4.8$ (1 H). Diese Verschiebung konnte nur durch einen elektronenziehenden Nachbarn verursacht werden. Die Aufspaltung zu einem Quartett entstand durch die Kopplung mit dem Dublett der Intensität 3 bei $\delta=1.60$. Eindeutig handelte es sich hierbei um das Signal einer Methylgruppe, die mit einer CH-Gruppe verbunden war. Im ESIMassenspektrum fand man das Signal der höchsten Intensität bei $m / z$ 190. Die Suche in AntiBase nach dem Strukturfragment Heteroatom- $\mathrm{CH}-\mathrm{CH}_{3}$ lieferte zuerst 7251 
Treffer. Eine weitere Einschränkung aufgrund der Masse lieferte drei Verbindungen. Alle vorliegenden spektroskopischen Befunde konnte letztendlich nur 2-(1Hydroxyethyl)-3H-chinazolin-4-on (47) erfüllen ${ }^{[96]}$.

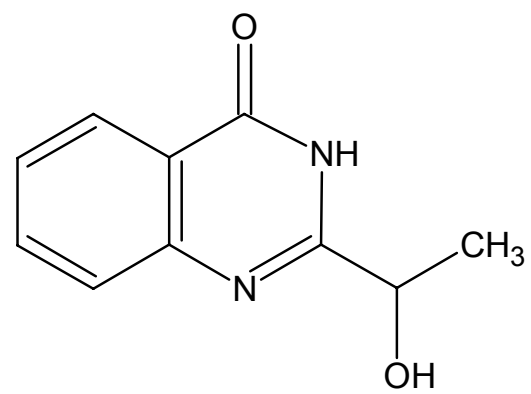

47

\section{Stamm GW 20/1811}

Der terrestrische Streptomycet GW20/1811wurde auf Malta gefunden und zeigte eine ausgeprägte Bioaktivität gegen Escherichia coli, Bacillus subtilis und Staphylococcus aureus. Zusätzlich wirkte der Rohextrakt als ein Chitinaseinhibitor und hemmt die Lyse von Chitin-Agar durch Shewanella frigidimarina. Auf der AgarPlatte wuchs er in kleinen weißgelblichen Kolonien. Die Anzucht des Mikroorganismus für die Vorkultur erfolgte auf Agar mit $\mathrm{M}_{2}$-Medium. Die Inkubationsdauer betrug 3 Tage. Danach wurden zwei Schüttelkolben unter der Sterilbank angeimpft und weitere drei Tage bei $20^{\circ} \mathrm{C}$ geschüttelt. Die so vorbereitete Vorkultur wurde für die Animpfung von 1550 ml-Erlenmeyer-Kolben verwendet. Die Zusammensetzung der jeweiligen Medien wurde mit dem Programm GALOP vorbereitet und über fünf Generationen optimiert. Das ergiebigste Medium wurde für die kontinuierliche Kultur eingesetzt (s. Tabelle 2). Die kontinuierliche Fermentation dauerte sieben Tage. Die Geschwindigkeit der Zuleitung des Nährmediums wurde so eingestellt, dass innerhalb der Fermentationszeit 10 Liter in die Sammelflasche durch den Fermenter geleitet wurden. Das verbrauchte Medium wurde dreimal mit je 1 Liter Essigester extrahiert und nach dem Einengen dreimal mit Cyclohexan entfettet. Durch die chromatographische Trennung an Kieselgel konnte man den Extrakt in drei Fraktionen. Laut Dünnschichtchromatogramm befanden sich in der farblosen Fraktion I zwei Hauptkomponenten, die im UV-Licht bei $254 \mathrm{~nm}$ stark fluoreszierten und bei 366 nm UV-löschende Flecken ergaben. Mit Anisaldehyd/Schwefelsäure verfärbten sich beide Zonen braun. Die Chromatographie an Sephadex LH-20 lieferte die Frak- 
tion Ia, aus der zwei reine Verbindungen durch präparative HPLC-Trennung isoliert werden konnten. Die Fraktionen II und III wurden durch präparative Dünnschichtchromatographie gereinigt. Aus der Fraktion III isolierte man nur eine UV-löschende Zone mit dem $R_{\mathrm{f}}$-Wert von $0.85\left(\mathrm{CH}_{2} \mathrm{Cl}_{2} / \mathrm{MeOH} ; 95: 5\right)$, die sich mit Anisaldehyd/Schwefelsäure braunviolett anfärben ließ. Die Fraktion II besaß eine auffallende, dunkelgrüne Farbe. Bei der präparativen Dünnschichtchromatographie an Kieselgel konnte man sie in drei weitere Unterfraktionen IIa bis IIc auftrennen. Aus der dunkelgrünen Fraktion IIb konnten jedoch keine für die NMR-Spektroskopie ausreichende Mengen an Substanz eluiert werden und sie wurde verworfen. Die ebenfalls stark grün verfärbte Fraktion IIa lieferte nach der zusätzlichen Reinigung an Sephadex LH-20 12 mg einer dunkelgrünen, kristallinen Substanz.

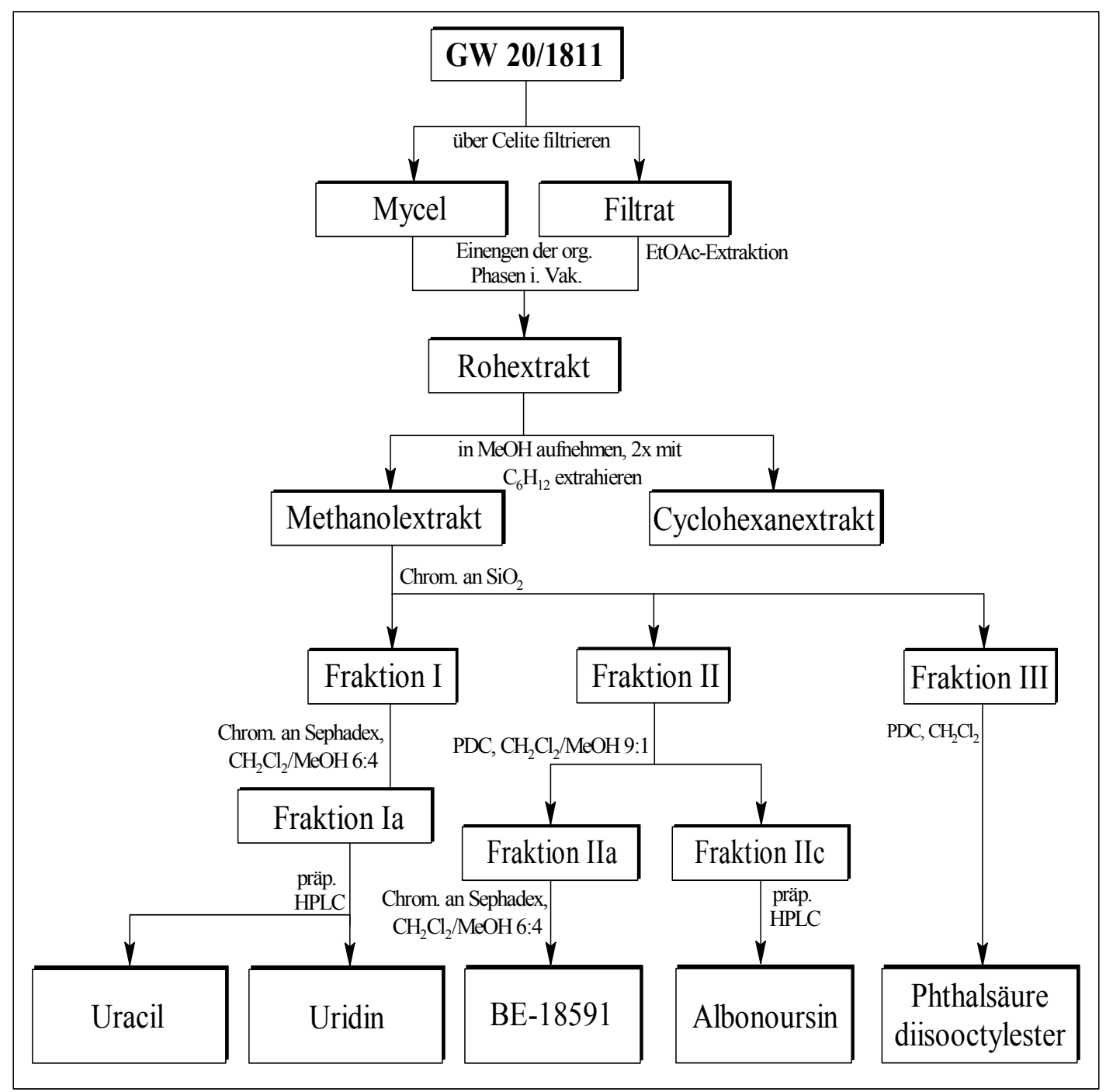

Schema 7: Aufarbeitung des Stammes GW 20/1811 


\section{Uracil}

Das ${ }^{1} \mathrm{H}-\mathrm{NMR}-$ Spektrum zeigte nur drei Signale. Bei $\delta=10.80$ beobachtete man ein breites Signal, welches sicherlich durch Anwesenheit austauschbarer Protonen entstanden war. Bei $\delta=7.36$ und 5.40 fand man zwei Dubletts, die miteinander koppelten. Die Molmasse (DCI-MS) betrug 112. Die Suche in AntiBase lieferte schnell eine plausible Lösung. Der endgültige Vergleich mit Referenzspektren bestätigte die Vermutung, dass es sich bei der gesuchten Verbindung um Uracil (48) handelte.<smiles>O=c1cc[nH]c(=O)cc1</smiles>

48

\section{Uridin}

Eine zweite Verbindung mit ähnlichen physikalischen Eigenschaften wie Uracil (48) und einem $R_{\mathrm{f}}$-Wert von $0.3\left(\mathrm{CH}_{2} \mathrm{Cl}_{2} / \mathrm{MeOH} 9: 1,48 R_{\mathrm{f}}=0.4\right)$ wurde ebenso aus der Fraktion I gewonnen. Die weiße pulvrige Substanz löste sich in Chloroform überhaupt nicht. Deswegen wurden die ${ }^{1}$ H-NMR-Spektren in Deuteriomethanol aufgenommen. Im ${ }^{1} \mathrm{H}-\mathrm{NMR}-\mathrm{Spektrum}$ fand man die charakteristischen und bereits bei $\mathbf{4 8}$ diskutierten zwei Dubletts. Allerdings fand man das eine Signal nicht mehr bei $\delta=$ 7.36, sondern tieffeldverschoben bei $8.00(1 \mathrm{H})$. Das zweite zugehörige Signal trat auch tieffeldverschoben bei $\delta=5.65(1 \mathrm{H})$ auf. Es handelte sich also um ein Uracilderivat mit elektronenziehenden Substituenten. Ein weiteres Dublett fand man bei $\delta$ $=5.85(1 \mathrm{H})$, das die gleiche Kopplungskonstante von $J=6 \mathrm{~Hz}$ besaß wie das Pentett bei $\delta=4.18$. Auffallend war die Häufung von Signalen im Bereich um $\delta=4$, in einem Bereich also, in dem man Protonen von Zuckerresten findet. Es fehlten jegliche Signale im aliphatischen Bereich, was die Anwesenheit von Seitenketten ausschloss.

Das ${ }^{13} \mathrm{C}$-NMR-Spektrum bestätigte zwei Carbonylkohlenstoff-Atome (Signale bei $\delta$ $=171$ und 166). Die Atome der Doppelbindung ergaben Signale bei $\delta=142$ und 
102. Ebenso fand man fünf Signale bei $\delta=90$ bis 60 , die dem später identifizierten Zucker-Rest zugeordnet werden konnten. Zusätzlich konnte das Signal bei $\delta=62$ anhand des APT-Spektrums dem Methylenkohlenstoff des Zuckers zugewiesen werden.

Die gefundene Molmasse von 244 erleichterte die Suche der Struktur. Der Vergleich der NMR-Daten und der Molmasse mit den Einträgen in AntiBase lieferte nur einen Treffer, Uridin (49). Durch den abschließenden Spektrenvergleich wurde die Struktur gesichert.

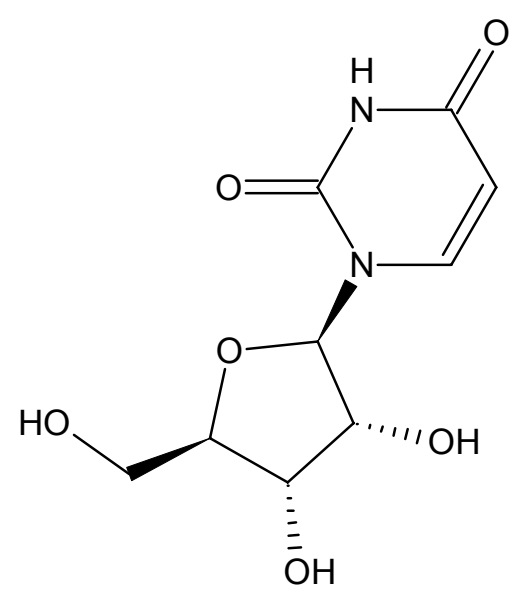

49

\section{Dodecyl-(4-methoxy-1'H-[1,2']bipyrrolyl-5-ylidenmethyl)-amin (BE-18591)}

Im ${ }^{1}$ H-NMR-Spektrum der aus der Fraktion IIa isolierten grünen Verbindung waren drei austauschbare Protonen bei $\delta=13.60(1 \mathrm{H}), 10.76(1 \mathrm{H})$ und $9.48(1 \mathrm{H}) \mathrm{zu}$ finden. Außerdem konnte man 5 Protonensignale im aromatischen Bereich erkennen. Das Signal bei $\delta=7.38(1 \mathrm{H})$ wurde zu einem Dublett mit der Kopplungskonstante $J$ $=15 \mathrm{~Hz}$ aufgespalten. Da die relativ große Kopplungskonstante nicht weiter im Spektrum auftrat, lag es nahe, dass es sich entweder um zwei Protonen handeln musste, oder der zweite Kopplungspartner ein austauschbares H-Atom war. Tatsächlich war die Kopplung nach dem $\mathrm{D}_{2} \mathrm{O}$-Austausch verschwunden. Die weiteren zwei aromatischen Protonen bei $\delta=7.04(1 \mathrm{H})$ und $6.70(1 \mathrm{H})$ waren als Triplett (nach dem $\mathrm{D}_{2} \mathrm{O}$ Austausch als Dublett) zu sehen. Bei $\delta=6.22(1 \mathrm{H})$ lag zuerst ein Quartett vor und bei $\delta=5.96(1 \mathrm{H})$ ein Dublett. Beide Signale wie auch das Triplett bei $\delta=$ $3.40(2 \mathrm{H})$ verloren nach dem $\mathrm{D}_{2} \mathrm{O}$ Austausch die Kopplungspartner. Die restlichen Signale veränderten sich nicht. Bei $\delta=3.90$ lag ein Singulett der Intensität 3 vor, das 
nur einer Methylengruppe zugeordnet werden konnte, die an ein elektronenziehendes Heteroatom gebunden war. Im aliphatischen Bereich fand man bei $\delta=2.40(2 \mathrm{H})$, $1.68(2 \mathrm{H})$ und 1.22 (Intensität nicht bestimmbar) weitere Signale, die nur einer langen aliphatischen Kette zugeordnet werden konnten, die mit terminalen Methylgruppe beendet werden musste (Triplett der Intensität 3 bei $\delta=0.80$ ). Anhand der aufgenommenen C-H-Korrelationsspektren konnte die Zuordnung der im ${ }^{13} \mathrm{C}-\mathrm{NMR}$ - und APT-Spektren sichtbaren Signale zu den entsprechenden Protonen erfolgen. Eine Information über die Molmasse der gesuchten Verbindung konnte leider nicht erhalten werden. Die mit den vorhandenen Angaben durchgeführte Datenbanksuche ergab nur eine plausible Lösung, laut der es sich bei der isolierten Substanz um Dodecyl(4-methoxy-1'H-[1,2']bipyrrolyl-5-ylidenmethyl)-amin (50) handeln muss. Der Vergleich der ${ }^{1} \mathrm{H}-\mathrm{NMR}$ - und ${ }^{13} \mathrm{C}-\mathrm{NMR}-$ Spektren mit den in der Literatur abgebildeten bestätigte die Struktur ${ }^{[92]}$. Überlegenswert ist jedoch das unerwartete Auftreten von drei austauschbaren Protonen im ${ }^{1}$ H-NMR-Spektrum, was an das ähnliche Verhalten der Prodigiosine erinnert. Zusätzlich überrascht die Aufspaltung des Signals bei $\delta=$ $7.38(1 \mathrm{H})$ zu einem Dublett. Da aber der Kopplungspartner nicht zu finden war und das Signal einem Proton der Seitenkette, der an die Doppelbindung gebunden ist, zugewiesen werden kann, handelt es sich wahrscheinlich um eine Mischung aus cisund trans-Isomeren.

Die Verbindung 50 wurde aus Streptomyces sp. BE-18591 zum ersten mal 1993 isoliert und zeigt beträchtliche Antitumoreigenschaften gegen die Zelllinien P388 und MKN-45. Es wirkt ebenfalls gegen Gram-positive und -negative Bakterien ${ }^{[107,108]}$.

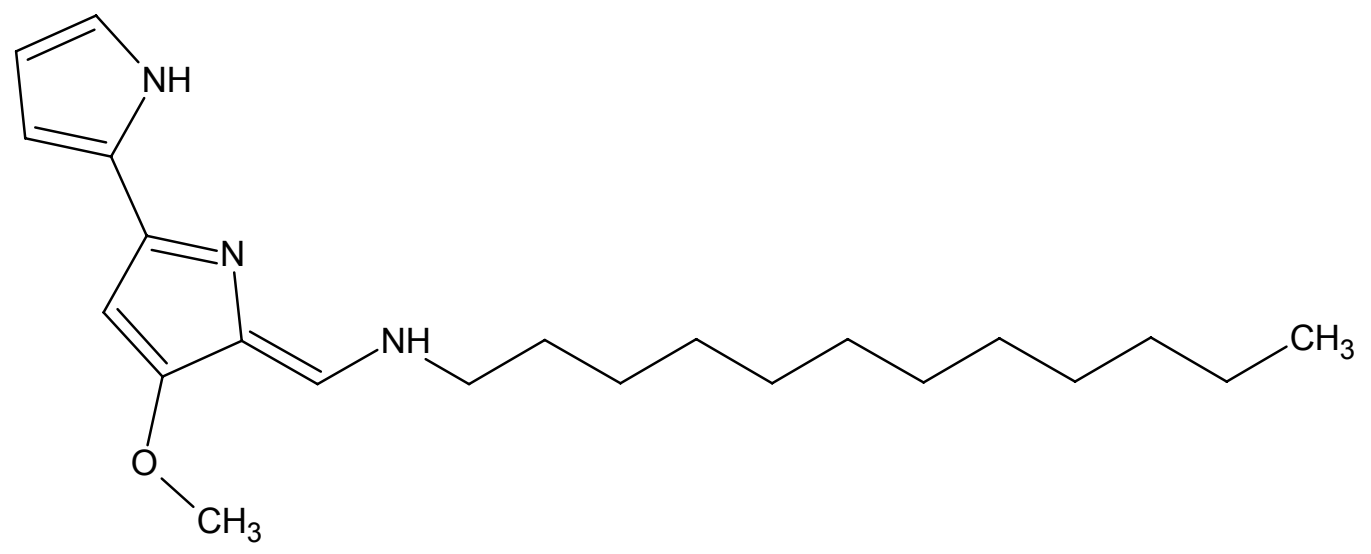




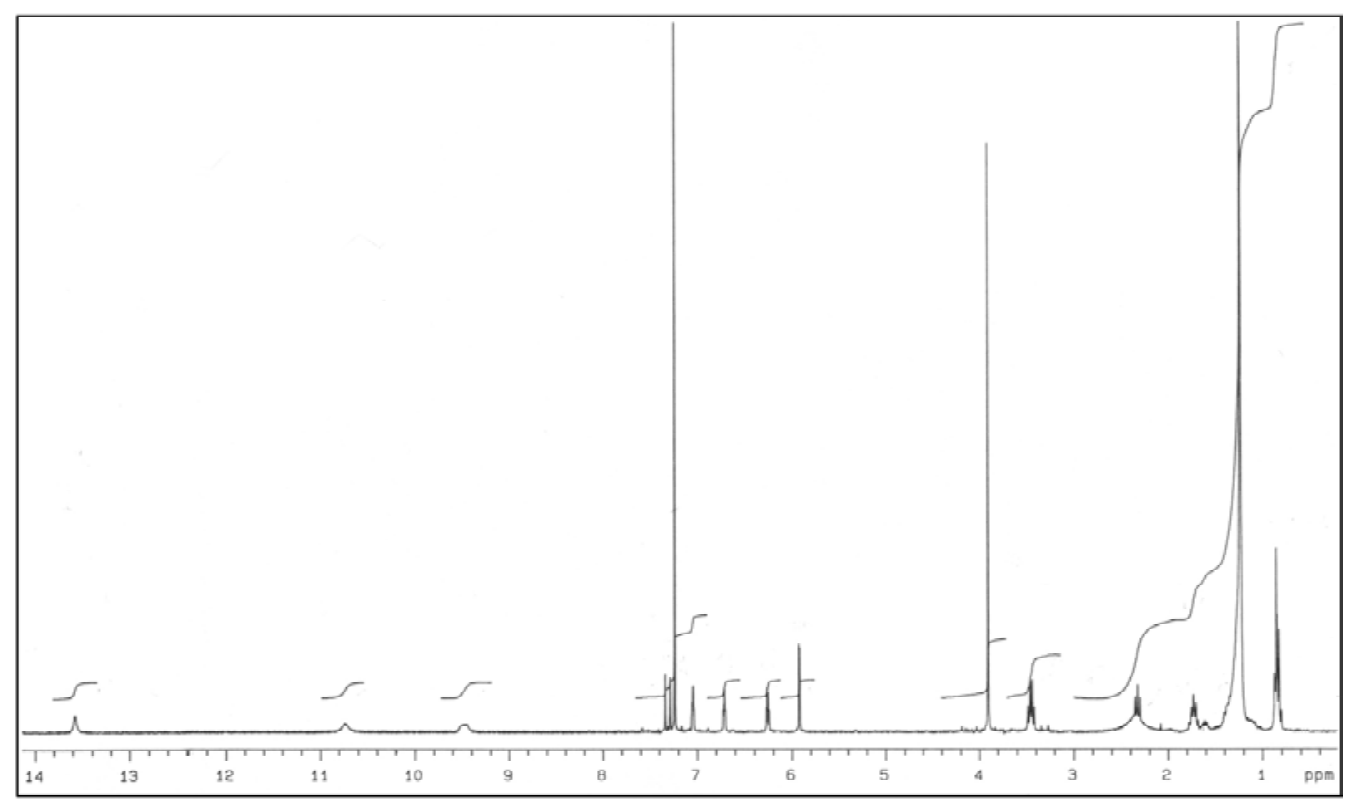

Abbildung 43: ${ }^{1} \mathrm{H}-\mathrm{NMR}-$ Spektrum von 50, aufgenommen in $\mathrm{CDCl}_{3}(300 \mathrm{MHz})$.

\section{(3Z, 6E)-1N-Methylalbonoursin (M145722)}

Die leicht gelbliche, kristalline Substanz aus der Fraktion IIc zeigte im ${ }^{1}$ H-NMRSpektrum ein breites Singulett $(1 \mathrm{H})$ bei $\delta=8.00$, das auf ein austauschbares Proton hindeutete. Ein Multiplett im aromatischen Bereich des Spektrums mit der Intensität fünf bei $\delta=7.40$ war ein Hinweis auf einen monosubstituierten Benzolring. Bei $\delta=$ 7.00 lag ein Singulett $(1 \mathrm{H})$ und bei $\delta=5.50$ ein Dublett $(1 \mathrm{H})$ vor. Ein weiteres Signal eines Protons war bei $\delta=3.85$ zu einem Dublett aufgespalten. Eine an ein Heteroatom gebundene Methylgruppe ergab ein Singulett bei $\delta=3.40$. Da es im ${ }^{13} \mathrm{C}$ NMR-Spektrum keine Signale im Bereich von 40 bis 60 gab, konnte man schließen, dass die Methylgruppe an ein Stickstoffatom gebunden ist.

Im aliphatischen Bereich des Spektrums konnte man bei $\delta=1.10$ ein Dublett der Intensität 6 erkennen, das durch zusätzliche Signale einer Verunreinigung überlagert war. Die Lage des Signals und die charakteristische Aufspaltung deutete auf eine Isopropylgruppe hin, bei der die beiden Methylreste durch die Kopplung mit einem Proton ein Dublett ergeben. Bei dem zugehörigen Proton musste es sich also um das durch einen elektronenziehenden Nachbarn tieffeldverschobene Multiplett bei $\delta=$ 3.85 handeln. Im ${ }^{13} \mathrm{C}-\mathrm{NMR}-$ Spektrum konnte man bei $\delta=157.7$ und 157.4 zwei weitere Signale erkennen, die nur einem Amid oder Ester zugeordnet werden konnten. Im DCI-Spektrum waren die Signale höchster Masse $m / z=271$ und 288 zu sehen, 
die den Clustern $[\mathrm{M}+\mathrm{H}]^{+}$und $\left[\mathrm{M}+\mathrm{NH}_{4}\right]^{+}$entsprachen. Die Suche in AntiBase nach der Verbindung mit der Molmasse von 270 konnte noch zusätzlich durch die Ergebnisse der Spektrenauswertung eingeschränkt werden und ergab nur $(3 \mathrm{Z}, 6 \mathrm{E})-1 \mathrm{~N}-$ Methylalbonoursin (34) als einzige Struktur. Durch den Vergleich der Spektren mit den Literaturwerten konnte das Suchergebnis bestätigt werden.

\section{Phthalsäure-di(2-ethylhexyl ester) (Diisooctyl-phthalat)}

Aus der Fraktion III eluierte man mit Essigester nach der präparativen Dünnschichtchromatographie 16 mg eines weißen, UV-löschenden Öls, das bei der Reaktion mit Anisaldehyd/Schwefelsäure braunviolette Flecken im Dünnschichtchromatogramm ergab.

Im ${ }^{1}$ H-NMR-Spektrum konnte man bei $\delta=7.70$ und 7.50 zwei symmetrische Multipletts erkennen, die nur von einem ortho-disubstituierten Aromaten verursacht werden konnten. Ferner musste es sich um zwei gleiche Substituenten handeln. Ein weiteres Signal bei $\delta=4.32$ war zu einem Dublett vom Dublett aufgespalten. Die restlichen Signale häuften sich alle im aliphatischen Bereich des Spektrums und besaßen eine Intensität von mehreren Protonen. Im DCI-Massenspektrum fand man zwei ausgeprägte Signale der Masse $m / z=279$ und 296, die den Clustern $[\mathrm{M}+\mathrm{H}]^{+}$und $[\mathrm{M}+$ $\left.\mathrm{NH}_{4}\right]^{+}$entsprachen. Daraus resultierte eine Molmasse der Verbindung von 278 Dalton. Die so beschränkte Suche in AntiBase führte zu Phthalsäure-di(2-ethylhexyl ester) (51). Da diese Substanz bei der Kunststoffproduktion als Weichmacher eingesetzt wird, besteht auch hier die Gefahr, ein nicht von den Bakterien produziertes Artefakt isoliert zu haben.<smiles>CCCCC(CC)COC(=O)c1ccccc1C(=O)OCC(CC)CCCC</smiles> 


\section{Stamm B 9020}

Der marine Streptomycet B9020 zeigte im chemischen Screening mehrere UVlöschende Zonen, die sich beim Ansprühen mit Anisaldehyd/Schwefelsäure violett anfärbten. Im biologischen Screening fand man eine mittlere Aktivität gegen Escherichia coli, Bacillus subtilis, Streptomyces viridochromogenes (Tü57), Staphylococcus aureus und Shewanella frigidimarina, sowie gegen zwei von drei der im Test eingesetzten Algen: Chlorella vulgaris und Chlorella sorokiniana. Auf der AgarPlatte wuchs er in weißen, hütchenförmigen Kolonien. Um die Naturstoffproduktion möglichst zu erhöhen, wurde das Medium mit Hilfe genetischer Algorithmen optimiert. Zu diesem Zweck wurden 15 kleine Erlenmeyer-Kolben (je $50 \mathrm{ml}$ ) mit den Bakterien angeimpft. Die Konzentration der Medienbestandteile (Glucose, Maltose, $\mathrm{CaCl}_{2}$ und Hefeextrakt) wurde bei der ersten Generation willkürlich bestimmt. Bei den vier darauf folgender Generation wurden die Konzentrationen aus der Elterngeneration abgeleitet (s. Tabelle 2).

Der Stamm wurde mit dem optimierten Medium im 20 Liter Batch-Reaktor angezogen. Mycel und Überstand wurden getrennt mit Essigester extrahiert. Die vereinigten Extrakte entfettete man mit Cyclohexan und trennte sie durch Chromatographie an Kieselgel in drei Fraktionen vor. Fraktion II enthielt zu wenig Material und wurde verworfen. Aus der Fraktion I wurden durch Chromatographie an Sephadex LH-20 zwei Substanzen gewonnen. Fraktion III wurde durch präparative HPLC an FlashKieselgel gereinigt und lieferte zwei weiße Verbindungen. 


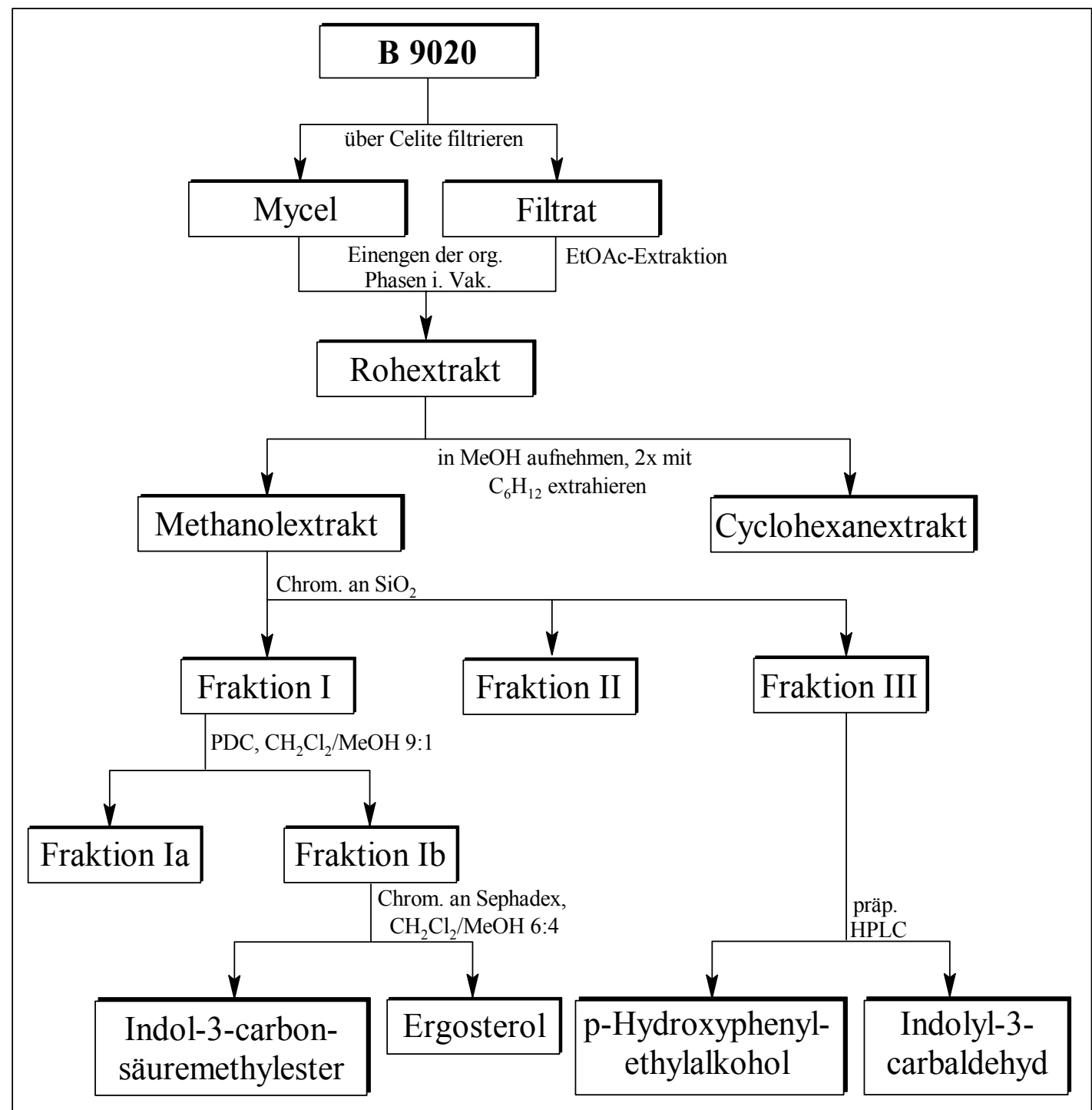

Schema 8: Aufarbeitung des Stammes B 9020

\section{4-(2-Hydroxy-ethyl)-phenol (Tyrosol) (39)}

Das ${ }^{1} \mathrm{H}-\mathrm{NMR}-S p e k t r u m$ war mit dem Spektrum des bereits aus dem marine Streptomyceten B7939 isolierten Tyrosols (39) identisch. Auch die physikochemischen Eigenschaften entsprachen der früher gefundenen Verbindung 39.

\section{Indolyl-3-carbaldehyd}

Bei der weißen Substanz mit dem $R_{\mathrm{f}}$-Wert von $0.3\left(\mathrm{CH}_{2} \mathrm{Cl}_{2} / \mathrm{MeOH}\right.$; 9:1) handelt es sich um eine zweite aus der Fraktion III isolierte Verbindung, die bei $291 \mathrm{~nm}$ ein Absorptionsmaximum zeigte. Das stark verrauschte ${ }^{1} \mathrm{H}-\mathrm{NMR}$-Spektrum zeigte bei $\delta$ $=10(1 \mathrm{H})$ ein scharfes Singulett, bei dem es sich um das Signal einer chelierten 
Hydroxygruppe oder um ein Aldehydproton handeln könnte. Nach dem $\mathrm{D}_{2} \mathrm{O}-$ Austausch verschwand das Signal nicht, was auf einen Aldehyd hindeutete.

Ein weiteres und austauschbares Proton ergab ein Signal bei $\delta=9.00(1 \mathrm{H})$. Weitere zwei Signale fand man bei $\delta=8.46(1 \mathrm{H})$ und $7.82(1 \mathrm{H})$. Das Kopplungsmuster der Signale war aus dem Spektrum nicht abzulesen. Weitere zwei Multipletts waren bei $\delta$ $=7.42(1 \mathrm{H})$ und $7.34(2 \mathrm{H})$ zu sehen. Insgesamt konnte es sich um ein in Position 3substituiertes Indolderivat handeln. Die Molmasse der gesuchten Verbindung von 129 wurde aus zwei Peaks der Masse $m / z=176$ und 193 errechnet, bei denen es sich eindeutig um die Cluster $[\mathrm{M}+\mathrm{H}]^{+}$und $\left[\mathrm{M}+\mathrm{NH}_{4}\right]^{+}$handelte. Die mit diesen Angaben in der Naturstoffdatenbank (AntiBase) durchgeführte Suche lieferte die Struktur von Indolyl-3-carbaldehyd (52). Ein bestätigender Spektrenvergleich der aufgenommenen Spektren und aus der internen Spektrensammlung fiel positiv aus.

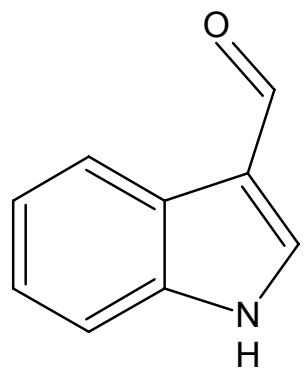

52

\section{Ergosterol (Provitamin $\mathbf{D}_{2}$ )}

Aus der Fraktion Ib wurde eine farblose Substanz isoliert, die den $R_{\mathrm{f}^{-}}$Wert von 0.8 $\left(\mathrm{CH}_{2} \mathrm{Cl}_{2} / \mathrm{MeOH}\right.$, 95:5) und UV-löschende Eigenschaften besaß. Das ${ }^{1} \mathrm{H}-\mathrm{NMR}-$ Spektrum zeigte allerdings im aromatischen Bereich keine Signale. Bei $\delta=5.58$ und 5.38 fand man zwei Multipletts (je $1 \mathrm{H}$ ) sowie bei $\delta=5.20$ ein Triplett $(2 \mathrm{H})$, die Protonen von mindestens zwei Doppelbindung zugeordnet werden konnten. Diese Vermutung schien das ${ }^{13} \mathrm{C}$-Spektrum zu unterstützen. Man fand Kohlenstoffsignale bei $\delta=141,139,135,131,119$ und 116. Ein weiteres Multiplett der Intensität 1 fand man im ${ }^{1} \mathrm{H}-\mathrm{NMR}-$ Spektrum bei $\delta=3.60$, das einem Proton in der Nachbarschaft eines Heteroatoms zugehörte. Die restlichen Signale fand man von $\delta=2.50$ bis 0.60 über den ganzen aliphatischen Bereich verteilt. Da sich bei diesen Signalen um mehrere überlagerte Multipletts handelte, lag die Schlussfolgerung nahe, dass die gesuchte Verbindung mehrere aliphatische Ringe enthielt. Lediglich bei $\delta=1.0$ konnte man 
ein Dublett der Intensität drei erkennen, das einem Methylrest an einer $\mathrm{CH}-$ Gruppe zugeordnet wurde. Ein weiteres Singulett bei 0.60 wurde einer quartären Methylgruppe zugeordnet. Die Molmasse der gesuchten Verbindung von 396 ergab sich aus den Peaks im DCI-Massenspektrum bei $m / z=397$ und 414, die den Clustern [M + $\mathrm{H}]^{+}$und $\left[\mathrm{M}+\mathrm{NH}_{4}\right]^{+}$entsprachen. Die Suche in AntiBase lieferte 28 mögliche Steroide. Den endgültigen Beweis der Struktur brachte ein Vergleich mit Spektren aus der internen Spektrensammlung. Bei der gesuchten Struktur handelt es sich um Ergosterol (53), das ein typischer Bestandteil von Pilzen und Hefen. Nur sehr selten wurde Ergosterol aus Bakterien isoliert ${ }^{[91]}$.

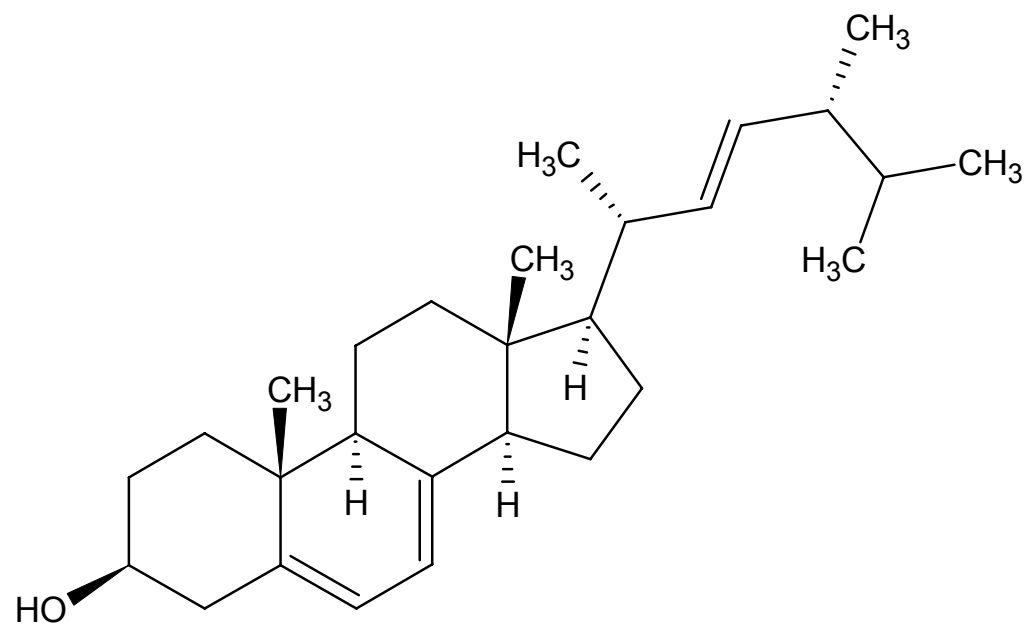

53

\section{3-Indolyl-carbonsäuremethylester}

Das ${ }^{1}$ H-NMR-Spektrum der aus Fraktion Ib isolierten Substanz zeigte im aromatischen Bereich ein Multiplett bei $\delta=8.16$ der Intensität 1 , bei $\delta=7.86$ ein Dublett ( 1 H) und zwei Multipletts bei $\delta=7.40(1 \mathrm{H})$ und $7.20(2 \mathrm{H})$. Zusätzlich fand man noch ein breites Signal $(1 \mathrm{H})$ bei $\delta=8.60$. Nach dem Vergleich mit den Spektren der mehrmals in der vorliegenden Arbeit isolierten Indolderivate kam man zu der Überzeugung, dass es sich bei der gesuchten Verbindung ebenfalls um ein in Position 3 substituiertes Indolderivat handeln musste. Im aliphatischen Bereich befand sich nur ein Singulett bei $\delta=3.82(3 \mathrm{H})$. Die Anwesenheit eines Esterrestes konnte man anhand des ${ }^{13} \mathrm{C}$-Spektrums annehmen. Bei $\delta=46$ fand man das Kohlenstoffsignal des Methylrestes. Die aus den DCI-Spektrum entnommene Molmasse betrug 175. Die auf diesen Informationen basierende Datenbanksuche in AntiBase führte $\mathrm{zu}$ 3Indolyl-carbonsäuremethylester (54). 


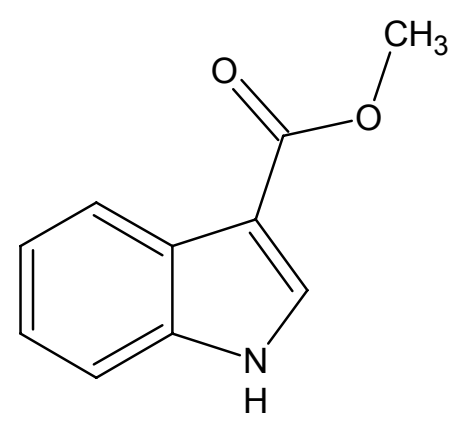

54

\section{Stamm B 8027}

Der marine Streptomycet B8027 fiel im biologischen Screening des Schüttelkulturextraktes durch wachstumshemmende Wirkung gegen Streptomyces viridochromogenes (Tü57), Staphylococcus aureus, Mucor miehei, Candida albicans sowie gegen die Algen Chlorella vulgaris, Chlorella sorokiniana und Scenedesmus subspicatus auf. Auf der Agar-Platte wuchs der Stamm in weißen, unregelmäßigen Kolonien. Die Medienzusammensetzung wurde mit Hilfe genetischer Algorithmen optimiert und der Fermenter wie beim Stamm B9020 vorbereitet.

Die kontinuierliche Fermentation dauerte sieben Tage. Nach Ablauf der Fermentation wurde das verbrauchte Nährmedium mit entsprechenden Mengen Essigester extrahiert. Nach dem Einengen wurde die Essigesterphase mehrmals mit Cyclohexan entfettet. Aus der Cyclohexanphase wurden mittels dünnschichtchromatographischer Trennung an Kieselgel (Cyclohexan) zwei Komponenten mit dem $R_{\mathrm{f}}$-Werten von 0.8 und eine UV-löschende mit $R_{\mathrm{f}}=0.8$ isoliert. Beide Zonen verfärbten sich bei der Reaktion mit Anisaldehyd/Schwefelsäure violett.

Die Vortrennung des entfetteten Rohextraktes erfolgte an einer Kieselgelsäule und lieferte mehrere Neben- und zwei Hauptfraktionen. Da die Nebenfraktionen nur geringe Substanzmengen enthielten, wurden sie nicht weiter bearbeitet. Die Fraktion I wurde im nächsten Schritt der Reinigung dünnschichtchromatographisch in weitere drei Zonen aufgeteilt (Ia bis Ic), die mit Essigester eluiert wurden. Fraktion Ic wurde durch Endreinigung an einer präparativen RP18-Säule chromatographisch mittels HPLC gereinigt und ergab drei ungesättigte (UV-löschende) Verbindungen. 


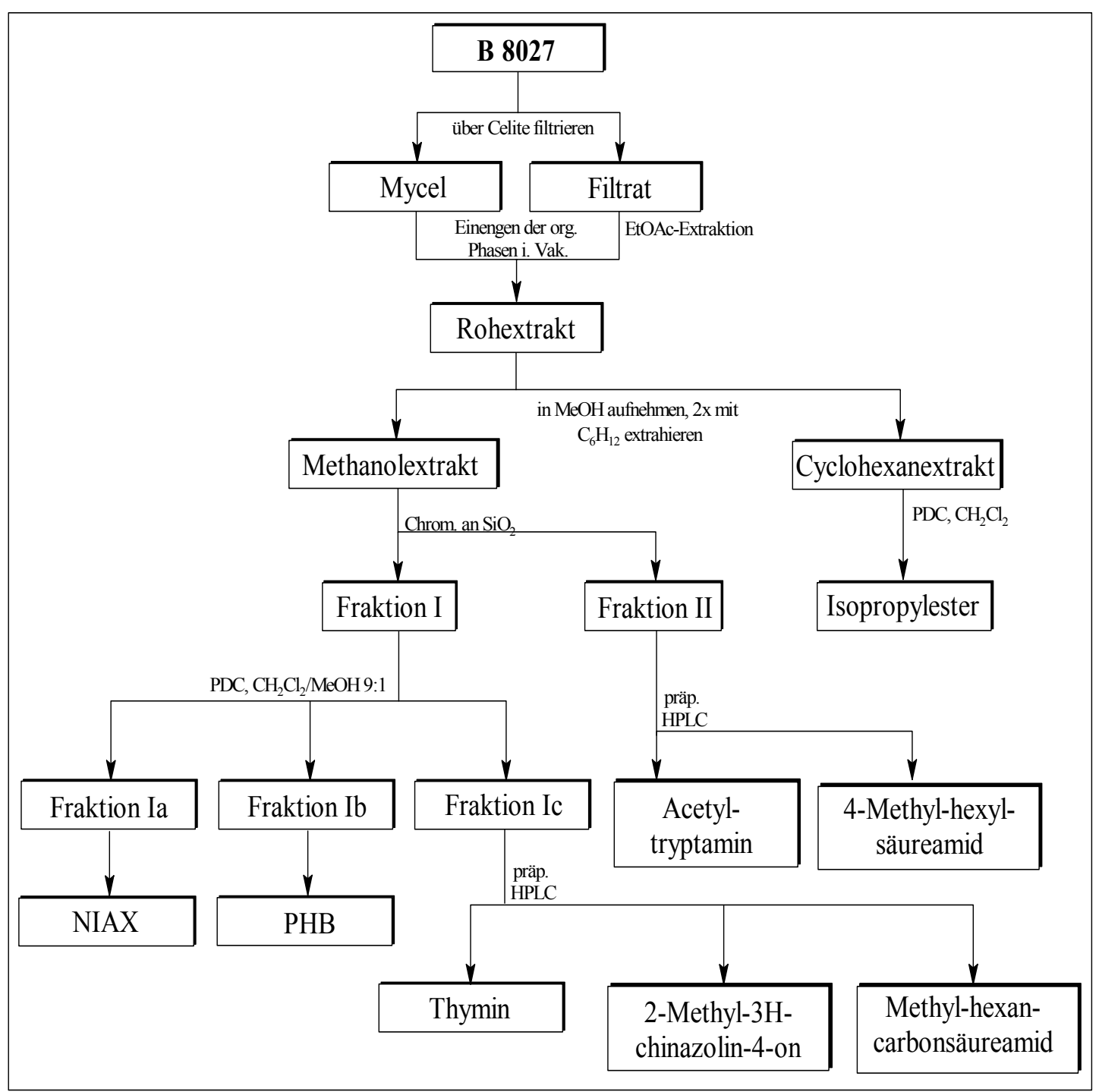

Schema 9: Aufarbeitung des Stammes B 8027.

\section{NIAX und Polyhydroxy-buttersäure}

Bei den aus der Fraktion I isolierten Verbindungen Ia und Ib handelt es sich um NIAX und möglicherweise die bereits bei dem marine Streptomyceten B 7939 diskutierten Dimeren oder Polymeren der Hydroxy-buttersäure (45) mit der scheinbaren Molmasse von 172 Dalton (CI-MS). NIAX ist eine oberflächenaktive Substanz, die als Antischaummittel bei der Fermentation eingesetzt wird. Da der marine Streptomycet B8027 bei der Fermentation stark schäumte, wurden mehrere Milliliter NIAX zufügt. Aus der Cyclohexanphase wurde ebenfalls der bei dem terrestrischen Streptomyceten GW 20/1811 besprochene Phthalsäure-di(2-ethylhexyl ester) (51) gewonnen. 


\section{N-Acetyltryptamin und 3-Methyl-hexyl-carbonsäureamid}

Aus der Fraktion II wurde eine UV-löschende Zone mit dem $R_{\mathrm{f}}$-Wert von 0.4 $\left(\mathrm{CH}_{2} \mathrm{Cl}_{2} / \mathrm{MeOH}\right.$; 95:5) mit Essigester eluiert. Das ${ }^{1} \mathrm{H}-\mathrm{NMR}-$ Spektrum zeigte jedoch, dass es sich um eine Mischung zweier oder mehrerer Substanzen handelt. Da sich die Signale nicht überlagerten, konnten die Peaks einer Komponente anhand des H,HCOSY-Korrelationsspektrums eindeutig zugewiesen werden. Die Signale im aromatischen Bereich bei $\delta=8.22$ bis 7.00 sowie bei $\delta=5.5,3.60,2.98$ und 1.90 konnten dem bereits aus dem marine Streptomyceten B7939 und dem terrestrischen Streptomyceten GW 19/2497 isolierten N-Acetyltryptamin (36) zugeordnet werden. Die übrig bleibenden Signale befanden sich ausschließlich im aliphatischen Bereich des Spektrums. Bei $\delta=2.20$ fand man ein Multiplett, das mit den Multipletts bei $\delta=1.60$ und 1.40 koppelt. Das Multiplett bei $\delta=1.20$ koppelt wiederum mit der endständigen Methylgruppe bei $\delta=0.80$, deren verzerrtes Signal auf ein Gemisch hindeutete. Im APT-Spektrum findet man außer drei Methylengruppen bei $\delta=34,32$, und 28, noch drei Signale von $\mathrm{CH}_{3^{-}}$oder $\mathrm{CH}-G r u p p e n$. Die Intensität der Signale im ${ }^{1} \mathrm{H}-\mathrm{NMR}-$ Spektrum deutet auf zwei Methylgruppen hin. Ein weiteres Signal im ${ }^{13} \mathrm{C}-\mathrm{NMR}$ Spektrum bei $\delta=170$ ließ vermuten, dass ein Carboxyl- oder Säureamid-Kohlenstoff vorhanden war. Die Molmasse der gesuchten Verbindung von 129 wurde anhand von zwei im DCI-Spektrum sichtbaren Signalen bei $m / z=130$ und 147 bestimmt, die den Clustern $[\mathrm{M}+\mathrm{H}]^{+}$und $\left[\mathrm{M}+\mathrm{NH}_{4}\right]^{+}$entsprachen. $\mathrm{Ob}$ es sich bei der gesuchten Verbindung um 5-Methyl-hexan-carbonsäureamid (55a) oder 4-Methyl-hexancarbonsäureamid (55b) handelt, kann aus den vorliegenden spektroskopischen Befunde nicht eindeutig entschieden werden. Möglicherweise liegt eine äquimolare Mischung beider Konstitutionsisomeren vor.<smiles>CC(C)CCCC(N)=O</smiles>

$55 \mathbf{a}$<smiles>CCC(C)CCC(N)=O</smiles>

$55 \mathbf{b}$ 


\section{2-Methyl-3H-chinazolin-4-on}

Aus der Fraktion Ic konnte durch präparative HPLC-Trennung eine farblose UVlöschende Substanz mit dem $R_{\mathrm{f}}$-Wert von $0.7\left(\mathrm{CH}_{2} \mathrm{Cl}_{2} / \mathrm{MeOH}\right.$; 9:1) isoliert werden. Mit Anisaldehyd/Schwefelsäure verfärbte sich die Zone braun. Nach einigen Minuten verschwand die braune Verfärbung und hinterlies einen dauerhaft gelben Fleck. Ein Verdacht, dass es sich bei der vorliegenden Verbindung um Anthranilsäure $\left(R_{\mathrm{f}}=\right.$ 0.5) handelt, die ebenfalls eine Gelbfärbung mit Anisaldehyd/Schwefelsäure ergibt, konnte anhand der unterschiedlichen Retentionszeiten nicht bestätigt werden. Die ${ }^{1} \mathrm{H}-\mathrm{NMR}-$ Spektren zeigten ein breites Singulett $(1 \mathrm{H})$ bei $\delta=12.28$, das nur von einem austauschbaren Protonen herrühren konnte. Im aromatischen Bereich bei $\delta=$ 8.06 und 7.76 konnte man zwei Multipletts $($ je $1 \mathrm{H}$ ) finden. Weiterhin befand sich bei $\delta=7.56(1 \mathrm{H})$ ein zu einem Dublett aufgespaltenes Signal mit einer Kopplungskonstanten von $J=8 \mathrm{~Hz}$. Das letzte aromatische Signal lag bei $\delta=7.42(1 \mathrm{H})$ und wurde durch die Kopplungspartner zu einem Dublett vom Triplett aufgespalten. Aus dem aliphatischen Singulett bei $\delta=2.34(3 \mathrm{H})$ konnte man auf einen Methylrest schließen. Die Tieffeldverschiebung war auf die Anwesenheit eines elektronenziehenden Partners zurückzuführen. Die Molmasse von 160 wurde anhand der zwei im DCISpektrum sichtbaren Signale bei $m / z=161$ und 178, die den Clustern $[\mathrm{M}+\mathrm{H}]^{+}$und $\left[\mathrm{M}+\mathrm{NH}_{4}\right]^{+}$entsprachen, gesichert. Die Suche in AntiBase lieferte nur eine Verbindung, das 2-Methyl-3H-chinazolin-4-on (56) als Möglichkeit, was durch den Vergleich mit Referenzspektren (SDBS-Spektrendatenbank) bewiesen werden konnte.

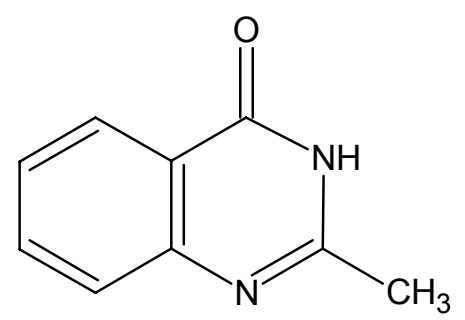

56

\section{2-Phenyl-acetamid}

Die aus der Fraktion II isolierte farblose Verbindung lief im Dünnschichtchromatogramm mit dem $R_{\mathrm{f}}$-Wert von $0.5\left(\mathrm{CH}_{2} \mathrm{Cl}_{2} / \mathrm{MeOH} ; 9: 1\right)$. Im ${ }^{1} \mathrm{H}-\mathrm{NMR}-\mathrm{Spektrum}$ konnte man in aromatischen Bereich bei $\delta=7.34$ mehrere überlagerte Protonensignale sehen. Leider konnte man die Intensität nicht eindeutig bestimmen, weil sie durch das Signal des Lösungsmittels verfälscht wurde. Da alle Signale fast bei der gleichen 
Verschiebung auftraten, konnte man daraus schließen, dass es sich um ein monosubstituierten aromatischen Ring handelt. Ein weiteres, sehr breites Signal trat bei $\delta$ $=5.37$ auf. Die gesuchte Verbindung musste also austauschbare Protonen besitzen. Im Aliphaten-Bereich fand man nur ein Singulett bei 3.6. Im DCI-Spektrum fand man Quasi-Molekülionen bei $m / z=136\left([\mathrm{M}+\mathrm{H}]^{+}\right)$und $153\left(\left[\mathrm{M}+\mathrm{NH}_{4}\right]^{+}\right)$. Daraus wurde die Molmasse von 135 abgeleitet. Die Datenbanksuche in AntiBase lieferte mit diesen Angaben 2-Phenyl-acetamid (57), was durch den Vergleich mit Referenzspektren bestätigt wurde. Das Amid 57 wurde in der Arbeitsgruppe bereits häufiger in Streptomyceten gefunden und kommt auch in dem Cyanobakterium Aphanizomenon flos-aquae vor $^{[93]}$.

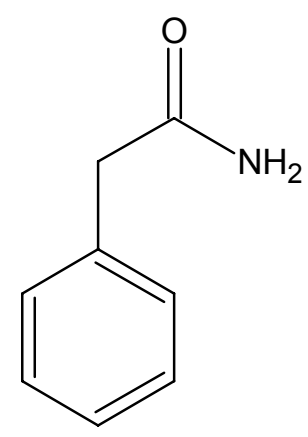

57

\section{Thymin}

Aus der Fraktion Ic erhielt man durch präparative HPLC Trennung an RP18 eine farblose Verbindung mit dem $R_{\mathrm{f}}$-Wert von 0.5. Im UV-Licht (366 nm) fluoreszierte sie stark und bei $254 \mathrm{~nm}$ wies sie eine löschende Zone auf. Da es sich hierbei um das bereits aus dem terrestrischen Streptomyceten GW 7/2495 isoliertes Thymin (33) handelt, wird an dieser Stelle auf weitere Diskussionen verzichtet.

\section{Mehrere homologe iso-Fettsäure-methylester}

Aus der Cyclohexanphase wurde durch präparative Dünnschichtchromatographie eine ölige Substanz isoliert, die den $R_{\mathrm{f}}$-Wert von $0.95\left(\mathrm{CH}_{2} \mathrm{Cl}_{2}\right)$ besaß und nur schwach UV-löschend war. Bei der Betrachtung des ${ }^{1}$ H-NMR-Spektrum fiel auf, dass keine Signale oberhalb von $\delta=4$ zu finden waren. Man konnte dadurch alle 
aromatischen Verbindungen ausschließen. Bei $\delta=3.60$ befand sich im Spektrum ein Singulett der Intensität 3 und bei $\delta=2.24$ ein Triplett der Intensität 2 . Im ${ }^{13} \mathrm{C}$ Spektrum fand man bei $\delta=174$ ein Signal, das als Carbonyl-Kohlenstoff eines Esters interpretiert wurde. Die starke Tieffeldverschiebung des Singuletts bei $\delta=3.60$ konnte nur durch eine elektronenziehende Gruppe verursacht werden. Demnach handelte es sich bei diesem Signal um ein Methylestersignal. Gleichzeitig konnte man mit der Anwesenheit der Carboxylgruppe das Signal bei $\delta=2.24$ plausibel erklären. Es musste der carbonylbenachbarten Methylengruppe zugehören.

Ein weiteres Multiplett fand man bei $\delta=1.60$. Ein ausgeprägtes Signal bei $\delta=1.20$ entsprach der Intensität mehrerer Protonen und war für lange aliphatische Ketten charakteristisch. Bei $\delta=0.80$ fand man das $6 \mathrm{H}$-Dublett eines Isopropylrestes. Bei der vorliegenden Substanz handelte es sich demnach um einen iso-Fettsäuremethylester (58). Da im ESI-Massenspektrum mehrere Peaks im Abstand von $\Delta m / z$ 14 zu sehen waren, lag offenbar ein Gemisch aus $C_{1}-C_{11}$-Säuren vor.

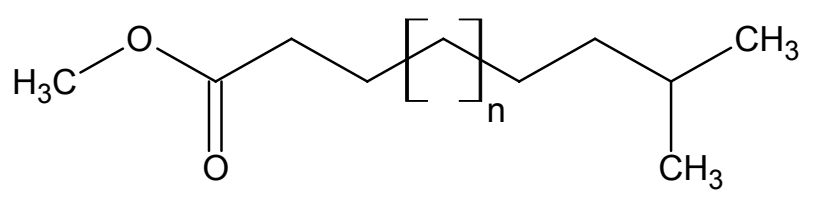

$58 n=1-11$

\section{Stamm GW 19/2497}

Der terrestrische Streptomycet GW19/2497 wuchs auf Agar in weißen Kolonien mit einem grauen Luftmycel. Im HPLC-Vorscreening zeigte der Extrakt mehrere Peaks von Substanzen, die nach den UV-Spektren farblos sein sollten. Die beträchtliche biologische Aktivität gegen Escherichia coli, Bacillus subtilis und Staphylococcus aureus sowie gegen Chlorella vulgaris war entscheidend bei der Auswahl dieses Stammes für die weitere Optimierung und Bearbeitung. Die Agar-Platte für die Vorkultur sowie auch die darauf folgenden fünf Schüttelkolben wurden mit $\mathrm{M}_{2}$-Medium vorbereitet. Die Zusammensetzung der jeweiligen Medien für die Optimierung wurde mit GALOP über fünf nächste Generationen optimiert. Das beste Medium für die kontinuierliche Kultur bestand aus Glucose, Maltose, Hefeextrakt und $\mathrm{CaCl}_{2}$ (s. 
Tabelle 2). Die kontinuierliche Fermentation dauerte sieben Tage. Die Extraktion der 101 Kulturbrühe erfolgte durch mehrmaliges Ausschütteln mit Essigester und anschließendes Eindampfen. Der Extrakt wurde wie üblich mit Cyclohexan entfettet und durch Chromatographie an Kieselgel, an Sephadex LH-20 und durch präparative HPLC getrennt.

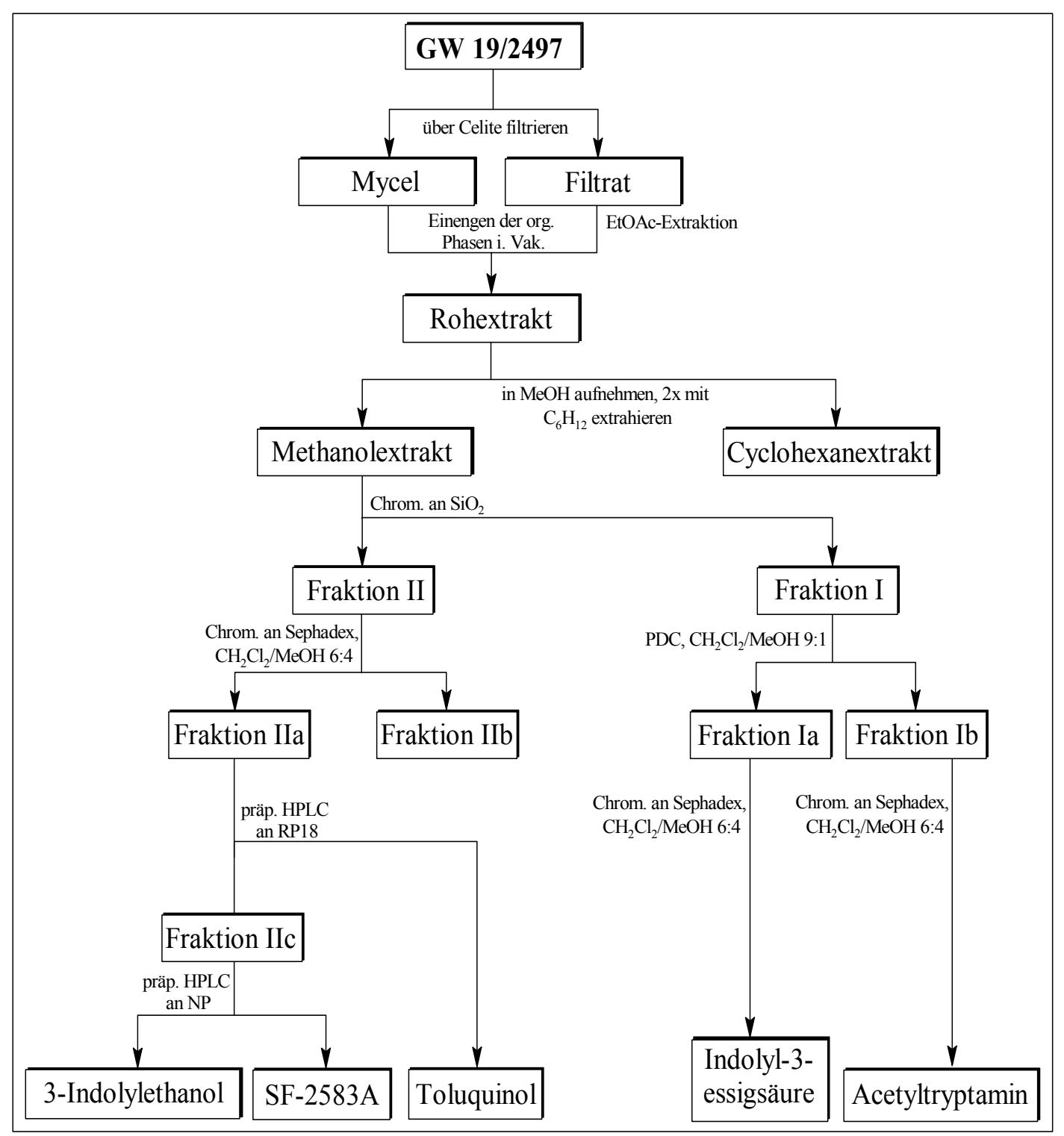

Schema 10: Aufarbeitung des Stammes GW 19/2497

\section{Indolyl-3-essigsäure}

Aus der Fraktion Ia (s. Schema 10) erhielt man eine weiße Verbindung, die im Dünnschichtchromatogramm den $R_{\mathrm{f}}$-Wert von $0.15\left(\mathrm{CH}_{2} \mathrm{Cl}_{2} / \mathrm{MeOH}\right.$ 95:5) besaß und sich mit Anisaldehyd/Schwefelsäure zuerst rosa, dann violett anfärben ließ. Das ${ }^{1} \mathrm{H}-$ NMR-Spektrums ließ das Kopplungsmuster eines in Position 3 substituierten Indols 
erkennen. Das Singulett der Intensität 2 bei $\delta=3.6$ wurde durch das APT-Spektrum als Methylengruppe erkannt. Außerdem sah man noch im ${ }^{13} \mathrm{C}-\mathrm{NMR}$-Spektrum das schwache Signal einer Carbonylgruppe bei $\delta=173$. Die Molmasse der gesuchten Verbindung lag nicht vor. Die Datenbanksuche führte zu Indolyl-3-essigsäure (59), was durch Vergleichsspektren bestätigt wurde. Das Indolderivat 59 ist sehr oft aus Bakterien und Pilzen isoliert worden ${ }^{[94]}$. Bei Pflanzen ist es als Wachstumshormon von Bedeutung.

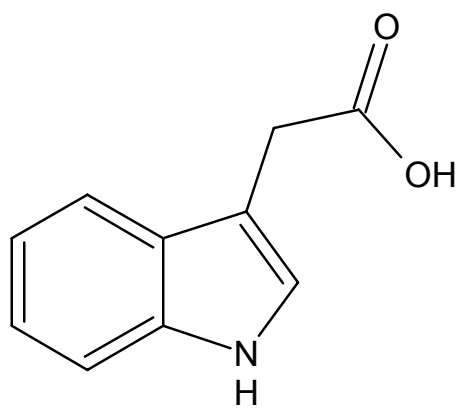

59

\section{4-Chlor-5-(3'-indolyl)oxazol (SF-2583A)}

Die mit Ehrlichs Reagenz besprühten Dünnschichtchromatogramme des GW19/2497-Extraktes zeigten bei mehreren weiteren Zonen die für Indole charakteristische violette Verfärbung. Das ${ }^{1} \mathrm{H}-\mathrm{NMR}-$ Spektrun einer dieser Verbindungen mit $R_{\mathrm{f}}=0.45\left(\mathrm{CH}_{2} \mathrm{Cl}_{2} / \mathrm{MeOH}\right.$ 9:1) bestätigte das Vorhandensein eines Indolderivates und zeigte die bereits bei $\mathbf{5 9}$ diskutierte Aufspaltung der Aromatsignale. Integration der Signale ergab außer dem NH-Signal bei $\delta=8.50$ allerdings sechs Aromat-Protonen; weitere Signale waren nicht sichtbar. Dass es sich um Indol selbst handelte, konnte ausgeschlossen werden. Zusätzlich fand man im APT-Spektrum statt der zwei für Indol erwarteten quartären Kohlenstoffatome im aromatischen Bereich fünf quartäre Kohlenstoffatome mit Signalen bei $\delta=135,124,123,121$ und 103 .

Im DCI-Spektrum waren zwei Signale bei $m / z=219$ und 236 zu sehen, die den Clustern $[\mathrm{M}+\mathrm{H}]^{+}$und $\left[\mathrm{M}+\mathrm{NH}_{4}\right]^{+}$entsprachen. Diese Signale wurden jeweils von M+2-Satelitensignalen mit etwa 1/3 der Intensität der Hauptsignale begleitet, was auf die Anwesenheit von Chlor hindeutete. Chlor tritt als Gemisch der Isotope ${ }^{35} \mathrm{Cl}$ (75.53\%) und ${ }^{37} \mathrm{Cl}(24.47 \%)$ auf. Die Suche in AntiBase mit diesen Strukturinfor- 
mationen führte zu einer einzigen Antwort, 4-Chlor-5-(3'-indolyl)oxazol (60). Die Struktur wurde durch den Vergleich mit Referenzspektren und Literaturdaten bestätigt ${ }^{[95]}$. Das Indol 60 wurde 1988 aus Streptomyces sp. SF2583 isoliert und ist aktiv gegen Nematoden, Staphylococcus aureus, Bacillus subtilis, Escherichia coli und Candida albicans.

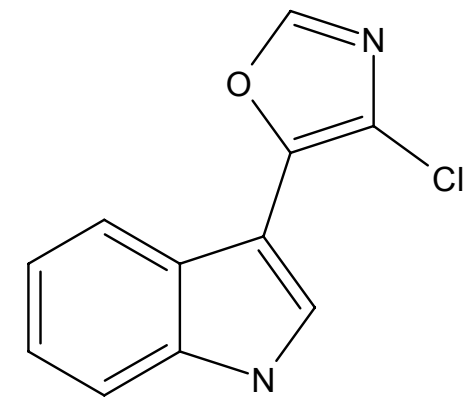

60

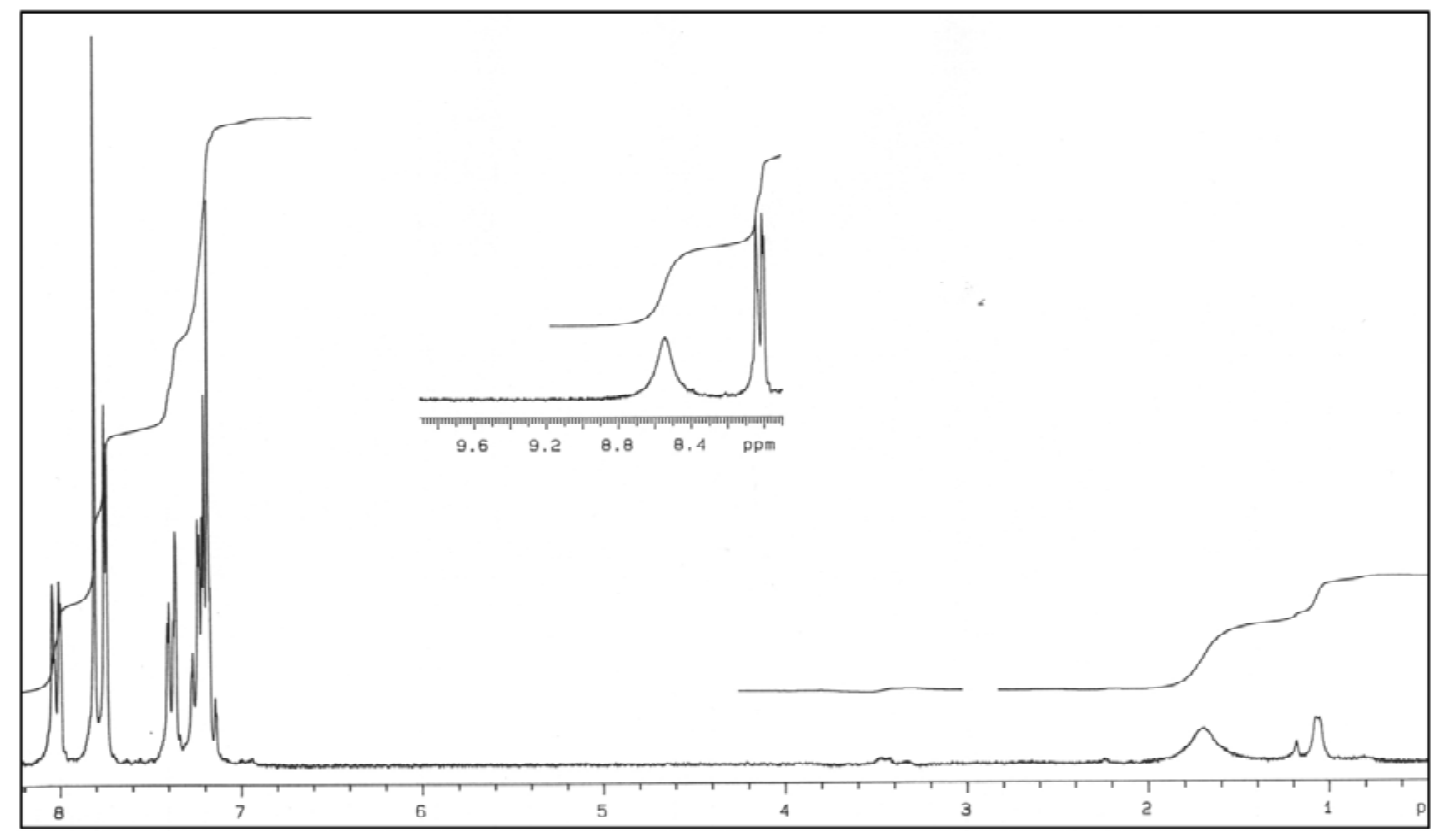

Abbildung 44: ${ }^{1} \mathrm{H}-\mathrm{NMR}-$ Spektrum von $\mathbf{6 0}$, aufgenommen in $\mathrm{CDCl}_{3}(300 \mathrm{MHz})$.

\section{1,4-Dihydroxy-2-methyl-benzol (Toluhydrochinon)}

Aus der Fraktion IIa gewann man $8 \mathrm{mg}$ einer gelblichen Substanz mit dem $R_{\mathrm{f}}$-Wert von $0.3\left(\mathrm{CH}_{2} \mathrm{Cl}_{2} / \mathrm{MeOH} ; 9: 1\right)$, die im Dünnschichtchromatogramm als UVlöschende, mit Anisaldehyd/Schwefelsäure braune Zone erkennbar war. Wegen der 
schlechten Löslichkeit im Chloroform wurden die ${ }^{1} \mathrm{H}-\mathrm{NMR}-\mathrm{Spektren}$ in [D $]$-DMSO aufgenommen. Die Substanz zeigte im ${ }^{1}$ H-NMR-Spektrum Signale vor allem im aromatischen Bereich. Bei $\delta=8.46$ und 8.40 lagen zwei Singuletts (je $1 \mathrm{H}$ ). Weitere aromatische Signale fand man hochfeldverschoben bei $\delta=6.58$ (Dublett, $1 \mathrm{H}$ ) mit einer großen Kopplungskonstante $(J=10 \mathrm{~Hz})$, die auf eine ortho-Kopplung hindeutete. Der zugehörige Kopplungspartner zeigte ein Dublett vom Dublett bei $\delta=6.38$ $(1 \mathrm{H})$, wobei es sich bei der zweiten Aufspaltung um eine kleinere Kopplungskonstante $(J=3 \mathrm{~Hz}$ ) handelte (meta-Kopplung). Bei $\delta=6.46$ fand man bei einem Dublett der Intensität 1 den gleichen Wert für $J$ (ortho- und meta-Kopplung). Ein weiteres Singulett der Intensität 3 lag bei $\delta=2.00$, was auf eine Methylgruppe hindeutete, die höchstwahrscheinlich an einen aromatischen Ring gebunden war. Im ${ }^{13} \mathrm{C}$-NMR-Spektrum fand man im aromatischen Beriech sechs Kohlenstoffatome. Das APT-Spektrum bestätigte, dass bei der gesuchten Verbindung ein trisubstituierter Benzolring vorlag. Außerdem fand man nur ein einziges Signal im Aliphatenbereich des ${ }^{13} \mathrm{C}$-NMR-Spektrums $\left(\mathrm{CH}_{3}\right.$-Gruppe) bei $\delta=16$. Die bei EI-MS gefundene Masse betrug 124. Die anhand der entstandenen Fragmentierungsmuster durchgeführte Datenbanksuche der EI-Signale ergab drei mögliche Strukturen 61a, 61b und 61c. Von den drei Molekülen erfüllte nur Toluhydrochinon (61a) die beobachteten Eigenschaften.<smiles>Cc1cc(O)ccc1O</smiles>

61a<smiles>Cc1c(O)cccc1O</smiles>

61b<smiles>Cc1ccc(O)cc1O</smiles>

61c

Aus der Fraktion Ib erhielt man 12 mg einer weißen Substanz, die mit EhrlichsReagenz eine Violettfärbung ergab. Die ${ }^{1} \mathrm{H}-\mathrm{NMR}-$ Spektren entsprachen den Spektren des aus dem marinen Stamm B 7939 isolierten N-Acetyltryptamins (36). 
Aus der Fraktion IIc wurde $6.3 \mathrm{mg}$ einer weißen Substanz isoliert, die ebenfalls eine violette Verfärbung mit Ehrlichs-Reagenz ergab. Es handelte sich um ein weiteres Indolderivat, 3-Indolylethanol (37).

\section{Stamm GW 71/2497}

Die aus der Sammlung des Instituts für Bodenkunde in Lora-Kirchvers stammende Bakterienkultur GW 71/2497 ist als Chartreusinproduzent bekannt und wurde in unserer Arbeitsgruppe bereits mehrmals untersucht.

Als A. Pusecker neben Chartreusin aus dem marinen Streptomyceten B5552 noch zwei neue Monoacetate von Chartreusin isolierte, fiel bei der Auswertung der spektroskopischen Daten eine ungewöhnliche Hochfeldverschiebung des Acetatsignals auf $^{[96]}$. Eine Nachuntersuchung von M. Speitling bestätigte die Befunde ${ }^{[97]}$. Es gelang jedoch nie wieder, diese Monoacetate in reiner Form aus dem Rohextrakt dieses Stammes zu bekommen, da sich der Stamm offensichtlich verändert hatte. Der terrestrische Streptomycet GW 71/2497 bildete jedoch außer Chartreusin (62) und (vielleicht) seinen Monoacetaten unter anderen Fermentationsbedingungen eine Reihe von Anthracyclinen und Angucyclinen ${ }^{[98]}$. Dieser Stamm war daher ein geeigneter Kandidat für eine Fermentationsoptimierung. Es stellte sich die Frage, ob man durch in der Arbeit verwendeten genetischen Optimierungsmethoden und kontinuierliche Anzucht, die Produktion dieser Verbindungen erzwingen kann.

Für die Vorkultur wurden mehrere Schüttelkolben mit $\mathrm{M}_{2}$-Medium vorbereitet. Da es aus Zeitgründen nicht möglich war, eine Optimierung mit GALOP über fünf Generationen durchzuführen, wurde das beste Medium bereits aus der dritten Generation ausgewählt (s. Tabelle 2). Die kontinuierliche Fermentation dauerte sieben Tage. Nach der Essigester-Extraktion der 101 Kulturbrühe erhielt man 2.1 g einer braungelben, öligen Flüssigkeit, die stark blaugrün fluoreszierte. Durch mehrere Trennschritte wurden aus dem Gemisch sechs Komponenten isoliert (s. Schema 11). 


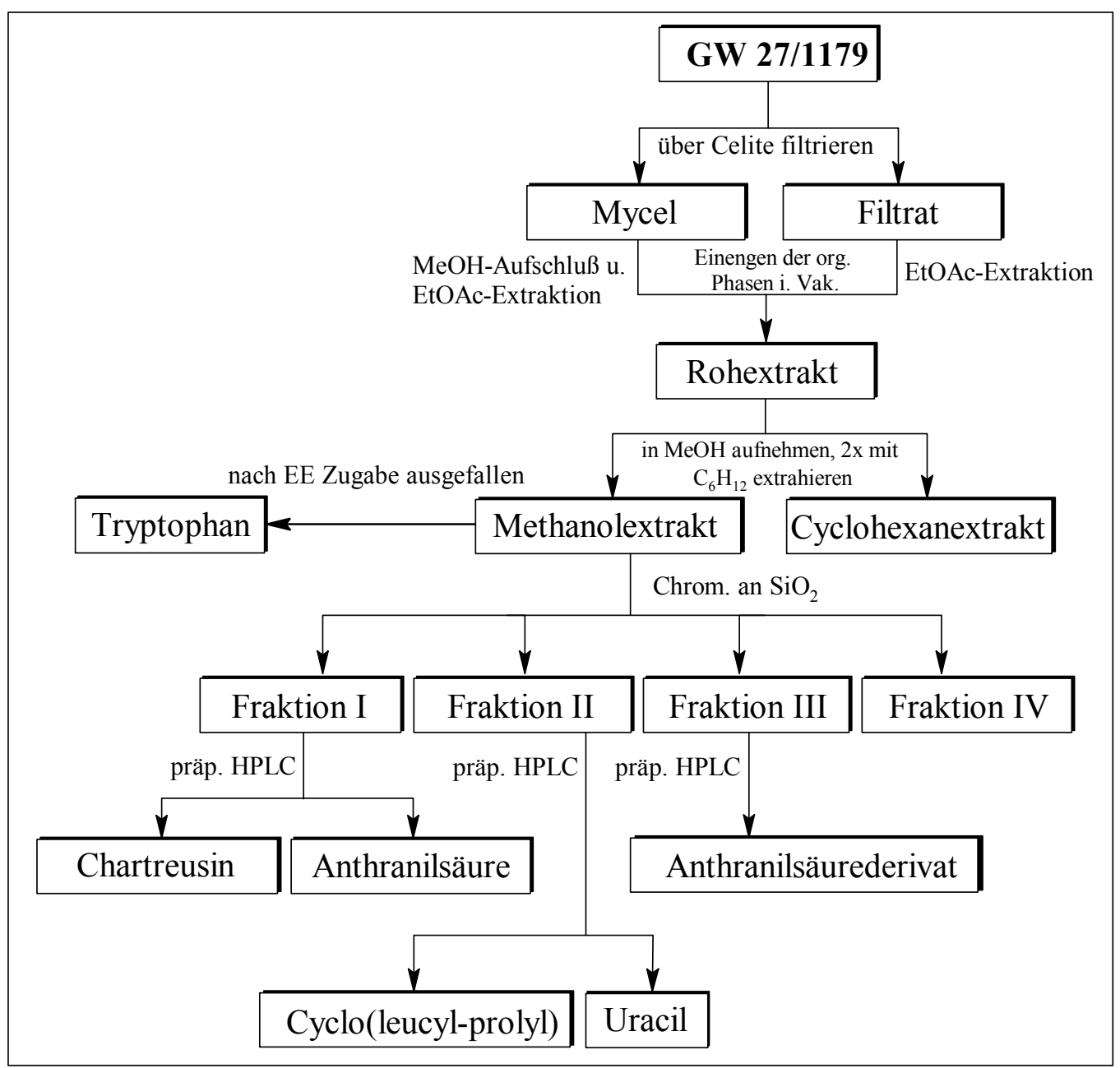

Schema 11: Aufarbeitung des Stammes GW 71/2497

\section{Chartreusin}

Die Fraktion I fiel durch ihre intensive gelbe Farbe auf. Die ${ }^{1} \mathrm{H}-\mathrm{NMR}-\mathrm{Spektren}$ der gelben Hauptkomponente zeigten die gleiche chemische Verschiebung und Aufspaltung der Signale wie das bereits aus diesem Stamm isolierte Chartreusin (62). Im ESI-Massenspektrum waren zwei Signale bei $m / z=1303$ und 663 zu sehen, die den Clustern $[2 \mathrm{M}+\mathrm{Na}]^{+}$und $[\mathrm{M}+\mathrm{Na}]^{+}$entsprachen. Die gesuchten Monoacetate wurden allerdings nicht gefunden, auch nicht die neuen Anthracycline.

Chartreusin wurde 1953 erstmals aus Streptomyces chartreusis isoliert ${ }^{[99-101]}$. Wegen seiner beträchtlichen antibakteriellen und Antitumorwirkung ist es das Objekt vieler Untersuchungen gewesen ${ }^{[102,103]}$. Wegen der geringen Wasserlöslichkeit gelang der große Durchbruch jedoch nie. Auch die Derivatisierung brachte keine zufriedenstellende Ergebnisse ${ }^{[104]}$. Die Biosynthese von 62 verläuft aus Acetateinheiten ${ }^{[105,106]}$. 


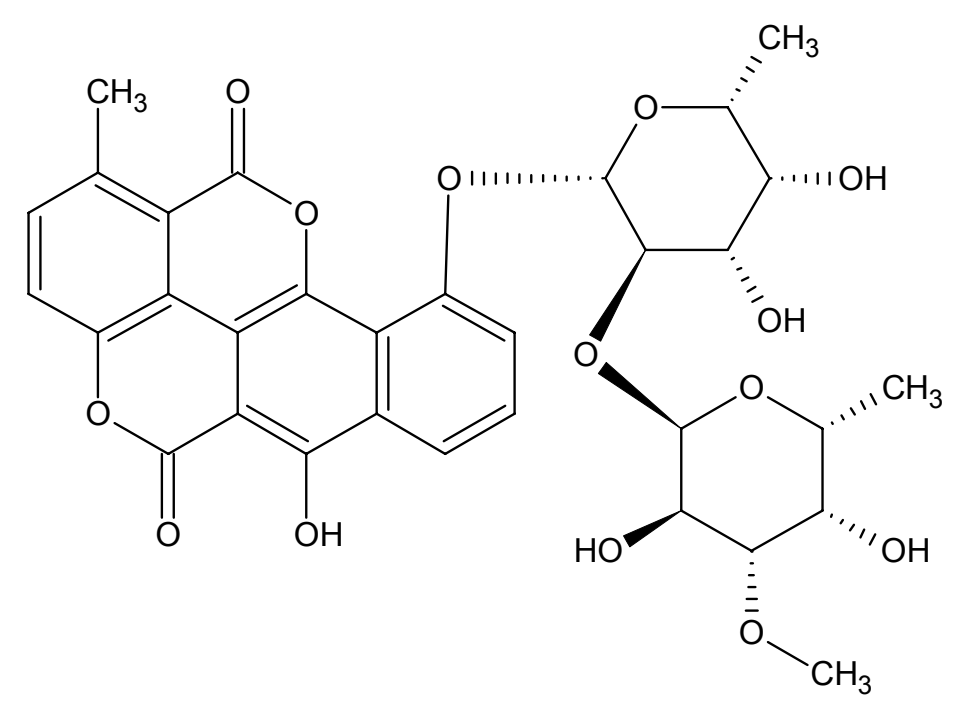

62

\section{Anthranilsäure, Cyclo(leucyl-prolyl) und Uracil}

Eine zweite aus der Fraktion I isolierte farblose Komponente fluoreszierte stark bei $256 \mathrm{~nm}$ und verfärbte sich mit Anisaldehyd/Schwefelsäure gelb. Im ${ }^{1} \mathrm{H}-\mathrm{NMR}-$ Spektrum fand man nur im aromatischen Bereich mehrere Signale. Die Kopplungsmuster deuteten darauf hin, dass es sich bei der vorliegenden Verbindung um Anthranilsäure (63) handelt, bestätigte endgültig der Spektrenvergleich mit den in der internen Sammlung vorliegenden Daten.

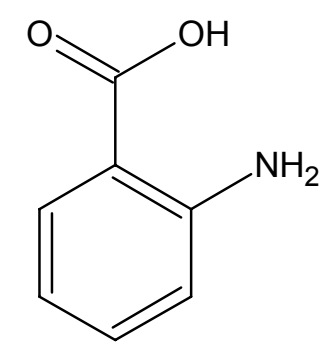

63

Bei der weißen Substanz aus der Fraktion II zeigten die spektroskopischen Daten keinen Unterschied zu den bereits bei der Aufarbeitung des Stammes B 7284 besprochenen Spektren von Cyclo(leucyl-prolyl) (35). Außerdem konnte man aus der Fraktion II Uracil (48) gewinnen. 


\section{Tryptophan}

Aus der methanolischen Fraktion fiel nach Zugabe von Ethylacetat ein weißer Niederschlag aus, der nach dem Umkristallisieren aus Methanol/Essigester farblose Plättchen bildete. Im Dünnschichtchromatogramm zeigte sich eine UV-löschende Zone mit $R_{\mathrm{f}}=0.05\left(\mathrm{CH}_{2} \mathrm{Cl}_{2} / \mathrm{MeOH} 8: 2\right)$, die sich mit Anisaldehyd/Schwefelsäure zuerst rosa, dann violett anfärben ließ. Das ${ }^{1} \mathrm{H}-\mathrm{NMR}-$ Spektrum zeigte das typische Bild eines 3- substituierten Indols. Die Signale des Substituenten zeigten eine Tieffeldverschiebung, die nur durch elektronenziehende Substituenten verursacht werden konnte. Das Kopplungsmuster ergab in Verbindung mit dem ${ }^{13} \mathrm{C}-\mathrm{NMR}-$ Spektrum ein - $\mathrm{CH}_{2} \mathrm{CHQ}$ - und ein zusätzliches CO-Fragment. Durch die Masse von 204 Dalton war damit Tryptophan (64) bewiesen.

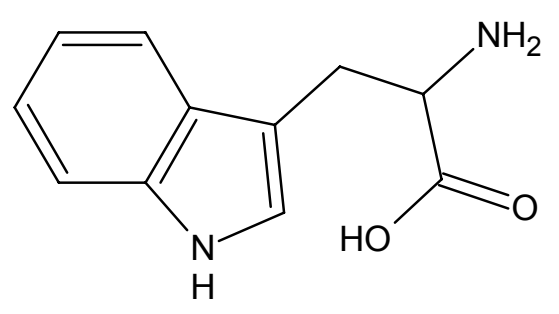




\section{Zusammenfassung}

Aufgrund der steigenden Multiresistenz pathogener Mikroorganismen ist die Suche nach neuen wirksamen Naturstoffen derzeit wieder ein hochaktuelles Thema. Auch mit dem zunehmenden Wissen über die unterschiedlichen Angriffsmechanismen der Krankheitserreger steigt der Bedarf nach neuen Leitstrukturen stetig, die man durch Derivatisierung an die Anforderungen optimal anpassen kann. Naturstoffe zeigen in dieser Hinsicht eine unübertroffene Diversität und Komplexität, die man durch synthetische Methoden kaum erreichen kann.

Aufgrund der steigenden Zahl nachisolierter bekannter Naturstoffe haben wir die Suche in der Vergangenheit auf Mikroorganismen aus marinen Habitaten erweitert, mit der Hoffnung, in ungewöhnlichen Lebensräumen und bei andersartigen Mikroorganismen auch neue Strukturen zu finden.

Aus diesem Grund wurden in dieser Arbeit 96 marine Pilzisolate untersucht, die von Dr. K. Schaumann am Alfred-Wegener-Institut für Polar- und Meeresforschung in Bremerhaven zur Verfügung gestellt wurden. Die Extrakte wurden einem mikrobiologischen, chemischen und HPLC/UV-Screening unterzogen. Die erste Bewertung fiel allerdings ernüchternd aus: Die untersuchten Pilze lieferten nur geringe Mengen an Rohextrakt und besaßen kaum biologische Aktivität.

Im Rahmen dieser Arbeit wurde der Meerespilz Lulworthia sp. Isolat KMPB H963 genauer untersucht. Dabei wurden nur Tyrosin (25) und die wohlbekannten Zucker $\mathrm{D}(+)$ Maltose (26), Mannose (27) und $\alpha, \alpha$-Trehalose (28a) gefunden. Mit einem HPLC/ESI-MS-Screening der Pilzextrakte konnte jedoch gezeigt werden, dass auch Metabolite mit Molmassen größer als 400 Dalton vorhanden sind. Wegen der geringen Produktion dieser Stoffe konnten sie aber nicht isoliert und aufgeklärt werden. 


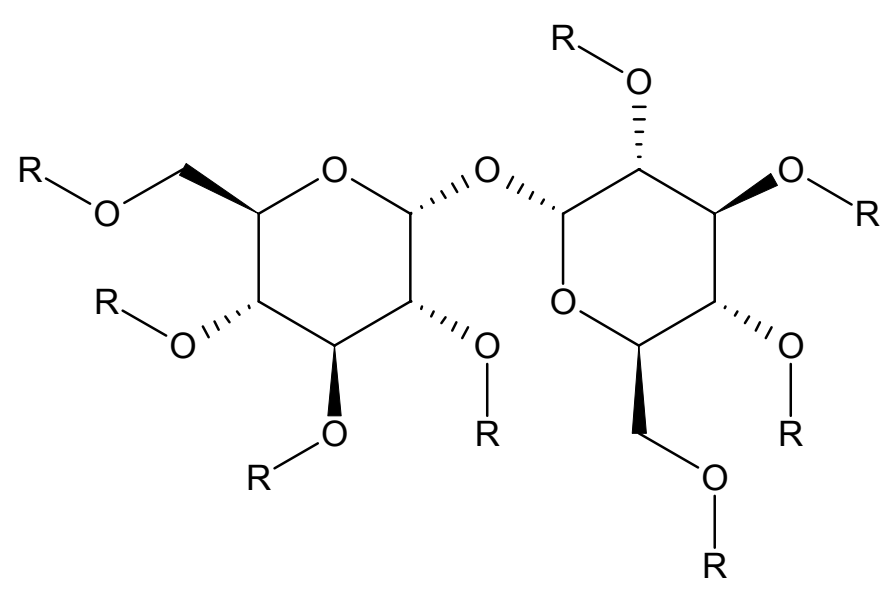

28a: $R=H$

Es ist allgemein bekannt, dass man durch Variation der Medienzusammensetzung oder der Kultivierungsbedingungen die Produktivität von Mikroorganismen um ein Mehrfaches steigern und auch Anlass zur Bildung geänderter Metabolitenspektren geben kann. Die Auswahl eines optimalen Nährmediums ist daher eine wesentliche Voraussetzung für eine erfolgreiche Suche nach neuen Metaboliten. Aus diesem Grunde sollten Methoden erprobt werden, die es ermöglichen, in möglichst kurzer Zeit entsprechende Optimierungsexperimente durchzuführen. Nach dem Entwurf und Eigenbau von 1 1-Durchfluß-Fermentern (personal fermentor) und der Entwicklung und Realisierung der gesamten zugehörigen elektronischen Steuerung einschließlich der speziellen Platinen konnte der praktische Einsatz einer auf Evolutionären Algorithmen basierenden Optimierungsmethode getestet werden.

Es wurden zehn bereits auf konventionelle Weise untersuchte Streptomyceten ausgewählt in der Hoffnung, durch Medienoptimierung die Produktion neuer Substanzen anzuregen oder die Ausbeute der bekannten Produkte zu steigern. Der Einsatz der Genetischen Algorithmen (Computerprogramm GALOP) lieferte für jeden der zehn untersuchten Stämme fünf Tochter-Generationen mit je 15 Individuen (unterschiedliche Medienzusammensetzungen). Anhand dieser Daten wurde für jeden Stamm eine optimale Medienzusammensetzung bezüglich der Produktion der Sekundärmetabolite ausgewählt. Das Spektrum der produzierten Metabolite konnte zwar nicht bedeutend verändert werden, aber durch die eingesetzten Optimierungsmethoden konnte schnell für jeden Stamm ein Medium gefunden werden, in dem die Produktion der Naturstoffe am effektivsten ausfiel. Durch den Einsatz von GALOP konnte z. B. eine Steigerung der Chartreusinproduktion um mehr als $700 \%$ erreicht werden. 
Die abschließende Isolierung der Substanzen brachte jedoch bei den ausgewählten Stämmen nur kleine und bekannte Moleküle.

Der marine Streptomycet B7284 lieferte nach der kontinuierlichen Fermentation in dem 21 Eigenbau-Fermenter nur Cyclo(leucyl-prolyl) (35) und (3Z, 6E)-Methylalbonoursin (34). Letzteres ist mit Signalstoffen strukturell verwandt, die bei Bakterien für die als ,quorum sensing“ bezeichnete Zell-Erkennung von Bedeutung sind.<smiles>CC(C)/C=c1/c(=O)[nH]/c(=C/c2ccccc2)c(=O)n1C</smiles>

34<smiles>CC(C)CC1NC(=O)C2CCCN2C1=O</smiles>

35

Der marine Streptomycet B7939 zeigte im HPLC-Screening nur wenige auffallende Peaks und geringe biologische Aktivität. Aus dem Rohextrakt konnten nach der Optimierung mehrere farblose Komponenten isoliert werden. Außer Vitamin MK-7 (43) konnten noch zwei Phthalsäureester (41) und (40), N-Acetyltryptamin (36), 3Indolylethanol (37), N-Acetyltyramin (38) und p-Hydroxyphenylethyl-alkohol (39) gewonnen werden. Die Phthalester stammen nicht aus den verwendeten Plastikmaterialien und können Biosyntheseprodukte sein.<smiles>CC(C)=CCC/C(C)=C/CC/C(C)=C/CC/C(C)=C/CC/C(C)=C/CC/C(C)=C/CC1=C(C)C(=O)c2ccccc2C1=O</smiles> 
<smiles>CC(C)COC(=O)c1ccccc1C(=O)OCC(C)C</smiles>

41<smiles>CCCCOC(=O)c1ccccc1C(=O)OCCCC</smiles>

40

Der terrestrische Streptomycet GW 20/1811 zeigte eine starke Aktivität gegen $E$ scherichia coli, Bacillus subtilis und Staphylococcus aureus. Außerdem inhibierte der Rohextrakt die Shewanella-Chitinase. Aus Agar oder in Lösung bildete der Stamm kleine gelb-grüne Kolonien. Die für die Verfärbung verantwortliche Verbindung wurde als das bereits bekannte gelbgrüne Pyrrol BE-18591 (50) identifiziert werden. Aus dem gleichen Rohextrakt wurden noch weitere farblose Verbindungen gewonnen, deren Molmasse 300 Dalton allerdings nicht überschritt: Uridin (49), Uracil (48), (3Z, 6E)-1-N-Methylalbonoursin (34) und - wahrscheinlich als Kontamination bei der Aufarbeitung - Diisooctylphthalat (51). BE-18591 zeigt hohe biologische Aktivitäten: Es wirkt gegen Gram-positive und Gram-negative Bakterien und hemmt das Wachstum der Zelllinien P388 und MKN-45 ${ }^{[107,108]}$.

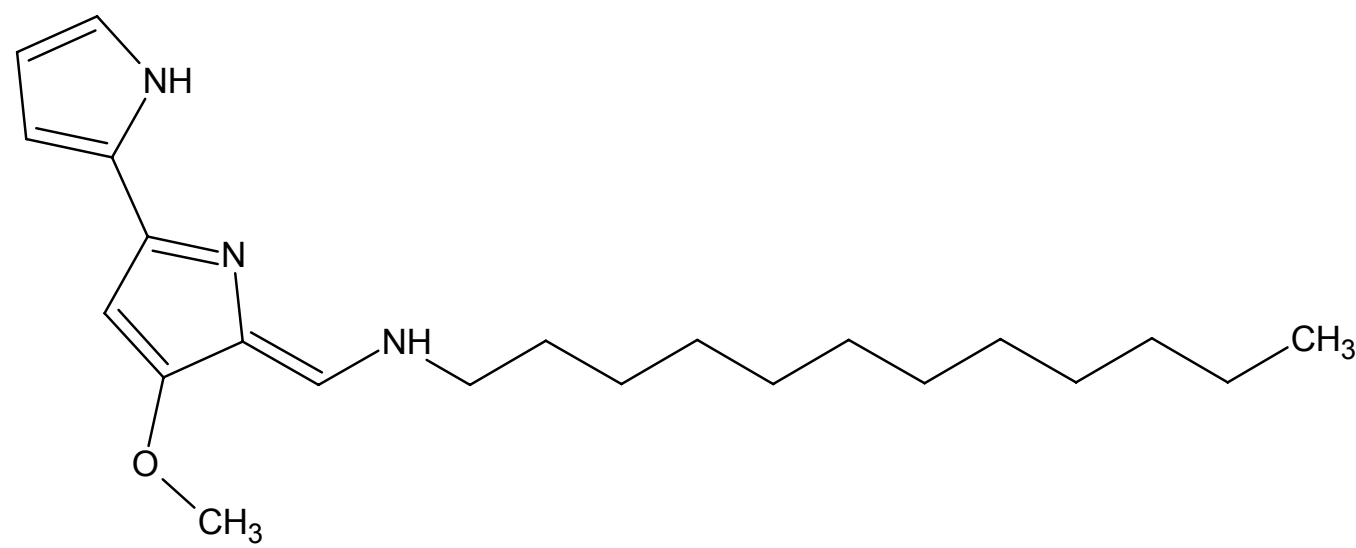

50

Ergosterol (53) ist ein ubiquitärer Bestandteil von Pilzen und kommt auch in Bierhefe vor. Nur selten wurde dieses Steroid aus Bakterien isoliert. Der marine Streptomycet B9020 bildet $\mathbf{5 3}$ allerdings in so großen Mengen, dass eine Herkunft aus Hefeex- 
trakt ausgeschlossen werden konnte. Außerdem fand man im Rohextrakt noch Indol3-carbonsäure-methylester (54), Tyrosol (39) und Indolyl-3-carbaldehyd (52).

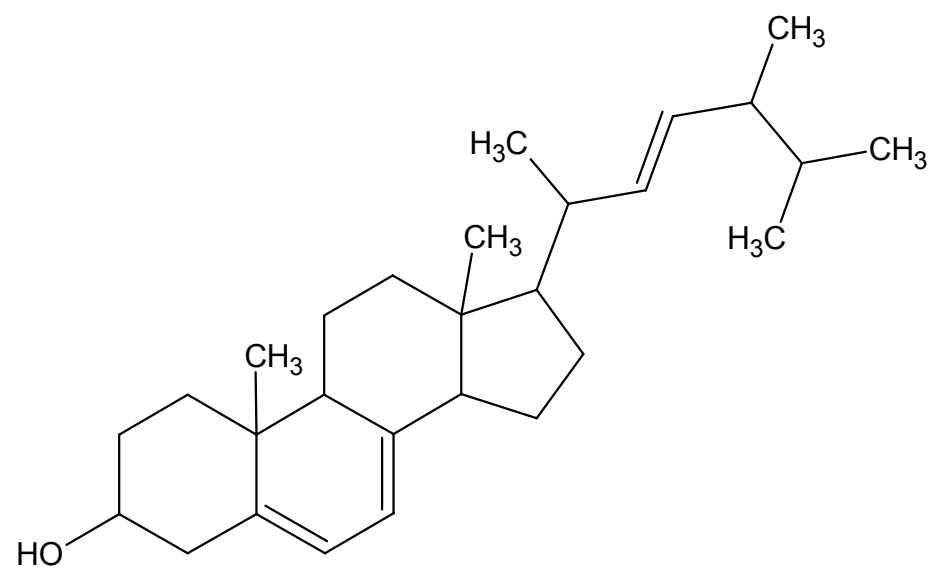

53

Der marine Streptomycet B2027 fiel im biologischen Screening des Schüttelkulturextraktes durch eine wachstumshemmende Wirkung gegen die meisten verwendeten Teststämme sowie gegen alle im Tests eingesetzten Algenarten auf. Die isolierten farblosen Sekundärmetabolite, wie 2-Methyl-3H-chinazolin-4-on (56) und 2Phenylacetamid (57), zeigten jedoch keine Aktivität. Aus dem Rohextrakt konnten zusätzlich N-Acetyltryptamin (36), 4-Methyl-hexan-carbonsäureamid (55) und Thymin (33) gewonnen werden. Die untersuchte Fettphase enthielt lediglich mehrere homologe iso-Fettsäuren, die als Methylester 58 identifiziert wurden.<smiles>Cc1nc2ccccc2c(=O)[nH]1</smiles>

56

Bei der Aufarbeitung des terrestrischen Streptomyceten GW 19/2497 isolierte man mehrere einfache Indolderivate, wie Indolyl-3-essigsäure (59), Acetyltryptamin (36), 3-Indolylethanol (37) und die Chlorverbindung 4-Chlor-5-(3'-indolyl)-oxazol (SF2583A) (60). Letzteres wurde 1988 aus Streptomyces sp. SF2583 isoliert und ist aktiv gegen Nematoden, Staphylococcus aureus, Bacillus subtilis, Escherichia coli und Candida albicans. 


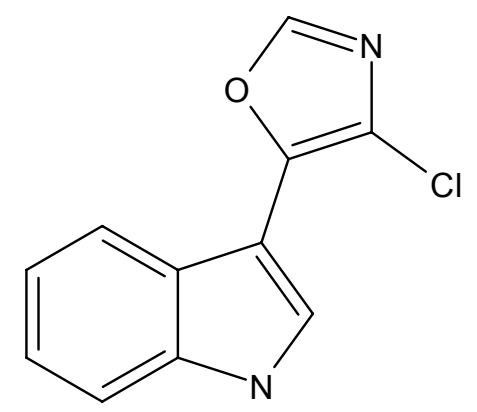

60

Eine Optimierung und anschließende Veränderung der Fermentationsbedingungen von diskontinuierlicher zur kontinuierlichen Anzucht brachte bei dem terrestrischen Streptomyceten GW 7/2495 eine Veränderung in der Sekundärmetabolitenproduktion. Es stellte sich heraus, dass bei der diskontinuierlichen Fermentation 2Methylfervenulon (29) als Hauptkomponente im Rohextrakt vorhanden war, bei der kontinuierlichen Anzucht jedoch nur in Spuren gefunden wurde. Man isolierte stattdessen $\varepsilon$ - und $\xi$-Pyrromycinone $(\mathbf{3 0}, \mathbf{3 1})$ und farblose 5-[2-(2-Hydroxy-3-methoxycarbonyl-propyl)-5-methyl-phenyl]-penta-2,4-diensäure (32), die in der Literatur noch nicht beschrieben wurde. Basierend auf der Biosynthese von Pyrromycinonen wurde ein Versuch unternommen, einen möglichen Biosyntheseweg für die neue Verbindung vorzuschlagen.<smiles></smiles><smiles>CCc1ccc2c(O)c3c(cc2c1C(=O)OC)C(=O)c1c(O)ccc(O)c1C3=O</smiles><smiles>CC[C@@]1(O)CCc2c(cc3c(c2O)C(=O)c2c(O)ccc(O)c2C3=O)C1C(=O)OC</smiles> 


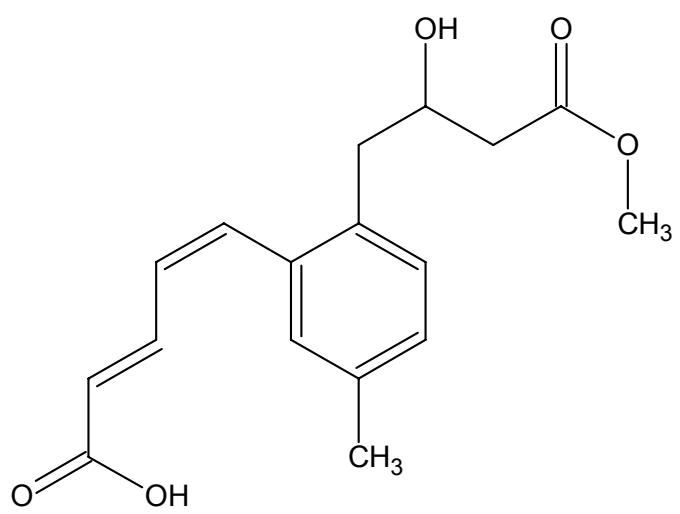

32

Die Umstellung der Kultivierungsbedingungen vom diskontinuierlichen auf kontinuierlichen Betrieb brachte bei dem Chartreusinproduzenten GW 71/2497 keine wesentliche Variation der produzierten Metabolite. Neben dem erwarteten Chartreusin (62) konnte man aus dem Rohextrakt nur Anthranilsäure (63), Cyclo(leucyl-prolyl) (35), Uracil (48) und Tryptophan (64) gewinnen.

Um die Bakterien im kontinuierlichen Betrieb züchten zu können, musste ein Trägermaterial zur Verankerung der Zellen im Bioreaktor gefunden werden. Nach der Erprobung mehrerer Trägermaterialien konnte eine optimale und wiederverwendbare Lösung in Form eines zusammengerolltes Stahlnetzes gefunden werden.

Zusätzlich wurde bei der kontinuierlichen Fermentation versucht, das Medium in einem geschlossenen Kreislauf von den bereits produzierten Sekundärmetaboliten zu befreien. $\mathrm{Zu}$ diesem Zweck wurde eine XAD-Säule mit dem Fermenter verbunden. Leider haben sich aber die im Medium frei schwimmenden Bakterien auf dem Harz aber weiterhin vermehrt und die Säule dadurch verstopft.

In der Zukunft wird bei der Untersuchung von Sekundärstoffen aus Mikroorganismen vor allem der Zeitaufwand bei der Fermentation der Bakterien und der Isolierung der Reinsubstanzen weiter reduziert werden müssen. In dieser Arbeit wurde der Versuch unternommen, dazu einige Alternativen zu entwickeln und sie praktisch zu erproben. Die Ergebnisse besonders bei der Fermentationsoptimierung sind vielversprechend; dennoch wird noch viel Forschungsarbeit auf diesem Gebiet zu leisten sein. 


\section{Experimenteller Teil}

\section{Geräte}

UV/VIS-Spektren: Perkin-Elmer Lambda 15 UV/VIS Spektrometer. - ${ }^{\mathbf{1} H-N M R-}$ Spektren: Varian VXR 200 (200 MHz), Varian Mercury 200 (200 MHz), Varian Unity 300 (300 MHz), Bruker AMX 300 (300 MHz), Varian INOVA 500 (500 $\mathrm{MHz})$.

Kopplungskonstanten $(J)$ in Hz. Abkürzungen: $\mathrm{s}=$ Singulett, $\mathrm{d}=$ Dublett, $\mathrm{dd}=$ Doppeldublett, $\mathrm{t}=$ Triplett, $\mathrm{q}=$ Quartett, $\mathrm{m}=$ Multiplett, $\mathrm{br}=$ breit. $-{ }^{13}$ C-NMRSpektren: Varian VXR 200 (50 MHz), Varian Mercury 200 (50 MHz), Varian Unity 300 (75 MHz), Bruker AMX 300 (75 MHz), Varian INOVA 500 (125 MHz). Abkürzungen: APT (Attached Proton Test): $\mathrm{CH} / \mathrm{CH}_{3}$-Signale stehen nach oben, $\mathrm{C} / \mathrm{CH}_{2}$ Signale nach unten. Chemische Verschiebungen in $\delta$-Werten relativ zu Tetramethylsilan als internem Standard. - Massenspektren: EI-MS mit Finnigan MAT 95 (70 eV), Finnigan LQC (70 eV) - HPLC-MS-Kopplung mit HPLC-Pumpe Rheos400 und UV-VIS-Detektor (Jasco). Hochauflösung: Perfluorkerosin als Vergleichssubstanz. DCIMS: Finnigan MAT 95 A, Reaktandgas: $\mathrm{NH}_{3}$; (+)-ESI-MS mit Quattro Triple Quadrupole Mass Spectrometer Finigan MAT-Incos 50. - HPLC: Analytische Anlage, Gerät I: Knauer HPLC-Anlage bestehend aus Spektral-Digital-Photometer A0293, zwei Pumpen Typ 64 A0307 und HPLC-Software V2.22, Mischkammer: A0285, Knauer Injektions-Schaltventil 6/1 A0263 (Typ Rheodyne); Probenschleife $20 \mu 1$. Gerät II: Jasco HPLC-Anlage bestehend aus Dioden-Array-Multiple Wavelength Detector Jasco MD-910, zwei Pumpen des Typs Jasco Intelligent Pump PU987 mit Mischkammer, Rheodyne-Ventil mit $20 \mu$ Probenschleife, Borwin HPLCSoftware; Präparative Anlage: analog der analytischen, nur mit (I) Knauer präparativer Durchflußküvette $(\mathrm{d}=0.5 \mathrm{~mm})$, Probenschleife $500 \mu \mathrm{l} \mathrm{bzw}$. (II) Jasco präparativer Durchflußküvette $(\mathrm{d}=1 \mathrm{~mm})$, Probenschleife $500 \mu$ l. Analytische Säulen: 1) Vertex $4 \times 250 \mathrm{~mm}$ mit $4 \times 4 \mathrm{~mm}$ Vorsäule, stationäre Phase: Eurochrom Eurospher RP 100-C18, $5 \mu \mathrm{m}, 2$ ) Vertex $4.6 \times 125$ mm, stationäre Phase: Hypersil RP 120-C18, $5 \mu \mathrm{m}, 3)$ Vertex $4.6 \times 250$, stationäre Phase: Nucleosil NP 100-C18, $5 \mu \mathrm{m}$; präparative Säulen: Vertex $16 \times 250 \mathrm{~mm}$ mit $16 \times 30 \mathrm{~mm}$ Vorsäule, stationäre Phase: Eurochrom Eurospher RP 100-C18, 5 m; Lösungsmittel für HPLC: Alle Eluenten wurden über Membranfilter (Wasser: Celluloseacetat, Porenweite $45 \mu \mathrm{m}$, Sartorius, Göttingen; Acetonitril: Filter RC-Vlies verstärkt, Porenweite $45 \mu \mathrm{m}$, Sartorius. Göttin- 
gen) filtriert und 15 min durch Ultraschall entgast; die Proben wurden durch ein PTFE-Filter (Minisart SRP15, Porenweite $0.20 \mu \mathrm{m}$, Sartorius, Göttingen) filtriert. Für die Trennung wurde durch fraktionierte Destillation zurückgewonnenes Acetonitril/Wasser-Azeotrop (83.7 \% Acetonitril / $16.3 \%$ Wasser, Siedepunkte: $78.5{ }^{\circ} \mathrm{C}$ ) eingesetzt. - Filterpresse: Schenk Niro 212 B40. - Gefriertrocknung: LOC-1M; Christ GmbH, - Harzextraktion: Eppendorf Schlauchpumpe 5260, Glassäule $3 \times$ $100 \mathrm{~cm}$ mit Hülse (NS 29) und Glasfritte (G1), Olive mit Kern (NS 29) und Glasfritte $(\mathrm{G} 1)$.

\section{Materialien}

Dünnschichtchromatographie (DC): DC-Folien Polygram SIL G/UV254 (Macherey-Nagel $\&$ Co., Düren). $R_{\mathrm{f}}$-Werte beziehen sich, wenn nicht anders angegeben, auf das bei der SC angegebene Laufmittel an neutralem Kieselgel. - Präparative Dickschichtchromatographie (PDC): Kieselgel P/UV254 (55 g/120 ml Wasser, Macherey-Nagel \& Co.) goss man auf waagerecht liegende Glasplatten $(20 \times 40 \mathrm{~cm}$ oder 2 je $20 \times 20 \mathrm{~cm}$ ), ließ an der Luft trocken und aktivierte $4 \mathrm{~h}$ bei $130{ }^{\circ} \mathrm{C}$. - Säulenchromatographie (SC): MN Kieselgel 60: 0.05 - 0.2 mm, 70 - 270 mesh (MachereyNagel \& Co.); Kieselgel für Flash-Chromatographie: 30 - 60 m (J. T. Backer); Modifiziertes Kieselgel für Flash-Chromatographie: $40-63 \mu \mathrm{m}$ LiChroPrepDIOL (Merck); die Säulen wurden nass gefüllt. - Ausschlusschromatographie: Sephadex LH-20 (Pharmacia). - Harzextraktion: Amberlite XAD-7, 20 - 50 mesh (FLUKA); Amberlite XAD-16, 13 - 80 mesh (Serva).

\section{Sprühreagenzien}

Anisaldehyd/Schwefelsäure als universales Sprühreagenz: $\mathrm{Zu} 100 \mathrm{ml}$ einer Stammlösung aus $85 \mathrm{ml}$ Methanol, $14 \mathrm{ml}$ Essigester und $1 \mathrm{ml}$ konz. Schwefelsäure fügte man $1 \mathrm{ml}$ Anisaldehyd hinzu. Die besprühten Chromatogramme werden mit dem Fön bis zur Farbentwicklung erwärmt. - Ehrlichs Reagenz: 1 g 4-Dimethylaminobenzaldehyd wurde in einer Mischung aus $25 \mathrm{ml}$ Salzsäure (36\%) und $75 \mathrm{ml}$ Methanol gelöst. Es färbt Indole beim Erwärmen rot bis violett, andere N-Heterocyclen meist grau auf gelblichem Untergrund an. - Ninhydrin: Sprühreagenz $0.1 \%$ Merck Art. 6758. 


\section{Mikrobiologische Materialien}

Fermenter: 20-1-Fermenter (Fa. Meredos GmbH, Göttingen) bestehend aus Kulturgefäß, Magnet-Propellerrührer, Kühlschlange mit Thermostat; Steuereinheit mit pHRegler, Antischaum-Regler, Fa. Meredos GmbH Göttingen. 2-1-Durchflußfermenter Eigenbau (s. Kap. Bau des Prototyps eines Bioreaktors).

Schüttler: Infors AG (CH 4103 Einbach) Typ ITE. - Autoklav: Albert Dargatz Autoklav, Volumen 119 1, Betriebstemperatur $121{ }^{\circ} \mathrm{C}$, Betriebsdruck $1.2 \mathrm{~kg} / \mathrm{cm}^{2}$. Stammhaltung: Tiefkühllagerung in Dewargefäß, Fa. 1'Air liquid Typ BT 37 A. Kapillaren zur Tiefkühllagerung: Durchmesser $1.75 \mathrm{~mm}$, Länge $80 \mathrm{~mm}$, Fa. Hirschmann Laborgeräte Eberstadt. - Erde für Erdkultur: Luvos Heilerde LUVOS JUST GmbH \& Co. Friedrichshof (aus dem Reformhaus bezogen). - Ultraturrax: Janke \& Munkel KG. - Antibiotika-Testplättchen: 9 mm Durchmesser, Schleicher \& Schüll No. 321261. - Nährbodenbestandteile: Glucose, Hefeextraktextrakt und Malzextrakt (Fa. Merck, Darmstadt), Bacto Agar, (Fa. Difco). - AntischaumLösung: Niax PPG 2025; Union Carbide Belgium N. V. (Zwiijndrecht). - Petrischalen: Durchmesser 94 mm, Höhe 16 mm, Fa. Greiner Labortechnik, Nürtingen. - Sterilfilter: Midisart 2000, $0.2 \mu \mathrm{m}$, PTFE-Filter, Sartorius, Göttingen. - Salinenkrebseier (Artemia salina): SERA Artemia Salinenkrebseier, SERA Heinsberg. Salinenkrebsfutter: Mikrozell DOHSE Aquaristik KG Bonn (Salinenkrebseier und -futter sind über den Aquarien-Fachhandel zu beziehen). - Mikrotiterplatten: $4 \times 6 \mathrm{~mm}$, h $20 \mathrm{~mm}$, Nunclon ${ }^{\mathrm{TM}}$ Delta (Nalge Nunc International, Rochester, USA).

\section{Rezepturen}

Alle Kultur-Medien wurden bei 1.2 bar und $121{ }^{\circ} \mathrm{C}$ autoklaviert. Die Verweildauer im Autoklav betrug für 1-1-Schüttelkulturen 33 min, für Fermenter-Medien in Form eines 2 1-Konzentrats 50 min und für den mit 161 Wasser gefüllten Fermentationsbehälter $82 \mathrm{~min}$.

\section{Künstliches Seewasser:}

Eisencitrat

$\mathrm{NaCl}$
$2 \mathrm{~g}$ (vorher zermörsert)

$389 \mathrm{~g}$ 


$\begin{array}{ll}\mathrm{MgCl}_{2} \cdot 6 \mathrm{H}_{2} \mathrm{O} & 176 \mathrm{~g} \\ \mathrm{Na}_{2} \mathrm{SO}_{4} & 68.8 \mathrm{~g} \text { (vorher in 2 1 Wasser aufgelöst) } \\ \mathrm{CaCl}_{2} & 36 \mathrm{~g} \\ \mathrm{Na}_{2} \mathrm{HPO}_{4} & 0.16 \mathrm{~g} \\ \mathrm{SiO}_{2} & 0.3 \mathrm{~g} \\ \mathrm{Spurenelement-Lösung}_{\text {Stamm-Lösung }} & 20 \mathrm{ml} \\ \text { Mit Leitungswasser auf 20 1 auffüllen. }\end{array}$

\section{Spurenelement-Lösung (Hoagland 1993):}

$\begin{array}{ll}\mathrm{H}_{3} \mathrm{BO}_{3} & 0.611 \mathrm{~g} \\ \mathrm{MnCl}_{2} & 0.389 \mathrm{~g} \\ \mathrm{CuSO}_{4} & 0.056 \mathrm{~g} \\ \mathrm{ZnSO}_{4} \cdot 7 \mathrm{H}_{2} \mathrm{O} & 0.056 \mathrm{~g} \\ \mathrm{Al}_{2}\left(\mathrm{SO}_{4}\right)_{3} \cdot 18 \mathrm{H}_{2} \mathrm{O} & 0.056 \mathrm{~g} \\ \mathrm{NiSO}_{4} \cdot 6 \mathrm{H}_{2} \mathrm{O} & 0.056 \mathrm{~g} \\ \mathrm{Co}\left(\mathrm{NO}_{3}\right)_{3} \cdot 6 \mathrm{H}_{2} \mathrm{O} & 0.056 \mathrm{~g} \\ \mathrm{TiO}_{2} & 0.056 \mathrm{~g} \\ \left(\mathrm{NH}_{4}\right)_{6} \mathrm{Mo}_{7} \mathrm{O}_{24} \cdot 4 \mathrm{H}_{2} \mathrm{O} & 0.056 \mathrm{~g} \\ \mathrm{LiCl}_{\mathrm{SnCl}_{2}} & 0.028 \mathrm{~g} \\ \mathrm{KI} & 0.028 \mathrm{~g} \\ \end{array}$

Die Spurenelementsalze wurden in 11 Leitungswasser gelöst.

\section{Stammlösung:}

$\begin{array}{ll}\mathrm{KCl} & 110 \mathrm{~g} \\ \mathrm{NaHCO}_{3} & 32 \mathrm{~g} \\ \mathrm{KBr} & 16 \mathrm{~g} \\ \mathrm{SrCl}_{2} \cdot 6 \mathrm{H}_{2} \mathrm{O} & 6.8 \mathrm{~g} \text { (getrennt aufgelöst) } \\ \mathrm{H}_{3} \mathrm{BO}_{3} & 4.4 \mathrm{~g} \\ \mathrm{NaF} & 0.48 \mathrm{~g} \\ \mathrm{NH}_{4} \mathrm{NO}_{3} & 0.32 \mathrm{~g}\end{array}$

Mit Leitungswasser auf 21 auffüllen. 
Nährmedien:

$M_{2}$-Medium:

$\begin{array}{ll}\text { Malz-Extrakt } & 10 \mathrm{~g} \\ \text { Glucose } & 4 \mathrm{~g} \\ \text { Hefeextrakt-Extrakt } & 4 \mathrm{~g} \\ \text { Leitungswasser } & 1000 \mathrm{ml}\end{array}$

Die Lösung wurde mit $2 \mathrm{~N} \mathrm{NaOH}$ auf pH 7.8 eingestellt. Bei festem Medium wurden zusätzlich 18 g Bacto Agar zugegeben.

$\mathrm{M}_{2}^{+}$mit Seewasser:

$\begin{array}{ll}\text { Malz-Extrakt } & 10 \mathrm{~g} \\ \text { Glucose } & 4 \mathrm{~g} \\ \text { Hefeextrakt-Extrakt } & 4 \mathrm{~g} \\ \text { künstliches Seewasser } & 500 \mathrm{ml} \\ \text { Leitungswasser } & 500 \mathrm{ml}\end{array}$

Die Lösung wurde mit $2 \mathrm{~N} \mathrm{NaOH}$ auf pH 7.8 eingestellt. Bei festem Medium wurden zusätzlich $18 \mathrm{~g}$ Bacto Agar zugegeben.

M-Test-Agar (für die Testkeime Escherichia coli, Bacillus subtilis (ATCC 6051), Staphylococcus aureus, Mucor miehei (Tü 284):

$\begin{array}{ll}\text { Malz-Extrakt } & 10 \mathrm{~g} \\ \text { Hefeextrakt } & 4 \mathrm{~g} \\ \text { Glucose } & 4 \mathrm{~g} \\ \text { Bacto Agar } & 20 \mathrm{~g} \\ \text { demineralisiertes Wasser } & 1000 \mathrm{ml} \\ \text { Die Lösung wurde mit 2 N NaOH auf pH 7.3 eingestellt. }\end{array}$

Sabouraud-Agar für den Testkeim Candida albicans:

$\begin{array}{lc}\text { Glucose } & 40 \mathrm{~g} \\ \text { Bacto Pepton } & 10 \mathrm{~g} \\ \text { Bacto Agar } & 20 \mathrm{~g}\end{array}$


demineralisiertes Wasser $1000 \mathrm{ml}$

Die Lösung wurde mit $2 \mathrm{~N} \mathrm{NaOH}$ auf pH 7.3 eingestellt.

\section{Nährlösung A}

Sojamehl entfettet $\quad 30 \mathrm{~g}$

Glycerin $30 \mathrm{~g}$

$\mathrm{CaCO}_{3} \quad 2 \mathrm{~g}$

künstliches Seewasser $\quad 750 \mathrm{ml}$

demineralisiertes Wasser $250 \mathrm{ml}$

\section{Nährlösung B}

Stärke

$10 \mathrm{~g}$

NZ-Amine

$5 \mathrm{~g}$

Fleischextrakt

$7.5 \mathrm{~g}$

Sojamehl

$2 \mathrm{~g}$

Hefeextraktextrakt

$5 \mathrm{~g}$

$\mathrm{KNO}_{3}$

$3 \mathrm{~g}$

Algenextrakt

$2.5 \mathrm{ml}$

künstliches Seewasser $\quad 750 \mathrm{ml}$

demineralisiertes Wasser $\quad 250 \mathrm{ml}$

\section{Stammlösungen und Medien zur Algenkultivierung:}

\section{Fe-EDTA}

Man löste $0.7 \mathrm{~g} \mathrm{FeSO}_{4} \cdot 7 \mathrm{H}_{2} \mathrm{O}$ und $0.93 \mathrm{~g}$ EDTA (Titriplex III) in $80 \mathrm{ml} \mathrm{H}_{2} \mathrm{O}$ unter Erwärmen. Diese Lösung wurde mit demineralisiertem Wasser auf $100 \mathrm{ml}$ aufgefüllt.

Spurenelement-Lösung II:

\section{Lösung A:}
$\mathrm{MnSO}_{4} \cdot \mathrm{H}_{2} \mathrm{O}$
$16.9 \mathrm{mg}$
$\mathrm{Na}_{2} \mathrm{MoO}_{4} \cdot 2 \mathrm{H}_{2} \mathrm{O}$
$13.0 \mathrm{mg}$ 
$\mathrm{Co}\left(\mathrm{NO}_{3}\right)_{2} \cdot 6 \mathrm{H}_{2} \mathrm{O} \quad 10.0 \mathrm{mg}$

Diese Salze wurden jeweils in $10 \mathrm{ml}$ demineralisiertem Wasser gelöst, die Lösungen dann vereinigt.

\section{Lösung B:}

$\begin{array}{ll}\mathrm{CuSO}_{4} \cdot 5 \mathrm{H}_{2} \mathrm{O} & 5.0 \mathrm{mg} \\ \mathrm{H}_{3} \mathrm{BO}_{3} & 10.0 \mathrm{mg} \\ \mathrm{ZnSO}_{4} \cdot 7 \mathrm{H}_{2} \mathrm{O} & 10.0 \mathrm{mg}\end{array}$

Die Salze wurden zusammengegeben und in $10 \mathrm{ml}$ demineralisiertem Wasser gelöst. Lösung A und Lösung B wurden vereinigt und mit demineralisiertem Wasser auf 100 $\mathrm{ml}$ aufgefüllt.

\section{Bold's Basal Medium (BBM):}

$\begin{array}{ll}\mathrm{NaNO}_{3} & 0.250 \mathrm{~g} \\ \mathrm{KH}_{2} \mathrm{PO}_{4} & 0.175 \mathrm{~g} \\ \mathrm{~K}_{2} \mathrm{HPO}_{4} & 0.075 \mathrm{~g} \\ \mathrm{MgSO}_{4} \cdot 7 \mathrm{H}_{2} \mathrm{O} & 0.075 \mathrm{~g} \\ \mathrm{NaCl} & 0.025 \mathrm{~g} \\ \mathrm{CaCl}_{2} \cdot 2 \mathrm{H}_{2} \mathrm{O} & 0.025 \mathrm{~g} \\ \mathrm{Fe}-\mathrm{EDTA} & 1.0 \mathrm{ml}\end{array}$

Spurenelement-Lösung II $1.0 \mathrm{ml}$

Die Salze wurden jeweils in $10 \mathrm{ml}$ Wasser gelöst. Diese Lösungen vereinigte man mit der Fe-EDTA- und der Spurenelement-Lösung II und füllte auf $1000 \mathrm{ml}$ mit demineralisiertem Wasser auf. 


\section{Mikrobiologische und analytische Arbeitsmethoden}

\section{Stammhaltung und Anzucht der Streptomyceten}

Die als Reinkulturen erhaltenen Stämme wurden auf Schrägagar mit $\mathrm{M}_{2}$ - für terrestrische bzw. $\mathrm{M}_{2}{ }^{+}$-Medium $\left(\mathrm{M}_{2}\right.$ mit $50 \%$ Seewasser) für marine Streptomyceten überimpft und $72 \mathrm{~h}$ bei $28^{\circ} \mathrm{C}$ inkubiert. Für die Glycerin-Dauerkultur schwemmte man das Mycel mit der entsprechenden Nährlösung ab. Von dieser Suspension wurden 0.5 $\mathrm{ml}$ in einem $2 \mathrm{ml}$ Schraubdeckelglas mit $0.5 \mathrm{ml}$ Nährlösung und $0.1 \mathrm{ml}$ Glycerin unter sterilen Bedingungen vermischt. Die Suspension wurde in Kapillaren aufgenommen, die mit einer abgeflammten Pinzette in der Mitte gehalten und in das Schraubdeckelgläschen getaucht wurden. Die zu 60 \% gefüllten Kapillaren wurden dann in der nichtleuchtenden Flamme eines Bunsenbrenners an beiden Enden zugeschmolzen. Dabei war darauf zu achten, dass die äußerste Spitze der Kapillare nur für möglichst kurze Zeit erhitzt wurde. Man ließ an der Luft etwas abkühlen und gab die Kapillare in eine mit Ethanol gefüllte Petri-Glasschale. Undichte Kapillaren saugen Ethanol auf und werden verworfen, die anderen zur Vermeidung weiteren Wachstums und daraus folgenden anaeroben Bedingungen sofort auf Eis gelegt. Nachdem die Kapillare $24 \mathrm{~h}$ bei $-20{ }^{\circ} \mathrm{C}$ gelagert wurde, bewahrte man sie in flüssigem Stickstoff auf. Zum Animpfen wurde die Kapillare bei Raumtemperatur aufgetaut, in Ethanol desinfiziert, kurz durch die Flamme gezogen und mit einer abgeflammten Pinzette an beiden Enden abgebrochen. Man fasste die Kapillare mit Daumen und Zeigefinger mittig und brachte den Inhalt durch Aufstauchen des Handballens auf die Arbeitsplatte auf den Agar auf.

Für die Erdkultur sporenbildender Organismen tropfte man $1 \mathrm{ml}$ der abgeschwemmten Suspension in ein zu 30 \% mit sterilisierter Heilerde gefülltes Reagenzglas. Zum Trocknen wurde es mit einer Kapsenberg-Kappe verschlossen und 2 Monate bei Raumtemperatur aufbewahrt, danach bei $4{ }^{\circ} \mathrm{C}$ eingelagert.

Für eine Wiederanzucht von der Erdkultur wurde etwas Material mit einem Spatel auf eine Agar-Platte gegeben, mit einer Impföse verteilt und $72 \mathrm{~h}$ bei $28^{\circ} \mathrm{C}$ inkubiert. Die gewachsenen Kulturen dienten als Impfmaterial für Schüttelkulturen. 


\title{
Biologisches und chemisches Screening
}

\section{Schüttelkulturen}

Fünf mit je 200 ml Nährmedium gefüllte und mit einem Baumwolltuch verschlossene 1 1-Schikanekolben (3 Schikanen) wurden $33 \mathrm{~min}$ bei $121{ }^{\circ} \mathrm{C}$ sterilisiert und mit Agarstückchen von einer $72 \mathrm{~h}$ bei $28^{\circ} \mathrm{C}$ gewachsenen Plattenagarkultur angeimpft. Die Schikanekolben inkubiert man $72 \mathrm{~h}$ bei $28^{\circ} \mathrm{C}$ mit ca. 95 Umdrehungen/min (Upm). Die Kulturbrühe wurde auf fünf 1 1-Rundkolben verteilt und gefriergetrocknet. Das Lyophilisat wurde dreimal mit Essigester extrahiert und die org. Phase i. Vak. bei $40{ }^{\circ} \mathrm{C}$ eingeengt.

\begin{abstract}
Aktivitätstests
Zur Bestimmung der antibiotischen Aktivität wurden Plattendiffusionstests mit Escherichia coli, Bacillus subtilis (ATCC 6051), Mucor miehei (Tü 284), Candida albicans, Streptomyces viridochromogenes (Tü 57), Staphylococcus aureus sowie den Mikrogrünalgen Chlorella vulgaris, Chlorella sorokiniana und Scenedesmus subspicatus durchgeführt. In die in $\mathrm{CHCl}_{3} / 13 \% \mathrm{MeOH}$ (= Azeotrop) gelösten Rohextrakte (Konzentration $100 \mu \mathrm{g} / \mathrm{ml}$ ) tauchte man Testplättchen ein, die nach dem Trocknen auf mit den Testkeimen beimpfte Agarplatten gelegt wurden. Nachdem die Kulturen $24 \mathrm{~h}$ bei $37{ }^{\circ} \mathrm{C}$ gewachsen waren, wurde der Hemmhofdurchmesser bestimmt. Die Algen wuchsen unter künstlicher Beleuchtung im Photoreaktor $72 \mathrm{~h}$ bei Raumtemperatur. Die im chemischen Screening eingesetzten Chromatogramme $(4 \times$ $10 \mathrm{~cm}$ ) wurden mit $\mathrm{CHCl}_{3} / 5 \% \mathrm{MeOH}$ und $\mathrm{CHCl}_{3} / 10 \% \mathrm{MeOH}$ entwickelt und mit Sprühreagenzien angefärbt oder unter Tages- und UV-Licht ausgewertet. Neben der Beschreibung der Art der Entwicklung und der benutzten Laufmittels in Vordrucken fotografierte man die DC-Platten.
\end{abstract}

\section{Toxizitätstest}

In einem $500 \mathrm{ml}$ Scheidetrichter gab man zu 400 ml künstlichem Seewasser ca. 100 mg getrocknete Artemia salina-Eier und ca. 100 mg Futter. Die Suspension wurde 24 bis $48 \mathrm{~h}$ bei Raumtemperatur von einem schwachen Luftstrom belüftet. Man ließ die Suspension $1 \mathrm{~h}$ stehen, wodurch nicht geschlüpfte Eier absanken. Der Scheidetrichter wurde einseitig abgedunkelt und die auf Grund ihrer positiven Phototaxis der Licht- 
quelle entgegenschwimmenden Tiere in einen $50 \mathrm{ml}$ Erlenmeyerkolben abpipettiert. Hiervon wurden je $1 \mathrm{ml}$ (ca. 30 bis 40 Tiere) in die Vertiefungen einer Mikrotiterplatte (Durchmesser ca. $1.8 \mathrm{~cm}$, Tiefe ca. $2 \mathrm{~cm}$ ) übertragen. Dann wurden $10 \mu 1$ Lösung des Rohextrakts in DMSO (10 mg/ml) zupipettiert. Als Kontrolle dienten 10 $\mu 1$ reines DMSO. Die Mikrotiterplatte wurde auf ein angefeuchtetes Filterpapier gestellt, mit einer Petrischale abgedeckt und bei Raumtemperatur (auf möglichst konstante Temperatur achten, da Schwankungen große Auswirkungen auf die Sterblichkeitsrate haben) vor direkter Sonnenstrahlung geschützt aufbewahrt. Nach 24 h ermittelte man den Prozentsatz der toten Larven, indem zunächst die Anzahl der toten Tiere und dann nach Abtöten der lebenden Tiere mit ca. $0.5 \mathrm{ml}$ Methanol die Gesamtzahl unter einem Lichtmikroskop bestimmt wurde.

\section{Ergebnisse des Vorscreenings (Bewertungsgrundlagen)}

Antibiotisches Screening (Plattendiffusionstest):

Bei Verwendung von Testplättchen mit einem Durchmesser von $9 \mathrm{~mm}$ wurden Hemmhöfe von 11 bis $20 \mathrm{~mm}$ Durchmesser als schwach aktiv (+), von 21 bis $30 \mathrm{~mm}$ als aktiv $(++)$ und über $30 \mathrm{~mm}$ als hochaktiv $(+++)$ bezeichnet.

Chemisches Screening: Bei standardisierter DC wurden Zonen, die in Größe und Anfärbung auffielen, positiv bewertet. - Toxizitätstest: bei Auszählung nach $24 \mathrm{~h}$ beurteilte man den Rohextrakt bei einer Sterblichkeitsrate unter $10 \%$ als unwirksam $(-)$, von 10 bis $59 \%$ als schwach wirksam (+), von 60 bis $95 \%$ als wirksam (++) und über $95 \%$ als stark wirksam $(+++)$.

\section{Standardgradient für die HPLC-Trennung:}

$\mathrm{H}_{2} \mathrm{O} / 10 \% \mathrm{MeCN}$ Anstieg auf $80 \% \mathrm{MeCN}$ in $30 \mathrm{~min}$, dann auf $100 \% \mathrm{MeCN}$ in 10 min, über 10 min $100 \% \mathrm{MeCN}$ und dann Abfall auf $10 \%$ in 1 min, Fluss 10.0 $\mathrm{ml} / \mathrm{min}$. 


\section{GALOP-Optimierung}

Für jede Generation wurden jeweils 15 Individuen erzeugt. Die durch GALOP angegebenen Medienzusammensetzungen wurden in $25 \mathrm{ml}$ destilliertem Wasser angesetzt. Sollten die Medien für marine Stämme verwendet werden, wurden zusätzlich $25 \mathrm{ml}$ künstliches Meerwasser zugesetzt; sollten die Medien für terrestrische Stämme verwendet werden, wurden stattdessen zusätzlich $25 \mathrm{ml}$ Trinkwasser zugegeben. Die mit den Nährmedien gefüllten $100 \mathrm{ml}$ Anzuchtkolben wurden $30 \mathrm{~min}$ bei $121^{\circ} \mathrm{C}$ autoklaviert.

Eine Kultur des entsprechenden Stammes wurde auf einer Agar-Platte kultiviert, mit $1 \mathrm{ml}$ Wasser überschichtet und die so entstandene Zellsuspension in einem Kolben mit Trinkwasser auf $25 \mathrm{ml}$ aufgefüllt. Die autoklavierten Anzuchtkolben wurden mit je genau einem Milliliter dieser Zellsuspension angeimpft und drei Tage bei $28^{\circ} \mathrm{C}$ mit einer Frequenz von $1 \mathrm{~Hz}$ auf einer Rührplatte kultiviert. Jedes Individuum (im Sinne von GALOP, nicht im mikrobiologischen Sinne) wurde nach Aussehen, Mycelbildung und Farbe beschrieben. Die Zellkulturen wurden mit $50 \mathrm{ml}$ Methanol versetzt und bei $40^{\circ} \mathrm{C}$ und $50 \mathrm{hPa}$ vollständig vom Lösungsmittel befreit. Die Trockensubstanzen wurden gewogen und je dreimal mit je $50 \mathrm{ml}$ Essigsäureethylester extrahiert. Die vereinigten Extrakte jedes Individuums wurden mittels HPLC untersucht, um die für die weitere Optimierung mit GALOP benötigten Werte vervollständigen zu können.

\section{Primärscreening der Pilzproben}

Die aus Bremerhaven (Alfred-Wegener Institut für Polar- und Meeresforschung) in 1 Liter Polyethylen-Flaschen erhaltenen und im Essigester abgetöteten 92 Pilzproben wurden mit dem Ultraturrax 3 min lang zerkleinert. Nach der mechanischen Behandlung wurden sie dreimal mit je $150 \mathrm{ml}$ Essigester extrahiert und die so erhaltenen Essigesterfraktionen vereinigt und eingeengt. Am Ende der Aufarbeitungsphase wurden die Proben dreimal mit je $50 \mathrm{ml}$ Cyclohexan entfettet und vom Lösungsmittel befreit. Die so erhaltenen Rohextrakte wurden für weitere Untersuchungen bei -39 ${ }^{\circ} \mathrm{C}$ aufbewahrt. Die Aktivitätstests basierten auf dem in der Arbeitsgruppe etablierten Plattendiffusionstests, die für alle untersuchten Mikroorganismen eingesetzt werden. Die Ergebnisse sind in den folgenden Tabellen 3 bis Tabelle 8 dargestellt. 
Tabelle 4: Aktivitäten der entfetteten Pilzextrakte im Plattendiffusionstest.

\begin{tabular}{|c|c|c|c|c|c|c|c|c|c|c|}
\hline Stamm & KMPB & Artname & Form & Medium & Tü57 & Cand. & Mucor & Subt & E.coli & Aur. \\
\hline P-1 & Н 622 & $\begin{array}{c}\text { Lulworthia } \\
\text { sp. }\end{array}$ & $\mathrm{St} / \mathrm{Sch}$ & $\mathrm{M} / 1$ & - & - & - & - & - & - \\
\hline P-2 & H 960 & $\begin{array}{c}\text { Lulworthia } \\
\text { sp. }\end{array}$ & $\mathrm{St} / \mathrm{Sch}$ & $\mathrm{M} / 1$ & - & - & - & - & - & - \\
\hline $\mathrm{P}-3$ & H 14648 & $\begin{array}{c}\text { Zalerion } \\
\text { maritimum }\end{array}$ & Sch & $\mathrm{M} / 1$ & - & - & - & - & - & - \\
\hline $\mathrm{P}-4$ & H 82 & $\begin{array}{c}\text { Zalerion } \\
\text { maritimum }\end{array}$ & $\mathrm{St}$ & $\mathrm{M} / 1$ & - & - & - & - & 14 & 11 \\
\hline $\mathrm{P}-5$ & H 150 & $\begin{array}{c}\text { Zalerion } \\
\text { maritimum }\end{array}$ & $\mathrm{St} / \mathrm{Sch}$ & $\mathrm{M} / 1$ & - & - & - & - & - & - \\
\hline P-6 & PP 4032 & $\begin{array}{c}\text { Lulworthia } \\
\text { uniseptata }\end{array}$ & Sch & $\mathrm{M} / 1$ & - & - & - & - & - & - \\
\hline P-7 & H 958A & $\begin{array}{c}\text { Lulworthia } \\
\text { sp. }\end{array}$ & $\mathrm{St} / \mathrm{Sch}$ & $\mathrm{M} / 1$ & - & - & - & - & - & - \\
\hline P-8 & H 82 & $\begin{array}{c}\text { Zalerion } \\
\text { maritimum }\end{array}$ & Sch & $\mathrm{M} / 1$ & - & - & - & - & - & - \\
\hline P-9 & Н 671 & $\begin{array}{c}\text { Corollospora } \\
\text { maritima }\end{array}$ & Sch & $\mathrm{M} / 1$ & - & - & - & - & - & - \\
\hline P-10 & PP 4033 & $\begin{array}{l}\text { Lulworthia } \\
\text { uniseptata }\end{array}$ & Sch & $\mathrm{M} / 1$ & - & - & - & - & - & - \\
\hline P-11 & PP 0148 & $\begin{array}{c}\text { Lulworthia } \\
\text { sp. }\end{array}$ & $\mathrm{St} / \mathrm{Sch}$ & $\mathrm{M} / 1$ & - & - & - & - & - & - \\
\hline P-12 & PP 2597 & $\begin{array}{c}\text { Lulworthia } \\
\text { sp. }\end{array}$ & Sch & $\mathrm{M} / 1$ & - & - & - & - & - & - \\
\hline $\mathrm{P}-13$ & H 671 & $\begin{array}{c}\text { Corollospora } \\
\text { maritima }\end{array}$ & St & $\mathrm{M} / 1$ & $\mathrm{NA}$ & - & NA & - & - & - \\
\hline P-14 & PP 1973 & $\begin{array}{c}\text { Lulworthia } \\
\text { medusa }\end{array}$ & Sch & $\mathrm{M} / 1$ & NA & - & NA & - & - & - \\
\hline P-15 & PP 4032 & $\begin{array}{l}\text { Lulworthia } \\
\text { uniseptata }\end{array}$ & $\mathrm{St}$ & $\mathrm{M} / 1$ & NA & - & - & - & - & 15 \\
\hline P-16 & H 957 & $\begin{array}{c}\text { Lulworthia } \\
\text { sp. }\end{array}$ & St/Sch & $\mathrm{M} / 1$ & - & - & NA & - & - & 11 \\
\hline P-17 & PP 4033 & $\begin{array}{l}\text { Lulworthia } \\
\text { uniseptata }\end{array}$ & $\mathrm{St}$ & $\mathrm{M} / 1$ & - & - & - & - & - & NA \\
\hline P-18 & Kontr. & Kontrolle & Kontr. & $\begin{array}{c}\text { Kontrol- } \\
\text { le }\end{array}$ & NA & - & NA & - & - & NA \\
\hline P-19 & H 970 & $\begin{array}{c}\text { Lulworthia } \\
\text { sp. }\end{array}$ & St/Sch & $\mathrm{M} / 1$ & - & - & - & - & - & - \\
\hline P-20 & H 992 & $\begin{array}{c}\text { Lulworthia } \\
\text { Sp. }\end{array}$ & St/Sch & $\mathrm{M} / 1$ & - & - & - & - & - & - \\
\hline P-21 & PP 1973 & $\begin{array}{c}\text { Lulworthia } \\
\text { Medusa }\end{array}$ & $\mathrm{St}$ & $\mathrm{M} / 1$ & - & - & - & - & - & NA \\
\hline $\mathrm{P}-22$ & H 485 & $\begin{array}{c}\text { Zalerion } \\
\text { Varium }\end{array}$ & St/Sch & $\mathrm{M} / 1$ & - & - & - & - & - & NA \\
\hline P-23 & H 994 & $\begin{array}{c}\text { Lulworthia } \\
\text { sp. }\end{array}$ & St/Sch & $\mathrm{M} / 1$ & - & - & - & - & - & NA \\
\hline P-24 & H 969 & $\begin{array}{c}\text { Lulworthia } \\
\text { sp. }\end{array}$ & $\mathrm{St} / \mathrm{Sch}$ & $\mathrm{M} / 1$ & - & - & - & - & - & NA \\
\hline P-25 & H 14648 & $\begin{array}{c}\text { Zalerion } \\
\text { maritimum }\end{array}$ & $\mathrm{St}$ & $\mathrm{M} / 1$ & - & - & - & - & - & - \\
\hline \multicolumn{11}{|c|}{$\begin{array}{c}* \text { Scenedesmus subspicatus, Cand } .=\text { Candida albicans, Mucor }=\text { Mucor miehei, E.coli }=\text { Es- } \\
\text { cherichia coli, Tü57 = Streptomyces viridochromogenes, Subt. }=\text { Bacillus subtilis, Aur. = Staphylo- } \\
\text { coccus aureus. }\end{array}$} \\
\hline
\end{tabular}


Tabelle 5: Aktivitäten der entfetteten Pilzextrakte im Plattendiffusionstest.

\begin{tabular}{|c|c|c|c|c|c|c|c|c|c|c|c|c|}
\hline Interne Nr. & $\begin{array}{c}\text { Stamm- } \\
\text { Nr. }\end{array}$ & Artname & Form & Kultur & $\begin{array}{l}\text { Menge/ } \\
\text { Farbe }\end{array}$ & \begin{tabular}{|c|} 
Algen- \\
aktivität
\end{tabular} & Cand. & Mucor & Ecoli & Tü57 & Subt. & Aur. \\
\hline 32 & H 950 & Lulworthia sp. & Stand & $\mathrm{M} / 1$ & Braun & 0 & 0 & 0 & 0 & 0 & 0 & 0 \\
\hline 04 & H 950 & Lulworthia sp. & Schüttel & $\mathrm{M} / 1$ & Hellbraun & 0 & 0 & 0 & 0 & 11 & 0 & $\mathrm{NA}$ \\
\hline 26 & H $951 \mathrm{~B}$ & Lulworthia sp. & Stand & $\mathrm{M} / 1$ & Dunkelbraun & 0 & 0 & 0 & 0 & 0 & 0 & $\mathrm{NA}$ \\
\hline 25 & H $951 \mathrm{~B}$ & Lulworthia sp. & Schüttel & $\mathrm{M} / 1$ & Hellbraun & 0 & 0 & 0 & 11 & 0 & 0 & NA \\
\hline 29 & H 952 B & Lulworthia $\mathrm{sp}$. & Stand & $\mathrm{M} / 1$ & Dunkelbraun & 0 & 0 & 0 & 0 & 0 & 0 & $\mathrm{NA}$ \\
\hline 27 & H 952 B & Lulworthia sp. & Schüttel & $\mathrm{M} / 1$ & Hellbraun & 0 & 0 & 0 & 12 & 0 & 0 & $\mathrm{NA}$ \\
\hline 30 & H 953 & Lulworthia sp. & Stand & $\mathrm{M} / 1$ & Dunkelbraun & 0 & 0 & 0 & 0 & 0 & 0 & $\mathrm{NA}$ \\
\hline 31 & H 953 & Lulworthia sp. & Schüttel & $\mathrm{M} / 1$ & Braun & 30* & 20 & 24 & 15 & 0 & 12 & 12 \\
\hline 14 & H 954 & Lulworthia sp. & Stand & $\mathrm{M} / 1$ & Dunkelbraun & 0 & 0 & 0 & 0 & 0 & 0 & $\overline{\mathrm{NA}}$ \\
\hline 07 & H 954 & Lulworthia sp. & Schüttel & $\mathrm{M} / 1$ & Braun & 0 & 0 & 0 & 0 & 0 & 0 & $\overline{\mathrm{NA}}$ \\
\hline 11 & H 955 & Lulworthia $\mathrm{sp}$. & Stand & $\mathrm{M} / 1$ & Dunkelbraun & 0 & 0 & 0 & 0 & 0 & 0 & $\mathrm{NA}$ \\
\hline 16 & H 955 & Lulworthia sp. & Schüttel & $\mathrm{M} / 1$ & Braun & 0 & 0 & 0 & 12 & 0 & 0 & $\mathrm{NA}$ \\
\hline 28 & H 956 & Lulworthia sp. & Stand & $\mathrm{M} / 1$ & Dunkelbraun & 0 & 0 & 0 & 0 & 0 & 0 & NA \\
\hline 34 & H 956 & Lulworthia sp. & Schüttel & $\mathrm{M} / 1$ & Hellbraun & 0 & 0 & 0 & 0 & 0 & 0 & 0 \\
\hline 06 & H 959 & Lulworthia sp. & Stand & $\mathrm{M} / 1$ & Braun & 0 & 0 & 0 & 0 & 0 & 0 & $\mathrm{NA}$ \\
\hline 10 & H 959 & Lulworthia sp. & Schüttel & $\mathrm{M} / 1$ & Braun & 0 & 0 & 0 & 0 & 0 & 0 & $\mathrm{NA}$ \\
\hline 21 & H 965 & Lulworthia $\mathrm{sp}$. & Stand & $\mathrm{M} / 1$ & Dunkelbraun & 0 & 0 & 0 & 0 & 0 & 0 & $\mathrm{NA}$ \\
\hline 22 & H 965 & Lulworthia sp. & Schüttel & $\mathrm{M} / 1$ & Hellbraun & 0 & 0 & 0 & 0 & 0 & 0 & NA \\
\hline & \multicolumn{12}{|c|}{$\begin{array}{c}* \text { Scenedesmus subspicatus, Cand. }=\text { Candida albicans, Mucor }=\text { Mucor miehei, Ecoli }=\text { Escherichia coli, } \text { Tü57 }=\text { Streptomyces viridochromogenes } \\
\text { Subt. }=\text { Bacillus subtilis, Aur. }=\text { Staphylococcus aureus. }\end{array}$} \\
\hline
\end{tabular}


Tabelle 6: Aktivitäten der entfetteten Pilzextrakte im Plattendiffusionstest.

\begin{tabular}{|c|c|c|c|c|c|c|c|c|c|c|c|c|}
\hline Interne Nr. & $\begin{array}{c}\text { Stamm- } \\
\text { Nr. }\end{array}$ & Artname & $\begin{array}{l}\text { Kuktur- } \\
\text { form }\end{array}$ & Kultur & Farbe & Algen & Cand. & Muc. & Ecoli & Tü57 & Subt. & Aur. \\
\hline 12 & H 966 & Lulworthia sp. & Stand & $\mathrm{M} / 1$ & Dunkelbraun & 0 & 0 & 0 & 0 & 0 & 0 & NA \\
\hline 01 & H 966 & Lulworthia sp. & Schüttel & $\mathrm{M} / 1$ & Braun & 0 & 0 & 0 & 0 & 0 & 0 & NA \\
\hline 19 & H 986 & Lulworthia sp. & Stand & $\mathrm{M} / 1$ & Dunkelbraun & 0 & 0 & 0 & 0 & 0 & 0 & NA \\
\hline 23 & H 993 & Lulworthia sp. & Stand & $\mathrm{M} / 1$ & Dunkelbraun & 0 & 0 & 0 & 0 & 0 & 0 & NA \\
\hline 02 & H 993 & Lulworthia sp. & Schüttel & $\mathrm{M} / 1$ & Hellbraun & 0 & 0 & 0 & 0 & 0 & 0 & NA \\
\hline 03 & H 997 & Lulworthia sp. & Stand & $\mathrm{M} / 1$ & Braun & 0 & 0 & 0 & 0 & 11 & 0 & NA \\
\hline 17 & PP 1249 & Lulworthia fucicola & Stand & $\mathrm{M} / 1$ & Braun & 0 & 0 & 0 & 0 & 0 & 0 & NA \\
\hline 15 & PP 2597 & Lulworthia sp. & Stand & $\mathrm{M} / 1$ & Dunkelbraun & 0 & 0 & 0 & 0 & 0 & 0 & NA \\
\hline 33 & IFO 7836 & Zalerion xylestrix & Stand & $\mathrm{M} / 1$ & Dunkelbraun & 0 & 0 & 0 & 0 & 0 & 0 & 0 \\
\hline 05 & IFO 7836 & Zalerion xylestrix & Schüttel & $\mathrm{M} / 1$ & Hellbraun & $13^{*}$ & 0 & 0 & 0 & 0 & 0 & NA \\
\hline 18 & $\begin{array}{c}\text { IFO } \\
32133\end{array}$ & Lulworthia crassa & Stand & $\mathrm{M} / 1$ & Braun & 0 & 0 & 0 & 0 & 0 & 0 & $\mathrm{NA}$ \\
\hline 08 & $\begin{array}{c}\text { IFO } \\
32133 \\
\end{array}$ & Lulworthia crassa & Schüttel & $\mathrm{M} / 1$ & Braun & 0 & 0 & 0 & 0 & 12 & 0 & 0 \\
\hline 09 & $\begin{array}{c}\text { IFO } \\
32135 \\
\end{array}$ & $\begin{array}{c}\text { Lulworthia lignoare- } \\
\text { naria }\end{array}$ & Stand & $\mathrm{M} / 1$ & Dunkelbraun & 0 & 15 & 0 & 12 & 0 & 0 & $\mathrm{NA}$ \\
\hline 20 & 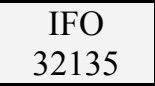 & $\begin{array}{l}\text { Lulworthia lignoare- } \\
\text { naria }\end{array}$ & Schüttel & $\mathrm{M} / 1$ & Hellbraun & 0 & 18 & 0 & 15 & 11 & 13 & NA \\
\hline 13 & $\begin{array}{c}\text { IFO } \\
32137 \\
\end{array}$ & Lulworthia uniseptata & Stand & $\mathrm{M} / 1$ & Braun & 0 & 0 & 0 & 0 & 0 & 0 & $\mathrm{NA}$ \\
\hline 24 & $\begin{array}{c}\text { IFO } \\
32137\end{array}$ & Lulworthia uniseptata & Schüttel & $\mathrm{M} / 1$ & Hellbraun & 0 & 0 & 0 & 0 & 0 & 0 & $\mathrm{NA}$ \\
\hline & \multicolumn{12}{|c|}{$\begin{array}{c}* \text { Scenedesmus subspicatus, } \text { Cand. }=\text { Candida albicans, } \text { Mucor }=\text { Mucor miehei, Ecoli }=\text { Escherichia coli, } \text { Tü } 57=\text { Streptomyces viridochromogene } \\
\text { Subt. }=\text { Bacillus subtilis, Aur. }=\text { Staphylococcus aureus. }\end{array}$} \\
\hline
\end{tabular}


Tabelle 7: Aktivitäten der entfetteten Pilzextrakte im Plattendiffusionstest.

\begin{tabular}{|c|c|c|c|c|c|c|c|c|c|c|c|c|c|c|}
\hline \begin{tabular}{|c|} 
Interne \\
$\mathrm{Nr}$.
\end{tabular} & $\begin{array}{c}\text { Stamm- } \\
\text { Nr. }\end{array}$ & Info & $\begin{array}{c}\text { PH } \\
\text { Wert } \\
\text { Anf. }\end{array}$ & $\begin{array}{l}\text { PH- } \\
\text { Wert } \\
\text { Ende }\end{array}$ & Kultur & Farbe & Algen & Cand. & Mucor & Ecoli. & Tü57 & Subt. & Aur. & $\begin{array}{c}\text { Salinen- } \\
\text { Krebse } \\
(\%)\end{array}$ \\
\hline 01 & 969 & M1 Test & 6.68 & 5.73 & $\mathrm{M} / 1$ & Hellbraun & 0 & 0 & 0 & 0 & 0 & 0 & 0 & 100 \\
\hline 02 & PP4033 & Sch 10 & & & $\mathrm{M} / 1$ & Dunkelbraun & 0 & 0 & 0 & 11 & 12 & 0 & 0 & 100 \\
\hline 03 & PP4032 & Sch & & & $\mathrm{M} / 1$ & Hellbraun & 0 & 0 & 0 & 11 & 11 & 11 & 0 & 19 \\
\hline 04 & 9.PP4032 & Test M1 & 5.40 & & $\mathrm{M} / 1$ & Dunkelbraun & 0 & 0 & 0 & 0 & 0 & 0 & 0 & 8 \\
\hline 05 & PP1973 & Sch 10 & & & $\mathrm{M} / 1$ & Hellbraun & 0 & 0 & 0 & 0 & 0 & 0 & 0 & 100 \\
\hline 06 & PDL Test & $\begin{array}{c}\text { P995/pp4032/ } \\
\text { PP1249/ } \\
\text { IFO32135 } \\
\end{array}$ & & & $\mathrm{M} / 1$ & Hellbraun & 0 & 0 & 0 & 0 & 0 & 0 & 0 & 100 \\
\hline 07 & PDL Test & $\begin{array}{c}\mathrm{C}(950 / 951 / \\
622)\end{array}$ & 5.37 & & $\mathrm{M} / 1$ & Hellbraun & 0 & 0 & 0 & 0 & 0 & 0 & 0 & 100 \\
\hline 08 & PDL 622 & Sch & & & $\mathrm{M} / 1$ & Braun & 0 & 0 & 0 & 14 & 0 & 13 & 0 & 100 \\
\hline 09 & 4033 & M1 Test & 6.12 & 6.17 & $\mathrm{M} / 1$ & Dunkelbraun & 0 & 0 & 0 & 0 & 0 & 0 & 0 & 100 \\
\hline 10 & $\begin{array}{c}\text { Test } \\
\text { PP1973 }\end{array}$ & Sch 1:10 & 6.47 & 5.77 & $\mathrm{M} / 1$ & Dunkelbraun & 0 & 0 & 0 & 0 & 0 & 0 & 0 & 8 \\
\hline 11 & H 969 & ST 10 & & & $\mathrm{M} / 1$ & Dunkelbraun & 0 & 0 & 0 & 0 & 0 & 0 & 0 & 15 \\
\hline 12 & 953 & Sch & & & PDL & Hellbraun & 0 & 0 & 0 & 0 & 0 & 0 & 0 & 100 \\
\hline 13 & 954 & Sch & & & PDL & Hellbraun & 0 & 0 & 0 & 0 & 0 & 0 & 0 & 4 \\
\hline 14 & 953 & $\mathrm{St}$ & & & PDL & Hellbraun & 0 & 0 & 0 & 0 & 0 & 0 & 0 & 8 \\
\hline 15 & 957 & G8YN1:1/St & & & & Hellbraun & 0 & 0 & 0 & 0 & 0 & 0 & 0 & 10 \\
\hline 16 & 955 & GPYN1:1/St & & & & Hellbraun & 0 & 0 & 0 & 0 & 0 & 0 & 0 & 20 \\
\hline 17 & PP2597 & $\mathrm{St}$ & & & PDL & Braun & 0 & 0 & 0 & 0 & 0 & 0 & 0 & 0 \\
\hline 18 & PDS Test & $\begin{array}{c}\text { P.969, 970, } \\
972,973\end{array}$ & 5.30 & 4.40 & & Hellbraun & 0 & 0 & 0 & 0 & 0 & 0 & 0 & 100 \\
\hline
\end{tabular}


Tabelle 8: Aktivitäten der entfetteten Pilzextrakte im Plattendiffusionstest.

\begin{tabular}{|c|c|c|c|c|c|c|c|c|c|c|c|c|c|c|}
\hline $\begin{array}{c}\text { Interne } \\
\text { Nr. }\end{array}$ & $\begin{array}{l}\text { Stamm- } \\
\text { Nr. }\end{array}$ & $\overline{\text { Info }}$ & $\begin{array}{l}\text { PH- } \\
\text { Wert } \\
\text { Anf. }\end{array}$ & $\begin{array}{l}\text { PH- } \\
\text { Wert } \\
\text { Ende }\end{array}$ & Kultur & Farbe & $\overline{\text { Algen }}$ & Cand. & Muc. & $\overline{\text { Ecoli }}$ & Tü57 & Subt. & Aur. & $\begin{array}{l}\text { Salinen- } \\
\text { Krebse } \\
\text { (\%) }\end{array}$ \\
\hline 19 & P14648 & $\begin{array}{c}\text { M1 Test / Sch } \\
1-10\end{array}$ & 6.00 & 5.70 & M1 & Hellbraun & 0 & 0 & 0 & 0 & 0 & 0 & 0 & 100 \\
\hline 20 & 954 & B1+1:1 / Sch & & & & Hellbraun & 0 & 0 & 0 & 0 & 0 & 0 & 0 & 6 \\
\hline 21 & 960 & $\mathrm{St}$ & & & PDL & Dunkelbraun & 0 & 0 & 0 & 0 & 0 & 0 & 0 & 3 \\
\hline 22 & 960 & Sch & & & PDL & Hellbraun & 10 & 0 & 0 & 0 & 0 & 0 & 0 & 0 \\
\hline 23 & 154 & $\mathrm{~B} 1+1: 1 / \mathrm{St}$ & & & & Hellbraun & 0 & 0 & 0 & 0 & 13 & 0 & 0 & 100 \\
\hline 24 & $957-955$ & $\begin{array}{c}\text { Test GPYN } \\
1: 1 \\
\end{array}$ & 7.71 & 6.69 & & Hellbraun & 0 & 0 & 0 & 0 & 0 & 0 & 0 & 100 \\
\hline 25 & 954 & $\mathrm{St}$ & & & PDL & Hellbraun & 0 & 0 & 0 & 0 & 0 & 0 & 0 & 5 \\
\hline 26 & 957 & $\begin{array}{l}\text { GPYN 1:1 } \\
\text { /Sch }\end{array}$ & & & & Hellbraun & 0 & 0 & 0 & 0 & 0 & 0 & 0 & 0 \\
\hline 27 & 955 & $\begin{array}{c}\text { GPYN 1:1 } \\
\text { /Sch }\end{array}$ & & & & Braun & 0 & 0 & 0 & 0 & 0 & 0 & 0 & 1 \\
\hline 28 & $\begin{array}{ll}\mathrm{H} 14648 \\
\end{array}$ & Sch & & & M1/PDL & Hellbraun & 0 & 0 & 0 & 0 & 0 & 0 & 0 & 3 \\
\hline 29 & PP2597 & Sch & & & PDL & Hellbraun & 0 & 0 & 0 & 0 & 0 & 0 & 0 & 100 \\
\hline 30 & 950 & Sch & & & PDL & Hellbraun & 0 & 0 & 0 & 0 & 0 & 0 & 0 & 5 \\
\hline 31 & PDL & & & & & Hellbraun & 0 & 0 & 0 & 0 & 0 & 0 & 0 & 1 \\
\hline 32 & $\mathrm{~B} 1+1: 1$ & $\begin{array}{c}\text { Test } \\
(950+954)\end{array}$ & 7.05 & 6.58 & & Braun & 0 & 0 & 0 & 0 & 0 & 0 & 0 & 18 \\
\hline \multirow[t]{2}{*}{33} & 950 & $\mathrm{~B} 1+1: 1 / \mathrm{St}$ & & & & Braun & 0 & 0 & 0 & 0 & 0 & 0 & 0 & 6 \\
\hline & \multicolumn{14}{|c|}{$\begin{array}{c}* \text { Scenedesmus subspicatus, Cand. }=\text { Candida albicans, Mucor }=\text { Mucor miehei, Ecoli }=\text { Escherichia coli, } \text { Tü } 57=\text { Streptomyces viridochromogenes, } \\
\text { Subt. }=\text { Bacillus subtilis, Aur. }=\text { Staphylococcus aureus. } \\
\text { DMSO-Kontr. } 0 \%\end{array}$} \\
\hline
\end{tabular}




\section{Stamm KMPB H963 (Lulworthia sp.)}

Der marine Pilz KMPB H963 (Lulworthia sp.) wurde im Rahmen eines BMBFProjektes in der Kooperation mit dem Alfred-Wegener-Institut für Polar- und Meeresforschung in Bremerhaven kultiviert.

\section{Primärscreening}

Der Pilzextrakt zeigte im biologischen Screening keine Aktivität. Im HPLCChromatogramm vom Rohextrakt fand man mehrere interessante Peaks, die ausschlaggebend für die Auswahl dieses Stammes für die Kultivierung im großen Maßstab waren (s. Kap. Primärscreening der Pilzextrakte).

\section{Fermentation und Aufarbeitung}

Der Pilz wurde auf Potato-Dextrose-Broth mit natürlichem Meerwasser innerhalb von 35 Tagen bei $20^{\circ} \mathrm{C}$ kultiviert. Die Biomasse wurde über $17 \mathrm{GF} / \mathrm{C}$-Filter abfiltriert und gefriergetrocknet. Man erhielt aus 20 Liter Kultur 6.3 g Biomasse.

Die Biomasse wurde in $200 \mathrm{ml} \mathrm{MeOH}$ aufgenommen, 5 min mit dem Ultraturrax behandelt und nach $12 \mathrm{~h}$ abfiltriert. Man extrahierte den Rückstand jeweils dreimal mit $100 \mathrm{ml}$ EtOAc. Nach dem Eindampfen der vereinigten org. Phasen wurde der Extrakt dreimal mit je $50 \mathrm{ml}$ Cyclohexan entfettet. Man erhielt $340 \mathrm{mg}$ eines hellbraunen Rohextraktes.

\section{Isolierung der Metabolite}

Der Rohextrakt wurde in $10 \mathrm{ml} \mathrm{CH}_{2} \mathrm{Cl}_{2} / \mathrm{MeOH}$ 8:2 aufgenommen und durch Säulenchromatographie an Kieselgel $(5 \times 60 \mathrm{~cm}$, Essigester/Dichlormethan-Stufengradient: $11 \mathrm{CH}_{2} \mathrm{Cl}_{2}, 11 \mathrm{CH}_{2} \mathrm{Cl}_{2} / 5 \%$ Essigester, $11 \mathrm{CH}_{2} \mathrm{Cl}_{2} / 10 \%$ Essigester, 11 $\mathrm{CH}_{2} \mathrm{Cl}_{2} / 20 \%$ Essigester, $11 \mathrm{CH}_{2} \mathrm{Cl}_{2} / 50 \%$ Essigester, 21 Essigester) vorgetrennt. Man erhielt zwei Fraktionen I und II.

Aus der Fraktion I erhielt man nach präparativer HPLC an RP18 (Standardgradient, Detektion im Bereich von 190 bis $620 \mathrm{~nm}) 18 \mathrm{mg} \mathrm{D}(+)$-Maltose $\left(\mathbf{2 6}, R_{\mathrm{f}}=16 \mathrm{~min}\right)$, 
$62 \mathrm{mg} \alpha, \alpha$-Trehalose $\left(\mathbf{2 8 a}, R_{\mathrm{f}}=21 \mathrm{~min}\right)$ und $19 \mathrm{mg}$ Mannose $\left(\mathbf{2 7}, R_{\mathrm{f}}=23 \mathrm{~min}\right)$; die Retentionszeiten beziehen sich auf den Gradienten.

Fraktion II wurde durch Chromatographie an Sephadex LH-20 $(3 \times 120 \mathrm{~cm}$, $\mathrm{CH}_{2} \mathrm{Cl}_{2} / \mathrm{MeOH}$; 4:6) gereinigt und ergab 9.6 mg L-Tyrosin (25).

L-Tyrosin (25): $\mathrm{C}_{9} \mathrm{H}_{11} \mathrm{NO}_{3}, \boldsymbol{R}_{\mathbf{f}}=0.20 ; \mathrm{CH}_{2} \mathrm{Cl}_{2} / \mathrm{MeOH}$, 95:5. ESI-MS: $m / z(\%)=181$ $\left(\mathrm{M}^{+}, 100\right), 183$ (35), 163 (12), 135 (20). ${ }^{1} \mathbf{H}-\mathbf{N M R}\left(\left[\mathrm{D}_{6}\right]-\mathrm{DMSO}, 300 \mathrm{MHz}\right): \delta=9.00$ (br s, H/D-austauschbar, $1 \mathrm{H}, \mathrm{OH}), 7.00\left(\mathrm{~d},{ }^{3} \mathrm{~J}=9 \mathrm{~Hz}, 2 \mathrm{H}, 2^{\prime}-\mathrm{H}, 6^{\prime}-\mathrm{H}\right), 6.62\left(\mathrm{~d},{ }^{3} \mathrm{~J}=\right.$ $\left.9 \mathrm{~Hz}, 2 \mathrm{H}, 3^{\prime}-\mathrm{H}, 5^{\prime}-\mathrm{H}\right), 4.05$ (dd, $\left.{ }^{3} J=8 \mathrm{~Hz},{ }^{3} \mathrm{~J}=8 \mathrm{~Hz}, 1 \mathrm{H}, 2-\mathrm{H}\right), 2.88-2.60$ (m, $2 \mathrm{H}$, $\left.\mathrm{CH}_{2}\right) .{ }^{13} \mathrm{C}-\mathrm{NMR}\left(\left[\mathrm{D}_{6}\right]-\mathrm{DMSO}, 300 \mathrm{MHz}\right): 175.1\left(\mathrm{C}_{\mathrm{q}}-1\right), 155.5\left(\mathrm{C}_{\mathrm{q}}-4\right)$ ') $130.6(\mathrm{CH}-$

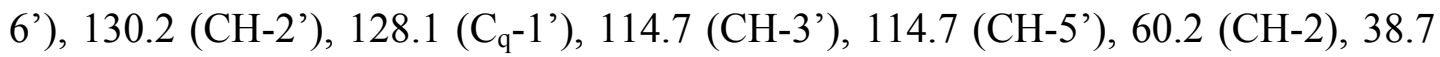
$\left(\mathrm{CH}_{2}-3\right)$.

D(+)-Maltose (26): $\mathrm{C}_{6} \mathrm{H}_{12} \mathrm{O}_{6},(+)$-ESI-MS: $m / z(\%)=707\left([2 \mathrm{M}+\mathrm{Na}]^{+}, 100\right), 365$ $\left([\mathrm{M}+\mathrm{Na}]^{+}, 27\right) .{ }^{1} \mathbf{H}-\mathbf{N M R}\left(\mathrm{D}_{2} \mathrm{O}, 300 \mathrm{MHz}\right): \delta=5.24\left(\mathrm{~d},{ }^{3} J=3 \mathrm{~Hz}, 1 \mathrm{H}\right), 5.06\left(\mathrm{~d},{ }^{3} J\right.$ $=3 \mathrm{~Hz}, 1 \mathrm{H}), 4.48\left(\mathrm{~d},{ }^{3} \mathrm{~J}=8 \mathrm{~Hz}, 1 \mathrm{H}\right), 3.82-3.04(\mathrm{br} \mathrm{m}, 12 \mathrm{H}), 3.26\left(\mathrm{t},{ }^{3} \mathrm{~J}=10 \mathrm{~Hz}, 2\right.$ $\mathrm{H}), 3.12\left(\mathrm{t},{ }^{3} \mathrm{~J}=9 \mathrm{~Hz}, 1 \mathrm{H}\right)$; Mischung aus $60 \% \alpha$ - und $40 \% \beta$-Form.

Mannose (27): $\mathrm{C}_{12} \mathrm{H}_{22} \mathrm{O}_{11},(+)$-ESI-MS: : $m / z(\%)=386\left([2 \mathrm{M}+\mathrm{Na}]^{+}, 100\right), 205$ $\left([\mathrm{M}+\mathrm{Na}]^{+}, 35\right) .{ }^{1} \mathbf{H}-\mathbf{N M R}\left(\mathrm{D}_{2} \mathrm{O}, 300 \mathrm{MHz}\right): \delta=3.80-3.55(\mathrm{~m} \mathrm{br}) .{ }^{13} \mathbf{C}-\mathbf{N M R}\left(\mathrm{D}_{2} \mathrm{O}\right.$, $300 \mathrm{MHz}): \delta=82.7(\mathrm{CH}-4), 70.9(\mathrm{CH}-5), 69.3(\mathrm{CH}-1,-2), 63.0\left(\mathrm{CH}_{2}-11\right), 49.0(\mathrm{CH}-$ $6)$.

$\alpha, \alpha$-Trehalose (28a): $\mathrm{C}_{12} \mathrm{H}_{22} \mathrm{O}_{11},{ }^{1} \mathbf{H}$-NMR $\left(\mathrm{D}_{2} \mathrm{O}, 300 \mathrm{MHz}\right): \delta=4.85\left(\mathrm{~d},{ }^{3} J=10\right.$ Hz, 2 H), 3.98-3.20 (br m,). ${ }^{13} \mathbf{C}-\mathbf{N M R}\left(\mathrm{D}_{2} \mathrm{O}, 300 \mathrm{MHz}\right.$ ): $\delta=93.1$ (CH-1, 1'), 72.8 (CH-5, 5'), 72.3 (CH-2, 2'), 71.6 (CH-3, 3'), 71.3 (CH-3'), 69.7 (CH-4, 4'), 63.8 $\left(\mathrm{CH}_{2}-6\right), 60.7\left(\mathrm{CH}_{2}-6\right)$.

Acetylierung von 28a: Eine Mischung aus $30 \mathrm{mg}$ des Disaccharids 28a, $5 \mathrm{ml}$ Acetanhydrid, $0.5 \mathrm{ml}$ Pyridin und einer katalytischen Menge 4-N,N-Dimethylaminopyridin wurde 60 min unter Rückfluss gerührt, wobei sich die Lösung leicht gelblich 
verfärbte. Man hydrolysierte vorsichtig unter Eiskühlung mit $30 \mathrm{ml}$ Methanol/Wasser (1:1). Dabei fiel ein weißer Niederschlag aus, der abfiltriert und mit 50 $\mathrm{ml}$ Wasser gewaschen wurde. Die Reinigung des Produktes erfolgte durch präparative HPLC an RP18 $\left(\mathrm{H}_{2} \mathrm{O} / 10 \% \mathrm{MeCN}\right.$ Anstieg auf $80 \% \mathrm{MeCN}$ in $30 \mathrm{~min}$, dann auf $100 \% \mathrm{MeCN}$ in 10 min, über 10 min $100 \% \mathrm{MeCN}$ und dann Abfall auf $10 \%$ in 1 min, Fluss $10.0 \mathrm{ml} / \mathrm{min}$, Detektion im Bereich von 190 bis $620 \mathrm{~nm}$ ). Man erhielt 40.2 mg (68 \%) $\alpha, \alpha$-Trehaloseperacetat (28b) $\mathrm{C}_{28} \mathrm{H}_{38} \mathrm{O}_{19}$, CI-MS: $701\left([\mathrm{M}+\mathrm{Na}]^{+}, 100\right)$. ${ }^{1}$ H-NMR $\left(\mathrm{CDCl}_{3}, 200 \mathrm{MHz}\right): \delta=5.42\left(\mathrm{t},{ }^{3} \mathrm{~J}=10 \mathrm{~Hz}, 2 \mathrm{H}\right), 5.21\left(\mathrm{~d},{ }^{3} \mathrm{~J}=3 \mathrm{~Hz}, 2 \mathrm{H}\right)$, $4.98(\mathrm{~m}, 4 \mathrm{H}), 4.18\left(\mathrm{dd},{ }^{3} \mathrm{~J}=6 \mathrm{~Hz},{ }^{3} J=6 \mathrm{~Hz}, 2 \mathrm{H}\right), 3.98(\mathrm{~m}, 4 \mathrm{H}), 2.01\left(\mathrm{~s}, 3 \mathrm{H}, \mathrm{CH}_{3}\right)$, 2.00 (s, $\left.3 \mathrm{H}, \mathrm{CH}_{3}\right), 1.99$ (s, $\left.3 \mathrm{H}, \mathrm{CH}_{3}\right), 1.98$ (s, $\left.3 \mathrm{H}, \mathrm{CH}_{3}\right) .{ }^{13} \mathbf{C}-\mathbf{N M R}\left(\mathrm{CDCl}_{3}, 300\right.$

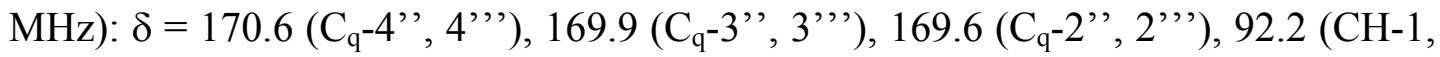
1'), 69.9 (CH-5, 5'), 69.8 (CH-2, 2'), 68.5 (CH-4, 4'), 68.1 (CH-3, 3'), 61.7 ( $\mathrm{CH}_{2}-6$, 6'), $20.6\left(8 \mathrm{CH}_{3}\right)$.

\section{Stamm GW 7/2495}

Bei dem Stamm GW 7/2495 handelt es sich um einen terrestrischen Streptomyceten, der bei der Firma bioLeads (Heidelberg) in Reinkultur gehalten wird. Auf Agar bildet der Stamm ein weißes Mycel. Der Agar wies in der Nähe der Bakterienkolonien keine Farbveränderung auf.

\section{Primärscreening}

Mit einer $\mathrm{M}_{2}$-Schrägagarkultur des Stammes wurden drei Agarplatten beimpft und $72 \mathrm{~h}$ bei $28^{\circ} \mathrm{C}$ inkubiert. Mit diesen Kulturen beimpfte man fünf mit je $200 \mathrm{ml}$ des gleichen Nährmediums gefüllte 1 1-Schikanekolben und züchtete $72 \mathrm{~h}$ bei $28{ }^{\circ} \mathrm{C}$ mit 95 Upm an. In den Hemmtests konnte lediglich eine schwache Aktivität gegen Streptomyces viridochromogenes (Hemmhofdurchmesser: $12 \mathrm{~mm}$ ) festgestellt werden.

\section{Diskontinuierliche Kultivierung von GW 7/2495}

Man fermentierte im 20-1-Maßstab in einem mit GALOP optimierten Nährmedium aus $6 \mathrm{~g}$ Maltose, $92 \mathrm{~g}$ Hefeextrakt, $60 \mathrm{~g}$ Glucose und $40 \mathrm{~g} \mathrm{CaCl}_{2}$ in 101 Leitungswas- 
ser. Beimpft wurde mit 21 einer $72 \mathrm{~h}$ bei $28{ }^{\circ} \mathrm{C}$ inkubierten Schüttelkultur $\left(\mathrm{M}_{2}\right.$ Nährmedium).

Die Fermentation führte man bei $28^{\circ} \mathrm{C}, 331 \mathrm{Luft} / \mathrm{min}, \mathrm{pH} 6.9 \pm 1.5(2 \mathrm{~N} \mathrm{NaOH}, 2 \mathrm{~N}$ $\mathrm{HCl}$ zur pH-Regulierung) und einer Rührgeschwindigkeit von $200 \mathrm{Upm}$ durch. Schaumbildung wurde durch regulierte Zugabe von insgesamt $20 \mathrm{ml}$ Niax verhindert. Die Kulturbrühe wurde 5 min mit dem Ultraturrax behandelt, mit der Filterpresse über Celite filtriert und das Filtrat mit EtOAc extrahiert.

Das Mycel wurde zunächst mit $\mathrm{MeOH}$ über $12 \mathrm{~h}$ aufgeschlossen. Danach digerierte man das Mycel dreimal mit jeweils 300 ml EtOAc. Nach dem Eindampfen der vereinigten org. Phasen erhielt man $1.7 \mathrm{~g}$ eines intensiv gelben Rohextraktes, der zweimal mit je $50 \mathrm{ml}$ Cyclohexan entfettet wurde.

Ein Bioautographie entwickelter Dünnschichtchromatogramme $\left(\mathrm{CH}_{2} \mathrm{Cl}_{2} / \mathrm{MeOH}\right.$, 95:5) der Essigester-Fraktion mit dem Testkeim Streptomyces viridochromogenes (Tü57) zeigte ausschließlich Aktivität bei einer gelbgefärbten Zone mit $R_{\mathrm{f}}=0.80$.

\section{Isolierung der Metabolite}

Den Rohextrakt löste man in $10 \mathrm{ml} \mathrm{CH}_{2} \mathrm{Cl}_{2} / \mathrm{MeOH}$ 8:2 und trennte durch Säulenchromatographie an Kieselgel $(5 \times 60 \mathrm{~cm}$, Essigester/Dichlormethan-Stufengradient: $11 \mathrm{CH}_{2} \mathrm{Cl}_{2}, 11 \mathrm{CH}_{2} \mathrm{Cl}_{2} / 5 \%$ Essigester, $11 \mathrm{CH}_{2} \mathrm{Cl}_{2} / 10 \%$ Essigester, $11 \mathrm{CH}_{2} \mathrm{Cl}_{2} / 20 \%$ Essigester, $11 \mathrm{CH}_{2} \mathrm{Cl}_{2} / 50 \%$ Essigester, 21 Essigester) vor. Aus der Fraktion I erhielt man nach der präparativen HPLC-Reinigung an RP18 (Standardgradient, Detektion im Bereich von 190 bis $620 \mathrm{~nm}) 84 \mathrm{mg}$ 2-Methylfervenulon (29).

2-Methylfervenulon (29): $\mathrm{C}_{8} \mathrm{H}_{9} \mathrm{O}_{3} \mathrm{~N}_{5}, \boldsymbol{R}_{\mathbf{f}}=0.80 ; \mathrm{CH}_{2} \mathrm{Cl}_{2} / \mathrm{MeOH}$, 95:5. EI-MS (70 $\mathrm{eV}): m / z(\%)=223.0705$ (100), 195 (36), 181 (32), 167 (10), 153 (28), 140 (15), 123 (18), 83 (23), 69 (10), 67 (34), 56 (13), 43 (63), 42 (10). ${ }^{1} \mathbf{H}-\mathbf{N M R}\left(\mathrm{CDCl}_{3}, 200\right.$ MHz): $\delta=3.76\left(\mathrm{~s}, 3 \mathrm{H}, \mathrm{CH}_{3}\right), 3.38\left(\mathrm{~s}, 3 \mathrm{H}, \mathrm{CH}_{3}\right), 3.36\left(\mathrm{~s}, 3 \mathrm{H}, \mathrm{CH}_{3}\right) .{ }^{13} \mathbf{C}-\mathbf{N M R}$ $\left(\mathrm{CDCl}_{3}, 300 \mathrm{MHz}\right): \delta=157.0\left(\mathrm{C}_{\mathrm{q}}-6 \mathrm{a}\right), 152.8\left(\mathrm{C}_{\mathrm{q}}-6\right), 148.8\left(\mathrm{C}_{\mathrm{q}}-4\right), 146.5\left(\mathrm{C}_{\mathrm{q}}-2 \mathrm{a}\right)$, $137.0\left(\mathrm{C}_{\mathrm{q}}-8\right), 42.1\left(\mathrm{CH}_{3}-1^{\prime}\right), 29.6\left(\mathrm{CH}_{3}-3^{\prime}\right), 28.6\left(\mathrm{CH}_{3}-5\right.$ '). IR (KBr): v = 3429 (br), $2925,2362,1731,1666,1618,1473,1437,1237,1032,763,742 \mathrm{~cm}^{-1}$. 


\section{Die kontinuierliche Kultur von GW 7/2495}

Man fermentierte im 10-1-Maßstab in einem mit GALOP optimierten Nährmedium aus $3 \mathrm{~g}$ Maltose, $46 \mathrm{~g}$ Hefeextrakt, $30 \mathrm{~g}$ Glucose und $20 \mathrm{~g} \mathrm{CaCl}_{2}$ in 101 Leitungswasser. Die Beimpfung des Durchflußfermenters erfolgte mit 21 einer $72 \mathrm{~h}$ bei $28{ }^{\circ} \mathrm{C}$ inkubierten Schüttelkultur.

Fermentation führte man kontinuierlich über sieben Tage bei $28{ }^{\circ} \mathrm{C}, 331 \mathrm{Luft} / \mathrm{min}$, pH $6.9 \pm 1.5(2 \mathrm{~N} \mathrm{NaOH}, 2 \mathrm{~N} \mathrm{HCl}$ zur pH-Regulierung) in dem 21 großen Durchflußfermenter durch.

Die Kulturbrühe wurde 3 min mit dem Ultraturrax behandelt und mit der Filterpresse über Celite filtriert. Man extrahierte den Überstand dreimal mit 21 EtOAc.

Das Mycel wurde über $12 \mathrm{~h}$ in $400 \mathrm{ml} \mathrm{MeOH}$ aufgeschlossen. Danach extrahierte man es dreimal mit jeweils $200 \mathrm{ml}$ EtOAc. Nach dem Eindampfen der vereinigten org. Phasen erhielt man $467 \mathrm{mg}$ eines rot gefärbten Rohextraktes, der zweimal mit je $50 \mathrm{ml}$ Cyclohexan entfettet wurde.

\section{Isolierung der Metaboliten}

Den Rohextrakt löste man in $10 \mathrm{ml} \mathrm{CH}_{2} \mathrm{Cl}_{2} / \mathrm{MeOH}$ 8:2 und trennte durch Säulenchromatographie an Kieselgel $(5 \times 60 \mathrm{~cm}$, Essigester/Dichlormethan-Stufengradient: $11 \mathrm{CH}_{2} \mathrm{Cl}_{2}, 11 \mathrm{CH}_{2} \mathrm{Cl}_{2} / 5 \%$ Essigester, $11 \mathrm{CH}_{2} \mathrm{Cl}_{2} / 10 \%$ Essigester, $11 \mathrm{CH}_{2} \mathrm{Cl}_{2} / 20 \%$ Essigester, $11 \mathrm{CH}_{2} \mathrm{Cl}_{2} / 50 \%$ Essigester, 21 Essigester) vor.

Aus der Fraktion I erhielt man nach der präparativen-HPLC Reinigung an RP18 (Standardgradient, Detektion im Bereich von 190 bis $620 \mathrm{~nm}$ ) $12 \mathrm{mg}$ Thymin (33) und eine zweite Mischfraktion Ia. Durch Chromatographie an Sephadex LH-20 (3 × $\left.120 \mathrm{~cm}, \mathrm{CH}_{2} \mathrm{Cl}_{2} / \mathrm{MeOH} ; 4: 6\right)$ wurden aus der Fraktion Ia zwei Substanzen isoliert, $\varepsilon$ Pyrromycinon (30) und $\zeta$-Pyrromycinon (31). Fraktion II chromatographierte man an präparativen Dickschichtplatten (3 Platten $20 \times 20 \mathrm{~cm}$; Kieselgel, $\mathrm{CH}_{2} \mathrm{Cl}_{2} / \mathrm{MeOH}$; 9:1) und erhielt $4.3 \mathrm{mg}$ 5-[2-(2-Hydroxy-3-methoxycarbonyl-propyl)-5-methylphenyl]-penta-2,4-dien-säure (32) als weiße Substanz mit $R_{\mathrm{f}^{-}}=0.65$.

ع-Pyrromycinon (30): $\mathrm{C}_{22} \mathrm{H}_{16} \mathrm{O}_{7}, \boldsymbol{R}_{\mathbf{f}}=0.60 ; \mathrm{CH}_{2} \mathrm{Cl}_{2},{ }^{1} \mathbf{H}-\mathbf{N M R}\left(\mathrm{CDCl}_{3}, 300 \mathrm{MHz}\right): \delta$ $=13.84(\mathrm{~s}, \mathrm{H} / \mathrm{D}$-austauschbar, $1 \mathrm{H}, \mathrm{OH}), 13.10(\mathrm{~s}, \mathrm{H} / \mathrm{D}$-austauschbar, $1 \mathrm{H}, \mathrm{OH})$, 
12.30 (s, H/D-austauschbar, $1 \mathrm{H}, \mathrm{OH}), 8.52\left(\mathrm{~d},{ }^{3} \mathrm{~J}=9 \mathrm{~Hz}, 1 \mathrm{H}, 7-\mathrm{H}\right), 8.25$ (s, $1 \mathrm{H}, 11$ H), $7.60\left(\mathrm{~d},{ }^{3} \mathrm{~J}=9 \mathrm{~Hz}, 1 \mathrm{H}, 8-\mathrm{H}\right), 7.28(\mathrm{~s}, 2 \mathrm{H}, 2-, 3-\mathrm{H}), 4.10\left(\mathrm{~s}, 3 \mathrm{H},-\mathrm{OCH}_{3}\right), 2.80$ (q, $\left.{ }^{3} \mathrm{~J}=7 \mathrm{~Hz}, 2 \mathrm{H},-\mathrm{CH}_{2}-\right), 1.30\left(\mathrm{t},{ }^{3} \mathrm{~J}=7 \mathrm{~Hz}, 3 \mathrm{H},-\mathrm{CH}_{3}\right)$.

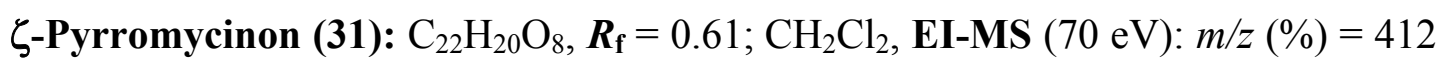
$\left(\mathrm{M}^{+}, 100\right), 394\left(\mathrm{M}^{+}-\mathrm{H}_{2} \mathrm{O}, 22\right), 380\left(\mathrm{M}^{+}-\mathrm{MeOH}, 13\right), 356$ (27), 335 (72), 323 (65), 295 (54), 281 (10). ${ }^{1} \mathbf{H}-\mathbf{N M R}\left(\mathrm{CDCl}_{3}, 300 \mathrm{MHz}\right): \delta=13.02$ (s, H/D-austauschbar, 1 $\mathrm{H}, \mathrm{OH}), 12.62$ (s, H/D-austauschbar, $1 \mathrm{H}, \mathrm{OH}), 12.28$ (s, H/D-austauschbar, $1 \mathrm{H}$, OH), 7.66 (s, 1 H, 11-H), 7.28 (br s, 2 H, 2-, 3-H), 3.96 (br s, 1 H, 10-H), 3.70 (s, 3 $\left.\mathrm{H},-\mathrm{OCH}_{3}\right), 3.05,2.86\left(2 \mathrm{~m}, 3 \mathrm{H}, 7-\mathrm{CH}_{2}\right), 2.30,1.90\left(2 \mathrm{~m}, 8-\mathrm{CH}_{2}\right), 1.60(\mathrm{~m}, 2 \mathrm{H},-$ $\left.\mathrm{CH}_{2}-\mathrm{CH}_{3}\right), 1.04$ (t, $\left.3 \mathrm{H},-\mathrm{CH}_{2}-\mathrm{CH}_{3}\right)$.

\section{5-[2-(2-Hydroxy-3-methoxycarbonyl-propyl)-5-methyl-phenyl]-penta-2,4-dien-}

säure (32): $\mathrm{C}_{17} \mathrm{H}_{20} \mathrm{O}_{5}, \boldsymbol{R}_{\mathbf{f}}=0.65 ; \mathrm{CH}_{2} \mathrm{Cl}_{2} / \mathrm{MeOH}, 95: 5$. EI-MS $(70 \mathrm{eV}): m / z(\%)=$ 304.1315 (M+, 28), 268 (13), 255 (15), 219 (10), 212 (13), 202 (64), 184 (20), 183 (16), 157 (100), 142 (67), 130 (30), 103 (24), 71 (24), 43 (14). CI-MS ( $\left.\mathrm{NH}_{3}\right): 626$ ([2 $\left.\left.\mathrm{M}+\mathrm{NH}_{4}\right]^{+}, 10 \%\right), 322\left(\left[\mathrm{M}+\mathrm{NH}_{4}\right]^{+}, 100\right) .{ }^{1} \mathbf{H}-\mathbf{N M R}\left(\mathrm{CDCl}_{3}, 300 \mathrm{MHz}\right): \delta=7.58$ (dd, $\left.{ }^{3} J=13 \mathrm{~Hz},{ }^{3} J^{\prime}=12 \mathrm{~Hz}, 1 \mathrm{H}, 3-\mathrm{H}\right), 7.05$ (m, $\left.2 \mathrm{H}, 3^{\prime}-\mathrm{H}, 4^{\prime}-\mathrm{H}\right), 7.02$ (d, ${ }^{3} J=12$ $\mathrm{Hz}, 1 \mathrm{H}, 5-\mathrm{H}), 7.00$ (s, $\left.1 \mathrm{H}, 6{ }^{\prime}-\mathrm{H}\right), 6.42$ (t, $\left.{ }^{3} J=11 \mathrm{~Hz}, 1 \mathrm{H}, 4-\mathrm{H}\right), 6.00$ (d, ${ }^{3} J=16$ Hz,1 H, 2'-H), 4.18 (m, 1 H, 2' '-H), 3.65 (s, 3 H, - $\mathrm{OCH}_{3}$ ), 3.42 (s, H/D-austauschbar, $1 \mathrm{H}, \mathrm{OH}), 2.84,2.70\left(2 \mathrm{~m}, 2 \mathrm{H}, 1\right.$ '’- $\left.\mathrm{CH}_{2}\right), 2.43$ (m, $2 \mathrm{H}, 3$ ' ' $\left.-\mathrm{CH}_{2}\right), 2.25$ (s, $3 \mathrm{H}, 5$ '$\left.\mathrm{CH}_{3}\right) .{ }^{13}$ C-APT-NMR $\left(\mathrm{CDCl}_{3}, 500 \mathrm{MHz}\right): \delta=173.1\left(\mathrm{C}_{\mathrm{q}}-4\right.$ '” $), 170.0(\mathrm{C}-1,-\mathrm{COOH})$, 142.4 (CH-3'’), 138.3 (CH-5), 136.5 (C $\left.\mathrm{C}^{-} 5^{\prime}\right), 135.4\left(\mathrm{C}_{\mathrm{q}}-2^{\prime}\right), 133.2\left(\mathrm{C}_{\mathrm{q}}-1^{\prime}\right), 131.1$ (CH-6'), 130.5 (CH-3'), 129.4 (CH-4'), 128.2 (CH-4), 122.4 (CH-2), 68.6 (CH-2'’), $51.8\left(\mathrm{OCH}_{3}-6\right.$ ') $), 40.3\left(\mathrm{CH}_{2}-3\right.$ '’), $39.9\left(\mathrm{CH}_{2}-1\right.$ ' '), $21.0\left(\mathrm{CH}_{3}-5\right.$ ') $)$ HMQC-NMR (inverses C,H-COSY, $\mathrm{CDCl}_{3}$, INVBTP, F1 125,7 MHz, F2 499.7 MHz) $(\mathrm{H} \rightarrow \mathrm{C})$ : Alle C-H-Konnektivitäten wurden durch Kopplungen bestätigt.

Thymin (5-Methyluracil, 33): $\mathrm{C}_{5} \mathrm{H}_{6} \mathrm{O}_{2} \mathrm{~N}_{2}, \boldsymbol{R}_{\mathbf{f}}=0.50 ; \mathrm{CH}_{2} \mathrm{Cl}_{2} / \mathrm{MeOH}, 9: 1$, CI-MS $\left(\mathrm{NH}_{3}\right): 270\left(\left[2 \mathrm{M}+\mathrm{NH}_{4}\right]^{+}, 8 \%\right), 253\left([2 \mathrm{M}+\mathrm{H}]^{+}, 2\right), 144\left(\left[\mathrm{M}+\mathrm{NH}_{4}\right]^{+}, 100\right), 127$ $\left([\mathrm{M}+\mathrm{H}]^{+}, 30\right) .{ }^{1} \mathrm{H}-\mathrm{NMR}\left(\left[\mathrm{D}_{6}\right]-\mathrm{DMSO}, 200 \mathrm{MHz}\right): \delta=10.92(\mathrm{~s}, \mathrm{H} / \mathrm{D}$-austauschbar, 
$1 \mathrm{H}, \mathrm{NH}), 10.51$ (s, H/D-austauschbar, je $1 \mathrm{H}, 2 \mathrm{NH}), 7.21$ (d, $\left.{ }^{3} \mathrm{~J}=15 \mathrm{~Hz}, 1 \mathrm{H}, 6-\mathrm{H}\right)$, 1. $73\left(\mathrm{~s}, 3 \mathrm{H}, \mathrm{CH}_{3}\right)$

\section{Stamm B 7284}

Der marine Streptomycet B 7284 stammt aus der Sammlung mariner Actinomyceten des Alfred-Wegener-Instituts für Polar- und Meeresforschung in Bremerhaven. Der Stamm bildet auf $\mathrm{M}_{2}^{+}$-Agar nach dreitägiger Inkubation bei $20^{\circ} \mathrm{C}$ ein weißes Mycel. Der Agar verfärbte sich in der Nähe der Bakterienkolonien leicht rosa.

\section{Primärscreening}

Im Dünnschichtchromatogramm des Rohextrakt beobachtete man einige UVlöschende Zonen, die sich mit Anisaldehyd/Schwefelsäure braun verfärbten.

Tabelle 9: Aktivitäten des entfetteten Extraktes im Plattendiffusionstest.

\begin{tabular}{|c|c|c|}
\hline & Organismus & Hemmhof $\varnothing$ (in mm) \\
\hline \multirow{4}{*}{} & Escherichia coli & 22 \\
\cline { 2 - 3 } & Bacillus subtilis & 22 \\
\cline { 2 - 3 } & Streptomyces viridochromogenes (Tü57) & 18 \\
\cline { 2 - 3 } & Candida albicans & 0 \\
\cline { 2 - 3 } & Mucor mehei & 16 \\
\cline { 2 - 3 } & Staphylococcus aureus & 16 \\
\cline { 2 - 3 } & Chlorella vulgaris & 0 \\
\cline { 2 - 3 } & Chlorella sorokiniana & 0 \\
\hline \multirow{2}{*}{} & Scenedesmus subspicatus & 0 \\
\hline
\end{tabular}

\section{Fermentation und Aufarbeitung}

Man fermentierte im 10-1-Maßstab mit GALOP optimierten Nährmedium aus $83 \mathrm{~g}$ Maltose, $98 \mathrm{~g}$ Hefeextrakt, $48 \mathrm{~g}$ Glucose und $16 \mathrm{~g} \mathrm{CaCl}_{2}$ in 5 Liter Leitungswasser und 5 Liter künstlichem Meerwasser. Beimpfung des kleinen Durchflußfermenters erfolgte mit 21 einer $72 \mathrm{~h}$ bei $28^{\circ} \mathrm{C}$ inkubierten Schüttelkultur. 
Die Fermentation führte man kontinuierlich über sieben Tage bei $28{ }^{\circ} \mathrm{C}, 331$ Luft/min und $\mathrm{pH} 6.9 \pm 1.5(2 \mathrm{~N} \mathrm{NaOH}, 2 \mathrm{~N} \mathrm{HCl}$ zur pH-Regulierung) in dem 21 großen Durchflußfermenter durch.

Die Kulturbrühe wurde 3 min mit dem Ultraturrax behandelt und über Celite filtriert. Man extrahierte den Überstand dreimal mit je 11 EtOAc.

Das Mycel wurde mit Methanol über $12 \mathrm{~h}$ aufgeschlossen, danach dreimal mit jeweils $200 \mathrm{ml}$ EtOAc extrahiert. Nach dem Eindampfen der vereinigten org. Phasen erhielt man $320 \mathrm{mg}$ eines braungefärbten Rohextraktes, der zweimal mit je $50 \mathrm{ml}$ Cyclohexan entfettet wurde.

\section{Isolierung der Metaboliten}

Der Rohextrakt wurde in $10 \mathrm{ml} \mathrm{CH}_{2} \mathrm{Cl}_{2} / \mathrm{MeOH}$ 8:2 aufgenommen und durch Säulenchromatographie an Kieselgel $(5 \times 60 \mathrm{~cm}$, Essigester/Dichlormethan-Stufengradient: $11 \mathrm{CH}_{2} \mathrm{Cl}_{2}, 11 \mathrm{CH}_{2} \mathrm{Cl}_{2} / 5 \%$ Essigester, $11 \mathrm{CH}_{2} \mathrm{Cl}_{2} / 10 \%$ Essigester, 11 $\mathrm{CH}_{2} \mathrm{Cl}_{2} / 20 \%$ Essigester, $11 \mathrm{CH}_{2} \mathrm{Cl}_{2} / 50 \%$ Essigester, 21 Essigester) vorgetrennt. Man erhielt zwei Fraktionen I und II. Fraktion II wurde verworfen.

Aus der Fraktion I wurden durch Chromatographie an Sephadex LH-20 $(3 \times 120 \mathrm{~cm}$, $\mathrm{CH}_{2} \mathrm{Cl}_{2} / \mathrm{MeOH}$; 4:6) zwei Fraktionen Ia und $\mathrm{Ib}$ gewonnen. Die Endreinigung fand durch präparative HPLC-Trennung an RP18 (Standardgradient, Detektion im Bereich von 190 bis $620 \mathrm{~nm}$ ) statt. Man erhielt $32 \mathrm{mg}$ (3Z,6E)-1-Methylalbonoursin (34) und $16 \mathrm{mg}$ Maculosin-6 (35).

(3Z,6E)-1-Methylalbonoursin (34): $\mathrm{C}_{16} \mathrm{H}_{20} \mathrm{O}_{2} \mathrm{~N}_{2}, \boldsymbol{R}_{\mathbf{f}}=0.80 ; \mathrm{CH}_{2} \mathrm{Cl}_{2} / \mathrm{MeOH}, 9: 1$, CI-MS $\left(\mathrm{NH}_{3}\right): 558\left(\left[2 \mathrm{M}+\mathrm{NH}_{4}\right]^{+}, 1 \%\right), 305\left(\left[\mathrm{M}+\mathrm{NH}_{4}+\mathrm{NH}_{3}\right]^{+}, 38\right), 288([\mathrm{M}+$ $\left.\left.\mathrm{NH}_{4}\right]^{+}, 100\right), 271\left([\mathrm{M}+\mathrm{H}]^{+}, 30\right) .{ }^{1} \mathbf{H}-\mathbf{N M R}\left(\mathrm{CDCl}_{3}, 200 \mathrm{MHz}\right): \delta=8.08$ (s br, H/Daustauschbar, $1 \mathrm{H}, \mathrm{NH}), 7.50-7.25\left(\mathrm{~m}, 5 \mathrm{H},-\mathrm{C}_{6} \mathrm{H}_{5}\right), 6.99(\mathrm{~s}, 1 \mathrm{H}, 3 \mathrm{a}-\mathrm{H}), 5.50\left(\mathrm{~d},{ }^{3} \mathrm{~J}=\right.$ $22 \mathrm{~Hz}, 1 \mathrm{H}, 1$ '’-H), 3.72 (m, $1 \mathrm{H}, 2$ ' '-H), 3.28 (s, $\left.3 \mathrm{H},-\mathrm{NCH}_{3}\right), 1.10$ (d, ${ }^{3} J=15 \mathrm{~Hz}, 6$ $\left.\mathrm{H}, 2 \times \mathrm{CH}_{3}\right) \cdot{ }^{13} \mathbf{C}-\mathbf{N M R}\left(\mathrm{CDCl}_{3}, 300 \mathrm{MHz}\right): \delta=157.8\left(\mathrm{C}_{\mathrm{q}}-2\right), 157.5\left(\mathrm{C}_{\mathrm{q}}-5\right), 134.5$ (CH-1'”), $133.1\left(\mathrm{C}_{\mathrm{q}^{-}}{ }^{\prime}\right.$ '), 129.8 (CH-2'-5'), 129.1 (CH-6'), 128.3 (CH-4'), $127.9\left(\mathrm{C}_{\mathrm{q}^{-}}\right.$ 6), $125.4\left(\mathrm{C}_{\mathrm{q}}-3\right), 115.8(\mathrm{CH}-3 \mathrm{a}), 30.9\left(-\mathrm{NCH}_{3}\right), 26.5(\mathrm{CH}-2$ '’), $23.2(\mathrm{CH}-3$ '’). 
Maculosin-6, [(3R,7aR)-Cyclo(leucylprolyl, 35]: $\quad \mathrm{C}_{11} \mathrm{H}_{18} \mathrm{O}_{2} \mathrm{~N}_{2}, \quad \boldsymbol{R}_{\mathbf{f}}=0.70$; $\mathrm{CH}_{2} \mathrm{Cl}_{2} / \mathrm{MeOH}, 9: 1$, CI-MS $\left(\mathrm{NH}_{3}\right): 421\left(\left[2 \mathrm{M}+\mathrm{NH}_{4}\right]^{+}, 4 \%\right), 228\left(\left[\mathrm{M}+\mathrm{NH}_{4}\right]^{+}\right.$, 100), $211\left([\mathrm{M}+\mathrm{H}]^{+}, 63\right) .{ }^{1} \mathrm{H}-\mathbf{N M R}\left(\mathrm{CDCl}_{3}, 200 \mathrm{MHz}\right): \delta=5.93$ (br s, H/Daustauschbar, $1 \mathrm{H}, \mathrm{NH}), 4.12\left(\mathrm{t},{ }^{3} J=7.8 \mathrm{~Hz}, 1 \mathrm{H}\right), 4.02\left(\mathrm{dd},{ }^{3} J=9.5,3.7 \mathrm{~Hz}, 1 \mathrm{H}\right)$, 3.70-3.48 (m, 2 H), 2.44-2.24 (m, 1 H), 2.23-1.64 (m, 5 H), 1.61-1.43 (m, 1 H), 1.01 $\left(\mathrm{d},{ }^{3} J=6.6 \mathrm{~Hz}, 3 \mathrm{H}, \mathrm{CH}_{3}\right), 0.95\left(\mathrm{~d},{ }^{3} J=6.4 \mathrm{~Hz}, 3 \mathrm{H}, \mathrm{CH}_{3}\right)$.

\section{Stamm B 7939}

Der marine Streptomycet B 7939 wird in der Sammlung mariner Actinomyceten des Alfred-Wegener-Instituts für Polar- und Meeresforschung in Bremerhaven in Reinkultur aufbewahrt. Nach drei Tagen Inkubationszeit bildete der Stamm auf Agar ein weißes Luftmycel. Der Agar wurde leicht gelb unter den Bakterienkolonien.

\section{Primärscreening}

Die Rohextrakte des Stammes zeigten im biologischen Screening eine Aktivität gegen den Teststamm Escherichia coli und gegen alle im Test verwendeten Algen. Im HPLC-Vorscreening zeigte der Rohextrakt nur wenige Signale. Im Dünnschichtchromatgramm konnte man nur wenige UV-löschende Zonen erkennen, die sich mit Anisaldehyd/Schwefelsäure braun verfärbten. 
Tabelle 10: Aktivitäten des entfetteten Extraktes im Plattendiffusionstest.

\begin{tabular}{|c|c|c|}
\hline & Organismus & Hemmhof $\varnothing$ (in mm) \\
\hline \multirow{4}{*}{} & Escherichia coli & 12 \\
\cline { 2 - 3 } & Bacillus subtilis & 0 \\
\cline { 2 - 3 } & Streptomyces viridochromogenes (Tü57) & 0 \\
\cline { 2 - 3 } & Candida albicans & 0 \\
\cline { 2 - 3 } & Mucor miehei & 0 \\
\hline \multirow{2}{*}{$\bar{*}$} & Staphylococcus aureus & 0 \\
\cline { 2 - 3 } & Chlorella vulgaris & 23 \\
\cline { 2 - 3 } & Chlorella sorokiniana & 11 \\
\cline { 2 - 3 } & Scenedesmus subspicatus & 13 \\
\hline
\end{tabular}

\section{Fermentation und Aufarbeitung}

Man fermentierte im 10-1-Maßstab in einem mit GALOP optimierten Nährmedium aus $27 \mathrm{~g}$ Maltose, $68 \mathrm{~g}$ Hefeextrakt, $51 \mathrm{~g}$ Glucose und $54 \mathrm{~g} \mathrm{CaCl}_{2}$ in 5 Litern Leitungswasser und 5 Litern künstlichem Meerwasser. Die Beimpfung des kleinen 21 Durchflußfermenters erfolgte mit 21 einer $72 \mathrm{~h}$ bei $28^{\circ} \mathrm{C}$ inkubierten Schüttelkultur.

Durchflußgeschwindigkeit des Nährmediums wurde so eingestellt, dass das Medium kontinuierlich über sieben Tage bei $28{ }^{\circ} \mathrm{C}, 331 \mathrm{Luft} / \mathrm{min}, \mathrm{pH} 6.9 \pm 1.5(2 \mathrm{~N} \mathrm{NaOH}, 2$ $\mathrm{N} \mathrm{HCl}$ zur pH-Regulierung) durch einen 21 großen Durchflußfermenter gepumpt wurde.

Die Kulturbrühe wurde 3 min mit dem Ultraturrax behandelt und über Celite filtriert. Man extrahierte den Überstand dreimal mit je 11 EtOAc. Das Mycel wurde mit Me$\mathrm{OH}$ über $12 \mathrm{~h}$ aufgeschlossen.

Danach extrahierte man das Mycel jeweils dreimal mit 200 ml EtOAc. Nach dem Eindampfen der vereinigten org. Phasen erhielt man $1.2 \mathrm{~g}$ einer braungefärbten öligen Flüssigkeit, die dreimal mit je $50 \mathrm{ml}$ Cyclohexan entfettet wurde.

\section{Isolierung der Metaboliten}

Der Rohextrakt wurde in $10 \mathrm{ml} \mathrm{CH}_{2} \mathrm{Cl}_{2} / \mathrm{MeOH}$ 8:2 aufgenommen und durch Säulenchromatographie an Kieselgel $(5 \times 60 \mathrm{~cm}$, Essigester/Dichlormethan-Stufengradienten: $11 \mathrm{CH}_{2} \mathrm{Cl}_{2}, 11 \mathrm{CH}_{2} \mathrm{Cl}_{2} / 5 \%$ Essigester, $11 \mathrm{CH}_{2} \mathrm{Cl}_{2} / 10 \%$ Essigester, 11 
$\mathrm{CH}_{2} \mathrm{Cl}_{2} / 20 \%$ Essigester, $11 \mathrm{CH}_{2} \mathrm{Cl}_{2} / 50 \%$ Essigester, 21 Essigester) vorgetrennt. Man erhielt zwei Fraktionen I und II.

Aus der Fraktion I erhielt man nach präparativer HPLC an $\mathrm{RP} 18\left(\mathrm{H}_{2} \mathrm{O} / 10 \% \mathrm{MeCN}\right.$ Anstieg auf $80 \% \mathrm{MeCN}$ in $30 \mathrm{~min}$, dann auf $100 \% \mathrm{MeCN}$ in $10 \mathrm{~min}$, über $10 \mathrm{~min}$ $100 \% \mathrm{MeCN}$ und dann Abfall auf $10 \%$ in $1 \mathrm{~min}$, Fluss $10.0 \mathrm{ml} / \mathrm{min}$, Detektion im Bereich von 190 bis $620 \mathrm{~nm}$ ) zwei Mischfraktionen (16 und $14 \mathrm{mg}$ ) mit Phthalsäuredibutylester (40) und Phthalsäure-diisobutyl-ester (41) in unterschiedlichen Mischungsverhältnissen, sowie $32 \mathrm{mg}$ Vitamin MK-7 (43).

Fraktion II chromatographierte man an präparativen Dickschichtplatten (12 Platten $20 \times 20 \mathrm{~cm}$; Kieselgel, $\mathrm{CH}_{2} \mathrm{Cl}_{2} / \mathrm{MeOH}$; 95:5) und erhielt die UV-löschenden Zonen IIa $\left(R_{\mathrm{f}}=0.32\right)$, IIb $\left(R_{\mathrm{f}}=0.41\right)$ und IIc $\left(R_{\mathrm{f}}=0.61\right)$.

Nach dem Eluieren der Fraktion IIa mit 100 ml EtOAc /MeOH (95:5) erhielt man 7.8 mg N-Acetyltyramin (38).

Aus der Fraktion IIb erhielt man nach der präparativen HPLC-Reinigung an RP18 (Standardgradient, Detektion im Bereich von 190 bis $620 \mathrm{~nm}$ ) $16 \mathrm{mg} \mathrm{N}$ Acetyltryptamin (36), $12 \mathrm{mg}$ Tyrosol (39) und $5 \mathrm{mg}$ 3-Indolylethanol (37), letzteres im Gemisch mit $60 \% 39$.

Aus Fraktion IIc enthielt man 23 mg niedermolekulare Polyhydroxybuttersäure (45), die durch Eluieren der von der Platten abgetrennten Zone mit 100 ml EtOAc gewonnen wurde.

N-Acetyltryptamin (36): $\mathrm{C}_{12} \mathrm{H}_{14} \mathrm{~N}_{2} \mathrm{O}, \boldsymbol{R}_{\mathbf{f}}=0.41 ; \mathrm{CH}_{2} \mathrm{Cl}_{2} / \mathrm{MeOH}$, 95:5, CI-MS $\left(\mathrm{NH}_{3}\right): 423\left(\left[2 \mathrm{M}+\mathrm{NH}_{4}\right]^{+}, 17 \%\right), 405\left([\mathrm{M}+\mathrm{H}]^{+}, 19\right), 220\left(\left[\mathrm{M}+\mathrm{NH}_{4}\right]^{+}, 100\right), 203.0$ $\left([\mathrm{M}+\mathrm{H}]^{+}, 19\right) .{ }^{1} \mathbf{H}-\mathbf{N M R}\left(\mathrm{CDCl}_{3}, 300 \mathrm{MHz}\right): \delta=8.08(\mathrm{~s}, \mathrm{H} / \mathrm{D}$-austauschbar, $1 \mathrm{H}$, Indol-NH), 7.59 (d, $\left.{ }^{3} J=7.6 \mathrm{~Hz}, 1 \mathrm{H}, 4-\mathrm{H}\right), 7.38\left(\mathrm{~d},{ }^{3} J=7.6 \mathrm{~Hz}, 1 \mathrm{H}, 7-\mathrm{H}\right), 7.20-7.05$ (m, 2 H, 5-H, 6-H), 7.02 (d, $\left.{ }^{3} J=4.7 \mathrm{~Hz}, 1 \mathrm{H}, 2-\mathrm{H}\right), 5.50$ (s, H/D-austauschbar, $1 \mathrm{H}$, $\mathrm{NH}), 3.59\left(\mathrm{dt},{ }^{3} J=7.8 \mathrm{~Hz},{ }^{3} J=7.0 \mathrm{~Hz}, 2 \mathrm{H}, 2^{\prime}-\mathrm{CH}_{2}\right), 2.94\left(\mathrm{t},{ }^{3} \mathrm{~J}=7.0 \mathrm{~Hz}, 2 \mathrm{H}, 1^{\prime}-\right.$ $\left.\mathrm{CH}_{2}\right), 1.90\left(\mathrm{~s}, 3 \mathrm{H}, \mathrm{CH}_{3}\right)$.

3-Indolylethanol (37): $\mathrm{C}_{12} \mathrm{H}_{14} \mathrm{~N}_{2} \mathrm{O}, \quad \boldsymbol{R}_{\mathbf{f}}=0.4 ; \mathrm{CH}_{2} \mathrm{Cl}_{2} / \mathrm{MeOH}, 95: 5,{ }^{\mathbf{1}} \mathbf{H}-\mathbf{N M R}$ $\left(\mathrm{CDCl}_{3}, 300 \mathrm{MHz}\right): \delta=8.05$ (s, H/D-austauschbar, $1 \mathrm{H}$, Indol-NH), $7.59\left(\mathrm{~d},{ }^{3} \mathrm{~J}=7.6\right.$ Hz, $1 \mathrm{H}, 4-\mathrm{H}), 7.38$ (d, $\left.{ }^{3} J=7.6 \mathrm{~Hz}, 1 \mathrm{H}, 7-\mathrm{H}\right), 7.25-7.10$ (m, 2 H, 5-H, 6-H), 7.08, 
$\left(\mathrm{d},{ }^{3} J=1 \mathrm{~Hz}, 1 \mathrm{H}, 2-\mathrm{H}\right), 3.90\left(\mathrm{t},{ }^{3} J=3.0 \mathrm{~Hz}, 2 \mathrm{H}, 2^{\prime}-\mathrm{CH}_{2}\right), 3.03\left(\mathrm{t},{ }^{3} J=3.0 \mathrm{~Hz}, 2 \mathrm{H}\right.$, $\left.1^{\prime}-\mathrm{CH}_{2}\right)$.

N-Acetyltyramin (38): $\mathrm{C}_{8} \mathrm{H}_{13} \mathrm{NO}, \boldsymbol{R}_{\mathbf{f}}=0.31 ; \mathrm{CH}_{2} \mathrm{Cl}_{2} / \mathrm{MeOH}, 95: 5$, CI-MS $\left(\mathrm{NH}_{3}\right)$ : $376\left(\left[2 \mathrm{M}+\mathrm{NH}_{4}\right]^{+}, 8 \%\right), 359\left([2 \mathrm{M}+\mathrm{H}]^{+}, 15\right), 214\left(\left[\mathrm{M}+\mathrm{NH}_{4}+\mathrm{NH}_{3}\right]^{+}, 5\right), 197([\mathrm{M}$ $\left.\left.+\mathrm{NH}_{4}\right]^{+}, 100\right), 180\left([\mathrm{M}+\mathrm{H}]^{+}, 20\right) .{ }^{1} \mathbf{H}-\mathrm{NMR}\left(\mathrm{CDCl}_{3}, 300 \mathrm{MHz}\right): \delta=7.02,6.76$ (AA'BB', ${ }^{3} J=9 \mathrm{~Hz}$, je $2 \mathrm{H}$ ), 5.46 (s, H/D-austauschbar, $\left.1 \mathrm{H}, \mathrm{NH}\right), 3.44$ (q, ${ }^{3} J=6$ $\left.\mathrm{Hz}, 2 \mathrm{H}, 2^{\prime}-\mathrm{H}_{2}\right), 2.72\left(\mathrm{t},{ }^{3} \mathrm{~J}=6 \mathrm{~Hz}, 2 \mathrm{H}, 1^{\prime}-\mathrm{H}_{2}\right)$.

4-(2-Hydroxyethyl)-phenol (Tyrosol, 39): $\mathrm{C}_{8} \mathrm{H}_{10} \mathrm{O}_{2}, \boldsymbol{R}_{\mathbf{f}}=0.40 ; \mathrm{CH}_{2} \mathrm{Cl}_{2} / \mathrm{MeOH}$, 95:5, CI-MS $\left(\mathrm{NH}_{3}\right): 294\left(\left[2 \mathrm{M}+\mathrm{NH}_{4}\right]^{+}, 27 \%\right), 173\left(\left[\mathrm{M}+\mathrm{NH}_{4}+\mathrm{NH}_{3}\right]^{+}, 50\right), 156$ $\left(\left[\mathrm{M}+\mathrm{NH}_{4}\right]^{+}, 100\right) .{ }^{1} \mathbf{H}-\mathrm{NMR}\left(\mathrm{CD}_{3} \mathrm{OD}, 300 \mathrm{MHz}\right): \delta=7.02,6.70\left(\mathrm{AA}^{\prime} \mathrm{BB}{ }^{\prime},{ }^{3} J=9\right.$ $\mathrm{Hz}$, je $2 \mathrm{H}), 3.70\left(\mathrm{t},{ }^{3} \mathrm{~J}=8 \mathrm{~Hz}, 2 \mathrm{H}, 2^{\prime}-\mathrm{H}_{2}\right), 3.72\left(\mathrm{t},{ }^{3} J=8 \mathrm{~Hz}, 2 \mathrm{H}, 1^{\prime}-\mathrm{H}_{2}\right)$.

Phthalsäuredibutylester (40): $\mathrm{C}_{16} \mathrm{H}_{22} \mathrm{O}_{4}, \boldsymbol{R}_{\mathbf{f}}=0.95 ; \mathrm{CH}_{2} \mathrm{Cl}_{2} / \mathrm{MeOH}, 9: 1,{ }^{\mathbf{1}} \mathbf{H}-\mathbf{N M R}$ $\left(\mathrm{CDCl}_{3}, 300 \mathrm{MHz}\right): \delta=7.70(\mathrm{~m}, 2 \mathrm{H}, 3-\mathrm{H}, 6-\mathrm{H}), 7.50(\mathrm{~m}, 2 \mathrm{H}, 4-\mathrm{H}, 5-\mathrm{H}), 4.24\left(\mathrm{t},{ }^{3} J\right.$ $=6 \mathrm{~Hz}, 4 \mathrm{H}, 4^{\prime}-\mathrm{H}, 4$ ' '-H), 1.70 (m, 4 H, 3'-H, 3' '-H), 1.40 (m, 4 H, 2'-H, 2' '-H), 0.98 (m, $6 \mathrm{H}, 1$ '- $\left.-\mathrm{CH}_{3}, 1{ }^{\prime \prime}{ }^{-} \mathrm{CH}_{3}\right) .{ }^{13} \mathbf{C}-\mathbf{N M R}\left(\mathrm{CDCl}_{3}, 300 \mathrm{MHz}\right): \delta=167\left(\mathrm{C}_{\mathrm{q}}-4\right), 167.4$ $\left(\mathrm{C}_{\mathrm{q}}-3\right), 132.3\left(\mathrm{C}_{\mathrm{q}}-2\right), 132.3\left(\mathrm{C}_{\mathrm{q}}-1\right), 130.9$ (CH-17), 130.9 (CH-18), $128.8(\mathrm{CH}-10)$, 128.8 (CH-9), $65.5\left(\mathrm{CH}_{2}-12\right), 65.5\left(\mathrm{CH}_{2}-11\right), 30.5\left(\mathrm{CH}_{2}-13\right), 30.5\left(\mathrm{CH}_{2}-14\right), 19.2$ $\left(\mathrm{CH}_{2}-16\right), 19.2\left(\mathrm{CH}_{2}-15\right), 13.7\left(\mathrm{CH}_{3}-20\right), 13.7\left(\mathrm{CH}_{3}-19\right)$.

Phthalsäure-diisobutyl-ester (41): $\mathrm{C}_{16} \mathrm{H}_{22} \mathrm{O}_{4}, \boldsymbol{R}_{\mathbf{f}}=0.80 ; \mathrm{CH}_{2} \mathrm{Cl}_{2},{ }^{1} \mathbf{H}-\mathbf{N M R}\left(\mathrm{CDCl}_{3}\right.$, $300 \mathrm{MHz}): \delta=7.70$ (m, 2 H, 3-H, 6-H), 7.50 (m, $2 \mathrm{H}, 4-\mathrm{H}, 5-\mathrm{H}), 4.04$ (d, ${ }^{3} J=6 \mathrm{~Hz}$, $4 \mathrm{H},-\mathrm{CH}_{2}{ }^{\prime},-\mathrm{CH}_{2}{ }^{\prime}$ ) $), 2.00$ (m, $2 \mathrm{H},-\mathrm{CH}^{\prime},-\mathrm{CH}^{\prime}$ '), 0.98 (d, $12 \mathrm{H}, 2$ Isopropyl-Reste).

Vitamin MK-7 (43): $\mathrm{C}_{46} \mathrm{H}_{64} \mathrm{O}_{2}, \boldsymbol{R}_{\mathbf{f}}=0.71 ; \mathrm{CHCl}_{3}$, EI-MS $(70 \mathrm{eV}): m / z(\%)=648$ $(\mathrm{M}+, 100), 396$ (34), 225 (76), 81 (55), 69 (82). ${ }^{1}$ H-NMR $\left(\mathrm{CDCl}_{3}, 300 \mathrm{MHz}\right): \delta=$ 8.05 (m, 2 H, 5-H, 8-H), 7.66 (m, 2 H, 6-H, 7-H), 5.11 (m; 7 H, 6'-H, 10'-H, 14'-H, 18'-H, 22'-H, 26'-H, 2-H), 3.37 (d, ${ }^{3} J=7$ Hz; 2 H, 1'- $\mathrm{H}_{2}$ ), 2.19 (s, 3 H, 3- $\mathrm{CH}_{3}$ ), 2.111.90 (m, 24 H, 4'- $\mathrm{H}_{2}, 5^{\prime}-\mathrm{H}_{2}, 8^{\prime}-\mathrm{H}_{2}, 9^{\prime}-\mathrm{H}_{2}, 12^{\prime}-\mathrm{H}_{2}, 13^{\prime}-\mathrm{H}_{2}, 16^{\prime}-\mathrm{H}_{2}, 17^{\prime}-\mathrm{H}_{2}, 20^{\prime}-\mathrm{H}_{2}, 21^{\prime}-$ 
$\left.\mathrm{H}_{2}, 24^{\prime}-\mathrm{H}_{2}, 25^{\prime}-\mathrm{H}_{2}\right), 1.69$ (s, $\left.3 \mathrm{H}, 3^{\prime}-\mathrm{CH}_{3}\right), 1.71$ (s br, $\left.6 \mathrm{H}, 27^{\prime}-\left(\mathrm{CH}_{3}\right)_{2}\right), 1.60$ (s, $15 \mathrm{H}$, 7'- $-\mathrm{CH}_{3}, 11^{\prime}-\mathrm{CH}_{3}, 15^{\prime}-\mathrm{CH}_{3}, 19^{\prime}-\mathrm{CH}_{3}, 23$ '- $\left.-\mathrm{CH}_{3}\right) .{ }^{13} \mathbf{C}-\mathbf{N M R}\left(\mathrm{CDCl}_{3}, 300 \mathrm{MHz}\right): \delta=$ $185.4\left(\mathrm{C}_{\mathrm{q}}-4\right), 184.5\left(\mathrm{C}_{\mathrm{q}}-3\right), 146.1\left(\mathrm{C}_{\mathrm{q}}-1\right), 143.3\left(\mathrm{C}_{\mathrm{q}}-2\right), 137.5\left(\mathrm{C}_{\mathrm{q}}-11\right), 135.2\left(\mathrm{C}_{\mathrm{q}}-15\right)$, $135.2\left(\mathrm{C}_{\mathrm{q}}-13\right), 135.2\left(\mathrm{C}_{\mathrm{q}}-12\right), 135.2\left(\mathrm{C}_{\mathrm{q}}-16\right), 135.2\left(\mathrm{C}_{\mathrm{q}}-14\right), 134.9$ (CH-48), 134.9 (CH-47), $133.3\left(\mathrm{C}_{\mathrm{q}}-5\right), 133.3\left(\mathrm{C}_{\mathrm{q}}-6\right), 131.2\left(\mathrm{C}_{\mathrm{q}}-17\right), 126.2(\mathrm{CH}-32), 126.1(\mathrm{CH}-31)$, 124.4 (CH-19), 124.2 (CH-20), 124.2 (CH-21), 124.2 (CH-22), 124.2 (CH-23), 124.1 (CH-18), $119.0(\mathrm{CH}-8), 39.7\left(\mathrm{CH}_{2}-38\right), 39.7\left(\mathrm{CH}_{2}-36\right), 39.7\left(\mathrm{CH}_{2}-35\right), 39.7\left(\mathrm{CH}_{2}-\right.$ 34), $39.7\left(\mathrm{CH}_{2}-33\right), 39.7\left(\mathrm{CH}_{2}-37\right), 29.7\left(\mathrm{CH}_{2}-30\right), 26.7\left(\mathrm{CH}_{2}-29\right), 26.7\left(\mathrm{CH}_{2}-28\right)$, $26.7\left(\mathrm{CH}_{2}-27\right), 26.7\left(\mathrm{CH}_{2}-25\right), 26.5\left(\mathrm{CH}_{2}-7\right), 25.7\left(\mathrm{CH}_{2}-26\right), 25.7\left(\mathrm{CH}_{3}-41\right), 25.7$ $\left(\mathrm{CH}_{3}-40\right), 17.7\left(\mathrm{CH}_{3}-46\right), 16.4\left(\mathrm{CH}_{3}-45\right), 16.2\left(\mathrm{CH}_{3}-44\right), 16.2\left(\mathrm{CH}_{3}-43\right), 16.2\left(\mathrm{CH}_{3}-\right.$ 42), $16.2\left(\mathrm{CH}_{3}-39\right), 12.7\left(\mathrm{CH}_{3}-24\right)$.

Polyhydroxybuttersäure (45) oder PHB: $\boldsymbol{R}_{\mathbf{f}}=0.61 ; \mathrm{CH}_{2} \mathrm{Cl}_{2} / \mathrm{MeOH}, 95: 5$, CI-MS $\left(\mathrm{NH}_{3}\right): 362\left(\left[2 \mathrm{M}+\mathrm{NH}_{4}\right]^{+}, 2 \%\right), 207\left(\left[\mathrm{M}+\mathrm{NH}_{4}+\mathrm{NH}_{3}\right]^{+}, 18\right), 190\left(\left[\mathrm{M}+\mathrm{NH}_{4}\right]^{+}\right.$, 100). ${ }^{1}$ H-NMR $\left(\mathrm{CDCl}_{3}, 300 \mathrm{MHz}\right): \delta=5.2$ (sext., $\left.{ }^{3} J=6 \mathrm{~Hz}, 1 \mathrm{H}, \mathrm{CH}\right), 2.60$ (dd, ${ }^{3} J=$ $\left.6 \mathrm{~Hz},{ }^{3} J=15.6 \mathrm{~Hz}, 1 \mathrm{H}, \mathrm{CH}_{2}\right), 2.40\left(\mathrm{dd},{ }^{3} \mathrm{~J}=6 \mathrm{~Hz},{ }^{3} J=15.6 \mathrm{~Hz}, 1 \mathrm{H}, \mathrm{CH}_{2}\right), 1.22(\mathrm{~d}$, $\left.{ }^{3} J=6 \mathrm{~Hz}, 3 \mathrm{H}, \mathrm{CH}_{3}\right)$.

\section{Stamm B 8927}

Der marine Streptomycet B 8927 aus der Sammlung mariner Actinomyceten des Alfred-Wegener-Instituts für Polar- und Meeresforschung in Bremerhaven bildet auf Agar ein leicht rosarot verfärbtes Mycel. Auch der Agar verfärbte sich in der Nähe des Mycels leicht rosa.

\section{Primärscreening}

Der Stamm wurde zunächst auf $\mathrm{M}_{2}{ }^{+}$-Schrägagar und anschließend auf Agarplatten jeweils $72 \mathrm{~h}$ bei $28^{\circ} \mathrm{C}$ inkubiert. Fünf mit je $200 \mathrm{ml}$ gefüllte 1-1-Schikanekolben beimpfte man mit den Agarkulturen und inkubierte $72 \mathrm{~h}$ bei $28^{\circ} \mathrm{C}$ und $95 \mathrm{Upm}$. Die Schüttelkultur wurde mit EtOAc extrahiert. 
Eine Bioautographie entwickelter Dünnschichtchromatogramme (Kieselgel, $\mathrm{CH}_{2} \mathrm{Cl}_{2}$ /MeOH, 95:5) des aus dem 20-1-Bioreaktors gewonnenen Rohextraktes zeigte jedoch nur eine schwache Aktivität bei einer UV-löschenden Zone mit $R_{\mathrm{f}}=0.62$.

Tabelle 11: Aktivitäten des entfetteten Extraktes im Plattendiffusionstest.

\begin{tabular}{|c|c|c|}
\hline & Organismus & Hemmhof $\varnothing$ (in mm) \\
\hline \multirow{4}{*}{} & Escherichia coli & 20 \\
\cline { 2 - 3 } & Bacillus subtilis & 20 \\
\cline { 2 - 3 } & Streptomyces viridochromogenes (Tü57) & 20 \\
\cline { 2 - 3 } & Candida albicans & 0 \\
\cline { 2 - 3 } & Mucor miehei & 11 \\
\cline { 2 - 3 } & Staphylococcus aureus & 12 \\
\cline { 2 - 3 } & Chlorella vulgaris & 12 \\
\cline { 2 - 3 } & Chlorella sorokiniana & 14 \\
\cline { 2 - 3 } & Scenedesmus subspicatus & 0 \\
\hline
\end{tabular}

\section{Fermentation und Aufarbeitung}

Man fermentierte im 20-1-Maßstab in einem mit GALOP optimierten Nährmedium aus $18 \mathrm{~g}$ Maltose, $43 \mathrm{~g}$ Hefeextrakt, $76 \mathrm{~g}$ Glucose und $45 \mathrm{~g} \mathrm{CaCl}_{2}$ in 10 Liter Leitungswasser und 10 Liter künstlichem Seewasser. Beimpft wurde mit 21 einer $72 \mathrm{~h}$ bei $28^{\circ} \mathrm{C}$ inkubierten Schüttelkultur.

Fermentation führte man diskontinuierlich bei $28^{\circ} \mathrm{C}, 331 \mathrm{Luft} / \mathrm{min}, \mathrm{pH} 6.9 \pm 1.5(2$ $\mathrm{N} \mathrm{NaOH}, 2 \mathrm{~N} \mathrm{HCl}$ zur pH-Regulierung) und einer Rührgeschwindigkeit von 200 Upm durch. Schaumbildung wurde durch Zugabe von $20 \mathrm{ml}$ Niax verhindert. Die Kulturbrühe wurde 5 min mit dem Ultraturrax behandelt und mit der Filterpresse über Celite. filtriert. Man extrahierte den Überstand mit EtOAc. Das Mycel wurde mit $\mathrm{MeOH}$ über $12 \mathrm{~h}$ aufgeschlossen, danach jeweils dreimal mit je $300 \mathrm{ml}$ EtOAc digeriert. Nach dem Eindampfen der vereinigten org. Phasen erhielt man $870 \mathrm{mg}$ eines braunen Rohextraktes, der zweimal mit je $50 \mathrm{ml}$ Cyclohexan entfettet wurde.

\section{Isolierung der Metaboliten}

Der Rohextrakt wurde in $10 \mathrm{ml} \mathrm{CH}_{2} \mathrm{Cl}_{2} / \mathrm{MeOH}$ 8:2 aufgenommen und durch Säulenchromatographie an Kieselgel $(5 \times 60 \mathrm{~cm}$, Essigester/Dichlormethan-Stufen- 
gradient: $11 \mathrm{CH}_{2} \mathrm{Cl}_{2}, 11 \mathrm{CH}_{2} \mathrm{Cl}_{2} / 5 \%$ Essigester, $11 \mathrm{CH}_{2} \mathrm{Cl}_{2} / 10 \%$ Essigester, 11 $\mathrm{CH}_{2} \mathrm{Cl}_{2} / 20 \%$ Essigester, $11 \mathrm{CH}_{2} \mathrm{Cl}_{2} / 50 \%$ Essigester, 21 Essigester) vorgetrennt. Man erhielt drei Fraktionen I bis III. Fraktion II wurde verworfen.

Aus der Fraktion I erhielt man durch Chromatographie an Sephadex LH-20 (3 × 120 cm, $\left.\mathrm{CH}_{2} \mathrm{Cl}_{2} / \mathrm{MeOH} ; 4: 6\right)$ die Fraktion Ia, die man weiterhin an präparativen Dickschichtplatten (3 Platten $20 \times 20 \mathrm{~cm}$; Kieselgel, $\left.\mathrm{CH}_{2} \mathrm{Cl}_{2} / \mathrm{MeOH} ; 9: 1\right)$ chromatographierte und erhielt Fraktion $\mathrm{Ib}\left(R_{\mathrm{f}}=0.2-0.5\right)$.

Die mit 100 ml EtOAc /MeOH (95:5) eluierte Fraktion Ib lieferte bei der präparativen HPLC-Reinigung an RP18 (Standardgradient, Detektion im Bereich von 190 bis $620 \mathrm{~nm}$ ) zwei weitere, noch stark verunreinigte Fraktionen Ic und Id. Die Endreinigung erfolgte durch Chromatographie an Sephadex LH-20 $\left(3 \times 120 \mathrm{~cm}, \mathrm{CH}_{2} \mathrm{Cl}_{2} /\right.$ $\mathrm{MeOH} ; 4: 6)$ und lieferte je Fraktion eine Reinsubstanz. Aus der Fraktion Id erhielt man $12 \mathrm{mg}$ 3-Isobutylpiperazin-2,5-dion (46). Fraktion Ic lieferte $4.7 \mathrm{mg}$ 2-(1Hydroxyethyl)-3H-chinazolin-4-on (47).

3-Isobutylpiperazin-2,5-dion (46): $\mathrm{C}_{8} \mathrm{H}_{14} \mathrm{~N}_{2} \mathrm{O}_{2}, \boldsymbol{R}_{\mathbf{f}}=0.3 ; \mathrm{CH}_{2} \mathrm{Cl}_{2} / \mathrm{MeOH}, 9: 1$, EIMS $(70 \mathrm{eV}): m / z(\%)=170(\mathrm{M}+, 100), 120(20), 86(38), 59$ (35). ${ }^{1} \mathbf{H}-\mathbf{N M R}\left(\mathrm{CDCl}_{3}\right.$, $300 \mathrm{MHz}): \delta=3.96$ (m, $1 \mathrm{H}, 3-\mathrm{H}), 3.62-3.36$ (br m, 2 H, 6-H), 1.80 (m, 2 H, 1'-H), $1.58\left(\mathrm{~m}, 1 \mathrm{H}, 2\right.$ ' -H), $0.86\left(\mathrm{~d},{ }^{3} J=6 \mathrm{~Hz}, 3 \mathrm{H}, 2\right.$ ' '- $\left.-\mathrm{CH}_{3}\right), 0.82\left(\mathrm{~d},{ }^{3} J=6 \mathrm{~Hz}, 3 \mathrm{H}, 3^{\prime}\right.$ $\left.\mathrm{CH}_{3}\right) .{ }^{13} \mathrm{C}-\mathrm{NMR}\left(\mathrm{CDCl}_{3}, 300 \mathrm{MHz}\right): \delta=168.9\left(\mathrm{C}_{\mathrm{q}}-2\right), 168.9\left(\mathrm{C}_{\mathrm{q}}-5\right), 53.3(\mathrm{CH}-3)$, $43.5\left(\mathrm{CH}_{2}-2\right), 29.7\left(\mathrm{CH}_{2}-1\right.$ '), $24.2\left(\mathrm{CH}-2\right.$ '), $23.24\left(\mathrm{CH}_{3}-3\right.$ '), $21.1\left(\mathrm{CH}_{3}-2^{\prime}{ }^{\prime}\right)$.

2-(1-Hydroxyethyl)-1 $\boldsymbol{H}$-chinazolin-4-on (47): $\mathrm{C}_{10} \mathrm{H}_{10} \mathrm{~N}_{2} \mathrm{O}_{2}, \boldsymbol{R}_{\mathbf{f}}=0.20 ; \mathrm{CH}_{2} \mathrm{Cl}_{2} /$ MeOH, 9:1, EI-MS (70 eV): m/z (\%) = $190(\mathrm{M}+, 100), 173$ (60), 147 (53), 119 (42), 43.1 (38). ${ }^{1} \mathrm{H}-\mathbf{N M R}\left(\mathrm{CDCl}_{3}, 300 \mathrm{MHz}\right): \delta=10.58$ (br s, H/D-austauschbar, $1 \mathrm{H}$, $\mathrm{NH}), 8.21\left(\mathrm{dd},{ }^{3} J=8 \mathrm{~Hz},{ }^{3} J=1 \mathrm{~Hz}, 1 \mathrm{H}, 7-\mathrm{H}\right), 7.67\left(\mathrm{dt},{ }^{3} J=7 \mathrm{~Hz},{ }^{3} J=1 \mathrm{~Hz}, 1 \mathrm{H}, 5-\right.$ H), $7.62\left(\mathrm{dd},{ }^{3} J=6 \mathrm{~Hz},{ }^{3} J=1 \mathrm{~Hz}, 1 \mathrm{H}, 4-\mathrm{H}\right), 7.42\left(\mathrm{dt},{ }^{3} J=1 \mathrm{~Hz},{ }^{3} J=7 \mathrm{~Hz}, 1 \mathrm{H}, 6-\right.$ H), $4.80\left(\mathrm{q},{ }^{3} \mathrm{~J}=6 \mathrm{~Hz}, 1 \mathrm{H}, 1\right.$ '-H), $1.60\left(\mathrm{~d},{ }^{3} \mathrm{~J}=6 \mathrm{~Hz}, 3 \mathrm{H}, \mathrm{CH}_{3}\right) .{ }^{13} \mathbf{C}-\mathbf{N M R}\left(\mathrm{CDCl}_{3}\right.$, $300 \mathrm{MHz}): \delta=158.3\left(\mathrm{C}_{\mathrm{q}}-8\right), 148.6\left(\mathrm{C}_{\mathrm{q}}-2\right), 134.76\left(\mathrm{C}_{\mathrm{q}}-3 \mathrm{a}\right), 127.0(\mathrm{CH}-5), 126.7(\mathrm{CH}-$ 4), 126.4 (CH-6), 126.4 (CH-7), $121.1\left(\mathrm{C}_{\mathrm{q}}-7 \mathrm{a}\right), 63.6\left(\mathrm{CH}-1\right.$ '), $22.5\left(\mathrm{CH}_{3}-2^{\prime}\right)$. 


\section{Stamm GW 20/1811}

Der auf Malta gefundene terrestrische Streptomycet GW 20/1811 wird bei der Firma bioLeads (Heidelberg) in Reinkultur gehalten. Auf Agar bildet er ein weißes Mycel. Man beobachtete eine leichte gelbe Verfärbung des Agars in der Nähe des Mycels.

\section{Primärscreening}

Extrakte des Streptomyceten GW 20/1811 zeigten im biologischen Screening die in der Tabelle 12 dargestellte Aktivität. Zusätzlich wirkte der Rohextrakt als Chitinaseinhibitor.

Tabelle 12: Aktivitäten des entfetteten Extraktes im Plattendiffusionstest.

\begin{tabular}{|c|c|c|}
\hline & Organismus & Hemmhof $\varnothing$ (in mm) \\
\hline \multirow{6}{*}{ 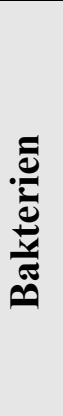 } & Escherichia coli & 20 \\
\hline & Bacillus subtilis & 24 \\
\hline & Streptomyces viridochromogenes (Tü57) & 0 \\
\hline & Candida albicans & 0 \\
\hline & Mucor miehei & 0 \\
\hline & Staphylococcus aureus & 24 \\
\hline \multirow{3}{*}{ 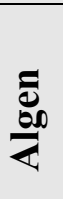 } & Chlorella vulgaris & - \\
\hline & Chlorella sorokiniana & - \\
\hline & Scenedesmus subspicatus & - \\
\hline
\end{tabular}

\section{Fermentation und Aufarbeitung}

Man fermentierte im 10-1-Maßstab in einem mit GALOP optimierten Nährmedium aus $66 \mathrm{~g}$ Maltose, $87 \mathrm{~g}$ Hefeextrakt, $68 \mathrm{~g}$ Glucose und $22 \mathrm{~g} \mathrm{CaCl}_{2}$ in 101 Leitungswasser. Der 2-1-Durchflußfermenters wurde mit 21 einer $72 \mathrm{~h}$ bei $28{ }^{\circ} \mathrm{C}$ inkubierten Schüttelkultur angeimpft.

Die Fermentation führte man kontinuierlich über sieben Tage bei $28{ }^{\circ} \mathrm{C}, 331$ Luft/min, pH $6.9 \pm 1.5$ (2 N NaOH, $2 \mathrm{~N} \mathrm{HCl}$ zur pH-Regulierung) durch.

Die Kulturbrühe wurde 3 min mit dem Ultraturrax behandelt und über Celite filtriert. Man extrahierte den Überstand dreimal mit 11 EtOAc und das Mycel behandelte man $12 \mathrm{~h}$ mit $\mathrm{MeOH}$. 
Danach extrahierte man das Mycel jeweils dreimal mit 200 ml EtOAc. Nach dem Eindampfen der vereinigten org. Phasen erhielt man $554 \mathrm{mg}$ eines braunen Rückstandes, der dreimal mit je $50 \mathrm{ml}$ Cyclohexan entfettet wurde.

\section{Isolierung}

Der Rohextrakt wurde in $10 \mathrm{ml} \mathrm{CH}_{2} \mathrm{Cl}_{2} / \mathrm{MeOH}$ 8:2 aufgenommen und durch Säulenchromatographie an Kieselgel $(5 \times 60 \mathrm{~cm}$, Essigester/Dichlormethan-Stufengradient: $11 \mathrm{CH}_{2} \mathrm{Cl}_{2}, 11 \mathrm{CH}_{2} \mathrm{Cl}_{2} / 5 \%$ Essigester, $11 \mathrm{CH}_{2} \mathrm{Cl}_{2} / 10 \%$ Essigester, 11 $\mathrm{CH}_{2} \mathrm{Cl}_{2} / 20 \%$ Essigester, $11 \mathrm{CH}_{2} \mathrm{Cl}_{2} / 50 \%$ Essigester, 21 Essigester) vorgetrennt. Man erhielt drei Fraktionen I bis III.

Aus der Fraktion I erhielt man durch Chromatographie an Sephadex LH-20 (3 × 120 $\left.\mathrm{cm}, \mathrm{CH}_{2} \mathrm{Cl}_{2} / \mathrm{MeOH} ; 4: 6\right)$ eine weitere Fraktion Ia, die in der präparativen HPLCReinigung an RP18 (Standardgradient, Detektion bei $210 \mathrm{~nm}$ ) in zwei Substanzen aufgeteilt wurde. Man erhielt $4.5 \mathrm{mg}$ Uracil (48) und $16 \mathrm{mg}$ Uridin (49).

Die dunkelgrüne Fraktion II chromatographierte man an präparativen Dickschichtplatten (6 Platten $20 \times 20 \mathrm{~cm}$; Kieselgel, $\mathrm{CH}_{2} \mathrm{Cl}_{2} / \mathrm{MeOH}$; 95:5) und erhielt die Fraktionen IIa $\left(R_{\mathrm{f}}=0.85\right)$ und IIc $\left(R_{\mathrm{f}}=0.60\right)$. Durch Endreinigung der Fraktion IIa an Sephadex LH-20 $\left(3 \times 120 \mathrm{~cm}, \mathrm{CH}_{2} \mathrm{Cl}_{2} / \mathrm{MeOH}\right.$; 4:6) gewann man $12 \mathrm{mg}$ des grünen BE-18591 (50). Fraktion IIc wurde mittels präparativer HPLC an RP18 (Standardgradient, Detektion bei $220 \mathrm{~nm}$ ) gereinigt und lieferte $11 \mathrm{mg}$ (3Z,6E)-1-Methylalbonoursin (34).

Fraktion III wurde präparativ an Dickschichtplatten (3 Platten $20 \times 20 \mathrm{~cm}$; Kieselgel, $\left.\mathrm{CH}_{2} \mathrm{Cl}_{2} / \mathrm{MeOH} ; 9: 1, R_{\mathrm{f}}=0.90\right)$ chromatographiert und ergab $16 \mathrm{mg}$ Phthalsäure-di(2-ethylhexylester, 51).

Uracil (1-H-Pyrimidin-2,4-dion) (48): $\mathrm{C}_{4} \mathrm{H}_{4} \mathrm{~N}_{2} \mathrm{O}_{2}, \boldsymbol{R}_{\mathbf{f}}=0.40 ; \mathrm{CH}_{2} \mathrm{Cl}_{2} / \mathrm{MeOH}, 9: 1$, CI-MS $\left(\mathrm{NH}_{3}\right): 242\left(\left[2 \mathrm{M}+\mathrm{NH}_{4}\right]^{+}, 8 \%\right), 147\left(\left[\mathrm{M}+\mathrm{NH}_{4}+\mathrm{NH}_{3}\right]^{+}, 42\right), 130([\mathrm{M}+$ $\left.\left.\mathrm{NH}_{4}\right]^{+}, 100\right), 113\left([\mathrm{M}+\mathrm{H}]^{+}, 28\right) .{ }^{1} \mathbf{H}-\mathbf{N M R}\left(\left[\mathrm{D}_{6}\right]-\mathrm{DMSO}, 300 \mathrm{MHz}\right): \delta=10.78(\mathrm{~s}$, H/D-austauschbar, $1 \mathrm{H}, \mathrm{NH}), 7.32\left(\mathrm{~d},{ }^{3} J=7.7 \mathrm{~Hz}, 1 \mathrm{H}, 4-\mathrm{H}\right), 5.42$ (d, ${ }^{3} J=7.7 \mathrm{~Hz}, 1$ H, 5-H). ${ }^{13}$ C-NMR ([D $]$-DMSO, $\left.300 \mathrm{MHz}\right): \delta=164.3\left(\mathrm{C}_{\mathrm{q}}-6\right), 151.5\left(\mathrm{C}_{\mathrm{q}}-2\right), 142.1$ (CH-5), $100.2(\mathrm{CH}-4)$. 
Uridin (49): $\mathrm{C}_{9} \mathrm{H}_{12} \mathrm{~N}_{2} \mathrm{O}_{5}, \boldsymbol{R}_{\mathbf{f}}=0.41 ; \mathrm{CH}_{2} \mathrm{Cl}_{2} / \mathrm{MeOH}, 9: 1$, CI-MS $\left(\mathrm{NH}_{3}\right): 506([2 \mathrm{M}+$ $\left.\left.\mathrm{NH}_{4}\right]^{+}, 1 \%\right), 279\left(\left[\mathrm{M}+\mathrm{NH}_{4}+\mathrm{NH}_{3}\right]^{+}, 18\right), 262\left(\left[\mathrm{M}+\mathrm{NH}_{4}\right]^{+}, 100\right), 245\left([\mathrm{M}+\mathrm{H}]^{+}\right.$, 10). ${ }^{1}$ H-NMR $\left(\mathrm{CD}_{3} \mathrm{OD}, 300 \mathrm{MHz}\right): \delta=8.01\left(\mathrm{~d},{ }^{3} J=8 \mathrm{~Hz}, 1 \mathrm{H}, 4-\mathrm{H}\right), 5.92\left(\mathrm{~d},{ }^{3} J=5\right.$ $\mathrm{Hz}, 1 \mathrm{H}, 4-\mathrm{H}), 5.64$ (d, $\left.{ }^{3} \mathrm{~J}=8 \mathrm{~Hz}, 1 \mathrm{H}, 1^{\prime}-\mathrm{H}\right), 4.18$ (m, 2 H, 2'-H, 3'-H), 4.00 (m, 1 H, 4'-H), 3.86-3.66 (br m, 2 H, - $\left.\mathrm{CH}_{2-}\right) .{ }^{13} \mathbf{C}-\mathbf{N M R}\left(\mathrm{CD}_{3} \mathrm{OD}, 300 \mathrm{MHz}\right.$ ): $\delta=166.2$ (C $\left.\mathrm{C}_{\mathrm{q}}-6\right), 156.9\left(\mathrm{C}_{\mathrm{q}}-2\right), 142.7$ (CH-4), 102.6 (CH-5), 90.7 (CH-1'), 86.4 (CH-4'), 75.7 (CH-2'), $71.3\left(\mathrm{CH}-3\right.$ '), $62.3\left(\mathrm{CH}_{2}-4^{\prime}{ }^{\prime}\right)$.

Dodecyl-(4-methoxy-1'H-[1,2']bipyrrolyl-5-ylidenmethyl)-amin (BE-18591, 50): $\mathrm{C}_{22} \mathrm{H}_{35} \mathrm{~N}_{3} \mathrm{O}, \boldsymbol{R}_{\mathbf{f}}=0.85 ; \mathrm{CH}_{2} \mathrm{Cl}_{2} / \mathrm{MeOH}, 95: 5,{ }^{1} \mathbf{H}-\mathbf{N M R}\left(\mathrm{CDCl}_{3}, 300 \mathrm{MHz}\right): \delta=13.60$ (s, H/D-austauschbar, 1 H, NH), 10.78 (s, H/D-austauschbar, 1 H, NH), 9.44 (s, H/Daustauschbar, $1 \mathrm{H}, \mathrm{NH}), 7.32$ (d, ${ }^{3} J=13 \mathrm{~Hz}, 1 \mathrm{H}, 1$ '’-H), 7.02 (m, $1 \mathrm{H}, 5$ '-H), 6.70 (m, 1 H, 3'-H), 6.22 (m, 1 H, 4'-H), 5.92 (d, $\left.{ }^{3} J=2 \mathrm{~Hz}, 3-\mathrm{H}\right), 3.90$ (s, $3 \mathrm{H},-\mathrm{OCH}_{3}$ ), 3.42 (q, ${ }^{3} J=6 \mathrm{~Hz}, 2 \mathrm{H}, 3$ ' '-H), 1.72 (m, 2-H, 4'”-H), 1.20 (m, 16 H, 5' -12'’), 0.82 $\left(\mathrm{m}, 5 \mathrm{H},-\mathrm{CH}_{2} \mathrm{CH}_{3}\right) .{ }^{13} \mathrm{C}-\mathrm{NMR}\left(\mathrm{CDCl}_{3}, 300 \mathrm{MHz}\right): \delta=163.7\left(\mathrm{C}_{\mathrm{q}}-2\right), 142.4\left(\mathrm{C}_{\mathrm{q}}-4\right)$, 140.2 (CH-5'), 124.2 (C $\mathrm{q}^{-2}{ }^{\prime}$ ), 122.5 (CH-1'’), 113.2 (CH-3'), 110.7 (CH-4'), 91.2 $\left(\mathrm{C}_{\mathrm{q}}-5\right), 91.2(\mathrm{CH}-3), 58.4\left(\mathrm{CH}_{3}-\mathrm{OCH}_{3}\right), 51.0\left(\mathrm{CH}_{2}-3{ }^{\prime \prime}\right), 33.7\left(\mathrm{CH}_{2}-4\right)$ ') $31.8\left(\mathrm{CH}_{2}-\right.$

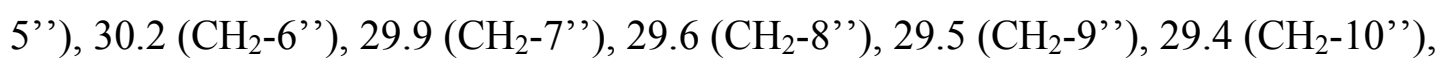

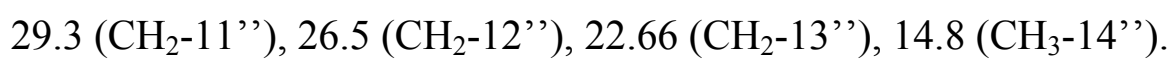

Phthalsäure-di(2-ethylhexyl ester) (Diisooctyl-phthalat) (51): $\mathrm{C}_{24} \mathrm{H}_{38} \mathrm{O}_{4}, \boldsymbol{R}_{\mathbf{f}}=$ 0.90; $\mathrm{CH}_{2} \mathrm{Cl}_{2}, \mathbf{C I}-\mathbf{M S}\left(\mathrm{NH}_{3}\right): 798\left(\left[2 \mathrm{M}+\mathrm{NH}_{4}\right]^{+}, 62 \%\right), 408\left(\left[\mathrm{M}+\mathrm{NH}_{4}\right]^{+}, 100\right), 391$ $\left([\mathrm{M}+\mathrm{H}]^{+}, 65\right) .{ }^{1} \mathbf{H}-\mathbf{N M R}\left(\mathrm{CDCl}_{3}, 200 \mathrm{MHz}\right): \delta=7.70(\mathrm{~m}, 2 \mathrm{H}), 7.50(\mathrm{~m}, 2 \mathrm{H}), 4.25$ (d, $\left.{ }^{3} J=5 \mathrm{~Hz}, 2 \mathrm{H}\right), 1.80-1.20$ (br m, $18 \mathrm{H}$ ), $1.00-0.75$ (br m, $12 \mathrm{H}$ ).

\section{Stamm B 9020}

Der Streptomyceten-Stamm B 9020 wird in der Sammlung mariner Actinomyceten des Alfred-Wegener-Instituts für Polar- und Meeresforschung in Bremerhaven in Reinkultur aufbewahrt. 


\section{Primärscreening}

Man inkubierte den Stamm erst auf $\mathrm{M}_{2}{ }^{+}$-Schrägagar und anschließend auf Agarplatten jeweils $72 \mathrm{~h}$ bei $28^{\circ} \mathrm{C}$. Mit den Agarkulturen wurden fünf mit je $200 \mathrm{ml}$ gefüllte 1-1-Schikanekolben beimpft und $72 \mathrm{~h}$ bei $28{ }^{\circ} \mathrm{C}$ und $95 \mathrm{Upm}$ inkubiert. Die Schüttelkultur wurde mit EtOAc extrahiert.

In biologischen Tests zeigte der marine Streptomycet eine in der Tabelle 13 dargestellte Aktivität sowohl gegen die Bakterien und Pilze als auch gegen die in den Tests eingesetzten Algen.

Dünnschichtchromatogramme des aus dem 20-1-Fermenter gewonnenen Rohextraktes zeigten mehrere UV-löschende Zonen, die sich mit Anisaldehyd/Schwefelsäure violett anfärben ließen $\left(R_{\mathrm{f}}=0.20, R_{\mathrm{f}}=0.42, R_{\mathrm{f}}=0.45\right.$ braune Zone, $\left.R_{\mathrm{f}}=0.81\right)$

Tabelle 13: Aktivitäten des entfetteten Extraktes im Plattendiffusionstest.

\begin{tabular}{|c|c|c|}
\hline & Organismus & Hemmhof $\varnothing$ (in mm) \\
\hline \multirow{6}{*}{ 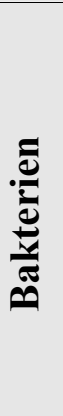 } & Escherichia coli & 16 \\
\hline & Bacillus subtilis & 16 \\
\hline & Streptomyces viridochromogenes (Tü57) & 17 \\
\hline & Candida albicans & 0 \\
\hline & Mucor miehei & 0 \\
\hline & Staphylococcus aureus & 15 \\
\hline \multirow{3}{*}{$\begin{array}{l}\frac{a}{20} \\
\frac{00}{2}\end{array}$} & Chlorella vulgaris & 11 \\
\hline & Chlorella sorokiniana & 13 \\
\hline & Scenedesmus subspicatus & 0 \\
\hline
\end{tabular}

\section{Fermentation und Aufarbeitung}

Man fermentierte diskontinuierlich im 20-1-Maßstab in einem mit GALOP optimierten Nährmedium aus $38 \mathrm{~g}$ Maltose, $55 \mathrm{~g}$ Hefeextrakt, $61 \mathrm{~g}$ Glucose und $19 \mathrm{~g} \mathrm{CaCl}_{2}$ in 101 Leitungswasser und 101 künstlichem Seewasser. Beimpft wurde mit 21 einer 72 $\mathrm{h}$ bei $25^{\circ} \mathrm{C}$ inkubierten Schüttelkultur.

Fermentation führte man in einem diskontinuierlichen 201 Fermenter bei $28{ }^{\circ} \mathrm{C}, 331$ Luft/min, pH $6.9 \pm 1.5$ (2 N NaOH, 2 N HCl zur pH-Regulierung) und einer Rührgeschwindigkeit von 200 Upm durch. Schaumbildung wurde durch Zugabe von $42 \mathrm{ml}$ Niax verhindert. Die Kulturbrühe wurde $5 \mathrm{~min}$ mit dem Ultraturrax behandelt und 
mit der Filterpresse über Celite. filtriert. Man extrahierte den Überstand dreimal mit je 21 EtOAc. Das Mycel wurde mit $300 \mathrm{ml} \mathrm{MeOH}$ über $12 \mathrm{~h}$ aufgeschlossen.

Danach digerierte man das Mycel dreimal mit jeweils $300 \mathrm{ml}$ EtOAc. Nach dem Eindampfen der vereinigten org. Phasen erhielt man 734 mg eines graubraunen Rohextraktes, der dreimal mit je $50 \mathrm{ml}$ Cyclohexan entfettet wurde.

\section{Isolierung der Metaboliten}

Der Rohextrakt wurde in $20 \mathrm{ml} \mathrm{CH}_{2} \mathrm{Cl}_{2} / \mathrm{MeOH}$ 8:2 aufgenommen und durch Säulenchromatographie an Kieselgel $(5 \times 60 \mathrm{~cm}$, Essigester/Dichlormethan-Stufengradient: $11 \mathrm{CH}_{2} \mathrm{Cl}_{2}, 11 \mathrm{CH}_{2} \mathrm{Cl}_{2} / 5 \%$ Essigester, $11 \mathrm{CH}_{2} \mathrm{Cl}_{2} / 10 \%$ Essigester, 11 $\mathrm{CH}_{2} \mathrm{Cl}_{2} / 20 \%$ Essigester, $11 \mathrm{CH}_{2} \mathrm{Cl}_{2} / 50 \%$ Essigester, 21 Essigester) vorgetrennt. Man erhielt drei Fraktionen I bis III. Fraktion II wurde als uninteressant eingestuft und verworfen.

Fraktion I chromatographierte man an präparativen Dickschichtplatten (8 Platten 20 $\times 20 \mathrm{~cm}$; Kieselgel, $\left.\mathrm{CH}_{2} \mathrm{Cl}_{2} / \mathrm{MeOH} ; 9: 1\right)$ und erhielt Fraktionen Ia und $\mathrm{Ib}\left(R_{\mathrm{f}}=0.60\right.$, $\left.R_{\mathrm{f}}=0.75-0.85\right)$. Die leicht UV-löschende Zone mit dem $R_{\mathrm{f}}$-Wert von 0.6 lieferte jedoch keine Substanz. Die Fraktion Ib wurde an Sephadex LH-20 $(3 \times 120 \mathrm{~cm}$, $\mathrm{CH}_{2} \mathrm{Cl}_{2} / \mathrm{MeOH}$; 6:4) chromatographiert und lieferte $34 \mathrm{mg}$ Ergosterol (53) sowie 14 mg Indolyl-3-carbonsäuremethylester (54).

Fraktion III ließ sich durch präparative HPLC an Flash-Kieselgel (30 - $60 \mu \mathrm{m}, \mathrm{J}$. T. Baker; Metallsäule $2 \times 40 \mathrm{~cm}, \mathrm{CH}_{2} \mathrm{Cl}_{2} / 2 \% \mathrm{MeOH}$ Anstieg auf $15 \% \mathrm{MeOH}$ in 30 min, dann auf $40 \% \mathrm{MeOH}$ in $10 \mathrm{~min}$, über $10 \mathrm{~min} 40 \% \mathrm{MeOH}$ und dann Abfall auf $2 \%$ in $1 \mathrm{~min}$, Fluss $10.0 \mathrm{ml} / \mathrm{min}$, Detektion bei $230 \mathrm{~nm}$ ) in zwei Substanzen auftrennen. Man erhielt $8.7 \mathrm{mg}$ Tyrosol (39) und $6.2 \mathrm{mg}$ Indolyl-3-carbaldehyd (52).

Indolyl-3-carbaldehyd (52): $\mathrm{C}_{9} \mathrm{H}_{7} \mathrm{NO}, \boldsymbol{R}_{\mathbf{f}}=0.30 ; \mathrm{CH}_{2} \mathrm{Cl}_{2} / \mathrm{MeOH}, 9: 1$, CI-MS $\left(\mathrm{NH}_{3}\right): 163\left(\left[\mathrm{M}+\mathrm{NH}_{4}\right]^{+}, 100 \%\right), 146\left([\mathrm{M}+\mathrm{H}]^{+}, 68\right) .{ }^{1} \mathbf{H}-\mathbf{N M R}\left(\mathrm{CDCl}_{3}, 300 \mathrm{MHz}\right)$ : $\delta=10.06$ (s, $1 \mathrm{H},-\mathrm{CHO}), 9.00$ (br s, H/D-austauschbar, $1 \mathrm{H}, \mathrm{NH}), 8.36$ (m, $1 \mathrm{H}, 4-$ H), $7.83\left(\mathrm{~d},{ }^{3} \mathrm{~J}=10 \mathrm{~Hz}, 1 \mathrm{H}, 2-\mathrm{H}\right), 7.42$ (m, $\left.1 \mathrm{H}, 7-\mathrm{H}\right), 7.32$ (m, $\left.2 \mathrm{H}, 6-\mathrm{H}, 5-\mathrm{H}\right)$. 
Ergosterol (53): $\mathrm{C}_{28} \mathrm{H}_{44} \mathrm{O}, \boldsymbol{R}_{\mathbf{f}}=0.80 ; \mathrm{CH}_{2} \mathrm{Cl}_{2} / \mathrm{MeOH}, 9: 1$, CI-MS $\left(\mathrm{NH}_{3}\right): 810([2 \mathrm{M}$ $\left.\left.+\mathrm{NH}_{4}\right]^{+}\right), 414\left(\left[\mathrm{M}+\mathrm{NH}_{4}\right]^{+}\right), 397\left([\mathrm{M}+\mathrm{H}]^{+}\right) \cdot{ }^{1} \mathbf{H}-\mathbf{N M R}\left(\mathrm{CDCl}_{3}, 300 \mathrm{MHz}\right): \delta=5.58$ $(\mathrm{m}, 1 \mathrm{H}), 5.38(\mathrm{~m}, 1 \mathrm{H}), 5.18\left(\mathrm{t},{ }^{3} \mathrm{~J}=8 \mathrm{~Hz}, 2 \mathrm{H}\right), 3.60(\mathrm{~m}, 1 \mathrm{H}), 2.24(\mathrm{~m}, 1 \mathrm{H}), 2.24$ (m, $1 \mathrm{H}), 2.04-1.20$ (br m, $18 \mathrm{H}), 1.0\left(\mathrm{~d},{ }^{3} \mathrm{~J}=8 \mathrm{~Hz}, 3 \mathrm{H}, \mathrm{CH}_{3}\right), 0.86\left(\mathrm{~s}, 3 \mathrm{H}, \mathrm{CH}_{3}\right)$, $8.85\left(\mathrm{~d},{ }^{3} \mathrm{~J}=8 \mathrm{~Hz}, 3 \mathrm{H}, \mathrm{CH}_{3}\right), 0.81\left(\mathrm{~d},{ }^{3} \mathrm{~J}=8 \mathrm{~Hz}, 3 \mathrm{H}, \mathrm{CH}_{3}\right), 0.80\left(\mathrm{~d},{ }^{3} \mathrm{~J}=8 \mathrm{~Hz}, 3 \mathrm{H}\right.$, $\left.\mathrm{CH}_{3}\right), 0.60\left(\mathrm{~s}, 3 \mathrm{H}, \mathrm{CH}_{3}\right) \cdot{ }^{13} \mathbf{C}-\mathbf{N M R}\left(\mathrm{CDCl}_{3}, 200 \mathrm{MHz}\right): \delta=141.4,\left(\mathrm{C}_{\mathrm{q}}-5\right), 139.7$ ( $\left.\mathrm{C}_{\mathrm{q}}-10\right), 135.6$ (CH-22), 131.9 (CH-23), 119.6 (CH-6), 116.2 (CH-7), 70.5 (CH-3), 55.7 (CH-17), 54.5 (CH-14), 46.2 (CH-9), 42.8 (C $\left.\mathrm{C}_{\mathrm{q}}-13\right), 42.8$ (CH-9), 40.7 (CH-20), $40.4\left(\mathrm{CH}_{2}-12\right), 39.1\left(\mathrm{CH}_{2}-2\right), 38.3\left(\mathrm{C}_{\mathrm{q}}-10\right), 37.2\left(\mathrm{CH}_{2}-1\right), 33.1(\mathrm{CH}-25), 32.0\left(\mathrm{CH}_{2}-\right.$ 1), $28.3\left(\mathrm{CH}_{2}-16\right), 23.0\left(\mathrm{CH}_{2}-15\right), 22.9\left(\mathrm{CH}_{2}-11\right), 21.1\left(\mathrm{CH}_{3}-26\right), 19.9\left(\mathrm{CH}_{3}-27\right), 19.5$ $\left(\mathrm{CH}_{3}-21\right), 17.6\left(\mathrm{CH}_{3}-28\right), 16.3\left(\mathrm{CH}_{3}-19\right), 12.1\left(\mathrm{CH}_{3}-18\right)$.

3-Indolyl-carbonsäuremethylester (54): $\mathrm{C}_{10} \mathrm{H}_{9} \mathrm{NO}_{2}, \boldsymbol{R}_{\mathbf{f}}=0.85 ; \mathrm{CH}_{2} \mathrm{Cl}_{2} / \mathrm{MeOH}, 9: 1$, CI-MS $\left(\mathrm{NH}_{3}\right): 368\left(\left[2 \mathrm{M}+\mathrm{NH}_{4}\right]^{+}, 4 \%\right), 351\left([\mathrm{M}+\mathrm{H}]^{+}, 1\right), 193\left(\left[\mathrm{M}+\mathrm{NH}_{4}\right]^{+}, 100\right)$, $176\left([\mathrm{M}+\mathrm{H}]^{+}, 60\right) .{ }^{1} \mathbf{H}-\mathbf{N M R}\left(\mathrm{CDCl}_{3}, 300 \mathrm{MHz}\right): \delta=8.58$ (s, H/D-austauschbar, 1 H, NH), 8.14 (m, 1 H, 6-H), 7.84 (d, $\left.{ }^{3} J=10 \mathrm{~Hz}, 1 \mathrm{H}, 2-\mathrm{H}\right), 7.38$ (m, $\left.1 \mathrm{H}, 7-\mathrm{H}\right), 7.20$ (m, $2 \mathrm{H}, 5-\mathrm{H}, 6-\mathrm{H}), 3.83\left(\mathrm{~s}, 3 \mathrm{H},-\mathrm{OCH}_{3}\right) .{ }^{13} \mathbf{C}-\mathbf{N M R}\left(\mathrm{CDCl}_{3}, 200 \mathrm{MHz}\right): \delta=165.6$ $\left(\mathrm{C}_{\mathrm{q}}-3^{\prime}\right), 136.0\left(\mathrm{C}_{\mathrm{q}}-7 \mathrm{a}\right), 130.9\left(\mathrm{C}_{\mathrm{q}}-3 \mathrm{a}\right), 125.7(\mathrm{CH}-2), 123.7(\mathrm{CH}-6), 122.0(\mathrm{CH}-5)$, $121.5(\mathrm{CH}-4), 111.3(\mathrm{CH}-7), 108.8\left(\mathrm{C}_{\mathrm{q}}-3\right), 51.0\left(\mathrm{CH}_{3}-\mathrm{OCH}_{3}\right)$.

\section{Stamm B 8027}

Der mariner Streptomycet B 8027 wird in der Sammlung der Actinomyceten des Alfred-Wegener-Instituts für Polar- und Meeresforschung in Bremerhaven in Reinkultur aufbewahrt. Auf Agar bildet er ein weißes Mycel. Man beobachtete keine Verfärbung des Agars in der Nähe des gebildeten Mycels.

\section{Primärscreening}

Der marine Streptomycet B8027 aus Bremerhaven wurde erst auf $\mathrm{M}_{2}^{+}$-Schrägagar und anschließend auf Agarplatten jeweils $72 \mathrm{~h}$ bei $28^{\circ} \mathrm{C}$ angezogen. Mit den Agarkulturen wurden fünf mit je $200 \mathrm{ml}$ gefüllte 1-1-Schikanekolben beimpft und $72 \mathrm{~h}$ bei $28{ }^{\circ} \mathrm{C}$ und $95 \mathrm{Upm}$ inkubiert. Die Schüttelkultur wurde mit EtOAc extrahiert. Die 
Ergebnisse des biologischen Screening sind in der Tabelle 14 dargestellt. Eine Bioautographie entwickelter Dünnschichtchromatogramme $\left(\mathrm{CH}_{2} \mathrm{Cl}_{2} / \mathrm{MeOH}, 95: 5\right)$ des aus dem kontinuierlichen 10-1-Bioreaktor gewonnenen Rohextraktes zeigte nur schwache Aktivität bei mehreren Zonen mit dem $R_{\mathrm{f}}$-Wert von $0.25,0.50,0.60$ und 0.70 .

Tabelle 14: Aktivitäten des entfetteten Extraktes im Plattendiffusionstest.

\begin{tabular}{|c|c|c|}
\hline & Organismus & Hemmhof $\varnothing$ (in mm) \\
\hline \multirow{4}{*}{$\approx$} & Escherichia coli & 0 \\
\cline { 2 - 3 } & Bacillus subtilis & 0 \\
\cline { 2 - 3 } & Streptomyces viridochromogenes (Tü57) & 11 \\
\cline { 2 - 3 } & Candida albicans & 15 \\
\cline { 2 - 3 } & Mucor miehei & 20 \\
\cline { 2 - 3 } & Staphylococcus aureus & 12 \\
\cline { 2 - 3 } & Chlorella vulgaris & 11 \\
\cline { 2 - 3 } & Chlorella sorokiniana & 35 \\
\cline { 2 - 3 } & Scenedesmus subspicatus & 12 \\
\hline
\end{tabular}

\section{Fermentation und Aufarbeitung}

Man fermentierte im 10-1-Maßstab mit GALOP optimierten Nährmedium aus $54 \mathrm{~g}$ Maltose, $77 \mathrm{~g}$ Hefeextrakt, $21 \mathrm{~g}$ Glucose und $37 \mathrm{~g} \mathrm{CaCl}_{2}$ in 51 Leitungswasser und 5 1 künstliches Seewasser. Beimpfung des kleinen Durchflußfermenters erfolgte mit 1.51 einer $72 \mathrm{~h}$ bei $28^{\circ} \mathrm{C}$ inkubierten Schüttelkultur.

Fermentation führte man kontinuierlich in einem 2-1-Durchflußfermenter über sieben Tage bei $28{ }^{\circ} \mathrm{C}, 331 \mathrm{Luft} / \mathrm{min}, \mathrm{pH} 6.9 \pm 1.5(2 \mathrm{~N} \mathrm{NaOH}, 2 \mathrm{~N} \mathrm{HCl}$ zur pHRegulierung) durch. Wegen der starken Schaumentwicklung wurde während der Kultivierung mehrmals Antischaummittel (Niax) in kleinen Portionen zugegeben.

Die Kulturbrühe wurde 3 min mit dem Ultraturrax behandelt und über Celite filtriert. Man extrahierte den Überstand dreimal mit 11 EtOAc und das Mycel behandelte man $12 \mathrm{~h}$ mit $\mathrm{MeOH}$.

Danach extrahierte man das Mycel jeweils dreimal mit 200 ml EtOAc. Nach dem Eindampfen der vereinigten org. Phasen erhielt man $928 \mathrm{mg}$ einer braunen öligen Mischung, die dreimal mit je $50 \mathrm{ml}$ Cyclohexan entfettet wurde. 


\section{Isolierung}

Der Rohextrakt wurde in $25 \mathrm{ml} \mathrm{CH}_{2} \mathrm{Cl}_{2} / \mathrm{MeOH}$ 7:3 aufgenommen und durch Säulenchromatographie an Kieselgel $(5 \times 60 \mathrm{~cm}$, Essigester/Dichlormethan-Stufengradient: $11 \mathrm{CH}_{2} \mathrm{Cl}_{2}, 11 \mathrm{CH}_{2} \mathrm{Cl}_{2} / 5 \%$ Essigester, $11 \mathrm{CH}_{2} \mathrm{Cl}_{2} / 10 \%$ Essigester, 11 $\mathrm{CH}_{2} \mathrm{Cl}_{2} / 20 \%$ Essigester, $11 \mathrm{CH}_{2} \mathrm{Cl}_{2} / 50 \%$ Essigester, 21 Essigester) vorgetrennt. Man erhielt zwei Fraktionen I und II. Aus der beim Entfetten erhaltenen Fettphase fiel beim Einengen ein weißer Niederschlag aus, der durch präparative Chromatographie an Dickschichtplatten (5 Platten $20 \times 20 \mathrm{~cm}$; Kieselgel, $\mathrm{CH}_{2} \mathrm{Cl}_{2}$ ) gereinigt wurde. Die Zone mit dem $R_{\mathrm{f}}$-Wert von 0.95 wurde mit Dichlormethan eluiert und lieferte $68 \mathrm{mg}$ einer farblosen, öligen Flüssigkeit, die unterschiedliche homologe isoFettsäure-methylester (58) beinhaltete.

Die Fraktion I chromatographierte man an präparativen Dickschichtplatten (14 Platten $\left.20 \times 20 \mathrm{~cm} ; \mathrm{CH}_{2} \mathrm{Cl}_{2} / \mathrm{MeOH} ; 9: 1\right)$ und erhielt die Fraktionen Ia, Ib und Ic $\left(R_{\mathrm{f}}=\right.$ $\left.0.85, R_{\mathrm{f}}=0.75, R_{\mathrm{f}}=0.50-0.65\right)$.

Fraktion Ia beinhaltete $43 \mathrm{mg}$ des Antischaummittels Niax. Fraktion Ib lieferte nach der Elution mit $50 \mathrm{ml}$ EtOAc $33 \mathrm{mg}$ niedermolekularen PHB (Polyhydroxybuttersäure).

Durch zusätzliche präparative HPLC-Trennung an RP18 (Standardgradient, Detektion bei $210 \mathrm{~nm}$ ) wurden aus der Fraktion Ic $16.8 \mathrm{mg}$ Thymin (33), $13 \mathrm{mg}$ 2-Methyl$3 H$-chinazolin-4-on (56) und $6.5 \mathrm{mg}$ 2-Phenyl-acetamid (57) isoliert.

Fraktion II lieferte nach der präparative HPLC-Trennung an RP18 (Standardgradient, Detektion bei $230 \mathrm{~nm}) 23 \mathrm{mg}$ einer Mischung aus N-Acetyltryptamin (36) und 5Methyl-hexan-carbonsäureamid (55).

5-Methyl-hexan-carbonsäureamid (55): $\mathrm{C}_{7} \mathrm{H}_{15} \mathrm{NO}, \boldsymbol{R}_{\mathbf{f}}=0.40 ; \mathrm{CH}_{2} \mathrm{Cl}_{2} / \mathrm{MeOH}, 95: 5$, CI-MS $\left(\mathrm{NH}_{3}\right): 259\left([2 \mathrm{M}+\mathrm{H}]^{+}, 8 \%\right), 164\left(\left[\mathrm{M}+\mathrm{NH}_{4}+\mathrm{NH}_{3}\right]^{+}, 16\right), 147([\mathrm{M}+$ $\left.\left.\mathrm{NH}_{4}\right]^{+}, 100\right), 130\left([\mathrm{M}+\mathrm{H}]^{+}, 20\right) .{ }^{1} \mathbf{H}-\mathbf{N M R}\left(\mathrm{CDCl}_{3}, 300 \mathrm{MHz}\right): \delta=5.48$ (br s, H/Daustauschbar, $\left.2 \mathrm{H}, \mathrm{NH}_{2}\right), 2.2(\mathrm{~m}, 2 \mathrm{H}, 1-\mathrm{H}), 1.62$ (m, $\left.1 \mathrm{H}, 2-\mathrm{H}\right), 1.45-1.25$ (m, 2 H, 3$\mathrm{H}), 1.20$ (m, $2 \mathrm{H}, 5-\mathrm{H}), 0.84$ (m, $\left.6 \mathrm{H}, 5-\mathrm{CH}_{3}, 2^{\prime}-\mathrm{CH}_{3}\right) .{ }^{13} \mathbf{C}-\mathbf{N M R}\left(\mathrm{CDCl}_{3}, 200 \mathrm{MHz}\right)$ : $\delta=176.1\left(\mathrm{C}_{\mathrm{q}}-\mathrm{CO}\right), 34.0(\mathrm{CH}-2), 33.6\left(\mathrm{CH}_{2}-1\right), 32.1\left(\mathrm{CH}_{2}-3\right), 29.1\left(\mathrm{CH}_{2}-4\right), 18.79$ $\left(\mathrm{CH}_{3}-5\right), 11.23\left(\mathrm{CH}_{3}-2^{\prime}\right)$. 
2-Methyl-3H-chinazolin-4-on (56): $\mathrm{C}_{9} \mathrm{H}_{8} \mathrm{~N}_{2} \mathrm{O}, \boldsymbol{R}_{\mathbf{f}}=0.62 ; \mathrm{CH}_{2} \mathrm{Cl}_{2} / \mathrm{MeOH}, 9: 1$, CIMS $\left(\mathrm{NH}_{3}\right): 321\left([2 \mathrm{M}+\mathrm{H}]^{+}, 15 \%\right), 178\left(\left[\mathrm{M}+\mathrm{NH}_{4}\right]^{+}, 22\right), 161\left([\mathrm{M}+\mathrm{H}]^{+}, 100\right) .{ }^{1} \mathbf{H}-$ NMR $\left(\mathrm{CDCl}_{3}, 300 \mathrm{MHz}\right): \delta=12.15$ (br s, H/D-austauschbar, $\left.1 \mathrm{H}, \mathrm{NH}\right), 8.06$ (m, 1 H, 7-H), 7.78 (m, $1 \mathrm{H}, 4-\mathrm{H}), 7.58\left(\mathrm{~d},{ }^{3} \mathrm{~J}=8 \mathrm{~Hz}, 1 \mathrm{H}, 5-\mathrm{H}\right), 7.40\left(\mathrm{dt},{ }^{3} \mathrm{~J}=8 \mathrm{~Hz},{ }^{3} \mathrm{~J}=1\right.$ $\mathrm{Hz}, 1 \mathrm{H}, 6-\mathrm{H}), 2.36\left(\mathrm{~s}, 3 \mathrm{H}, \mathrm{CH}_{3}\right) .{ }^{13} \mathbf{C}-\mathbf{N M R}\left(\mathrm{CDCl}_{3}, 200 \mathrm{MHz}\right): \delta=161.7\left(\mathrm{C}_{\mathrm{q}}-8\right)$, $154.25\left(\mathrm{C}_{\mathrm{q}}-2\right), 148.9\left(\mathrm{C}_{\mathrm{q}}-3 \mathrm{a}\right), 134.2(\mathrm{CH}-5), 126.5\left(\mathrm{C}_{\mathrm{q}}-7 \mathrm{a}\right), 125.8(\mathrm{CH}-7), 125.8(\mathrm{CH}-$ 6), $125.6(\mathrm{CH}-4), 21.4\left(\mathrm{CH}_{3}-2\right.$ ').

2-Phenyl-acetamid (57): $\mathrm{C}_{8} \mathrm{H}_{9} \mathrm{NO} .\left(\boldsymbol{R}_{\mathbf{f}}=0.65 ; \mathrm{CH}_{2} \mathrm{Cl}_{2} / \mathrm{MeOH}, 9: 1\right)$, CI-MS $\left(\mathrm{NH}_{3}\right)$ : $288\left(\left[2 \mathrm{M}+\mathrm{NH}_{4}\right]^{+}, 1 \%\right), 153\left(\left[\mathrm{M}+\mathrm{NH}_{4}\right]^{+}, 100\right), 136\left([\mathrm{M}+\mathrm{H}]^{+}, 11\right) .{ }^{1} \mathbf{H}-\mathbf{N M R}$ $\left(\mathrm{CDCl}_{3}, 300 \mathrm{MHz}\right): \delta=7.39-7.32(\mathrm{~m}, 5 \mathrm{H}, 2-3-4-5-6-\mathrm{H}), 5.38$ (br s, H/Daustauschbar, $\left.2 \mathrm{H}, \mathrm{NH}_{2}\right), 3.59$ (s, $2 \mathrm{H}, 1$ ' $\left.-\mathrm{CH}_{2}\right) .{ }^{13} \mathbf{C}-\mathbf{N M R}\left(\mathrm{CDCl}_{3}, 200 \mathrm{MHz}\right): \delta=$ $172.7\left(\mathrm{C}_{\mathrm{q}}-2^{\prime}\right), 134.7\left(\mathrm{C}_{\mathrm{q}}-1\right), 129.4(\mathrm{CH}-5), 129.4(\mathrm{CH}-3), 129.1(\mathrm{CH}-6), 129.1(\mathrm{CH}-$ 2), $127.5(\mathrm{CH}-4), 43.3\left(\mathrm{CH}_{2}-1^{\prime}\right)$.

Homologe iso-Fettsäure-methylester (58): $\boldsymbol{R}_{\mathbf{f}}=0.95 ; \mathrm{CH}_{2} \mathrm{Cl}_{2}$, EI-MS (70 eV): $\mathrm{m} / z$ $(\%)=\mathrm{C}_{8} \mathrm{H}_{16} \mathrm{O}_{2}, 143(\mathrm{M}+, 20), \mathrm{C}_{11} \mathrm{H}_{22} \mathrm{O}_{2}, 185(\mathrm{M}+, 10), \mathrm{C}_{12} \mathrm{H}_{24} \mathrm{O}_{2}, 199(\mathrm{M}+, 20)$, $\mathrm{C}_{13} \mathrm{H}_{26} \mathrm{O}_{2}, 213(\mathrm{M}+, 10), \mathrm{C}_{14} \mathrm{H}_{28} \mathrm{O}_{2}, 228(\mathrm{M}+, 15), \mathrm{C}_{15} \mathrm{H}_{30} \mathrm{O}_{2}, 242(\mathrm{M}+, 10)$, $\mathrm{C}_{16} \mathrm{H}_{32} \mathrm{O}_{2}, 256(\mathrm{M}+, 30), \mathrm{C}_{17} \mathrm{H}_{34} \mathrm{O}_{2}, 270(\mathrm{M}+, 26), \mathrm{C}_{18} \mathrm{H}_{36} \mathrm{O}_{2}, 284(\mathrm{M}+, 10), \mathrm{C}_{19} \mathrm{H}_{38} \mathrm{O}_{2}$, $298(\mathrm{M}+, 1) .{ }^{1} \mathbf{H}-\mathbf{N M R}\left(\mathrm{CDCl}_{3}, 300 \mathrm{MHz}\right): \delta=3.61\left(\mathrm{~s}, 3 \mathrm{H},-\mathrm{OCH}_{3}\right), 2.24\left(\mathrm{t},{ }^{3} J=7\right.$ $\left.\mathrm{Hz}, 2 \mathrm{H}, \mathrm{CH}_{2}\right), 1.62-1.40\left(\mathrm{~m}, 2 \mathrm{H}, \mathrm{CH}_{2}\right), 1.36-1.00$ (br m, $\left.20 \mathrm{H}, 10 \times \mathrm{CH}_{2}\right), 0.82(\mathrm{~d}$, ${ }^{3} J=6 \mathrm{~Hz}, 6 \mathrm{H}$, isoprop. $\left.\mathrm{CH}_{3}\right)$.

\section{Stamm GW 19/2497}

Der terrestrische Streptomycet GW 19/2497 stammt aus der Sammlung des Instituts für Bodenkunde in Lohra-Kirchvers und wird in der Arbeitsgruppe als Erd- und Stickstoffkultur gehalten. Auf Agar bildet er ein weißes Mycel. Eine Verfärbung des Agars wurde nicht beobachtet. 


\section{Primärscreening}

Man inkubierte den Stamm erst auf $\mathrm{M}_{2}$-Schrägagar und anschließend auf Agarplatten jeweils $72 \mathrm{~h}$ bei $28^{\circ} \mathrm{C}$. Mit den Agarkulturen wurden sechs mit je $250 \mathrm{ml}$ gefüllte 11-Schikanekolben beimpft und $72 \mathrm{~h}$ bei $28^{\circ} \mathrm{C}$ und $95 \mathrm{Upm}$ inkubiert. Die Schüttelkultur wurde gefriergetrocknet und anschließend mit EtOAc extrahiert.

In biologischen Tests zeigte der marine Streptomycet die in der Tabelle 15 dargestellte Aktivität sowohl gegen Teststämme als auch gegen in den Tests eingesetzten Algen.

Tabelle 15: Aktivitäten des entfetteten Extraktes im Plattendiffusionstest.

\begin{tabular}{|c|c|c|}
\hline & Organismus & Hemmhof $\varnothing$ (in mm) \\
\hline \multirow{6}{*}{ } & Escherichia coli & 19 \\
\hline & Bacillus subtilis & 26 \\
\hline & Streptomyces viridochromogenes (Tü57) & 20 \\
\hline & Candida albicans & 0 \\
\hline & Mucor miehei & 0 \\
\hline & Staphylococcus aureus & 25 \\
\hline \multirow{3}{*}{ 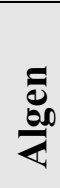 } & Chlorella vulgaris & 17 \\
\hline & Chlorella sorokiniana & 0 \\
\hline & Scenedesmus subspicatus & 20 \\
\hline
\end{tabular}

\section{Fermentation und Aufarbeitung}

Man fermentierte im 10-1-Maßstab mit GALOP optimierten Nährmedium aus $67 \mathrm{~g}$ Maltose, $60 \mathrm{~g}$ Hefeextrakt, $41 \mathrm{~g}$ Glucose und $18 \mathrm{~g} \mathrm{CaCl}_{2}$ in 101 Leitungswasser. Die Beimpfung des kleinen Durchflussfermenters erfolgte mit 1.61 einer $72 \mathrm{~h}$ bei $28^{\circ} \mathrm{C}$ inkubierten Schüttelkultur.

Fermentation führte man kontinuierlich über sieben Tage bei $28{ }^{\circ} \mathrm{C}, 331 \mathrm{Luft} / \mathrm{min}$, pH $6.5 \pm 1(2 \mathrm{~N} \mathrm{NaOH}, 2 \mathrm{~N} \mathrm{HCl}$ zur pH-Regulierung) durch.

Die Kulturbrühe wurde 3 min mit dem Ultraturrax behandelt und mit der Filterpresse über Celite filtriert. Man extrahierte den Überstand dreimal mit je 31 EtOAc. Das Mycel wurde mit $500 \mathrm{ml} \mathrm{MeOH}$ über $12 \mathrm{~h}$ aufgeschlossen. 
Danach extrahierte man das Mycel dreimal mit jeweils 200 ml EtOAc. Nach dem Eindampfen der vereinigten org. Phasen erhielt man 1.2 g eines leicht braunen Rohextraktes, der zweimal mit je $50 \mathrm{ml}$ Cyclohexan entfettet wurde.

\section{Isolierung der Metabolite}

Der Rohextrakt wurde in $25 \mathrm{ml} \mathrm{CH}_{2} \mathrm{Cl}_{2} / \mathrm{MeOH}$ 7:3 aufgenommen und durch Säulenchromatographie an Kieselgel $(5 \times 60 \mathrm{~cm}$, Essigester/Dichlormethan-Stufengradient: $11 \mathrm{CH}_{2} \mathrm{Cl}_{2}, 11 \mathrm{CH}_{2} \mathrm{Cl}_{2} / 5 \%$ Essigester, $11 \mathrm{CH}_{2} \mathrm{Cl}_{2} / 10 \%$ Essigester, 11 $\mathrm{CH}_{2} \mathrm{Cl}_{2} / 20 \%$ Essigester, $11 \mathrm{CH}_{2} \mathrm{Cl}_{2} / 50 \%$ Essigester, 21 Essigester) vorgetrennt. Man erhielt zwei Fraktionen I und II.

Fraktion I chromatographierte man an präparativen Dickschichtplatten (10 Platten 20 $\times 20 \mathrm{~cm}$; Kieselgel, $\left.\mathrm{CH}_{2} \mathrm{Cl}_{2} / \mathrm{MeOH} ; 9: 1\right)$ und erhielt die Fraktionen Ia und $\mathrm{Ib}\left(R_{\mathrm{f}}=\right.$ $\left.0.15, R_{\mathrm{f}}=0.30-0.45\right)$. Die Zone Ia wurde mit $100 \mathrm{ml} \mathrm{EtOAc/MeOH} \mathrm{(9:1)} \mathrm{eluiert} \mathrm{und}$ an Sephadex LH-20 $\left(3 \times 120 \mathrm{~cm}, \mathrm{CH}_{2} \mathrm{Cl}_{2} / \mathrm{MeOH} ; 6: 4\right)$ chromatographiert. Man erhielt 16 mg Indolyl-3-essigsäure (59). Die Zone Ib wurde ebenfalls mit 100 ml EtO$\mathrm{Ac} / \mathrm{MeOH}$ (9:1) eluiert und an Sephadex LH-20 (3 × $\left.120 \mathrm{~cm}, \mathrm{CH}_{2} \mathrm{Cl}_{2} / \mathrm{MeOH} ; 6: 4\right)$ endgereinigt. Man erhielt $12 \mathrm{mg} \mathrm{N}$-Acetyltryptamin (36).

Fraktion II wurde an Sephadex LH-20 (3 × $120 \mathrm{~cm}, \mathrm{CH}_{2} \mathrm{Cl}_{2} / \mathrm{MeOH}$; 6:4) chromatographiert und lieferte die Fraktionen IIa und IIb. Fraktion IIb wurde verworfen.

Fraktion IIa lieferte in der präparativen HPLC-Trennung an RP18 (Standardgradient, Detektion bei $230 \mathrm{~nm}) 8 \mathrm{mg}$ Toluhydrochinon (61a) und eine weitere Fraktion IIc, die schließlich durch präparative HPLC-Trennung an Flash-Kieselgel $\left(\mathrm{CH}_{2} \mathrm{Cl}_{2} / 2 \%\right.$ $\mathrm{MeOH}$ Anstieg auf $15 \% \mathrm{MeOH}$ in $30 \mathrm{~min}$, dann auf $40 \% \mathrm{MeOH}$ in $10 \mathrm{~min}$, über 10 min $40 \% \mathrm{MeOH}$ und dann Abfall auf $2 \%$ in $1 \mathrm{~min}$, Fluss $10.0 \mathrm{ml} / \mathrm{min}$, Detektion

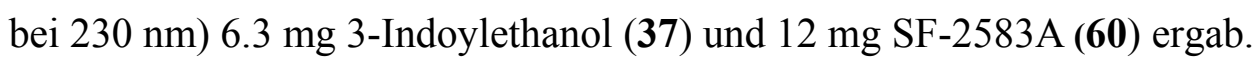

Indolyl-3-essigsäure (59): $\mathrm{C}_{10} \mathrm{H}_{9} \mathrm{NO}_{2}, \boldsymbol{R}_{\mathbf{f}}=0.25 ; \mathrm{CH}_{2} \mathrm{Cl}_{2} / \mathrm{MeOH}, 9: 1, \mathbf{C I}-\mathbf{M S}\left(\mathrm{NH}_{3}\right)$ : $193\left(\left[\mathrm{M}+\mathrm{NH}_{4}+\mathrm{NH}_{3}\right]^{+}, 100 \%\right), 176\left([\mathrm{M}+\mathrm{H}]^{+}, 15\right) .{ }^{1} \mathbf{H}-\mathbf{N M R}(\mathrm{CDCN}, 300 \mathrm{MHz})$ : $\delta=9.00($ br s, H/D-austauschbar, $1 \mathrm{H}, \mathrm{NH}), 7.40\left(\mathrm{~d},{ }^{3} J=5 \mathrm{~Hz}, 1 \mathrm{H}, 4-\mathrm{H}\right), 7.26\left(\mathrm{~d},{ }^{3} J\right.$ $=5 \mathrm{~Hz}, 1 \mathrm{H}, 7-\mathrm{H}), 7.00\left(\mathrm{~d},{ }^{3} \mathrm{~J}=2 \mathrm{~Hz}, 1 \mathrm{H}, 2-\mathrm{H}\right), 6.98-6.8$ (m, $\left.2 \mathrm{H}, 5-\mathrm{H}, 6-\mathrm{H}\right), 3.54$ (s, $\left.2 \mathrm{H}, \mathrm{CH}_{2}\right) .{ }^{13} \mathbf{C}-\mathbf{N M R}(\mathrm{CDCN}, 200 \mathrm{MHz}): \delta=173.6\left(\mathrm{C}_{\mathrm{q}^{-}}{ }^{\prime}{ }^{\prime}\right), 137.6\left(\mathrm{C}_{\mathrm{q}^{-}}-7 \mathrm{a}\right), 128.4$ 
$\left(\mathrm{C}_{\mathrm{q}}-3 \mathrm{a}\right), 124.8(\mathrm{CH}-2), 122.6(\mathrm{CH}-6), 120.1(\mathrm{CH}-5), 119.6(\mathrm{CH}-4), 112.4(\mathrm{CH}-7)$, $109.1\left(\mathrm{C}_{\mathrm{q}}-3\right), 31.3\left(\mathrm{CH}_{2}-1{ }^{\prime}\right)$.

4-Chlor-5-(3'-indolyl)oxazol $\quad$ (SF-2583A) $\quad(60): \quad \mathrm{C}_{11} \mathrm{H}_{7} \mathrm{~N}_{2} \mathrm{OCl}, \quad \boldsymbol{R}_{\mathbf{f}}=0.45$; $\mathrm{CH}_{2} \mathrm{Cl}_{2} / \mathrm{MeOH}, 9: 1$, CI-MS $\left(\mathrm{NH}_{3}\right): 454\left(\left[2 \mathrm{M}+\mathrm{NH}_{4}\right]^{+}, 3 \%\right), 437\left([2 \mathrm{M}+\mathrm{H}]^{+}, 8\right)$, $253\left(\left[\mathrm{M}+\mathrm{NH}_{3}+\mathrm{NH}_{4}\right]^{+}, 15\right), 236\left(\left[\mathrm{M}+\mathrm{NH}_{4}\right]^{+} 100\right), 219\left([\mathrm{M}+\mathrm{H}]^{+}, 83\right) .{ }^{1} \mathbf{H}-\mathbf{N M R}$ $\left(\mathrm{CDCl}_{3}, 300 \mathrm{MHz}\right): \delta=8.47$ (br s, H/D-austauschbar, $\left.1 \mathrm{H}, \mathrm{NH}\right), 8.22\left(\mathrm{~d},{ }^{3} J=6 \mathrm{~Hz}, 1\right.$ H, 4'-H), 7.81 (s, 1 H, 2-H), 8.78 (d, ${ }^{3} J=2$ Hz, 1 H, 2'-H), 7.40 (m, 1 H, 5'-H), 7.457.30 (m, 2 H, 6'-H, 7'-H). ${ }^{13} \mathbf{C}-\mathbf{N M R}\left(\mathrm{CDCl}_{3}, 200 \mathrm{MHz}\right): \delta=147.6\left(\mathrm{C}_{\mathrm{q}}-5\right), 147.6$ (CH-2), $135.6\left(\mathrm{C}_{\mathrm{q}}-7 \mathrm{a}\right), 124.4\left(\mathrm{CH}-5^{\prime}\right), 123.4\left(\mathrm{C}_{\mathrm{q}}-3 \mathrm{a}\right), 123.2\left(\mathrm{C}_{\mathrm{q}}-3\right), 121.2\left(\mathrm{CH}-4{ }^{\prime}\right)$, 120.8 (CH-6'), 120.8 (CH-7'), 111.4 (C $\left.\mathrm{C}_{\mathrm{q}}-4\right), 111.4$ (CH-2').

1,4-Dihydroxy-2-methyl-benzol (Toluhydrochinon, 61a): $\mathrm{C}_{7} \mathrm{H}_{8} \mathrm{O}_{2}, \boldsymbol{R}_{\mathbf{f}}=0.30$; $\mathrm{CH}_{2} \mathrm{Cl}_{2} / \mathrm{MeOH}, 9: 1$, EI-MS (70 eV): $m / z(\%)=124(\mathrm{M}+, 100), 123$ (40), 95 (20), 77 (8). ${ }^{1}$ H-NMR $\left(\mathrm{CDCl}_{3}, 300 \mathrm{MHz}\right): \delta=8.46$ (s, H/D-austauschbar, $\left.1 \mathrm{H}, \mathrm{OH}\right), 8.40$ (s, H/D-austauschbar, $1 \mathrm{H}, \mathrm{OH}), 6.53\left(\mathrm{~d},{ }^{3} J=9 \mathrm{~Hz}, 1 \mathrm{H}, 6-\mathrm{H}\right), 6.46\left(\mathrm{~d},{ }^{3} J=4 \mathrm{~Hz}, 1 \mathrm{H}\right.$, 3-H), $6.38\left(\mathrm{dd},{ }^{3} J=9 \mathrm{~Hz},{ }^{3} J=4 \mathrm{~Hz}, 1 \mathrm{H}, 5-\mathrm{H}\right), 2.00\left(\mathrm{~s}, 3 \mathrm{H}, \mathrm{CH}_{3}\right) .{ }^{13} \mathbf{C}-\mathbf{N M R}$ $\left(\mathrm{CDCl}_{3}, 200 \mathrm{MHz}\right): \delta=149.7\left(\mathrm{C}_{\mathrm{q}}-1\right), 147.9\left(\mathrm{C}_{\mathrm{q}}-4\right), 124.6\left(\mathrm{C}_{\mathrm{q}}-2\right), 117.3(\mathrm{CH}-3)$, 115.3 (CH-5), 112.8 (CH-6), $16.1\left(\mathrm{CH}_{3}-2^{\prime}\right)$.

\section{Stamm GW 71/2497}

\section{Primärscreening}

Der terrestrische Streptomycet GW 71/2497 stammt aus der Sammlung des Instituts für Bodenkunde in Lohra-Kirchvers und war bekannt als ein Chartreusinproduzent. Im chemischen Vorscreening fielen mehrere blaugrün fluoreszierende Zonen auf. Die nach der Optimierung aufgenommenen HPLC-Spektren wiesen mehrere farblose Komponenten auf und einen charakteristischen Peak mit dem für Chartreusin charakteristischen Absorptionsmuster. 


\section{Fermentation und Aufarbeitung}

Mit $72 \mathrm{~h}$ bei $28^{\circ} \mathrm{C}$ vorkultivierten Agarplatten impfte man 5 Schüttelkolben an und kultivierte drei Tage bei $28{ }^{\circ} \mathrm{C}$ mit $105 \mathrm{Upm}$. Mit der so erhaltenen Vorkultur wurde ein kleiner 2 1-Durchflußfermenter angeimpft und bei $28^{\circ} \mathrm{C}$ zwei Tage kultiviert.

Man fermentierte im 10-1-Maßstab mit GALOP optimierten Nährmedium aus $29 \mathrm{~g}$ Maltose, $93 \mathrm{~g}$ Hefeextrakt, $33 \mathrm{~g}$ Glucose und $17 \mathrm{~g} \mathrm{CaCl}_{2}$ in 101 Leitungswasser. Fermentation führte man kontinuierlich über sieben Tage bei $28{ }^{\circ} \mathrm{C}, 331 \mathrm{Luft} / \mathrm{min}$, pH $6.5 \pm 1(2 \mathrm{~N} \mathrm{NaOH}, 2 \mathrm{~N} \mathrm{HCl}$ zur pH-Regulierung) durch.

Die so erhaltene Kulturbrühe wurde 3 min mit dem Ultraturrax behandelt und mit der Filterpresse über Celite filtriert. Man extrahierte den Überstand dreimal mit je 31 EtOAc. Das Mycel wurde mit $300 \mathrm{ml} \mathrm{MeOH}$ über $12 \mathrm{~h}$ aufgeschlossen.

Danach extrahierte man das Mycel dreimal mit jeweils 200 ml EtOAc. Nach dem Eindampfen der vereinigten org. Phasen erhielt man $2.1 \mathrm{~g}$ einer braungelben, öligen Flüssigkeit, die zweimal mit je $50 \mathrm{ml}$ Cyclohexan entfettet wurde.

\section{Isolierung der Metabolite}

Der Rohextrakt wurde in $25 \mathrm{ml} \mathrm{CH}_{2} \mathrm{Cl}_{2} / \mathrm{MeOH}$ 1:1 aufgenommen und durch Säulenchromatographie an Kieselgel $(5 \times 60 \mathrm{~cm}$, Essigester/Dichlormethan-Stufengradient: $11 \mathrm{CH}_{2} \mathrm{Cl}_{2}, 11 \mathrm{CH}_{2} \mathrm{Cl}_{2} / 5 \%$ Essigester, $11 \mathrm{CH}_{2} \mathrm{Cl}_{2} / 10 \%$ Essigester, 11 $\mathrm{CH}_{2} \mathrm{Cl}_{2} / 20 \%$ Essigester, $11 \mathrm{CH}_{2} \mathrm{Cl}_{2} / 50 \%$ Essigester, 21 Essigester, 1 Essigester/MeOH 9:1) vorgetrennt. Man erhielt vier Fraktionen I bis III.

Die erhaltenen Fraktionen wurden weiterhin durch präparative HPLC an RP18 gereinigt (Gradient : $\mathrm{H}_{2} \mathrm{O}$ Anstieg auf $80 \% \mathrm{MeOH}$ in $40 \mathrm{~min}$, dann auf $100 \% \mathrm{MeOH}$ in 10 min, über 10 min $100 \% \mathrm{MeOH}$ und dann Abfall auf $100 \% \mathrm{H}_{2} \mathrm{O}$ in 1 min, Fluss $10.0 \mathrm{ml} / \mathrm{min}$., Detektion bei $220 \mathrm{~nm}$ ). Aus der Fraktion I erhielt man $18 \mathrm{mg}$ Chartreusin (62) und $62 \mathrm{mg}$ Anthranilsäure (63). Fraktion II lieferte $22 \mathrm{mg}$ Uracil (48) und 31 mg Cyclo(leucyl-prolyl, 35). Aus der methanolischen Phase ist bei dem Versetzen mit Essigsäureester ein weißer Niederschlag ausgefallen. Nach dem Umkristallisieren aus Methanol/Essigester bekam man $32 \mathrm{mg}$ Tryptophan (64). 
Chartreusin (62): $\mathrm{C}_{32} \mathrm{H}_{32} \mathrm{O}_{14}, \boldsymbol{R}_{\mathbf{f}}=0.24 ; \mathrm{CH}_{2} \mathrm{Cl}_{2} / \mathrm{MeOH}, 95: 5$, (+)-ESI-MS: 1303 $\left([2 \mathrm{M}+\mathrm{Na}]^{+}, 100\right), 663\left([\mathrm{M}+\mathrm{Na}]^{+}, 28\right) .(-)-\mathbf{E S I}-\mathbf{M S}: 1301\left([2 \mathrm{M}-2 \mathrm{H}+\mathrm{Na}]^{-}, 92\right)$, $639\left([\mathrm{M}-\mathrm{H}]^{-}, 100\right) .{ }^{1} \mathrm{H}-\mathrm{NMR}\left(\mathrm{CDCl}_{3}, 300 \mathrm{MHz}\right): \delta=11.63$ (s, $\mathrm{D}_{2} \mathrm{O}$ austauschbar, $10-\mathrm{OH}), 8.22\left(\mathrm{~d},{ }^{3} J=8 \mathrm{~Hz}, 1 \mathrm{H}, 4-\mathrm{H}\right), 7.60\left(\mathrm{t},{ }^{3} J=8 \mathrm{~Hz}, 1 \mathrm{H}, 3-\mathrm{H}\right), 7.49$ (dd, ${ }^{3} J=8$ $\left.\mathrm{Hz},{ }^{3} J=15 \mathrm{~Hz}, 2 \mathrm{H}, 8-\mathrm{H}, 9-\mathrm{H}\right), 7.40$ (d, $\left.{ }^{3} \mathrm{~J}=8 \mathrm{~Hz}, 1 \mathrm{H}, 2-\mathrm{H}\right), 5.68$ (d, ${ }^{3} J=4 \mathrm{~Hz}, 1 \mathrm{H}$, $\left.1^{\prime}-\mathrm{H}\right), 5.30\left(\mathrm{~d},{ }^{3} J=6 \mathrm{~Hz}, 1 \mathrm{H}, 1\right.$ ' $\left.-\mathrm{H}\right), 4.26\left(\mathrm{t},{ }^{3} J=8 \mathrm{~Hz}, 1 \mathrm{H}, 3^{\prime}-\mathrm{H}\right), 4.20$ (d, ${ }^{3} J=6$ Hz, $1 \mathrm{H}, 3$ '’-H), 3.90 (m, 4 H, 4'-5'-2' '-5' '-H), 3.78 (dd, ${ }^{3} J=4 \mathrm{~Hz},{ }^{3} J=8 \mathrm{~Hz}, 1 \mathrm{H}$, 4' '-H), 3.37 (s, $3 \mathrm{H},-\mathrm{OCH}_{3}{ }^{\prime \prime}$ ), 3.30 (dd, ${ }^{3} \mathrm{~J}=8 \mathrm{~Hz},{ }^{3} \mathrm{~J}=3 \mathrm{~Hz}, 1 \mathrm{H}, 2^{\prime}-\mathrm{H}$ ), 2.82 (s, 3 $\left.\mathrm{H}, \mathrm{Ar}-\mathrm{CH}_{3}\right), 1.41\left(\mathrm{~d},{ }^{3} \mathrm{~J}=6 \mathrm{~Hz}, 3 \mathrm{H},-\mathrm{CH}_{3}{ }^{\prime}\right), 1.40$ (s, $\left.3 \mathrm{H},-\mathrm{CH}_{3}{ }^{\prime}{ }^{\prime}\right)$.

Anthranilsäure (63): $\mathrm{C}_{8} \mathrm{H}_{10} \mathrm{O}_{2}, \boldsymbol{R}_{\mathbf{f}}=0.70 ; \mathrm{CH}_{2} \mathrm{Cl}_{2} / \mathrm{MeOH}, 9: 1$, EI-MS $(70 \mathrm{eV}): \mathrm{m} / z$ $(\%)=\mathrm{C}_{7} \mathrm{H}_{7} \mathrm{O}_{2} \mathrm{~N}: 137(\mathrm{M}+, 78 \%), 119$ (100), 92 (56), 65 (18). ${ }^{1} \mathbf{H}-\mathbf{N M R}\left(\mathrm{CD}_{3} \mathrm{OD}\right.$, $300 \mathrm{MHz}): \delta=7.94\left(\mathrm{dd},{ }^{3} J=7 \mathrm{~Hz},{ }^{3} J=1 \mathrm{~Hz}, 1 \mathrm{H}, 6-\mathrm{H}\right), 7.32\left(\mathrm{dt},{ }^{3} J=7 \mathrm{~Hz},{ }^{3} J=1\right.$ $\mathrm{Hz}, 1 \mathrm{H}, 4-\mathrm{H}), 6.63$ (m, 2 H, 3-5-H).

Tryptophan (64): $\mathrm{C}_{8} \mathrm{H}_{10} \mathrm{O}_{2}, \boldsymbol{R}_{\mathbf{f}}=0.40 ; \mathrm{CH}_{2} \mathrm{Cl}_{2} / \mathrm{MeOH}, 95: 5$, (+)-ESI-MS: 227 ([ M $\left.+\mathrm{Na}]^{+}, 40 \%\right), 205\left([\mathrm{M}+\mathrm{H}]^{+}, 100\right) .{ }^{1} \mathbf{H}-\mathbf{N M R}\left(\left[\mathrm{D}_{6}\right]-\mathrm{DMSO}, 300 \mathrm{MHz}\right): \delta=11.02$ (s, $\mathrm{D}_{2} \mathrm{O}$ austauschbar, Indol-NH), 7.59 (d, $\left.{ }^{3} \mathrm{~J}=6 \mathrm{~Hz}, 1 \mathrm{H}, 4-\mathrm{H}\right), 7.39$ (d, ${ }^{3} \mathrm{~J}=6 \mathrm{~Hz}, 1$ H, 7-H), 7.22 (d, $\left.{ }^{3} J=1 \mathrm{~Hz}, 1 \mathrm{H}, 2-\mathrm{H}\right), 7.04\left(\mathrm{t},{ }^{3} J=5 \mathrm{~Hz}, 1 \mathrm{H}, 6-\mathrm{H}\right), 6.98\left(\mathrm{t},{ }^{3} J=5\right.$ $\mathrm{Hz}, 1 \mathrm{H}, 5-\mathrm{H}), 4.30$ (br s, $\left.3 \mathrm{H},-\mathrm{NH}_{2},-\mathrm{COOH}\right), 3.58\left(\mathrm{dd},{ }^{3} \mathrm{~J}=3 \mathrm{~Hz},{ }^{3} \mathrm{~J}=6 \mathrm{~Hz}, 1 \mathrm{H}\right.$, $\left.2^{\prime}-\mathrm{H}\right), 3.37\left(\mathrm{dd},{ }^{3} J=12 \mathrm{~Hz},{ }^{3} J=3 \mathrm{~Hz}, 1 \mathrm{H}, 1^{\prime}-\mathrm{H}\right), 3.02\left(\mathrm{dd},{ }^{3} J=6 \mathrm{~Hz},{ }^{3} J=12 \mathrm{~Hz}, 1\right.$

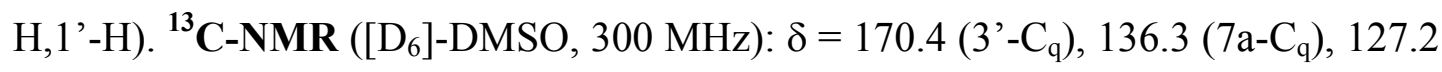
$\left(3 \mathrm{a}-\mathrm{C}_{\mathrm{q}}\right), 124.0(2-\mathrm{CH}), 120.8(6-\mathrm{CH}), 118.3$ (4-CH), $118.1(5-\mathrm{CH}), 111.2(7-\mathrm{CH})$, $109.5\left(3-\mathrm{C}_{\mathrm{q}}\right), 54.71\left(2^{\prime}-\mathrm{CH}\right), 27.1\left(1^{\prime}-\mathrm{CH}_{2}\right)$. 


\section{Anhang A}

Bestückungsplan der Platine zur Auswahl und Steuerung der Geschwindigkeit der Pumpen.

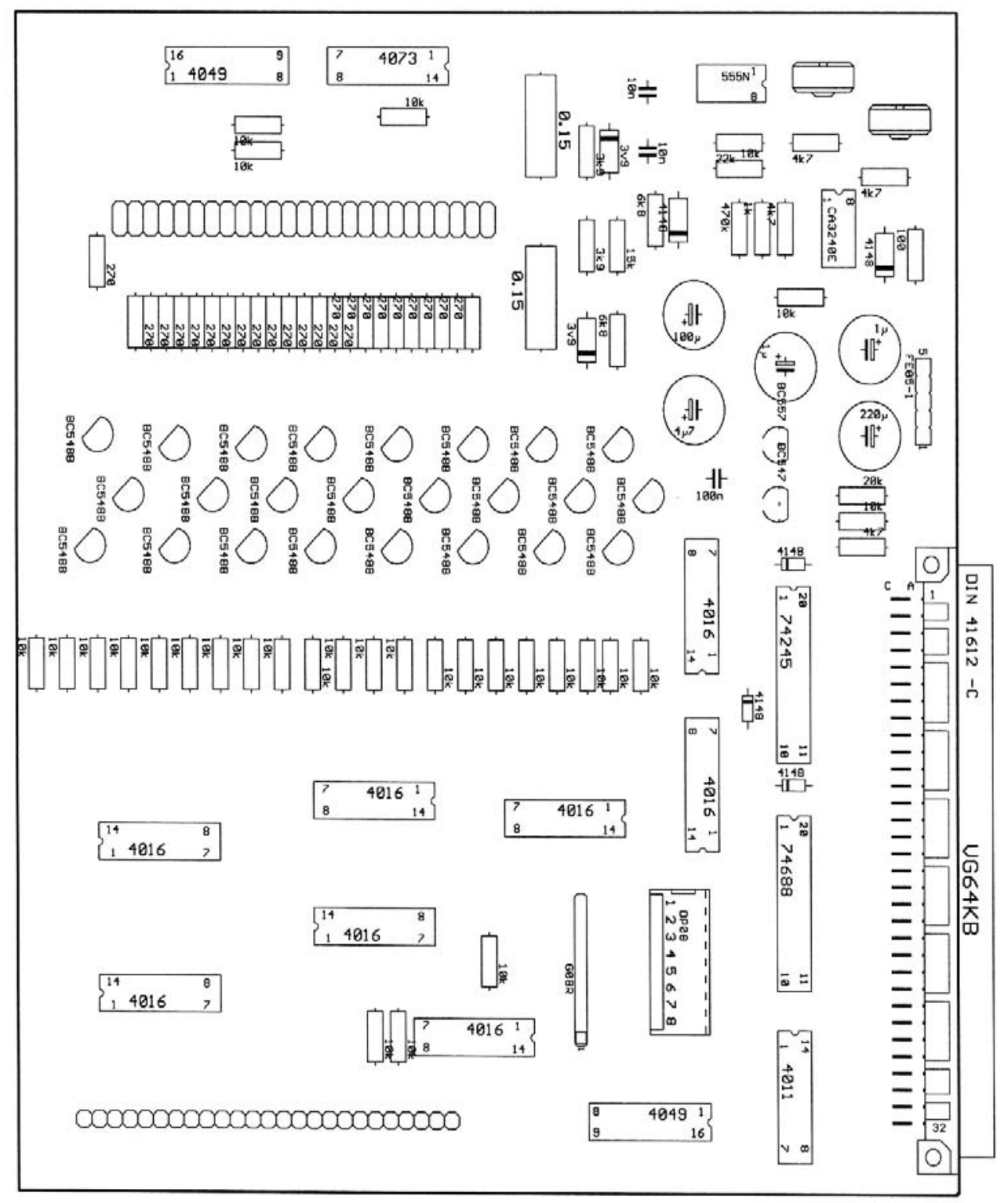


Layout der Platine zur Auswahl und Steuerung der Geschwindigkeit der Pumpen.

Seite A.

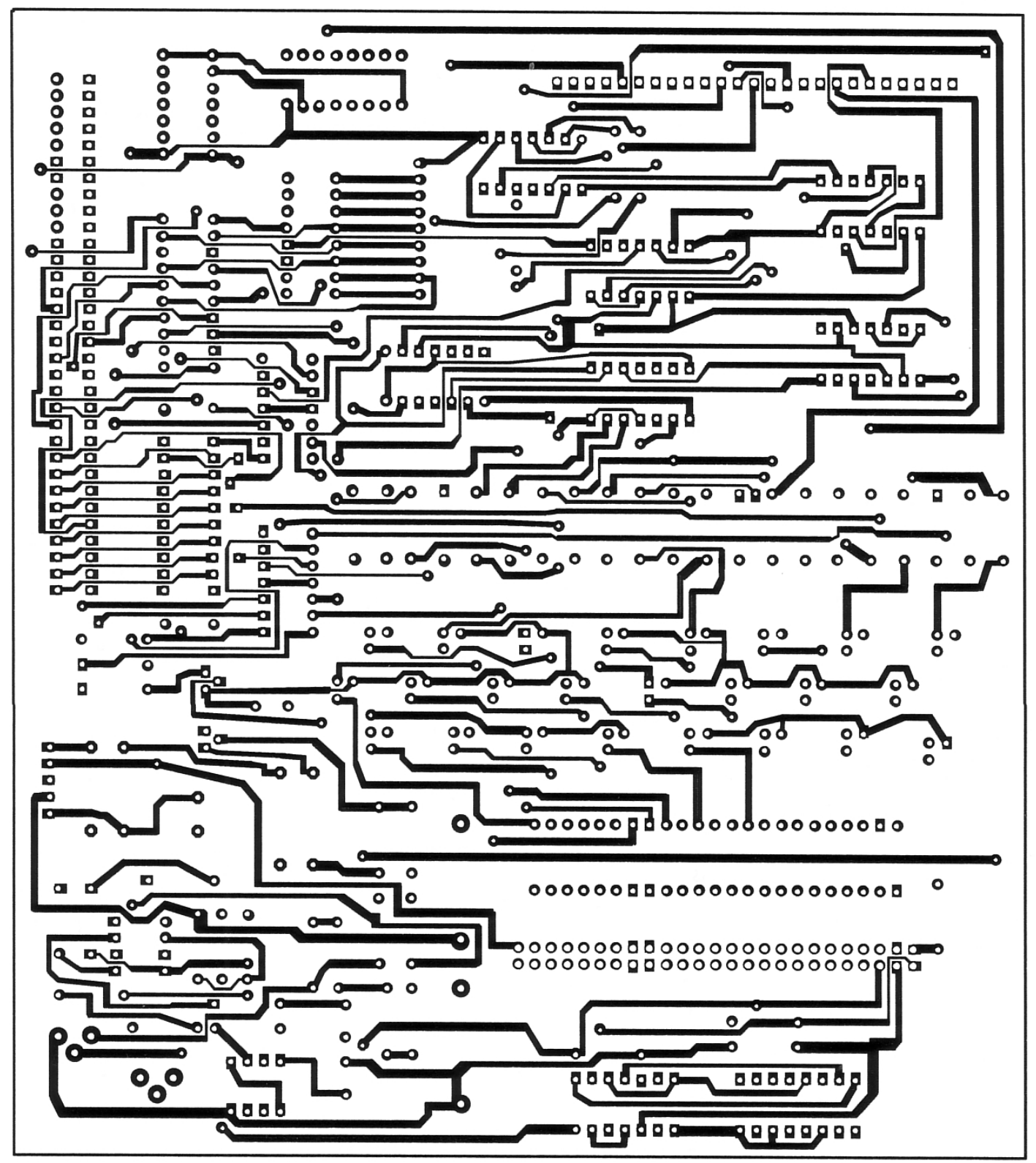


Layout der Platine zur Auswahl und Steuerung der Geschwindigkeit der Pumpen. Seite B.

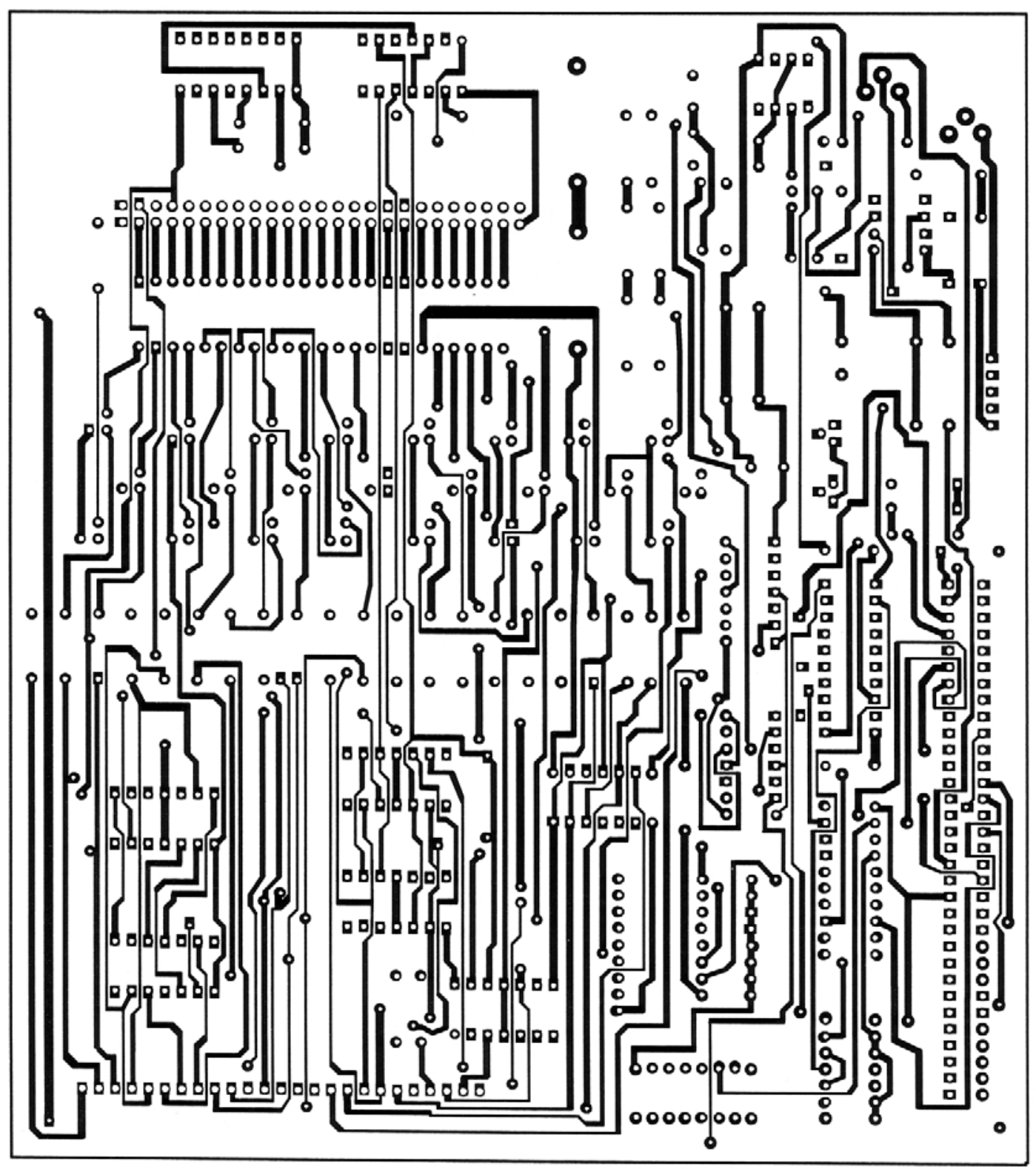




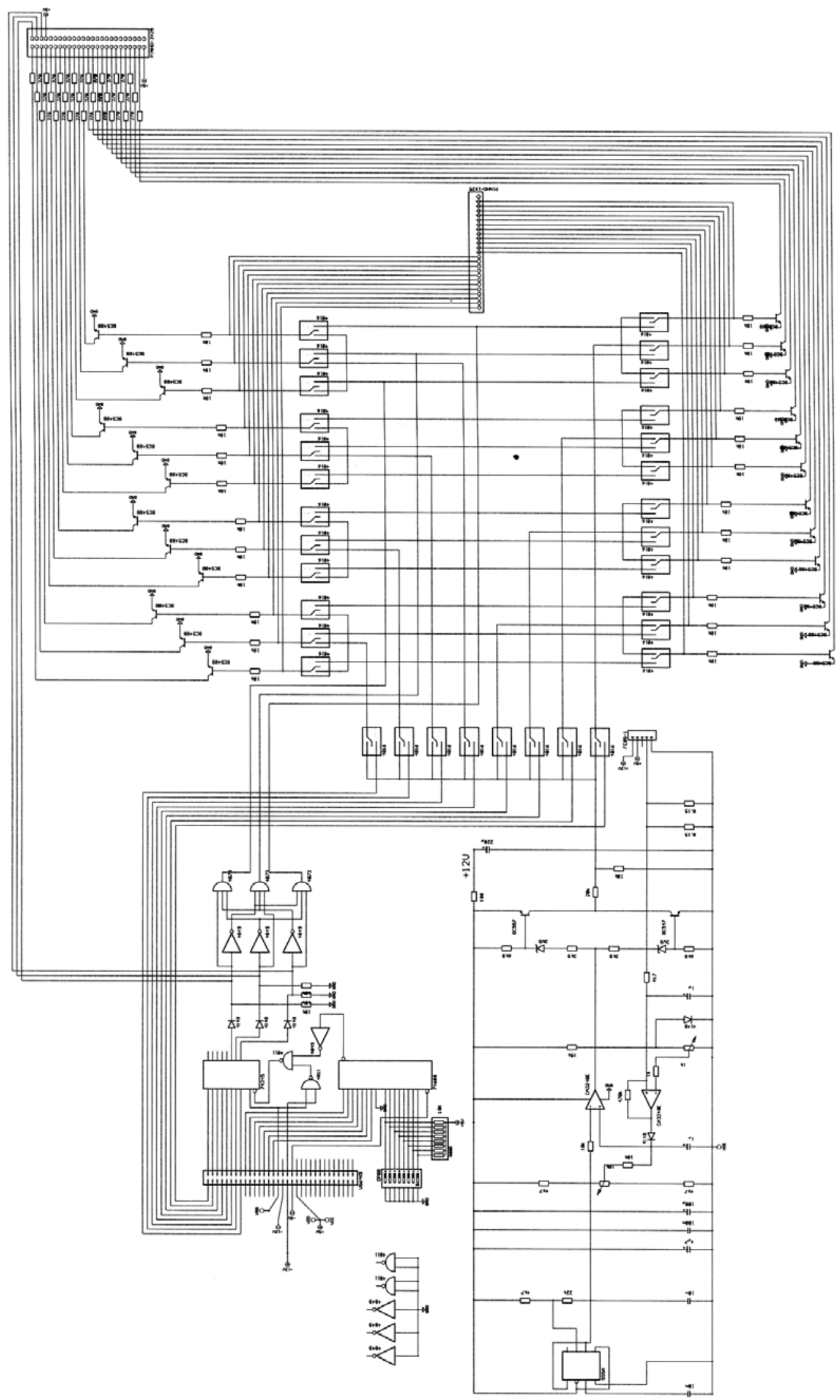

Schematische Darstellung der Steuerung der Geschwindigkeit der Pumpen. 
Bestückungsplan der Platine zur Fermenterauswahl.

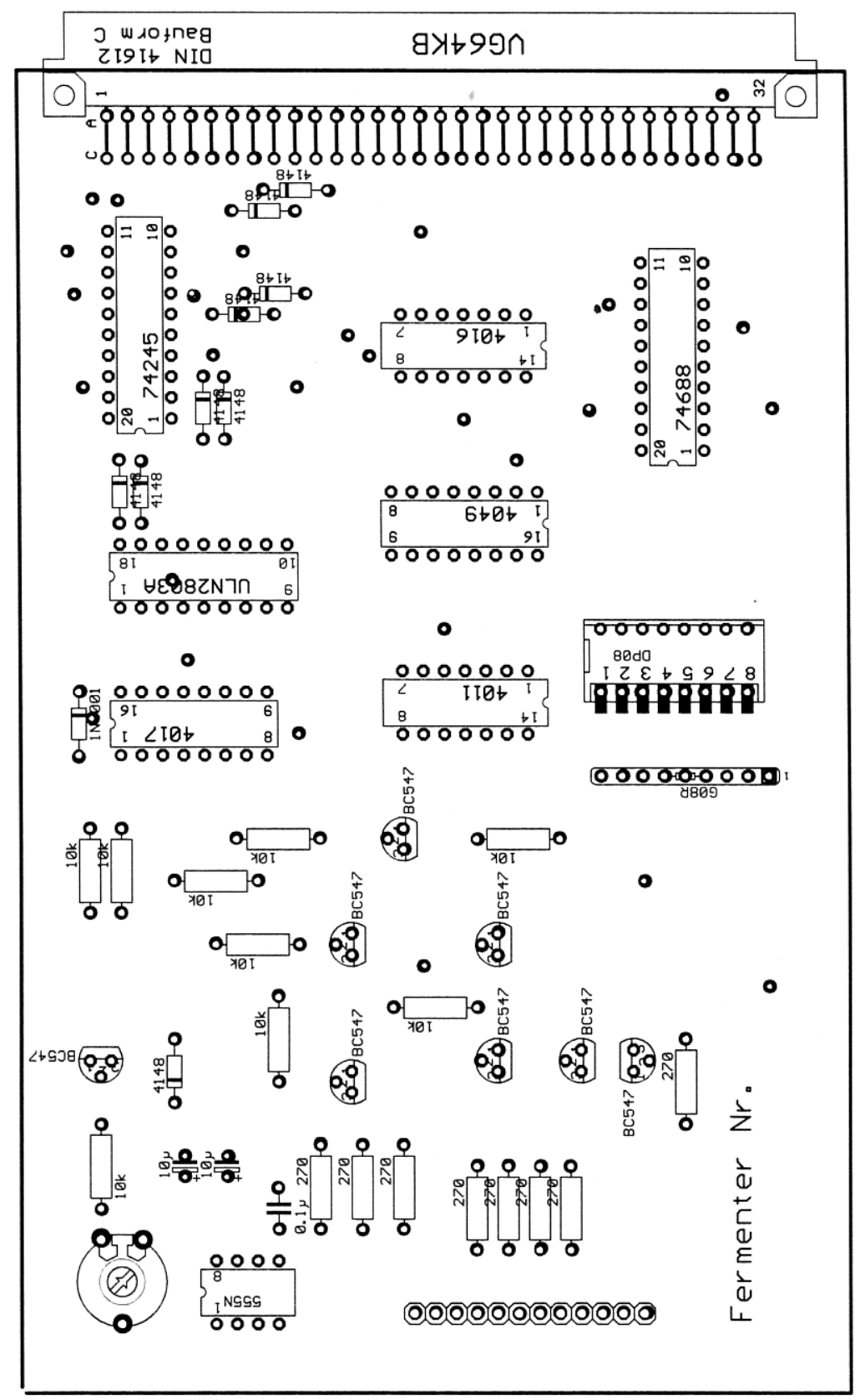


Layout der Platine zur Fermenterauswahl. Seite A.

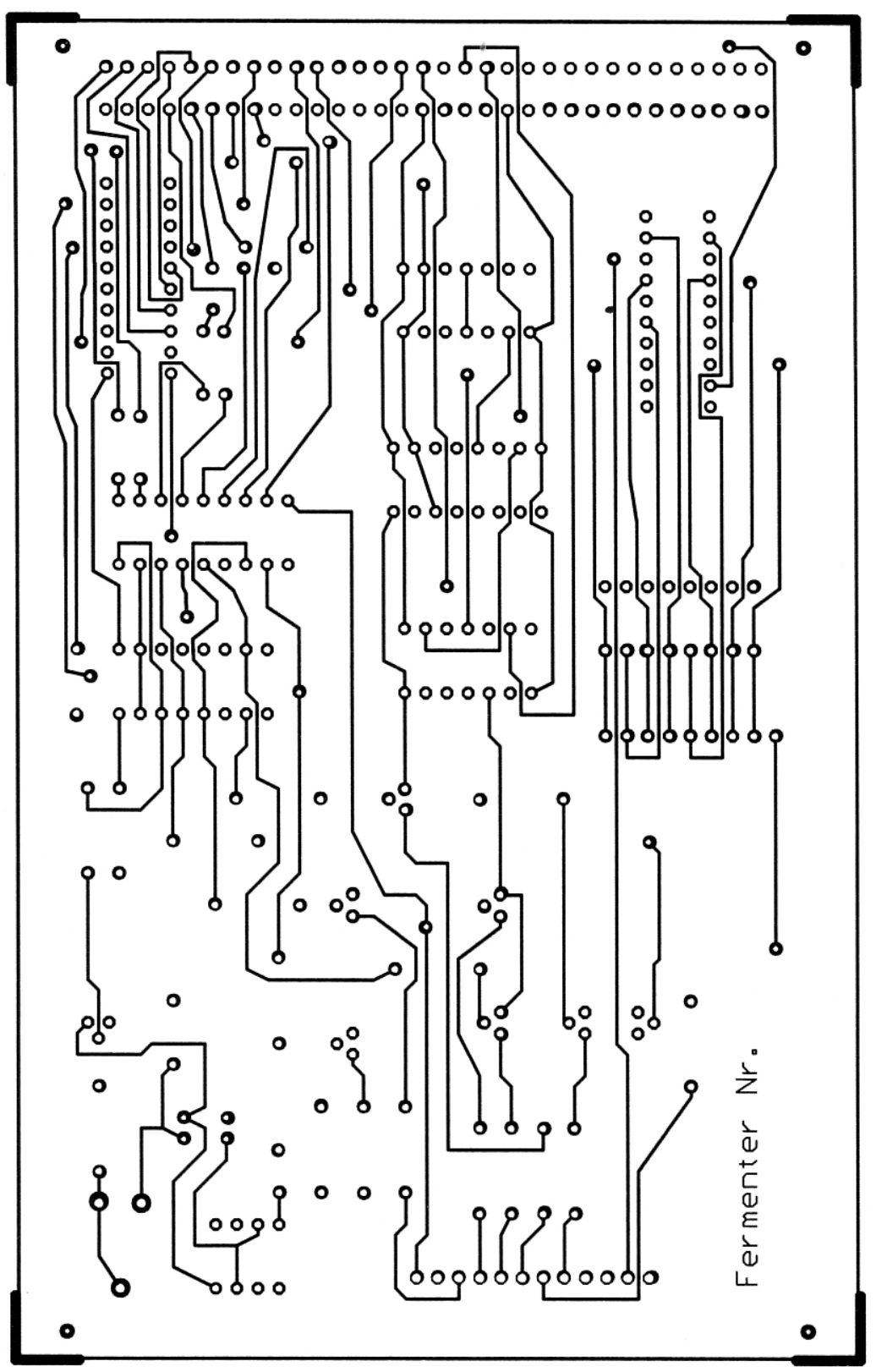


Layout der Platine zur Fermenterauswahl. Seite B.

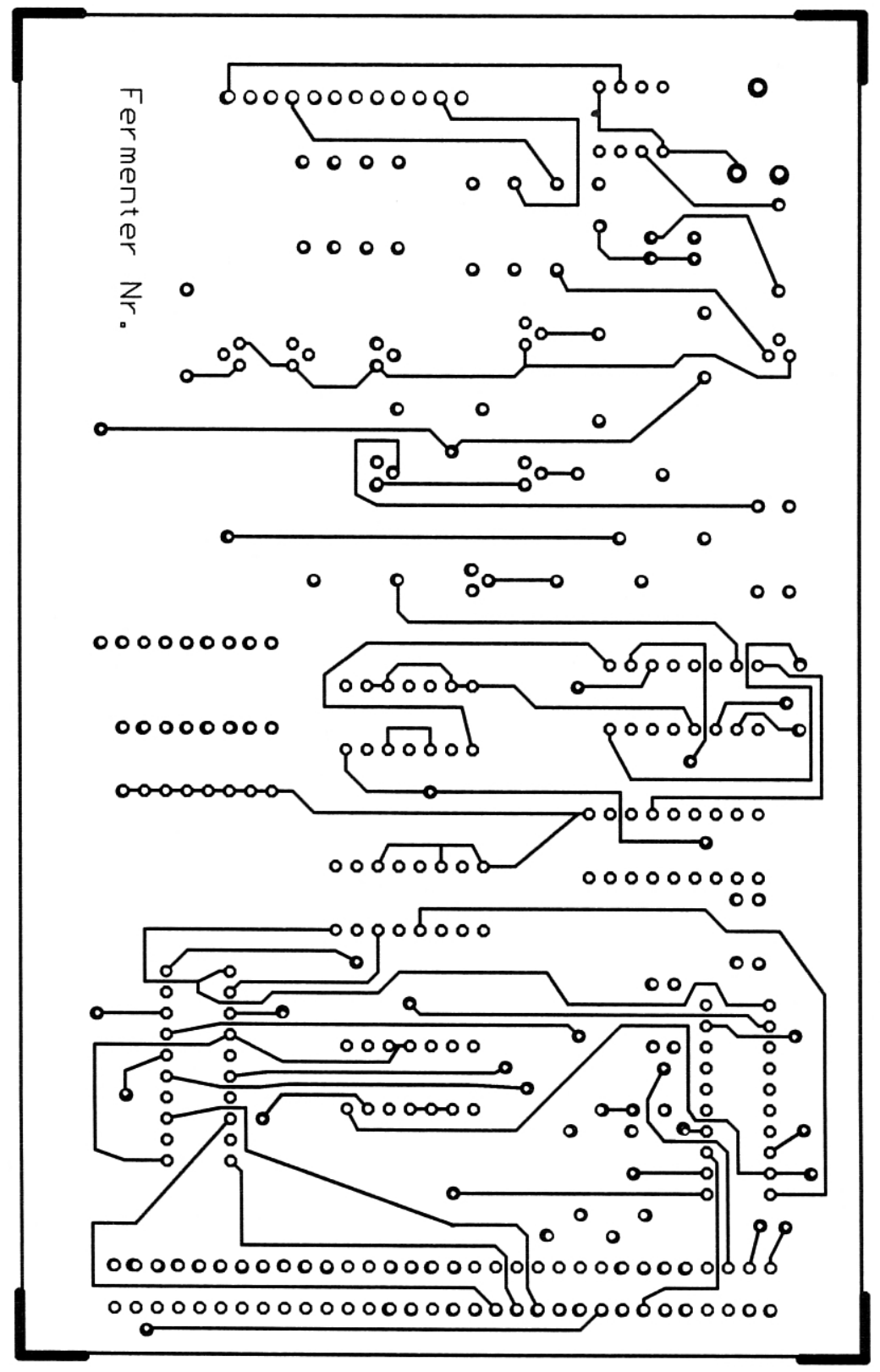



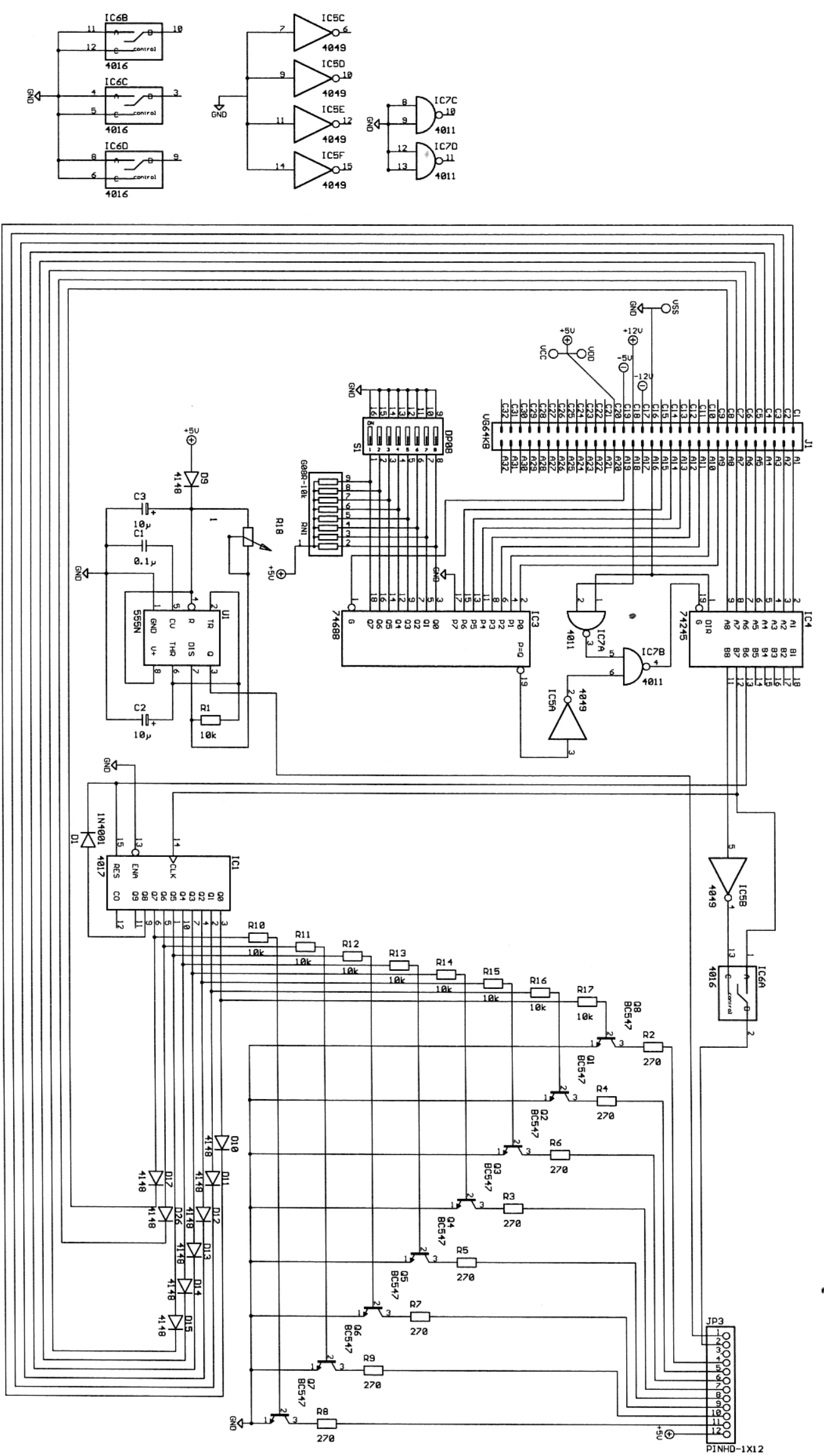

Schematische Darstellung der Platine zur Fermenterauswahl. 
Bestückungsplan der Platine zur Antischaumkontrolle.

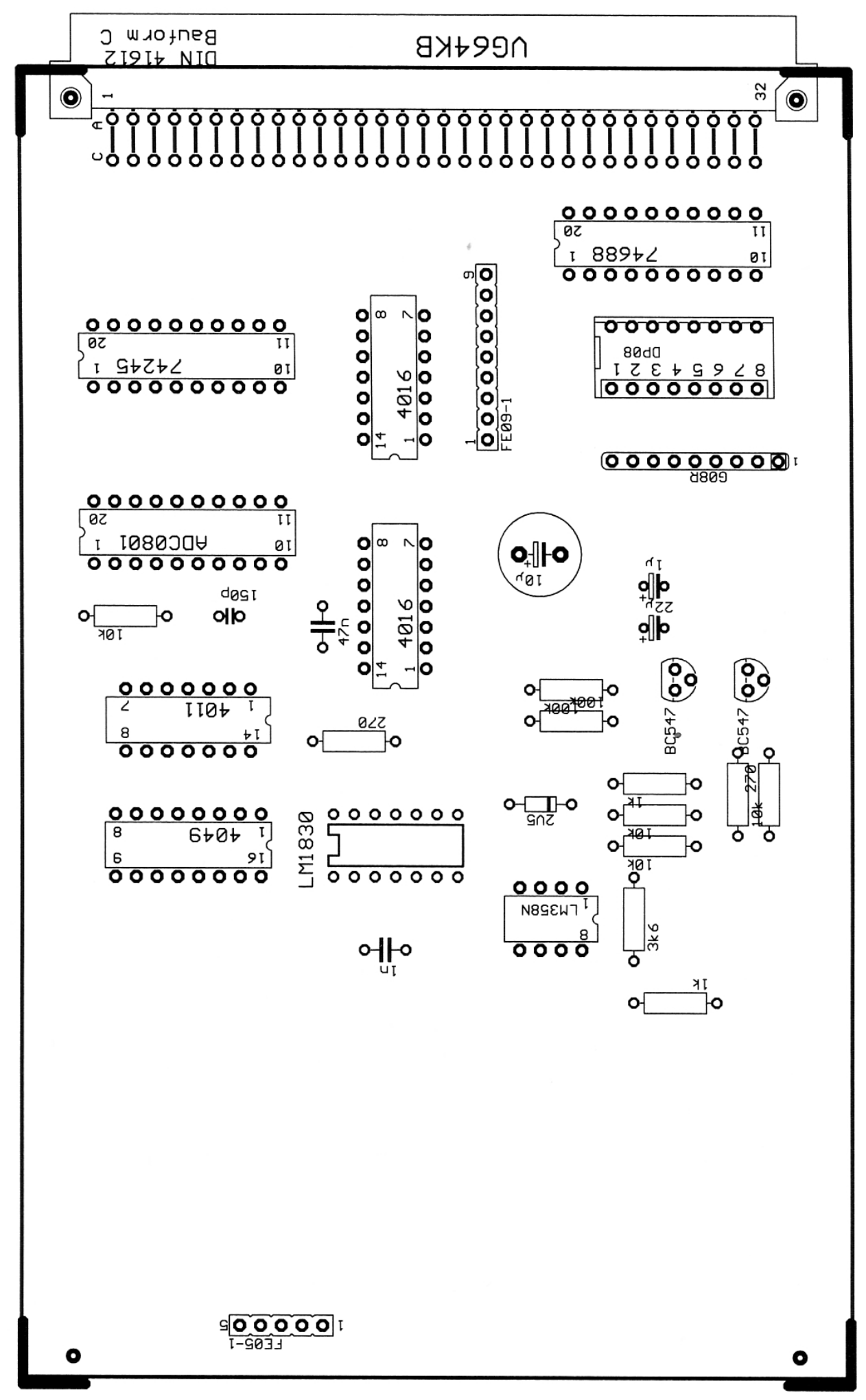


Layout der Platine zur Antischaumkontrolle. Seite A.

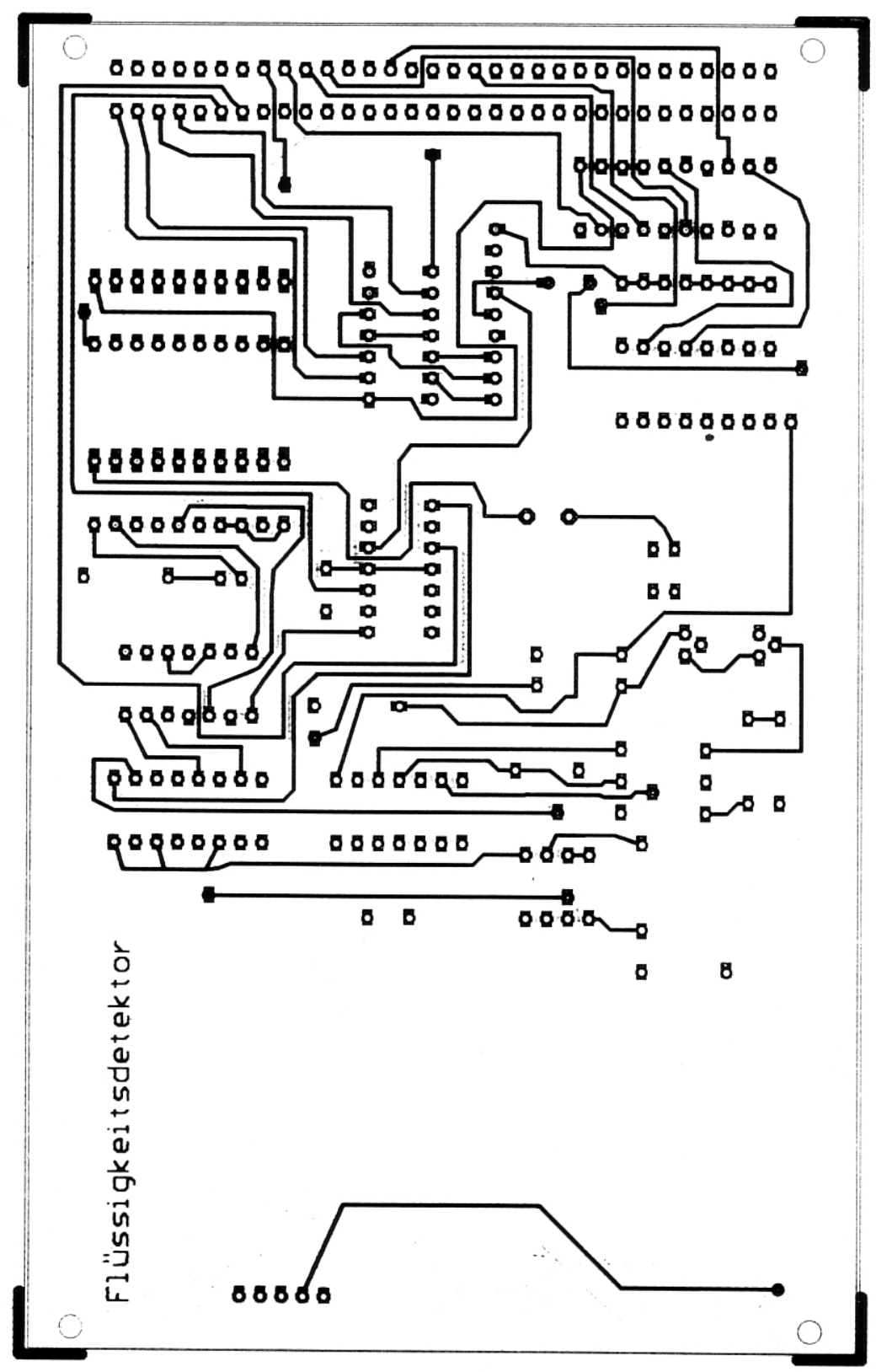


Layout der Platine zur Antischaumkontrolle. Seite B.

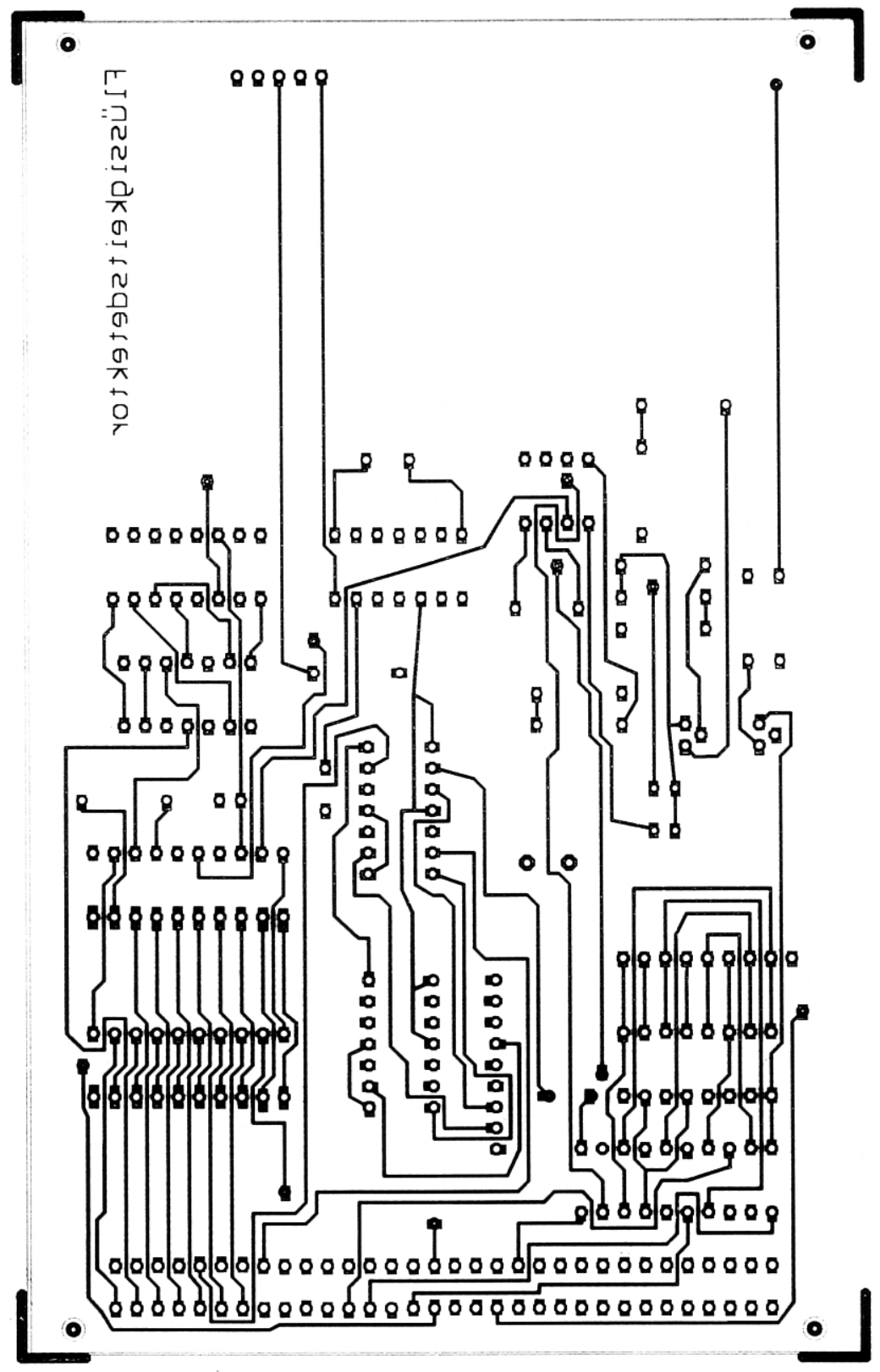




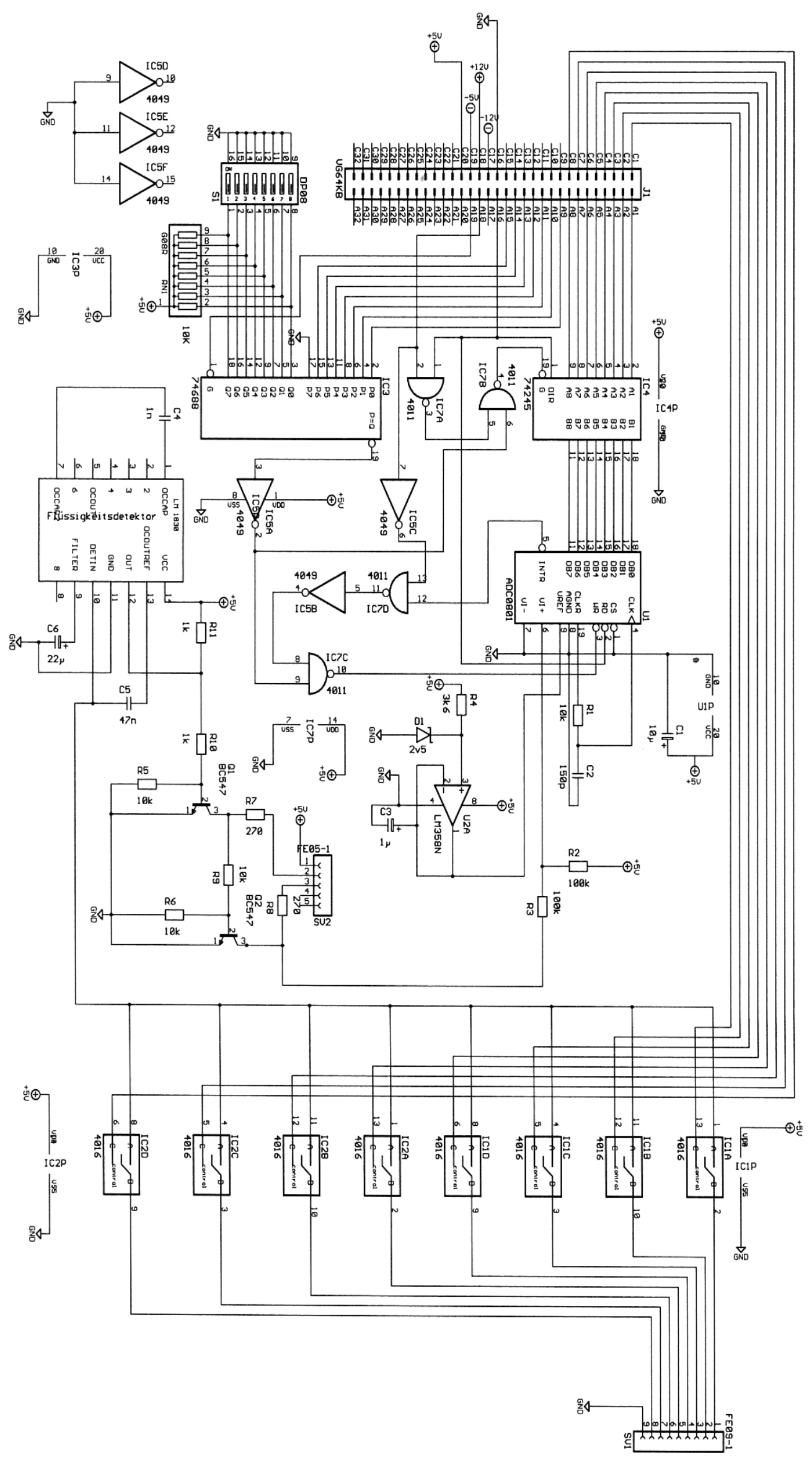

Schematische Darstellung der Platine für die Antischaumkontrolle. 
Bestückungsplan der Platine des AD - Wandlers für die pH - Kontrolle.

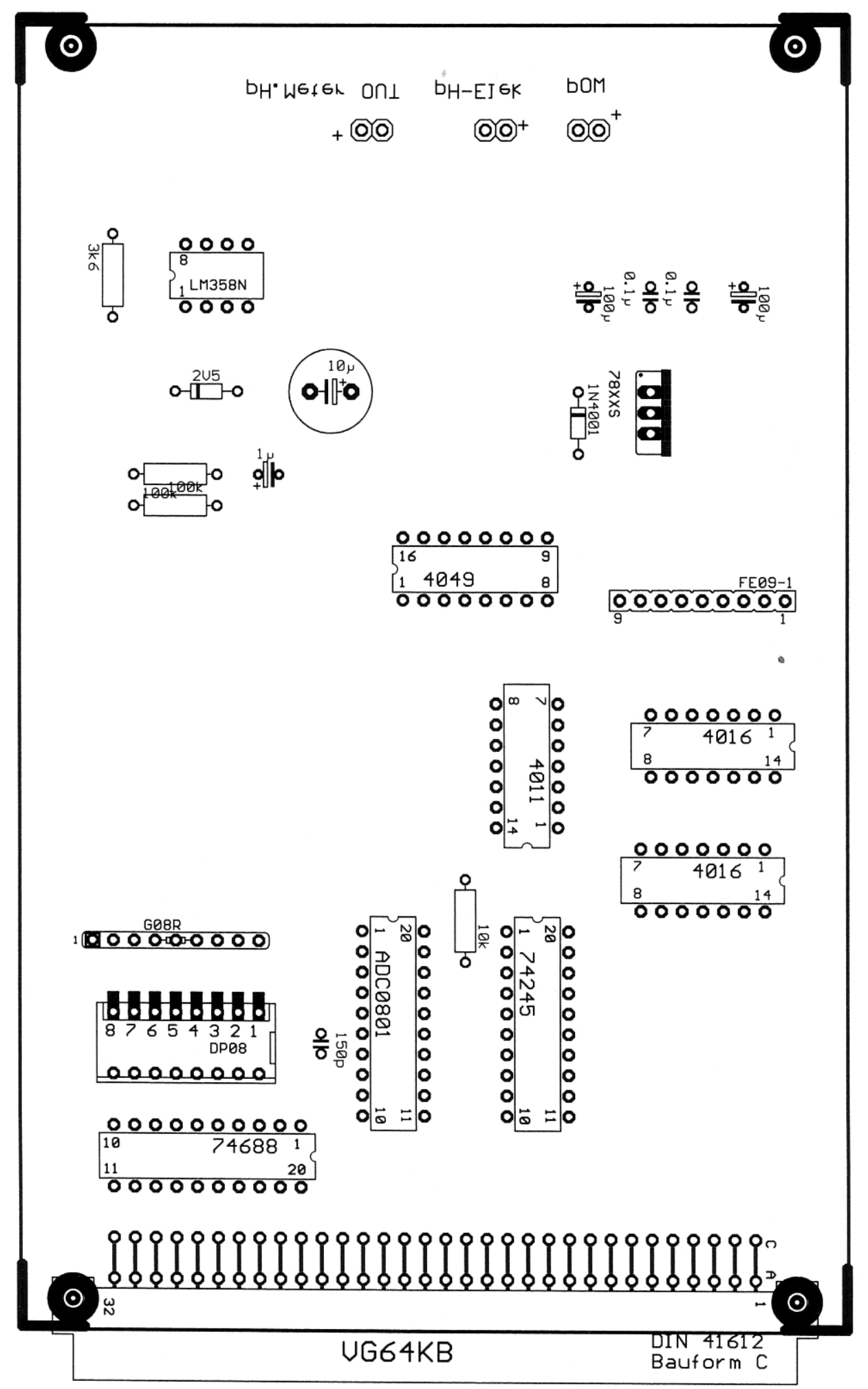


Layout der Platine des AD - Wandlers für die pH - Kontrolle. Seite A.

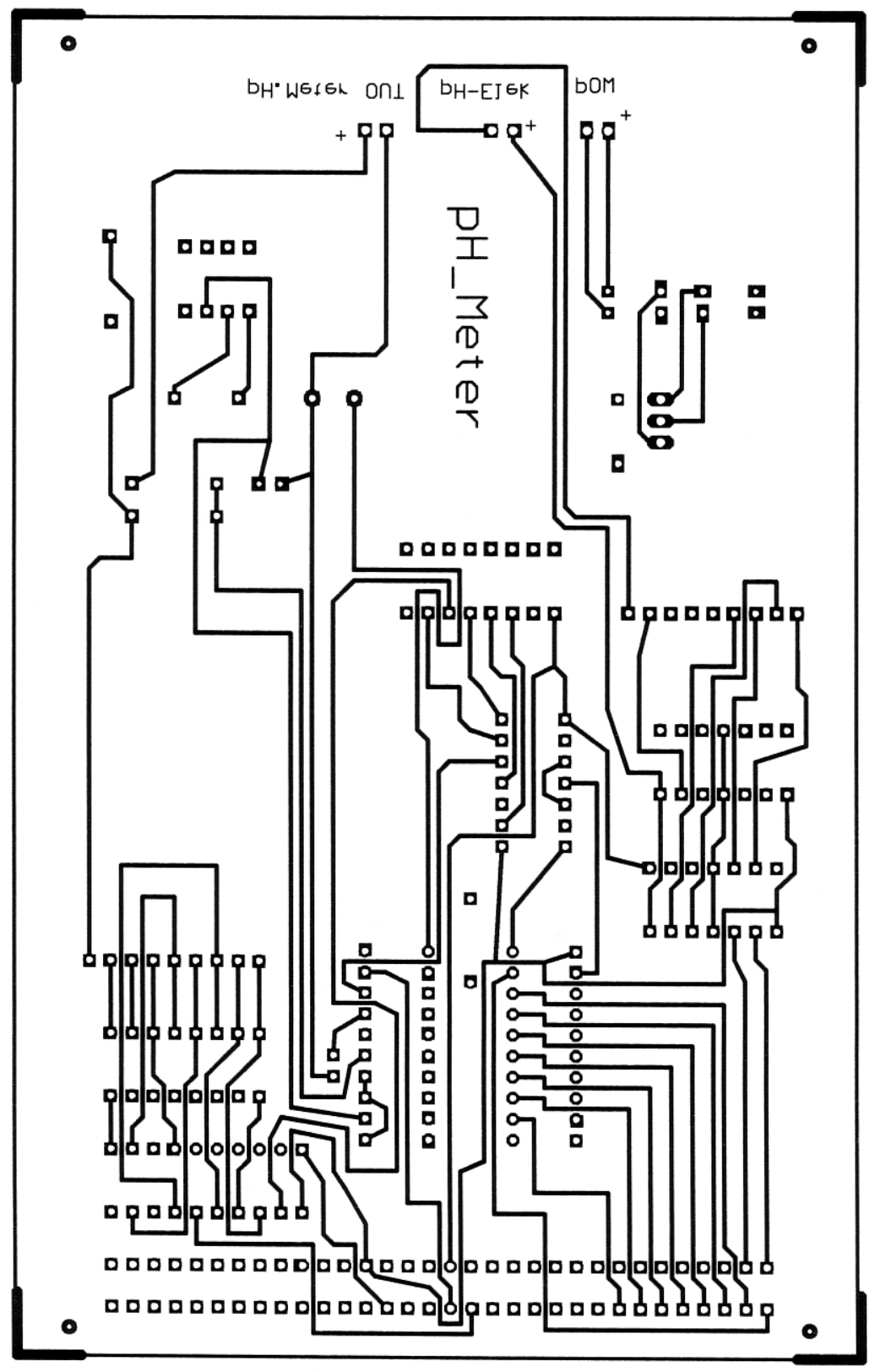


Layout der Platine des AD - Wandlers für die pH - Kontrolle. Seite B.

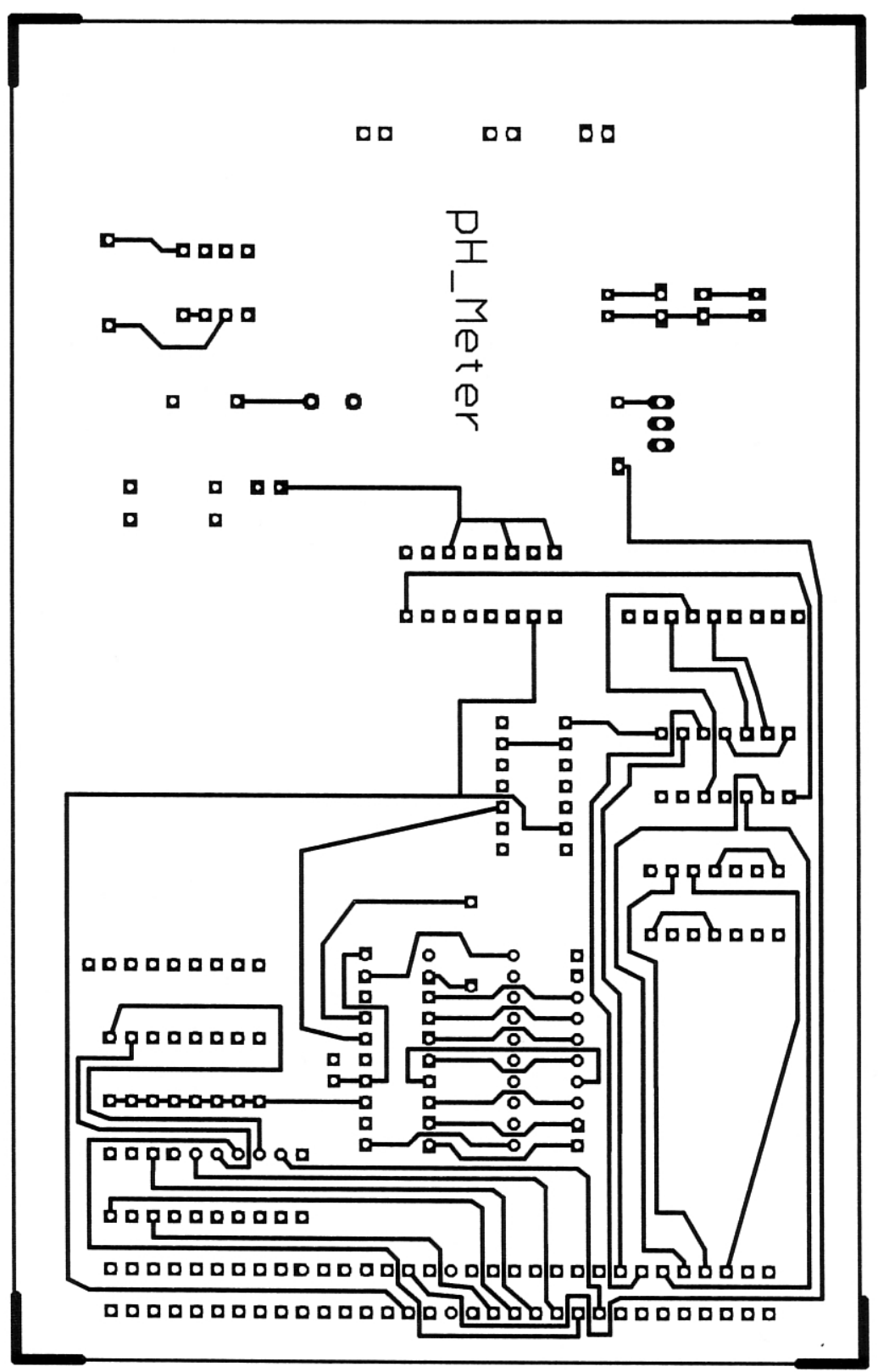




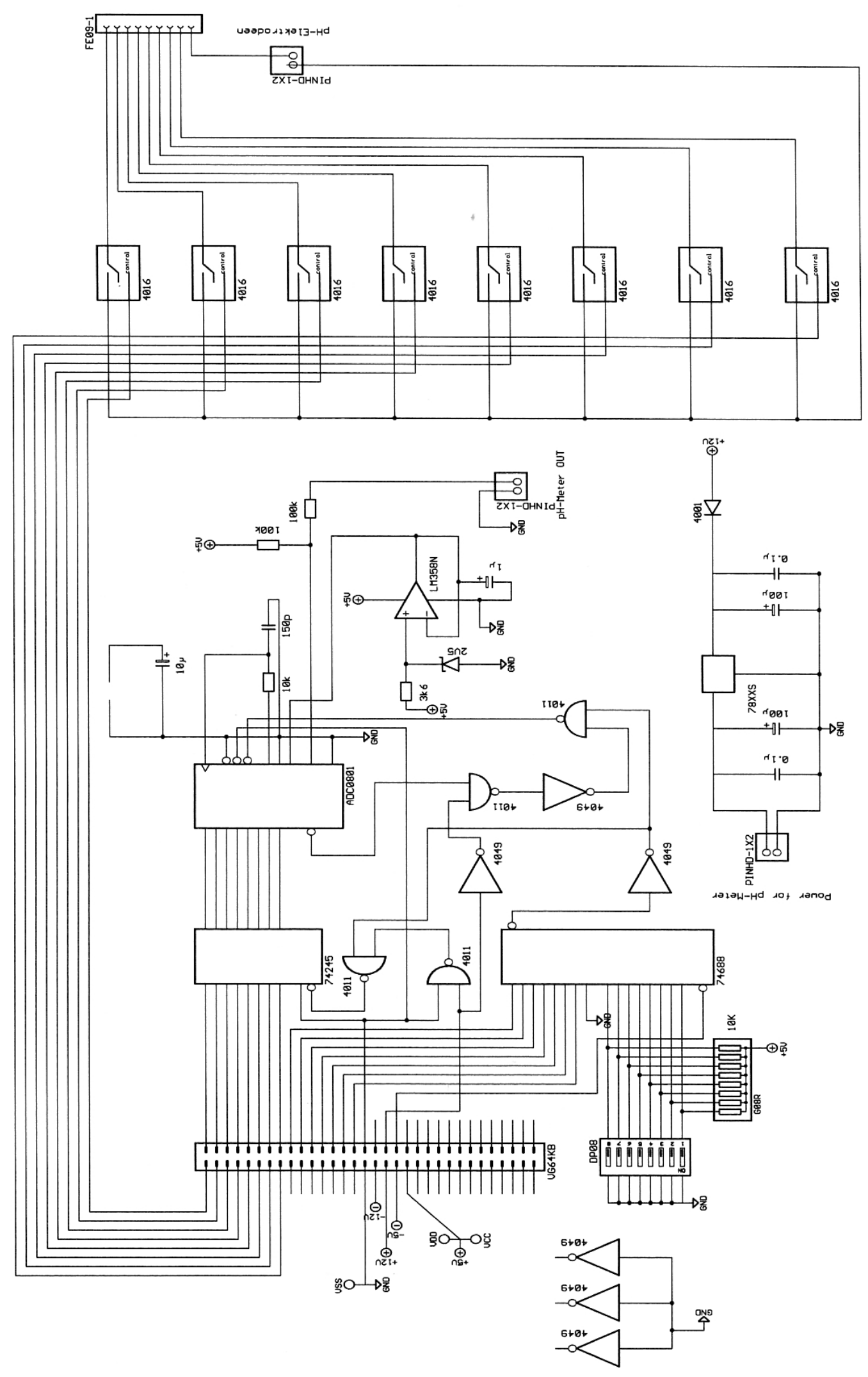

Schematische Darstellung des AD-Wandlers. 
Bestückungsplan der Platine der Platine für die Kommunikation des Gerätes mit dem PC.

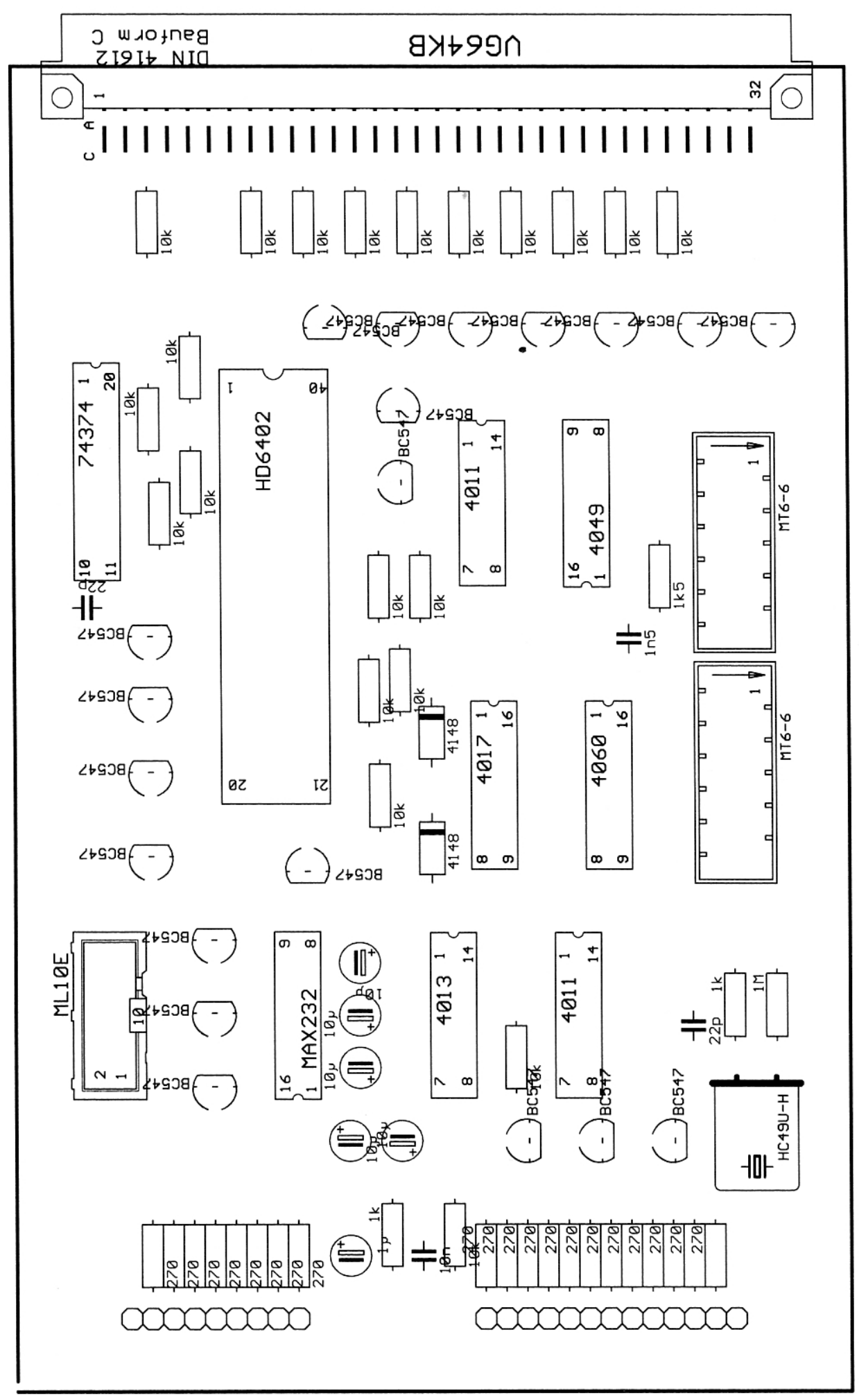


Layout der Platine der Platine für die Kommunikation des Gerätes mit dem PC. Seite A.

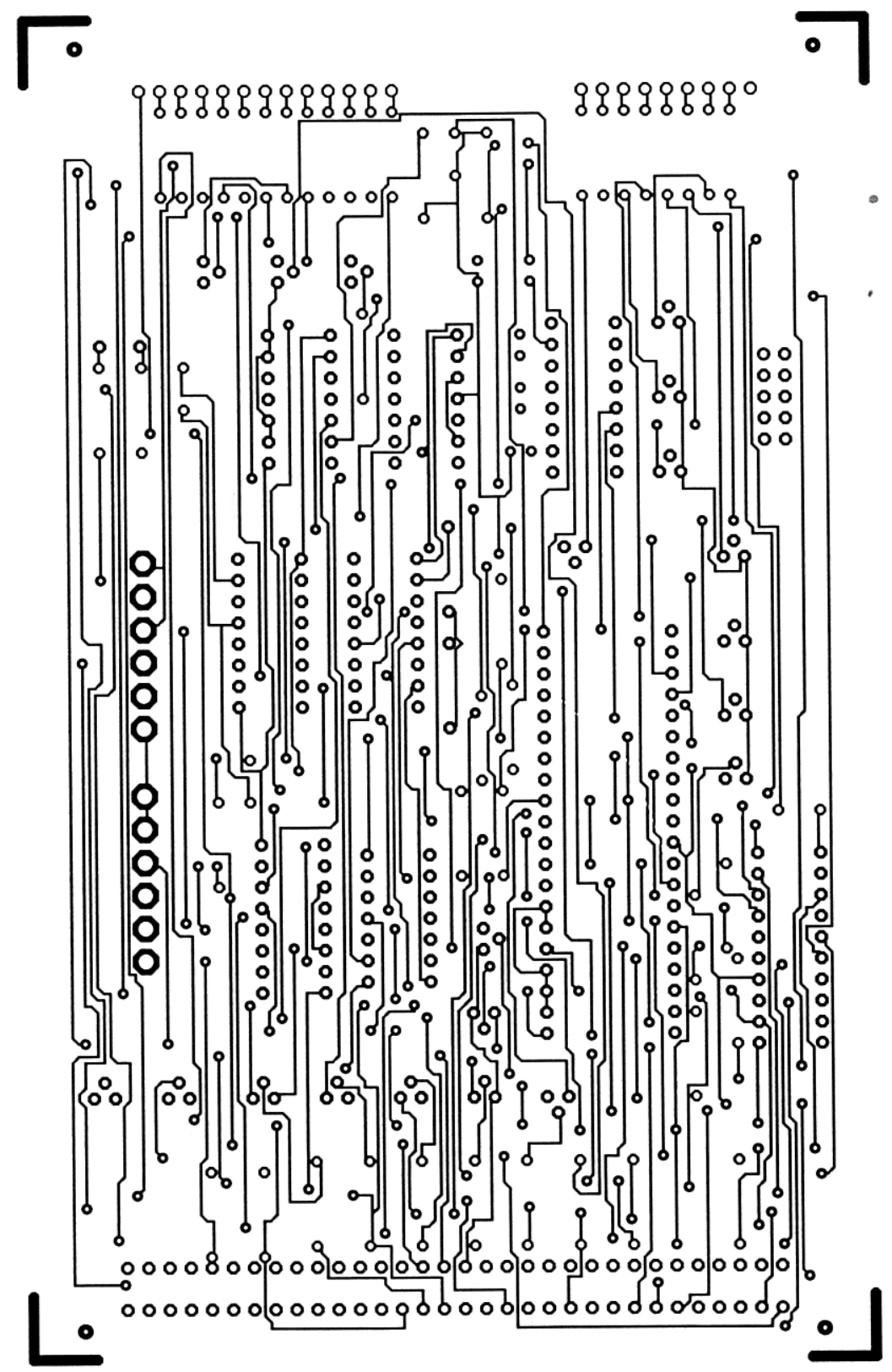


Layout der Platine der Platine für die Kommunikation des Gerätes mit dem PC. Seite B.

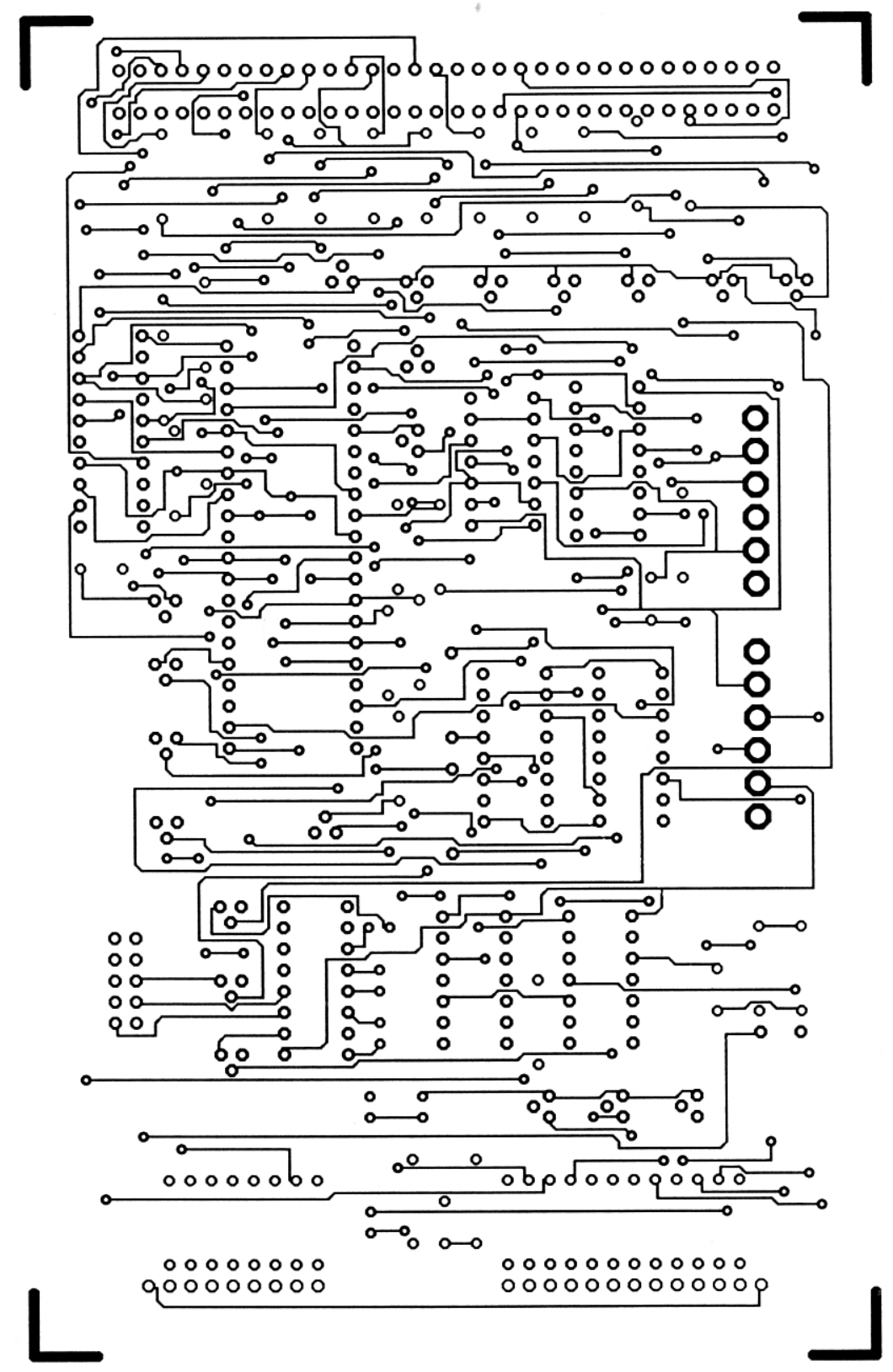




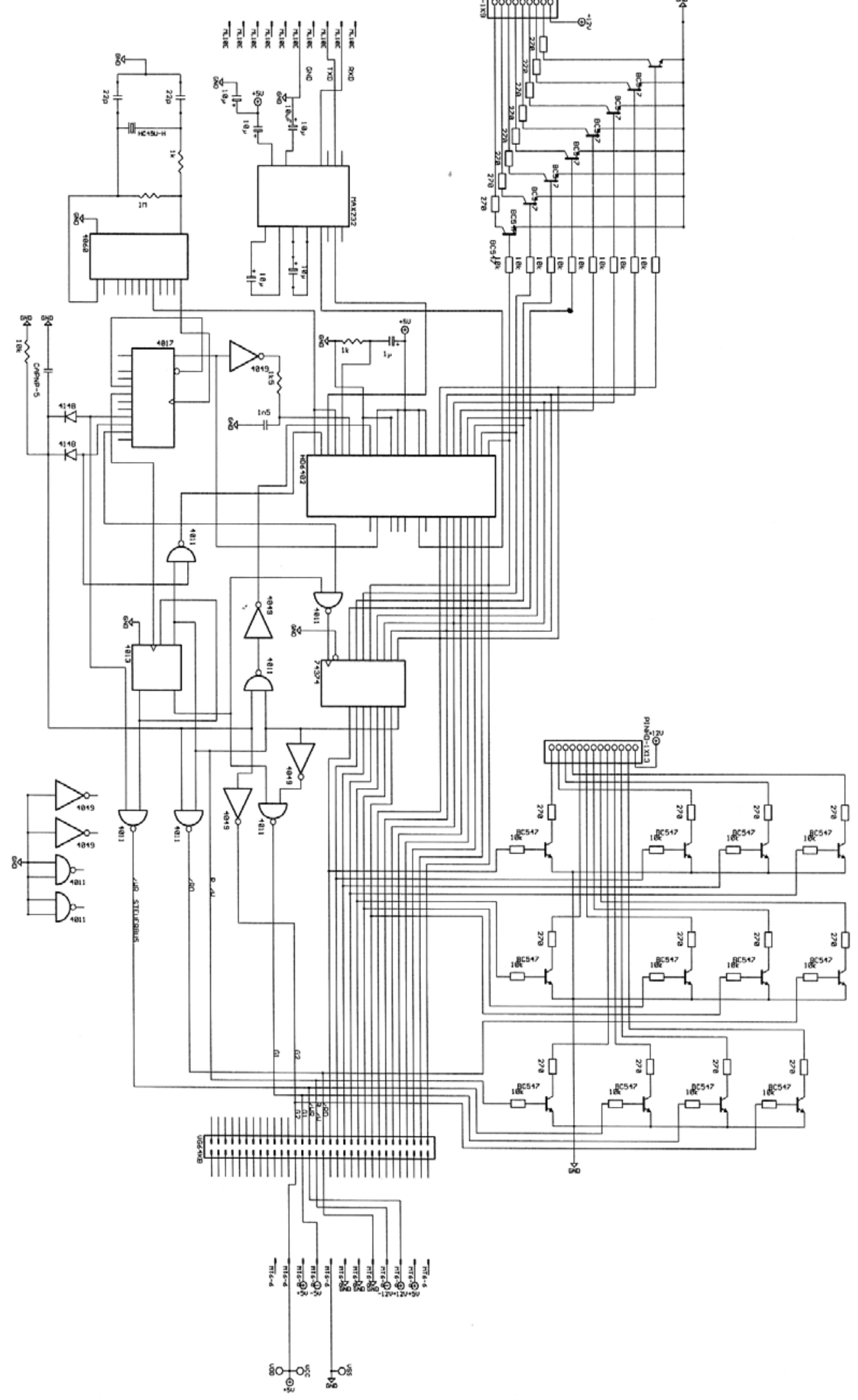

Schematische Darstellung der Platine der Platine für die Kommunikation des Gerätes mit dem PC 
Bestückungsplan der Platine des Motorentreibers.

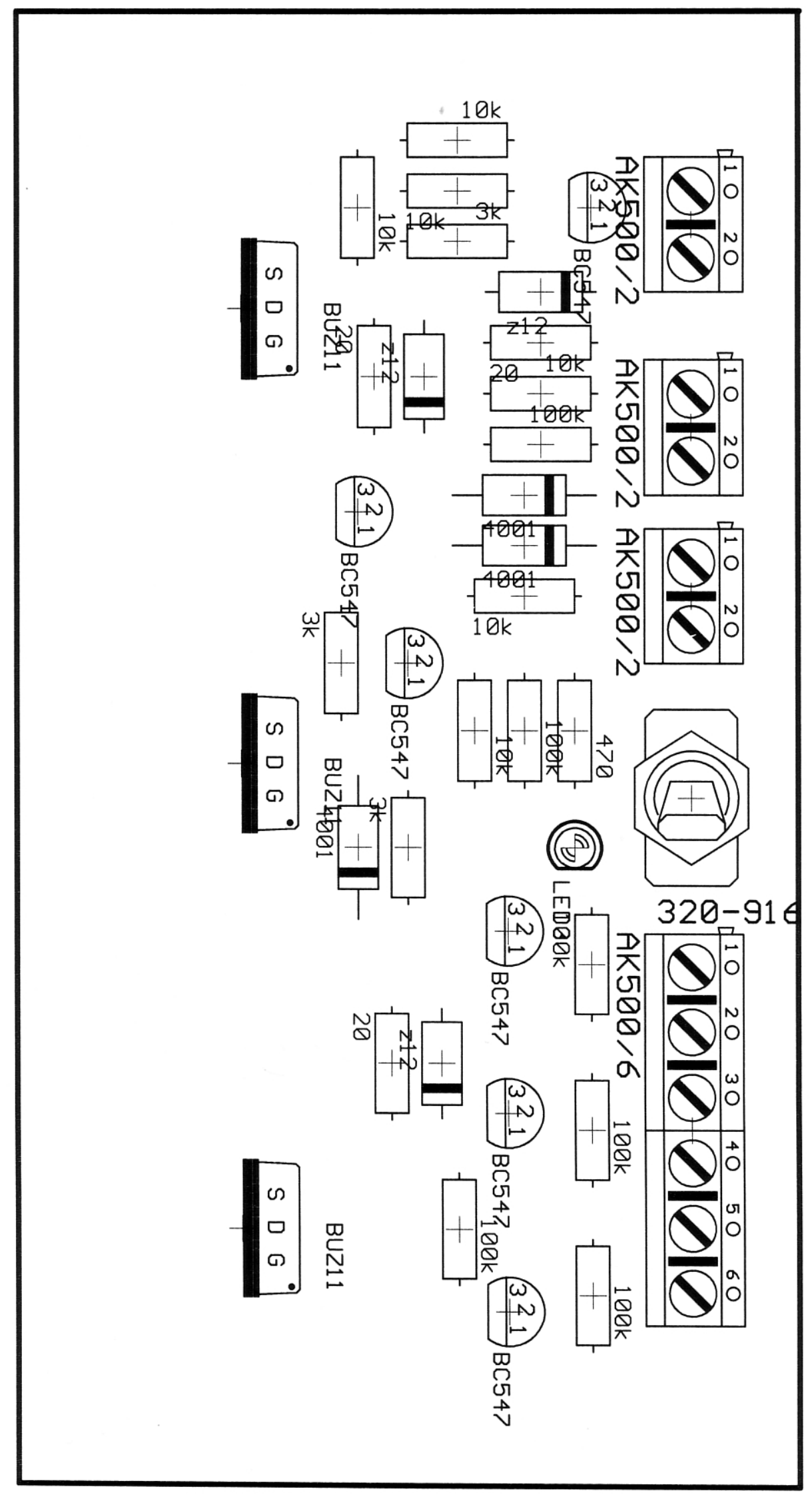


Layout der Platine des Motorentreibers. Seite A.

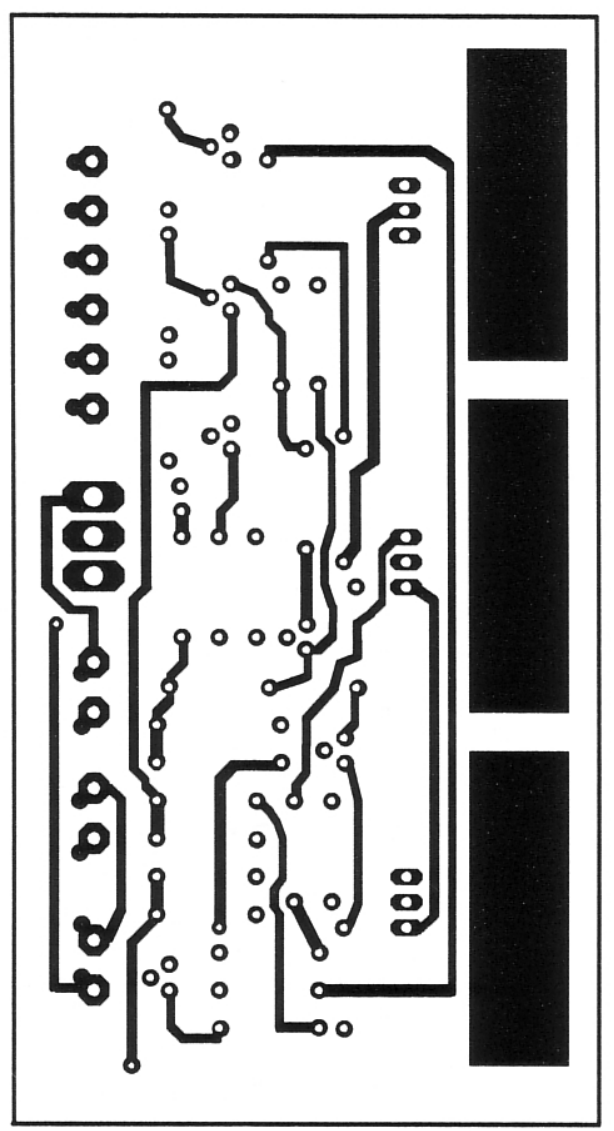


Layout der Platine des Motorentreibers. Seite B.

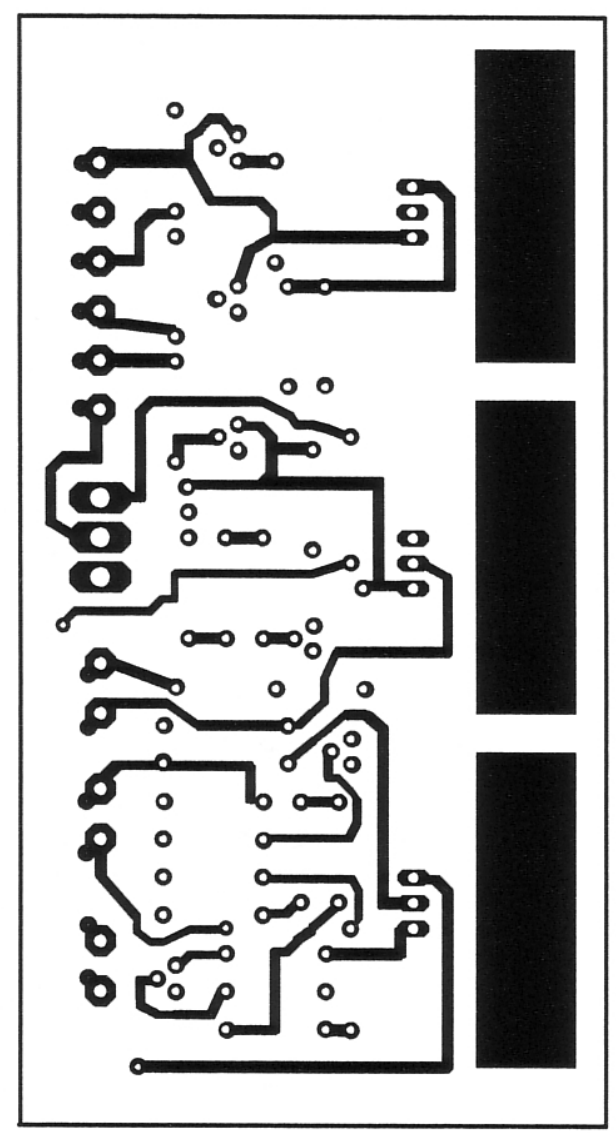




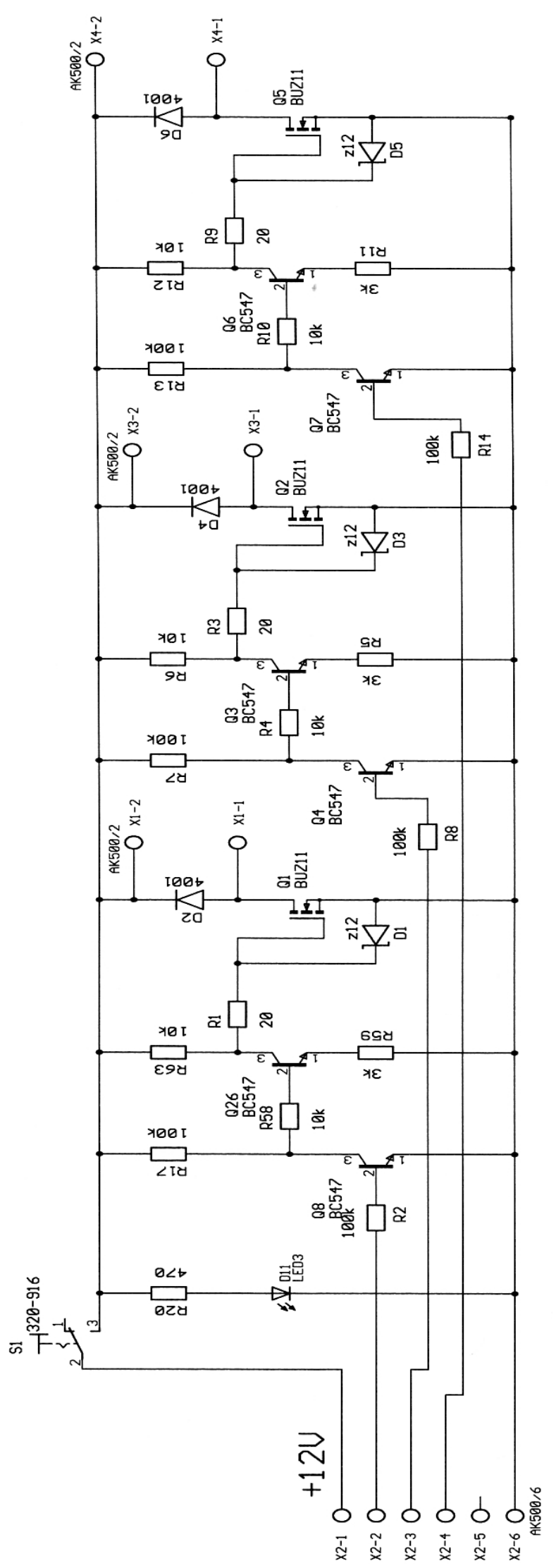

Schematische Darstellung der Platine des Motorentreibers. 
Bestückung der Anzeigeplatine.

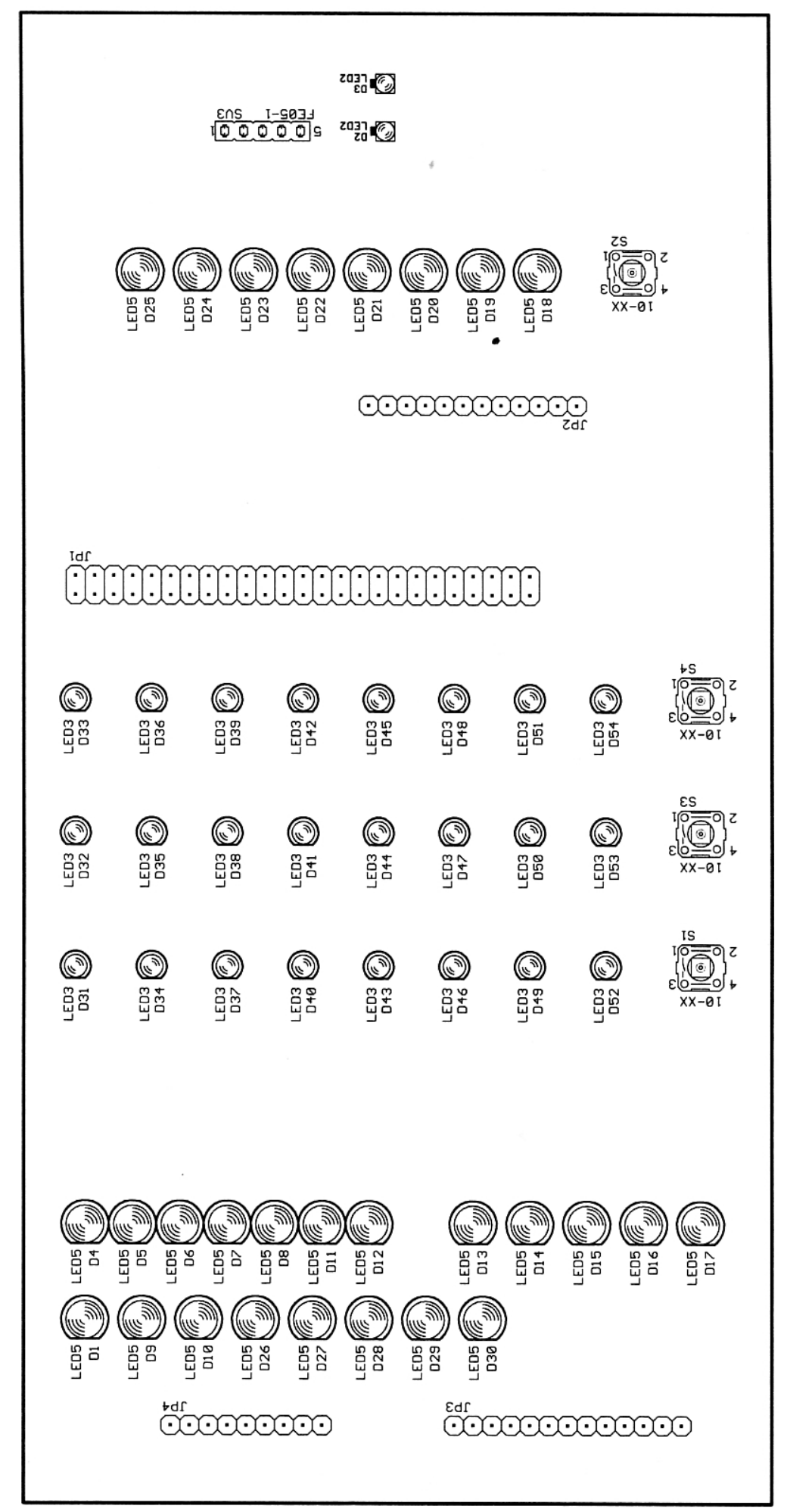


Layout der Anzeigeplatine. Seite A.

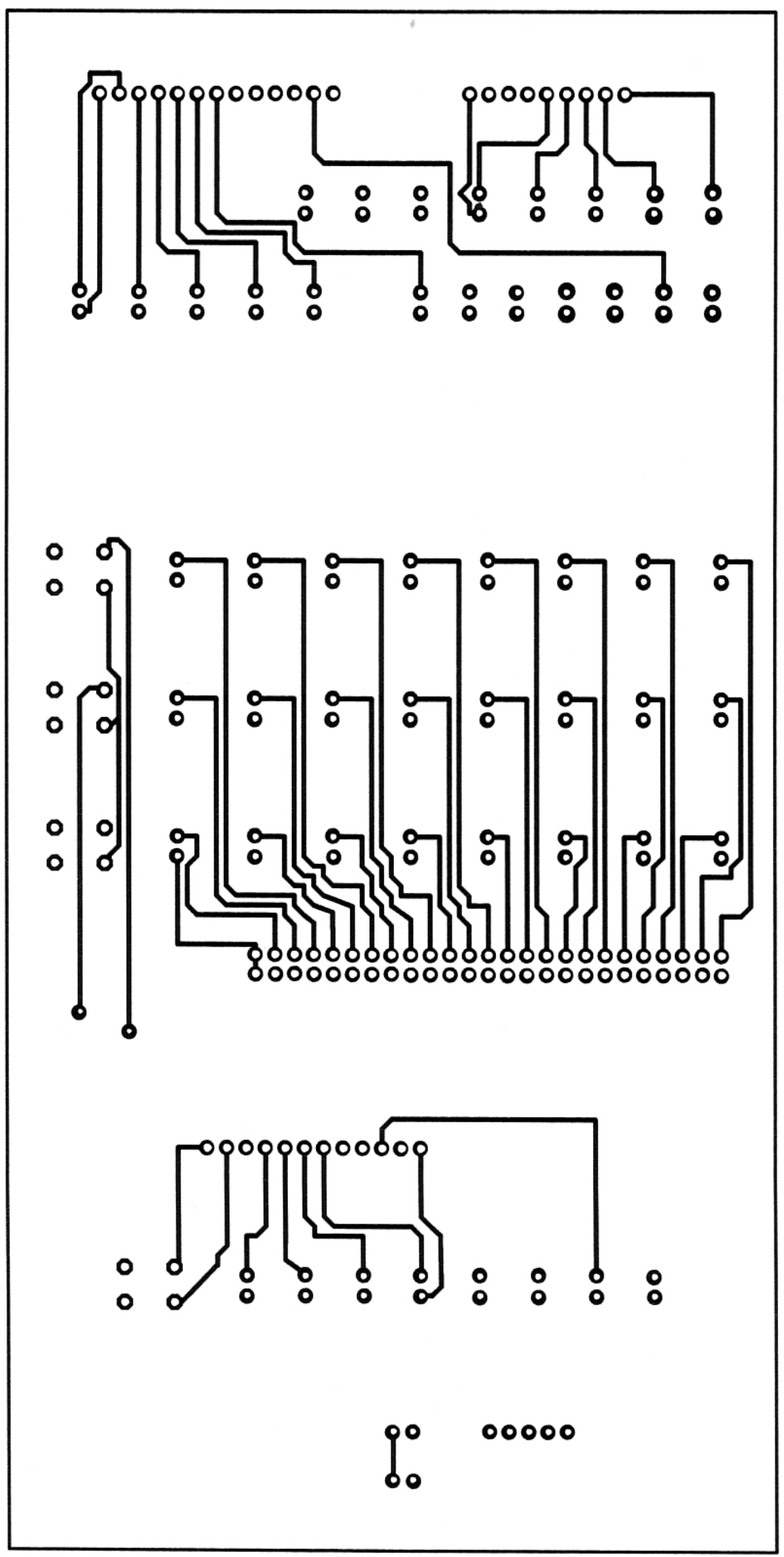


Layout der Anzeigeplatine. Seite B.

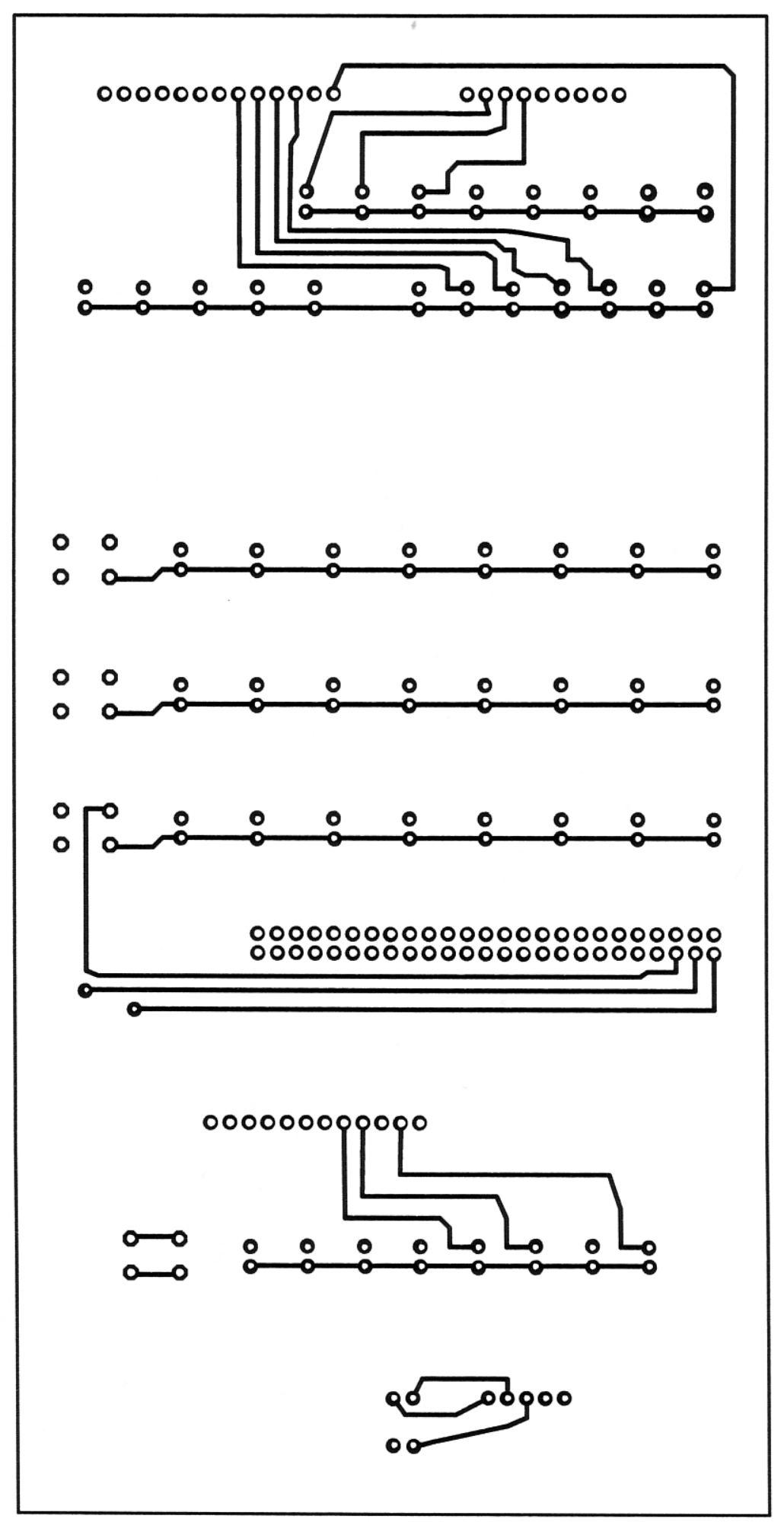



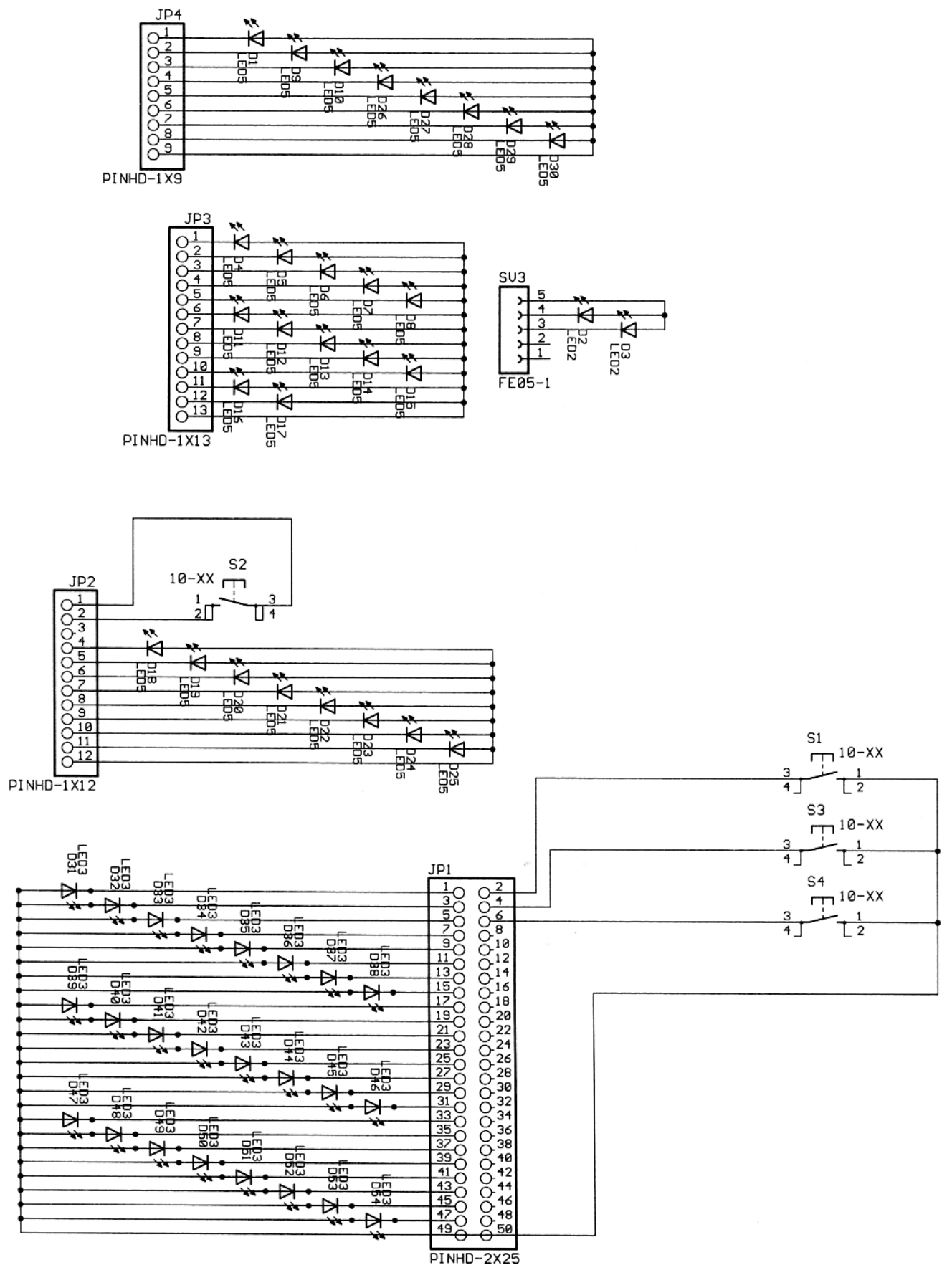

Schematische Darstellung der Platinen der Anzeige. 


\section{Literatur}

[1] U. Brunner, GA-Kongress „Ethnobotanik und Naturstoffscreening“, in: http://www.pharmazeutische-zeitung.de/pza/2000-37/pharm2.htm.

[2] A. Krüger, Berliner Heilpraktikernachrichten 1997, 4, 6.

[3] LIBRA - Verantwortungsvoller Einsatz von Antibiotika - Resistenz, Faktoren, die Resistenzen begünstigen. http://www.librainitiative.com/de/ap/re/li_ap_re_fr.html.

[4] M. Teuber, Antibiotikaresistenzen - Ausbreitung und Konsequenzen, http://www.vdbiol.de/artikel/teuber.html.

[5] Resistenzentwicklung - Probleme der Antibiotikatherapie, http://www.mww.de/pharmakologie/arzneimittel/antiifektiva/resistenz.htm.

[6] W. Lenz, N. Blümel, G. Bierbaum, B. Herding-Sotzeck, P. M. Shah, Chemother. J. 2001, 5, 174-180.

[7] M. Kresken, D. Hafner, W. Witte, R. R. Reinert, Chemother. J. 1999, 4, 136145.

[8] Zusammenhang zwischen Antibiotikaresistenz und Tiermast, http://www.ekoline.de/mednews/n2005d.htm.

[9] Stumpfe Waffen: 60 Jahre nach Einführung der Antibiotika sind immer mehr Erreger resistent, http://www.uni-heidelberg.de/presse/news/2210stumpf.html.

[10] BUKO Pharma-Brief Nr. 7, Oktober 1998, http://www.epo.de/bukopharma/phbf9807.htm.

[11] C. Werckenthin, S. Schwarz, Skriptum zum Vortrag: Molekulare Grundlagen der Resistenzentwicklung dargestellt an den Beispielen Florfenicilresistenz bei Escherichia coli und Resistenz gegenüber Makroliden, Lincosamiden und Streptogramin B-Antibiotika bei Staphylococcus spp., bei der: 20. AVIDTagung, Kloster Banz, 26.-28. September 2001.

[12] I. Klare, Übertragung von resistenten Bakterien und Resistenzgenen zwischen Masttier und Mensch, http://www.antiinfectivesintelligence.de/honnef2001/klare.htm. 
[13] K. Ohlsen, Bedeutung niedriger Antibiotikakonzentrationen in der Umwelt, http://www.antiinfectives-intelligence.de/honnef2001/ohlsen.htm.

[14] H. Tschäpe, Populationsstruktur und Resistenzentwicklung, http://www.antiinfectives-intelligence.de/honnef2001/tschaepe.htm.

[15] LIBRA - Verantwortungsvoller Einsatz von Antibiotika - Resistenzen überwinden, http://www.librainitiative.com/de/ap/or/li_ap_or.html.

[16] LIBRA - Verantwortungsvoller Einsatz von Antibiotika - Resistenz, http://www.librainitiative.com/de/ap/re/li ap re.html.

[17] H. Lüllmann, K. Mohr, Pharmakologie und Toxikologie, Thieme Verlag, New York, 1999.

[18] U. Flückiger, Schweiz. Med. Wochenschr. 1999, 129, 2005-9.

[19] W. Schunack, Erreger unterschätzt, auf: http://www.pharmazeutischezeitung.de/pza/201-50/pharm6.htm.

[20] LIBRA - Verantwortungsvoller Einsatz von Antibiotika - Resistenz, Folgen von Resistenz, http://www.librainitiative.com/de/ap/re/li ap_re_cr.html.

[21] P. Heisig, Neue Optionen in der Antibiotikatherapie, http://www.antiinfectivesintelligence.de/honnef2001/heisig.htm.

[22] G. Antranikian, Extremophile Mikroorganismen im praktischen Einsatz, http://www.vdbiol.de/muenster/antranikian.html.

[23] Online Archiv der TuTecH,Hamburg, Extremophile Biotechnologie, http://tutech.de/archiv/biobuero/23-25.html.

[24] D. Schröder, Diss., Univ. Göttingen, 2001.

[25] http://www.welt.de/daten/2002/01/28/0128vtr310589.htx.

[26] Wissen Online, Metall-Pyranhas im Vitrol-Fluss, http://www.g-o.de/.

[27] H. Laatsch, Meeresbiotechnologie in Deutschland: Stand und Perspektiven der Sekundärstofforschung an marinen Mikroorganismen., Abstracts eines Vortrags in Hannover, 6.10.1998, http://www.merc.de/german/services/bioprocessing/laatsch.html.

[28] U. Fittschen, Diss., Univ. Hamburg, 2001. 
[29] M. Maier, Diss., Univ. Braunschweig, 2003.

[30] J. Harms, Europäische Konferenz über marine Naturstoffe auf Schloss Elmau in: Apothekennachrichten 2002, 11.

[31] U. Hanefeld, G. Floss, H. Laatsch, J. Org. Chem. 1994, 59, 3604.

[32] P. Burkholder, R. Pfister, F. Leitz, App. Microbiol. 1966, Vol. 14, 649.

[33] M. Hüners, Diss., Univ. Braunschweig, 2002.

[34] http://science.orf.at/science/news/29662.

[35] R. Emmerich, Forscher suchen neue Arzneistoffe in Schwämmen, Mitteilungen, 1998, http://www.idw-online.de/public/pmid-8290/zeige pm.html.

[36] XIX. Internationale Projektwoche des IAAC, http://www.brg.traun.ac.at/IAAC/VIS/vortrag/pharma, CC 17.01.2003.

[37] T. Scheper, Unimagazin 2001, 3-4, Univ. Braunschweig.

[38] U. Höller, Diss., Univ. Braunschweig, 1999.

[39] G. Kirsch. Diss., Univ. Braunschweig, 2000.

[40] Presseinformationen, BRAIN AG, Wirkstoffe aus marinen Pilzen, www.brainbiotech.de.

[41] K. Schaumann, Marine Mykologie, http://www.awibremerhaven.de/Pelagic/Sections/MarineChemistry/Schaumann index-d.html.

[42] K. Torssell, Natural Product Chemistry, Apotekarsocieteten-Swedish Pharmaceutical Society, Swedish Pharmaceutical Press 1997, Stockholm, Sweden.

[43] M. Klose. Diss., Univ. München, 2000.

[44] U. Hauptmann, Diss., Univ. Gießen, 2000.

[45] H. Giese, Diss., Univ. Gießen, 1999.

[46] F. Bergter, Wachstum von Mikroorganismen - Experimente und Modelle, Verlag Chemie, Weinheim, 1983.

[47] Labor Zellkulturtechnik, http://www.zellkultur.fh-aachen.de/fermentation.htm.

[48] G. König, Kultivierung und Aufarbeitung mariner Bakterien, Arbeitsbericht, 1998, http://www.tu-bs.de/institute/pharm.biol/GAWK.html. 
[49] T. Stonier, Information und die innere Struktur des Universums, Springer Verlag Berlin, 1991.

[50] I. Hosp, Evolution, Entwicklung und Organisation in der Natur, Rowohlt Taschenbuch Verlag GmbH, Reinbek bei Hamburg, 1994.

[51] L. M. Rocha, Form Artifical Life to Semiotic Agent Models, Review and Research Directions, 1999, Los Alamos National Laboratory Technical Report LA-UR-99-5475, http://www.lanl.gov/la-pubs/00460075.pdf

[52] V. Nissen, Einführung in Evolutionäre Algorithmen, Friedrich Vieweg \& Sohn Verlagsgesellschaft mbH, 1997.

[53] B. Goodwin, Der Leopard, der seine Flecken verliert - Evolution und Komplexität, Piper Verlag GmbH, München, 1997.

[54] M. Gerhardt, H. Schuster, Das digitale Universum, Zelluläre Automaten als Modelle der Natur, Vieweg Verlag Braunschweig, 1995.

[55] H. Reevers, Schmetterlinge und Galaxien, dtv Verlag München, 1994.

[56] F. Kursawe, Diss., Univ. Dortmund, 1999.

[57] R. Merkl, S. Waack, Bioinformatik Aktiv-Algorithmen und Praxis, Wiley-VCH, Weinheim, 2003.

[58] P. Coveney, R. Highfield, Frontiers of Complexity- The Search for Order in a Chaotic World, R. Highfield Verlag, 1995.

[59] S. Adolf, Genetische Algorithmen und Evolutionsstrategien, THF, Berlin 2001, http://www.stadolf.de.

[60] D. McNeill, P. Freiberger, Fuzzy Logic - die "unscharfe" Logik erobert die Technik, Knaur Verlag München, 1994.

[61] J. Albertz, Evolution und Evolutionsstrategien in Biologie, Technik und Gesellschaft, Wiesbaden, 1989.

[62] T. Bäck, Evolutionary Algorithms in Theory and Practice, New York-Oxword, 1996.

[63] Genetische Algorithmen, Evolutionsstrategie, http://www.wachtler.de. 
[64] Genetische Algorithmen, Seminararbeit, 1998, http://miss.wuwien.ac.at/ mitloehn/trader/archive/9250969/dokumentation/semarbeit.htm.

[65] C. Clemens, T. Riechmann, Diskussionspapier Nr. 195, Fachbereich Wirtschaftswissenschaften der Universität Hannover, Juni 1996, S. 1-23, Evolutionäre Optimierungsverfahren und ihr Einsatz in der ökonomischen Forschung. Miteilungen der TFH Berlin, 13.07.01, S. Adolf, Genetische Algorithmen und Evolutionsstrategien, S. 1-10.

[66] M. Möllney, S. Freuyer, W. Wichert, D. Weuster-Botz, Programmdokumentation GALOP 2.2, Forschungszentrum Jülich GmbH.

[67] B. Goodwin, C. Briere, A Mathematical Model of Cytoskeletal Dynamics and Morphogenesis in Acetabularia, 1992, Boca Raton, Fla.: CRC Press, 219-238.

[68] B. Goodwin, S. Pateromichaelakis, Planta, 1979, 145, 427-435.

[69] B. Goodwin, L. Tailor, J. Theor. Biol. 1985, 117, 79-106.

[70] M. Ichiba, S. Nishigaki, K. Senga, J. Org. Chem. 1978, 43, 3.

[71] K. Tatsuta, M. Kitagawa, Heterocycles 1994, 38, 8, 1747-1750.

[72] K. Tatsuita, M. Kitagawa, J. Antibiot. 1994, 47,3, 388-390.

[73] K. Tatsuta, M. Kitagawa, Heterocycles 1994, 38, 6, 1233-1235.

[74] K. Eckardt, Chem. Ber. 1967, 100, 2561-2568.

[75] Z. Siemion, Organic Magnetic Resonance 1971, Vol. 3, 545-550.

[76] F. Huth, Diss., Univ. Göttingen, 1998.

[77] F. Ehrlich, Ber. Dtsch. Chem. Ges. 1912, 45, 883-889.

[78] A. A. Willium, M. Lois, F. Meow-Chen, O. Helena, S. Hossein, Can. J. Chem. 1996, 64, 904 .

[79] W. Ayer, Can. J. Chem. 1986, 64, 904-909.

[80] B. Cross, M. Lechevalier, H. Lechevalier, J. Chem. Soc. 1963, Part II, 29372943.

[81] M. Devys, Phytopob. Z. 1976, 85, 176-178.

[82] G. Schneider, H. Anke, O. Sterner, Z. Naturforsch. 1996, 51c, 802-806. 
[83] M. Savard, Mycopathologia, 1994, 127, 19-27.

[84] F. El-Beih, Al-Azhar, Bull. Sci. 1993, 579-592.

[85] M. Collins, J. Farrow, A. Phillips, S. Ferusu, D. Jones, FEMS Microbiol. Lett. 1987, 43, 307-312.

[86] R. Kroppenstedt, E. Grund, Int. J. Syst. Bacteriol. 1989, 39, 304-308.

[87] O. Isler, Helv. Chim. Acta 1958, 41, 786 - 807.

[88] Y. Yamada, Agric. Biol. Chem. 1980, 44, 1693; 1982, 46, 1105.

[89] R. Maskey, I. Kock, M. Shaaban, I. Grün-Wollny, E. Helmke, F. Mayer, I. Wagner-Döbler, H. Laatsch, Polymer Bull. 2002, 49, 87-93.

[90] D. Seebach, A. Brunner, H. Brüger, J. Schneider, R. Reusch, Eur. J. Biochem., 1994, 224, 317-328.

[91] CD Römpp Chemie Lexikon - Version 1.0, Stutgart/New York: Georg Thieme Verlag 1995.

[92] J. Warren, J. Shusterman, E. Nelson, The Molecular Modelling Workbook for Organic Chemistry, Wavefunction, Inc, 1998.

[93] V. Dembitsky, I. Shkrob, O. Lev, J. Chem. Ecology 2000, 6, 1359-1365.

[94] A. Barrero, J. Oltra, J. Poyatos, Phythochemistry 1996, Vol. 42, 5, 1427-1433.

[95] H. Watabe, S. Amano, J. Yoshida, Y. Takase, S. Miyadoh, T. Sasaki, M. Hatusu, Y. Takeuchi, Y. Kodama, K. Kajii, T. Shomura, M. Sezaki, Sci. Reports of Meiji Seika Kaisha, 27, 55-62, 1998.

[96] R. Maskey, Dssertation, Univ. Göttingen 2003.

[97] M. Speitling, Dissertation, Univ. Göttingen 1998.

[98] R. P. Maskey, I. Grün-Wollny, H. Laatsch, J. Antibiot. 2003 im Druck.

[99] B. E. Leach, K. Colhoun, L. Johnson, C. Teeters, W. Jackson, J. Am. Chem. Soc. 1953, 75, 4011-4012.

[100] E. Simonitsch, W. Eisenhuth, O. Stamm, H. Schmid, Helv. Chim. Acta, 1960, $43,58-64$. 
[101] E. Simonitsch, W. Eisenhuth, O. Stamm, H. Schmid, Helv. Chim. Acta, 1964, 47, 1459-1484.

[102] K. Sugawara, M. Tsunakawa, M. Konishi, H. Kwaguchi, H. Cun-heng, J. Clardy., J. Org. Chem. 1987, 52, 996-1000.

[103] J. Beisler, Prog. Med. Chem., 1982, 19, 247-268.

[104] K. Kon, H. Sugi, K. Tamai, Y. Ueda, N. Yamada, J. Antibiot., 1990, 43, 372382.

[105] P. Cahnham, L. Vining, A. McInnes, J. Walter, J. Wright, J. Chem. Soc. Chem. Commun. 1976, 67, 319-320.

[106] P. Cahnham, L. Vining, A. McInnes, J. Walter, J. Wright., Can. J. Chem. 1977, $55,2450-2457$.

[107] K. Kojiri, S. Nakajima, H. Suzuki, A. Okura, H. Suda, J. Antibiot. 1993, Vol. 46, No. $12,1799-1803$.

[108] K. Kojiri, S. Nakajima, H. Suda, J. Antibiot. 1993, Vol. 46, No. 12, 1993-1996. 


\section{Danksagung}

Sich bei jemandem zu bedanken bedeutet manchmal gleichzeitig vom jemanden Abschied zu nehmen. Auch in diesem Fall geht eine bestimmte Konfiguration des Lebens unausweichlich zu Ende. Nun greife ich die letzte Gelegenheit auf, um all diesen Menschen mit den ich gearbeitet habe und von den ich so viel gelernt habe, „bye“ zu sagen. Ein wenig überrascht bin ich doch, denn so viel Hilfsbereitschaft, sei es bei wissenschaftlichen Diskussionen oder bei der Lösung technischer Probleme habe ich nicht erwartet.

Der Mechanischen Werkstatt danke ich vor allem für die Geduld mit mir sowie bei der Herstellung diverser, bescheuerten Maschinenteile und das Fachwissen, das ich von diesen Leuten erworben habe. Stets bereit waren die Menschen, sich Gedanken zu machen und sie mit mir zu teilen, wie man eine Idee noch besser und praktischer umsetzen kann.

Bei der Elektrowerkstatt bedanke ich mich ebenfalls herzlich für die Unterstützung bei den nicht so ganz ungefährlichen Spielereien mit dem Strom und Lötkolben.

Nun ist es an der Zeit die Menschen zu erwähnen, die ständig im Hintergrund versteckt, aber doch aktiv an der Entstehung der Arbeit beteiligt waren. Fleißig nahmen sie meine Spektren auf, druckten aus und diskutierten sie mit mir durch. Der ganzen NMR- und MS-Abteilung danke ich für die hervorragende Dienstleistung und Verständnis bei nicht immer messbaren Größen.

Bei dieser Reise durch das Institut komme ich schließlich zu den Menschen, die tagtäglich dazu beitrugen, dass die Arbeit in einer angenehmen und sauberen Umgebung verlief. Der Abteilung danke ich für die nette Atmosphäre und den duftenden Kaffee.

Felix, Michael, Thomas und Jörg waren quasi dabei, als ich meine ersten, selbstständigen Schritte im Labor gemacht habe. Björn, Nizar und Dirks waren dabei, als ich dann vernünftig zu gehen lernte. Ahja.. .bei der netten Cafeteriaunterhaltung waren sie auch doch dabei.

Zum Schluss bleibt es nur noch sich bei den Menschen zu bedanken, die mich im privaten Leben unterstützt haben. Eva - danke Dir, dass es dich gibt. Meinen Eltern danke ich ebenfalls, weil sie es mir ermöglicht haben nicht nur diesen Text zu 
schreiben aber überhaupt Texte zu schreiben. Falls ich jemanden vergessen sollte, tut es mir Leid....bin eben so ein wenig chaotisch in den letzten Tagen....Sorry.

Diesen Menschen widme ich ebenfalls diese Arbeit.

Nun die vielen Namen.....

Dr. Eva Kansy, Dr. Björn Torp-Patragst, Dr. Nizar El-Hachach, Dr. Dirk Schröder. Dr. T. Schrapel, Dr. F. Huth, D. Fechner, Dipl. Pharm. K. Vossler, Dipl. Geol. F. Lissy. Dr. R. Maskey, K. Vogel, S. Höfner, Dr. R. Asolkar, Dr. P. Monecke, Frau Lackner, A. Godawa, U. Leonard, Dipl. Chem. R. Machinek, G. Udvarnoki, Dr. G. Remberg, Dr. H. Frauendorf. Dr. E. Helmke, Dr. I. Wagner-Döbler, F. Brandes, H. Renziehausen, T. Nguyen, U. Henschke, Ing. K. Jung, K. Manegold, M. Pohl, liebe Ewa Bialkowski, Dipl. Kauf. L. Szlesak, R. Stoppa. 